\title{
ROLE OVERLOAD APPRAISAL and COPING: A Case Study of Female Health Care Workers
}

\author{
by \\ Margaret Stevenson, M.B.A.
}

A thesis submitted to the Faculty of Graduate Studies and Postdoctoral Affairs in partial fulfillment of the requirements for the degree of

\author{
Doctor of Philosophy \\ in \\ Management \\ Carleton University \\ Ottawa, Ontario \\ (C) 2014 \\ Margaret Stevenson
}




\begin{abstract}
Role overload is an important form of role stress that can overwhelm individuals and has been linked to a wide range of physical and psychological symptoms of stress. Research on role overload has, however, been fragmented and there has been little attempt to develop the associated theory. Inconsistent results linking role overload and perceived stress and a growing interest in positive and negative outcomes from stressful encounters suggest a different approach is warranted to examine how and why stress should arise from mismatching role expectations and how and why it might vary.

This exploratory case study applies qualitative methodology to examine the relationship between role overload and perceived stress. Using stress, appraisal and coping theory (Lazarus and Folkman, 1984) as a guiding lens, this study seeks to model the process and the contextual conditions that contribute to variable stress reactions to role overload at work and at home. Semi-structured interviews were conducted with twenty female health care workers from four hospitals in a major city. The interviews explored the nature of two contrasting role overload experiences recalled by these individuals.

The findings from this research suggest that whilst the role overload appraisal and coping process maps closely to the cognitive stress model of Lazarus and Folkman (1984), important areas of difference also exist. The differences include situation properties that are specific and unique to role overload; an internal role pressure that plays an important part in the perception of role overload; modified interpretations of challenge and threat appraisals; a focus on demand reduction versus demand management coping strategies; coping strategies specific to role overload; and four distinct types of role overload episodes.

The case study concludes with the development of a framework of the role overload appraisal and coping process. The framework includes a taxonomy of role overload situation properties and a typology of role overload coping strategies, Finally, the study identified factors that distinguish overwhelming from not-overwhelming episodes of role overload, as well as the factors that distinguish the role overload episodes described by individuals with high levels of stress from those described by people with low stress levels.
\end{abstract}




\section{ACKOWLEDGEMENTS}

This thesis is dedicated to my parents, Roy and Mary Stevenson

This thesis could not have been completed without the support and encouragement of a large number of people. First, I would like to express my sincere gratitude to the health care workers who took the time from their busy schedules to share their experiences of role overload with me.

I would particularly like to thank my supervisor, Dr. Linda Duxbury for her insightful guidance throughout my $\mathrm{PhD}$. Her energy and enthusiasm are inspirational and I could not have imagined having a better mentor for my $\mathrm{PhD}$ studies. I would also like to thank the internal members of my doctoral dissertation committee, Dr. Steven Murphy and Dr. Greg Sears, for their advice, feedback, and their willingness to answer my questions. Thanks also to my external examiner Dr. Rekha Karambayya for her thorough review of this dissertation.

I received a lot of support from my fellow $\mathrm{PhD}$ candidates throughout my studies and I would like to mention two in particular. Dr. Rob Smart worked with me on a number of research projects. These would not have been as successful or as enjoyable without his input. Throughout my studies, my dear friend Dr. Aareni Uruthirapathy was also there in good times and bad to give me the benefit of her wise words. Thanks to both of you.

Thanks go to to my friends and family, in Canada, USA and the UK, whose friendship, love and support kept me going throughout my PhD. The person I must thank the most, however, is my husband, Tom. He endured many hours alone whilst I worked on my dissertation but was always there for me with his unwavering encouragement, patience, love and support. I could not have completed this journey without Tom by my side. 


\section{TABLE of CONTENTS}

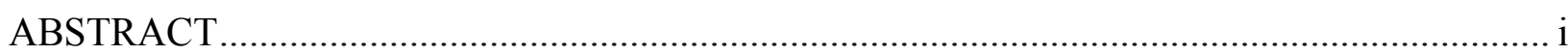

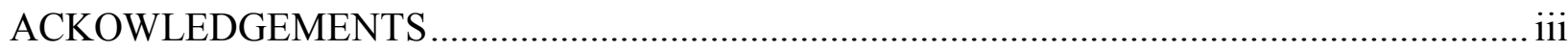

TABLE of CONTENTS ………………......................................................................... iv

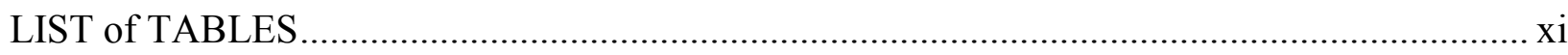

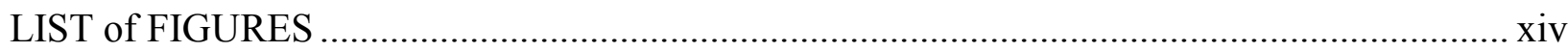

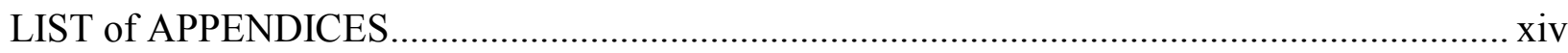

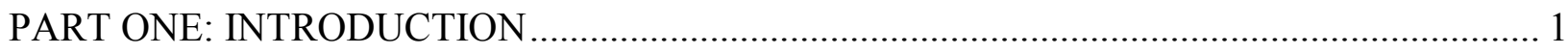

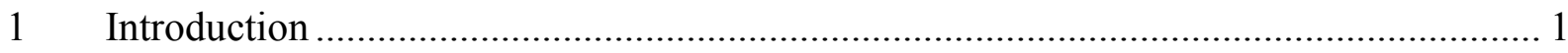

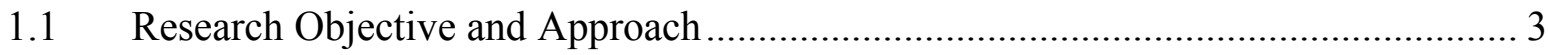

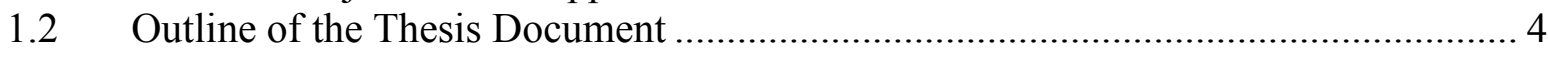

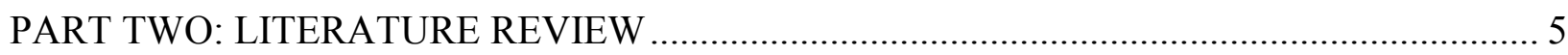

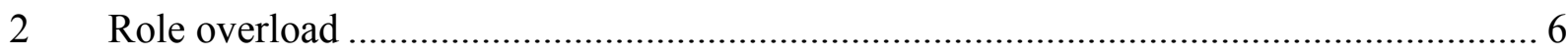

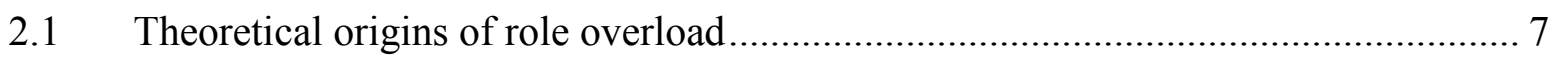

2.1.1 Developments in role theory ...................................................................... 8

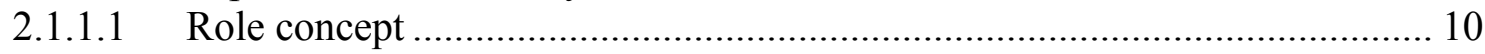

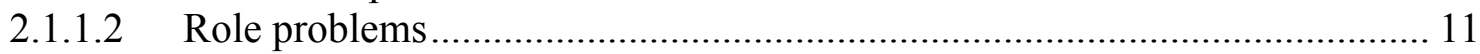

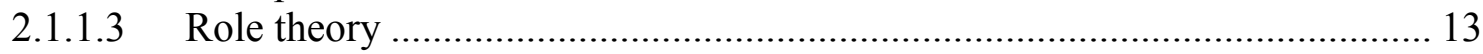

2.1.2 Organizational role theory ........................................................................ 15

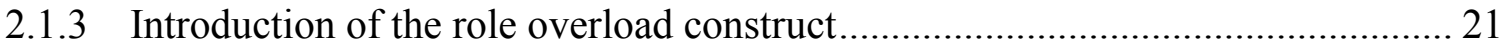

2.1.3.1 Role overload and role conflict formulation................................................. 23

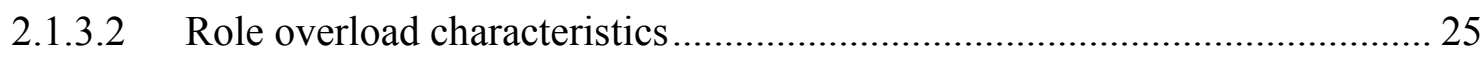

2.1.3.3 Role overload and role ambiguity ................................................................ 30

2.1.4 Critique and implications for the current research............................................... 31

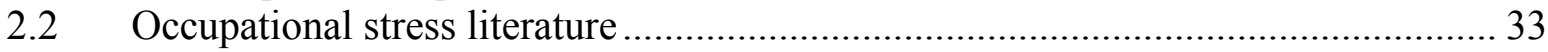

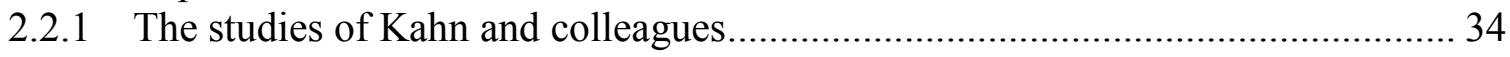

2.2.2 Role overload - the forgotten work role stress construct …………………......... 37

2.2.3 Role overload as an independent work stressor .................................................. 44

2.2.4 Critique and implications for the current research............................................ 48

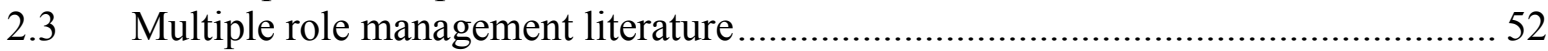

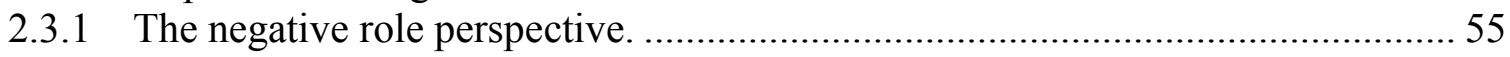

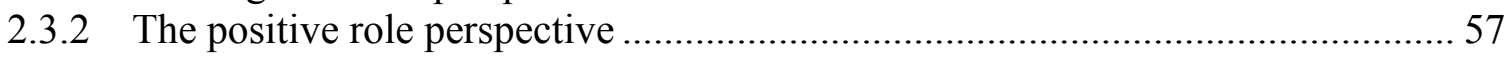

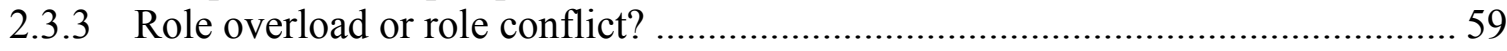




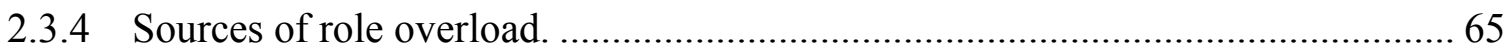

2.3.5 Critique and implications for the current research.............................................. 69

2.4 Summary of the role overload literature review .................................................... 74

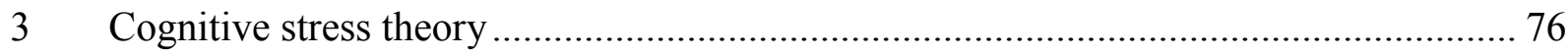

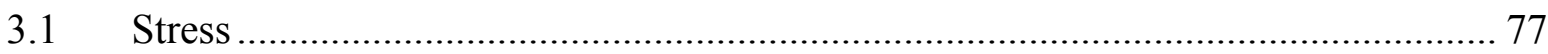

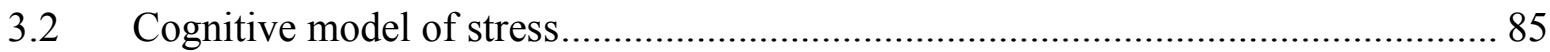

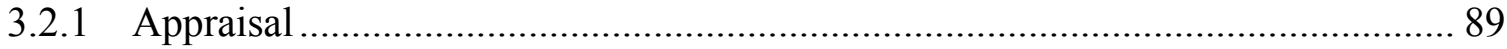

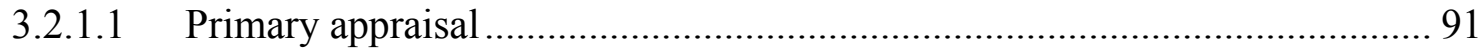

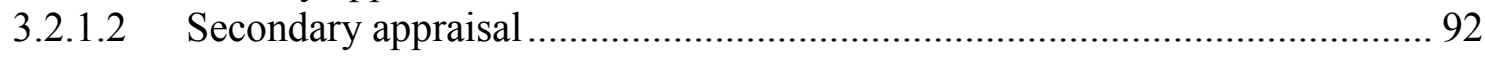

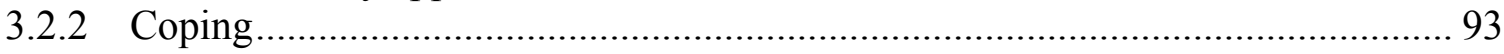

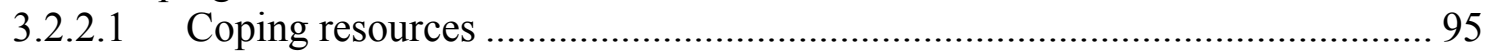

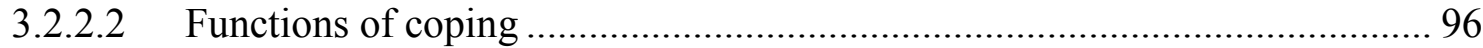

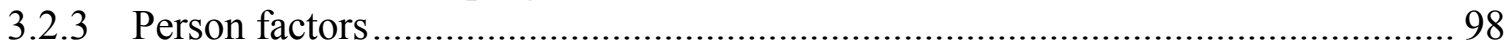

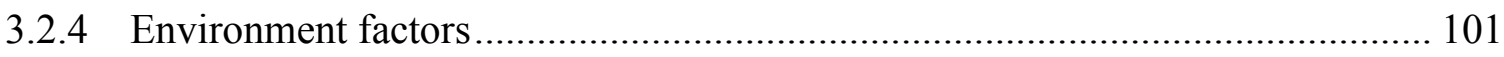

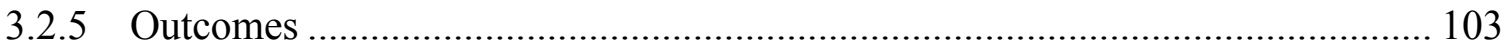

3.3 Strengths and weaknesses of the cognitive model of stress ................................... 105

3.3.1 Key benefits of the cognitive model of stress .................................................. 106

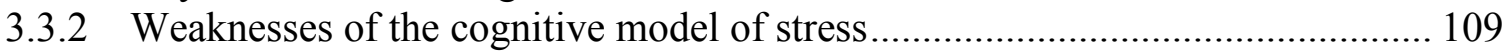

3.4 Implications for current research .................................................................. 110

PART THREE: PRELIMINARY CONCEPTUAL FRAMEWORK and RESEARCH

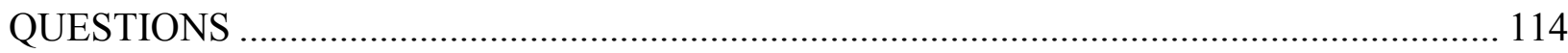

4 Conceptual framework and research questions ......................................................... 115

$4.1 \quad$ Conceptual framework .................................................................................. 115

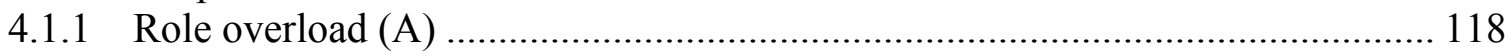

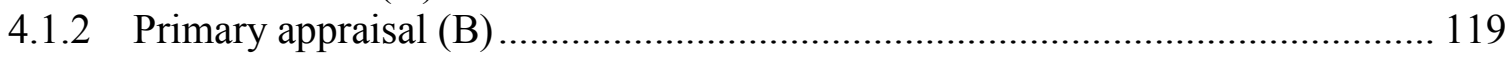

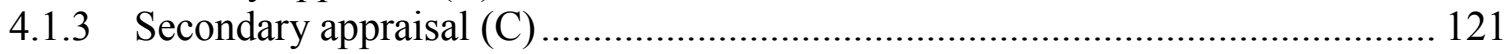

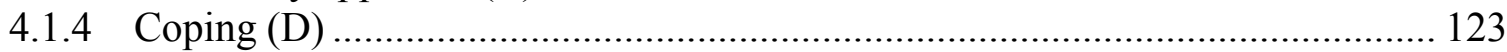

4.1.5 Immediate outcomes (E) and perceived stress (F) ......................................... 126

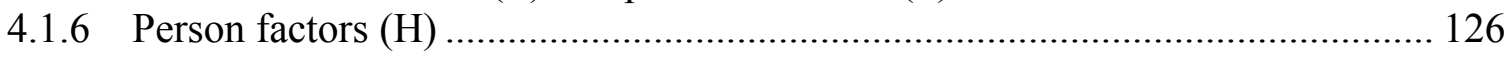

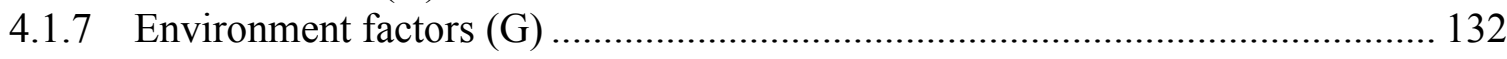

$4.2 \quad$ Research questions .......................................................................................... 133

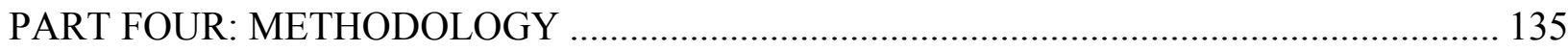

$5 \quad$ Building theory from a case study ........................................................................ 138

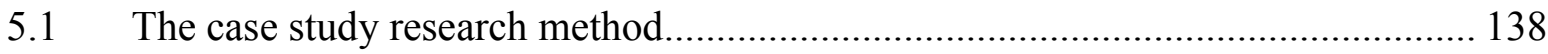

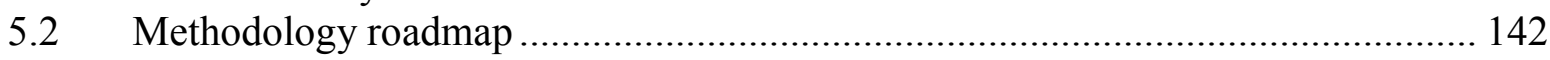

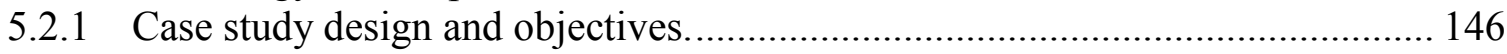

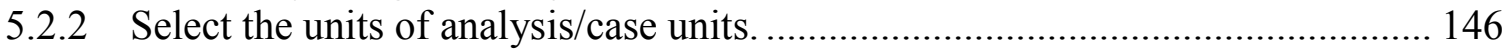

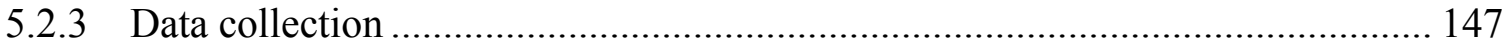

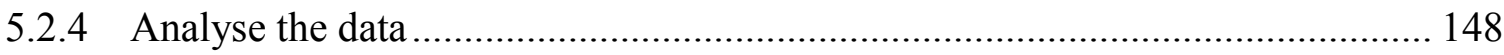




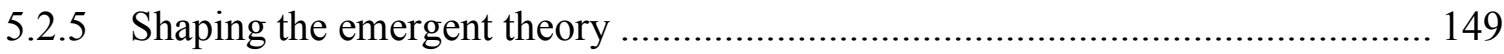

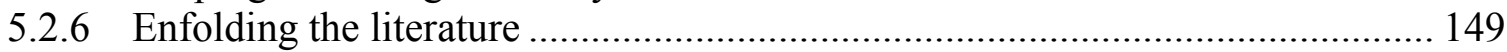

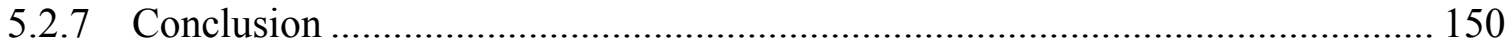

6 Case study setting, units of analysis and sample selection ........................................ 151

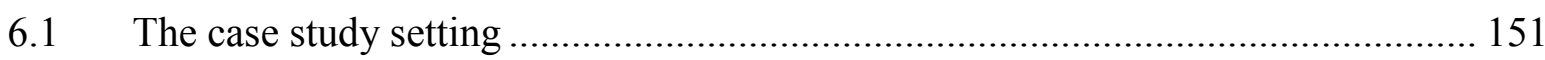

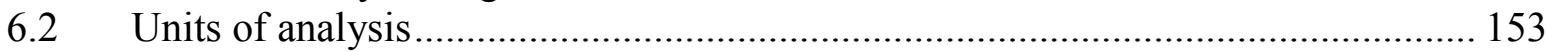

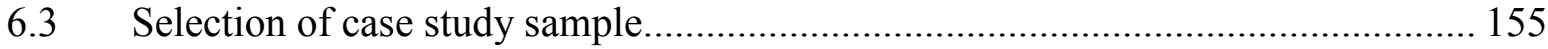

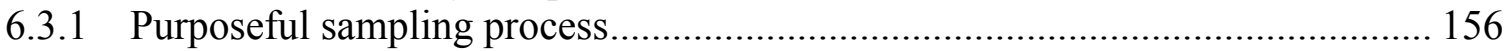

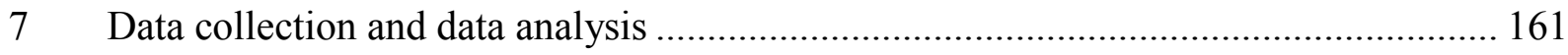

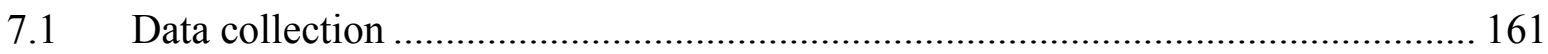

7.1.1 Qualitative data collection - Interview protocol ............................................... 162

7.1.1.1 Part I - Background information............................................................... 165

7.1.1.2 Part II - Role overload scenarios ............................................................. 166

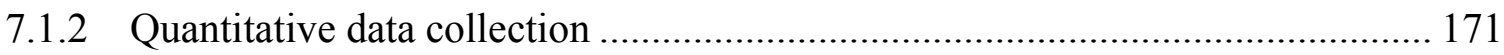

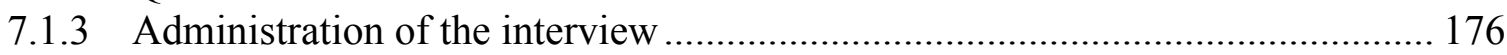

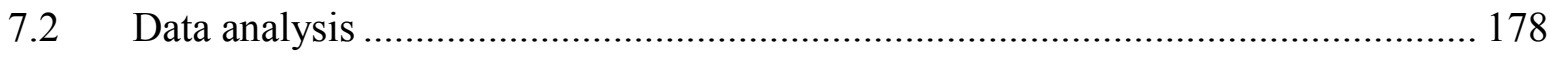

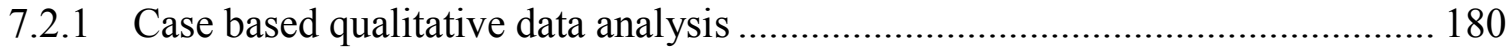

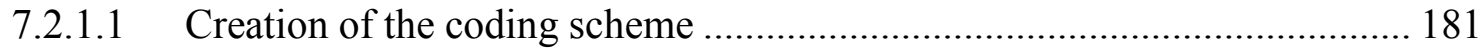

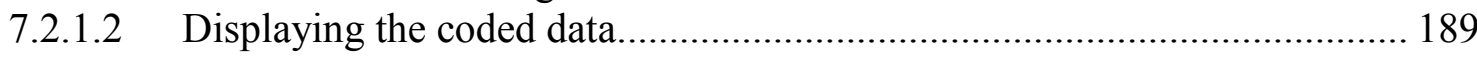

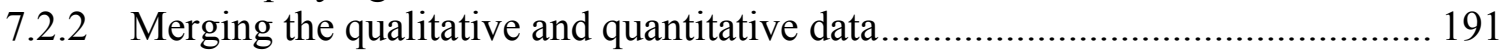

7.2.3 Interpreting the results .................................................................................... 193

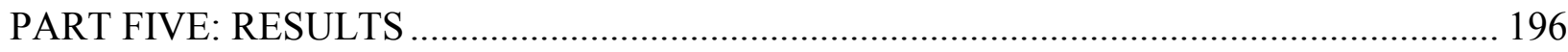

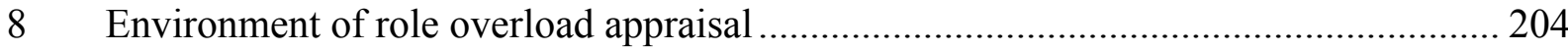

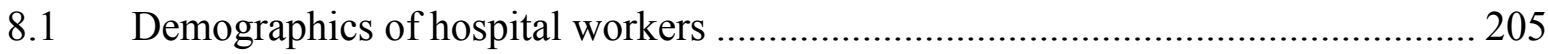

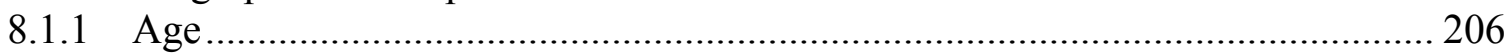

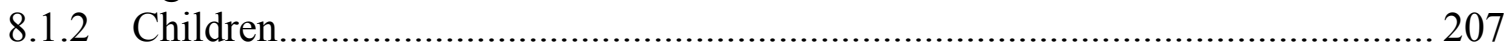

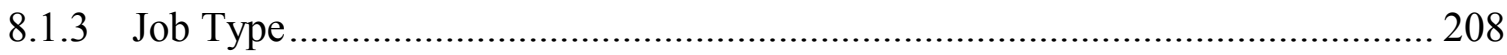

8.2 Event types triggering role overload ..................................................................... 209

8.2.1 Descriptions of event types triggering role overload......................................... 209

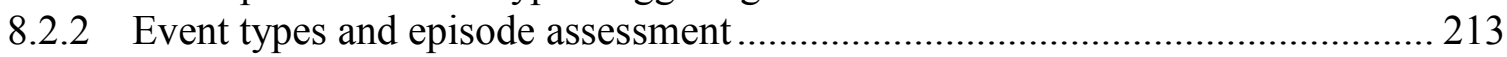

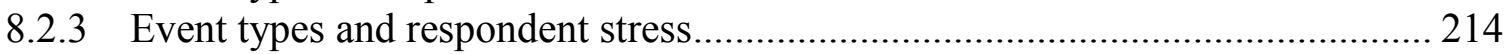

8.2.4 Event types by respondent stress and episode assessment................................ 215

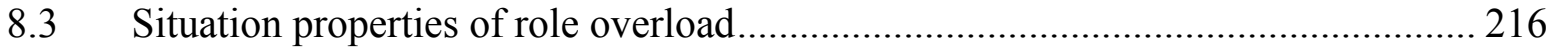

8.3.1 Descriptions of role overload situation properties .......................................... 216

8.3.2 Situation properties and episode assessment ………...................................... 225

8.3.3 Situation properties and respondent stress ...................................................... 226

8.3.4 Situation properties by respondent stress and episode assessment .................... 227

8.4 Other role factors in the role overload environment............................................. 228

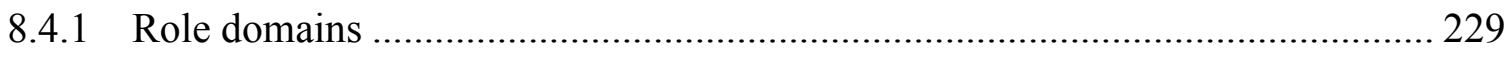

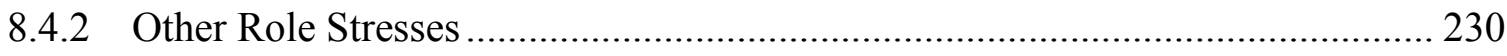




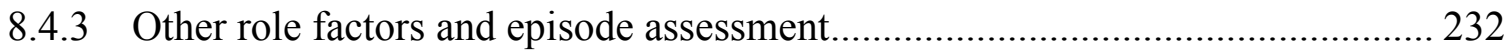

8.4.4 Other role factors and respondent stress ....................................................... 233

8.4.5 Other role factors by respondent stress and episode assessment ...................... 234

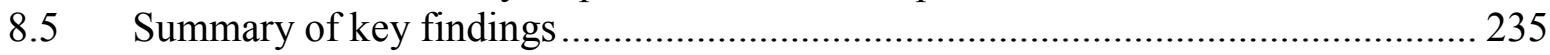

8.5.1 Commonality of findings for the role overload environment ........................... 236

8.5.2 Key environment findings by role overload episode assessment ....................... 238

8.5.3 Key role overload environment findings by respondent stress level ................. 240

8.5.4 Key role overload environment findings in episode groupings ......................... 242

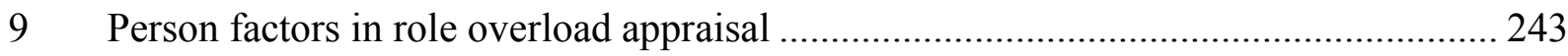

9.1 Person factors cited in role overload recollections ............................................... 243

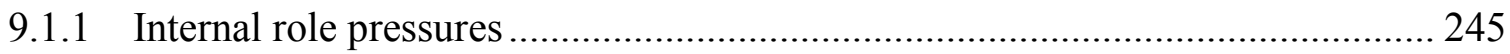

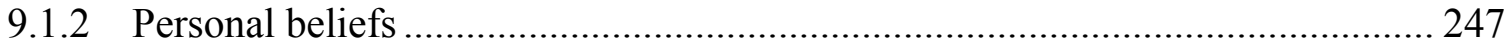

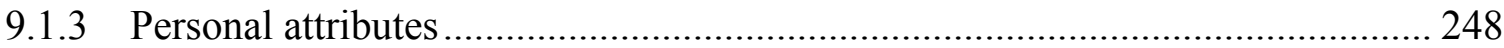

9.1.4 Person factors and episode assessment ........................................................ 248

9.1.5 Person factors and respondent stress level...................................................... 249

9.1.6 Person factors by respondent stress and episode assessment.............................. 250

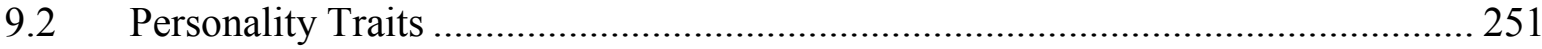

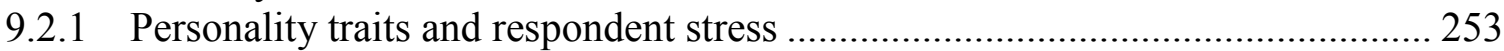

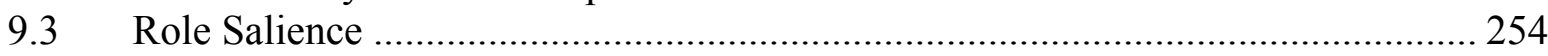

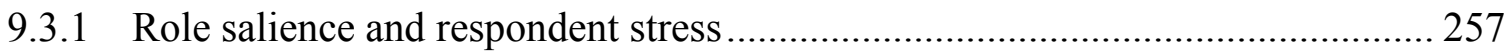

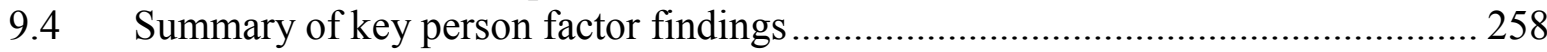

9.4.1 Key person factor findings for all episodes ................................................... 258

9.4.2 Key person factor findings by role overload episode assessment....................... 259

9.4.3 Key person factor findings for respondents with low and high

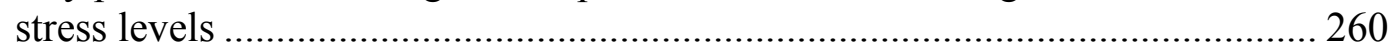

9.4.4 Key person factor findings by episode groupings............................................. 261

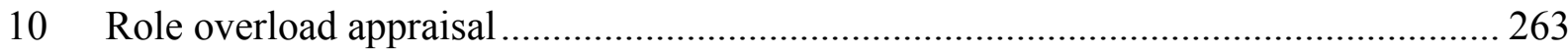

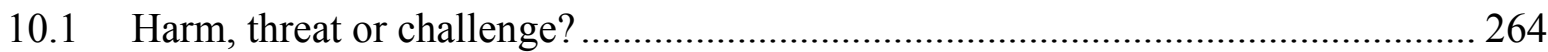

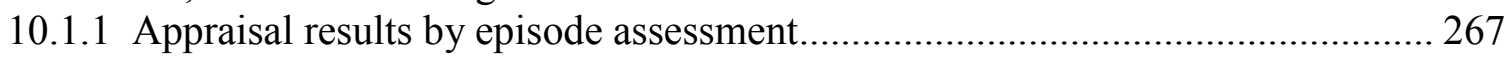

10.1.2 Appraisal results by respondent stress ......................................................... 267

10.1.3 Appraisal results by respondent stress and episode assessment ........................ 268

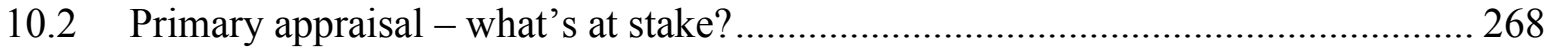

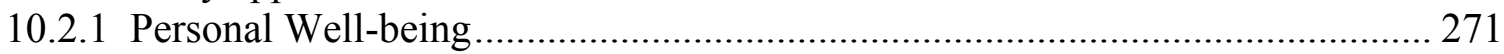

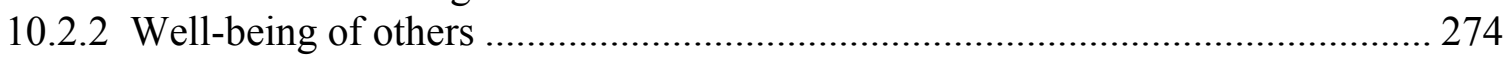

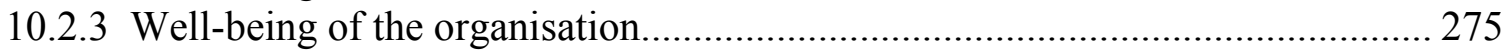

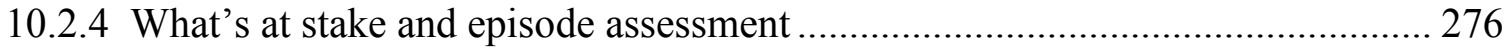

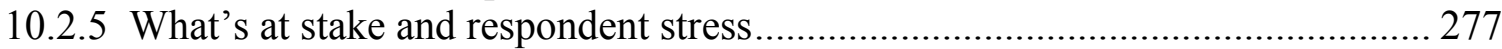

10.2.6 What's at stake by respondent stress level and episode assessment................... 278

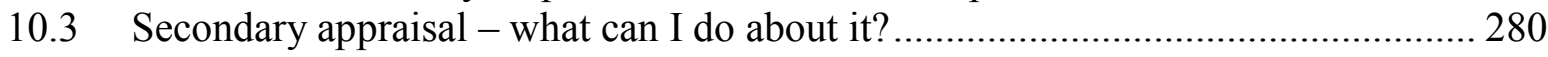

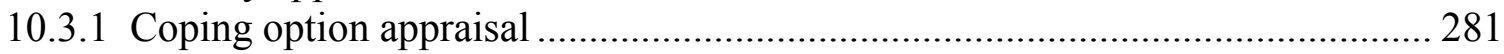

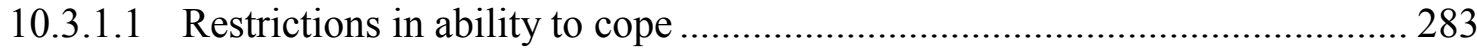

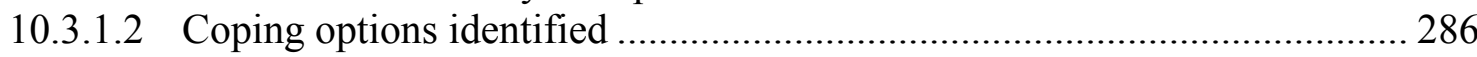




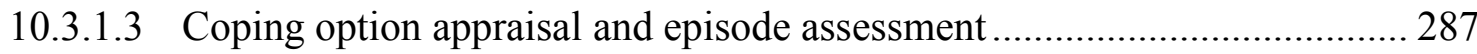

10.3.1.4 Coping option appraisal and respondent stress.......................................... 288

10.3.1.5 Coping option appraisal by respondent stress and episode

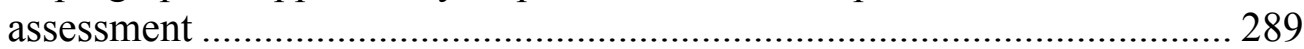

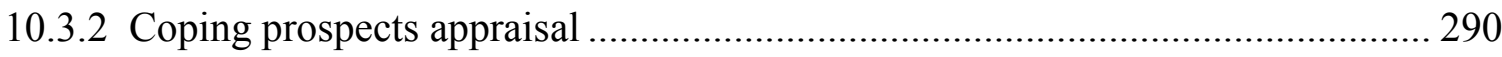

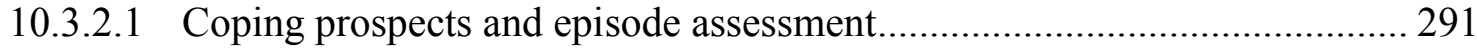

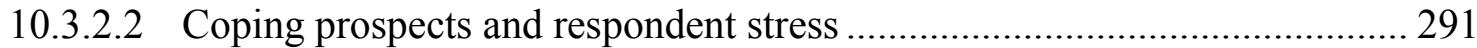

10.3.2.3 Coping prospects by episode assessment and respondent stress ................ 292

10.4 Emotional outcomes from role overload appraisal ................................................. 293

10.4.1 Appraisal emotional outcomes and episode assessment .................................... 296

10.4.2 Appraisal emotional outcomes and respondent stress ……………………........ 297

10.4.3 Appraisal emotional outcomes by respondent stress and episode

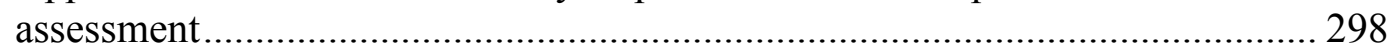

10.5 Summary of role overload appraisal key findings ................................................ 299

10.5.1 Key appraisal findings for all episodes ................................................................ 300

10.5.2 Key appraisal findings by role overload episode assessment ............................. 301

10.5.3 Key appraisal findings for respondents with low and high stress

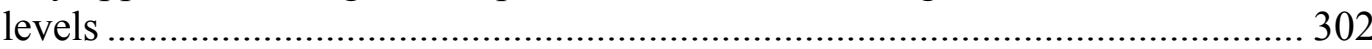

10.5.4 Key appraisal findings by episode groupings ………………………………..... 303

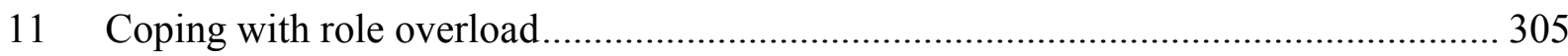

11.1 Coping Strategies for Role Overload Episodes....................................................... 306

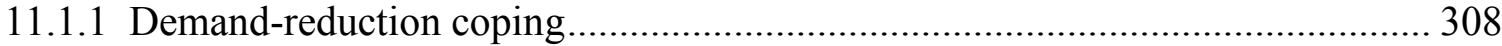

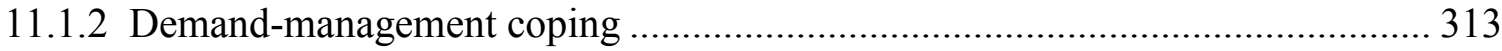

11.1.3 Role overload coping strategies and episode assessment ...................................319

11.1.4 Role overload coping strategies and respondent stress ......................................... 319

11.1.5 Role overload coping strategies by respondent stress and episode

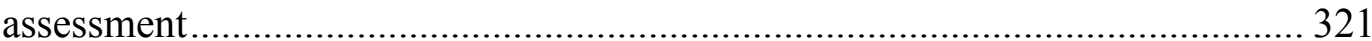

11.2 Summary of key findings for coping with role overload ......................................... 323

11.2.1 Key role overload coping strategy findings for all epsiodes................................ 324

11.2.2 Key coping strategy findings by role overload episode assessment .................... 325

11.2.3 Key coping strategy findings for respondents with low and high

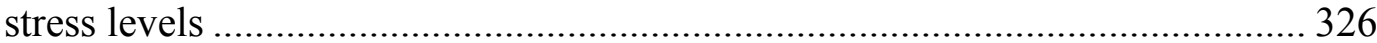

11.2.4 Key coping strategy findings by episode groupings ......................................... 327

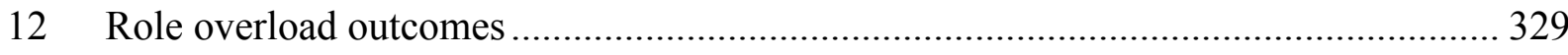

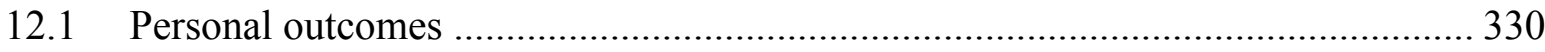

12.1.1 Positive personal outcomes........................................................................... 330

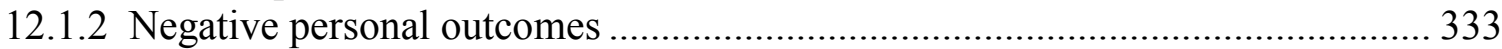

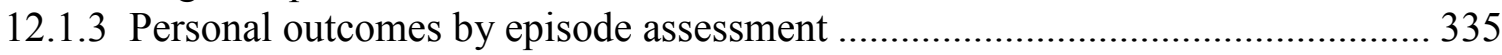

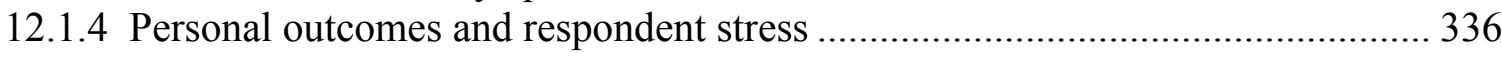

12.1.5 Personal outcomes by respondent stress level and episode

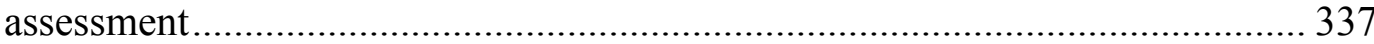

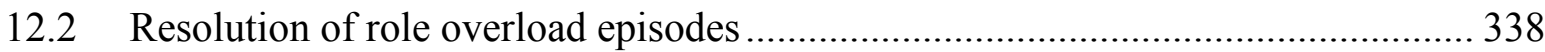




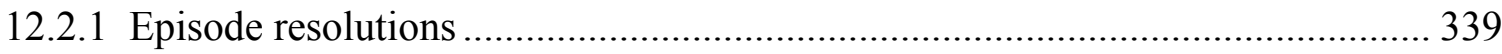

12.2.2 Episode resolution and episode assessment..................................................... 340

12.2.3 Episode resolution and respondent stress ........................................................ 341

12.3 Summary of key findings for role overload outcomes.......................................... 341

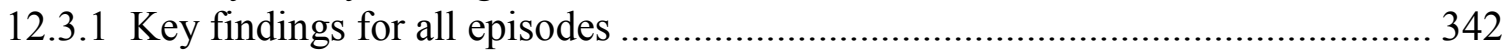

12.3.2 Key role overload outcome findings by episode assessment.............................. 343

12.3.3 Key role overload outcome findings by respondent stress level......................... 344

12.3.4 Key findings by episode grouping …………………….................................... 345

PART SIX: DISCUSSION and REFINEMENT of CONCEPTUAL FRAMEWORK .............. 346

13 Development of a conceptual framework to explain the relationship

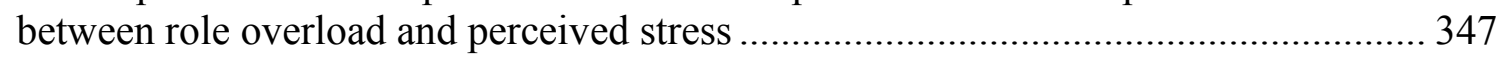

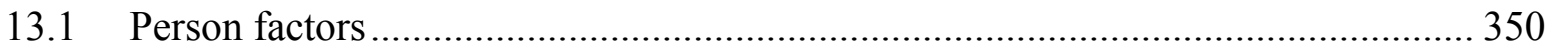

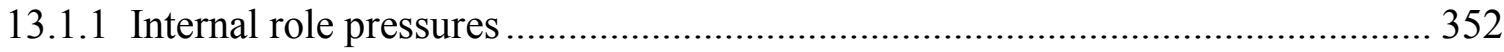

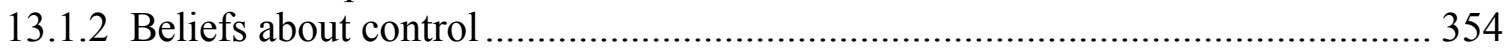

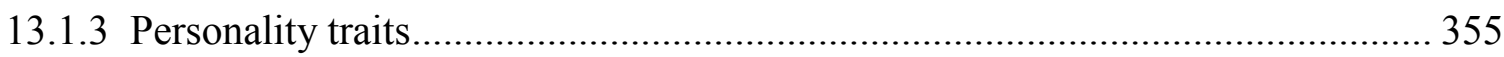

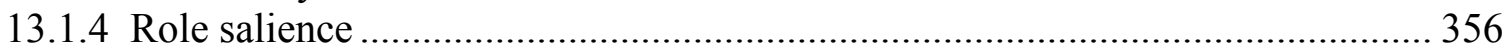

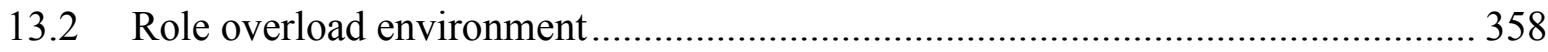

13.2.1 Role overload properties consistent with situation properties

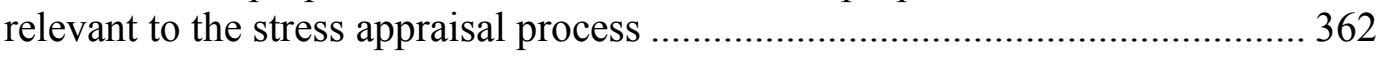

13.2.2 Role overload properties sharing some characteristics with situation properties influential in the stress appraisal process ..............................366

13.2.3 Unique role overload properties described as influential in the

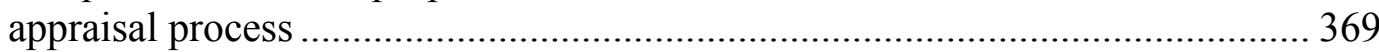

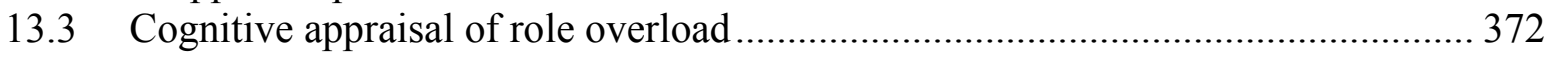

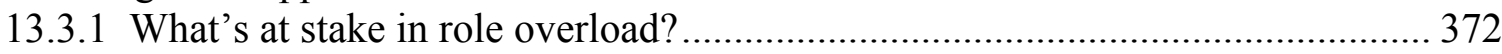

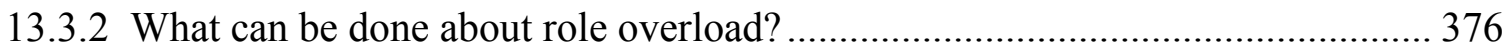

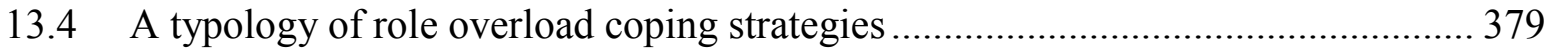

13.4.1 Dimensions of the role overload coping strategy typology ................................ 380

13.4.2 Coping strategies of the role overload coping typology ..................................... 384

13.4.2.1 Problem-focused role overload coping strategies used to

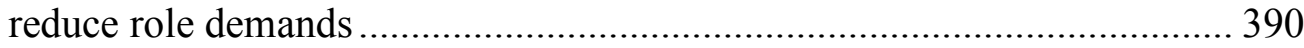

13.4.2.2 Emotion focused role overload coping strategies used to

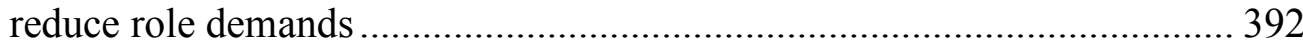

13.4.2.3 Problem focused role overload coping strategies focused on

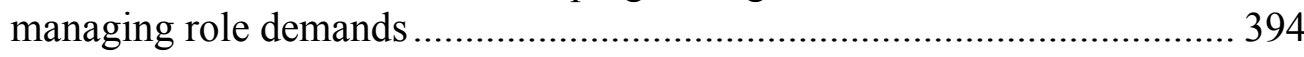

13.4.2.4 Emotion focused role overload coping strategies focused on

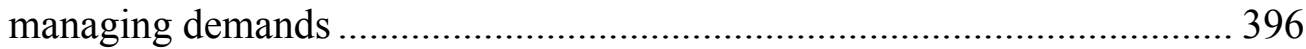

13.5 Outcomes from the role overload appraisal and coping process ........................... 399

13.5.1 A typology of emotions and feelings associated with role overload appraisal and role overload episode resolutions 
14 Why do some overloaded workers experience greater levels of stress than others?

14.1 Underlying factors of the role overload appraisal and coping process .................... 406

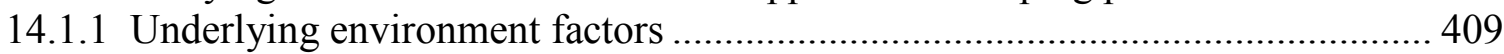

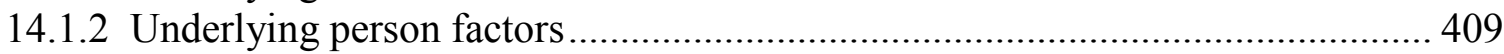

14.1.3 Underlying primary and secondary appraisal factors …………....................... 410

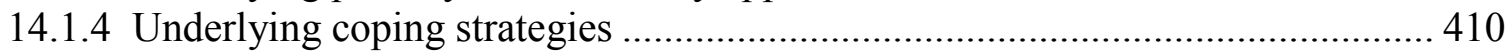

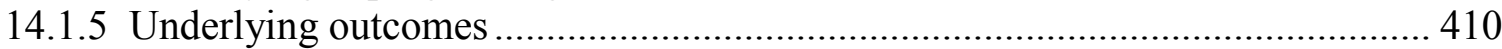

14.2 Role overload appraisal and coping by episode assessment and by respondent stress

14.2.1 Not-overwhelming role overload episodes described by Anna, a hospital worker who reported low levels of stress.

14.2.2 Overwhelming role overload described by Beth, a hospital worker with low levels of stress

14.2.3 Not-overwhelming episodes described by Cate, a hospital worker who reported high levels of stress.

14.2.4 Overwhelming role overload episodes described by Dawn, a hospital worker with high levels of stress.

14.3 What distinguishes role overload appraisal and coping in notoverwhelming episodes from that in overwhelming episodes.

14.3.1 Environment factors of not-overwhelming and overwhelming role overload

14.3.2 Person factors of not-overwhelming and overwhelming role overload.

14.3.3 Primary and secondary appraisal in not-overwhelming and overwhelming role overload

14.3.4 Coping strategies in not-overwhelming and overwhelming role overload.....

14.3.5 Outcomes in not-overwhelming and overwhelming role overload...................... 446

14.4 What distinguishes role overload appraisal and coping described by hospital workers with lower levels of stress from that described by workers with higher stress

14.4.1 Environment factors in role overload recalled by low stress and high stress hospital workers.

14.4.2 Person factors in the role overload recalled by low stress and high stress hospital workers

14.4.3 Primary and secondary appraisal in role overload recalled by low stress and high stress hospital workers

14.4.4 Coping strategies used in role overload recalled by low stress and high stress respondents 460

14.4.5 Outcomes in role overload recalled by low stress and high stress hospital workers 


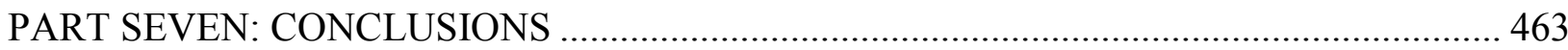

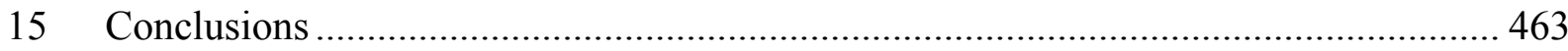

15.1 Conclusions and contribution of this research ...................................................... 463

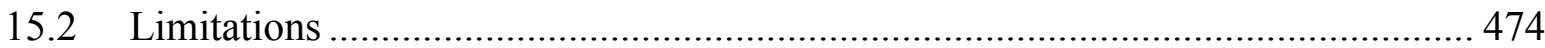

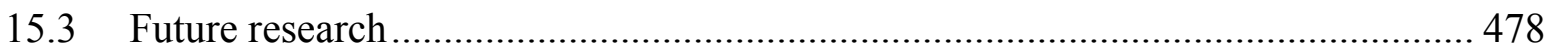

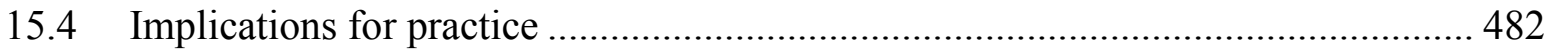

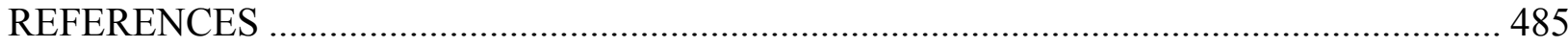

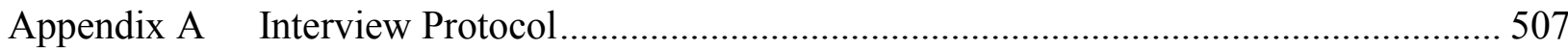

Appendix B: Full Data Sets for Results by Respondent Stress Level and Episode

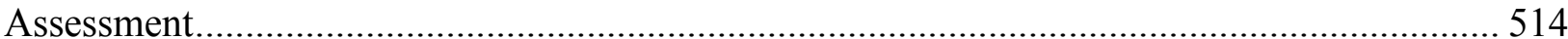

\section{LIST of TABLES}

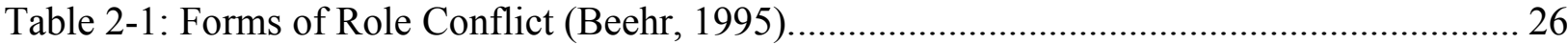

Table 3-1: A theoretical schematisation of the cognitive stress model (Lazarus \&

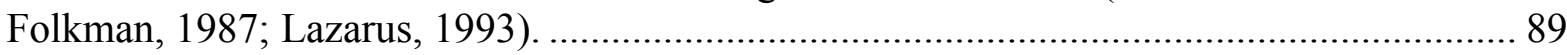

Table 3-2: Environment factors affecting cognitive appraisal (Lazarus \& Folkman, 1984)

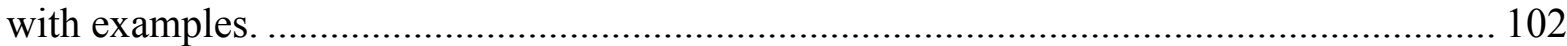

Table 5-1: Steps for building theory from case study research (Source: Eisenhardt, 1989

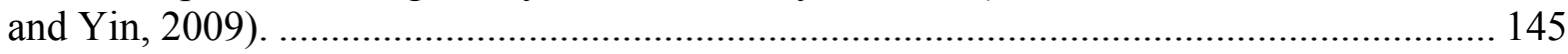

Table 7-1: Mapping of Part II interview questions to conceptual model and research questions.

Table 7-2: Mapping of Gosling et al. (2003) descriptor pairs representing Big Five personality dimensions.

Table 7-3: Derivation and outcomes of personality measure used in Part III of interview protocol (Source: Gosling et al., 2003; Costa and McCrae, 1992).................................... 175

Table 7-4 Illustration of part of the NVivo node hierarchy created during within-case and cross-case analysis of role overload episodes ................................................................ 185

Table 7-5 Top two levels of final NVivo node hierarchy completed after first two stages of analysis

Table 7-6 Example data display showing role factor results for the total sample and by episode assessment

Table 7-7 Example display of quantitative data for personality trait scores of low and high stress level respondents

Table 7-8 Example of first table type used to display results...

Table 7-9 Example of second table type used to display results 
Table 7-10 Example of third table type used to display results .......................................... 199

Table 8-1 Age range of respondents by stress level .......................................................... 206

Table 8-2 Number of children and age of youngest child of respondents by stress level ......... 207

Table 8-3 Job type of respondents by stress level............................................................ 208

Table 8-4 Event types triggering role overload by episode assessment. ............................... 210

Table 8-5 Event types triggering role overload by stress level of respondent......................... 214

Table 8-6 Event types triggering role overload by episode assessment and stress level of

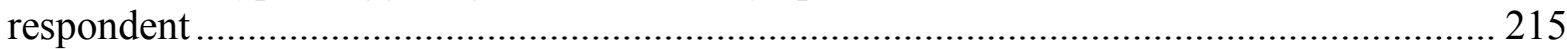

Table 8-7 Situation properties of role overload by episode assessment ............................... 217

Table 8-8 Situation properties of role overload: by stress level of respondent ....................... 226

Table 8-9 Situation properties of role overload by episode assessment and stress level of

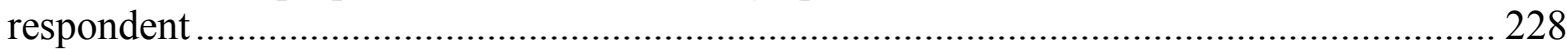

Table 8-10 Role factors described in role overload episodes by episode assessment. .............. 229

Table 8-11 Role factors described in role overload episodes by respondent stress level .......... 233

Table 8-12 Role factors described in role overload by episode assessment and stress level of respondent.................................................................................................. 234

Table 8-13 Key environment factors of role overload assessed as overwhelming and notoverwhelming disregarding the stress level of the respondents..................................... 238

Table 8-14 Key environment factors of role overload for low and high stress workers, without regard to how episodes were assessed ......................................................... 241

Table 8-15 Summary of role overload environment factors by episode grouping ................... 242

Table 9-1 Person factors described in role overload episodes by episode assessment .............. 245

Table 9-2 Person factors described in role overload episodes by stress level of respondent ..... 250

Table 9-3 Person factors described in role overload episodes by episode assessment and stress level of respondent ....................................................................................... 251

Table 9-4 Personality trait mean scores by stress level of respondent ................................... 253

Table 9-5 Role salience scores for the occupational, parental and marital roles by stress level of respondent ............................................................................................. 256

Table 9-6 Key person factors described in role overload episodes assessed as overwhelming and not-overwhelming, disregarding the stress level of the respondents ..... 260

Table 9-7 Key person factors for role overload of low and high stress respondents, without regard to how the role overload episodes were assessed .................................... 261

Table 9-8 Summary of role overload person factors by episode grouping ............................. 262

Table 10-1 Appraisal of role overload by episode assessment .............................................. 265

Table 10-2 Appraisal of role overload by respondent stress level ....................................... 267

Table 10-3 Appraisal of role overload by respondent stress level and episode assessment ....... 268

Table 10-4 What is at stake in role overload by episode assessment?................................. 270

Table 10-5 What's at stake in role overload by respondent stress level ................................ 277

Table 10-6: What's at stake in role overload by respondent stress level and episode

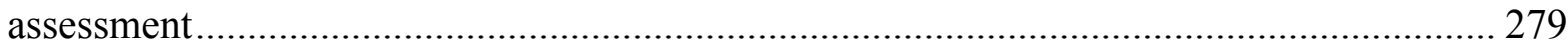

Table 10-7 Appraisal of coping options for role overload by episode assessment................... 282

Table 10-8 Appraisal of coping options for role overload by respondent stress level ............. 289

Table 10-9 Appraisal of coping options for role overload by respondent stress level and episode assessment 
Table 10-10 Appraisal of prospects for role overload coping success by episode assessment

Table 10-11 Appraisal of prospects for role overload coping success by respondent stress level and episode assessment.

Table 10-12 Emotions and feelings following role overload appraisal by episode assessment

Table 10-13 Emotions and feelings following role overload appraisal by respondent stress..... 298

Table 10-14 Emotions and feelings following role overload appraisal by respondent stress level and episode assessment

Table 10-15 Key appraisal factors and results for role overload assessed as overwhelming and not-overwhelming without regard to respondent stress level

Table 10-16 Key appraisal factors and results for role overload of high and low stress respondents without regard to how the episode was assessed 302

Table 10-17 Summary of role overload appraisal factors by episode grouping ...................... 304

Table 11-1 Coping strategies used for role overload by episode assessment

Table 11-2 Coping strategies used for role overload by stress level of respondents

320

Table 11-3 Coping strategies used for role overload by respondent stress and episode assessment

Table 11-4 Key coping strategies for role overload assessed as overwhelming and notoverwhelming without regard to respondent stress level.....

Table 11-5 Key coping strategies for role overload of high and low stress respondents, without regard to how the episodes were assessed

Table 11-6 Summary of role overload coping strategies by episode grouping ...................... 328

Table 12-1 Role overload personal outcomes by episode assessment.................................... 332

Table 12-2 Role overload personal outcomes by respondent stress level ............................. 336

Table 12-3 Role overload personal outcomes by respondent stress level and episode assessment

Table 12-4 Episode resolutions by episode assessment

Table 12-5 Episode resolutions by respondent stress level and episode assessment

Table 12-6 Key outcomes for role overload assessed as overwhelming and notoverwhelming without regard to respondent stress level.

Table 12-7 Key outcomes for role overload of high and low stress respondents without regard to episode assessment

Table 12-8 Summary of role overload outcome factors by episode grouping

Table 13-1 Taxonomy of role overload situation properties that create the potential for stressful appraisals

Table 13-2 Typology of role overload coping strategies 


\section{LIST of FIGURES}

Figure 2-1: Role Episode Model (Source: Kahn et al. 1964) .................................................. 18

Figure 4-1: Preliminary conceptual framework for the cognitive model of role overload ........ 116

Figure 7-1 Example display of respondent and associated role overload episodes stored as

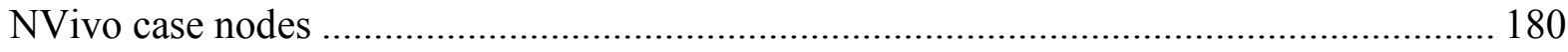

Figure 13-1 Conceptual framework of role overload appraisal and coping process ................ 348

Figure 13-2 Person factors in the role overload appraisal and coping process....................... 351

Figure 13-3 Primary appraisal in the role overload appraisal and coping process .................... 373

Figure 13-4 Secondary appraisal in the role overload appraisal and coping process ............... 377

Figure 13-5 Typology of role overload coping strategies (Element 5 of Figure 13-1)............. 380

Figure 13-6 Emotions and feelings cited during recollections of appraisal and post

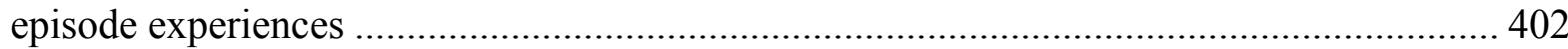

Figure 14-1 Common underlying factors of the role overload appraisal and coping

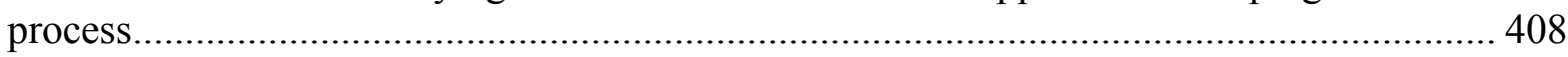

Figure 14-2 Not-overwhelming role overload appraisal and coping of low stress hospital

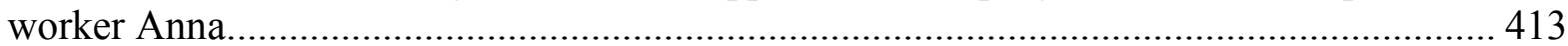

Figure 14-3 Overwhelming role overload and coping for low stress hospital workers........... 420

Figure 14-4 Not-overwhelming role overload and coping process of high stress hospital

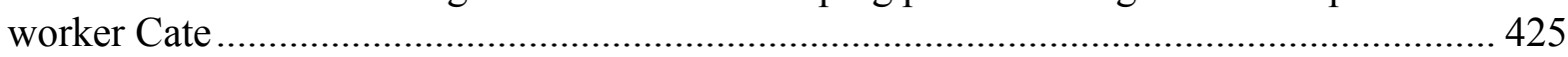

Figure 14-5 Overwhelming role overload appraisal and coping for high stress hospital

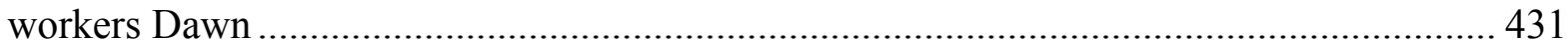

Figure 14-6 Key factors identified in role overload episodes assessed as notoverwhelming and overwhelming

Figure 14-7 Key factors of role overload appraisal and coping as described by respondents with low and high levels of perceived stress

\section{LIST of APPENDICES}

Appendix A Interview Protocol.....

Appendix B: Full Data Sets for Results by Respondent Stress Level and Episode Assessment. 


\section{PART ONE: INTRODUCTION}

\section{Introduction}

Every day individuals juggle the demands of multiple roles: parent, spouse, worker, and friend are just a handful of the social roles that people take on. Some people positively thrive on the challenges of fulfilling many different roles; for others, at times, it can be overwhelming and result in role stress. Role stress is the collective name for environmental demands, constraints, and events that affect an individual's role fulfillment (Parasuraman and Alutto, 1984). A particularly important but under studied form of role stress (Fournier et al. 2010) is referred to as role overload. Role overload arises from the perception that "the collective demands imposed by multiple roles are so great that time and energy resources are insufficient to adequately fulfill the requirements of the various roles to the satisfaction of self or others" (Duxbury, Lyons and Higgins, 2008 p. 130).

Role overload was empirically affirmed as an issue for employees in a 1964 U. S. study by Kahn, Wolfe, Quinn, Snoek and Rosenthal. Over the years since the concept was introduced, role overload has persistently, though often sporadically, been the subject of research in two significant fields of stress based research: occupational, and work and family. Unfortunately, however, much of the work on role overload in these two fields has either seen role overload subsumed into the role conflict or the work-family conflict constructs, or confused role overload with other role stress concepts (Biddle, 1986; 
Newton and Keenan, 1987; Coverman, 1989; King and King, 1990; Hecht, 2001;

Duxbury et al., 2008; Gilboa, Shirom, Fried and Cooper, 2008; Fournier, 2010).

The lack of research in this area and the confusion between this construct and others is unfortunate as the research that has been done indicates that role overload is both prevalent and consequential. Recent studies continue to reinforce the literature demonstrating that role overload can show a strong negative relationship to psychological health (Santos et al., 2003; Pearson, 2008; Glynn, Maclean, Forte and Cohen, 2009). Role overload has also been linked to a range of physical and psychological symptoms of stress (Beehr, King and King, 1986; Osipow and Davis, 1988; Lease, 1999; Perrewé et al. 2005) as well as negative organisational outcomes such as increased absenteeism from work and reduced job performance (Gupta and Beehr, 1979; Thompson and Blau, 1993; Gilboa et al. 2008). Of particular relevance to this thesis are data showing that role overload is one of the main reasons for nurses leaving the workforce (Chang, Hancock, Johnson, Daly and Jackson, 2005).

According to Beehr and Glazer (2005), writing in their summary of role stress research in the Handbook of Work Stress, the effects of role stressors such as role overload can, in fact, be either positive or negative, although generally, the role stress literature focuses on negative outcomes such as increased levels of anxiety, fatigue, burnout and depression. Very little research has been done exploring the other side of the coin: the link between role overload and positive outcomes. Nor have researchers attempted, either theoretically or empirically, to explain why in some circumstances of role overload, the stress symptoms are negligible. These shortfalls in the literature and the 
overall neglect of this increasingly important role stress construct are the main drivers for this thesis. This part of the thesis document introduces the objectives and approach of this thesis and explains the overall structure of the remaining parts of the document.

\subsection{Research Objective and Approach}

The aim of this study is to investigate the underlying factors influencing the appraisal of stress by individuals facing role overload at work and at home. More specifically, this thesis seeks first to develop a theoretical framework describing the relationship between role overload and perceived stress, and second to determine why and how some people who suffer role overload, experience symptoms of stress and others do not. The theoretical underpinnings of this study come from cognitive stress appraisal theory, as developed by Lazarus and Folkman in 1984. According to this theory, psychological stress arises from a potentially stressful episode when it is appraised as threatening or harmful or challenging in some way. Harmful and threatening appraisals are strongly associated with negative feelings. Situations that are appraised as challenging, on the other hand, are believed to result in positive emotions. The coping process that follows, to deal with the issues that arise from the stressful episode, may result in changes in subsequent reappraisals or in the associated emotions and feelings. This study uses Lazarus and Folkman's model as a guiding lens, and seeks to model the process and the contextual conditions that contribute to variable stress reactions to role overload. An exploratory, embedded case study approach will be used to gain an insight into the relationship between stress and role overload. Qualitative inquiry will provide the 
information rich data set that will best support this deep and focused research. To strengthen the study, the qualitative data will be supplemented with quantitative data where appropriate (Patton, 2002). Quantitative data for the case study was collected from individuals who participated in Duxbury et al.'s (2010) survey study on role overload within Canadian hospitals. Qualitative data was collected from individuals who volunteered at the end of the survey to take part in extensive follow up interviews.

\subsection{Outline of the Thesis Document}

This thesis comprises seven separate parts of which this introduction, outlining the objectives, is the first. Part two of this thesis is dedicated to summarising the literature on the main constructs explored in this study, role overload and perceived stress. Part three of this thesis details the research questions and the preliminary conceptual framework that guides the current research program whilst part four describes the methodology that is used to conduct the research. Part five presents the results and describes the key findings. In part six the initial conceptual framework is refined in light of the research findings. Part six also includes a discussion of the results with reference to the thesis objectives outlined above. Finally, part seven discusses the contributions and the limitations of the study, both practically and theoretically. 


\section{PART TWO: LITERATURE REVIEW}

The overall purpose of the literature review is not to find answers to fulfill the research objectives, but rather to create a firm foundation for knowledge advancement (Webster and Watson, 2002) and to facilitate the development of "sharper and more insightful questions" (Yin, 2009, p.14). To begin this task a thorough re-examination of the role overload literature, both theoretical and empirical, is required before we can explore its impact on psychological stress. Chapter 2 therefore investigates previous role overload research, to discover the theories behind this concept, the extent of existing research, and gaps that this thesis can rectify. Chapter 3 introduces the theoretical framework upon which this thesis is grounded: the cognitive model of stress, introduced by Lazarus and Folkman in 1984. In this thesis, this model is used to understand how individuals might theoretically respond to episodes of a specific stressor, role overload. 


\section{Role overload}

The research literature for role overload has a long and complex history. It is widely accepted (see Newton and Keenan, 1987; Fineman and Payne, 1981; King and King, 1990; Beehr and Glazer, 2005; Duxbury et al. 2008) that the source for the role overload concept lies in the 'theory of role dynamics' formulated by Kahn and his colleagues in the mid 1960's (i.e. Kahn, Wolfe, Quinn, Snoek and Rosenthal, 1964 and Katz and Kahn, 1966). Their pioneering work on role stress and the social psychology of organisations, undertaken at the Institute for Social Research in the University of Michigan, ultimately became known as organisational role theory (Biddle, 1986; Wickham and Parker, 2007). Their work in the area stimulated over forty years of research into role stress across multiple scholarly fields, which actively continues today. Whilst Kahn et al.'s work was the first to explicitly name and define the role overload concept, the study of society in terms of the roles that individuals play in it, the issues they face in fulfilling these roles, and how it relates to personal stress started much earlier, at the beginning of the twentieth century. As this review will reveal, these early developments of theories based around social roles also have important implications for role overload research today.

To cover the full gamut of role overload research, chronologically and thematically, the review of this literature in this chapter is divided into four parts. The first section reviews the theoretical foundations of role overload in the sociological theory of social roles and one of its prime sub-branches, organisational role theory. The second 
section critically reviews the role overload literature within the occupational stress field, whilst the third section does the same for the research literature examining stress arising from multiple roles. Together, these two areas of research have generated a sizable volume of literature concerning role stress. The main aim of these two sections is to trace the developments from the renowned work of Kahn and his associates in order to discover how these developments have influenced the progress of role overload research and hence identify shortfalls in the literature that the current research can address. More specifically, these sections seek to untangle the meaning of the role overload concept and to identify where and why confusion about the construct still remains. Chapter 2 concludes with a summary and critique of the role overload research, identifying key areas and their relevance to this study.

\subsection{Theoretical origins of role overload}

Before we delve into the history of role overload it is useful to recall the definitions presented in the introduction to this thesis proposal. Role overload is defined to be a "time-based form of role conflict in which an individual perceives that the collective demands imposed by multiple roles are so great that time and energy resources are insufficient to adequately fulfill the requirements of the various roles to the satisfaction of self or others" (Duxbury et al., 2008 p.130). Role overload and role conflict are both examples of role stress. Role stress consists of stressors that have been defined as environmental demands, constraints, and events that affect an individual's role fulfillment (Parasuraman and Alutto, 1984). Underpinning these definitions is the idea of 
the social role, a concept that has been well established as a research tool since the late 1940's (Nieman and Hughes, 1951; Biddle, 1986). The objective of this initial section of the role overload literature review is to examine the theoretical and empirical developments that resulted in the development of the framework of organisational role behaviour as defined in Kahn et al.'s (1964. 1966) theory of role dynamics formulated for their studies of role stress.

The first part of this section covers the early discussions and contentious debates about the notion of 'role theory' and its many related concepts. The remaining subsections focus on the important work of Kahn and his colleagues in giving substance and definition to the role overload concept. Specifically, in the second subsection the background and objectives behind the development of the theory of role dynamics are discussed and a detailed discussion of the role based behaviours that make up an organisational role system are provided. In the third subsection Kahn et al.'s (1964) construct, role overload, is defined and analysed. This examination of the early formulation of role overload concludes with a summary and critique of the theories underpinning the concept, which will thus provide the context and starting point for the current research study.

\subsubsection{Developments in role theory}

According to Biddle (1986) in his review of role theory developments, the use of the term 'role' in behavioural sciences began its life as a theatrical metaphor in the 1920 's. In the 1930's the term was used for the first time in academic writing with 
specific reference to role problems (Biddle and Thomas, 1966) and by the end of the 1940's the role concept was "an integral part of sociological vocabulary" albeit a concept that was "still rather vague, nebulous and non-definitive" (Nieman and Hughes, 1951, p.149). The first part of this introductory piece of the role overload literature review briefly explores how early developments in the use of the term 'role' in scholarly research established the concept as one of the most popular ideas in the behavioural sciences whilst debates about its formal definition continued. The second part examines the research approach taken to study role fulfillment during the period before Kahn et al.'s (1964) seminal work on role stress. The last part takes an overall view of role theory development in order to establish the context for the subsequent research into role overload.

The primary goal of this brief review of early role theory is to elucidate definitions of, and relationships between, the role based concepts crucial to the subsequent theoretical development of role overload (i.e. role, role strain, role conflict, role theory and organisational role theory). As mentioned in the introductory sections to this thesis proposal, role overload has been confused with other role constructs. Special attention will therefore be paid, in this initial subsection of the role overload literature review, to the link between nascent conceptualisations of role overload, role strain and role conflict in order to seek early indicators as to how this conceptual confusion arose. 


\subsubsection{Role concept}

From the beginning, the concept of role was applied in many different contexts based on differences in assumptions about its meaning (Nieman and Hughes, 1951; Biddle and Thomas, 1966). At the same time operational definitions of associated constructs also suffered from lack of agreement (Gross, Mason and McEahern, 1958; Biddle, 1979, 1986). This was, in part because historically, developments in this field stemmed from two theoretical trends in sociology: symbolic interactionism and structural-functionalism. The symbolic-interactionists followed the early work of Mead (1934) and focused on how individuals develop their sense of self to become members of society though their interactions with others. For these researchers, roles are particular behaviours and expectations tied to a specific positional label (e.g. mother, nurse, or patient) that we learn and can call up in a particular context (Knowles, 1982). The structural-functionalists (e.g. Linton, 1936; Parsons, 1951; and Merton, 1957), on the other hand, defined a social system as a collection of positions structured to perform the functions of society. According to Linton (1936) a social position or status "is simply a collection of rights and duties", and roles are the patterns of behaviours enacted by an individual to fulfill those rights and duties (p.113). According to Parson (1951), how an individual interacts with others and plays out her role will be seen in terms of its significance for the functioning of the social system.

Even within these two groups, dissension was more common than agreement on such basic concepts as role, status, and position (Gross et al., 1958). Whilst the diversity in the orientations of the role language and the lack of agreed theoretical boundaries and 
assumptions for this body of work placed limitations on its suitability for systematic investigations, these same characteristics also increased the applicability of the ideas to a wide range of research (e.g. for studies of small groups, families, communities, classrooms, kinship systems and formal organisations). As a result, subsequent developments in role theory arose from products of interdisciplinary work and the use of the role concept spread quickly, becoming common throughout the fields of sociology, anthropology, and social psychology (Biddle, 1979; Knowles, 1982; Hardy \& Hardy,1988).

With the benefit of hindsight, we can look back and observe that the core focus of these role concept theories were (and still are) with "characteristic behaviours, parts to be played, and scripts for behaviour or, in more familiar language, roles, social positions, and expectations" (Biddle, 1986 pp. 69). A generic definition that encompasses these core concepts can be found in Gross et al. (1958) - "roles are expectations for or evaluative standards employed in assessing the behaviour of occupants of specific social positions. A social position is simply a location in a system of social relationships."

\subsubsection{Role problems}

An examination of sociology and psychology journals prior to 1964 produces no evidence of research on the specific concept of role overload, yet the idea that individuals could encounter stress when faced with too many roles had been argued by a (small) number of theorists (e.g. Merton, 1957; Goode, 1960; Slater, 1963 cited in Biddle, 1979). During this early period in the development of role language and theories, a glimpse of 
what the role overload concept might look like came in Goode's (1960) article "The Theory of Role Strain". He used the term role strain to refer to the difficulty of performing multiple roles, but his references to time constraints and his conclusion that "the individual's total role obligations are over-demanding" (p.485) highlight key characteristics of the construct role overload as defined by Kahn and his colleagues. It is no coincidence that in 1974, Sieber (p. 567) argued that although Goode did not specifically mention overload, "many of his observations can be subsumed under this notion". Goode also acknowledged the significant potential for conflict between roles. His paper has proven to be influential in that he assumed the experience of role strain to be the normal state of affairs and thus piqued the interest of social scientists. It was also probably the first to discuss the concept of role overload (albeit indirectly) and role conflict as coexisting in a multi role environment.

Despite Goode's article, prior to 1964 the majority of academic work studying role problems focused largely on conflict experienced by multi-role players. Like Goode, many of these researchers assumed that in any role system, conflict was inescapable. For example, Parson's (1951, p. 280) suggested that “there is a certain endemic potentiality of role conflict inherent in the fact that any actor has a plurality of roles". Merton (1957, p.370) believed that anyone occupying a particular position has role partners with different values and moral beliefs and therefore the individual will be "readily subject to conflicting role-expectations". As was the case for other role constructs, there were, however, wide disparities in the conceptualization of the role conflict construct. Gross et al. (1958), now considered a renowned source for a number of role construct definitions, 
provided important and influential clarification of the role conflict concept (King and King, 1990; Scott, 1998). Their definition states that role conflict occurs when a role incumbent feels that he or she is faced with incompatible expectations. With this definition they incorporated conflict between legitimate and/or illegitimate expectations, within single roles (intra-role) and across multiple roles (inter-role), but limited it to conflict as perceived by the individual subject to the expectations in question.

\subsubsection{Role theory}

Given the breadth of discussions surrounding role concepts, it is not surprising that the question of whether there exists a singular role theory was a frequent subject of debate for many years (Biddle and Thomas, 1966; Stryker and Statham, 1985; Biddle, 1986; Hardy and Hardy, 1988a). A more realistic view is presented by Hardy and Hardy (1988a) in their contribution to the book "Role Theory Perspectives for Health Professionals". They argue that the body of knowledge that is commonly called role theory consists of "clusters of concepts, emerging sub-theories, and a diverse set of empirical research findings that address specific aspects of social behaviour" (pp 32). The fact that they also felt the way these ideas have been used to create theoretical tools to examine problems in social systems are more variable than common, suggested little progress had been made in this area in the 30 years since Gross et al. made similar observations in 1958.

Apart from Hardy and Hardy's (1988a) book, a search of the social science databases fails to identify further reviews of role theory since Biddle's 'Recent 
Developments in Role Theory' (1986). Also of note is the observation that latterly, academics have shied away from trying to develop an integrative theory of role concepts (George, 1993; Wickham and Parker, 2007). Despite these limitations, a large amount of research literature has built up over the years and role concepts and their associated theories play an important part in behavioural sciences (King and King, 1990; Beehr and Glazer, 2005). Conventional wisdom now accepts that within this body of knowledge multiple perspectives exist, and the work by Kahn and his colleagues $(1964,1966,1978)$ and the studies by Gross, Mason and MacEarchen (1958), are together, credited with being the impetus for a particularly influential perspective of role theory, namely organisational role theory (Biddle, 1986; George, 1993; Wickham and Parker, 2007). Building on Gross et al.'s (1958) renowned studies and also consolidating a broad spectrum of role related theory and research, Kahn et al. (1964) developed the theory of role dynamics in order to examine role stress in organisational settings. In taking this approach, they combined the structural-functionalist and symbolic-interactionist perspectives as a means for obtaining a fuller comprehension of social behaviour (Knowles, 1982; Hardy and Hardy, 1988). This allowed them to turn their attention to the study of role behaviours in complex everyday life. This branch of so-called role theory expanded quickly and continues to be the most prolific in terms of empirical research (Biddle, 1986; King and King, 1990; Wickham and Parker, 2007). The next subsection will begin the overview of this far-reaching theory. 


\subsubsection{Organizational role theory}

Kahn and his team of fellow researchers included members from two groups, the Survey Research Centre and the Research Centre for Group Dynamics, both of which were based at the University of Michigan in the early 1960s (Newton and Keenan, 1987; Frantilla, 1998). The joint program of research, undertaken by these Michigan groups, under the umbrella of the university's Institute for Social Research, was aimed at understanding the effect of the social environment, particularly that of a large organisation, on the mental and physical health of individuals. Two major pieces of published work resulted from the research of these groups. The first title published in 1964 was concerned with the nature, causes, and the consequences of organisational role stress. The second book 'The Social Psychology of Organizations', published two years later, was aimed at providing a more general theoretical treatment of organizational processes.

In formulating a theory of organisation, Kahn and his associates saw great merit in the concept of role but like others (Nieman and Hughes, 1951; Gross et al. 1958; Biddle and Thomas, 1966) were cognisant of weaknesses reported in the literature regarding inadequate operational definitions and a lack of systematic research. As social psychologists, their intent was also to apply a psychological approach to the study of problems in the social world and more specifically, to research the mechanisms that can help us understand how individual behaviour is affected by social organisations. In an article examining the interplay between the fields of sociology and psychology, Thoits (1995b) explains that sociologists tend to focus their attention on which social 
phenomena have effects on individuals, whilst psychologists generally spend their time investigating the processes through which the social phenomena have their effect on individuals. Kahn et al. were aiming to combine both these approaches. Katz and Kahn (1966) explain their approach to social organisations as follows:-

"Social systems have structure, but it is a structure of events rather than physical parts, a structure therefore inseparable from the functioning of the system......This aspect of social structure reminds us....that social organizations are contrived systems, held together by psychological bonds. The contrived quality of social systems and their quality of event-structure mean that they require control mechanisms .... to keep their component parts together and functioning in the required interdependent fashion.” (p. 69)

"The concept of role is proposed as the major means of linking the individual and organizational level......; it is at once the building block of social systems and the summation of the requirements with which such systems confront their members as individuals.” (p.197)

Kahn and his colleagues thus gave the role concept a "central place" in their theory of organizations (Kahn et al., 1964, p. 11). Accordingly, as social systems, organisations are defined and controlled by the patterned behaviours and socially constructed relationships of their members. The set of activities expected of an individual, located in a given position, constitutes the role required to be performed. Observing the role behaviour of the members of an organisation therefore provides an opportunity to study the impact of an organisation upon the individual (Kahn et al. 1964). Although their focus was on studying formal organisations, Kahn et al. also recognised that individual behaviour is influenced by all the groups and organisations to which a person belongs and thus their initial stated objective was to define a "common set of concepts for characterizing all roles in all these organizations" (Kahn et al., 1964, p. 12). 
Acknowledging Linton's (1936) nascent use of the role concept in the social sciences and the important work on structure and social action by Parsons (1951), Kahn and his fellow researchers drew directly from a number of other sources to systematically describe how role incumbents interact with other members of generic organisational systems. From Merton (1957), they borrowed the concept of role-set to indicate the complement of relationships that individuals have by virtue of occupying a particular social position. (For example, one or more patients, doctors, hospital administrative staff, orderlies, or medical technicians could all form part of a nurse's role-set.) Each member of the role-set develops beliefs and attitudes about what should or should not be done to fulfill that role and these expectations are communicated, or sent, to the focal person in an attempt to influence the role incumbent. Referring to role expectations as sent was originally proposed by Rommetveit in 1954 . The key point, as argued by Kahn et al. $(1964,1966)$, is that activities that define a role are upheld through the expectations of role-set members, and these expectations are constantly communicated in the form of role pressures. From Gross et al. (1958) we learn that role pressures vary along a number of dimensions, such as magnitude, intensity or direction, and in Kahn et al.'s model, when perceived and cognitively processed by the focal person, they take the form of received role forces. The sent role represents the objective view of required role activities. The received role consists of the perceptions of what was sent and is thus the "immediate influence" for role behaviour (Katz and Kahn, 1966, p. 177). This process by which the expectations of role set members are linked to role behaviours is described by 
Kahn and his colleagues as a role episode. A diagrammatic representation of the role episode model is presented in Figure 2-1.

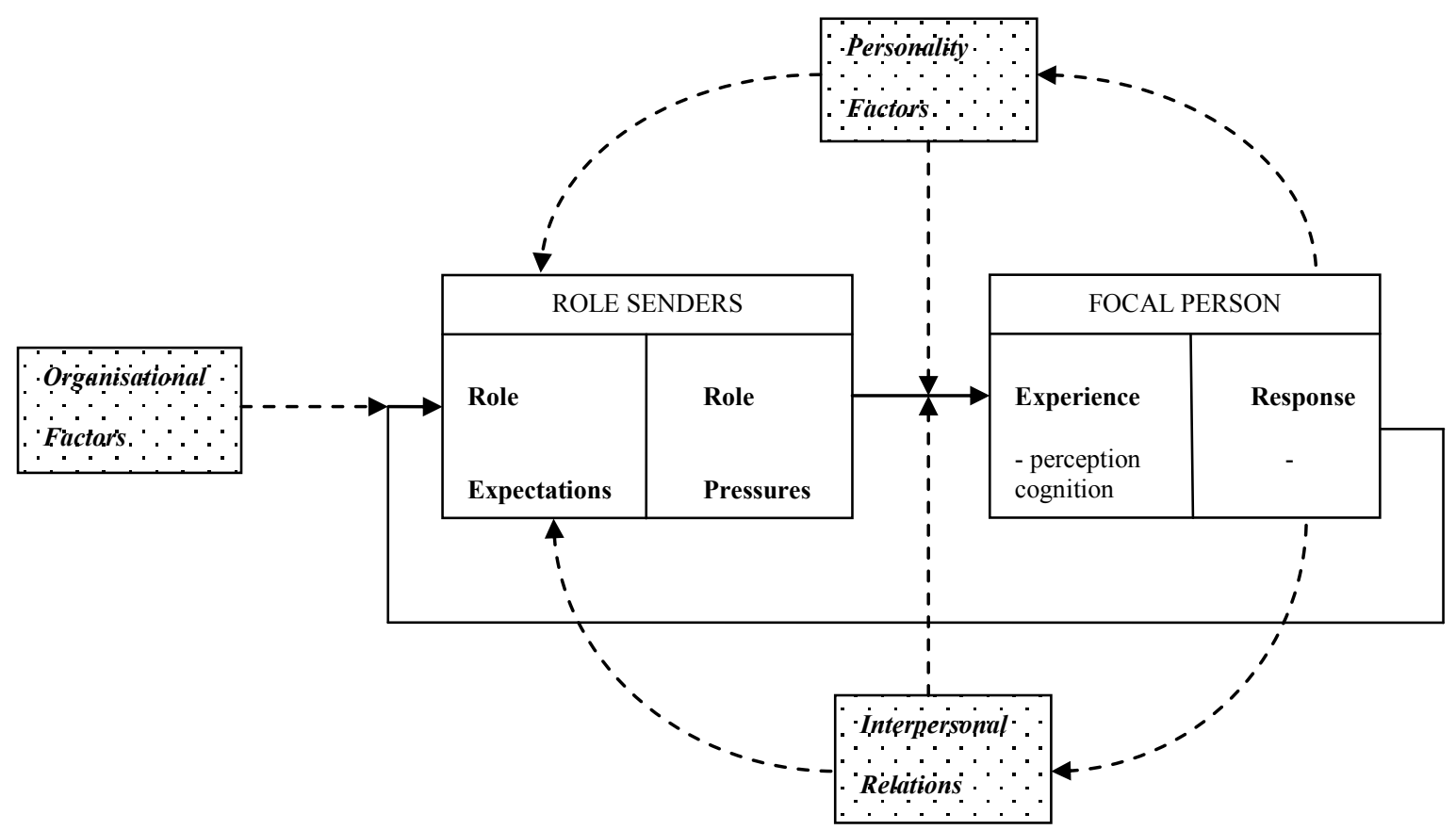

Figure 2-1: Role Episode Model (Source: Kahn et al. 1964)

A role episode is conceived of as cyclical and the cycle is completed by the response of the focal person and the effect of that response on the role senders' future expectations (as represented in the diagram by the solid feedback arrow from the focal person to the role sender). Role episodes do not, however, take place in isolation, unaffected by the environment or the characteristics of the people involved. As Katz and Kahn (1966) noted, "every act of role-sending and role-receiving is in part determined by the context within which it occurs" (p. 184). To complete their model, Kahn et al. thus proposed three additional classes of variables as influencing a role episode: 
organisational factors, personality factors, and characteristics of the interpersonal relationships between the focal person and role senders. These "enduring states of the organization, the person, and the interpersonal relations" (Kahn et al. 1964, p.31) are represented in the role episode diagram by three patterned boxes surrounding the process linking the role sender and the role incumbent.

The personality traits of the focal person, and the interpersonal relations with their role set are both seen as important conditioning variables in the relationship between the role as sent and the role as received. Kahn et al. also noted in their second publication that some personality attributes of the role incumbent may even be modified over time by recurring role behaviour (Katz and Kahn, 1966), although they did not elaborate on this process. Kahn and colleagues further argued that the third set of contextual variables, organisational factors, have a direct influence on the role expectations held about and sent to any particular position. Kahn et al. (1964) explain that organisational factors include properties such as size, number of echelons, rate of growth, or internal reward structures, as well as ecological factors pertaining to the characteristics of the role-set. For an example of ecological factors, consider that some role-set members may have the same status and be in the same department as the focal person, whilst others could be from separate units and be of a different rank. Each will produce unique role pressures by virtue of their different positions in the organisation. Despite the recognition that the organisational context will influence the role senders expectations, in Kahn et al.'s model there is no acknowledgement of organisational factors having a direct influence on the focal persons perceptual and cognitive processes that ultimately determine the response 
in a role episode. Moreover, by restricting the focus to formal organisations, in terms of environmental influences at least, Kahn et al.'s model becomes less applicable to other types of groups in which individuals undertake roles.

According to Kahn and his associates, in the ongoing life of a social group or organisation, role incumbents will be involved in numerous role episodes, which may therefore impose pressures towards a variety of behaviours, which in turn may also be affected directly or indirectly according to the context of the situation. There is another important factor that potentially influences the focal person of the role episodes. Each person has a personal set of values and beliefs about what behaviours are appropriate for their role and what abilities they possess. In other words, individuals are role-senders to themselves, thereby creating internal pressures and forces for role performance (Kahn et al., 1964). To the extent that these external and internal role pressures collectively or individually give rise to perceived role forces to fulfill expectation, the role incumbent may experience psychological stress. Specifically, the theory of role dynamics hypothesizes that it is the perception of the expectation that determines the nature of the role pressures, regardless of whether the communicated demand was correctly interpreted (Beehr and Glazer, 2005).

It is these specific forces experienced by individuals in undertaking their roles, collectively termed role stress (Kahn, 1980), that were the subject of Kahn and his colleagues research in their first published work, 'Organizational Stress' (1964). Using the role episode model as the framework for their studies, Kahn et al. (1964) focused their attention on the impact of role stress on the psychological well-being of the 
individual, in particular on the impact of role conflict and role ambiguity ${ }^{1}$, as well as role overload. The three sets of contextual variables affecting the role episode, described in the previous paragraph, illustrate Kahn et al.'s attempt, as social psychologists, to explain how society is tied to the individual as well as illustrating the impact of social phenomena on role relationships (Thoits, 1995b). As such they are also an important part of the theory of role dynamics. The next subsection will detail how Kahn et al. (1964) positioned role overload in their theory of role dynamics, and specifically within the context of the role episode model of role stress.

\subsubsection{Introduction of the role overload construct}

The population of a role incumbent's role set (i.e. all the people who are associated with that person because of the role they hold), will themselves occupy a variety of roles within a social system. For example, in a hospital setting a nurse has a role-set that could include patients, doctors, medical technicians, administrative staff, hospital management, other nurses, and allied health professionals such as physiotherapists. This role-set may extend outside the hospital boundary to include patient family members and community health professionals, such as social workers or general practitioners. Moreover, the nurses role-set may also include members of groups who are role related in other ways, such as volunteer based community organisations seeking free health advice, as well as close friends and family who are, as Kahn et al. (1964) suggested, "also concerned with his [or her] activity and behaviour in his [or her] organizational role" (p. 14). Such a vast role-set

\footnotetext{
${ }^{1}$ Role ambiguity occurs when sent expectations are unclear to an individual (Kahn et al., 1964)
} 
could well be replicated for each member of the nurse's role-set for their own personal roles. Inevitably, role senders will have differing viewpoints on how a role should be performed and may communicate their expectations without regard to the volume or content of sent expectations from other role-set members. The situation is made more complex when we consider that individuals have multiple roles, each with its own roleset potentially spanning multiple overlapping groups and organisations. Consequently, the opportunity for an excess (or overload) of expectations or for conflict between expectations to arise is real and significant.

According to Kahn et al (1964), role overload is a specific but complex and composite type of role conflict in which it becomes impossible to perform the required tasks within expected time limits. Whilst seemingly straight forward, this definition firmly ties role overload to the concept of role conflict. In order to fully understand the nature of role overload we therefore need to examine and analyse the complete typology of role conflict presented by Kahn et al. $(1964,1966)$ to explain this sub-type of role stress. This will be achieved in two stages. The development of the role overload entity from within Kahn et al.'s typology of role conflict is first examined in detail to reveal what separates this construct from other types of role conflict. The characteristics of role overload is then explored to reveal additional important characteristics of Kahn et al.'s formulation.

Alongside role overload and role conflict Kahn et al. (1964) also defined and studied a third type of role stress, role ambiguity. In this section introducing the role 
overload construct, a third subsection also briefly examines role ambiguity and its relationship with role overload as described by Kahn et al. (1964).

\subsubsection{Role overload and role conflict formulation}

Kahn and colleagues defined role conflict generically as "the simultaneous occurrence of two (or more) role sendings such that compliance with one would make more difficult compliance with the other" (Katz and Kahn, 1966 p. 184). In this, as already noted, they are following the formulation of Gross et al. (1958) in focusing on the perceptions of the role incumbent occupying either a single or multiple positions, without regard to the legitimacy or otherwise of the expectations sent. Based upon the source of the role pressures, Kahn et al. identified five types of role conflict as follows:

i. Intra-sender conflict - incompatible expectations emanating from a single member of a role set.

ii. Inter-sender conflict - incompatible expectations from two or more members of the same role set.

iii. Inter-role conflict - incompatible expectations from members of two or more different role sets.

iv. Role-person conflict-incompatibilities between role sent pressures and the needs, values or capacities of the role incumbent.

v. Role overload - logically compatible sent expectations that cannot be completed within given time limits and quality requirements.

In line with their objectives to facilitate comparisons across the environments of all individuals by describing concepts common to all roles, Kahn et al.'s typology was an attempt to capture the broad spectrum of role conflict faced by individuals in social 
organisations. Based on the descriptions, the first three (i.e. intra-sender, inter-sender and inter-role conflict) involve conflicts in the content of the role as sent, and as such "they exist as conflicts in the objective environment of the focal person" (Katz and Kahn, 1966 p. 185). The fourth type of role conflict (i.e. role-person conflict) arises from "a combination of externally sent role expectations and internal forces or role expectations" (Katz and Kahn, 1966 p. 185), and the final form, role overload, they point out, comprises combined elements of the other "basic types" (Kahn et al. 1964 p. 20).

Katz and Kahn (1966) explain that role overload is likely to be experienced as a conflict between priorities or as a conflict between quality and quantity. In other words, role demands received from one or more role senders that are not in themselves logically incompatible, cannot all be completed to the level of excellence requested ( as perceived by the role incumbent) before the specified deadlines. The priority of an expectation may be specified by the role sender or determined independently by the role occupant. The result is that to avoid being taxed beyond personal limits, role incumbents must thereafter respond by deciding which pressures to comply with, and/or by reducing quality or performance levels for some or all tasks. Role overload was regarded by Katz and Kahn (1966, p. 185) as combining "aspects of conflict between role senders with conflict between senders and [the] focal person" (i.e. in their terminology; it is a mixture of intersender and person-role conflict). In theory, however, role overload could also arise from a single demand from one role sender that involves multiple sub tasks (i.e. a mixture of intra-sender and person-role conflict). 
In summary, the key aspects of role overload can be said to be excessive role demands or expectations that are not necessarily logically incompatible; lack of available time; and limits in the personal capacities of the role incumbent. Based on the specificity of these definitions and observations, role overload can be considered to be a unique and complex type of role conflict.

\subsubsection{Role overload characteristics}

At the broadest level, Kahn et al. conceptualised role conflict, and hence by definition role overload, as either intra-role or inter-role. As King and King (1990) explain, intra-role conflict is a term first used by Gross et al. (1958) to describe conflicting expectations associated with a single position or status, whilst inter-role conflict results from demands arising from multiple roles. Beehr (1995) used the same classification as one of the categorising elements in a two dimensional table listing types of role conflict based on a combination of who sends the role expectations to the role incumbent (division by sender) and what role the expectation is about (division by role). Using Kahn et al.'s labels, which indicate who sends the role expectations, Beehr (1995) specified three basic forms of role conflict (i.e intra-sender, inter-sender, person). Each of these three basic forms can apply to conflict within a single role (intra-role) or across multiple roles (inter-role). Hence, six types of role conflict as shown in Table 2-1: 


\begin{tabular}{|l|c|c|}
\hline & \multicolumn{2}{|c|}{ Division by Role } \\
\hline Division by sender & Intra-role & Inter-role \\
\hline Intra-sender & A & B \\
\hline Inter-sender & C & D \\
\hline Person-role & E & F \\
\hline
\end{tabular}

Table 2-1: Forms of Role Conflict (Beehr, 1995).

Intra-sender conflict involves conflicting demands that one role sender has for the focal person. Kahn et al.'s (1964) example referred to a manager demanding that a worker obtain material unavailable from normal sources whilst prohibiting violations of normal channels. Intuitively, it is obvious that intra-sender conflict would usually be a form of intra-role conflict as labeled in Beehr's (1995) table as 'A'. Beehr (1995, p. 71), however, also noted that intra-sender conflict can occur between two or more roles when "the same person is a member of two or more of the focal person's role sets". For example, a family member may also be a co-worker and could theoretically send conflicting expectations based on their different roles. In Beehr's table this is represented by 'B'.

Demands from two or more people can obviously conflict (inter-sender conflict) whether from within one role ( C) or from across multiple roles (D). The third type of role conflict, person-role conflict, focuses on who sends the demand. This type of conflict was intended by Kahn et al. (1964) to reflect conflicts between demands the role incumbent receives from others and (internal) demands from themselves based on personal values and standards. Kahn et al.'s (1964) example of pressure from senior 
management to use price fixing strategies that trouble a workers personal ethics is focused on the singular work role (i.e. E in the Table 2-1). Beehr (1995) argues that individuals will be role senders to themselves for all their roles and therefore, the potential exists for their own expectations for one role to conflict with their expectations of themselves for another. The obvious example is the conflict between working long hours to fulfill personal commitments to their chosen profession whilst expecting themselves to put in many hours as an active family member, thereby creating inter-role conflict ('F' in Table 2-1).

Beehr's (1995) table is intended to show all the possible simple types of role conflict and he argues that theory and empirical research has overlooked some of these forms of role conflict. Whilst, role overload, as a complex type of role conflict and therefore a 'special case' (Beehr, 1995 p. 70), was excluded from the table, the concepts from this typology can be used to help us understand the construct. Role overload can, for example, also be specific to the domain of a single role or can be experienced across multiple roles. Using the letter based labeling of Beehr's (1995) table, as complex forms of role conflict, intra-role overload would be represented by $\mathrm{AE}$ or $\mathrm{CE}$, whilst inter-role overload would be BF or DF. In other words role overload could arise from demands from a single role sender in one role set, from multiple demands from a single role sender from multiple role sets, from multiple demands from multiple role senders from the same role-set, or from multiple demands from role senders from different role sets.

As noted by Gross et al. (1958), many earlier studies took the position that role conflict arises from incompatible expectations from multiple positions (inter-role), often 
ignoring the possibility that incompatible demands can also be associated with a single position (i.e. intra-role). For example, a nurse may find that her (or his) work role demands prevent her from fulfilling her (or his) role as a parent (i.e. inter-role role overload or role conflict). In her (or his) work role, however, the same nurse may find that demands from a doctor prevent her (or him) giving necessary care to a patient (i.e. intra-role role overload or role conflict). From the data collected for their studies of role stress, Kahn et al. (1964) observed that role overload typically arises when too many expectations are sent from the various members within a single role set. Thus, as this analysis has revealed, whilst Kahn et al. specifically incorporated the broader view, which acknowledges intra and inter-role conflict and overload into their typology, because they were primarily interested in the role of the worker in large organisations, their research, and hence examples, were restricted to intra-role stress, contrary to earlier research. .

This focus on the large industrial organisation limited Kahn et al.'s research in other ways too. In accordance with their model, the role overload construct has an objective or environmental component as well as a subjective or psychological component, represented by the sent role and the received role within a role episode, respectively. For example, this means that objective role overload is a verifiable condition in the organisational environment whilst subjective role overload is an internal state of the focal person (King and King, 1990) mediated, according to Kahn et al., by personality, interpersonal factors, and indirectly, by organisational factors. As such, subjective role overload may not mirror objective role overload. This disparity can, 
according to Kahn et al. (1964) lead to psychological stress. In their role episode model, Kahn et al. hypothesize that organisational factors have a direct affect on the expectations and associated pressures which can lead to role overload. Within the social systems where most people work, role expectations and pressures are largely "reinforced by the formalities of organization" (Kahn et al., 1964). In other groups, such as families, volunteer organisations and social clubs, there is less emphasis on formally agreed policies and procedures and, as a result, role expectations and pressures are largely based on tradition and personal relationships. In such dissimilar organisations, the impact of the generated role expectations and pressures on the perception of role overload is also likely to be quite different.

Kahn et al. (1964) focused on behaviour caused by forces generated in the work situation itself, but also acknowledged that role behaviour in any role can be "determined by forces and pressures originating outside the organization in which the behaviour occurs" (p.18). This suggests that the type of organisation in which role expectations arise will be one of the factors influencing the perception of role overload, as will the organisation in which the expectations are received. More importantly, this is another indicator that role overload and other forms of role stress, as defined by Kahn et al. (1964), can arise from an involvement in multiple roles as well as from the singular role position: work. In their study, however, they did not investigate the impact of these multiple roles. Such approaches came later in the history of organisational role theory. This distinction between the effects of the single role versus the effects of multiple roles is the basis for the categorisation of the review of the role overload literature arising from 
Kahn et al.'s theories that follows this section. First however, the final part of the review of the theoretical origins of role overload will discuss the implications of Kahn et al.'s work and its history, for the proposed research.

\subsubsection{Role overload and role ambiguity}

Role ambiguity was defined by Kahn et al. (1964) as a lack of information required by a role incumbent for adequate role performance. Ambiguity may result either because information does not exist or because information has been inadequately communicated. An individual's experience of ambiguity is based on personal perception of the environment and thus may not reflect the real world. Kahn et al. (1964) suggest, however, that the correspondence between objective and experienced ambiguity is considerable, noting that there are several frequently encountered areas of ambiguity within organisations. For example, individuals in organizations often do not know what they are supposed to do or how they are supposed to go about doing it. Such ambiguities can arise if expectations defining a role are vague and/or inconsistent. Regardless of the extent of ambiguity in the role environment Kahn et al. note that the intensity of the experience of ambiguity can vary according to individual differences.

Kahn et al. (1964) make no specific reference to a link between role ambiguity and role overload, but they do discuss the possible relationship between ambiguity and conflict, suggesting that there are several reasons for them to be associated, all of which apply equally to overload. For example, conflicting role pressures may create ambiguity for the role incumbent. If various people press the role incumbent to do multiple tasks 
that cannot be completed in the time available, she may not know which requests to comply with first.

\subsubsection{Critique and implications for the current research.}

Critiques of Kahn et al.'s theory of role dynamics have commended the detailed description of the role stress constructs and the logical development of the role episode model but criticised the overwhelming focus on role conflict (King and King, 1990). For example, although Kahn et al. reported that a significant number of the workforce experience role overload (i.e. $43 \%$ of 1300 respondents from a variety of large organisations from across the U.S.), their results from the follow up intensive interviews did not attempt to link any conclusions about role stress specifically to role overload even though the reported narrative included a number of instances of role overload. The results of their study of the labour force in the US also concluded that role conflict and role ambiguity are independent sources of stress although the sources of ambiguity are also sources of conflict. Although Kahn et al. (1964) observed role overload as one of the dominant forms of role conflict in their data they did not offer examples that included both overload and ambiguity.

The strength of the theoretical work of Kahn and his colleagues overcame many of the weaknesses previously identified in the immature role stress field of the 1950's and early 1960s, and it has been acknowledged as the stimulus for the bulk of the research into role stress that has followed since (Newton and Keenan, 1987; King and King, 1990; Wickham and Parker, 2007). Nevertheless, their work continued the bias towards role 
conflict already prevalent in early role theory research. Role overload is clearly identified by Kahn et al. (1964) as an important and unique type of role stress but no attempt was made to empirically differentiate role overload from role conflict at this time, even though preceding researchers had already identified the close relationship between the two concepts (e.g. Goode, 1960).

As social psychologists, Kahn et al. (1964) recognised the importance of context and its influence on the stress and strain relationship. They specifically included three sets of contextual variables in their role episode model as being important to this association. Nevertheless, their model stops short of explaining the process by which role stress is perceived by the individual, how it relates to the responses, and how the different contexts from the multitude of roles that individuals undertake can give rise to different role pressures.

Subsequent studies based on their work have tended to ignore the dynamic influence of these contextual variables in favour of focusing on the relationship between role pressures and the physical and psychological responses to them (Newton and Keenan, 1987; King and King, 1990). Fineman and Payne (1981) argue that Kahn et al.'s role theory "masks much of the idiographic nature of stress" and does not make clear why stress should arise from mismatching role expectations between senders and receivers. Although Kahn et al. (1964) provide a clear definition for role overload and recognise the importance of an individual's subjective perceptions of role pressures, their framework is inadequate for explaining variable psychological stress levels. Addressing these gaps in the literature is the objective of this thesis research. 
Within the ensuing research that grew out of organisational role theory, research into role overload has been strongly influenced by the impact of organisational and social developments on role stress research overall. The next section will discuss the impact of these influences in the occupational stress literature.

\subsection{Occupational stress literature}

The program of research undertaken by Kahn et al. $(1964,1966)$ at the University of Michigan was, according to Cooper and Dewe (2007), largely responsible for encouraging the then emerging field of industrial psychology to focus on work stress. Subsequently, the study of work stress was "to generate volumes of research and create an enthusiasm for stress that has continued unabated to the present day" (Cooper and Dewe, 2007 p. 24). As a result, role overload along with role conflict and role ambiguity, known collectively as 'role stress', have become the most widely studied of occupational stressors (Beehr and Glazer, 2005). Researchers in the field of occupational stress tend to use a number of terms to talk about the subject (see Beehr, 1995; Cooper, Dewe and O'Driscoll, 2001). This thesis proposal will follow a similar pattern and use the terms job stress, work stress, organisational stress and occupational stress interchangeably for any reference to stress arising in an individual's workplace.

This section begins by reviewing the studies undertaken by the cohort of Kahn and his collaborators immediately after the introduction of the theory of role dynamics, as they continued their interest in exploring the impact of corporate organisations on the individual. The discussion in the second subsection then examines how the use of certain 
measures of the role stress construct negatively impacted the subsequent development of role overload research in the occupational stress field. This is followed by an exploration of how role overload evolved from a forgotten construct to be accepted as an independent and important work role stress construct. Nascent research highlighting the positive as well as negative effects of role overload will also be discussed in the third subsection. The section ends with a critique of the occupational stress literature that will highlight the key developments of, and gaps in, role overload research within the work stress literature.

\subsubsection{The studies of Kahn and colleagues}

The research groups at the Institute of Social Research, Michigan University, whose work gave rise to organisational role theory, continued to study the effects of role stress in large, formal organisations for some time after Kahn et al. published their seminal work. The frequent occurrence of role overload as an issue for respondents in Kahn et al.'s (1964) study was of particular interest to the doctoral student Sales who reanalysed Kahn et al.'s data with the specific purpose of differentiating the concept of role overload (Kahn, 1980) from other forms of role conflict. Sales (1969, p.425) selected three items from the original study, namely "the amount of pressure felt to do more work; the feeling of not being able to finish one's work in an ordinary day; and the feeling that the amount of work interferes with how well it gets done", to formulate an "improvised" index for role overload. He found that these items explained much of the effects previously attributed to role conflict in general. Further independent studies emanating from the University of Michigan researchers, and reported in French, Tupper and Mueller 
(1965 cited in Kahn, 1980), Sales (1969 cited in Kahn, 1980), and French and Caplan (1972), confirmed the findings on the prevalence of role overload and its independent relationship to symptoms of psychological stress. Such differentiation, Kahn (1980) concluded is "as necessary for understanding as for solving the pragmatic problems of job stress". In a review in the work and health section of their anthology, "The Study of Organisations", Katz, Kahn and Adams (1980) commented on these findings:

\footnotetext{
"The reinterpretation of much (of) role conflict as role overload is not an assertion that work situations are conflict free; it is rather a specification of what most role conflict in work situations is about-quantity versus quality, time pressures, quotas, magnitude of assignments, and the like.” (p.416).
}

The items selected to measure role overload by Sales (1969) and the comments by Katz et al. (1980) once again emphasise time constraints and volume of work as key to role overload, as well as their potential impact for individuals. Also repeated in their study and subsequent analysis is the acknowledgement that either quality or quantity must be sacrificed in such a situation. This latter observation is likely what prompted French et al. (1965), French and Caplan (1972) and Caplan, Cobb, French, Harrison and Pinneau $(1975,1980)$ to distinguish, in their own studies in the area, between two different types of role overload. When a person has more work than can be done in a given period of time, they designate this as quantitative overload, whilst work that is overloading because it exceeds the skills, abilities and knowledge of the individual is termed qualitative overload. Such a categorisation reflects the choices involved in dealing with role overload as suggested by Katz and Kahn (1966, p. 185) when they said 
that role overload is likely to be experienced by a role incumbent as "a conflict between quality and quantity". Katz and Kahn (1966) concluded that the role incumbent "must decide which to comply with and which to hold off". In terms of their role episode model, this choice is reflected in the role incumbent's perception of the sent role pressures (i.e., the received role forces) and the subsequent responses. Individuals may simply feel that their capabilities and resources are not adequate to fulfill all the work asked of them. In other words both qualitative and quantitative role overload are at play, suggesting that these concepts should not be disengaged.

Ilgen and Hollerback (1990) explored the qualitative versus quantitative overload concept further suggesting that each behaviour in any set of expectation can be described along two dimensions; a quality dimension and a quantitative one, such that roles are bounded on any particular qualitative dimension by the finite amount of time and effort that the individual can spend on the total set of behaviours constituting the role. Pienaar, Sieberhagen and Mostert (2007) have suggested also that the relationship between these two dimensions of role overload will be dependent on the nature of the work itself as well as the characteristics and abilities of the individual.

In the recent review of organisational role stress in the Handbook of Work Stress, Beehr and Glazer (2005) point out that the more usual definition for role overload is that used for quantitative overload. Other work stress researchers confirm this (see Newton and Keenan, 1987; Elloy and Smith, 2003). Certainly, studies in which qualitative and quantitative overload are distinguished and differentiated, appear much less frequently in the PsychInfo and Business Source Complete databases. Nevertheless, the level of 
interest in qualitative role overload within the relatively small pool of role overload research suggests an open mind is still required as to the nature of the relationship between quantity and quality of perceived expectations in a role overload situation. Although French and his colleagues and others more recently (eg. Pienaar, Sieberhagen and Mostert, 2007) have shown that the distinction can be empirically demonstrated, the views of the researchers described above suggest that the outcomes of role overload are dependent on the relationship between the two. In addition, although measures of both are typically based on subjective self assessment, quantitative overload is a measure against two environmental demands i.e. workload and specified deadlines, whilst qualitative overload assesses perceived personal abilities. In other words, in terms of the role episode model, qualitative overload is likely to generate a greater amount of internal role pressure. How internal and external role pressures interact is not well described in the role episode model. This suggests a closer examination of how role overload comes to be perceived is required to understand the relationship between quantitative and qualitative role overload. Examinations of the personal and organisational contexts of role overload will also help to understand this relationship. These suggestions provide additional support for the objectives of this thesis research.

\subsubsection{Role overload - the forgotten work role stress construct}

In the sixties and early seventies of the last century, the joint research program of the two groups from the Institute of Social Research, Michigan University, who continued the work of Kahn et al. $(1964,1966)$, was focused on work stress and the 
exploration of its impact on people's health. By the end of the seventies, however, the groups' attention shifted to job-related transitions, social support as a buffer against stress, and other factors that play a part in the management of stress (Frantilla, 1998). In other words, away from the effects of the individual role stresses: role overload, role conflict and role ambiguity. Despite the observed prevalence of role overload in a number of work situations and its clear differentiation from the role conflict construct demonstrated by these researchers, when meta-analytic reviews of work role stress studies appeared 20 or so years after Kahn et al. (1964), they portrayed a set of literature in which role conflict and role ambiguity were the only constructs of interest to researchers (see Fisher and Gitelson, 1983; Jackson and Schuler, 1985; Abramis, 1994). The early interest in role overload appeared to have fizzled out.

Of these three reviews, Jackson and Schuler (1985) do make passing reference to overload as a "form" (p. 42) of role conflict and also suggest future value in differentiating the multiple types of role conflict. Nevertheless, their selection criteria for the studies used in their meta-analysis omitted role overload research. Another metaanalytic review of the work role stress literature, which appeared in 2000, this time by Tubre and Collins, stated that their objective was to repeat the meta-analysis of Jackson and Schuler. Thus they followed the same selection process, again ignoring any research that had explored role overload. By excluding research on role overload from their metaanalysis sample selections, these reviews implied that role overload was either embedded within role conflict or was not an issue worthy of research. 
The reason for this apparent sharp drop of interest in role overload within the work stress field can be traced to researchers' choice of role stress measurement scales during this time period. It is not the primary purpose of this paper to investigate the validity or otherwise of role stress scales in the work stress field. The goal in this part of the literature review, nevertheless, is to examine how their use may have impacted role overload research. The role stress scales developed for the seminal study of Kahn et al. (1964) have been criticised because they specifically asked about job related tensions and were thus more likely to assess aspects of work that "bother" the individual themselves (Beehr, 1995 p. 110). In other words, they tended to assess the reactions to the role stressors as well as the actual stressors themselves. Rizzo, House and Lirtzman (1970) recognised this shortfall and noted the "lack of systematic measurement and empirical testing" of role constructs in studies of work stress (p. 150), which subsequently motivated their own development of measures of role stress.

Rizzo et al. (1970) drew on the work of Kahn et al. (1964) and Gross et al. (1958) to develop the items for their measures of role conflict and role ambiguity. They used the five components of role stress (see 2.1.1.2), as originally defined by Kahn et al. (1964), to develop and classify their role conflict scale. Whilst, these authors acknowledged that role overload was a type or "component" (Rizzo et al., 1970 p.155) of role conflict, they did not, however, make any attempt to differentiate between the types of role conflict by creating separate scales, as Caplan et al. $(1975,1980)$ and his colleagues from the Institute for Social Research would later do. Nor did Rizzo et al. (1970) differentiate quantitative from qualitative role pressures (Newton and Keenan, 1987). 
In the February 1983 Citation Classic review of the article that launched these measurement scales, Rizzo attributed the ongoing frequent use of the scales to their explanatory power and to the fact that role stress, in the form of role conflict and ambiguity, was pervasive in complex organisations. Their paper was published just as the interest in work related stress was rising (see Beehr, 1995) and, as Rizzo (1983) noted, researchers "needed the scale" ( $\mathrm{p}$ 22). An early literature review of the field by Van Sell, Brief and Schiler in 1981, reported that at least 50\% of work role stress research literature used the role conflict and role ambiguity scales developed by Rizzo et al. Four years later, the Jackson and Schuler meta-analysis claimed the figure was closer to $85 \%$. Many other role stress researchers since have confirmed the popularity of these measures (e.g. Newton and Keenan, 1987; Hardy and Hardy, 1988; Ilgen and Hollerbeck, 1990; King and King, 1990; Beehr, 1995; Tubre and Collins, 2000; Gilboa et al. 2008).

The scales created by Rizzo et al. in 1970 were the first to describe role stressors in terms of the demands and constraints produced by social interactions and as such were an improvement on earlier versions (Beehr and Glazer, 2005). Nevertheless, a careful examination of the development of the scales and the resulting measures reveals that Rizzo and his colleagues did not fully understand Kahn et al.'s $(1964,1966)$ idea of role overload or the other forms of role conflict. Rizzo et al. used a list of thirty questions to develop their role conflict and role ambiguity scales.

Included in this list of questions are items related to key elements of role overload already identified in this thesis. The items are:

\section{I have enough time to complete my work;}


15. I receive assignments that are within my training and capability; and

17. I have just the right amount of work to do.

Whilst these items are similar to those chosen by Sales (1969) to separate role overload from the broader role conflict construct, Rizzo et al. (1970) did not identify them with role overload, referring to them only as examples of person-role conflict. Items 1 and 17 were the only questions in the question set to refer to the amount of time available or the work load directly, but neither they nor item 15 were ultimately included in the final role conflict scale because of their weak loading on the role conflict factor in the factor analysis. Their weak association with role conflict should not be surprising given the results demonstrated by Sales (1969).

Rizzo et al.'s (1970, p.155) confusion about the definition of role overload is further illustrated when they referred to the following questions as "examples of role overload and inter-role conflict":

7. I am able to act the same regardless of the group I am with; and

19. I work with two or more groups who operate quite differently. Neither of these items reflect role overload as described by Kahn et al. (1964).

A number of other mismatches between Kahn et al.'s definition and Rizzo et al.'s item content were highlighted by King and King (1990) in their appraisal of role construct validity. Based on the inability of the item set to capture the full meaning of role conflict as intended, King and King concluded that the "scales seem to be lacking in the degree to which they represent the breadth of the role conflict (and role ambiguity) constructs" (p. 53). The clear misinterpretation of Kahn et al.'s (1964) definition of role 
overload and the absence of any quantitative items for an important type of role conflict, role overload, in the final scale, only confirms this view. Perhaps it was the confusion about the five components of role conflict as well as the urgency to create meaningful scales for the work stress research community that contributed to the decision to focus only on role conflict and role ambiguity.

Over the years, the Rizzo et al. (1970) role conflict and role ambiguity scales have increasingly come under scrutiny, largely because of the over dependency on these scales amongst the work stress research community (Netemeyer Johnston and Burton, 1990; Beehr, 1995). Criticisms have been levied at their psychometric properties, at the degree to which the items represent designated components (for examples see above), and at the difference in wording styles between them (i.e., items in the role conflict scale are positively worded whilst the role ambiguity items are all negative). This latter issue is seen as a potential source of method variance that may impact observed relationships (Ilgen and Hollenbeck,1991). Amongst the reviewers of the scales, Schuler, Aldag and Brief (1977), House, Schuler and Levanoni (1983), Kelloway and Barling (1990), and Gonzalez-Roma and Lloret (1998) all examined and re-confirmed the validity of the measures. Tracy and Johnson (1981), Netemeyer et al. (1990), King and King (1990), Ilgen and Hollenbeck (1991), Harris and Bladen (1994), and Gilboa et al. (2008), however, all suggested that these scales should be used with caution. The chronology of the rich literature debating the validity of these scales suggests that the prevailing view is against the use of the role stress measures of Rizzo et al. (1970) for work stress research. 
It is clear from these review articles that after their introduction by Kahn et al. (1964), role conflict and role ambiguity held centre stage in work stress research for many years. Furthermore, this review of the role overload literature has already demonstrated that role overload is not fully represented, if at all, by the items in Rizzo et al.'s (1970) role conflict scale. Soon after the Rizzo et al. scales were published, two of the three authors published a related article with the emphatic title "Role conflict and ambiguity as critical variables in a model of organizational behaviour" (House and Rizzo, 1972). This paper described the identical development process for creating the two work stressor scales as in their earlier paper. In other words, whilst House and Rizzo's (1972) definition of role conflict, once again identified the five components as defined by Kahn et al. (1964), as we know, role overload is not represented in this scale. Moreover, the choice of title for this paper was to position role conflict and role ambiguity as the only two intervening variables in their model.

In his 1995 book, reviewing psychological stress in the workplace, Beehr (p. 55) argued that this follow up paper by House and Rizzo and the "relatively unquestioned acceptance" of the Rizzo et al. scales for the two constructs (role conflict and role ambiguity) "helped keep researchers' attention on them for almost two decades". In other words, Beehr (1995) supports the idea put forward in this thesis that role conflict and role ambiguity dominated as the main causal variables of interest in the occupational stress literature, and that the role overload construct was either ignored or misrepresented. Another consequence of the focus on these two constructs at this time is that research examining the relationship between role overload and the other two role stresses was also 
discouraged. It is also true, however, as Beehr (1995) noted, that there were a small number of researchers who took the broader view to include role overload (and other workplace stressors) in their work stress studies during this time period. The next subsection will review the development of this work, and outline how role overload became a recognised work stressor in its own right.

\subsubsection{Role overload as an independent work stressor}

Beehr was another alumnus of the Institute for Social Research at the University of Michigan, who began a research career in organisational psychology in the mid 1970's. In his research, he made use of the Kahn et al.'s (1964) role stress concepts, and considered role overload as an independent construct. His research included the development of a self-report measure of role overload and found that it was related to a number of organisationally valued outcomes (Beehr, Walsh and Taber, 1976). Beehr (1995) explained the difference between the three role stress constructs (conflict,

ambiguity, and overload) using the language of organizational role theory (i.e. in terms of the activities expected of the role incumbents' by role set members). He postulates that the three constructs are clearly differentiated since role conflict and role ambiguity are caused by conflicting and ambiguous expectations, whilst role overload is "the result of a sum of expectations of activities that exceeds what is reasonable or possible" (Beehr, 1995 p. 86). This definition eloquently and implicitly encompasses the concepts of excessive workload, time restrictions, and skill and resource limitations. 
Beehr and other occupational stress researchers have since demonstrated relationships between role overload and work and coronary heart disease (Cooper and Marshall, 1976; Beehr and Newman, 1978); burnout (Beehr, King and King, 1986); somatic complaints (Osipow and Davis, 1988); absenteeism (Gupta and Beehr, 1979); tension at work (Keenan and McBain, 1979); individual coping strategies (Lang and Markowitz, 1986); job dissatisfaction (Beehr, 1981) and psychological stress (Lease, 1999).

Increasingly job stress researchers began to acknowledge role overload as "a central component of role stress" (Kelloway and Barling, 1990, p.738;) and also argued that role overload should be treated as a separate construct (Newton and Keenan, 1987; King and King, 1990; Beehr, 1995; Gonsalez et al. 1998). In their paper on work processes, Bacharach, Bamberger and Connolly (1990) argued strongly that without empirical evidence it should not be assumed that the predictors of role overload and role conflict are the same. In a rare study treating role conflict and role overload as dependent variables, they confirmed these two role stresses are associated with different work process variables and therefore "a distinction must be drawn between role overload and role conflict” (p. 221). Drawing on Kahn et al.'s (1964) definition of role overload as a separate factor of role stress, Schaubroek, Cotton and Jennings (1989) were one of the few to discuss and demonstrate role overload as an antecedent to role conflict in the workplace. Their study also demonstrated a link between role ambiguity and high levels of psychological strain. However, their findings on the relationship between role 
overload and role ambiguity were inconclusive. Schaubroek et al. (1989, p. 53) also affirmed role overload as a construct "distinct from role conflict".

In the 1990's the pervasiveness of role overload for a wide variety of employees became more evident in the role stress research literature, with many studies reporting detrimental effects for employee well-being (Jones, Chonko, Rangarajan and Roberts, 2007). Role overload began to be studied alongside role conflict and ambiguity. For example, Chang and Hancock's (2003) study of role overload and role ambiguity in new nursing graduates provides empirical evidence for the coexistence of these two role stresses as defined by Kahn et al. (1964). An examination of stress in the workplace found that work overload was mentioned more frequently by respondents as a source of strain than either role ambiguity or role conflict (Narayanan, Menon and Spector, 1999). More importantly, two recent meta-analytic reviews of literature summarising the research of role stress in the work environment (Örtqvist and Wincent's, 2006 and Gilboa et al.'s, 2008) purposely included role overload studies in their samples, affirming its significance as a role stress construct, and also that such research did not completely disappear. Furthermore, the results from theses meta-analyses confirm that the consequences of role overload vary across a spectrum of variables for each role stress type (i.e. role conflict, role ambiguity, and role overload each have different consequences) thus supporting the conclusion that they should be individually studied.

A final important development in role overload research in the occupational stress field is highlighted in the recent meta-analysis on work stressors by Gilboa et al. (2008), when their results revealed an inconsistent relationship between role overload and 
different aspects of job performance. The researchers suggest that this finding may indicate that, in certain circumstances, role overload could be viewed as a challenge and hence in a positive light. They also noted that these circumstances may be influenced by the life cycle of the organisation, suggesting that organisational context may impact how role overload is perceived. Gilboa et al.'s findings are consistent with Beehr (1995), who also noted, particularly in her research on family businesses, instances of role overload that did not seem to have stressful effects. In Beehr's 1995 book, she posed the following research question: "under what conditions do individuals take on extra work and not suffer negative consequences" (p. 86). The answer to this question, which is still unknown, is sought in this thesis.

A few more researchers have suggested other plausible reasons for why work role overload has been linked to positive as well as negative outcomes. In a study of the relationship between role stress (role overload, conflict and ambiguity) and work related outcomes for middle managers Keenan and McBain (1979) observed high levels of role overload. High role overload was not, however, associated with lowered levels of job satisfaction as predicted. The researchers proposed that managers may see overload as challenging and more specifically, for some, overload may be self-created rather than an inherent characteristic of the work environment. The association between Type A persons and role overload in the same study supports this view, in that the competitive outlook to work of such individuals may encourage overload.

Another example has been brought to light by Virick, Lilly and Casper's (2007) examination of how increased work overload of layoff survivors is related to job and life 
satisfaction. Contrary to their hypothesis they found a positive relationship between role overload and life satisfaction (after controlling for work life balance). Given the layoff circumstances, they suggested that being asked to perform a greater variety of tasks and the fact that the respondents were the only individuals available to do the work may lead to increased feelings of being 'needed' (p.474). An alternative explanation suggested was that after multiple waves of layoffs, extra work demands may increase the sense of job security and hence life satisfaction. Furthermore, additional responsibilities can elevate individual skills and thereby enrich job characteristics. The implication from these studies is that investigating the context of role overload of the individual experiencing role overload can provide more information about the experience and why it is different from person to person, and from situation to situation.

Before discussing how such answers might be found this review must complete the story of developments in role overload research to include research in multiple role situations. First, however, this review of work stress research will end with a critique and summary of the key factors affecting role overload.

\subsubsection{Critique and implications for the current research}

Early research of occupational stress tended to focus on the impact of the physical environment on the individual. Following the research conducted by Kahn et al. (1964), however, interest shifted to role stressors such as role overload, role conflict and role ambiguity. This emphasis still dominates the occupational stress domain today (Cooper, Dewe and O'Driscoll, 2001). Examining the literature published on occupational role 
stress as far back as 1960, Örtqvist and Wincent (2006) noted that apart from the few attempts to summarise the body of knowledge on occupational role stress (i.e., Fisher and Gitelson, 1983; Jackson and Schuler, 1985; Abramis, 1994; Tubre and Collins, 2000) the field appeared to be rather fragmented and short of cumulated research findings. They specifically observed that individual studies have tended not to use all three facets of role stress (i.e. role overload, role ambiguity and role conflict), but they ended their metaanalysis with the conclusion that role stress has "incrementally developed" to include all three (pp. 401). This review of the work stress research literature for role overload has established the reasons for this fractured pattern of research in the study of occupational role stress.

After an initial burst of research, which confirmed role overload as a distinct construct, the development and prolific use of Rizzo et al.'s (1970) scales for role conflict and role ambiguity had the effect of creating confusion about the relationship between the components of role conflict as defined by Kahn et al. (1964), whilst implicitly encouraging researchers to ignore the effects of role overload in the work stress arena for at least twenty years. With this in mind, the apparent disappearance and re-emergence of role overload as an independent construct in the set of role stressors represents a divergence from the tenets of organisational role theory, and a temporary loss of focus on a widely occurring phenomenon. The result is that role stress research, at least in the occupational stress field, has not rigorously followed Kahn et al.'s referenced theoretical formulation. Role overload research in particular, has been fragmented, and empirical results may have been confounded because of the failure to distinguish role overload 
from role conflict. Given the recognition by Katz et al. (1980) that much of the conflict in the workplace can be categorised as role overload, the focus should remain on this important form of role stress.

As well as the fragmented characteristics of the empirical research within the work stress field over time, a number of other factors impacting role overload research should be noted. First, the progress of role overload research in the occupational stress literature has been impeded by an overall lack of theoretical development of organisational role theory. Since its inception in the 1960's, the role episode model and its associated constructs have not been subject to any significant level of reconceptualization (George, 1993; Wickham and Parker, 2007). So comprehensive is its applicability that researchers appear complacent, failing to question or challenge its use in differing circumstances. Ilgen and Hollenbeck, (1990, p.198) recommended more "theory driven research", noting that frequently in work role stress research literature, there is little theoretical justification given for the constructs, and sometimes, they are introduced without definitions. George (1993, p. 355) observed that "the breadth and generality of (organisational) role theory may be both its major attraction and its greatest flaw". Second, although the analysis of role overload has highlighted excessive role demands; lack of available time; and limits in the personal capacities of the role incumbent to be key aspects of this phenomenon, how the quantitative factors and the qualitative factors interact when role overload is perceived as stressful is not clear. This requires a deeper understand of the stress process as it relates to role overload, which this thesis is proposing to reveal. Third, the recent reviews of work role stress research 
confirming differential relationships between the three role stressors (overload, conflict and ambiguity) and various outcomes, also indicate a need for further theorising and examinations of the causes for these variations. Fourth, the tentative signs that role overload at work can be seen in a positive as well as a negative light warrants further investigations. Finally, Newton and Keenan, (1987) noted that the vast majority of role stress research studies have essentially been of a quantitative nature, and that the contribution of qualitative information to our understanding of role stress, since the study of Kahn et al. (1964), has been very limited. Qualitative data offers greater opportunities to add more depth to existing theories or to develop new ones (Schonfeld and Farrell, 2009). A more open-ended exploratory approach is recommended particularly with regard to the conceptual clarification of the different forms of role stress (Newton and Keenan, 1987). This study will use open ended questions to collect qualitative data on individuals' experiences of role overload. In this way this research will hopefully contribute to a much needed re-examination of the theories underlying this important construct.

Kahn et al.'s (1964) typology of role stress was carefully crafted to accommodate one or many roles. Conflict between two or more roles, i.e. inter-role conflict, was explicitly ignored in their pioneering study of role stress and in subsequent work in the organization arena. It was suggested by Beehr (1995) that this was because of reluctance on the part of researchers in this area to move away from their key area of expertise, the work setting. Ironically, despite this substantive body of work, focussing on stress from the singular work role (i.e. intra-role conflict), it is inter-role conflict that has 
subsequently been the subject of most research in the role stress literature overall (Beehr, 1995). The progress of role overload research in the literature examining multiple role management is the subject of the next section.

\subsection{Multiple role management literature}

In 1977, in a policy review of work and family in the United States, Kanter challenged the prevailing "myth of separate worlds" (p. 115) when she argued that the boundaries between home and work life are permeable. According to Kanter it is important to be attentive to relationships outside of work as well as inside, in order to motivate and retain employees. Such realisations have been gradually reinforced by the continuing changes in demographics that western society has seen in the past thirty years in terms of the structure of families and the gender composition of the workforce. As a result, there has been an exponential rise in literature focusing on the relationship between work roles and roles occupied outside of work, in particular those within the family. The consequences of integrating work-role demands with the increasingly complex array of non-work roles enacted by employees became a focus of interest for many researchers (Wickham and Parker, 2007), with much of the research in the so called 'work-family' field having its roots in Kahn et al.' s (1964) theory of role dynamics (Bellavia and Frone, 2005).

It should be noted at this point that many scholars believe that the work and family literature, in which a substantive portion of the role overload research literature resides, should consider "all the work and non-work domains of life, not just work and 
family". Nevertheless, the name work-family is persistently used as a descriptor for this body of work (Pitt-Catsouphes, Kossek and Sweet, 2006). Given the focus on role analysis in this thesis, the broader concept will be assumed and therefore whenever the term work-family is used, unless specifically stated otherwise, it should be considered synonymise with multiple roles. The predominant focus on only work and family roles has also been criticized in reviews of organisational role theory (see Biddle, 1986, George, 1993, Wickham and Parker, 2007). Where this restriction has affected role overload research it will be specifically highlighted in the review below.

As discussed in the earlier sections, before the rise in academic interest in role stress at work, most studies and articles exploring role theory concepts, explicitly or implicitly, examined role stress and strain across multiple roles. Fundamentally, everyone has more than one role in life and therefore it is to be expected that researchers from many disciplines would be interested in the effects of excessive demands from life's major roles. Research into role overload experienced by individuals managing multiple roles has, however, been patchy (Duxbury et al., 2008) and inconclusive (Hardy and Hardy, 1986; Coverman, 1989; Hecht, 2001). Notwithstanding the general bias towards role conflict already noted in role stress research overall, there are also specific reasons for the patchy role overload research in the work family literature. One of these has been the confusion between the concepts role overload and role conflict, brought to light by Coverman in 1989. Much of this confusion is fuelled by the complexity of the interaction between role demands in a multi-role environment, firstly in terms of the close relationship between these two role constructs but also with regard to the role domains 
from which overload might arise. These issues have received little attention in research literature.

The work family literature is vast. "Its study embraces every social science and nearly every professional discipline" (Kanter, 2006). It is beyond the scope of this review to provide a complete analysis of this extensive body of work. This section, therefore, concentrates on developments that have affected or could affect role overload research in order to identify areas where the current research may be able to contribute. Recent reviews and overviews of the work family literature consistently refer to two opposing role perspectives (one negative, the other positive) in the approach to research within this field (e.g. Eby et al. 2005; Barnett, 1998; Perry-Jenkins, Repetti and Crouter, 2000; Barnett and Gareis, 2006; Thompson, Beauvais and Allen, 2006; LePine, LePine and Saul, 2007; Carlson and Grzwacz, 2008; Pleck, 1995). This section therefore begins by briefly reviewing these two points of view and their general effect on role overload research in the work family field. Following the overview of the two opposing role perspectives that have shaped research within the work family field, the third and fourth subsections explore to what extent the conceptual confusions mentioned above have been resolved in the work family literature, and how and why role overload research has been impacted. A fifth and final subsection critiques the current state of the multiple role research literature, summarising the key points as they relate to this thesis, drawing implications for how the proposed research should proceed. 


\subsubsection{The negative role perspective.}

The first role perspective within the work family field, which has overwhelmingly dominated the literature, assumes that "conflict is the inevitable consequence of multiplerole occupancy" (Barnett, 1998, p.146). This approach is grounded in the early work of social role theorists (eg. see references from Parsons (1951) and Merton (1957) in section 2.1.1). The most powerful factor provoking this point of view, however, was the persistence of the sex-role attitudes prevalent in the 1950s and 1960s regarding the primacy of men's role as the breadwinner and women's role as the homemaker and carer of the children. Thus, when married women's labour force participation rose sharply in the 1970s and 1980s, the assumed potential for greater conflict between work and nonwork roles, particularly for women, became a focus for social scientists and gave rise to the phrase work-family conflict (Barnet and Gareis, 2006). The result was a large body of literature now institutionalised as a unique field of research (Pitt-Catsouphes et al., 2005).

Kahn et al.'s (1964) role episode model and their typology of role conflict provided the ideal theoretical foundation for researchers following this perspective. As Kahn et al. (1964) predicted, work-family conflict is significantly correlated "with many indicators of well-being, in particular to indicators of psychological stress" (MacDermid and Harvey, 2006, p. 568). However, studying social roles as distinguishable sets of obligations and responsibilities subject to incompatible role pressures, focused research onto a very specific type of role conflict and thus in general, away from role overload. The work family conflict construct is thus fundamental to this role perspective in which work and family are considered as distinct and conflicting spheres. The construct has 
been further broken down into two components, work to family and family to work, indicating the direction or interference of the conflict. These two components have been found to be differentially related to outcome variables (Frone, Yardley, and Markel, 1997) suggesting each will have a unique relationship with role overload.

According to the negative perspective, individuals have a finite amount of time and energy available to them, a factor that will be of particular importance to the study of role overload. The consequence of this limitation is that role demands from participation in one role will deplete the resources available to the others, thereby negatively affecting them (LePine et al. 2007). The negative perspective seems to be entrenched in most people's psyche (Carlson and Grzywacz, 2008). The idea that role resources are finite is referred to as the scarcity hypothesis (see Sieber, 1974; Marks, 1977) and was originally derived from Goode's (1960) paper on role strain (see section 2.1.1.1). Goode (1960) also postulated that individuals will tend to over-perform at work and under-perform in other roles because of the relative value placed on work by society, further emphasising the influence of social attitudes in the growth of this field. The focus on scarce resources not surprisingly led researchers to position role overload as one of the (many) antecedents and consequences of work family conflict (see Byron, 2005 for a review of work family conflict antecedents).

The overall approach of the negative role perspective draws on organisational stress research, and as a consequence one of the most popular research themes within the conflict literature explores how role stress at work (including role overload) impacts 
employee behaviour and well being off the job (Perry-Jenkins et al. 2000; Eby et al. 2005).

\subsubsection{The positive role perspective}

Supported by a growing body of research that indicates in general multiple roles are beneficial to individuals (see Barnet and Hyde, 2001 and Greenhaus and Powell, 2006 for reviews of this literature), the second role perspective in the work family field contrasts sharply with the conflict approach described above. It was the in depth analysis of Goode's (1960) article (first by Sieber, 1974 then by Marks, 1977), to counter Goode's focus on role system resource limits, that stimulated research into resource expansion and the potential benefits of multiple roles. Marks (1977) argues that it is the individual's relative level of commitment to roles that will determine the level of role strain, and also how energy and time are utilized, rather than the influence of some external cultural ranking system. Individuals will decide how to use their time and energy, and will manipulate roles and resources to find extra energy for each role according to their role commitment and their desire to limit role overload (Marks, 1977).

According to this positive perspective (also referred to as role enhancement, work family enrichment, positive spillover, and work family facilitation), multiple roles can produce positive outcomes in three ways. Firstly, work and non-work experiences can independently increase well being (Barnett and Hyde, 2001); second positive experiences in one role can buffer the mental health consequences of another (e.g. Barnett and Baruch,1985) and third, positive experiences from one role can be transferred to a 
different role (e.g. Marks, 1977; Greenhaus and Parasuraman, 1999). In a recent review of measurement in work-family research, Carlson and Grzwacz (2008) suggest that these three concepts need to be clearly defined and distinguished to enable the theory development and measurement that is currently lacking for this perspective. Notwithstanding this criticism, research has shown consistently that men and women appear to benefit from undertaking multiple roles (Barnett \& Gareis, 2006). Cause and effect are not clear, however, since mentally healthy people may take on more work simply because they have the capacity to do so (Doress-Worters, 1994 cited in Wells and de Vaus, 2003).

In terms of the type of extra role demands that can improve well being, research in this set of the work family literature clearly shows a contrast with the traditional sex role assumptions that initially accelerated research in the conflict perspective (i.e. adding the worker role appears to be beneficial for women, whilst engaging in family roles is good for men (Barnett and Hyde, 2001)). Other research, on quantity versus quality of roles, suggests that subjective role quality (i.e. the degree to which role conditions are perceived as rewarding or problematic) is a greater predictor of mental and physical health than the number of roles (Barnet and Gareis, 2006). Lease (1999) and recently Pearson (2008) found that role overload was a powerful predictor of psychological health and more significantly, in line with the findings regarding subjective role quality, both authors also concluded that the subjective appraisal of being overwhelmed was likely to be more important than an objective measure of the number of roles and demands. Despite these findings, Barnett and Hyde (2001) surmise that there are limits to the 
benefits of multiple roles. More specifically, they posit that overload may lead to distress when the number of roles is too much or the number of demands is excessive. Thus, in this second role perspective of the work family literature, role overload is seen as an indicator that the benefits of multiple role occupancy are failing or diminishing. Based on this viewpoint of role overload, the construct is commonly used as a measure for role stress or strain along with role conflict (eg. Barnett and Baruch, 1985; Marks \& MacDermid, 1996). In a field where the analysis of multiple role benefits is the focus, little attention has been paid to role overload and the nature of its relationship with these positive outcomes.

\subsubsection{Role overload or role conflict?}

Within the body of literature that resulted from the upsurge in academic interest for work and family issues, the definition of work-family conflict most often cited is that of Greenhaus and Beutell (1985). Their article is also the most referenced in the work family field (Masson, 2001). Using Kahn et al.'s (1964) work as the basis for their denotation, Greenhaus and Beutell (1985) describe work-family conflict as "a form of inter-role conflict in which the role pressures from the work and family domains are mutually incompatible in some respect. That is, participation in the work (family) role is made more difficult by virtue of participation in the family (work) role" (p. 77). This definition has guided most of the research on the friction between work and family life since (Bellavia and Frone, 2005; MacDermid and Harvey, 2006; Carlson and Grzywacz, 
2008). It has also been the source for much of the confusion between role overload and role conflict.

Citing the potential competition for an individuals' time from multiple roles, Greenhaus and Beutell (1985) identified time-based conflict as one of the three major types of work-family conflict. Moreover, Greenhaus and Beutell (1985, p. 77) observed that time-based conflict is "consistent with .... role overload identified by Kahn et al. (1964)". The implication is that time based work-family conflict is indistinguishable from role overload thereby contradicting Kahn et al.'s definitions. The other types of workfamily conflict proposed by Greenhaus and Beutell (1985) are strain based (from anxiety, fatigue, and depression) and behaviour-based (due to incompatible behavioural expectations). Although these other aspects of work-family conflict have been identified in a number of studies, most research within the literature focuses on competing time demands (Barnett and Gareils, 2006). As a result, the concept of role overload was closely linked to Greenhaus and Beutell's definition of work-family conflict and was thus often buried within this fundamental construct in the work-family literature. By examining the terminology in more detail we can see how this has led to confusion in the field.

According to Greenhaus and Beutell (1985), time-based conflict generally arises when "time-pressures" within one role make it difficult to meet the demands of other roles (p.78). The phrase 'time pressures' strongly suggests deadlines and time limits, both of which are indicative of role overload. However, if an individual has too many role demands (from one or many roles) and too little time to fulfill them this will lead to 
conflict between roles if, and only if, the requirements of one leave no time to deal with demands of the other(s). In other words, role overload may or may not lead to inter-role (or specifically, work family) conflict, which supports the idea that these phenomenon are different and separate concepts (Coverman, 1989). The conceptual confusion is further exacerbated because of the tendency of work family researchers to use the term 'role conflict' as a synonym for 'work-family conflict' often with no explanation or clarification (e.g. see Pearson, 2008). This is likely to be because of the lack of interest in intra-role conflict within work-family research where the management of multiple roles is the fundamental focus. Given that role overload is also a unique type of 'role conflict' however, if the specificity of work-family conflict is not highlighted or clarified, then this may lead to further misconceptions.

Their ongoing research later led Greenhaus and Beutell to clarify the nature of the relationship between work-family conflict with role overload. Specifically, in an investigation of the antecedents of work family conflict, Greenhaus, Parasuramen, Granrose, Rabinowitz and Beutell (1989) included the work stressor, role overload. Although they failed to explicitly define the concept, they described it as "pervasive across organisations and occupational groups" (p. 135). Using items from the role overload measure developed by the associates of Kahn (Caplan et al., 1975), Greenhaus et al.'s (1989) results demonstrated that quantitative work role overload is a significant source of time based and strain based work family conflict. Nevertheless, these researchers still persist in referring to work role overload as "an example" of time based work family conflict (Parasuramen and Greenhaus, 1997, p. 4) rather than as an important 
construct in its own right. The result is more confusion, which continues in contemporary literature.

Not surprisingly, in the search to understand the relationship between multiple role demands and stress, role overload and role conflict have been frequently confused and sometimes used interchangeably (Coverman 1989; Hecht, 2001; Duxbury et al., 2008). Coverman (1989) and Hecht (2001) further report that results from studies of these two role stress concepts have been inconsistent and therefore clarifying the different processes is "essential to the task of resolving those inconsistencies" (Hecht, 2001, p. 112). Duxbury et al. (2008) have noted that the term role overload has, at times, also been transposed with 'role strain' and 'role stress'. The confusion between role strain and role overload arises directly from Goode's (1960) allusion to role overload in his description of the broader concept, role strain. A brief scan of the literature reveals other instances of imprecise terminology in reference to role overload. For example, Voydanoff (1988) uses 'role overload' and 'workload pressure' interchangeably in the article in which she reports on her study of work role characteristics and work family conflict, whilst in a recent meta-analysis of work family models, Michel, Mitchelson, Kotrba, LeBreton and Baltes (2009) report that the conceptual definition for time demands in both the work and family domains, is "often confused" (p. 201) with role overload, and Repetti, Matthews and Waldron (1989) refer to role overload across life's roles as 'multiple role strain'. In another meta-analytic review, this time of work-family conflict and its antecedents, Byron (2005) reports that role overload and role ambiguity are routinely combined in measures of overall job stress. This continuing confusion about terminology further 
hinders the development of role overload as a separate and independent construct (Duxbury et al., 2008) and is also masking the important nature of the relationship between role overload and role conflict.

To clarify the difference between role overload and role conflict, Coverman (1989) explains that whilst a person may experience incompatible demands from two or more roles, "unless time pressure is an issue, he or she will not necessarily encounter role overload" (p.968). Logical compatibility of demands was key to Kahn et al.'s original definition of role overload and is also emphasised by Duxbury et al. (2008) who argue that role overload occurs "even when role demands are compatible, simply because the individual does not have sufficient time and energy to fulfil them all” (p.129). Hecht (2001) also argues that the experience of role overload arises from a different source to that of role conflict (i.e. work family conflict) because of the unique and overarching temporal limitations impacting the experience of role overload. The studies of both Coverman and Hecht confirm varying consequences for role overload and role conflict whilst Hecht's (2001) study results indicate that each of the two constructs are affected by different contextual factors. In this way, Hecht's work illustrates the "importance of precise hypotheses driven by theory" (MacDermid and Harvey, 2006, p. 571). Both these studies confirm that when role overload is carefully and clearly distinguished from role conflict, the two concepts can be empirically differentiated.

With regard to the links between the two concepts, unlike the occupational stress literature, in the work family literature a substantive number of articles have explicitly investigated the relationship between work-family conflict and role overload. For 
example, in a study of over 1000 married men and women, Voydanoff, 1988 demonstrated that role overload at work was one of the work role characteristics most strongly related to work family conflict. Other authors that have demonstrated an association between work role overload and heightened work family conflict include Greenhaus et al. (1989), Bacharach, Bamberger and Conley (1991), Parasuraman, Purohit, Godshalk and Beutell (1996), Wallace (1999), and Fu and Schafer, (2001). In a study of role overload amongst nurses with diverse role sets, role overload was found to be a function of role conflict and frequency of interaction (Arndt and Laeger, 1970 cited in Hardy and Hardy,1988). Most comprehensively, Frone Yardley and Markel (1997) developed and tested an integrative model of the work-family interface through which they argue that work role overload and family role overload have direct and indirect effects on work to family conflict and family to work conflict respectively. Furthermore, each of these bi-directional types of work-family conflict was shown to have a between domain relationship with intra-role overload in the other domain (i.e. work to family conflict is directly related to family overload, and family to work conflict is linked to work overload).

Byron's (2005) meta-analytic review of the antecedents of work-family conflict selected only those articles that investigated both work to family and family to work conflict, to ensure consistent comparison of samples. She identified ten studies (from a total of sixty) that included the antecedent work role overload, and concluded that this type of job stress is impacting employees' lives outside work through work to family conflict. Although Frone et al. (1997) demonstrated the complementary effects from the 
family to the work domain, Byron (2005) was unable to identify sufficient studies (defined as five or more) to support this finding. Similarly, Byron's review failed to find any support for overload from the sum total of all roles as an antecedent to work family conflict. Overall however, this body of work briefly reviewed in these two paragraphs, confirms the importance of the relationship between work-family conflict and domain based role overload, particularly that of the work domain.

\subsubsection{Sources of role overload.}

Another important issue to arise from the discussions about the representation of role overload in the work and family literature is that few researchers have differentiated the sources of role overload or acknowledged that there may be a difference between role overload within a single role and the total effects of too many demands across an individual's multiple roles.

In their discussion of role theories, Hardy and Hardy (1986) identified role-set diversity (i.e. too many demands from the role set of one role) and multiple roles as the two primary sources for role overload. This reflects Kahn et al.'s (1964) theories that originally proposed role overload as a special type of either intra-role or inter-role conflict. This implies that overload can arise in the separate domains of work or family, or may result from the accumulation of demands from several roles. In the ensuing research literature, Coverman (1989) and Hecht (2001) for example, both suggest that role overload is most likely to arise from fulfilling several roles, but used a generic definition without reference to the source (i.e. having too many role demands and too 
little time to fulfill them). Given their objective of differentiating role overload from work family conflict, their focus was on role issues arising from two domains. However, their secondary objective, to reduce inconsistencies in research results by clarifying the constructs, may also benefit from differentiating between the sources of role overload. When first describing the sources of conflict between work and family roles, Greenhaus and Beutell (1985), omitted a specific definition for role overload, but emphasised the concept as emanating from a single role, a viewpoint that is consistent with their later work and the work of others investigating antecedents of work family conflict. Many of the studies in the work family field that include the variable role overload focus on the domain based construct, particularly in research from the conflict perspective. Even Frone et al. (1997), whose study was intended to create a model of the interface between two roles and therefore investigated antecedents and consequences arising from family and work, specifically include only domain based overload in their model. As Duxbury et al. (2008) point out, Frone et al. (1997) failed to consider role overload in its totality. As noted above, this variable also failed to turn up in any meaningful numbers in Byron's (2005) meta-analytic review of work family conflict antecedents studied in the work family literature. Duxbury et al. (2008) explain that "total role overload" can result from "too many roles, including non-work and non-family roles, or excessive demands in any number of roles" (p. 138). Moreover, they argue that even if intra-role overload is not present, the sum of the demands across multiple roles could cause (total) role overload. 
The concept of excessive demands from an individual's total complement of roles was, as we know, the subject of discussion in the literature before Kahn et al. (1964) introduced the role overload term. This review has already explained that Goode (1960), although not explicit in his reference, was an early proponent of this concept. MacDermid and Harvey (2006) argue that Greenhaus and Beutell (1985) and their followers focus on the relationship between specific roles in studies of role stress, whereas Goode (1960) focused on the role system as a whole, and therefore from his perspective, total role overload is more significant for psychological well-being. Another paper co-authored by MacDermid, this time with Mark in 1996, suggested that Goode's holistic approach has been neglected in the literature. Mark and MacDermid (1996) propose that examining an individual's approach to their role system as a whole could provide a greater understanding of the relative experience of role strain (as measured in the study by total role overload). Their research confirmed that people who consciously and actively maintain balance across their entire system of roles experience less role strain and more feelings of well being, thus highlighting the potential significance of role overload to the total role system. The role enhancement perspective is very much in keeping with Goode's role system approach, and thus within the associated research, where role overload has been included as a variable, it tends to be defined in terms of the sum total of demands because of its use to measure strain in the role system.

This review identified very few articles in the work family literature that explicitly acknowledge that role overload can arise from a single role domain and/or from the total role system. Moreover, including measures of more than one type of role 
overload, based on their role domain source, is rare. For example, in their article examining the predictors of job involvement (and its association with absenteeism), Thompson and Blau (1993, p. 636) expanded "the usual definition" of role overload to refer instead to "the perceptions that there is not enough time for work and non-work roles". They chose to extend the domain of the variable because of the increasing evidence that individuals are suffering role stress across work and non work roles, and thus they inferred that role overload is typically assumed to occur in our work role only. Thompson and Blau's (1993) study did not, however, examine the effects of domain based role overload for the work role domain or any other.

In another paper, this time reviewing the empirical literature covering the impact of employment on women's health, Repetti, Matthews and Waldron (1989) included role overload from two sources (i.e. the individual's total set of roles and the work role) as important mediators potentially linking their two variables of interest. They referred to the two role overload constructs as "multiple role strain" and "heavy job demands" (p.1394). Using these terms, the paper also contributes to the confusion surrounding the definition of the role overload construct, as discussed in the previous section. Repetti et al. (1989) were able to conclude that overload from heavy work demands increases health risks for women. The evidence was, however, mixed regarding the impact of role overload from the total set of roles - i.e. 'multiple role strain'. In their analysis of these inconclusive results about the impact of total role overload on employed women's health, they referenced an earlier study of women in mid-life by, Barnet and Baruch (1985). Barnet and Baruch's objective was to identify which role types contribute to total role 
overload and was thus also one of the few studies examining the source of the construct. They found that specifically, the occupancy of the parental role was linked to role overload. Nevertheless, Repetti, Matthews and Waldron (1989) reported that the other cross-sectional and longitudinal studies selected for their meta-analysis had failed to confirm Barnet and Baruch's (1985) finding that employment has less beneficial effects for mothers. Repetti et al. (1989) concluded that study numbers in their review were too small to make firm conclusions about the relationship between role type, employment and multiple role strain. The overall lack of research, and the inconclusive evidence from the few studies that have explored the nature and impacts of role overload arising from variable sources, suggests that future studies should acknowledge the potential difference between the source of role overload. This research hopes to address this shortfall.

\subsubsection{Critique and implications for the current research.}

The predominant focus of research as outlined for the two role perspectives described at the beginning of this section has drawn research in the work family field to role conflict first, then to role enhancement. The demonstrated research bias throughout the field, in the research of both role perspectives, strongly supports the notion that the role overload construct has been neglected and sidelined as a significant construct in studies of multiple role stress. The term 'role overload' is not even indexed in the 2006 Work and Family Handbook and 'overload' is discussed only briefly to illustrate the differing effects of role conflict and overload as demonstrated by Hecht (2001). Although she championed role overload as a distinct construct, Hecht's (2001) study has even been 
interpreted as downplaying its effect because role conflict was shown to be more difficult to manage (MacDermid and Harvey, 2006). The current role overload research will help address this imbalance as it focuses research on individual experiences of role overload. Another broader criticism that can be made of the work family field is that work family researchers continue to pay little attention to developing or testing theoretical models of the work-family interface (Eby et al., 2005). In particular, Eby et al. (2005) noted a limited number of exploratory studies. Duxbury et al. (2008) also argued that few, if any, work family researchers have attempted to develop a theoretical model of role overload, and the concept tends to be subsumed in the models of related constructs such as work family conflict and role strain. This is consistent with the dominant research paradigms in the literature as described above. Using an exploratory approach, the current research is intended to contribute to the theoretical development of role overload as a concept, and hence the theoretical development of an important part of the relationship between work and family.

Despite the conceptual confusion surrounding key constructs, the lack of theoretical development of the work family interface, and the research bias within the two role perspectives, role overload has nevertheless proved to be an important variable in certain areas of work and non-work life research such that it cannot easily be dismissed when considering the effects of multiple roles on individuals. First, in multiple role environments, role overload has been shown to be an independent construct separable from simple forms of role conflict. Second, work role overload has been shown, in a 
number of studies, to be an antecedent of work family conflict. Initial results indicate family role overload also has a similar effect.

Much of this section has dealt with the inconsistencies and deficiencies in the conceptualisation of role overload in the work family literature, which have continued to plague the role stress literature and hindered the overall progress of role overload research. When considered in the context of the themes driving the two work family perspectives, the failure to rigorously distinguish role overload from role conflict is not so surprising. Notwithstanding the ongoing debate about the role conflict approach versus the role enhancement approach, as Hecht (2001) concluded, making careful distinctions between role overload and role conflict is important for "building and testing theories aimed at understanding the processes by which multiple roles influence psychological well-being”.

In her paper differentiating role overload and role conflict, Hecht (2001) suggested that role conflict was more difficult to manage because conflict is often the result of simultaneous incompatible demands. On the other hand, she also correctly predicted that time spent in roles is more strongly related to overload than conflict. This strongly suggests that role overload is a chronic condition, which, as MacDermid and Harvey (2006) acknowledge, will remain untouched until work hours are reduced. The connection between this chronic condition and volume of demands also suggests that a reduction in workload could reduce role overload. Moreover, given the relationship between role overload and role conflict across work and family domains demonstrated by 
Frone et al. (1997) and others, understanding the circumstances that lead to role overload could help reduce the incidence of work family conflict as an acute condition.

Two key interrelated points arise from these issues that must be incorporated into any investigation of role overload. They are expressed concisely and collectively by Coverman (1989 p. 968,) when she explains that role overload and role conflict (i.e. interrole conflict) "overlap, but it is important to maintain their conceptual and analytical distinctions" because of the differences in their sources and effects. The latter point mirrors the conclusions already reached in reviewing role overload research within the occupational stress literature. It is important, however, particularly for the qualitative research strategy proposed for this study, to be cognisant of the relationship between the two concepts. Nevertheless, to minimise inconsistencies in empirical results regarding the outcomes of work family conflict and role overload, the constructs and sources must be clearly defined and agreed upon by the community of researchers. The research proposed in this paper is based on a multiple role based definition of role overload as presented in the introduction to the role overload literature review. The case study research proposed will involve in depth investigation of role episodes. Given the close links between role overload and work family conflict illustrated in this section of the literature review, it is possible that this form of role conflict may also be an issue. With the clear definitions presented here, this research may lead to a better understanding of the nature of the relationship between these important constructs.

The third way in which role overload demonstrates its potential value to the work family field is that role overload is an important indicator of the health of a role system 
and in the research literature of the role enhancement genre, role overload is, as Goode (1960) suggested, considered to be more important to the overall system than role conflict. Related to this, but significant in its own right, is the indication that the source of role overload could have a differential effect on an individual's well being. Evidence is growing that research focusing on the relationship between total role overload and general life outcomes is on the increase (Glyn et al. 2009; Pearson, 2008).

Overwhelmingly, however, in the field of work family research, researchers have not taken into consideration that domain based role overload and total role overload could be independent, or separate but related concepts. Whilst these omissions do not detract from the results of the studies into work-family conflict antecedents, multiple role management, or the importance of distinguishing between role overload and role conflict, they do raise questions about the general approach to role overload research. The failure to acknowledge the potential for multiple sources of role overload suggests that when the construct is included in work family research, the conceptualisation may be incomplete. The result is that the nature and context of role overload, which may vary with the source, is not accounted for in studies of the work family interface, or in research into the management of multiple roles.

The roles of individuals, both men and women, are increasingly seen as overlapping, rather than separate spheres (MacDiarmid \& Harvey 2006; Barnett, 1998) and therefore, evaluating the totality of an individual's role system would also make more sense, particularly for the study of role overload. Bearing in mind the shortfalls in research identified for role overload within the occupational stress field and the intended 
exploratory approach, the current research will assume a total role system approach to ensure that the full impact of this type of role stress is accounted for.

A final point to highlight in the multiple role management literature is that the role enhancement perspective raises the notion again that the subjective view of the role environment is particularly important to the experience of role overload. This paper has already noted that further investigation of how role overload is perceived as stressful is required. Before this literature review begins the investigation of stress research, the last section of the role overload literature review will examine instances of role overload that have been linked to positive outcomes.

\subsection{Summary of the role overload literature review}

There is sufficient empirical research spread across the past 45 years to illustrate that role overload is an important factor in people's individual role sets as well as across the multiple work and non-work roles that people now regularly juggle. In a recent Canadian study, Glynn, Maclean, Forte and Cohen (2009) demonstrated the importance of role overload to the totality of role experiences for an individual, whilst in a large European study, Schulz, Wang and Olsen (2009) confirm that role overload remains an issue within the single role domain of work. The review of role overload research has confirmed that role overload is a singularly important type of role stress. Early research on role theory development also revealed that role conflict dominated before role overload was explicitly defined. This established a research culture in which role overload was the second class role stress variable and helps to explain why role overload 
was often misrepresented in the role stress research that followed. Despite the fragmented nature of role overload research, a clear picture of the characteristics of this phenomenon has emerged from this literature review. Based on Kahn et al.'s definition and the subsequent research reviewed here, role overload can be said to be characterised by time and energy constraints, excessive role demands, framed by the subjective views of the individual about the situational and personal context.

Since the work of Kahn and his fellow researchers from Michigan University was published, the focus of subsequent research on role stress and role overload has been predominantly empirical and there has been little attempt to develop the related theory (Hardy and Hardy, 1988; King and King, 1990; Eby, 2005). Unfortunately, existing approaches to role analysis are increasingly seen as inadequate for explaining the complexity of the relationship between role overload and stress. The importance of the environmental and personal context in shaping the subjective perception of excessive role demands has also been largely ignored - yet these are areas that can help explain how and why role overload affects people differently. To gain the insight into the role episode not afforded by Kahn et al., this review strongly suggests that an alternative theoretical framework is required to reveal how stress can arise from mismatching role expectations. The next section will review the evidence in the stress literature for an alternative approach to role overload research. 


\section{Cognitive stress theory}

Thoits (1995b) argues that the theoretical mechanisms to connect social structural variables, such as role overload and other role stresses, to cognitions, emotions and actions are typically supplied by the work of psychologists. The cognitive model of stress, as originally proposed by Lazarus (1966) and developed fully in the book "Stress, Appraisal and Coping" by Lazarus and Folkman (1984), has guided stress research for over twenty years (Amatea and Fong, 1991; Thoits, 1991; Cohen, Kessler and Underwood Gorden. 1995; Monroe and Kelley, 1995; Cooper, Dewe and O’Driscoll, 2001; Ice and James, 2006). Furthermore, the definitions of stress, cognitive appraisal, and coping put forward by Lazarus and Folkman are the most widely quoted in the literature (Tennen et al., 2000). This widespread influence of the cognitive model of stress is not, however, reflected in role stress research. The objective of this section is to examine how the theories underpinning cognitive stress can help explain the process enacted in a role overload episode.

The review of this literature is divided into four parts. The first section briefly covers the history of stress research. This part clarifies important concepts relevant to this thesis as well as explaining the basis for the thinking behind the current stress paradigm. The second section explores in detail the theory behind the cognitive model of stress and its potential to explain individual differences in response to stressors such as role overload. The third section of the literature review summarises the strengths and 
weaknesses of the cognitive model of stress. Chapter 3 concludes by returning briefly to the role stress literature to examine proposed applications of the cognitive stress model.

\subsection{Stress}

Stress has been called one of the most important medical, psychological, and social phenomena of our time (Fink, 2000). Certainly, we only have to open the newspaper to realise that there is significant interest in the subject, both amongst the public and academics. A search of the ISI Web of Knowledge Social Sciences Citation Index database, using 'stress' as the topic, uncovered over 84,000 journal articles published since 1956, with some 30,000 published since 2005 alone. This supports the idea that stress research has been and still is a fruitful area of research. Historically however, researchers have frequently disagreed as to the precise meaning of stress (Cohen, Kessler and Gordon, 1995; Levi, 1998; Cooper, Dewe and O’Driscoll, 2001; Monat, Lazarus and Reevy, 2007). Even in common use and as defined in the Oxford English Dictionary, stress is both a noun and a verb. As a noun it can refer to an external event or an internal state, and as a verb it can either be active, i.e. 'to stress', or passive, i.e. 'to be stressed' (Aldwin, 2007).

In academia, the confusion over terminology is exacerbated by the breadth of application of the stress concept across the fields of medicine, behavioural and social sciences. Some have defined stress as a stimulus, others as a response, and still others as the result of an interaction between the two (Cox, 1978 cited in Cooper et al., 2001). In their anthology of strategies for measuring stress, Cohen et al. (1995, p. 3) argue that 
despite the variety of approaches across the multiple research fields, all share a common interest in "a process in which environmental demands tax or exceed the adaptive capacity of an organism, resulting in psychological and biological changes that may place persons at risk for disease". A review of how stress research has developed over the years will help explain the apparent diversity of approaches and provide clarification as to the meaning of stress.

Detailing the research history of the stress concept as a human problem, Levi (1998) attributes its source to clinical experiments undertaken by the young AustroHungarian scientist Seyle in the 1930's, in which he had noted a base similarity in the physiological reactions of rats subject to a wide variety of stimuli. Levi (1998) further explains that disagreements about the definition of stress initially arose because of a misinterpretation of engineering terminology by Selye when he was seeking a suitable description for his newly discovered biological phenomenon. In engineering terminology, stress is described as 'a force which deforms bodies' whilst what happens in the bodies in question is generically called 'strain'. Focusing on this latter concept, Seyle compared the non-specific responses that he observed in his experiments to the tension in the girders of a bridge when subject to variable forces from crossing trains, lorries, or cars. He believed, incorrectly however, that this tension was called 'stress' and named the phenomenon accordingly. Thus to Seyle, stress is the "lowest common denominator in an organism's reaction to every conceivable kind of stressor, challenge and demand" (Levi, 1998, p. vi). In other words the stressor is the stimulus and stress is the reaction. Seyle argued that pathogens, physical stressors and psychosocial stressors all produce the same pattern of 
physiological response (Cohen et al., 1995). In his book on the "Stress of Life', Selye (1956, p. viii) also referred to stress as "the rate of all the wear and tear caused by life". Seyle's focus was clearly medical and from this early work he developed a model describing the stress related response pattern as the general adaptation syndrome or GAS (see Seyle, 1956). He described three stages of response within the GAS: alarm, resistance and collapse. The alarm stage is the initial psycho-physiological response, in which defense mechanisms such as the well known 'fight or flight' responses are activated to prepare the body for action. For example, due to changes in hormonal levels, the heart rate and blood pressure increases, and blood supplies are directed to the brain and skeletal muscles. The second stage is resistance, or the "ability to stay healthy during intense stress" (Seyle, 1956, p. 187). If the alarm reaction is too intense or continues over an extended period, the energy required for adaptation becomes depleted leading to the third stage, collapse, exhaustion, or even death. It is interesting to note that in his later work Seyle (1976) suggested that stress reactions are not automatically bad. He suggested that there may be a pleasant stress of fulfillment (eustress) without the harmful consequences of damaging stress (distress). His hypothesis is, however, vague and has not been supported by empirical research (Cohen et al. 1995; Lazarus, 1999)

Although Selye is often acknowledged to be the first to systematically research stressful life events, the general adaptation syndrome model has been criticised for being too simplistic (Cohen et al. 1995; Cooper et al. 2001; Monat and Lazarus, 2007). Critics have also argued that responses to stimuli do not always follow the GAS pattern whilst circumstances that might not typically be considered stressful, such as heavy exercise, 
can produce a similar pattern of responses to those described in the first stage of GAS. Furthermore, it does not address psychological responses, the relationship between psychological and physiological reactions, or the possibility that responses can become the stimulus for other responses. Pearlin, Lieberman, Menaghan, and Mullan, (1981) argue that stress can have many manifestations, and dissent exists as to where in the organism stress is manifested: at single cell, organ, system, or organism level; in terms of biochemical, physiological or emotional functioning; or in particular diseases, either physical or psychological. Such a variety in stress indications is not reflected in Seyle's approach. The response based approaches have also been criticised for ignoring "the stimulus dimension of stress experiences", such as environmental factors that elicit these responses (Cooper et al., 2001, p.7)

Notwithstanding the criticisms, Seyle's work inspired other researchers to examine the links between life events and psychiatric illness, and also had considerable influence in the rise of interest in stress and its effect on psychological and physical health (Cohen et al. 1995; Cooper and Dewe, 2007). The attentiveness to stress was growing concomitantly during World War II after 'battle fatigue' was recognised as a combat induced psychological disorder (Lazarus, 1999). After World War II, stress became a subject of interest to the wider community, not just to the military. Lazarus, (2007) explained that there were two reasons for this ongoing growth in the interest in stress research. Firstly, technological advances in warfare during and after WWII meant that civilians also became potential victims of war, and therefore potentially subject to the same psychological stress as soldiers. Secondly, everyone (both the public and 
academics) slowly began to realise that stress could be a problem in other arenas of life. As the name 'battle fatigue' implies, an external force (the battle) is the cause of the symptoms. The research community subsequently recognised that major life events such as family deaths or environmental disasters could also result in harmful psychological and physical symptoms.

The idea that stress can be defined as a destructive environmental demand became the dominant academic thought through to the 1960's (Lazarus, 1999). Such a stimulus based definition of stress followed the classic engineering model described above, in which stress is an exerted force that subsequently results in some reaction or distortion in the organism. Early research into blue-collar stress also focused on this stimulus based stress model in the attempt to identify sources of stress in the work environment, particularly physical and task based circumstances (Cooper et al. 2001). As Cooper et al. (2001 p. 8) explain, however, focusing on the "objective measures of environmental conditions" is a major weakness of the stimulus based approach to the study of stress, since it cannot account for the fact that two individuals may react in different ways to the same objective conditions.

According to Lazarus (2007, p. 34), questions asked by military psychologists in the aftermath of World War II (WW II), and particularly during the Korean and Vietnam wars, about the suitability of individuals to face and cope with the stresses of combat could not be fully answered using the stimulus approach because of the "complexities that result from individual differences in the conditions that arouse stress". Stress researchers in the social and biological sciences also began to challenge the limiting 
focus on major life events as stress stimuli, when the stresses of everyday life (i.e. at work, at home and at school) were shown to be as significant to health outcomes (Cooper and Dewe, 2007). The variability of individual responses was also noted to be greater for ordinary every day stressors (Lazarus and Folkman, 1984) than for major life events, further highlighting the inadequacy of a stress theory focused on the objective presence of extreme environmental conditions.

To this point in the development of stress research, definitions of stress were set conceptually within the simple stimulus-response paradigm. Two problems with these definitions have already been noted. First, whether the focus is on the stimuli or the responses, they reflect only one aspect of the stress process, to the neglect of the other. Second, they do not explain why what is stressful for one individual may not be stressful for another, since they fail to take account of individual differences. According to Cooper et al. (2001), a third problem exists in that the focus on one component of stress also detracts attention from the nature of the relationship between the stimulus and the response. Even models that acknowledge an interaction between the two components failed to explain the intricacies of such a relationship (Cooper et al. 2001).

A response to these criticisms came with the re-emergence of cognitive psychology as a major force in social and biological sciences in the 1960s and 1970s. This cognitive movement within the field of psychology argues that behaviour is dependent on the subjective cognitive processing of information rather than on objectively measurable external stimuli and encouraged the view that the inner experience is important to the study of human behaviour. As a result of this change in 
focus, the stimulus response models of stress were gradually abandoned in favour of a cognitive transactional model of stress that focused on the dynamics of the relationship between the individual and environment in a stressful encounter. Debates in the stress research community about critical life events versus daily hassles and their relative importance to the stress experience became a more fundamental debate about stress theory and the nature of the psychological processes that habitually link the individual to the environment whatever the circumstances (Cooper and Dewe, 2007). Stress researchers also began to move away from laboratory based research, typical for stress research up to that time, to field research, in the drive to understand, identify and describe how cognitive processes affect the stress, the associated emotions, and coping in the course of daily living. The result was a fundamental change in stress research pioneered by Lazarus and his colleagues.

During the 1970's and early 1980's, Lazarus and his colleagues at Berkley University undertook a program of field based research that was to ultimately lead to the publication of the seminal book, 'Stress, Appraisal and Coping' (Lazarus and Folkman, 1984), which introduced the cognitive model of stress. Their research program can be traced in publications from this time period. For example: Lazarus, Averill, and Opton, 1974; Lazarus and Launier, 1978; Coyne and Lazarus, 1980; Lazaus, Kanner and Folkman, 1980; Lazarus, 1981; and Lazarus, Coyne and Folkman, 1982. Lazarus continued his work on stress, emotions and coping until his death in 2002. Examples of his later published work include: Folkman and Lazarus, 1985; Folkman, Lazarus, Dunkel-Schetter, DeLongis and Gruen (1986); and Lazarus (1990, 1991, 1996, 1998a, 
1998b, 1999, 2000). The cognitive model of stress (Lazarus and Folkman, 1984), which still dominates the stress field today, is described in the next section.

For physiologists, psychologists, sociologists and social workers who were already using a variety of conceptually related terms such as "conflict, frustration, trauma, anomie, alienation, anxiety, depression, and emotional distress" for this phenomenon, 'stress' became the favoured term to unite the overlapping concepts (Lazarus, 2007, p.35). Although debates about the meaning of stress still abound, as Lazarus (1966, p. 27) stated, in his first book on stress:

It seems wise to use "stress" as a generic term for the whole area of problems that includes the stimuli producing stress reactions, the reactions themselves, and the various intervening processes. Thus, we can speak of the field of stress, and mean the physiological, sociological,and psychological phenomena and their respective concepts. It could then include research and theory on group or individual disaster, physiological assault on tissues and the effects of this assault, disturbances or facilitation of adaptive functioning produced by conditions of deprivation, thwarting or the prospect of this, and the field of negatively toned emotions such as fear, anger, depression, despair, hopelessness, and guilt. Stress is not any one of these things, nor is it a stimulus, response, or intervening variable, but rather a collective term for an area of study.

Lazarus (1999) continued, throughout his life's work, to argue that how the terminology is employed to label the stimulus and the reactions in stress analysis is not important, as long as we are clear about what they are referring to. Thus, unless specifically defined, this dissertation uses the terms that are now in common use within the field of psychology, in which they speak of stress stimulus or stressor as the external input, and stress response or reaction as the output (Lazarus, 1999). 


\subsection{Cognitive model of stress}

Lazarus and Folkman (1984) define psychological (or perceived) stress as a particular relationship between an individual and the environment that is "appraised by the person as taxing or exceeding his or her resources and endangering his or her wellbeing" (Lazarus and Folkman, 1984, p.19). Rejecting the simplistic notion that only certain situations are normatively stressful, their definition is based on the belief that most of the stresses experienced by the average person are not in the form of major life events or unusual situations, but consist of minor daily hassles that plague them, day in and day out. Major life events will affect the pattern of daily hassles in a person's life, but with this definition of psychological stress, the scope of its meaning is broadened to include all potential stressors (Lazarus 1998b).

From the many field studies that they undertook with their colleagues at Berkeley, Lazarus and Folkman (1984) noted a wide variety in the responses to stressful situations. More importantly, they observed that what "is stressful for some is not for others", suggesting that the stress response is the result of a specific encounter between the person and the environment (Lazarus and Folkman, 1984, p. 19). They concluded that to understand the stress process and its outcomes, it is important, therefore, to examine the nature of the relationship between the environmental conditions and the person characteristics.

Lazarus and Folkman's (1984 p. 325) cognitive theory of stress rests on a transactional model that views the person and the environment in a "mutually reciprocal, bi-directional relationship". An important corollary of a transactional model is the 
concept of process, defined in the Oxford English Dictionary to be a series of actions or steps towards achieving a particular end. According to Lazarus and Folkman (1984, p. 19) there are two important processes that "mediate the person environment relationship: cognitive appraisal and coping".

Cognitive appraisal is the "process of categorizing an encounter, and its various facets, with respect to its significance for well-being" (Lazarus and Folkman, 1984 p. 31). During cognitive appraisal individuals evaluate the degree to which a situation is a threat to self (primary appraisal) and what resources are available for dealing with the situation (secondary appraisal). Shaped by the characteristics, or factors, of the person and the environment, this transactional process plays a central role in evaluating and determining whether a particular person-environment relationship is stressful.

Coping is the process by which an individual manages the demands and the emotions generated by the stressful transaction. Cognitive appraisal is a critical determinant of the coping process as well as in the subsequent evaluation of coping outcomes (as a reappraisal) (Folkman and Lazarus 1980), and is seen by many to be at the heart of their influential stress model (Snyder and Pulvers, 2001; Ptacek and Pierce, 2003).

A stressful encounter is thus a dynamic on-going process that involves individuals transacting with their environment, making appraisals of encounters, and attempting to cope with the issues that arise. Stress is neither in the person nor in the environment but in the relationship between the two (Lazarus, 1990). At any given phase of a stressful encounter there are "substantial individual differences in emotion, and these, in large 
part, reflect individual differences in cognitive appraisal and coping" as the stress process unfolds (Folkman and Lazarus, 1985 p. 168). These emotions and the quality of the encounter represent short term outcomes of the stress process. Longer term outcomes are hypothesised to include somatic health or illness.

The principle of process was crucial to the development of Lazarus and Folkman's cognitive model of stress. Later Lazarus (1990b, 1998a) admitted that his single minded efforts to generate interest in a process approach were driven by a desire to improve the balance in psychology research, which had previously always tended to be structural in emphasis. The result initially was, however, the development of a model focused on situational characteristics and context, to the exclusion of dispositional influences, which were largely neglected.

Lazarus $(1990 b, 1993)$ readdressed this issue in reviews of the stress and coping literature to explain that complex stress transactions between the person and the environment may be influenced by the unique context of the person environment relationship and by stable relationships between the person and environment. A key influencer of these stable relationships is the dispositional traits that each person brings to the stress transaction as part of the set of person factors (Lazarus, 1993; Folkman, 1997; Ptacek, and Pierce, 2003). As well as acknowledging the weakness in the original model, Lazarus (1993) was also responding to the renewed interest amongst researchers in the role of personality in the appraisal and coping processes. Current research based on the cognitive model of stress acknowledges the role of both the environmental context and the person, including stable personality dispositions, in the stress and coping process 
(Ptacek and Pierce, 2003; Folkman and Moskowitz, 2004; Thompson, Poelmans, Allen and Andreassi, 2007).

A theoretical representation of the Lazarus and Folkman's (1984) cognitive model of stress is shown in Table 3-1 below. In the remainder of this section, each of the key elements of Lazarus and Folkman's model is discussed in more detail. The discussions focus first on the mediating processes listed in Table 3-1, beginning with the three forms of cognitive appraisal. The process of coping is explored next, followed by overviews of the person and environment factors that act as causal antecedents to these key processes. More specifically, the subsection on person factors discusses the characteristics that an individual brings to a potentially stressful encounter, and includes dispositional traits to reflect the extensions to the cognitive model of stress suggested by Lazarus in 1993. The subsection on environmental factors discusses the formal properties of stressful situations, and their influence in the appraisal process. In their model, Lazarus and Folkman (1984) specified that personal and environmental resources are the two main types of coping resource. These items will, however, be included for discussion in the coping subsection (rather than with other personal and environmental factors), because the coping process is heavily dependent on these resources. The detailed overview of the cognitive model of stress is completed with a discussion of the short and long term outcomes of the stress process. 


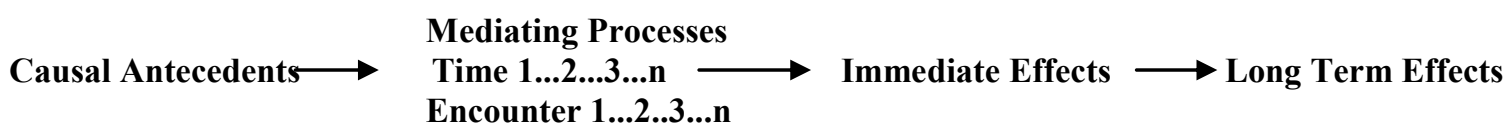

$\begin{array}{llll}\text { Person Factors } & \text { Primary Appraisal } & \text { Physiological changes } & \text { Somatic health/illness } \\ \text { Environment Factors } & \text { Reappraisal } & \begin{array}{l}\text { Positive or negative } \\ \text { feelings } \\ \text { Quality of encounter }\end{array} & \text { Well being } \\ \text { Coping } & \text { Social functioning }\end{array}$

Table 3-1: A theoretical schematisation of the cognitive stress model (Lazarus \& Folkman, 1987; Lazarus, 1993).

\subsubsection{Appraisal}

According to the Oxford English dictionary, appraisal means 'to evaluate the worth of'. It is now well accepted that as humans we have developed sophisticated cognitive processes with which we are constantly evaluating or appraising what happens to us in terms of its potential impact on, and relevance to our well-being (Lazarus and Folkman, 1984). Given a potentially threatening situation, the magnitude of a stress reaction depends on how the individual construes what is happening i.e. what they tell themselves about it (Lazarus 1998b). This process is labelled by Lazarus and Folkman (1984) as appraisal. That this cognitive appraisal reflects the unique and changing relationship taking place between a person and their environment is a central tenet of Lazarus and Folkman's (1984) stress and coping model. In this relationship, the person and environmental elements join together to form new meaning via the transactional appraisal process. In one of their supporting research papers, Lazarus and Folkman and 
their research colleagues explain that an environmental event cannot be identified as a stressor independently of its appraisal by the individual concerned because there are "no environmental stressors without vulnerable people whose agendas and resources influence whether they will experience stress, the form it will take, and its short and longrun outcomes" (Lazarus, DeLongis, Folkman, and Gruen, 1985 p. 776). Moreover, any given stressor cannot have the same reality across people because the 'same' stressful event will not be interpreted by two people in the same way. As such, a stressor is a unique and phenomenological ${ }^{2}$ personal matter (Snyder and Pulvers, 2001). From a phenomenological perspective, it is the individual's own appraisal of the demands and nature of the situation that is important to the prediction of the behavioural and psychological outcomes and coping strategies, not how others objectively assess an event (Terry, 1991).

Lazarus and Folkman (1984) define three basic types of appraisal: primary appraisal, secondary appraisal and reappraisal. Primary and secondary appraisal are the two main evaluative appraisal forms. Primary appraisal asks the question "Am I in trouble or being benefited, now or in the future, and in what way?" (Lazarus and Folkman, 1984 p. 31) or put more simply 'What's at stake?'. In secondary appraisal the person asks, "What, if anything can be done about it?" (Lazarus and Folkman, 1984, p. 31). It should be noted that the terminology used does not indicate relative importance or temporal order. Nor does it suggest what these two forms of appraisal might comprise (Lazarus and Folkman, 1984). The last type of cognitive appraisal discussed by Lazarus

\footnotetext{
${ }^{2}$ Pertaining to the science of phenomena as opposed to being (ontology) (Oxford English Dictionary)
} 
and Folkman (1984) is a reappraisal, and this they explain is simply an appraisal that follows and modifies an earlier appraisal for the same encounter. Primary appraisal and secondary appraisal are discussed in more detail in the next two subsections.

\subsubsection{Primary appraisal}

Primary appraisal is the evaluation of what is at stake for the person in a particular encounter with the environment. The stakes relevant to that person are partly defined by person factors such as values, commitments, goals and beliefs about oneself and partly by environmental characteristics such as novelty, predictability, ambiguity and temporal factors (Lazarus and Folkman, 1984). More enduring traits such as extraversion or neuroticism, for example, also have an effect on the appraisal process (Lazarus 1993, 1999). Snyder and Pulvers (2001) remind us that because of the extremely personspecific meaning of any given stressor, a stressor does not need to be large to elicit a response. However, a stressor must have personal significance or meaning to an individual for it to be construed as requiring coping (Lazarus 1998a).

Through primary appraisal, an individual first decides whether an encounter is irrelevant, benign-positive, or stressful. An irrelevant appraisal carries no implications for the well-being of the individual. A benign-positive appraisal occurs if an encounter is construed to preserve or enhance well-being. Stressful appraisals can be characterized as 'harm-loss', 'threat', or 'challenge'. In harm/loss, injury to the individual has already occurred, whilst threat concerns harm or losses that are anticipated. A challenge refers to the potential of gain, mastery, or personal growth. Although all three types of stressful 
appraisal call for coping efforts, the opportunity for gain from a challenge elicits positive emotions, such as excitement or eagerness. Threat and harm/loss appraisals, on the other hand, revolve around potential or actual harm, leading to negative emotions such as anxiety or anger.

Threat and challenge appraisals are not necessarily mutually exclusive. Whilst they are distinguished by "their cognitive component (the judgement of potential harm or threat versus mastery or gain) and their affective component (negative versus positive emotion), they can occur simultaneously or at different times during the same stressful event (Lazarus and Folkman, 1984 p. 33). The mix of emotions and different appraisals was noted in the study by Folkman and Lazarus (1985). Lazarus and Folkman (1984) argue that a challenge appraisal is, however, more likely to occur when the individual has a sense of control over the troubled person-environment relationship. By definition, a challenge will not occur, if what has to be done does not call for significant effort.

\subsubsection{Secondary appraisal}

Secondary appraisal relates to the individual's perception of their capabilities for dealing with the situation. It is also a complex process that evaluates the coping options and resources available (both personal and environmental), the ease with which they can be applied, and their potential effectiveness in light of other demands or constraints. Furthermore, the possibility that coping activities will create new problems for the individual experiencing the stressful event must also be considered. Secondary appraisal, like primary appraisal is influenced by person factors, both enduring and transitory. The 
relationship between appraisal, coping and person factors will be discussed further in the sections below. Together, the primary and secondary appraisals form an iterative process that ultimately shapes the stress experienced. Lazarus and Folkman (1984) argue that secondary and primary appraisal are interdependent, interacting with each other to create the degree of stress and the strength and content of the emotional reaction, illustrating the transactional nature of the process. For example, if an individual finds that there are no coping resources available, feelings of stress will be higher because a threatened harm or loss cannot be overcome.

\subsubsection{Coping}

Lazarus and Folkman (1984) define coping as "constantly changing cognitive and behavioural efforts to manage specific external and/or internal demands that are appraised as taxing or exceeding the resources of the person" (p.141). Folkman, Lazarus, Dunkel-Schetter, DeLongis and Gruen (1986), explain that a key feature of this definition is that coping is defined without reference to its outcome (i.e. success or failure). This enables researchers to understand what helps and what hurts an individual, and in what way, without the confounding of coping and outcome. Furthermore, "to equate coping with successful outcomes also implies that all effective coping results in mastery", and, effective coping actually might involve coming to terms with unpleasant outcomes rather than mastering them (Folkman et al. 1991 p. 243). Another important feature of this definition of coping is that, like cognitive appraisal, coping is process oriented. In fact, the concept of coping is indelibly interwoven with the appraisal process in that actual 
coping activities will affect the ongoing appraisal process and vice versa. Theoretically though, it is important to keep the two concepts, appraisal and coping, separate (Cohen et al. 1995; Lazarus and Folkman, 1984). Coping is the outcome of the appraisal process and in Lazarus and Folkman's cognitive model of stress, cognitive appraisals of a potential threat, are hypothesised to function as a mediating link between life stressors and the individual's coping responses (Holahan, Moos and Schaefer, 1996). Thus, coping is determined by cognitive appraisal (i.e. the combination of primary and secondary appraisal) of the situation and, for any one stressful episode, can change following one or more reappraisals.

Lazarus and Folkman $(1984,1987)$ identify three main characteristics of the process approach to coping. Firstly, coping is concerned with what the individual actually thinks, or does. Secondly, coping thoughts and actions are always directed at a particular situation and therefore, as the authors elucidate, to understand and evaluate coping, we need to know what the person is coping with. Coping as a process can only be studied if the opportunity exists to compare what happened at one moment, or in one context, with another (Lazarus and Folkman, 1987). This leads to the third feature, which is that the term coping process means we must assume change in coping thoughts and actions "as the stressful encounter unfolds" (Lazarus and Folkman, 1984, p. 142). According to Folkman and Lazarus (1985), ignoring the changes taking place during a stressful encounter would be ignoring the way people change troubled person-environment relationships through coping. Summarising the features and components of the coping process Lazarus and Folkman (1984) write:- 
Changes in coping and other aspects of the psychological state as the [stressful] encounter unfolds may occur within a few moments, as in an argument that is quickly resolved, or may continue to occur for hours, days, weeks, or even years, as in grieving. In both short and long-term cases there is an unfolding, shifting pattern of cognitive appraisal and reappraisal, coping, and emotional processes (p. 143).

The answer to the question 'What can I do?', raised in secondary appraisal is, as Lazarus and Folkman (1984) explain, a key determinant of what a person will actually do to cope. Certain contextual features have particular relevance to how this question is answered as well as to the coping strategies that result. The first is the availability of resources. Another important consideration of coping strategy choice relates to their intended purpose. Each of these issues is discussed briefly to complete this overview of coping.

\subsubsection{Coping resources}

As the definition of coping suggests, the way people cope will be heavily dependent on the resources available to an individual and the constraints that inhibit the use of these resources. Coping resources come in a myriad of forms but can, according to Lazarus and Folkman, be broadly categorised into resources that are primarily properties of the person, and those that are more environmental in nature. Examples of personal resources discussed in Lazarus and Folkman's book, 'Stress Appraisal and Coping', include health and energy; positive beliefs; commitments; social skills and problem solving skills. A particularly important type of coping resource, according to Lazarus and Folkman (1984) is the environmental resources of social support. The social environment 
can provide social relationships, which can help sustain individuals during times of stress. Environmental resources also include material resources such as money.

These characteristics of the person or the environment may "increase an individual's potential for dealing effectively with stress" by affecting such factors as the range of responses considered and the effort expended (Wheaton, 1983, p.210). In this way, resources reflect a "latent dimension of coping because they define a potential for action, but not action itself' (Gore 1985, p. 266 cited in Thoits, 1995a). Sometimes however, that potential is constrained by features of the environment or characteristics of the person. Personal constraints or agendas that can restrict the way a person makes use of resources include the internalised cultural values, and the beliefs of the individual. Environmental constraints include demands that compete for the same resources or institutions that restrict the effective use of resources (Lazarus and Folkman, 1985). Lazarus and Folkman (1984) argue that high levels of threat can also prevent a person from using coping resources effectively.

\subsubsection{Functions of coping}

There have been numerous efforts to classify the many different types of coping responses into a practical set of coping dimensions (Schwarzer, 1998). (For a review, see Parker and Endler, 1996 and Skinner, Edge, Altman and Sherwood, 2003). In a recent comprehensive review of coping category systems, Skinner, Edge, Altman and Sherwood (2003) counted 15 higher order coping distinctions, which they grouped into 3 types: 1) functions of coping, e.g. problem-focused versus emotion-focused; 2) topological 
features e.g. active, passive, cognitive, or behavioural; and 3) higher order action types, e.g. primary versus secondary control. Schwarzer (1998) also noted that coping can have a temporal aspect, in that coping can occur before, during or after a stressful event.

Despite the abundance of systems for classifying coping it is important to note, that emotion-focused versus problem-focused coping, as defined by Lazarus and Folkman (1984), is still the most widely used way of categorising coping responses (Parker and Endler,1992 cited in DeRidder, 1997; Skinner et al. 2003). According to Lazarus and Folkman (1984) there is an overriding distinction that can be made about coping functions, namely between coping that is directed at altering the situation itself, and coping that is directed at managing the negative emotional reactions that go with such situations. They refer to the former as problem-focused coping, and the latter as emotion-focused coping. Both problem focused and emotion focused coping can be either behavioural or cognitive. Lazarus and Folkman (1984) also remind us that coping functions should not be confused with coping outcomes; explaining that a coping function refers to the purpose a strategy serves, whilst an outcome refers to the effect a strategy has. During the research program that supported the further development of the cognitive model of stress, Folkman and Lazarus $(1980,1985)$ developed a comprehensive checklist of coping thoughts and actions that they called 'Ways of Coping'. Thay also identified several separate examples of emotion focused coping: wishful thinking, distancing, emphasizing the positive, self-blame, tension reduction and self-isolation, and also defined 'seeking social support' as a mix of problem and emotion focused coping. 
Research undertaken by Folkman and Lazarus (1980) and others since (see Thoit 1995a) have found that emotion-focused coping is more likely following an appraisal that determines nothing can be done to modify the stressful situation, whilst problem focused coping is more probable when an appraisal reveals a changeable condition. Research also indicates that, in any given stressful situation, individuals tend to use a variety of both problem-focused and emotion-focused coping responses (Tennen, Affleck, Armeli, and Carney, 2000), with each type influencing the other throughout a stressful encounter.

\subsubsection{Person factors}

Person factors include all the characteristics that an individual brings to the potentially stressful situation, such as commitments, beliefs, other personality traits, and personal resources. Person factors transact with environment factors to form new meaning via appraisal, which consequently affects the coping process. Personal resources, assessed specifically by the secondary appraisal process, are a key determinant of the coping process, and have already been reviewed in section 3.2.2.1. Commitments, beliefs and other personality traits will be discussed below.

According to Lazarus and Folkman (1984) the most important person factors affecting cognitive appraisal are commitments and beliefs. Commitments and beliefs are also referred to as motivational personality traits (Lazarus, 1990). Commitments are expressions of what is important or salient to individuals. They reflect the values, choices and goals that we wish to maintain, but also imply an "enduring motivational quality" (Lazarus and Folkman, p.56) that can sustain efforts in stressful situations. Commitments 
will guide people away from or toward harmful or beneficial situations, and will also determine the perception of what is at stake in a situation (Lazarus and Folkman, 1984; Schwarzer, 1998). For example, the more salient a role is to an individual the more vulnerable an individual is likely to be if that role is threatened (Thompson et al. 2007). Beliefs are expectations and convictions of the ability to meet situational requirements (Lazarus and Folkman, 1984; Schwarzer, 1998). Lazarus and Folkman (1984) argue that beliefs about personal control and existential beliefs are particularly relevant to stress theory. More specifically, their research suggests that appraising a situation as controllable is stress reducing, whilst existential beliefs, such as faith in God, can help individuals maintain hope and therefore reduce stress in difficult situations.

Rotter's (1966) concept of internal versus external locus of control (i.e., the extent to which one's achievements in life are due one's own actions or to factors outside of one's control), has also been associated with aspects of the stress and coping process (Lazarus and Folkman, 1984; Thompson et al. 2007). For example, research has shown that an individual who has a strong perception of control is less likely to appraise a specific situation as harmful or threatening (Rotter, 1975). Barnett and Baruch (1985) also reported that women perceiving high levels of personal control report fewer psychological and physical strain symptoms than women with lower personal control levels.

Self-esteem is an example of a belief about control that has been shown to be an important resource influencing cognitive appraisal and coping (Carver, Scheier, and Weintrub, 1989; Jerusalem and Schwarzer, 1989: Smith and Wallston, 1992; Lazarus, 
1993; Cooper et al. 2001; Caste and Burke, 2002). Other researchers have studied particular constellations of personal characteristics, which include aspects of both personal control and commitment. For example, hardiness, defined as a combination of commitment, control and challenge, has been shown to be linked to stress related illness in men (Kobasa, Maddi and Kahn, 1982).

One of the factors responsible for the resurgence of interest in the link between personality and the stress process, noted in the overview of the cognitive model of stress above, was the development of a taxonomy of broad, theoretically meaningful, personality dimensions referred to as the Big Five, namely, neuroticism, extraversion, openness to experience, agreeableness, and conscientiousness (Suls, David, and Harvey, 1996). This taxonomy and the associated measures also provided a more systematic way of examining personality in the stress and coping process. A significant amount of research into links between the Big Five personality variables and stress and coping followed these developments, with neuroticism and extraversion receiving the greatest attention (Thompson et al. 2007). Costa and McCrae (1985) were the primary developers of the Big Five measure, and in their subsequent research in 1990 they found evidence that neuroticism can influence perceptions of stress, ways of coping, psychological wellbeing, and physiological complaints.

With the renewed interest in the role of personality in the coping process Suls et al. (1996) also noted that researchers became more open to the premise that the stress and coping process can, in turn, influence certain aspects of an individual's personality, either positively or negatively. By and large, studies into the links between person factors and 
stress and coping have focused on the impact of the person on the process, however, Lazarus and Folkman (1984 p. 181) made an oblique reference to a potential for personal growth when they acknowledged that some people can "gain strength from stress that can be used in subsequent crisis". This was likely based on the work of Pearlin et al. (1981) who observed increases in mastery in adults who underwent stressful experiences. Aldwin et al. (1996) specifically differentiate between enduring personality traits and what they term personality processes, such as mastery and self-esteem, suggesting that the latter are more amenable to change as a result of coping. Lazarus and Folkman (1984) also suggested that well being may be related to a tendency to appraise encounters as challenges.

As Lazarus and Folkman (1984) explain, person factors are not sufficient to explain appraisal and coping since they transact with environment factors to determine the level of stress and the coping strategies to be applied. Environment factors affecting these processes will be discussed next.

\subsubsection{Environment factors}

Environment factors include both resources and situation properties.

Environmental resources are those features and constituents of the environment that can be used by an individual for coping, and as such they were included in the discussion of the coping process above (section 3.2.2.1). 


\begin{tabular}{|c|c|c|}
\hline Property & Description & Example \\
\hline Novelty & $\begin{array}{l}\text { Situations that the individual has not } \\
\text { directly confronted before. }\end{array}$ & $\begin{array}{l}\text { First major project } \\
\text { management role; First shift in } \\
\text { emergency. }\end{array}$ \\
\hline Predictability & $\begin{array}{l}\text { When established expectancies can no } \\
\text { longer be met }\end{array}$ & $\begin{array}{l}\text { Emergency situations. } \\
\text { Unexpected additional } \\
\text { responsibilities }\end{array}$ \\
\hline Event Uncertainty & $\begin{array}{l}\text { Not knowing if an event is going to } \\
\text { occur }\end{array}$ & Threat of layoffs \\
\hline Imminence & $\begin{array}{l}\text { How much time there is before an } \\
\text { event occurs }\end{array}$ & $\begin{array}{l}\text { Anticipation before an } \\
\text { important deadline }\end{array}$ \\
\hline Duration & How long a stressful event persists & $\begin{array}{l}\text { Extended periods of overtime. } \\
\text { Extended family sickness }\end{array}$ \\
\hline Temporal Uncertainty & $\begin{array}{l}\text { Not knowing when an event is going } \\
\text { to happen }\end{array}$ & $\begin{array}{l}\text { Unscheduled but planned } \\
\text { event. }\end{array}$ \\
\hline Ambiguity & $\begin{array}{l}\text { When information is unclear or } \\
\text { insufficient }\end{array}$ & $\begin{array}{l}\text { Details of project objectives } \\
\text { are unclear or unavailable }\end{array}$ \\
\hline Life Cycle Timing & $\begin{array}{l}\text { Event occurs simultaneously with } \\
\text { other stressful personal events }\end{array}$ & $\begin{array}{l}\text { Job interview just before } \\
\text { important family event }\end{array}$ \\
\hline
\end{tabular}

Table 3-2: Environment factors affecting cognitive appraisal (Lazarus \& Folkman, 1984) with examples.

Although stress appraisals are the result of a transaction between the person and environment it is not unreasonable to conceive of environmental conditions that would be more likely to result in stress than others (Schwarzer, 1998). Lazarus and Folkman (1984) identified eight underlying properties of stress stimuli that have the potential to be harmful, threatening or challenging: novelty, predictability, event uncertainty, temporal factors (imminence, duration, temporal uncertainty), ambiguity, and timing in relation to life cycle. Each of the eight factors are defined, with examples, in Table 3-2 above. According to Lazarus and Folkman (1984) these properties are not mutually exclusive and any episode can feature more than one property. Schwarzer (1998 p. 533) referred to them as antecedents of stress and coping, explaining that "demands that are difficult, ambiguous, unannounced, not preparable, and/or to be worked on both for a long time 
and under time pressure are more likely to induce threat perceptions than easy tasks that can be prepared for thoroughly and can be solved under convenient pace and time conditions".

\subsubsection{Outcomes}

As discussed in the overview of stress research history, researchers have long been driven by the desire to explain how stress impacts the human condition, physically, psychologically and socially. Lazarus and Folkman (1984 p. 181) were no different and their theory of stress posits that "the quality of life and what we usually mean by mental and physical health are tied up with the ways people evaluate and cope with the stresses of living". Given the dynamic, unfolding nature of the stress process, Lazarus and Folkman (1984) categorise outcomes into two broad temporal types: short term and long term, explaining that to understand the long term impacts on physical, psychological and social health it is important to understand the short term outcomes of stress encounters. It is not the intention of this thesis study to examine long term outcomes of role overload and therefore the emphasis of discussion in this subsection will be on short term outcomes of the stress process, recognising that these can include instantaneous emotional reactions as well as the overall outcome of a specific stressful event that may last for many days.

Short term outcomes occur at every step of the stress process in the form of positive and negative emotions. Furthermore, emotions in a specific stressful event have 
been shown to vary as the situation progresses (Folkman and Lazarus, 1985). The authors explain:

"The emotions one experiences in a stressful encounter... are characterized by flux .... The sequence of feelings reflects the changing meanings or significance of what is happening as the encounter unfolds" (p. 150).

To explain these variations it is useful to consider the possible combinations of cognitive appraisal and coping processes, based on the discussions of Lazarus and Folkman's model in this section. Primary appraisals of stressful situations as challenges, which indicate the possibility of mastery or gain, are characterized by positively toned emotions such as eagerness, excitement, and confidence, whilst situations appraised as a threat may result in anxiety, fear or anger. The result of the associated secondary appraisal could intensify or reduce these emotions depending on the availability of coping resources. Adaptive and maladaptive coping strategies will also change felt emotions differently. The mix of potential emotions at any stage of the stress process is further complicated by the fact that challenge and threat appraisals can co-exist, as can adaptive and maladaptive coping strategies.

At the outcome of a specific encounter the mix of emotions will reflect appraisals of how satisfied an individual is with the outcome and how well goals were achieved. A favourable outcome is likely to result in positive emotions, feelings of well being, and the conclusion of coping activity, whilst an unsatisfactory outcome can lead to high levels of perceived stress and more coping activity (Folkman, 1997). Feelings of well being or low 
levels of perceived stress will depend on "the tendency to appraise encounters as challenges, to cope with negative outcomes by putting them in a positive light, and, overall effectively managing a wide range of demands" (Lazarus and Folkman, 1984 p. 224). According to Lazarus and Folkman (1984), the quality of the immediate experience can also have short term physiological impacts. Examples of physiological effects include sleeplessness or persistent headaches (Lazarus and Folkman, 1984). Conversely, a satisfactory outcome may bring a sense of relief (see Taylor, Helgeson, Reed, and Skokan, 1991 cited in Folkman and Moskowitz, 2004), which may also bring immediate improvements in physical health.

Lazarus and Folkman (1984) emphasised that stress is not inherently maladaptive and damaging and that some people can gain strength from stress. In the short term the tone of perceived stress will depend on the mix of challenge and threat appraisals. Over time, it is coping strategies that will determine whether individuals are able to adapt to or resolve stressful situations. These will, in-turn, affect longer-term outcomes, such as somatic health or illness, well-being, and social functioning (Lazarus \& Folkman, 1984).

\subsection{Strengths and weaknesses of the cognitive model of stress}

The change in direction in stress theory and research that occurred at the end of the 1970's was an important one, not least because of the sheer volume of stress and coping research that followed. This new stress research paradigm is forever linked with Lazarus and Folkman. At a time when stress research was somewhat in flux, their book 'Stress, Appraisal, and Coping' published in 1984, was highly influential in laying out the 
priorities for stress research at that time. Moreover, their landmark work set the direction for ongoing stress and coping research throughout the 1990's (Carpenter, 1992; Compass et al., 1997; Snyder and Dynoff, 1999) and into the new millennium (Moos and Holahan, 2003).

The cognitive model of stress, proposed by Lazarus and Folkman (1984), is a comprehensive explanation of the stress process, applicable to a broad range of everyday as well as unusual stressors. This makes it suitable for the study of role overload and how it affects the psychological health of individuals who suffer this increasingly common phenomenon. This section in the review of cognitive stress theory is intended to discuss the strengths and weaknesses of the cognitive model of stress. It is divided into two parts. The first part highlights the key benefits of Lazarus and Folkman's (1984) approach and briefly suggest how they relate to the cognitive appraisal of role overload. The second part of this piece in the cognitive stress theory literature review discusses the weaknesses in the cognitive stress model.

\subsubsection{Key benefits of the cognitive model of stress}

One of the key features of Lazarus and his colleagues' approach to the stress process is that cognitive appraisal and coping are treated as mediators of short-term emotional reactions. In other words, according to their model, stress, coping and the associated emotions are dependent on how individuals evaluate, or appraise, their personal transactions with the environment (Lazarus, 1999, 2007). Coping arises from an appraisal of harm, threat or challenge, and as such, it can alter that appraisal and hence 
the emotional response. What is important and unique about this conceptualisation, is that it "clearly demarcated the appraisal process from subsequent coping responses" (Snyder and Dynoff, 1999). This suggests that studying these processes as independent concepts will increase the understanding of stress and coping for specific stressors such as role overload.

The second key factor of the cognitive model of stress, which has had a significant impact on stress and coping research, is the distinction that Lazarus and Folkman (1984) made between coping aimed at the perceived source of stress (problemfocused) and coping aimed at regulating one's emotions (emotion-focused). This distinction is important because it indicates that reducing stress can be achieved in other ways besides directly reducing the stress response. In addition to acknowledging the significance of indirect means of stress reduction, the distinction between the two functions of coping also helps to emphasis the important relationship between appraisal and coping. This classification of coping into problem and emotion focused has guided research for over twenty years (Tennan et al. 2000). Furthermore Lazarus and Folkman's (1984) definition of coping has been widely accepted and many tools were developed to measure these coping thoughts and behaviours (Folkman and Moskowitz, 2004), which had a direct impact on the increase in stress and coping research (Snyder and Dinoff, 1999)

The third key benefit of the cognitive model of stress is that it provides a theoretical framework that acknowledges the possibility that negative and positive outcomes can result and in some cases co-exist. Lazarus and Folkman (1984) argued that 
stress is not inherently maladaptive and damaging. Positive outcomes and personal growth can result from stressful experiences. Historically, however, research has focused on what can be done to regulate the negative symptoms of stress (Folkman and Moskowitz, 2004). Research by Folkman and Moskowitz $(2000,2004)$ has refocused attention on the potential benefits of positive outcomes in the stress process as originally proposed by Lazarus and Folkman (1984). The potential for positive outcomes to reduce perceived stress is a particular interest for this thesis and the current research will pay close attention to this aspect of the cognitive model of stress.

Finally, Lazarus and Folkman's model emphasises that the outcomes from cognitive appraisal and coping are the unique result of the interplay between the person and the environment. Despite the resurgence of studies focused on the impact of personality dispositions on coping, researchers recognise the importance that context, both environmental and personal, plays in the overall stress process (Folkman and Moskowitz, 2004). Folkman and Moskowitz (2004) recommend that coping efforts are best evaluated in the specific situation that created the stress, since what is effective in one stressful situation might not be effective in another.

These four key elements of the cognitive model of stress can help further the understanding of the behavioural and psychological reactions to role overload. In their formulation of the role overload episode (see Section 2.1), Kahn et al. (1964) reflects some of the features of Lazarus and Folkman's model. Specifically, Kahn et al. (1964) recognised that an individual, confronted with demands from the environment (sent role pressures), perceived as role overload because of lack of available time and energy, may 
experience stress and therefore must use coping responses to deal with these received role forces. Kahn and his colleagues also acknowledged the part played by internal values and beliefs in the creation of additional internal role forces for individuals. Finally, they recognised that coping responses to role overload and other role stresses would include direct attempts to solve the problem, including persuading role senders to modify demands, and indirect methods to avoid and minimise anxiety. As Thoits (1995b) suggested, the work of psychologists is often used to explain how social structures are connected to cognitions, emotions and behaviours. For a role overload situation, the psychological process of primary and secondary appraisal can help to explain the complexity of the relationship between the sent role pressures and the received role forces and the responses to these demands.

\subsubsection{Weaknesses of the cognitive model of stress}

The first weakness of the cognitive stress model, developed by Lazarus and Folkman (1984), is its complexity and the difficulty this poses for researchers. Because of its complexity and transactional nature, the overall conceptual framework is difficult to investigate and empirically test as a whole model (Schwarzer and Taubert, 2002). Lazarus and Folkman (1984) recommended that the best way to research stress is through the use of longitudinal studies and in depth interviews. Recently, however, Coyne and Racioppo (2000) have criticised stress and coping research for its reliance on questionnaires in cross sectional studies. In a response to this criticism Lazarus (2000) noted that Coyne and Racioppo (2000) have ignored an increasing number of important 
stress and coping research programs which are making use of in depth interview and observations rather than questionnaires. Folkman and Moskowitz (2004) suggest that retrospective accounts can address complexity and a great deal can be learned by asking people to provide narratives about stressful events, including what happened, the emotions they experienced, and what they thought and did as the situation unfolded. Although a longitudinal study is not possible for this thesis, the proposed case study approach, based on in depth interviews, will contribute to this growing number of qualitative studies of stress.

The second weakness of the cognitive model of stress is its lack of clarity regarding the relationship between context and dispositional traits particularly in the coping processes. There is equally strong evidence that coping is determined by the context of the stressful encounter (Folkman and Lazarus, 1985) and that coping choice is strongly linked to personality traits (Costa and McCrae, 1990). Although researchers are beginning to study how context and personality traits interact in the coping process, the research is still limited (Bourchard et al. 2004; Lee-Baggley et al. 2005). The proposed case study thesis is an opportunity to contribute to this literature.

\subsection{Implications for current research}

Despite the prevalence of employee role overload and the links to poor mental health reported in the introduction to this thesis proposal (e.g. Duxbury and Higgins, 2003a; Glyn et al., 2009; Pearson, 2008), some of the research on role overload appearing in the work family and organisational stress literatures, report only a weak link between 
overload and negative outcomes (e.g. Keenan and McBain, 1979; Coverman, 1989; Virick, Lilly and Casper, 2007; Gilboa et al., 2008). In other words, having too much to do may not always lead to high levels of psychological stress. Progress by researchers in both of these fields to differentiate between role overload and role conflict has not eliminated these mixed results.

This conundrum is at the heart of this thesis proposal. The broad applicability of the cognitive model of stress, its wide support and extensive use in many fields of research, and the notion that positive and negative outcomes can result from appraisal and coping suggest that the cognitive model of stress might be a useful way to examine role overload. This approach is consistent with the small but growing number of scholarly articles where the cognitive model of stress has been proposed as a tool to examine role stress. It is worthwhile briefly reviewing this literature to explore how their discussions can inform the planned approach for this research.

Our review of the role overload literature revealed a growing interest in the positive and negative effects of role stress and role overload in particular. In the work stress literature, observations of mixed outcomes have raised question, which so far remain unanswered (see 2.2.3). In the work family field the debate surrounding the benefits versus the harm resulting from multiple roles (see Section 2.3) is leading to calls for a more integrative approach. In the 2006 'Work and Family Handbook', Barnett and Gareis, MacDermaid and Harvey, and Thompson, Beauvais and Allen, (2006) all recommend, in separate articles, that further research is needed to determine the process by which both positive and negative outcomes occur. Collectively, these authors have 
proposed the following research questions: Under what conditions are the effects of combining work and family roles positive? Under what conditions are they negative? How do work and family roles influence one another, and why are the effects stronger for some people than for others? It is not surprising that some researchers have turned to stress theory to attempt to answer the questions raised in both fields of literature.

In the organisational stress field, Gilboa et al. (2008) used Lazaurus and Folkman's, (1984) cognitive model of stress to suggest why, in certain situations, role overload may be associated with positive well being. Their meta-analysis of work related stressors and job performance showed that the relationship between excessive role demands and job performance was variable. Gilboa et al. hypothesised that in certain circumstances employees may appraise role overload as a challenge, and hence view it in a positive light leading to higher levels of job performance.

In the work family literature MacDermaid and Harvey (2006) and Thompson et al. (2007) have made specific recommendations about how cognitive stress theory can be used to enhance the study of role stress. MacDermaid and Harvey (2006) recommend that by paying greater attention to the circumstances that produce different outcomes in different individuals it will allow researchers to explain more variability, and avoid overlooking important predictors. Their recommendations were made specifically with regard to one of the most commonly studied examples of role stress, the conflict between work and family. On the basis that this is essentially a special case of stress, MacDermaid and Harvey argue that it is important to examine cognitive appraisals of demands posed by multiple roles, the ways individuals choose to cope, and the emotions generated by 
these processes. Thompson et al. (2007 p. 93) took this approach one step further and presented an extension of Lazarus and Folkman's (1984) model of the stress and coping process tailored to work-family conflict explaining:

"the model proposes that when work family conflict occurs, individuals first appraise the situation in terms of whether it is a threat (or not), then secondly, they appraise the situation in terms of what they can do to resolve the conflict. Thirdly, they choose a method with which they attempt to either solve the conflict or manage the emotions associated with the conflict"

These examples of how the cognitive stress model has been applied to role stress questions in the literature give support to the use of this model in this thesis.

The process by which individuals appraise the risks associated with role overload, evaluate resource availability to deal with the overload, and choose the method of coping with the overload, and how these processes relate to psychological stress has not been studied in depth before and is therefore not well understood. The next section will present a framework based on Lazarus and Folkman's stress and coping model that was used in this thesis to examine role overload in more depth from the role incumbent's point of view. 


\section{PART THREE: PRELIMINARY CONCEPTUAL FRAMEWORK and RESEARCH QUESTIONS}

The review of the role overload and stress literature has provided the essential platform of knowledge on which the research is built. Although role overload is an important and independent form of role stress, the literature review has confirmed that research of this construct has been relatively sparse in both the work stress and work family fields. More specifically research into, how and why role overload and stress are related has been neglected. The thesis research intends to address this gap in our knowledge by examining this relationship in detail.

The use of a theoretical framework for a case study is a valuable element of research design, and the subsequent data collection and analysis (Yin, 2003, 2009). This approach orients the study to its objectives (Patton, 2002) and set limits to avoid data overload. The first step in the research design of a case study is the formulation of the research questions (Yin, 2009). In an exploratory case study where the existing theory is inadequate to explain the phenomenon of interest, an initial conceptual framework also provides a structure from which precise research questions can be developed (Miles and Huberman, 1994; Yin, 2009). Chapter 4 describes the development of the conceptual framework and research questions based on this approach. 


\section{Conceptual framework and research questions}

There are two goals to this part of the thesis. The first is to theoretically justify the conceptual framework used in this study. The second is to develop the research questions that will guide the study. This chapter is structured as follows. Section 4.1 describes the conceptual framework that will support the case study research. Section 4.2 presents the research questions that will guide the data collection and analysis. The methodology used to address research questions is the subject of Part 4 of this thesis.

\subsection{Conceptual framework}

The primary objective of this thesis, as outlined in section 1.1, is to develop a theoretical framework that describes the relationship between role overload and perceived stress. Based on the cognitive appraisal and coping process, the conceptual framework described in this section will form the initial version for this theoretical framework. In this way the conceptual framework becomes the "researcher's first cut at making some explicit theoretical statements" (Miles and Huberman, 1994, p.91).

The preliminary conceptual framework that guided this study is presented in Figure 4-1 below. The key constructs of the conceptual framework in Figure 4-1 have been labeled A through $\mathrm{H}$. Although the key elements of the cognitive stress model (primary and secondary appraisal, and coping, outcomes and perceived stress) frequently overlap, for clarity they have been represented in the conceptual framework as a sequential process. Whilst the framework is based on Lazarus and Folkman's cognitive 
model of stress, the framework is also consistent with the recommendations of

MacDermaid and Harvey (2006) and Thompson et al. (2007) that the cognitive model of stress can be applied to special cases of stress to expand the level of analysis. In this thesis the model is applied to role overload.

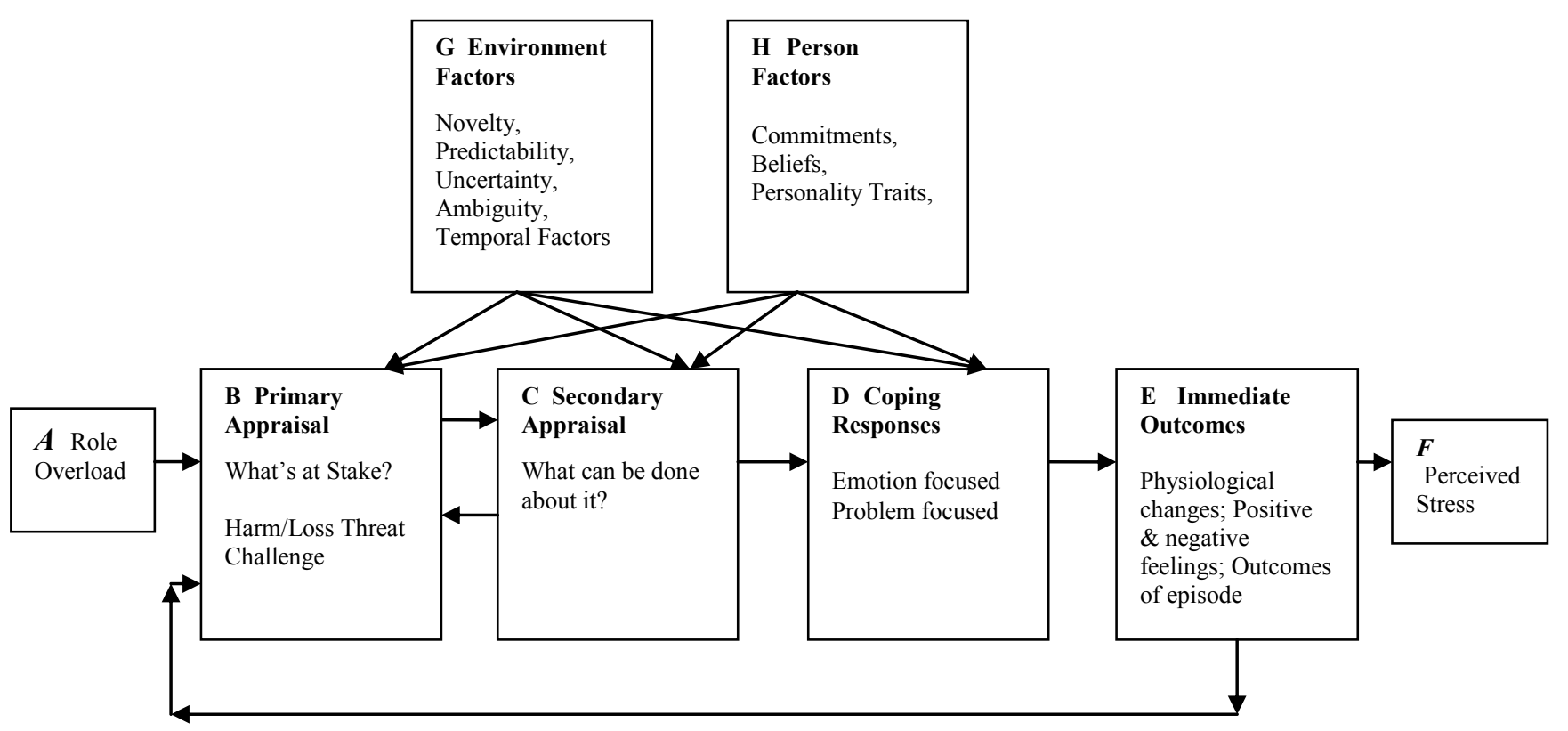

Figure 4-1: Preliminary conceptual framework for the cognitive model of role overload 
In the conceptual framework, role overload is a special case of stress where the mismatch between demands and resources specified in Lazarus and Folkman's stress and coping definitions is represented by the gap between role expectations and time and energy constraints. In Kahn et al.'s role episode model, the disparity between objective and subjective role overload (as represented by external and internal sent role demands and received role forces) can lead to psychological stress for the role incumbent. The "judgement" that this particular person-environment relationship is stressful depends on cognitive appraisal, as defined by Lazarus and Folkman (1984, p. 21). The role responses arising during the role episode reflect the behavioural and cognitive efforts to manage the role overload demands and the immediate psychological stress (Lazarus and Folkman, 1984).

Stepping through the cognitive model of role overload from left to right, the conceptual framework views the relationship between role overload (A) and perceived stress (F) as follows. The first step is hypothesised to be the primary appraisal (B), in which the individual will evaluate the role overload event in terms of the degree to which it is harmful, threatening, or challenging. In the secondary appraisal (C), the individual evaluates what if anything can be done to overcome or prevent the harm or improve the prospects for gain. The two way arrows between primary appraisal (B) and secondary appraisal (C) indicate that the two processes are posited to be interdependent, and that they can reinforce each other. The outcome of the primary (B) and secondary appraisals (C) is expected to vary for each individual and is posited to be determined by 
environment factors $(\mathrm{G})$ specific to role overload, and the person factors $(\mathrm{H})$ of the role incumbent.

The appraisal of role overload is hypothesised to give rise to coping (D), the efforts to reduce or manage the role overload and the associated emotions. Immediate outcomes (E) of the cognitive appraisal and coping processes include physiological changes, positive and negative emotions and feelings and outcomes of the role overload episode.

In the sections below each of the seven constructs in the framework will be defined, beginning with (A) Role Overload, and (following Figure 4-1 from left to right) ending with $(\mathrm{H})$ Person Factors. At the same time, supporting evidence for the hypothesised constructs will be presented. This evidence will be drawn from the empirical work in the area of role overload and will focus on concepts included in the model.

\subsubsection{Role overload (A)}

In the conceptual framework, Role Overload $(A)$ represents the constantly communicated expectations sent from role set members to the role incumbent (sent role pressures) In the literature review of this thesis, role overload has been defined as the perception "that the collective demands imposed by multiple roles are so great that time and energy resources are insufficient to adequately fulfill the requirements of the various roles to the satisfaction of self or others" (Duxbury et al., 2008 p.130). It will be recalled that in Kahn et al.'s (1964) model of a role episode, role overload has an objective and 
subjective component, represented by the sent role pressures and the received role forces (see section 2.1.2 an 2.1.3). In the conceptual framework of the cognitive model of role overload, the primary (B) and secondary appraisal (C) constructs are hypothesised to be the two processes that will result in this subjective perception of role overload, role responses and degree of stress.

\subsubsection{Primary appraisal (B)}

The cognitive appraisal processes are key elements in the conceptual framework. Primary Appraisal $(B)$ is the cognitive process by which individuals will assess the nature of the demands (A) and degree of risk that the situation $(\mathrm{G})$ presents in terms of the impact for their own personal belief and commitment systems (H). Harm/loss appraisals refer to an evaluation that injury has already taken place, whilst a threat appraisal applies when harm or loss is a potential outcome. A challenge appraisal occurs if the demanding situation has the potential for personal growth whilst still requiring considerable effort. For example, the assignment of additional work to an employee is hypothesised to become a source of role overload for an employee when he perceives that he is threatened and he has a stake in the outcome of his performance (Perrewe and Zellars, 1999). The growing recognition that role overload can produce mixed outcomes also indicates threat and challenge appraisals may be combined when assessing role overload (Gilboa et al., 2008).

The literature review noted that few studies have been conducted on the process of appraisal of role stress. Researchers addressing this gap in the literature often have different academic motivations and as a consequence have taken different approaches. 
For example, research by Siegall (2000) was aimed at improving role stress measures by adding threat appraisals to the traditional measures of role ambiguity and conflict. He achieved some success in his attempts and recommended future efforts to incorporate other stages of the stress and coping process. Amatea and Fong, (1991) claimed utilisation of Lazarus and Folkman's (1984) cognitive appraisal approach to stress, in their study of the contributions of role stress and personal resources in predicting strain symptoms for professional women managing multiple roles. Amatea and Fong reported that appraisal of personal resources in role stress situations has a greater impact on the level of stress experienced from role stress than role stress alone. Their approach was, however, more an acknowledgement that both personal and situational contexts transact to affect strain symptoms, rather than an attempt to analyse the appraisal processes. They concluded that the primary unique contribution of their study lies in the attempt to apply the cognitive, transactional model to the study of women's role stress.

Van Steenbergen, Ellemers, Haslam and Urlings (2008) proposed that the way a stressful situation is cognitively appraised makes a fundamental difference to both short and long term outcomes. Based on Lazarus and Folkman's cognitive model of stress they argue that the way a person thinks and feels about, deals with, and performs in a potentially stressful situation depends on whether they cognitively appraise the situation as a threat or a challenge (Haslam, O'Brien, Jenen, Vormedal, and Penna, 2005 cited in Steenbergen et al., 2008). Steenbergen et al. used an experimental approach to examine the effect of informational support on cognitive appraisals of the work family interface. Their study draws inspiration from the work of Tomaka and his colleagues (1993, 1994, 
1997) whose experimental research has given further support to the central role of cognitive appraisals in the elicitation of threat and challenge responses to potentially stressful situations. Steenbergen et al. predicted and, from their field experiment, were able to demonstrate that it is possible, using informational support, to influence the way in which individuals appraise their own ability to combine work and family. Notwithstanding the focus on work family conflict, the research demonstrates the potential outcomes from analysing cognitive appraisals.

\subsubsection{Secondary appraisal $(C)$}

The Secondary Appraisal (C) of a role overload situation involves the identification and assessment of options for coping. During this process the individual will evaluate both personal and environmental resources in terms of their capability and availability for dealing with the situation. A realisation that it may not be possible to cope can strengthen an already threatening appraisal (Folkman and Lazarus, 1985, Tomaka, Blascovitch, Kelsey and Leiten, 1993), demonstrating the interdependent relationship between secondary and primary appraisals represented by the bidirectional arrows between these two processes (B and C) in the conceptual framework.

Time is an obviously important resource that is assessed during the secondary appraisal in a role overload situation. By definition, the role incumbent's immediate assessment is that there is insufficient time to complete the sent demand as requested with the resources available. Other resources that could facilitate better management of the limited time will, therefore, be critical in an individual's evaluation. Controllability and 
social support have been noted as factors that may affect the relationship between role stress and psychological strain (Aryee, Luk, Leung and Lo, 1999; Beehr and Glazer, 2005; Thompson et al., 2007). A controllability factor such as job autonomy could have a significant impact on an individual's perceived ability to keep to, or even reduce, project timelines. In contrast, lack of support where time is tight is intuitively more likely to reinforce a threat appraisal than weaken it.

According to the reviews by Beehr and Glazer (2005) and Thompson et al. (2007), one of the most commonly tested moderators of the effects of role stress is social support. As well as being a strategy used for coping (D) (see Section 4.1.4 below), according to Lazarus and Folkman, (1984) social support is also an environmental resource and may therefore be significant in the secondary appraisal (C) of what can be done in a harmful, threatening or challenging situation (see Section 4.1.3 above). Contrary to the assumed logic, the effect of social support on the relationship between stressor and strain can, however, be variable (Lazarus and Folkman, 1984; Beehr and Glazer, 2005). Conceptually, in a role overload situation, when time is short, the availability of social support and good interpersonal relationships, across the multiple roles an individually occupies, would be expected to lessen the chance of a stress appraisal. This is supported by Duxbury et al. (2008) who report that the most important aspect of work culture for the prediction of role overload is the level of support for work life balance; specifically, the more supportive the environment the lower was the incidence of role overload. 
Finally, energy is a particularly important resource in situations of role overload as noted in the definition of role overload in Kahn et al. (1964) and Duxbury et al. (2008) In particular, energy levels may be impacted by physical health. Other individual qualities, such as personal skills, also represent coping resources that may be assessed during secondary appraisal.

\subsubsection{Coping (D)}

Coping $(D)$ is the process by which an individual facing role overload manages the demands and the emotions generated by the stressful transaction. The conceptual framework hypothesises that when faced with role overload a variety of coping strategies will be used. Research in the area supports the idea that these coping strategies will be either targeted at reducing the overload or designed to deal with the emotional effects of the stress arising from role overload. In other words they will be problem focused or emotion focused (Lazarus and Folkman, 1998). In a recent review of stress and coping literature in Australia, nurses were found to suffer a variety of stresses of which a heavy workload was the most prevalent (Lim, Bogossian and Ahern, 2010). To counter these stresses, Lim et al. report that Australian nurses cope by including seeking support, problem solving and self-control in their coping repertoires. Lang and Markowitz (1986) developed a role overload coping scale based on a pretest in their study of short-term role overload in an academic environment. Coping with role overload were categorized as task reduction and task management. Higgins, Duxbury and Lyon's (2010) reported that men and women in dual earner families relied most on scaling back and family role 
restructuring to cope with total role overload. Whilst overall the coping literature is extensive (Folkman and Moskowitz, 2004), Dewe et al (2010) noted a lack of coping research in the role overload literature. The limited research on coping and role overload has, however, highlighted the importance of coping resources and strategies in reducing the negative impacts of stress.

Researchers have for some time been divided as to whether coping resources and strategies act directly on the strain symptoms, or if they act as a buffer to moderate the relationship between the stressor and stress symptoms (Mak and Mueller, 2000). Lang \& Markowitz (1986) and later Lang (1992), for example, investigated the effectiveness of task management coping in preventing strain resulting from role overload. The results of their studies demonstrated that task management techniques significantly lower the levels of anxiety but not depression. This coping strategy did not, however, moderate the relationship between role overload and any of the measured symptoms of strain.

Higgins, Duxbury and Lyon's (2010) study of men and women in dual earner families, found family role restructuring moderated the relationship between role overload and stress for both men and women, whereas work role restructuring moderated the relationship between role overload and stress only for men.

In an investigation of coping strategies that moderated the relationship between role overload and burnout Yip, Rowlinson and Oi Ling Sui (2008) found that only rational problem solving significantly moderated the relationship between role overload and all three dimensions of burnout,( i.e. emotional exhaustion, cynicism and reduced professional efficacy). The moderating effect of resigned distancing and seeking 
support/ventilation, on the other hand, was significant only on emotional exhaustion and cynicism respectively. Other researchers have found that the negative effects of role stress and job burnout can also be mitigated by a healthy lifestyle, mediated by its effect on vitality and psychological well-being (Jones, Norman and Weir, 2010).

An important coping strategy used in many stressful situations, and also specifically for role stress, involves the use of social support (Dewe et al, 2010; Lazarus and Folkman, 1984; Beehr and Glazer, 2005). Social support is used to describe the potential effects of many different interpersonal variables (Lazarus and Folkman, 1984; Beehr and Glazer, 2005), and typically the functional support provided by other people is the topic most studied in role stress research. Kahn et al. (1964) also recognised the importance of interpersonal relationships in managing role demands, which makes social support an obvious coping topic for role stress researchers.

A final example of role overload coping research was discussed in the early work on dual career families by Rapoport and Rapoport (1976). They reported that couples often modify their usual standards with regard to their home life in order to cope with feelings of overload. More recently, Somech and Drach-Zahavy (2007), reported that in work family conflict situations, gender role ideologies determined whether individual cope by taking a less active role at home or vice versa. These latter results highlight the relationship between personal values and chosen coping strategies. 


\subsubsection{Immediate outcomes (E) and perceived stress ( $F)$}

Based on Lazarus and Folkman's (1984) model of cognitive stress, perceived stress $(\mathrm{F})$ indicates the quality of the stressful encounter and is thus dependent on the person and environment factors as well as the appraisal and coping processes (Cohen, Kamarck and Mermelstein, 1983). Lazarus and Folkman (1984) describe the following immediate effects: physiological changes, positive or negative feelings, and the quality of the episode outcome. Role overload has been linked to high levels of perceived stress and other measures of psychological health (Lease, 1999; Duxbury and Higgins, 2003a; Glynn et al.. 2009) and low levels of psychological stress (Coverman, 1989; Gilboa et al., 2008) in a number of empirical studies.

\subsubsection{Person factors $(\mathrm{H})$}

Person Factors $(\mathrm{H})$ are the characteristics that individual bring to the role overload situation. They include commitments, beliefs and other more enduring personality traits, as well as personal resources available for coping, such as energy and skills. Commitments reflect what is important to a person, and include personal values and goals. Beliefs are pre-existing notions that reflect an individual's opinions and evaluations of the self, and also his or her view of the world (Tomaska and Blascovitch, 1994).

The literature review has highlighted that the influence of personal characteristics on the stress and coping process has long been the subject of research and discussion. This is reflected in the amount of existing research focused on person factors $(\mathrm{H})$ and 
stress, and person factors (H) and coping (D), compared with other components of the framework. The absence of research focused specifically on cognitive appraisal (B and/or C) in the stress process, also means that much of the research into the influence of person factors in the stress process concentrates on the broader relationship between stressors (role overload and the other role stressors, conflict and ambiguity) and strain (psychological, physiological and behavioural), or on coping strategy choice.

The conceptual framework posits that person factors $(\mathrm{H})$ can influence primary appraisal (B), secondary appraisal (C) and the choice of coping strategies (D). Many of these relationships have been touched on briefly in previous parts of this section and will not be re-iterated here. Instead, this section will provide the reader with information on the person factors found to be most important to the relationship between role overload and perceived stress as represented by the model in Figure 4-1.

The contention by Lazarus and Folkman (1984) that commitments and beliefs are the most important person factors to influence appraisal, has been translated into studies of many different forms of these two person factor categories. For example, in the early years of organisational role theory research, Sales's (1970) laboratory work demonstrated evidence that the effects of stress from role overload are most severe for individuals with the specific person characteristic labelled fear of failure. Following the path of Kahn et al. (1964) and Sales's $(1969,1970)$ work, Lang and Markowitz (1986) predicted that Type A behaviours, alienation, and perfectionism would increase strain (i.e. short-term negative emotions such as anxiety) and moderate its relationship with overload. Only alienation, described as the belief that life lacks purpose or the power to change, intensified the 
effect of overload on strain in their sample of students. Recent reviews of potential moderators of the role stress-strain relationship in the job stress and work family literatures have included self efficacy, self esteem, internal locus of control, type A behaviour patterns, and negative affectivity as individual difference moderators (Beehr and Glazer, 2005; Thompson et al. 2007). Beehr and Glazer (2005) specifically noted that self-efficacy has been reported as a moderator of role overload and psychological strain. Perceived self-efficacy is concerned with "people's judgments of their capabilities to execute given levels of performance", suggesting that individuals high in self efficacy may be more likely to initiate and persist at coping efforts (Bandura 1977). Beehr and Glazer (2005) further suggested that this will increase the chance that such individuals will be better at reducing their own psychological stress.

According to Lazarus and Folkman (1984) personal belief about control are the most important beliefs affecting cognitive appraisals of stress and the subsequent emotional reactions and behaviour. The degree to which an individual believes they have control over the functioning of their environment has mostly been studied in the work stress field. According to Thompson et al. (2007) it will affect the secondary appraisal, the choice of coping strategy and the extent to which a strategy might be affective. Beehr and Glazer (2005) suggest that controllability of the environment may also be relevant to a number of variables popular in workplace research, such as autonomy, participation, participative decision making, and empowerment. They note that while Ganster, Fox and Dwyer (2001) found job control to be a moderator of the relationship between role 
overload and the organisational variable, health care costs, in other research the link between role stress and psychological strain was weak.

Another person factor $(\mathrm{H})$ construct the literature suggests may be relevant to the experience surrounding role overload is the concept of role salience. An important dimension of role salience is "the extent to which the person demonstrates a willingness to commit personal resources to assure success in the role or to develop the role" (Amatea et al. 1986 p. 832). This suggests that role salience may influence the primary appraisal (B) of role overload. Much of the research on role salience and role stress, however, focuses on the impact on multiple role management, in general, and work family conflict in particular. This research on role salience and work family conflict is based on the contention that participation in a highly salient role may reduce participation in a less salient role (Greenhaus and Beutell, 1985; Frone et al 1992; Powell and Greenhaus, 2006) increasing the likelihood of conflict between roles. Although role overload has been noted as an antecedent of work family conflict, the relationship between role overload and role salience has not been examined in these studies. Early work by Rappaport and Rappaport (1976) on dual career families did note, however, that overload increased when having children and a family life became increasingly more important that just being married. Whether this translates into psychological stress will depend on appraisal. Applying the logic of Lazarus and Folkman's model, Thompson et al. (2007) suggested, that because more time and energy are invested in highly salient roles, role salience will negatively influence primary appraisal of role stress since stakes 
are more likely to be perceived to be higher in a domain that is more salient to an individual.

As discussed in the review of this cognitive stress model, the development of the Five Factor measure (Costa and McCrae, 1985) of personality led to a resurgence of personality based research that was also reflected in the stress and coping literature, most specifically with regard to the choice of coping strategy. Research into the influence of person factors in the cognitive model of stress was broadened to include other personality traits, particularly those represented in the Five Factor measure (ie, extraversion, neuroticism, conscientiousness, agreeableness, and Open to experience) often referred to as the Big Five (Goldberg, 1990).

Research into the relationship between the Big Five personality variables and stress has rarely focused on role overload. Other research has shown, however, that personality plays an important role in many aspects of the stress and coping process (LeeBaggley, Preece and DeLongis, 2005). For example, after defining and developing the model to measure the Big Five personality characteristics, Costa and McCrae (1985, 1990) subsequently found evidence that neuroticism can influence perceptions of stress, ways of coping, psychological well-being, and physiological complaints. Nakano (1992) and Watson and Hubbard (1996) both reported that extraverts are more likely to seek social support to cope with stressful situations, whilst the latter authors also found that the other three items from the Big Five were related to choice of coping strategy. LeeBaggley et al. (2005) have noted recently that much of the research examining the relationship between personality traits and the stress and coping process tends to focus on 
only one or two of the Big Five dimensions in any one study. They suggest that, based on a review of this literature, examining all of the Big Five dimensions can add to our knowledge of the stress and coping process.

Role overload has been the specific focus of a small number of recent studies searching for other personality based moderators of the relationship with psychological strain. Negative affectivity (NA) refers to the tendency to experience negative thoughts, perceptions and feelings. According to Watson and Clarke (1992), it is strongly associated with neuroticism as defined in the Five Factor model and it has even been suggested that neuroticism and NA are alternative labels for the same construct (Burke et al., 1993). Watson and Clarke (1984) had already reported that individuals high in NA are more likely to experience distress. On finding that three of four studies showed NA to moderate the work role overload / psychological stress relationship, Beehr and Glazer (2005) concluded that NA is a promising individual difference moderator for role overload.

Other dispositional attributes have also been examined with regard to their positive or negative moderating influence on coping, many of which have been found to influence the appraisal process as well (for a review see Snyder and Pulvers, 2001). In his later book on stress and emotion, Lazarus (1999) also noted that with regard to coping there are differences among people in terms of socioeconomic status, intelligence, education, marital/relationship status, age, race, gender, and physical health. 


\subsubsection{Environment factors (G)}

Environment factors $(\mathrm{G})$ include those properties of the role overload situation that will influence the primary $(\mathrm{B})$ and secondary appraisal $(\mathrm{C})$ of role overload. According to Lazarus and Folkman (1984) they can be categorised according to the eight underlying situational conditions detailed in the literature review (i.e. novelty, predictability, event uncertainty, imminence, duration, temporal uncertainty and timing of life events; see 2.2.2.4 for more details).

A search of the role stress literature has been unable to identify any research specifically examining role overload or other forms of role stress according to the properties of the environment. In terms of the definition of role overload, it would be expected that novelty and ambiguity would impact the appraisal of role overload if an individual lacked the necessary skills, knowledge or energy required to complete the sent role demands.

Temporal factors are also considered to be one of the most important parameters of situations that can contribute to stress appraisal (Lazarus and Folkman, 1984). Specifically, the cognitive appraisal process (B and C) is heavily influenced by the amount of time available for anticipation of harm (Lazarus and Folkman, 1984).

There has also been a significant amount of research into the relationship between time spent occupying roles and role stress. Most of this research focused on work family conflict (e.g. Frone, Yardley and Markel, 1997). The specific relationship between role overload and hours worked is, according to Barnett and Hyde (1985), not linear, as more time spent in multiple roles can initially bring benefits, and only when certain time limits 
are exceeded will role overload and distress result. Lazarus and Folkman's (1984) also contend that how long a stressful event persists can lead to increased psychological and physiological stress as in chronic diseases and psychopathologies. Coverman's (1989) research, comparing the outcomes of role overload and role conflict, indicates that overload is more likely to be a chronic condition that may lead to increasing instances of acute role conflict. Together, this body of research supports the notion that event duration will be related to stress appraisals (B and C) in role overload conditions.

Lastly, the notion of controllability (a person factor discussed in Section 4.1.6 above) could also be related to elements in Lazarus and Folkman's (1984) typology of environment factors affecting appraisal. Specifically, not knowing if and when an event is likely to occur (i.e., event uncertainty and temporal uncertainty) suggests a lack of control over the environment and therefore may lead to harm, threat or challenge stress appraisals.

\subsection{Research questions}

The conceptual framework reviewed and presented above began the fulfillment of the first objective of this thesis, to develop a theoretical framework describing the relationship between role overload and perceived stress. The second objective of this thesis study is to determine why some people who suffer role overload experience symptoms of stress and others do not. The conceptual framework allows us to identify the research questions which will guide the research undertaken to complete both these objectives. The research questions are also intended to help define the necessary 
boundaries of the case study, as well as to assist in the selection of the appropriate unit of analysis. The following research questions are addressed in this study:

1. What environment factors help us to understand the differential stress response under role overload conditions?

2. What person factors help us to understand the differential stress response under role overload conditions?

3. How does primary appraisal impact the differential stress response under role overload conditions?

4. How does secondary appraisal impact the differential stress response under role overload conditions?

5. What impact do coping strategies have on the differential stress response under role overload conditions?

Differential stress response is defined in two ways in this thesis, according to whether the role overload episodes is assessed as overwhelming or not, and according to whether the respondents reported high or low levels of perceived stress. Details are given in the methodology chapters that follow below. 


\section{PART FOUR: METHODOLOGY}

A qualitative research methodology was used in this thesis. The review of the literature in Part 2 of this thesis suggests that the use of a qualitative methodology to meet the thesis objectives is particularly appropriate. The overall objective of this thesis is to explain the relationship between role overload and perceived stress. The secondary objective is to determine why some people experience more stress than others during role overload. The role overload literature review has revealed that role overload research has been neglected and there has been little attempt to develop the associated theory (i.e., organizational role theory) since role overload was first formally defined in the 1960's. More importantly, organizational role theory has proved inadequate for explaining how perceived stress arises from role overload and why it might vary.

Furthermore, although the stress literature is extensive and complex, the conditions that lead to perceived stress when individuals are faced with role overload is an area where knowledge is also limited. Nor are we clear on the process involved during role overload appraisal and coping. Finally, the stress literature, and also the role overload literature, has revealed that the relationship between role overload and perceived stress is highly dependent on the personal and situational context in which it arises.

These findings from the literature suggest that an in-depth examination of the meanings people make of their role overload experiences and the associated context is required in order to develop an alternative theoretical framework to meet the objectives 
of this thesis. Qualitative methodologies, which are used to study the feelings, thoughts, perceptions or behaviors of individuals during a particular experience (Patton, 2002; Creswell, 2009; Yin, 2011) are recommended in situations such as this when researchers seek new knowledge that can be used to add depth to existing theories or to develop new ones (Schonfeld \& Farrell, 2009) as they facilitate a deeper understanding of real life situations and how they can vary. The fact that role overload research to date has been predominantly quantitative also supports the use of a qualitative methodology for this study.

The qualitative methodological approach chosen to meet the objectives of this thesis is the case study. Case studies are rich empirical descriptions of particular instances of real-life situations that typically draw on data from a variety of sources (Eisenhardt and Graebner, 2007). The drive to capture the holistic characteristics and contextual conditions of complex contemporary events is a distinctive feature of case study research (Feigin, Orum and Sjoberg, 1991; Yin, 2009). This makes this methodological approach particularly relevant when an in-depth understanding of a phenomenon is required and where the relationship between the context and the phenomenon is unclear (Patton, 2002: Yin, 2009). Using a case study methodology, therefore, facilitates a thorough exploration of the role overload cognitive appraisal and coping process, and the identification of the important contextual factors that combine to influence the psychological outcomes. This part of the thesis details the case study methodology used to conduct this research.

The first chapter (Chapter 5) discusses how a case study research strategy is particularly suitable for building theory. Chapter 5 also includes a description of the steps 
required to conduct case studies for theory development as laid out by Eisenhardt (1989) and Yin (2009). The remaining chapters of this part of the thesis describe the application of the theory building methodology to this research. Chapter 6 details the setting for this case study, the units of analysis and the selection of the sample for this thesis. Chapter 7 discusses the data collection and data analysis techniques and procedures aimed at addressing the research questions developed in Part 3 of this thesis. 


\section{Building theory from a case study}

This research employs an exploratory embedded case study approach, using data from multiple sources to support the data collection and analysis. This chapter discusses how this approach allows us to fulfill the theory building objectives outlined in the introduction to this thesis. Section 5.1 discusses the different types of case study methodological approaches, focusing on why the chosen methodology is best suited to the thesis objectives. Section 5.2 presents the overall plan for the case study research and details the steps of the methodology that supports a theory building approach.

\subsection{The case study research method}

Case study research design can vary according to the type of case study. Firstly, like other research methods, the case study research approach can serve many purposes, the most typical being exploration, description, and explanation (Post and Andrew, 1982; Babbie, 2001; Yin, 2003). A descriptive case study provides a complete description of a phenomenon within its setting, whilst an explanatory case study seeks to explain specifically why an event occurs (Post and Andrew, 1982; Yin, 2003). Exploratory studies are undertaken when a researcher seeks to gain a better understanding of a previously unexplored phenomenon (Babbie, 2001). Given the lack of research investigating the relationship between perceived stress and role overload, this research uses an exploratory case study approach. 
Exploratory research has a more deliberate analytical purpose than a descriptive focus and is aimed at developing structures suitable for future, larger analysis (not necessarily by case study), or for determining the feasibility of undertaking a more extensive study (Post and Andrew, 1982; Babbie, 2001; Yin, 2003). Through the identification of relevant variables and contextual conditions related to the phenomenon of interest, and the isolation of relationship patterns and recurring themes, exploratory case study research is particularly useful for theory building (Babble 2001; Eby et al. 2005). The case study approach, notably the immersion in rich data from multiple sources, has also been acknowledged as a valuable research strategy for developing emergent theory, or for sharpening existing theory (Post and Andrew, 1982; Babbie, 2001; Voss et al. 2002; Eisenhardt and Graebner, 2007; Siggelkow, 2007; Weick, 2007). The theory building strengths of the case study research strategy give further support to the decision to use this approach for this exploratory research.

Case study research can be based on either a single case study or on multiple case studies (Yin, 2009; Eisenhardt, 1989). Single cases are the most common approach for case study research and are often chosen to represent unusual, rare or critical circumstances (Yin, 2009). The review of literature has confirmed that role overload is linked to negative outcomes in many organizations, including the health sector. The circumstance of role overload in the health sector is the case being studied in this thesis. Studying a phenomenon such as role overload in the context in which it exists is another benefit of a case study (Yin, 2009). Understanding the context of a stressor such as role overload is an important element of understanding stress appraisal and coping (Lazarsus 
and Folkman, 1984), making a case study a particularly suitable methodology to fulfill the objectives of this thesis.

Case studies can also be holistic or embedded by design (Eisenhardt, 1989; Yin, 2009). Holistic case studies examine the case as a single unit, whereas embedded case studies involve the study of multiple sub-units, which can be analysed at different levels. Results from these units or case-units are gathered together to provide an overall picture of the case in question, or a view of the differences across particular groups of case units. For the current thesis, an embedded approach enlists the use of multiple units of analysis at two levels: (1) overloaded female hospital workers and (2) role overload episodes described by these workers. Description of the case study setting and selection of the units of analysis is detailed in Chapter 6.

This research will also draw data from multiple data sources. According to Patton (2002), a mixed approach allows the researcher to gather data most relevant to the particular setting and questions. For a theory building case study, the use of both quantitative and qualitative data is recommended to provide richer and stronger evidence from which new theories can arise (Eisnhardt, 1989; Siggelkow, 2007). Whilst a combination of research techniques can be particularly potent (Babbie, 2001, Yin, 2009), the nature and degree to which qualitative and quantitative data and methods are mixed, depends on the case study objectives and research questions.

The emphasis on an inductive approach for the proposed case study means that qualitative data collection and analysis will be the primary method that will guide this thesis. This decision is supported by Eisenhardt (1989), who notes that, qualitative data is 
useful for exploring and understanding the underlying relationships in the phenomenon of interest (in this case, the cognitive appraisal of, and subsequent efforts to deal with, role overload).

Quantitative data can be used to provide support for qualitative analysis in a number of ways. For example, Creswell (2009) suggests that quantitative data can be used to identify the participants for the qualitative data collection. Creswell (2009) also suggests that quantitative evidence collected alongside the qualitative data may be used to answer specific research questions, or to provide a different level of analysis within the overall study. In this way the quantitative data can be integrated into the qualitative analysis as part of the overall assessment of the problem. When both sets of data are collected concurrently, quantitative data can also be used to temper misleading impressions arising from the qualitative analysis. It can also reinforce findings by "corroborating those findings from qualitative evidence" (Eisenhardt, 1989 p. 538). In this thesis, quantitative data will be used to support both the qualitative data collection strategy, and data analysis.

Yin (2009) argues that triangulation is the most important benefit of using multiple sources of evidence. Triangulation has been broadly defined as a combination of qualitative and quantitative methods used to capture a more holistic or contextual description of a phenomenon under study (Jick, 1979). Patton (2002) points out, however, that there are at least four types of triangulation: data, investigator, theoretical, and methodological. According to Yin (2009), it is the first of these types that is pertinent to case study research. Data triangulation encourages the use of multiple collection methods 
aimed at substantiating, or corroborating, the same fact or phenomenon. This narrower definition of the triangulation concept focuses on the use of mixed data resources that can be used to provide clarification and fuller explanations of results in a complementary fashion (Sale, Lohfield and Brazil, 2002; Yauch and Steudal, 2003). In the data analysis for this thesis, data triangulation will provide support for the case based qualitative data analysis.

The remainder of this section will complete the discussion of the planned methodology approach and present a more detailed roadmap for the rest of this section and the subsequent results presentation and discussions.

\subsection{Methodology roadmap}

Case studies have long been used for research in social sciences and the management field (Feigin et al. 1991; Locke, 2001; Eisenhardt and Graebner, 2007; Noor, 2008). In recent years, the use of the case study for building and testing theory has received increasing attention in management research (Gibbert, Ruigrok and Wicki, 2008). According to Dooley (2002) and Patton and Appelbaum (2003), one of the reasons for this growth has been the development of clearer roadmaps for conducting case study research, by authors such as Eisenhardt (1989) and Yin (1984, 1994, 2003, 2009).

Yin and Eisenhardt are noted proponents of the case study methodology and have wide experience in its application (Leonard-Barton, 1990; Paré, 2004; Brown, 2008). Yin's $(1984,1994,2003,2009)$ approach to case study research is focused on the desire "to build and maintain validity and rigour in the data", and his contributions to this aim 
derive particularly from his technical definitions of the components of case studies, and from the design logic specified to enhance case study theory development (Brown, 2008 p. 6; Eisenhardt, 1989). As Gibbert et al. (2008) warn, lack of rigour in the development of new theory will impact any subsequent studies, and diminish claims of relevance to management research. Eisenhardt's (1989) contribution to the increased use of the case study approach, was to synthesize the principles of building theory from case studies from a number of researchers already active in the field (including Yin), in order to present a comprehensive framework for the process.

At the core of Eisenhardt's (1989) framework for building theory from case studies is a method based on the grounded theory technique. Originally developed by Glaser and Strauss in 1967, the grounded theory technique has as its focus an ordered process of generating theory from the data. Glaser and Straus (1967) place great emphasis on becoming immersed in the data so that hypothesis and concepts can emerge. Moreover, these relationships and meanings are systematically formulated in relation to the data through the process of cross-case analysis (Ibid). Eisenhardt (1989) combined these principles of grounded theory with Yin's (2009) focus on case study research design and also Miles and Huberman's (1994) work on qualitative research methods and extended them in specific areas such as a prior specification of constructs and the use of existing literature.

Eisenhardt (1989) and Yin (2009) lay out well-defined steps of what activities are required to conduct case studies for theory development. Their approaches share many commonalities. This thesis will follow these steps, drawing from both authors, to achieve 
the objectives of the proposed research. As a recent example of theory building case study research in the management field based on Eisenhardt's approach, Johnson's (2007) study of the boundary spanning role has also provided inspiration for the current thesis. Johnson's (2007) highly methodical approach, tailored specifically to the theory building objectives of the research, is exemplary in terms of justifying theory building, the careful selection of the units of analysis, and the presentation of the empirical evidence. Eisenhardt and Graebner (2007) noted that these three areas are particularly important for differentiating theory building case studies from other research strategies; a challenge that is important for the increasing number of researchers following this approach to address.

The key methodology steps for this thesis, which closely follow the work of Eisenhardt (1989) and Yin (2003, 2009), are presented in Table 5-1. Included on this table are the key activities and objectives for each step of the methodology, as well as a reference to the section number of this document where the practical implementation of this step is described for this thesis. For information purposes, the final column of Table 5-1 provides the label used by Eisenhardt (1989) for the equivalent step in her methodology roadmap A key aspect of Eisenhardt's (1989) and Yin's (2009) recommendations for the case study methodology is that much of the process is essentially iterative involving overlapping steps that are repeated until marginal improvements are very small. Each step is described consecutively for convenience below. 


\begin{tabular}{|c|c|c|c|c|}
\hline $\begin{array}{c}\text { Thesis } \\
\text { Methodology Steps }\end{array}$ & Activity & Objectives & Thesis Section & $\begin{array}{l}\text { Eisenhardt } \\
\text { (1989) }\end{array}$ \\
\hline $\begin{array}{l}\text { Case Study Design } \\
\text { \& Objectives }\end{array}$ & $\begin{array}{l}\text { Definition of conceptual } \\
\text { framework, research } \\
\text { questions \& possible a priori } \\
\text { constructs. } \\
\text { Determine levels of analysis }\end{array}$ & $\begin{array}{l}\text { Focuses design \& data collection effort } \\
\text { Improves support for emerging theory } \\
\text { Design to support objectives. Data } \\
\text { collected at lowest level }\end{array}$ & $\begin{array}{l}\text { Ch } 4 \text { \& Ch } 6 \text { Section } \\
6.1 \& 6.2\end{array}$ & Getting Started \\
\hline $\begin{array}{l}\text { Select the Unit(s) } \\
\text { of Analysis/ case } \\
\text { units }\end{array}$ & Purposeful Sampling & Select theoretically useful case units & $\begin{array}{l}\text { Ch 6: Section } 6.2 \& \\
6.3\end{array}$ & Selecting Cases \\
\hline $\begin{array}{l}\text { Prepare for Data } \\
\text { Collection }\end{array}$ & $\begin{array}{l}\text { Identify multiple data sources } \\
\& \text { investigators Develop } \\
\text { protocols }\end{array}$ & $\begin{array}{l}\text { Develop converging lines of inquiry. } \\
\text { Increase data reliability }\end{array}$ & Ch 7: & $\begin{array}{c}\text { Crafting } \\
\text { Instruments \& } \\
\text { Protocols } \\
\end{array}$ \\
\hline Collect Data & $\begin{array}{l}\text { Versatile data collection } \\
\text { Overlap with analysis Formal } \\
\text { documentation }\end{array}$ & $\begin{array}{l}\text { Allows adjustments to data collection to } \\
\text { take advantage of emerging themes }\end{array}$ & $\begin{array}{l}\text { Ch 7: Sections 7.1, } \\
7.2,7.3\end{array}$ & $\begin{array}{l}\text { Entering the } \\
\text { Field }\end{array}$ \\
\hline Analyse the Data & $\begin{array}{l}\text { Within-case analysis; } \\
\text { Cross-case analysis }\end{array}$ & $\begin{array}{l}\text { Preliminary theory generation. Confirm } \\
\& \text { sharpen theory. Build internal validity }\end{array}$ & Ch 7: Section 7.4 & $\begin{array}{l}\text { Analyze the } \\
\text { Data }\end{array}$ \\
\hline $\begin{array}{l}\text { Shaping the } \\
\text { Emergent Theory }\end{array}$ & $\begin{array}{l}\text { Iterative comparisons of the } \\
\text { evidence for the emerging } \\
\text { theory using tables \& } \\
\text { diagrams }\end{array}$ & $\begin{array}{l}\text { Focus presentation on theory rather than } \\
\text { detailed narrative }\end{array}$ & $\begin{array}{l}\text { Ch 7: Section } 7.4 \& \\
\text { Ch's } 8 \text { to } 12\end{array}$ & $\begin{array}{l}\text { Shaping } \\
\text { Hypothesis }\end{array}$ \\
\hline $\begin{array}{l}\text { Enfolding the } \\
\text { literature }\end{array}$ & $\begin{array}{l}\text { Compare with existing } \\
\text { literature }\end{array}$ & $\begin{array}{l}\text { Improve construct definition and } \\
\text { generalizability. Builds internal validity }\end{array}$ & $\begin{array}{l}\text { Ch's } 8 \text { to } 12 \& \text { Ch } 13 \\
\text { to } 14\end{array}$ & $\begin{array}{l}\text { Enfolding the } \\
\text { Literature }\end{array}$ \\
\hline Conclusion & $\begin{array}{l}\text { Theoretical satuaration when } \\
\text { possible }\end{array}$ & $\begin{array}{l}\text { Process ends when marginal } \\
\text { improvement is small }\end{array}$ & Ch 13 to 14 & $\begin{array}{l}\text { Reaching } \\
\text { Closure }\end{array}$ \\
\hline
\end{tabular}

Table 5-1: Steps for building theory from case study research (Source: Eisenhardt, 1989 and Yin, 2009). 


\subsubsection{Case study design and objectives.}

The first step in the theory building framework is to develop an initial orienting framework for the research. This framework focuses the researcher on the objectives of the study, otherwise it is easy to "become overwhelmed by the volume of data" (Eisenhardt, 1989 p. 536). The framework also facilitates the second part of the case study design, the development of research questions that encourage a systematic data collection process. A prior specification of constructs is also permitted, although no such construct is guaranteed a place in the final emerging theory. Although it is impossible to achieve the ideal state of no theory under consideration, it is important that researchers should avoid thinking about specific relationships between variables especially at the beginning of the study (Ibid). The tentative conceptual framework and the research questions for this thesis were presented in Part 3 of this thesis.

Case study design also includes determining the levels of analysis to be used in the case study. According to Eisenhardt (1989), case studies can "employ an embedded design". In other words, multiple levels of analysis can be used in a single case study. The levels of analysis will depend on the objectives of the case study but data will be collected at the lowest level of analysis.

\subsubsection{Select the units of analysis/case units.}

Identification of the units of analysis and their subsequent selection is an important part of the theory building process (Eisenhardt, 1989; Yin, 2009). Units of 
analysis are mini-cases selected purposefully to support the theoretical objectives of the research to study a phenomenon in depth. This contrasts with traditional random sampling whose purpose is to support generalization from the sample to the population. In purposeful sampling, cases are chosen for their potential to contribute to the emerging theory.

\subsubsection{Data collection}

Preparation for data collection is as important as the collection itself. Although case studies are often considered synonymous with qualitative data, as argued earlier in this thesis, theory building research is best supported by multiple sources of data. The triangulation made possible by multiple sources of data helps substantiate the emerging constructs and hypothesis in the subsequent analysis (Eisenhardt, 1989; Yin, 2009). Multiple investigators are also recommended for case study data collection. Different interviewers can bring complementary insights to the collection process, which adds to the richness of qualitative data. In addition, the convergence of findings from data collected by different interviewers strengthens the confidence in the findings. The protocol used by the investigators to collect the data should be driven by the research questions and the initial framework. Nevertheless, adjustments can be made to the protocol during implementation to allow flexibility for any special situations that might arise. Eisenhardt (1989 p. 539) referred to this as "controlled opportunism". 


\subsubsection{Analyse the data}

Yin (2009) recommends that the preferred overall strategy to use for analysing case study data is to follow the conceptual framework that led to the case study. He also acknowledges, however, that the analysis will be driven by the unique combination of qualitative and quantitative data. Through the use of a systematic coding process, Yin (2009) and Eisenhardt (1989) both recommend a combination of within-case analysis (i.e., analysis of data of each individual cases, on a case by case basis) and cross-case analysis (i.e., analyzing and comparing the data across multiple cases) Within-case analysis first allows unique characteristics and patterns to emerge from the data. This process enables the researcher to become closely acquainted with each case unit. This is necessary before beginning the comparative cross-case analysis process. Cross-case analysis makes the researcher look at the data in divergent ways, as suggested by the theory, as suggested by the literature, as suggested by themes arising during analysis, or by randomly comparing pairs or groups of case units. The objective is to look for within group similarities, across group differences, as well as areas where there are no discernible patterns. In this way patterns across the case units are captured through a process of constant comparison. Such patterns illustrate concepts identified by the researcher that occur substantially in the data. According to Eisenhardt (1989), this approach will challenge any impressions developed during within-case analysis, and ultimately is more likely to lead to "theory with a close fit to the data". The outcome of this iterative process is a structured and coded representation of the data ready for further analysis. 


\subsubsection{Shaping the emergent theory}

Shaping the emerging constructs, involves repeated modifications of tentative hypothesis through systematic comparisons between the emerging theory and the evidence from each case unit or between groups of case units. Typically this involves tabulations of evidence from multiple sources initially for the constructs arising from within and cross-case analysis. The use and examination of data displays continues at higher inference levels as the researcher begins to develop initial theories, which can subsequently be validated against other case units or case unit groups, in an iterative process of constant comparison, as the researcher shapes and reshapes hypothesis. Eisenhardt (1989) argues that this process will be more likely to yield an empirically valid theory. A note making approach as each pattern code, variable, relationship, and hypothesis is formulated, helps to maintain a chain of evidence for the new theory, and hence increases the reliability of the case study (Yin, 2009).

\subsubsection{Enfolding the literature}

Researchers are encouraged to return to the literature to identify similarities or differences to the research findings. Examining the differences between the emergent theory and the extant literature can deepen the insight of both, and also gives more precision to limitations on generalizability. Examining the similar findings is also important because it draws together similarities in different contexts that might otherwise go unnoticed. 


\subsubsection{Conclusion}

The theory building process ends when marginal changes to the emerging theory are very small. 


\section{Case study setting, units of analysis and sample selection}

Like other research methodologies, case study research comprises "an allencompassing method - covering the design logic, data collection techniques, and specific approaches to data analysis" (Yin, 2009, p.18). The development of the conceptual framework and the research questions in Part 3 of this thesis began the design logic for this case study research. This chapter completes the description of the design phase of our research methodology by presenting an overview of the setting for this research (Section 6.1). The next step in the research methodology focuses on determining the units of analysis (Section 6.2) and the selection of a sample (Section 6.3) that specifically supports the theory building approach, as outlined in the previous chapter.

\subsection{The case study setting}

The setting for this study is the Canadian health care sector delineated by four major hospitals operating across nine sites in Ottawa and the surrounding areas of eastern Ontario. The four hospitals have a total workforce of approximately 20,000. The use of the health care sector for a case study about role overload and stress can be justified by data indicating that health care workers in Canada are particularly prone to high levels of work intensification, overload, and psychological stress (Duxbury and Higgins, 2003, 2009; Shield and Wilkins, 2005). Research focused on the Australian health care system has also reported that role overload is the number one reason for nurses leaving the workforce (Chang, Hancock, Johnson, Daly and Jackson, 2005). The health care sector is 
thus highly likely to provide the opportunity to observe the phenomenon of interest for this case study, (i.e. cognitive appraisal of role overload and subsequent coping efforts), a factor that is also important when selecting cases for case study research (Yin, 2009). Focusing the research on a particular setting also minimizes the risk that sources of extraneous variation will conflate the findings (Eisenhardt, 1989).

The data for this thesis was provided by individuals from these four hospitals who participated in a survey study of hospital workers initiated by Duxbury, Higgins and Lyons (2010). The survey was developed to test a model of the antecedents, moderators, and consequences of role overload (see Duxbury et al., 2010). At the end of the survey, respondents were asked to indicate if they were interested in taking part in follow-up interview exploring the role overload construct. The sample frame for this thesis consists of all the survey respondents who volunteered to participate in the interview process.

Included in the data collected from the survey respondents, and specifically relevant to this thesis, were measures of three types of role overload (work, family, and total role overload) and a measure of individual mental health (perceived stress). 'Work role overload' was measured using a 12 item scale developed by Caplan et al. (1980). Measurement of the 'family role overload' construct was based on the same 12 item scale by Caplan et al.'s, modified for the family domain by Duxbury et al. (2010). The 'total role overload' construct was measured with a five-item scale developed by Bohen and Viveros-Long (1981). A complete list of the constructs measured in this survey study along with the demographic data collected, together with the relevant references, is available from the author on request. 
The survey data collected represents a comprehensive pool of quantitative data available for use in this case study. The ability to incorporate additional data during a case study can be an important feature of theory building research. If a new line of thinking arises during the data analysis it makes sense to take advantage of the available survey data, if it can add further insight (Eisenhardt, 1989). Details of how this survey data was used for this case study will be discussed in the data analysis section of Chapter 7.

In total, over 1300 employees responded to the survey, which was made available on-line and by paper for those without access to the Internet. The sample was well distributed across all job types and life cycle stages. Consistent with the demographics of the sector (see Galameau, 2003), however, $84 \%$ of this sample was female. Of those employees surveyed, 252 (18\% of the total sample) agreed to take part in further interviews. These 252 volunteers represent the set of entities from which the case study research sample was drawn. Details of how this sample was drawn are given in Section 6.3 .

\subsection{Units of analysis}

According to Yin (2009), after defining the research questions, the next key component of case study design is to identify the units of analysis. Once defined, the process of selecting the case units must follow. Careful attention to both these tasks will

improve the results of case studies aimed at theory building (Patton, 2002; Eisenhardt and Graebner, 2007; Yin, 2009). 
As a general guide, the identification of the unit(s) of analysis is closely related to the objectives of the research and the way in which the research questions have been defined (Yin, 2003). The choice of the unit of analysis suggested by the research questions, and the conceptual framework, for the current thesis, leads us first to suggest the individual as the unit of analysis. For example, the research question - What impact do coping strategies have on the level of stress people experience under role overload conditions? - is directed at examining the results of targeted behaviours on the role overload experiences of the individual.

This case study involves more than one level of analysis. Whilst some refer to this as a nested design (Patton, 2002), Eisenhardt (1989) and Yin (2009) refer to it as an embedded approach. The description of the interview protocol in Chapter 7 will reveal that the hospital employee interviewees were asked to recall and recount two instances of role overload, one that was assessed as not-overwhelming and another that was overwhelming for the respondent. These requests are aimed at the role overload episode level rather than at the individual, indicating an embedded sub-case, below that of the individual. Examination of the differences in role overload behaviours, actions, and experiences between two sub-cases for the same individual or between groups of subcases will add evidence to support more accurate development of the individual case theory for the role overload appraisal and coping process. This approach is also consistent with advice from Patton (2002, p. 448), who reminds the researcher that larger case units can be created from smaller units for the purpose of analysis, and therefore it is advisable to "collect data at the lowest level unit of analysis possible". 


\subsection{Selection of case study sample}

A distinguishing characteristic of all qualitative methodologies is the purposeful sampling process that involves the careful selection of specific cases or case units that will yield the most relevant and plentiful data for answering the questions under study (Patton, 2002; Yin, 2011). Explaining that random sampling is "neither necessary, nor even preferable" Eisenhardt (1989, p. 537) notably emphasises the importance of deliberately selecting the sample to the process of building theory from a case study. Whilst there are a number of different strategies for purposeful sampling, according to Eisenhardt (1989) and Yin (2009), overall, the notion of replication is vital to the selection of the necessary information rich case units. Each unit must be selected carefully so that they either:

a) predict similar results (literal replication), or

b) predict contrasting results for anticipatable reasons (theoretical replication).

This deliberate, purposeful sampling ensures that case units are chosen according to what they can contribute to theory development, rather than for statistical reasons (Eisenhardt and Graebner, 2007).

The individuals to be interviewed for this case study were selected to provide information rich data about the issues of central importance to this thesis: role overload and differential stress levels. More specifically, individuals were sought who regularly experienced intense levels of role overload whilst also ensuring a broad range of perspectives from the health care workers of the four hospitals. Secondly, units were 
selected according to a deliberate theoretical sampling plan, namely to highlight role overload experiences under differential stress levels. The step by step process of purposeful sampling used for this thesis is detailed in Section 6.3.1 below.

\subsubsection{Purposeful sampling process}

For this study individuals for the case study were selected from the 252 hospital employees who volunteered to take part in follow up interviews about their experiences of role overload. The survey data for this population provides the following a priori knowledge that can be used to select the sample for this case study: the measures of three types of role overload (work, family and total), perceived stress, and gender (see references above in Section 6.1). All the individual items in each measure were answered using a 5-pont Likert scale. For the role overload and perceived stress constructs, respondent scores were calculated as the summed average of the scores given to the individual items making up the measure. Items bolded in these measures were reverse coded before being summed. For each construct the scores were then classified as being 'High' or 'Low' using population norms (see Duxbury et al. 2010) as follows. Those with mean scores less than or equal to 3 were classified as having 'low' scores. Those with mean scores greater than 3 were classified as having 'high' scores.

There are a number of different approaches that can be taken to purposeful sampling. These approaches are not mutually exclusive and can legitimately be combined during the sampling process. The objective of all approaches is to select embedded cases from which we can learn the most about the matters of importance 
(Patton, 2002) (i.e. cognitive appraisal of role overload). A four stage process was used in this thesis to create the sample frame:

$\underline{\text { Step } 1}$ : The first sample selection strategy involves intensity sampling. Intensity sampling logic seeks the selection of cases that exhibit the dimension of interest intensely, so as to maximise the availability of rich information on the phenomenon (Patton, 2002). Role overload is the primary phenomenon of interest for this study. Individuals with high levels of total (scores of 3.6 or greater), work (scores of 3.3 or greater), and family role overload (scores of 3.0 or greater) were selected in this first step. Sixty two (62) individuals from the population of 252 potential interviewees fulfilled these criteria (i.e. scored high on all three measures of overload).

Step 2: The second sample selection strategy involves homogeneous sampling. This technique ensures the selection of a homogeneous sample to allow in depth analysis of a sub group. In this thesis, the decision was taken to focus only on the role overload experiences of women. In the work family literature, much of the research on role stress focuses on women, largely because of the historical background to the rise in interest in work family conflict (see section 2.3.1). In the work stress field, research is more balanced across the genders. In Canada, the health sector is female dominated. As such it is not surprising that the majority of individuals in the survey sample and the interviewee sample were women. Of the 62 individuals measuring high on all three types of role overload, only $7(11 \%)$ are men. The lack of men in the sample suggests that cross-case 
analysis by gender will not be theoretically meaningful because of the disproportionate size of the groups. We therefore decided to omit men from the thesis sample frame. This left 55 female health care workers scoring high in all forms of role overload in our initial pool of possible respondents.

Step 3: The third step in the sampling strategy involved 'two-tail' sampling. This strategy involves the selection of cases representative of both extremes (i.e., polar opposites) of some important theoretical condition, such as good or bad outcomes (Yin, 2009). One objective of this thesis is to explain the differential stress levels experienced by overloaded individuals. To fulfill this objective we divided the cases into two groups, those scoring high levels of all three forms of role overload but low levels of perceived stress and those scoring high levels of all three forms of role overload and high levels of perceived stress. The first group (high overload low stress) contained 13 individuals, the second (high overload, high stress) 42 individuals. These two groups formed the preliminary sampling frame from which the individuals were selected for building the model of role overload cognitive appraisal. Choosing these cases, which are intense examples, or polar opposites, of important constructs, should make it easier to observe similar or contrasting patterns in the data (Eisenhardt, 1989; Patton, 2002; Yin, 2009).

We then set out to conduct the interviews. Participation in the interview was voluntary and there was a time lag between when the survey was conducted and when we attempted to contact the individuals to schedule the interviews. During this time period individuals left their posts, others became unavailable for other reasons, and some 
changed their minds about participating. All thirteen (13) of the female health care sector workers in the high overload low stress group were contacted and interviewed. Those in the high overload high stress group proved more difficult to contact and only thirty (30) out of the forty three (43) were interviewed. Nevertheless, the number of cases in the high stress group remains far in excess of the number of replications in the low stress group.

$\underline{\text { Step 4: }}$ To ensure extreme polar opposite groups of individuals with the highest and lowest levels of perceived stress additional further individuals were eliminated from the sample frame as follows. According to Eisenhardt (1989) there is no ideal sample size for case study research, although she recommends that with larger sample sizes it can become difficult to manage the complexity and volume of data. To complete the two-tail sampling strategy and with the sample size recommendations in mind, the final step of the sampling process was to create two extreme polar opposite groups of comparable yet manageable size based on stress scores. The perceived stress scores recorded for the 43 female health care workers interviewed (i.e. the 13 with high overload low stress and the 30 with high overload high stress) were revisited. The ten (10) informants with the highest stress scores were selected for the final sample as were the ten (10) respondents with the lowest stress scores. All the respondents in the high stress group had perceived stress scores greater than 3.44, whilst all those in the low stress group had scores less than 2.75. Individuals in each of these two groups came from all four hospital and worked in a wide variety of jobs (i.e., nursing, allied health, management and administrative support 
roles). These two groups provide the maximum contrast for the secondary phenomenon of interest in this thesis: perceived stress, whilst ensuring that the complexity and size of the data is kept to manageable proportions. 


\section{Data collection and data analysis}

This thesis uses a combination of qualitative and quantitative data, as recommended for building theory from a case study. This chapter details the methodology used for the collection and analysis of this data for this thesis. Section 7.1 describes the data collection whilst Section 7.2 covers data analysis.

\subsection{Data collection}

The instrument used for data collection was a telephone interview. Interviews are a "highly efficient way to gather rich, empirical data" (Eisenhardt and Graebner, 2007) and an essential source of case study information (Yin, 2009). Although a qualitative approach will predominate in this thesis, the literature review identified a set of well defined constructs of particular relevance to one of the research questions in this thesis (i.e., respondent personality traits). Given the intensive nature of the respondents' work environment and the fact that they were pre-identified as suffering from high levels of role overload, the decision was made to use a single instrument to collect data from the volunteers. The interview was therefore used to collect the qualitative data and this important piece of quantitative data. This approach minimised the impact to the respondents and maximised the efficiency of the data collection.

The first subsection of this part of the methodology discusses the interview protocol that was used to collect the qualitative data. Specifically, this subsection explains how the objectives of the research are supported by the chosen interview 
approach, and describes the various components of the interview. The second subsection describes the quantitative data measure, which was also included as part of the interview instrument. The last section discusses the details of how the interview was administered.

\subsubsection{Qualitative data collection - Interview protocol}

The qualitative data collection protocol for this thesis aims to capture the context, activities, thoughts, and inner experiences of an overloaded individual, as the literature reviewed earlier supports the idea that it is the meanings that are formed in role overload circumstances that will determine the level of perceived stress for that person. Lazarus and Folkman (1984, p.143) explain that the formation of these meanings involves an "unfolding and shifting pattern" of emotions, behaviours, and cognitive evaluations that can occur in a short span of time, or can last for a lengthy period. In short, it is a process. Researching process presents specific challenges.

A key requirement in studying process is to address the structural issue of the time or sequence in which the process unfolds (MacDermaid and Harvey, 2006). Ideally longitudinal data are required; however, practical limitations' regarding the agreed mode of access to the volunteer respondents, precluded a longitudinal approach for the current research. There are strategies available, however, that allow researchers to make judgments about such a process, even if only cross sectional data are available. Within an in depth interview, asking people to recall past events is the most common way of approximating observations over time (Babbie, 2001). It is also a strategy common in stress research (Thoits, 1995a). Moreover, according to Folkman and Moskowitz (2004) 
retrospective accounts can help address the problems of complexity in studies of stress and coping.

When using an interview to capture qualitative data based on past events, researchers must be aware that people have faulty memories and they can lie (Babbie, 2001). To mitigate for the possibility of retrospective sense-making that increases the chance of data bias, this research uses numerous respondents who "view the phenomenon from diverse perspectives", as recommended by Eisenhardt and Graebner (2007, p. 28). While the respondents selected for this thesis (as described in section 5.3 above) were all involved in delivering health care, either directly or indirectly, from a hospital environment, they also worked in a wide variety of roles (six types of job), in (4) different hospitals, and on (5) different sites.

According to Patton (2002 p. 159), in-depth qualitative inquiry has a number of features that make it "highly appropriate for studying process". Firstly, depicting process requires detailed description of how individuals engage with the environment. Second, the experience of process typically varies for different people; therefore their experiences need to be captured in their own words. Lastly, the perceptions of the participants are important to the outcomes of the process. It is noteworthy and particularly relevant to this thesis that Lazarus (1995) specifically recommended in-depth interviewing as an efficient approach to examining the appraisal process.

An example of an approach that used in depth interviews to capture retrospective processes is found in Dutton, Ashford, O’Neil and Lawrence's (2001) study of issue selling and its use in the management of change. Through a qualitative and systematic 
examination of descriptions of issue selling episodes, these researchers were able to extract lessons about the elements thought to be important to successful implementation of this key component of effective management. To collect the descriptive data, Dutton et al. (2001) asked each respondent to describe a successful personal attempt to bring an issue to the attention of top management. Through a partially directed protocol the respondents were also asked to describe the major milestones in the process and how they interpreted them, whilst specific probes were used to ask additional questions about the overall context, what worked well, and what could have been done differently. Their goal was to capture, as completely as possible, a retrospective account of the process as it unfolded over time, together with the elements that seemed most important to the interviewees. The protocol was repeated after respondents were also asked to describe an unsuccessful attempt at issue selling. This account of an unsuccessful issue selling attempt offered insights into those factors contributing to successful techniques.

For the current thesis, the design of the interview protocol developed for collecting the qualitative data was based on Dutton et al.'s approach. A semi-structured design was used to strike the necessary balance between focusing the respondents on a consistent and specific line of inquiry, whilst allowing individual perspectives and experiences to emerge (Patton, 2002; Yin, 2009). The objective of the qualitative interview protocol is to capture retrospective accounts of role overload episodes. This was achieved by carefully wording the questions to encourage open ended data, as the interviewers used the questions to step the respondents through the process as it unfolded. The interview protocol also included probes to allow the investigators the flexibility to 
add questions if necessary. This is particularly important for theory building research when unexpected responses require clarification (Johnson, 2007). Using a semistructured interview protocol means that valuable interview time is used efficiently, and the subsequent analysis process will be improved because responses can be located more quickly, and multiple cases become easier to compare (Patton, 2002; Yin, 2009). The semi-structured interview design also minimises the variation between different interviewers.

The complete interview script can be found in Appendix A. The interview is divided into three parts with twenty-four questions overall, comprising a mixture of short answer and open-ended questions. The items in Part I set the scene for Part II. Part II is the core of the qualitative data collection protocol, whose overall aim was to capture, in detail, the thoughts and behaviours arising during two specific role overload episodes. An overview of Parts I and II of the interview script is presented below. Details of Part III of the data collection instrument, which contains items for collecting quantitative data, will be presented in Section 7.1.2 below. In each of the three parts of the data collection protocol, the relationship between the items in the interview and the thesis research questions will be flagged as appropriate.

\subsubsection{Part I - Background information}

This part of the interview was aimed at collecting pertinent background information about the respondent. Specifically, this includes asking respondents to 
describe their role at work, how long they have been in the role, and how much of their work involves direct patient care. The responses to these questions serve two purposes. Firstly, this contextual information was used by the investigator to make initial interpretations of the descriptions of role overload situations as they were collected in Part II. As a result, interviewers were able to prompt for more information or ask additional questions where necessary during the interview, in order to answer the research questions. This is illustrative of the notion of overlapping data collection and analysis, a feature of building theory from case studies, as described by Eisenhardt (1989). The second use for the Part I responses will be during analysis, to help understand the impact of environment factors on the cognitive appraisal of role overload (Research Question 6)

\subsubsection{Part II - Role overload scenarios}

This part of the interview asked the respondent to recall and recount two specific episodes of role overload, one with a positive outcome and one with a negative outcome. As already discussed, this approach follows a similar form to that used in Dutton et al.'s (2001) research on issue selling, in which they collected descriptions of successful and unsuccessful issue selling episodes from each respondent. To collect the data about the role overload scenarios, the interviewer started with the following preamble:

"In this section of the interview we are going to focus on your experiences of role overload. Role overload is defined as having too much to do and not enough time to do it. In order to understand how people respond and cope with overload I would like you to think about a situation in the past six months where 
you felt overloaded - you just had too much to do, and not enough time. This situation can be at work or at home, whatever comes to mind. I'm going to walk you through the experience by asking you a number of questions to help me understand the situation, how it unfolded, how you felt about the situation, what you did to try and reduce the overload, and how you now evaluate your actions."

The respondent was then asked a series of ten questions about the situation. At the end of these questions, based on an initial judgment of the role overload experience described, the interviewer then suggested one of the following:

Thanks for sharing this example with me. It was very useful. You described an overload situation that (EITHER you were able to overcome so that you felt comfortable with at the end of the day $\mathrm{OR}$ was stressful for you and that resulted in your feeling overwhelmed and you were not happy with how it turned out).

The interviewer and respondent discussed and agreed on a role overload assessment of either overwhelming or not-overwhelming, and then, depending on the assessment of the first episode, the interviewer asked the respondent one of the following two questions:

Can you describe a situation within the past six months that went the other way: (EITHER where despite feeling overloaded you were able to resolve the situation in the end in a more favourable way, OR the situation resulted in you feeling very overwhelmed, and you were not happy with how it had turned out). Again, this could be at work or home. I am going to ask you the same set of questions as before, but in this case I want you to focus on this second situation.

In this way, Part II of the interview protocol was designed to capture descriptions of two contrasting role overload episodes, which, together, are intended to highlight factors associated with high and low perceptions of being overwhelmed from role overload. 
Although this format increased the length of the interview, the research benefits from being able to make comparisons between two role overload episodes for a single individual.

For each of the two role overload scenarios, details were sought about the thoughts and perceptions involved in the primary and secondary appraisals that resulted in differing psychological outcomes (i.e the role overload was subsequently assessed to be overwhelming or not). These questions were also intended to elicit descriptions of the environmental and person factors that influenced the cognitive appraisals of role overload in each episode. The questions developed to capture this data were primarily based on the definitions and descriptions of primary and secondary appraisal in the book by Lazarus and Folkman (1984), and the associated body of research (e.g. Folkman and Lazarus, 1980; Folkman, Lazarus, Gruen and DeLongis et al. 1986; and Folkman, Lazarus, Dunkel-Schetter, DeLongis and Gruen, 1986). Other studies that examined either primary and or secondary appraisal in detail (Dewe, 1993; Stephens and Gwinner, 1998) were also sources for this set of questions.

Although cognitive appraisal (i.e. primary and secondary appraisal) is not necessarily a linear process (Lazarus and Folkman, 1984), the set of questions about the retrospective role overload scenarios were, out of necessity, designed to expose the key features in a step by step manner, to ensure as much detail was recorded as possible. Although this risked duplication of data, this approach made it easier for the respondent to understand the questions, and for the interviewer to administer them. 
The set of ten questions asked for each role overload episode can be loosely grouped to target four areas of the cognitive appraisal and coping process. The four areas are: context (i.e. person and environment factors), primary and secondary appraisal, coping, and outcomes. Each of the four target areas with the corresponding (part II) interview question numbers, the thesis research questions they relate to, and a brief description of their intention are presented in Table 7-1. It was not necessary to make the distinction between these four areas in the protocol for the respondents or the interviewers. They are explained here to help the reader understand the interview protocol. These ten questions were repeated for the second retrospective role overload episode identified by the respondent to complete Part II of the interview. 


\begin{tabular}{|c|c|c|}
\hline MODEL & $\frac{\text { INTERVIEW QUESTIONS for }}{\text { Part II }}$ & $\frac{\text { PURPOSE/INTENT }}{\text { Ouestion) }}$ \\
\hline $\begin{array}{c}\text { Context - } \\
\text { Environment } \\
\text { \&Person }\end{array}$ & $\begin{array}{l}\text { 1. Please give this event a brief title } \\
\text { 2. What made you feel overloaded? } \\
\text { What was it about the situation } \\
\text { itself? }\end{array}$ & $\begin{array}{l}\text { Research Question } 1 \& 2 \\
\text { These questions were intended to draw out } \\
\text { descriptions of the various environment } \\
\text { factors or person factors pertinent to the } \\
\text { role overload episode as it was initially } \\
\text { perceived. }\end{array}$ \\
\hline $\begin{array}{l}\text { Primary \& } \\
\text { Secondary } \\
\text { Appraisal }\end{array}$ & $\begin{array}{l}\text { 3. What were your initial thoughts } \\
\text { about the situation? } \\
\text { 4. Did you feel that the situation was } \\
\text { harmful to you, or potentially } \\
\text { threatening in any way, or } \\
\text { challenging to you (if none of these } \\
\text { how would you describe it)? In } \\
\text { what way? } \\
\text { 5. What was your overriding feeling } \\
\text { about the situation? } \\
\text { I want to identify the potential } \\
\text { consequences of the situation: } \\
\text { a. What did it mean (to your goals } \\
\text { \& values)? } \\
\text { b. How was it likely to affect your } \\
\text { organisation/ boss/ colleague/ } \\
\text { patients/ family and what did that } \\
\text { mean to you? } \\
\text { This question is to determine how } \\
\text { well prepared you felt to respond to } \\
\text { the situation? } \\
\text { a. To what extent did you believe } \\
\text { the situation could be resolved } \\
\text { successfully? } \\
\text { b. Describe your initial thoughts } \\
\text { about how you could overcome } \\
\text { the situation? } \\
\text { What was the single most important } \\
\text { factor that made this situation } \\
\text { potentially overwhelming or } \\
\text { stressful for you? }\end{array}$ & $\begin{array}{l}\text { Research Questions } 3 \& 4 \\
\text { Q3-Q8 This set of questions seek to } \\
\text { discover the respondents perceptions } \\
\text { formed during the primary and secondary } \\
\text { appraisal processes, before the respondents } \\
\text { began to deal with the situation. } \\
\text { Q3 is aimed at capturing immediate } \\
\text { primary appraisals and any immediate } \\
\text { emotional response } \\
\text { Q4 \& } 6 \text { are intended to draw out the } \\
\text { specific components of primary appraisal } \\
\text { (i.e., harm, threat or challenge, and what is } \\
\text { at stake). Q6 is also intended to draw out } \\
\text { person factors affecting the appraisals. } \\
\text { Q } 5 \text { is aimed at capturing the emotional } \\
\text { outcome of the cognitive appraisal process } \\
\text { Q } 7 \text { is aimed at discovering the outcome of } \\
\text { the initial secondary appraisal (i.e. } \\
\text { availability and suitability of coping } \\
\text { resources, and initial plans for coping). } \\
\text { Q8 seeks to identify the key person or } \\
\text { environment factor contributing to the } \\
\text { overall appraisal. } \\
\text { All these questions and Q6\&8 in particular, } \\
\text { will also provide more information about } \\
\text { what and how environmental and personal } \\
\text { contexts influence the results of the } \\
\text { cognitive appraisal process (i.e. Research } \\
\text { Questions } 1 \text { \& 2). }\end{array}$ \\
\hline
\end{tabular}




\begin{tabular}{|c|c|c|}
\hline MODEL & $\frac{\text { INTERVIEW QUESTIONS for }}{\text { Part II }}$ & $\frac{\text { PURPOSE/INTENT }}{\text { Ouestion) }}$ \\
\hline Coping & $\begin{array}{l}\text { 9. I now want to ask what you did to } \\
\text { cope with the situation? } \\
\text { a. What actions did you take? } \\
\text { b. How did your actions differ from } \\
\text { your initial plan? } \\
\text { c. Why these action? Did they work } \\
\text { or not? In what way? } \\
\text { d. What actions did others take? Did } \\
\text { they work or not? In what way? } \\
\text { e. What did you do to deal with the } \\
\text { emotional aspects of the } \\
\text { situation? Why this? Did it work } \\
\text { or not? }\end{array}$ & $\begin{array}{l}\text { Research Question } 5 \\
\text { Q9 This question is intended to capture the } \\
\text { coping strategies undertaken to handle the } \\
\text { role overload. With the use of prompts, care } \\
\text { was taken to capture practical as well as } \\
\text { emotional coping responses, and personal } \\
\text { as well as actions by others. To capture the } \\
\text { notion of an unfolding process, respondents } \\
\text { were asked how initial plans differed from } \\
\text { actual events. This questions seeks to } \\
\text { capture coping outcomes, in terms of the } \\
\text { impact on the role overload, on personal } \\
\text { emotions and also on other person factors }\end{array}$ \\
\hline Outcomes & $\begin{array}{cl}10 . & \\
\text { a. How did the situation end? } \\
\text { b. Was this what you expected to } \\
\text { happen or not? } \\
\text { c. Why do you say this? } \\
\text { d. How did you feel at the end of } \\
\text { the situation? }\end{array}$ & $\begin{array}{l}\text { Research Question 1-5 } \\
\text { Q10 The last question of this set asks how } \\
\text { the role overload situation ended, how the } \\
\text { respondent felt, and how it fit with their } \\
\text { expectations. The responses to these } \\
\text { questions will be used to reveal any } \\
\text { moderating effects coping might have on } \\
\text { perceived stress from overload. }\end{array}$ \\
\hline
\end{tabular}

Table 7-1: Mapping of Part II interview questions to conceptual model and research questions.

\subsubsection{Quantitative data collection}

Part III of the interview protocol contains a ten item quantitative data instrument for measuring the personality traits of the respondents. Whilst research of cognitive appraisal of role overload has overall been scant, the literature review for this thesis revealed that in studies of stress, including role stress, there has been substantive research focused on the relationships between personality traits and stress, and personality traits and coping. From the literature review, we also know that dispositional personality traits are an important element in the cognitive appraisal process alongside other, more changeable, person factors (e.g. self esteem or beliefs about control), which also 
influence this process and hence the resultant level of perceived stress (Lazarus and Folkman, 1984; Aldwin et al. 1996).

The rise in interest in personality in the stress literature (as noted by Suls et al. 1996) was largely driven by the development of empirical personality trait measures based on the five factor model of personality (Costa and McCrae, 1985, 1992) also referred to as the Big-Five (Goldberg, 1990). Costa and McCrae's (1985) comprehensive 240 item measure, and even their shorter 60 item (NEO-FFI) measure developed in 1992, are restrictive for many types of research because of the time it takes to complete them. More recently, brief measures have been developed that are suitable for research where personality is just one element under examination, time is tight, and conciseness is a high priority (Saucier, 1994; Gosling, Rentfrow and Swann Jr., 2003; Donnellan, Oswald, Baird and Lucas, 2006). This offers the potential to collect additional data that can be used to complement the qualitative data collected to answer Research Question 5 (i.e., What person factors help us to understand the cognitive appraisal of/ differential stress response under role overload conditions). The literature has also suggested that including all five dimensions of personality in a single study can improve our understanding of the relationship between personality and the stress and coping process.

The Big Five model is a hierarchical framework that posits that most individual differences in personality can be categorized into five domains: Extraversion; Neuroticism; Openness to Experience; Agreeableness; and Conscientiousness. The ten item personality measure used for this thesis is based on Gosling et al.'s (2003) "Very brief measure of the Big Five personality domains". The remainder of this subsection will 
explain how Gosling et al. (2003) created and tested their measure, how it relates to the Big Five dimensions of personality, how it was modified for the interview protocol for this research, and how the final measure is scored and interpreted.

Gosling et al. (2003) selected ten pairs of descriptors, where each pair represents one pole of the five factor model. For example the two poles for the 'extraversion' dimension were Extraverted/Enthusiastic and Reserved/Quiet.

\begin{tabular}{|l|l|}
\hline $\begin{array}{c}\text { Dimension of } \\
\text { Personality }\end{array}$ & \multicolumn{1}{c|}{$\begin{array}{c}\text { Gosling et al. (2003) } \\
\text { Pairs of descriptors representing poles of personality } \\
\text { dimension }\end{array}$} \\
\hline Extraversion & Extraverted/Enthusiastic (1) \& Reserved/Quiet (6) \\
\hline Agreeableness & Sympathetic, warm (2) \& Critical, Quarrelsome (7) \\
\hline Conscientiousness & Dependable, self-disciplined (3) \& Disorganised, careless (8) \\
\hline Neuroticism & Anxious, easily upset (4) \& Calm, emotionally stable (9) \\
\hline Open to Experience & Open to new experiences, complex (5) \& Conventional, uncreative (10) \\
\hline
\end{tabular}

Table 7-2: Mapping of Gosling et al. (2003) descriptor pairs representing Big Five personality dimensions.

To create the 10 item measure Gosling et al. culled descriptors from existing measures, drawing most heavily from Goldberg's (1990) list of Big Five markers, which were in turn derived from Costa and McCrae's (1992) long measure. Gosling et al.'s (2003) objective was to retain breadth of coverage, and to represent both poles of each dimension whilst avoiding items that were extreme, and that were simply negations. The pairs of descriptors, representing the two poles of each personality dimensions, is shown in Table 7-2. The resulting 10 item personality inventory consisted of each of the pairs of descriptors, preceded by the phrase 'I see myself as". 
In the interview protocol for this thesis, each pair of Gosling et al.'s descriptors was replaced with the equivalent statements from Costa and McCrae's 60 item NEO-FFI measure combined as a single statement. For example, the Disorganized, careless descriptor pair (No. 8 in the Gosling et al. scale), representative of the low pole of the Conscientiousness factor, is represented in Costa and McCrae's NEO-FFI scale by the two statements "I never seem to be able to get organised / I sometimes make a mess of things". These two statements were combined in the interview protocol for this research as "I never seem to be able to get organised and I sometimes make a mess of things". This approach was considered to be a more appropriate and meaningful way to present the personality measure in a semi-structured telephone interview, whilst maintaining the brevity and design approach of Gosling et al.'s (2003) scale. The resulting personality dimension measure used in Part III of the interview protocol is shown in Table 7-3 alongside the equivalent Gosling et al. (2003) descriptors.

The scale was administered according to Gosling et al.'s instructions. A 7 point Likert scale was used to ask the extent to which the respondent agreed with each statement. Respondents were instructed to rate the extent to which the pair of traits applied to them, even if one characteristic applied more strongly than the other. The Likert scale used was: (1) Disagree Strongly; (2) Disagree Moderately; (3) Disagree a little; (4) Neither agree or disagree; (5) Agree a little; (6) Agree Moderately; (7) Agree Strongly.

Table 7-3 also shows the outcomes and question pairs for scoring each personality dimension. 


\begin{tabular}{|c|c|c|c|c|}
\hline $\begin{array}{l}\text { Dimension of } \\
\text { Personality }\end{array}$ & $\begin{array}{c}\text { Part III } \\
\text { Question No. } \\
\text { measuring } \\
\text { dimension }\end{array}$ & $\begin{array}{l}\text { Gosling et al. } \\
\quad(\mathbf{2 0 0 3 )} \\
\text { I see myself as: }\end{array}$ & $\begin{array}{c}\text { Part III Questions } \\
\text { measuring dimension } \\
\text { (using Costa and } \\
\text { McCrae (1992) } \\
\text { equivalent items) } \\
\end{array}$ & $\begin{array}{c}\text { Interpretation } \\
\text { of high score }\end{array}$ \\
\hline \multirow{3}{*}{ Extraversion } & Q1 & $\begin{array}{l}\text { Extraverted, } \\
\text { enthusiastic }\end{array}$ & $\begin{array}{l}\text { In groups I have belonged } \\
\text { to, I am the most } \\
\text { enthusiastic and often take }\end{array}$ & \multirow{3}{*}{$\begin{array}{l}\text { The individual is } \\
\text { highly extravert }\end{array}$} \\
\hline & & & the leadership role. & \\
\hline & Q6 (R) & Reserved, quiet & $\begin{array}{l}\text { Many people think of me as } \\
\text { quieter and somewhat } \\
\text { reserved }\end{array}$ & \\
\hline \multirow{3}{*}{ Agreeableness } & Q2 (R) & $\begin{array}{l}\text { Critical. } \\
\text { quarrelsome }\end{array}$ & $\begin{array}{l}\text { I am not interested in any } \\
\text { other people's problems } \\
\text { and pride myself on my }\end{array}$ & \multirow{3}{*}{$\begin{array}{l}\text { The individual is } \\
\text { highly agreeable }\end{array}$} \\
\hline & & & $\begin{array}{l}\text { shrewdness in handling } \\
\text { people. }\end{array}$ & \\
\hline & Q7 & $\begin{array}{l}\text { Sympathetic, } \\
\text { warm }\end{array}$ & $\begin{array}{l}\text { I go out of my way to help } \\
\text { or sympathise with people } \\
\text { if I can }\end{array}$ & \\
\hline \multirow{2}{*}{ Conscientiousness } & Q3 & $\begin{array}{l}\text { Dependable, self- } \\
\text { disciplined }\end{array}$ & $\begin{array}{l}\text { I like order and plan ahead } \\
\text { carefully for major events } \\
\text { in my work and home life. }\end{array}$ & \multirow[b]{2}{*}{$\begin{array}{l}\text { The individual is } \\
\text { highly } \\
\text { conscientious }\end{array}$} \\
\hline & Q8 (R) & $\begin{array}{l}\text { Disorganised. } \\
\text { careless }\end{array}$ & $\begin{array}{l}\text { I never seem to be able to } \\
\text { get organised and } \\
\text { sometimes make a mess of } \\
\text { things }\end{array}$ & \\
\hline \multirow[t]{2}{*}{ Neuroticism } & Q4 & $\begin{array}{l}\text { Anxious, easily } \\
\text { upset }\end{array}$ & $\begin{array}{l}\text { I often get upset or worry } \\
\text { about things that might go } \\
\text { wrong. }\end{array}$ & \multirow[t]{2}{*}{$\begin{array}{l}\text { The individual is } \\
\text { highly neurotic }\end{array}$} \\
\hline & Q9 (R) & $\begin{array}{l}\text { Calm, } \\
\text { emotionally } \\
\text { stable }\end{array}$ & $\begin{array}{l}\text { It takes a lot to make me } \\
\text { mad and I rarely feel fed } \\
\text { up. }\end{array}$ & \\
\hline \multirow{2}{*}{$\begin{array}{l}\text { Open to } \\
\text { Experience }\end{array}$} & Q5 & $\begin{array}{l}\text { Open to new } \\
\text { experiences, } \\
\text { complex }\end{array}$ & $\begin{array}{l}\text { I am open to new } \\
\text { experiences and have a } \\
\text { vivid imagination }\end{array}$ & \multirow{2}{*}{$\begin{array}{l}\text { The individual is } \\
\text { highly open to } \\
\text { experience }\end{array}$} \\
\hline & Q10 (R) & $\begin{array}{l}\text { Conventional, } \\
\text { uncreative }\end{array}$ & $\begin{array}{l}\text { I am not interested in, and } \\
\text { sometimes have difficulty } \\
\text { understanding abstract } \\
\text { ideas }\end{array}$ & \\
\hline
\end{tabular}

Table 7-3: Derivation and outcomes of personality measure used in Part III of interview protocol (Source: Gosling et al., 2003; Costa and McCrae, 1992). 
Each of the five personality trait constructs was calculated as the summed score of the two items in the scale, with one of the items in each pair being reversed coded. In each case higher scores reflect a higher level for the personality trait construct being measured.

Gosling et al. (2003) tested their scale against the measures of Costa and McCrae (1992) and Goldberg (1990). The 10 item instrument reached adequate levels in each of the criteria against which it was evaluated: convergent and discriminant validity, testretest reliability, and patterns of external correlates. Gosling et al. (2003) concluded that whilst the long measures are undoubtedly more psychometrically sound the relative efficiency of the brief scale makes it a very attractive research tool when respondent availability is restricted and personality is not the primary focus of the research.

\subsubsection{Administration of the interview}

The interview was administered to the volunteer health care employees by three PhD students from Carleton University, including the primary researcher, over a period of five months. Such use of multiple interviewees can, according to Eisenhardt (1989 p. 538)) "enhance the creative potential" of the study.

The interview protocol was developed iteratively in three stages. Before starting the interviews the three interviewees met to become familiar with the research objectives, under the directive of the primary researcher. Each interviewee then pre-tested the interview on family members working in the health sector but outside the setting for this 
research. This procedure was used to test the logic, flow and length of the interview. Lastly, each of the three interviewers completed two interviews with volunteer respondents from the Ottawa hospitals, and then met to evaluate the protocol. At this point, the primary researcher made adjustments to the prompts embedded in the questions to improve the breadth and relevance of data capture.

After asking the permission of each respondent, the interviews were recorded. The recordings were subsequently transcribed to ensure that a complete record was captured for the analysis process. This dual approach also improves the opportunity for systematic analysis. To protect the anonymity of the respondents, names were not mentioned during the recorded interview. All the interviewees agreed to be recorded and also gave permission for the transcription process. According to Yin (2009), audio taping provides a more accurate rendition of an interview than any other method, as long as permission is obtained, and the interviewers do not view it as a substitute for close listening and intervention, where necessary, to gain additional information.

The pre interview discussions and testing were aimed at ensuring consistent and appropriate interviewer attention during the data collection. Each interviewer also took notes during the process and randomly checked the recordings after the interviews to check for missing data. Notes collected by multiple investigators also benefited the theory building research approach, as they provided additional and, moreover, diverse viewpoints on emerging theory, giving further support to analysis results (Eisenhardt, 1989). The length of the interviews for the respondent sample selected for the thesis varied between 40 and 100 minutes. On average, the interviews of the respondents with 
high stress scores were marginally shorter (by 4 minutes) than the interviews of individuals with low stress.

\subsection{Data analysis}

Although both qualitative and quantitative data were collected for this thesis, qualitative data is the primary data source, and a case based qualitative analysis approach is critical to achieving this study's research objectives. As intimated in the descriptions of theory building analysis in Chapter 5, there are specific underlying qualitative data analytic techniques that together form an iterative and cyclical process as the researcher moves from a vast collection of words towards a theory that fits the data. Both Eisenhardt (1989) and Yin (2009) have acknowledged Miles and Huberman $(1984,1994)$ as the formulator of a comprehensive methodology for the analysis of case based qualitative data. Miles and Huberman's approach is used for this study.

One of Miles and Huberman's (1994) initial recommendations is that every case study requires a systematic way to store the analysed data. Yin (2009) also recommends that a suitable software application be used to support qualitative data analysis. NVivo data analysis software was chosen as a data storage and analysis tool for this thesis. NVivo is a computer assisted qualitative data analysis software tool developed by QSR International Pty Ltd. QSR recently released the $10^{\text {th }}$ version of this software and NVivo is now well established and commonly used for qualitative research. For example, a recent search of the Business Source Complete database for the term "NVivo" in the abstract of the articles, produced 83 journal article references. 
In preparation for data analysis, each of the two digitally recorded role overload episodes (i.e., one episode assessed as overwhelming the other not) recalled by the 20 respondents in our sample were transcribed and saved as Microsoft Word documents and then imported into NVivo to form the source documents for the thesis analysis. Using NVivo, these source documents were saved as individual case nodes. An NVivo node is a collection of references about a specific theme, concept or other area of interest. Case nodes are special types of nodes for which attributes can also be recorded. One attribute that can be recorded for these episodes, for example, is the stress level of the respondent describing the role overload. Case nodes thus reference all the data associated with a case or sub-case. The thesis case nodes reference the raw data describing an episode of role overload. Each pair of case nodes, referencing the two episodes described by a single respondent, were then linked together in the NVivo file system to a parent case node named for the respondent (with a made up moniker to ensure anonymity). Examples of how these source documents were stored and displayed in the NVivo case file system is shown in Figure 7-1. The less than signs in front of the episode case nodes indicate connection to the parent nodes representing the respondent cases in the left hand column. With this structure the data can be easily examined and hence analysed at the lowest case unit of analysis, the role overload episode, as well as at the respondent level as discussed in Section 6.2.

How NVivo was used to analyse the data for this thesis is woven into the description of Miles and Huberman's (1994) data analysis methodology presented in the two subsections to follow. Section 7.2.1 will detail the qualitative analysis approach as 
proposed by Miles and Huberman (1994) and specifically how it was implemented for this study. Section 7.2.2 will explain how the quantitative data was combined with the qualitative data to support the analysis. How case node attributes are implemented and used in NVivo to reference quantitative data will also be discussed in this section. In some areas, the terminology used in NVivo differs to that used by Miles and Huberman (1994). The relationship between the two nomenclatures will be clearly stated as the methodology steps are explained. Section 7.2.3 will explain how results are interpreted during analysis.

\begin{tabular}{|c|c|}
\hline $\begin{array}{c}\text { Respondent } \\
\text { Cases }\end{array}$ & $\begin{array}{c}\text { Role Overload Episode } \\
\text { Cases }\end{array}$ \\
\hline Anna & $<$ Not-overwhelming episode \\
& $<$ Overwhelming Episode \\
& $<$ Not-overwhelming episode \\
Beth & $<$ Overwhelming Episode \\
& $<$ Not-overwhelming Episode \\
Cate & $<$ Overwhelming Episode \\
& $<$ Not-overwhelming Episode \\
& $<$ Overwhelming Episode \\
\hline Dawn & \\
&
\end{tabular}

Figure 7-1 Example display of respondent and associated role overload episodes stored as NVivo case nodes

\subsubsection{Case based qualitative data analysis}

The first stage in Miles and Huberman's (1994 p. 56) data analysis method is to reduce the large volume of qualitative data by developing codes to serve as "retrieval and organizing devices" that subsequently allow the analyst to manipulate the data more 
easily. Miles and Huberman (1984) describe codes as abbreviations, or tags, for assigning concise meanings to a segment of descriptive data. The outcome of this iterative process is a structured representation of the coded data, commonly called a coding scheme. Assigning codes in this way means that it becomes easier to identify, and gather together, all data segments relevant to a particular theme, construct, or research question. Moreover, in coded form the data can be counted, organised, and displayed to support theoretical inferences (Johnson, 2007). Section 7.2.1.1 details the creation of the coding scheme. Section 7.2.1.2 describes how the coded data is displayed to support the final stages of analysis.

\subsubsection{Creation of the coding scheme}

There are multiple types of codes that can be selected when creating a coding scheme, and which types are chosen and how they are used are driven by the research objectives as espoused in the conceptual framework and research questions (Miles and Huberman, 1994; Yin, 2009). Initially, the types of codes assigned to segments of text are descriptive. As the analysis and coding continues on a within-case basis, the researcher becomes more knowledgeable about the dynamics of the research site (in this thesis the four hospitals), and may also note patterns emerging across the multiple case units. Codes

are assigned to highlight these patterns and to add elements of interpretation to the coding scheme as they are confirmed (Miles and Huberman, 1994). This is the beginning of the second stage of the process, cross-case analysis, which effectively overlaps with the first stage of within-case analysis. Making notes in the database as the coding proceeds makes 
the identification of patterns more efficient and easier. If too many segments of data get the same code, sub-codes are created and the coding scheme revised accordingly. In this way the coding scheme is continually updated and refined as the researcher analyses the interview data (Miles and Huberman, 1994).

Using the NVivo software the analysis process was enacted as follows. The source of each role overload episode captured and imported into NVivo for this thesis was thoroughly scanned by the researcher. Any and all descriptive data segments thought relevant to the thesis objectives and research questions were coded at independent, and initially unrelated, 'free nodes' created with the NVivo software. Within NVivo, coding involves: 1) highlighting the piece of text of interest, 2) either creating a new NVivo node with a suitable name to reflect the data referenced, or selecting an existing node whose name reflects the highlighted data, and 3) saving the 'free node' now linked to the piece of descriptive text. This NVivo process is equivalent to within-case analysis as described above.

As the within-case analysis is repeated for each role overload episode stored in NVivo, the second step of the analysis process began. As other pieces of descriptive data, in other role overload episodes, were coded to existing 'free nodes' or, to new 'free nodes' created as required, the researcher noted similarities between certain independent 'free nodes', both within and across cases. These nodes were, therefore, gathered together and linked to a new 'parent node'. The 'parent node' was given a name (or label) to reflect the diverse but related nature of the captured 'child nodes' underneath. As the analysis of the role overload episodes continued, other related 'free nodes' were 
identified and linked together to create more new 'parent nodes'. This NVivo process formed the initial stages of cross-case analysis as described by Miles and Huberman (1994). The NVivo coding technique described above and used repetitively in within and cross-case analysis to compare each piece of descriptive text in the raw data to existing codes, is also illustrative of the constant comparison technique described by Eisenhardt (1989) in their recommendations for theory building case studies.

The cross-case analysis process described began to add structure and elements of interpretation to the growing coding scheme. In NVivo, when parent and child nodes are linked in this way, this is described as a node hierarchy. Node hierarchies can have multiple levels of 'tree nodes' (as 'parent nodes' and 'child nodes' are collectively referred to, to distinguish them from singular and independent 'free nodes'). As also suggested by Miles and Huberman (1994), notes were periodically taken during crosscase analysis (using NVivo 'annotations' and 'memos') to aid the researcher during analysis. 'Annotations' are akin to scribbled notes in the margins of a document. In NVivo, annotations are used to make short comments about particular content linked to a node. 'Memos' are a type of document within NVivo that allows the researcher to record interpretations and growing ideas about underlying theory. Memos can be used to help document the analysis process after completion. 'Annotations' and 'memos' are automatically linked to the node about which the note is written for future perusal or amendment.

As the cross-case analysis continued for this thesis, the researcher observed similarities across multiple 'tree nodes' within NVivo, which were therefore collected 
together at another new 'parent node' in the node hierarchy, with a name to reflect the characteristics of the observed connections. Similarly, when too many segments of data were collected at a single node (either a free node, or a tree node), the data was reexamined and appropriate 'child nodes' were created to represent sub-categories. Each segment of data was re-coded to one of the new child nodes (according to the new level of classification embodied in the child node name) and un-coded from the original node. As this process continued larger node hierarchies were developed within NVivo, to form a network of connected data representative of the initial within and cross-case analysis of the data.

An example of part of the NVivo node hierarchy created from the thesis data is presented in Table 7-4 to illustrate the analysis process and their results. In this example, the table highlights (using italic font) the step by step creation of one specific branch of the NVivo node hierarchy that has five layers. Each step (and therefore each hierarchical layer) is represented by the columns of the table.

The first column in Table 7-4 represents the raw data and gives three examples of comments made by respondents in separate role overload episodes. Each describes details of an event that triggered the role stress recalled. Each of these segments of descriptive text was coded using NVivo to three separate and independent 'free nodes'. The names given to the nodes are displayed in column two. This coding process exemplifies the first stage within-case analysis process described above. As the analysis progressed, other episodes revealed similar text referring to 'family illness or crises', and other interviews described more occurrences of 'personal illnesses'. The associated descriptive text from 
these other episodes was also coded to the appropriate 'free nodes'. Thus begins the second stage cross-case analysis, in which similar data from multiple case units (i.e. episodes) are gathered together under a single code (i.e. NVivo node). The result, at this point, was three, as yet unrelated and independent, 'free nodes' (listed in column two of Table 7-4), two of which contained multiple references to raw text in multiple episodes (number of references not shown).

\begin{tabular}{|c|c|c|c|c|}
\hline $\begin{array}{l}\text { Descriptive text from } \\
\text { interviews describing } \\
\text { events in role overload } \\
\text { episodes }\end{array}$ & $\begin{array}{l}\text { Initial free nodes } \\
\text { referencing types of } \\
\text { personal or family } \\
\text { illnesses or crises related } \\
\text { to role overload in the } \\
\text { recalled role episodes }\end{array}$ & $\begin{array}{l}\text { Tree nodes referencing } \\
\text { types of event trigger }\end{array}$ & $\begin{array}{l}\text { Tree nodes } \\
\text { referencing } \\
\text { environment } \\
\text { themes }\end{array}$ & $\begin{array}{c}\text { Tree node } \\
\text { representing } \\
\text { element of } \\
\text { role } \\
\text { overload } \\
\text { appraisal } \\
\text { process } \\
\end{array}$ \\
\hline $\begin{array}{c}\text { "mother in law needed } \\
\text { intervention - had to be put in a } \\
\text { home" } \\
\text { "I had a huge operation in } \\
\text { which I wasn't able to do } \\
\text { anything for } 3 \text { weeks" } \\
\text { "Our furnace blew" }\end{array}$ & $\begin{array}{c}\text { Family Illness or Crises> } \\
\text { Personal Illness }> \\
\text { Crises at home }>\end{array}$ & $\begin{array}{r}\text { Everyday demands }> \\
\text { Personal or Family Crises }> \\
\\
\text { Extracurricular Task > } \\
\text { High Profile Corporate Task > } \\
\text { Interpersonal Issues > } \\
\text { Client/Patient Issues } \\
\text { Staffing Shortages > } \\
\text { Other> }\end{array}$ & $\begin{array}{c}\text { Event Trigger > } \\
\text { Situation Properties > }\end{array}$ & Environment \\
\hline
\end{tabular}

Table 7-4 Illustration of part of the NVivo node hierarchy created during within-case and cross-case analysis of role overload episodes 
With growing numbers of nodes referencing other data relevant to the thesis objectives and research questions, the researcher noted the similarities between these three nodes. The researcher therefore brought them together as 'child nodes' jointly connected to a new 'parent node' labeled 'personal/family crises'. The node 'personal/family crises' appears in the third column of Table 7-4 with links indicated by arrows from the three child nodes. The title of this third column is 'Tree nodes referencing types of event trigger'. The additional 'tree nodes' listed in this column (e.g. 'everyday demands' and 'staffing shortages') reference other role overload event triggers created through an analysis process that was virtually identical to that just described. The various analyses undertaken for this thesis ran simultaneously with the process exemplified in Table 7-4, illustrating the overlapping and iterative nature of within-case and cross-case analysis. Child nodes of these other event triggers are not shown in the table, however, all eight different types of event were subsequently gathered together at a second level tree node and collectively labeled 'event triggers', as shown in the fourth column of the table.

Role overload event triggers were identified by the researcher as one facet or theme of the role overload environment (hence the fourth column title "tree nodes referencing environment themes). Another theme was the situation properties of role overload events associated with perceived stress. Continuing the cross-case analysis, the researcher collected additional references to these role overload properties (not shown) at the node labeled 'situation properties' (listed in column four of Table 7-4). The role overload environment themes were collected together under the highest level tree node 
labeled simply, 'environment', and shown in the final column of Table 7-4. The 'environment' node also represents one of the boxes of the conceptual framework guiding this thesis and as such is linked to research question 1 and 6 . Table 7-4 and the accompanying description thus illustrate the analysis process from raw data to a structured set of connected data for one element of the conceptual framework that is available for the next stages of theory development.

The top two levels of the complete multi-layered node hierarchy resulting from these early stages of the analysis process is shown in Table 7-5, as it is displayed in NVivo, with all the highest hierarchical level tree nodes listed in the left most column in alphabetical order and the tree nodes of the second level shown in the second column (with arrows indicating the link back to the appropriate parent node). The highest level hierarchical NVivo nodes are named for all five key elements of the cognitive stress process shown in the conceptual framework in Figure 4.1 (with primary and secondary appraisal collected together under a single node named 'appraisal') and hence link to the research questions presented in Part 3 of this thesis.

While the questions of the respondent interview were designed to provide answers to the research questions and therefore target the key elements of the cognitive stress process (see Table 7-1 in Section 7.1.1), the semi-structured nature of the phone interview meant that many concepts emerged randomly throughout the interview. To ensure that all information was captured, such data was coded to new or to existing relevant NVivo nodes wherever they occurred in the interview. This approach to 
analyzing raw data allows concepts to arise that are potentially outside the guiding framework reflected in the top level hierarchy of nodes presented in Table 7-5.

\begin{tabular}{|c|c|}
\hline \multicolumn{2}{|c|}{ NVivo Node Hierarchy } \\
\hline $\begin{array}{c}\text { Tree node } \\
\text { representing element } \\
\text { of role overload } \\
\text { appraisal process }\end{array}$ & $\begin{array}{c}\text { Second level tree nodes related to } \\
\text { elements of role overload appraisal } \\
\text { process }\end{array}$ \\
\hline \multirow{3}{*}{ Appraisal } & $<$ Appraisal Outcomes \\
\hline & $<$ Primary Appraisal - What's at stake \\
\hline & $<$ Secondary Appraisal - What can I do about it \\
\hline \multirow[t]{2}{*}{ Coping } & $<$ Demand-reduction strategies \\
\hline & $<$ Demand-management strategies \\
\hline \multirow{3}{*}{ Environment } & $<$ Event Triggers \\
\hline & $<$ Situation Properties \\
\hline & $<$ Other Role Factors \\
\hline \multirow[t]{3}{*}{ Outcomes } & $<$ Personal Outcomes \\
\hline & $<$ Role Overload Outcomes \\
\hline & $<$ Attributes \\
\hline \multirow[t]{2}{*}{ Person Factors } & $<$ Beliefs \\
\hline & $<$ Internal Role Pressures \\
\hline
\end{tabular}

Table 7-5 Top two levels of final NVivo node hierarchy completed after first two stages of analysis

Other concepts related to role overload appraisal and coping, but previously unidentified in the literature, were also coded during these initial stages of the analysis process. These concepts will be presented under the appropriate broad headings related to the thesis's conceptual framework in the results chapters in the next part of this thesis. In this way, the flexibility and ease of use of NVivo supports the creation of top level tree nodes that allowed the researcher to ostensibly collect the data together for presentation in results chapters (which are both relevant to the research questions and more easily read by the reader), whilst also allowing new concepts to emerge at any level. 


\subsubsection{Displaying the coded data}

The next stage of analysis involves the use of systematically created data displays. Presenting empirical evidence for embedded case studies is a particular challenge for the theory building case study approach. According to Eisenhardt and Graebner (2007) to deal with this challenge it is best to develop theory in sections, organise the results around the theory, and make extensive use of tables and other visual devices to summarise case evidence. In particular, they argue that evidence for theoretical constructs should be presented in separate tables, confirming the approach recommended by Miles and Huberman (1994).

The format of the displays in this thesis are driven by the research questions developed in Chapter 4 and the concepts represented in the emerging NVivo node hierarchy of coded data pertinent to role overload episodes. Data displays allowed the researcher to develop initial hypothesis to explain role overload appraisal by observing the relationships between possible variables (Miles and Huberman, 1994). The data displays were also used by the researcher to compare results across the role overload episode cases to confirm or refute the validity of observed relationships.

During the creation of the coding scheme, NVivo automatically kept count of the number of role overload episodes in which any piece of coded data featured. Having recorded the codes in a database management system it was therefore easy to produce role overload episode counts for each coded response for the whole data set, for a particular question, or for a group of cases. NVivo offers a number of alternative ways to search the data captured in the node hierarchies (e.g. text searches, word frequencies, 
coding, matrix coding, and compound queries). For this thesis, matrix coding queries were used extensively to create specific coded data displays with the associated role overload episode counts. In these matrix data displays, results for specific collections of coded data (usually equivalent to the second level node hierarchy themes identified in Table 7-5 above) are presented for the whole set of 40 role overload episodes and for researcher specified groups of cases (i.e., episodes assessed as overwhelming or notoverwhelming; or by the respondent stress level groups: high or low). All the parent child nodes for the specific collection of coded data are represented in the data display. An example data display is provided in Table 7-6. These displays of counts enables the researcher to firstly get a clearer picture of what the coded data is showing by indicating the relative importance of a particular coded response to role overload episodes in a group. Counts are also used to observe patterns and highlight data that suggested leads for further investigation. According to Miles and Huberman (1994) these are some of the key benefits of counting qualitative data codes.

How the results in these matrix data displays are presented, and how they are used to continue the cross-case analysis process, form an important and inseparable part of the results and discussion part of this thesis. For this reason, the detailed formats of the qualitative data matrix tables created using NVivo queries and the step by step explanation of the results presentation protocol will be explained at the beginning of part five of this thesis rather than in this, the methodology chapter. 


\begin{tabular}{|c|c|c|c|c|c|c|}
\hline \multirow[b]{4}{*}{ Other Role Factors } & \multicolumn{4}{|c|}{ Role Overload Episode Assessment } & \multirow{2}{*}{\multicolumn{2}{|c|}{$\begin{array}{l}\text { Total Episodes } \\
\qquad(\mathrm{n}=\mathbf{4 0})\end{array}$}} \\
\hline & \multicolumn{2}{|c|}{$\begin{array}{c}\text { Not- } \\
\text { overwhelming } \\
\text { Episodes }(n=20)\end{array}$} & \multicolumn{2}{|c|}{$\begin{array}{l}\text { Overwhelming } \\
\text { Episodes }(n=20)\end{array}$} & & \\
\hline & \multicolumn{6}{|c|}{ \# \& \% of episodes in which this characteristic was mentioned } \\
\hline & \# & $\%$ & \# & $\%$ & \# & $\%$ \\
\hline Role Domain & 20 & 100 & 20 & 100 & 40 & 100 \\
\hline Work Situation & 12 & 60 & 13 & 65 & 25 & 62.5 \\
\hline Family - Home Situation & 4 & 20 & 4 & 20 & 8 & 20 \\
\hline Work and Home & 4 & 20 & 3 & 15 & 7 & 17.5 \\
\hline Other Role Stresses & 8 & 40 & 7 & 35 & 15 & 37.5 \\
\hline Intra-Role Conflict & 4 & 20 & 3 & 15 & 7 & 17.5 \\
\hline Work Family Conflict & 4 & 20 & 3 & 15 & 7 & 17.5 \\
\hline
\end{tabular}

Table 7-6 Example data display showing role factor results for the total sample and by episode assessment

\subsubsection{Merging the qualitative and quantitative data}

As discussed in this thesis earlier, quantitative data can be used in a variety of ways in a case study where the primary source of data is qualitative data. Quantitative data collected in the survey that preceded this thesis has already been used to purposefully select the cases for this study. An additional piece of quantitative data was collected in the interview for each of the respondents, providing measures of five personality traits. In a theory building case study, in which qualitative data is the primary source of data, quantitative data is intended to provide an additional source of data to triangulate qualitative data at the case level (Yin, 2009). In other words, the quantitative data provides extra information about the individual suffering role overload, in much the same manner as the descriptive data. Yin (2009) further argues that it is not pertinent to aggregate data across case units to arrive at group means. The respondent sample was not chosen to support statistical analysis and therefore data should remain focused at the case 
level. The stress level scores collected in the survey were calculated for each respondent and used to determine whether individuals had high levels or low levels of perceived stress and then used in the sample selection process (see Section 6.3). The scores for each personality trait measure were calculated for each respondent, as described in Section 7.1.2. To facilitate a simplified display of this data each of the five trait scores for each respondent were also categorized, this time as high, medium, or low scores, according to a three way proportionate division of the 7 point scale used to measure the traits (i.e. mean scores less than 2.33 were coded 'low'; 2.33 to 4.66 , 'moderate'; and greater than 4.66, 'high').

The stress level and the five personality trait level scores are all examples of descriptive data that can be stored as attributes for each respondent case node within NVivo. Through the use of NVivo queries, data can then be examined by attribute values as well as for specific coded qualitative data, as already described. These high, medium, low scores for personality traits can then be compared across cases in the same manner as the presence or absence of a piece of descriptive text represented by a qualitative code. Personality traits (or for that matter coded qualitative data) can also be examined for the two groups of cases determined by the high or low values of the stress level attributes also recorded at the case nodes. An example quantitative data display is provided in Table 7-7. 


\begin{tabular}{|c|c|c|c|c|c|c|}
\hline \multirow{4}{*}{$\begin{array}{c}\text { Personality Trait } \\
\text { Score }\end{array}$} & \multicolumn{6}{|c|}{ Hospital Workers - Stress Level } \\
\hline & \multicolumn{2}{|c|}{$\begin{array}{c}\text { Low } \\
(n=10)\end{array}$} & \multicolumn{2}{|c|}{$\begin{array}{c}\text { High } \\
(n=10)\end{array}$} & \multicolumn{2}{|c|}{$\begin{array}{c}\text { Total } \\
(n=20)\end{array}$} \\
\hline & \multicolumn{6}{|c|}{$\#$ and $\%$ of hospital workers with this level of the personality trait } \\
\hline & \# & $\%$ & $\#$ & $\%$ & \# & $\%$ \\
\hline Low & 2 & 20 & 6 & 60 & 8 & 40 \\
\hline Medium & 4 & 40 & 2 & 20 & 6 & 30 \\
\hline High & 4 & 40 & 2 & 20 & 6 & 30 \\
\hline
\end{tabular}

Table 7-7 Example display of quantitative data for personality trait scores of low and high stress level respondents

For this table, the individual personality trait scores are grouped into the three categories.

The display show how many respondents scored low, medium, and high on this trait across the whole respondent sample, as well within the respondents groups with the lowest stress level scores and those with the highest.

The quantitative survey data, previously collected from the respondents of this study, can also be merged with the qualitative data in the same way as described above. Constructs that were relevant to this analysis are identified in the results section of this thesis.

\subsubsection{Interpreting the results}

The criteria for interpreting case study data is the final key component of research design for case studies and also the least developed area of case study research according to Yin (2009). There is no precise way to interpret findings in case study data analysis and statistical techniques are inappropriate where the sample has been purposefully selected to support theory development (Ibid.), as they are in this case study research. Proponents of case study and qualitative research specifically recommend that findings 
be judged by their substantive significance (Miles and Huberman, 1994; Patton, 2002;

Yin, 2009). Attaching significance to what has been found allows the researcher to then offer explanations, draw conclusions and extrapolate lessons (Patton, 2002). The argument for substantive significance is made by presenting findings and conclusions that are judged to deepen the understanding of the phenomenon, to be sound and consistent in support of the findings, and to be related to other findings in the study (Ibid).

In this study, data displays typically present frequency counts of the number of role overload episodes in which the specified coded data is cited. Such frequency counts are presented in descending order for the total sample first. Tables comparing the findings by episode assessment (overwhelming versus not-overwhelming) and stress level (high versus low) are also used. Such displays are a convenient way to view the masses of data that qualitative analysis produces and allows the researcher to identify constructs and seek patterns and connections in the data that are important to the research objectives of the thesis. Presenting the coded data in tables also allows readers to make their own judgments about patterns in the data. The difficulty lies in judging to what extent constructs, patterns or connections are important (i.e., substantive)

Miles and Huberman (1994) warn against quantitative modes of thinking when interpreting data displays showing frequency counts and Yin (2009) suggests that speculating on subtle patterns be avoided in favour of gross patterns whose interpretations are less likely to be challenged. In this thesis the extent to which constructs and observed patterns are judged to be important is determined firstly by the relative ordering of the episode frequency counts presented in the tables. In other words, 
the rank of the episode frequency counts of the coded data presented in any table is more important than the quantitative difference between the counts. Similarly, the relative ranking of episode frequency counts within compared groups of cases is used to suggest connections or relationships between coded data and groups of cases important to the research objectives of the thesis. For example, with reference to the data in the example display in Table 7-6, we would judge that 1) work is the most salient role overload domain for respondents in this case study, and 2) is equally important in overwhelming and not-overwhelming episodes. As relationships are identified, they are examined in the light of previously noted connections. Previous interpretations are thus rejected, modified, or solidified as the researcher moves back and forth between the descriptions of what has happened and the interpretations of those descriptions. This approach is consistent with Eisenhardt's (1989) and Yin's (2009) iterative approach to building theory from case studies. 


\section{PART FIVE: RESULTS}

This part of the thesis presents the results of the role overload appraisal case study of female health care workers. The data come primarily from the qualitative analysis of the in-depth interviews in which respondents recalled and answered questions about personal role overload incidents. When relevant, the results also incorporate quantitative data on personality (from a respondent measure completed at end of the interview) and role salience (which was collected in a survey that preceded the interviews). The first objective of this case study is to develop a theoretical framework describing the relationship between role overload and perceived stress. In order to understand the role overload stress process we will examine the relationship between the environment and the person, how role overload is appraised, what actions were taken to cope with the role overload and how the outcomes were re-appraised.

The structure of this part of the thesis mirrors the key elements of this cognitive appraisal and coping process and hence the research questions. The decision was taken to look at the environment and person factors first as, according to Lazarus and Folkman's (1984) model (the starting point for this thesis), it is the transaction that takes place between these two elements that will determine how role overload is appraised. More specifically, environment and person factors are always interdependent as "their significance for stress and coping derives from the cognitive processes that give weight to one in the context of the other" (Ibid, p.116). 
Chapter 8 examines which features of the environment impact the level of perceived stress under role overload conditions. Chapter 9 discusses what personality traits and other person factors of the individual hospital workers help us to understand the role overload episode and any differential stress results. Appraisal, both primary and secondary, is the subject of Chapter 10. More specifically, this chapter reviews how appraisal impacts the differential stress response to role overload. Chapter 11 presents findings on the coping actions taken to deal with role overload and how they relate to the level of perceived stress and Chapter 12 assesses the outcomes of the role overload events and their relationship to stress experiences.

The second objective of this thesis is to explain the differential stress experience of individuals who suffer role overload. Achievement of this objective requires a specific presentation style of results in each of the chapters listed above, to support these later stages of the analysis process. The rest of this introduction will explain the tactics used to make the results chapters more meaningful and relevant to the objectives of the thesis. These explanations also provide a deeper insight into the qualitative analysis and theory building process used in this thesis.

\section{Display of thesis results}

Having analysed the raw qualitative data and created the complete NVivo node hierarchy representing the coded and structured qualitative data (the top two levels of which are displayed in Table 7-5, Section 7.2.1.2), the next stage is to systematically display the data to support the continued cross-case analysis and theory development. 
The display tables thus, not only present the results, they are also used to continue the analysis process to answer the research questions and fulfill the thesis objectives. The second level tree nodes listed in the second column of Table 7-5 become (in most cases) the subject of sections within each results chapter to follow. The researcher used NVivo queries to create the qualitative data displays for these sections. Displays of quantitative data were created by hand. Three types of tables will be used to display the results discussed in this thesis. Examples of the three table types are shown in Tables 7-8, 7-9 and 7-10 and discussed below.

\begin{tabular}{|c|c|c|c|c|c|c|}
\hline \multirow[b]{4}{*}{ Other Role Factors } & \multicolumn{4}{|c|}{ Role Overload Episode Assessment } & \multirow{2}{*}{\multicolumn{2}{|c|}{$\begin{array}{c}\text { Total Episodes } \\
(n=40)\end{array}$}} \\
\hline & \multicolumn{2}{|c|}{$\begin{array}{c}\text { Not- } \\
\text { overwhelming } \\
\text { Episodes }(n=20)\end{array}$} & \multicolumn{2}{|c|}{$\begin{array}{l}\text { Overwhelming } \\
\text { Episodes }(n=20)\end{array}$} & & \\
\hline & \multicolumn{6}{|c|}{ \# \& \% of episodes in which this characteristic was mentioned } \\
\hline & \# & $\%$ & \# & $\%$ & \# & $\%$ \\
\hline Role Domain & 20 & 100 & 20 & 100 & 40 & 100 \\
\hline Work Situation & 12 & 60 & 13 & 65 & 25 & 62.5 \\
\hline Family - Home Situation & 4 & 20 & 4 & 20 & 8 & 20 \\
\hline Work and Home & 4 & 20 & 3 & 15 & 7 & 17.5 \\
\hline Other Role Stresses & 8 & 40 & 7 & 35 & 15 & 37.5 \\
\hline Intra-Role Conflict & 4 & 20 & 3 & 15 & 7 & 17.5 \\
\hline Work Family Conflict & 4 & 20 & 3 & 15 & 7 & 17.5 \\
\hline
\end{tabular}

Table 7-8 Example of first table type used to display results

The first table classifies the data according to the respondent assessment of the role overload experience (overwhelming versus not-overwhelming). The three columns in this table display findings as follows: for episodes assessed as not-overwhelming $(n=20)$; for episodes assessed as overwhelming $(n=20)$; and for the full set of 40 episodes.

The second table in each section will be used to present the data according to the stress level of the respondents. In this case, the three columns in the table display findings 
for the 20 role overload episodes recalled by low stress respondents, the 20 episodes recalled by those with higher levels of reported stress, and the results using the full set of 40 episodes.

\begin{tabular}{|c|c|c|c|c|c|c|}
\hline \multirow[b]{4}{*}{ Other Role Factors } & \multicolumn{6}{|c|}{ Role Overload Episodes by Respondent Stress Level } \\
\hline & \multicolumn{2}{|c|}{ Low $(n=20)$} & \multicolumn{2}{|c|}{ High $(n=20)$} & \multicolumn{2}{|c|}{$\begin{array}{c}\text { Total Episodes } \\
\qquad(n=40)\end{array}$} \\
\hline & \multicolumn{6}{|c|}{ \# \& \% of episodes in which this characteristic was mentioned } \\
\hline & \# & $\%$ & \# & $\%$ & \# & $\%$ \\
\hline Role Domain & 20 & 100 & 20 & 100 & 40 & 100 \\
\hline Work Situation & 14 & 70 & 11 & 55 & 25 & 62.5 \\
\hline Family - Home Situation & 4 & 20 & 4 & 20 & 8 & 20 \\
\hline Work and Home & 2 & 10 & 5 & 25 & 7 & 17.5 \\
\hline Other Role Stresses & 12 & 60 & 11 & 55 & 23 & $\mathbf{5 7 . 5}$ \\
\hline Intra-Role Conflict & 6 & 30 & 2 & 10 & 8 & 20 \\
\hline Work Family Conflict & 3 & 15 & 4 & 20 & 7 & 17.5 \\
\hline
\end{tabular}

Table 7-9 Example of second table type used to display results

\begin{tabular}{|c|c|c|c|c|c|c|c|c|c|c|}
\hline \multirow{5}{*}{$\begin{array}{c}\text { Other Role } \\
\text { Factors }\end{array}$} & \multicolumn{8}{|c|}{ Role Overload Episodes by Respondent Stress Level } & & \\
\hline & \multicolumn{4}{|c|}{ Low $(N=10)$} & \multicolumn{4}{|c|}{ High $(\mathbf{N}=10)$} & & \\
\hline & \multicolumn{2}{|c|}{$\begin{array}{c}\text { Not- } \\
\text { overwhelming } \\
\text { Episodes } \\
(\mathbf{n}=\mathbf{1 0}) \\
\end{array}$} & \multicolumn{2}{|c|}{$\begin{array}{l}\text { Overwhelming } \\
\text { Episodes } \\
(\mathbf{n}=10)\end{array}$} & \multicolumn{2}{|c|}{$\begin{array}{c}\text { Not- } \\
\text { overwhelming } \\
\text { Episodes } \\
(\mathbf{n}=\mathbf{1 0})\end{array}$} & \multicolumn{2}{|c|}{$\begin{array}{l}\text { Overwhelming } \\
\text { Episodes } \\
(\mathbf{n}=10)\end{array}$} & \multicolumn{2}{|c|}{$\begin{array}{c}\text { Total } \\
\text { Episodes } \\
(n=40)\end{array}$} \\
\hline & \multicolumn{10}{|c|}{ \# \& \% of episodes in which this characteristic was mentioned } \\
\hline & \# & $\%$ & \# & $\%$ & \# & $\%$ & \# & $\%$ & \# & $\%$ \\
\hline \multicolumn{11}{|l|}{ Role Domain } \\
\hline Work and Home & 2 & 20 & 0 & 0 & 2 & 20 & 3 & 30 & 7 & $\mathbf{1 7 . 5}$ \\
\hline Other Role Stresses & 7 & 70 & 5 & 50 & 6 & 60 & 5 & 50 & 23 & 57.5 \\
\hline Intra-Role Conflict & 4 & 40 & 2 & 20 & 1 & 10 & 1 & 10 & 8 & 20 \\
\hline
\end{tabular}

Table 7-10 Example of third table type used to display results

The third table will display the findings obtained when the data is categorized using both stress levels and role overload episode assessment (see Table 7-10). To avoid repetition only those row items showing differences across the four groups will be shown. 
Where the number of data items to be considered is small (i.e., a small number of rows) the second table will be omitted and all items will be presented in the third table type format. In these cases, data results for low and high stress respondents can be calculated by summing the appropriate columns.

The three types of tables will follow a common row display format, showing parent NVivo nodes, along with their children to fully illustrate themes in the data represented by the node hierarchy coded for the subset of data being examined. Two types of data are shown in each column: the number of episodes in which respondents gave a particular response and the percentage of the particular episode group in which the response was cited. Aggregated totals for parent nodes may not always add up to 100 per cent as multiple child node items can appear in any one episode. To increase transparency all the data is displayed in the first two tables for each parent and child node coded for the subset of data under examination. The full set of data for the third table type is provided in the Appendix. Exceptions to the display formats described here will be noted where appropriate.

\section{Reporting of thesis results}

For these final steps of the analysis process, a strict protocol was applied to the narrative presentation of the three display tables in order to focus on the thesis objectives. The analysis process described in Section 7.2.1 produced very large amounts of data for this thesis. It is not possible, therefore, to present all the data and all the stages of the analysis in detail. Rather we must focus on providing the data and analysis to answer the 
research questions and the objectives of this thesis. Moreover, a mechanism was required to make the thesis readable. Accordingly, not all results displayed in the tables will be discussed in the text. This does not mean that the findings not discussed are unimportant but instead that they are not what the researcher would view as key to the objectives of the thesis. Readers are free, nevertheless, to peruse all the data and make their own judgments'. Further details on the mechanism used in this thesis to improve readability are given below.

For each subset of data under consideration, the results for the full set of 40 role overload episodes are presented first (Total Column, Table Type One). This approach provides the reader with an overview of what the node hierarchy is showing whilst examining the prevalence and, hence, the relative importance of the coded responses within the total group of role overload episodes. The narrative will move from those row items mentioned most often to those mentioned least often within any level. The rows presenting findings for the parent nodes will be examined first. Findings for the individual child node items beneath these broad parent themes will then be presented. Example respondent comments will be used to illustrate the meaning of the node labels (i.e., the codes for collections of descriptive data segments). Items mentioned in fewer than 4 out of 40 episodes will not be discussed. The next stage in the analysis process is to examine the data according to the assessment of the role overload episode. Again, the first table type is used (e.g. Table 7-8) but the focus will be on the first two columns that display results for the 20 not-overwhelming episodes and the 20 overwhelming episodes respectively. Similarities between these two role overload episode assessment groups will 
be examined first before turning to the differences. As a rule of thumb, only items in the two role overload experience groups where the relative counts differ by at least three episodes will be highlighted. This does not mean that other data patterns are unimportant, but rather reflects the idea that larger differentials are more relevant given our research objectives.

Next, the findings according to the stress level of the respondents (i.e. high and low) will be examined. The first two columns in table type two are used for this stage. Similarities between the stress level groups will be identified first. This will be followed by the identification of any substantive differences between the episodes of the high and low stress respondent groups. Finally, data patterns related to both episode assessment and respondent stress level will be examined using table type three. Only new information will be identified and discussed in these sections.

On a final note, as acknowledged in Chapter 7, judgments about the importance of emerging relationships identified in qualitative data are based on substantive rather than statistical significance. Great efforts are made in this thesis to describe only the relative ranking of frequency counts between groups for specific concepts, as recommended by Miles and Huberman, (1994), and to avoid using language that infers statistical inference. Unfortunately, when comparing results across groups of role overload episodes identified in any of the three tables used, the volume of data and the complexity of the role overload appraisal and coping process as described by respondents often means that the narrative required to explain differences between groups can be awkward, lengthy, and cumbersome. To simplify the writing, when a particular concept is mentioned more often 
in one group of episodes than another, it is often easier and more meaningful to say that the concept is more likely to be cited in the role overload episodes of one group than the other. The reader should keep in mind, however, that wherever the phraseology 'more or less likely' has been used in this thesis, it is not intended to infer statistical significance. Rather these terms are used to make the results and discussions sections more readable. 


\section{Environment of role overload appraisal}

This chapter will examine the environmental context of the role overload episodes chosen for recollection by overloaded female hospital workers, guided by the following research question: 'What environment factors help us to understand the differential stress experience under role overload conditions?'

Four environment factors of relevance to our understanding of role overload were identified when analysing the data for this study: event types, situation properties, other role factors and demographic data. Event types and situation properties were identified primarily from the responses to the following two interview questions respectively: 'Give a title to the event to be described', and 'What was it about the situation that made you feel overloaded?' (see Table 7-2 in Section 7.1.1).

Role factors in the role overload environment were identified as follows. The researcher noticed that many of the responses throughout the interviews were related to the role or roles undertaken by the hospital employees in their work and family lives. More specifically, factors, such as role stressors, arising from demands are part of the objective environment according to organisational role theory, as are the role domains from which role overload episodes are drawn (Kahn et al. 1964, 1966). Where they were identified, they were included as environment factors in this chapter, either as role overload situation properties or as other role factors.

A fourth environment factor in the form of demographic data collected by a survey instrument prior to the thesis interviews is also available to this thesis. The survey 
data can add important details to the environmental context of the role overload episodes experienced by these differentially stressed hospital workers. Moreover, this demographic data provides contextual information of relevance to the other environmental facets of role overload. For this reason the demographic data is presented first in this chapter (Section 8.1). The other three facets of the role overload environment, event types, situation properties, and other role factors are presented in Section 8.2, 8.3, and 8.4 respectively. The final section of this chapter summarises key findings from the environment chapter.

\subsection{Demographics of hospital workers}

Of the available data collected during the survey undertaken at the four Ottawa hospitals prior to the thesis interviews, measures of three types of role overload (work, family and total), perceived stress, and gender were, as discussed in Section 6.3, used to select the sample for this thesis. Of the remaining demographic data from the survey, the items thought most likely to influence the environment surrounding role overload stress appraisals of the respondents are: age of respondent (Section 8.1.1); number of children and age of youngest child (Section 8.1.2); and job type (Section 8.1.3). The analysis of the interviews as presented in this thesis also suggested other survey data that is worthy of examination. Such items will be discussed and presented as the need arises.

Only one set of data tables (those showing the breakdown by the stress level of the respondents) are shown in this section since demographic data is irrelevant to how role overload episodes were assessed. 


\subsubsection{Age}

The age groups of the twenty female hospital workers interviewed for this thesis are presented in Table 8-1. Overall, whilst the ages of the respondents range from twentysix (26) to sixty (60), a plurality (9 individuals), are in the forty-six to fifty (46-50) age range. More interestingly, however, the spread of ages differs notably between those with low stress and those with high stress. High stress workers have ages spread widely across the total age range whilst low stress workers are more concentrated in the older age brackets (i.e., $80 \%$ of the low stress employees are 46 years old or older, compared with only $50 \%$ of the highly stressed respondents).

\begin{tabular}{|c|c|c|c|c|c|c|}
\hline & \multicolumn{6}{|c|}{ Respondent Stress Level } \\
\cline { 2 - 7 } & Low & $(\mathbf{n}=\mathbf{1 0})$ & High & $\mathbf{( n = 1 0 )}$ & Total & \multirow{2}{*}{$(\mathbf{n = 2 0})$} \\
\cline { 2 - 7 } Age Range & $\#$ & $\mathbf{6}$ & $\#$ & $\mathbf{\%}$ & $\#$ & $\mathbf{\%}$ \\
\cline { 2 - 7 } & \multicolumn{7}{|c|}{ \# and \% of hospital workers in each age range } \\
\hline $26-30$ & 0 & 0 & 1 & 10 & $\mathbf{1}$ & $\mathbf{5}$ \\
\hline $31-35$ & 1 & 10 & 2 & 20 & $\mathbf{3}$ & $\mathbf{1 5}$ \\
\hline $36-40$ & 1 & 10 & 1 & 10 & $\mathbf{2}$ & $\mathbf{1 0}$ \\
\hline $41-45$ & 0 & 0 & 1 & 10 & $\mathbf{1}$ & $\mathbf{5}$ \\
\hline $46-50$ & 5 & 50 & 4 & 40 & $\mathbf{9}$ & $\mathbf{4 5}$ \\
\hline $51-55$ & 2 & 20 & 0 & 0 & $\mathbf{2}$ & $\mathbf{1 0}$ \\
\hline $56-60$ & 1 & 10 & 1 & 10 & $\mathbf{2}$ & $\mathbf{1 0}$ \\
\hline
\end{tabular}

Table 8-1 Age range of respondents by stress level

The other half of this latter group is spread evenly in age from the lowest age bracket (2630) up to the age of forty-five. This age difference between the two respondent groups suggests that it may be the presence of young families at home that brings particular challenges to highly overloaded hospital employees. 


\subsubsection{Children}

Table 8-2 presents data on the number of children and the age of the youngest child for the respondents. Only three workers ( $15 \%$ of the total sample) do not have children. The majority (60\%) have two children and only two workers (10 \%) have larger families. A plurality of the respondents (40\%) are mothers to children between the ages of $11-17$, whilst $30 \%$ have offspring under 11 . This means that $70 \%$ of the total sample has at least one child under 18 and only $15 \%$ have adult children $(18+)$.

\begin{tabular}{|c|c|c|c|c|c|c|}
\hline \multirow[b]{4}{*}{ Number of children } & \multicolumn{6}{|c|}{ Respondent Stress Level } \\
\hline & Low & $(\mathrm{n}=10)$ & High & $(n=10)$ & Total & $(n=20)$ \\
\hline & \multicolumn{6}{|c|}{$\#$ and $\%$ of hospital workers in specified category } \\
\hline & \# & $\%$ & $\#$ & $\%$ & $\#$ & $\%$ \\
\hline 0 & 1 & 10 & 2 & 20 & 3 & 15 \\
\hline 1 & 2 & 20 & 1 & 10 & 3 & 15 \\
\hline 2 & 6 & 60 & 6 & 60 & 12 & 60 \\
\hline 3 & 1 & 10 & 0 & 0 & 1 & 5 \\
\hline 4 & 0 & 0 & 1 & 10 & 1 & 5 \\
\hline \multirow{2}{*}{$\begin{array}{c}\text { Age of Youngest } \\
\text { Child } \\
\end{array}$} & & & & & & \\
\hline & \# & $\%$ & \# & $\%$ & \# & $\%$ \\
\hline No Children & 1 & 10 & 2 & 20 & 3 & 15 \\
\hline Under 5 & 1 & 10 & 2 & 20 & 3 & 15 \\
\hline 5 to 10 & 1 & 10 & 2 & 20 & 3 & 15 \\
\hline 11 to 17 & 5 & 50 & 3 & 30 & 8 & 40 \\
\hline 18 or over & 2 & 20 & 1 & 10 & 3 & 15 \\
\hline
\end{tabular}

Table 8-2 Number of children and age of youngest child of respondents by stress level

There is one difference of note between the high and low stress groups with respect to parental status. Specifically, those in the high stress group are more likely than their counterparts in the low stress group to have children under 10 at home (40\% versus $20 \%$ ). These figures give some support to the idea that the highly stressed hospital workers have younger families. 


\subsubsection{Job Type}

The job types of the twenty female employees interviewed for this thesis are presented in Table 8-3 below. Overall, the largest group of workers by job type (comprising $30 \%$ of the sample), are allied health professionals such as social workers, counsellors and physiotherapists. The smallest group are allied health technicians (only one respondent worked in this type of job). The remaining sample of hospital employees is spread relatively evenly across the other job types: management, clinical staff and nursing, union and non-union support.

\begin{tabular}{|c|c|c|c|c|c|c|}
\hline \multirow[b]{4}{*}{ Job Type } & \multicolumn{6}{|c|}{ Respondent Stress Level } \\
\hline & Low & $(\mathrm{n}=10)$ & High & $(\mathrm{n}=10)$ & & \\
\hline & \multicolumn{6}{|c|}{ \# and $\%$ of hospital workers in specified job type } \\
\hline & $\#$ & $\%$ & \# & $\%$ & $\#$ & $\%$ \\
\hline Allied health professional & 2 & 20 & 4 & 40 & 6 & 30 \\
\hline Union support & 3 & 30 & 1 & 10 & 4 & 20 \\
\hline Management & 3 & 30 & 1 & 10 & 4 & 20 \\
\hline Clinical staff/nursing & 1 & 10 & 2 & 20 & 3 & 15 \\
\hline Non- U support & 0 & 0 & 2 & 20 & 2 & 10 \\
\hline Allied health technician & 1 & 10 & 0 & 0 & 1 & 5 \\
\hline
\end{tabular}

Table 8-3 Job type of respondents by stress level

Examination of the data in Table $8-3$ shows that $60 \%$ of the low stress employees are in two job types: management and union support. On the other hand, $60 \%$ of the highly stressed group work in the following two types of job: clinical staff and nursing, and allied health. Thus, most of the highly stressed group are clinically involved with patients on a regular basis, whilst the low stress group hold positions that focus more on patient administration. 


\subsection{Event types triggering role overload}

The objective of this section is to identify the types of event that trigger role overload. According to Lazarus and Folkman (1964), event type is not a particularly reliable indicator of stress appraisals, since some people will find an event stressful whilst others will not. Event type can, however, provide important indicators of the situations that hospital workers associate most with role overload and hence provide context for the subsequent analysis.

The fact that hospital employees interviewed for this study work in a variety of jobs and live in a number of different family situations suggests that the role overload environments will therefore also vary between respondents. The objective of the initial question asked for each of the two role overload episodes discussed by the respondent interviewees (Give the event a brief title or description) was to classify these differences. The event types triggering role overload identified by respondents are examined in Section 8.2.1. How these event types are related to role overload episode assessment is examined in Section 8.2.2 whilst Section 8.2.3 examines the event types selected by high and low stressed workers. Finally, Section 8.2.4 examines the event types by respondent stress level and episode assessment.

\subsubsection{Descriptions of event types triggering role overload}

The eight role overload event types emerging from the data analysis are displayed in Table 8-4, in descending order by episode count. Of the eight types of event triggering role overload for the hospital employees, three pertain specifically to the work 
environment (high profile corporate task, staffing shortages, client-patient issues and night shifts). The rest can occur in either the work or home domain.

\begin{tabular}{|c|c|c|c|c|c|c|}
\hline \multirow[b]{4}{*}{ Event Types } & \multicolumn{6}{|c|}{ Role Overload Episode Assessment } \\
\hline & \multicolumn{2}{|c|}{$\begin{array}{l}\text { Not-overwhelming } \\
(\mathbf{n}=\mathbf{2 0})\end{array}$} & \multicolumn{2}{|c|}{$\begin{array}{l}\text { Overwhelming } \\
(\mathbf{n}=\mathbf{2 0})\end{array}$} & \multicolumn{2}{|l|}{ Total } \\
\hline & \multicolumn{6}{|c|}{$\begin{array}{l}\# \text { and \% of episodes for which the following situations were } \\
\text { chosen }\end{array}$} \\
\hline & $\#$ & $\%$ & $\#$ & $\%$ & $\#$ & $\%$ \\
\hline Everyday demands & 8 & 40 & 5 & 25 & 13 & 32.5 \\
\hline Personal or Family Crisis & 6 & 30 & 5 & 25 & 11 & 27.5 \\
\hline High Profile Corporate Task & 3 & 15 & 3 & 15 & 6 & 15 \\
\hline Interpersonal Issues & 1 & 5 & 4 & 20 & 5 & 12.5 \\
\hline Staffing Shortages & 1 & 5 & 3 & 15 & 4 & 10 \\
\hline Extracurricular Task & 2 & 10 & 1 & 5 & 3 & 7.5 \\
\hline Client/ Patient Issues & 2 & 10 & 1 & 5 & 3 & 7.5 \\
\hline Other & 1 & 5 & 3 & 15 & 4 & 10 \\
\hline
\end{tabular}

Table 8-4 Event types triggering role overload by episode assessment.

The most common type of event that hospital workers said resulted in role overload was given the descriptor 'everyday demands' since the events being described reflect normal day to day demands faced and accepted by these respondents as part of their role responsibilities, whether they take place in the home or work domain. Of the 40 episodes discussed in total by respondents, one third featured this type of situation. These numbers suggest that exposure to conditions causing high levels of role overload is a part of life for many of these hospital workers. The following quotes, illustrating this event type, also suggest that role overload triggered by everyday demands often happens on a daily basis:-

It was almost daily or weekly at work with our case load that I felt overloaded.

Well typically on a normal day coming into work the minute you get in you are on and you are basically running 
When I pick up my two young children from our care giver at the end of the day and walk in the door to my house and have an hour and a half or so in which to make dinner and eat dinner and clean up after dinner and get my children in bed. The second most common kind of role overload event involved a 'personal or

family crisis' (mentioned in $27.5 \%$ of the episodes). These situations typically revolved around major illnesses or traumatic events imposing additional demands on top of the everyday workload, as well as introducing the potential for personal harm, or harm to a loved one. For example:

My husband is disabled, I have two teenagers and I work full time. My son had a car accident.

Mother in law needed intervention, needed to be put in nursing home, but she had to stay with us for a while.

These comments from hospital employees also indicate that these personal or family crises were not necessarily limited in their impact to the home domain but have the potential to affect work.

High profile corporate tasks were the subject of $15 \%$ of the role overload episodes described. One worker recalled:-

(I was asked to be put on) the OMA (Ontario Medical Association) negotiation team;

...presentation to senior management about social work service delivery needs; ...rolling out a new system in two of the staffing offices;

The 'high profile corporate tasks' label signifies that the individuals involved were put under the scrutiny of senior management and the wider hospital community. The following quotes suggest that these events brought additional pressures from a broad role set. 
It's almost like well, we can't handle what we're doing now so why are we bringing up another one live, so this will be a difficult time.

We had two major policy changes within the organization that had to be rolled out by July first. So simultaneous to this which is already a huge workload issue for me I also had people demanding my time and energy to other major corporate initiatives as well.

Role overload episodes describing 'interpersonal issues', involving disagreements with close colleagues or family members, or difficult interpersonal atmospheres at work or at home, were the fourth most common event chosen for discussion during the interviews (cited in $12.5 \%$ of the episodes). Typically these incidents were precipitated by either a prior breakdown in a personal relationship or an ongoing personality clash with a known role set member. The following quotes illustrate the personal strain indicative of these interpersonal issues:

I went to my supervisor and I said I can't work with this person anymore I'm talking about situations I don't feel there's any respect and I feel that I'm at my wits end I don't wanna have to deal with it anymore.

My husband has a chronic illness and is unable to work. His condition flares up periodically, and it is usually easy to cope with but sometimes it can cause strains between he and I, as well as between he and my 15 year old son. This puts strain on me, because I try to jump in and help the situation.

The last event type, triggering role overload, involved staff shortages.

Understaffing at year end when deadlines must be met, on night shifts, and when covering for others on leave or away sick were examples of staff shortages mentioned by these workers. The following comment illustrates the difficulties that such staffing issues created:

My manager was on holidays, there were supposed to be three of us supposed to be covering and we found out that one colleague was now on sick leave for a 
month long period so we had to re-shuffle while at the same time I realized I had to cancel my holidays. It was a situation that lasted over a month.

The remaining event categories ('extracurricular tasks' not normally part of the typical role duties but not applicable to any other category, 'client issues' involving sick or difficult patients, and other singular issues) were each mentioned by less than $10 \%$ of the sample and will not be discussed further.

Finally, it is important to note that some episodes were triggered by more than one identified event type as illustrated in the quote below:

I had a big presentation, our computer systems were being merged, there was a lot of expansion going on, and my dad had a heart attack all in the same day.

This quote indicates that the chosen episode was set at work and at home and involved a family crisis in the form of a family illness (father's heart attack), and revolved around two high profile corporate tasks (big presentation and merging computer systems). Of the 40 episodes recalled by the respondents six featured two or more of these event types (not shown).

\subsubsection{Event types and episode assessment}

Examination of the data in Table 8-4 suggests that episode assessment (overwhelming versus not overwhelming) is not associated with the occurrence of the following events: personal family crises, high profile corporate tasks and staffing shortages. The data in Table 8-4 also show that everyday demands were more likely to trigger role overload assessed as not-overwhelming ( 8 episodes versus 5 ) whilst 
interpersonal issues were cited more often in role overload deemed to be overwhelming (4 episodes to 1).

\subsubsection{Event types and respondent stress}

Table 8-5 presents data linking respondent stress level and role overload event types.

\begin{tabular}{|c|c|c|c|c|c|c|}
\hline \multirow[b]{3}{*}{ Event Types } & \multicolumn{6}{|c|}{ Role Overload Episodes by Respondent Stress Level } \\
\hline & \multicolumn{2}{|c|}{$\operatorname{Low}(n=20)$} & \multicolumn{2}{|c|}{ High $(n=20)$} & \multicolumn{2}{|c|}{$\begin{array}{c}\text { Total Episodes } \\
(n=40)\end{array}$} \\
\hline & \multicolumn{6}{|c|}{ \# and \% of episodes for which this task was chosen } \\
\hline & \# & $\%$ & \# & $\%$ & $\#$ & $\%$ \\
\hline Everyday demands & 4 & 20 & 9 & 45 & 13 & 32.5 \\
\hline Personal or Family Crisis & 6 & 30 & 5 & 25 & 11 & 27.5 \\
\hline High Profile Corporate Task & 3 & 15 & 3 & 15 & 6 & 15 \\
\hline Interpersonal Issues & 2 & 10 & 3 & 15 & 5 & 12.5 \\
\hline Staffing Shortages & 3 & 15 & 1 & 5 & 4 & 10 \\
\hline Client Issues & 3 & 15 & 0 & 0 & 3 & 7.5 \\
\hline Extracurricular Task & 3 & 15 & 0 & 0 & 3 & 7.5 \\
\hline Other & 1 & 5 & 3 & 15 & 4 & 10 \\
\hline
\end{tabular}

Table 8-5 Event types triggering role overload by stress level of respondent

From the results in Table 8-5 three observations are made. First, stress level is not associated with the frequency with which the hospital employees in this sample identified the following event types in their discussions of role overload: personal or family crises, interpersonal issues, or high profile corporate tasks. Second, role overload episodes involving everyday demands were described more often by highly stressed hospital workers (9 episodes to 4). In fact nearly half of the episodes chosen for discussion by the highly stressed respondents featured everyday demands. Finally, although the numbers are small, it is also worth noting that only workers with low stress described role overload 
episodes triggered by client issues or extracurricular tasks.

\subsubsection{Event types by respondent stress and episode assessment}

Table 8-6 displays data showing event types by episode assessment and respondent stress level for those cases featuring notable between group differences. Only one event type met this criteria: everyday demands. Although we know that everyday demands characterised more of the role overload episodes that were assessed as notoverwhelming (see Section 8.2.2), further examination of the data (in Table 8-6) shows that in most cases these everyday demands assessed as not-overwhelming were recalled by high stress workers (6 vs. 2 episodes). In other words, whilst nearly half of the workers with higher levels of stress $(n=9)$ routinely reported role overload on a regular (often daily) basis, more often than not these individuals assessed such events as notoverwhelming.

\begin{tabular}{|c|c|c|c|c|c|c|c|c|c|c|}
\hline \multirow{5}{*}{ Event Types } & \multicolumn{8}{|c|}{ Role Overload Episodes by Respondent Stress Level } & & \\
\hline & \multicolumn{4}{|c|}{$\operatorname{Low}(\mathbf{N}=10)$} & \multicolumn{4}{|c|}{ High $(\mathrm{N}=10)$} & & \\
\hline & \multicolumn{2}{|c|}{$\begin{array}{c}\text { Not- } \\
\text { overwhelming } \\
\text { Episodes } \\
(\mathbf{n}=\mathbf{1 0}) \\
\end{array}$} & \multicolumn{2}{|c|}{$\begin{array}{l}\text { Overwhelming } \\
\text { Episodes } \\
(\mathbf{n}=\mathbf{1 0})\end{array}$} & \multicolumn{2}{|c|}{$\begin{array}{c}\text { Not- } \\
\text { overwhelming } \\
\text { Episodes } \\
(\mathbf{n}=\mathbf{1 0}) \\
\end{array}$} & \multicolumn{2}{|c|}{$\begin{array}{l}\text { Overwhelming } \\
\text { Episodes } \\
(\mathbf{n}=10)\end{array}$} & \multicolumn{2}{|c|}{$\begin{array}{c}\text { Total } \\
\text { Episodes } \\
(\mathbf{n}=\mathbf{4 0})\end{array}$} \\
\hline & \multicolumn{10}{|c|}{ \# and \% of episodes which this event triggered } \\
\hline & \# & $\%$ & \# & $\%$ & \# & $\%$ & \# & $\%$ & \# & $\%$ \\
\hline Everyday demands & 2 & 20 & 2 & 20 & 6 & 60 & 3 & 30 & 13 & 32.5 \\
\hline
\end{tabular}

Table 8-6 Event types triggering role overload by episode assessment and stress level of respondent 


\subsection{Situation properties of role overload}

The previous section identified the role overload events faced by these female hospital workers who are suffering from high levels of overload in all areas of their lives. Overall, the data confirmed that these workers have very busy work and home lives. Apart from the persistent nature of daily overload, the high levels of responsibility faced by many workers, and the intense nature of some issues, however, these event categories give little indication of what it is that makes these events potentially stressful.

The probing nature of the two part second interview question (i.e., 'What made you feel overloaded? What was it about the situation itself?') was designed to reveal the underlying properties of the role overload situation that made the event personally burdensome and potentially stressful.

The role overload situation properties are described in Section 8.3.1 below. The role overload situation properties described by respondents in the two episode assessment types are explored in Sections 8.3.2, whilst those cited by the two stress level groups are examined in Section 8.3.3. Finally, Section 8.3.4 examines the role overload situation properties by respondent stress and by episode assessment.

\subsubsection{Descriptions of role overload situation properties}

Thirteen situation properties of role overload were abstracted from the data, although one (predictability) was mentioned in fewer than four episodes. The frequencies for all role overload situation properties identified are given in Table 8-7 for the total sample and by episode assessment. Descriptions of the role overload situation properties 
are given below. It should be noted that as for event types, multiple situation properties may underlie each episode.

\begin{tabular}{|c|c|c|c|c|c|c|}
\hline \multirow[b]{4}{*}{ Situation Properties } & \multicolumn{6}{|c|}{ Role Overload Episode Assessment } \\
\hline & \multicolumn{2}{|c|}{$\begin{array}{c}\text { Not- } \\
\text { overwhelming } \\
(\mathrm{n}=\mathbf{2 0})\end{array}$} & \multicolumn{2}{|c|}{$\begin{array}{c}\text { Overwhelming } \\
(n=20)\end{array}$} & Total & $(n=40)$ \\
\hline & \multicolumn{6}{|c|}{ \# and \% of episodes with identified property } \\
\hline & $\#$ & $\%$ & $\#$ & $\%$ & $\#$ & $\%$ \\
\hline Time Pressures & 10 & 50 & 10 & 50 & 20 & 50 \\
\hline Volume of demands & 9 & 45 & 7 & 35 & 16 & 40 \\
\hline Ambiguity & 7 & 35 & 5 & 25 & 12 & 30 \\
\hline Familiarity & 5 & 25 & 5 & 25 & 10 & 25 \\
\hline Duration & 1 & 5 & 7 & 35 & 8 & 20 \\
\hline Synchronicity of demands of a different nature & 3 & 25 & 5 & 20 & 8 & 20 \\
\hline Synchronicity of additional demands to existing workload & 6 & 30 & 2 & 10 & 8 & 20 \\
\hline Synchronicity of simultaneous demands & 2 & 20 & 3 & 20 & 5 & 12.5 \\
\hline Regularity & 3 & 15 & 2 & 10 & 5 & 12.5 \\
\hline Complexity & 4 & 20 & 0 & 0 & 4 & 10 \\
\hline Novelty & 2 & 10 & 2 & 10 & 4 & 10 \\
\hline Event uncertainty & 0 & 0 & 4 & 20 & 4 & 10 \\
\hline Predictable & 0 & 0 & 3 & 15 & 3 & 7.5 \\
\hline
\end{tabular}

Table 8-7 Situation properties of role overload by episode assessment

Time pressures The most prevalent role overload property observed in half the set of 40 episodes recalled in this study sample refers to deadlines and time constraints. In many of these episodes the weight of the time pressures was produced by strict time constraints or by specific deadlines set for the hospital workers by role set members. The following quotes also suggest that only by extending timelines would role overload pressures from multiple demands subside.

I guess just sort of chronically feeling time pressed, and if we had the luxury of four hours I don't know I would feel so pressured. 
I had probably 10 or 15 other issues that were equally as pressing to get done, from timely charting to people that I had to phone back, but yet I had a deadline and this had to get done by Friday.

Workers also appeared to have very little spare time to respond to any changes in demands or expectations:

I really don't have any grey area or any play time to deal with a crisis.

These comments also suggest that in many cases hospital employees were bound by rigid time based targets that compounded already heavy workloads.

Volume of demands was the second most mentioned property of role overload (cited in $40 \%$ of the episodes recalled). In many episodes workers often found the sheer volume of demands facing them quite daunting:

It was the sheer volume of looking at ... the amount of work that needed to be done.

I just had like a million things I wanted to get done at home.

For a number of hospital employees this heavy workload specifically involved patient numbers, suggesting an additional level of burden that could not be avoided:

The sheer volume of patient related issues in addition to the number of physical calls that I had to take and also work preparation as far as things that were either hadn't been done yet and still needed to be done in order for the day to run smoothly.

Ambiguity Mentioned in close to a third (30\%) of the episodes presented by the hospital workers, ambiguity is characterised by a lack of clear guidelines for the role demands faced, particularly with regard to priorities. For example, mixed messages made 
it difficult for respondents facing role overload, particularly when having to decide on priorities in overload situations:

I wish that admin could do a better job at considering or setting priorities...sometimes when someone says what's a higher priority: the client or the report ... admin of course say the report because when things happen at a higher level (you) can't move forward without that happening but from the front line perspective we are client centred - the client comes first so there are conflicting messages.

Hospital employees also reported that not fully understanding what is expected of them in their role contributed to role overload because it made it difficult to respond to demands.

This scenario has the potential to create more work and therefore more overload:

We were asked to put a presentation together for senior management. We were unaware of who would be in attendance. I had little information from my boss on what her agenda for this presentation was. (This led to ) frustration at the lack of clarity of what was required and the lack of assurance that there would be some benefit or positive outcome as a result of doing all this work.

Respondents also talked of a lack of information in some situations:

...there is always a question of what kind of group I am going to get and how varied are they in terms of their knowledge bases

This property of the role overload episodes experienced by hospital workers matches the concept of the same name described by Lazarus and Folkman (1984). According to these authors ambiguity arises when the information necessary for appraisal is unclear or insufficient. The descriptions given by respondents are also consistent with Kahn et al.'s (1964) description of role ambiguity, defined as the lack of information required for adequate role performance. 
Familiarity Descriptions of role overload indicate that a quarter of all the recalled episodes involved situations well known to the hospital workers. As one worker commented:

It had been a situation I had to deal with before and I think from previous experience... just knowing how demanding it can be...,

This comment further suggests that this familiarity formed the basis for role overload appraisal.

Duration Role overload episodes that lasted for a long time, or for which workers could not see an end were linked to feelings of overload in a fifth of the episodes recalled. When workers talked of the duration of role overload they described the recurrent, long lasting or chronic nature of the problem, for example:

It had been close to 3 years of me saying the same thing and the same complaints going to the right people,

Hospital employees facing ongoing role overload situations sometimes expressed a degree of frustration, complaining that often there was no foreseeable resolution to the issues:

I couldn't see an end, even after I refocused and looked to see what I could do. It wasn't happening.

It is also notable that the role overload situation properties of duration and time pressures were never mentioned in the same episode.

This property of role overload situations is consistent with the situation property of the same name as identified by Lazarus and Folkman (1984). 
Synchronicity of demands of a different nature Respondents who mentioned this property of role overload (cited in $20 \%$ of episodes) spoke about multiple demands of a different nature that required effort over a period of time (usually hours or several days). These demands often arose from major projects or crises making them very different in nature to the simultaneous short requests described below. When surmising what made her feel overloaded, one worker characterized her examples of role overload as: ...multiple demands of a different nature These competing demands were not necessarily received at the same instant. Nor did they have to be completed immediately. That being said, the role incumbents still had to process and manage the requests:

A week in May where I had several of my clinical clients needing immediate attention whilst... and I sit on a management committee for the rehab program at the Ottawa hospital, and I had a large report to prepare \& deliver at that, as well as... I am chairing a number of committees and task forces where major professional practices are being created and done, as well as having day to day things that have to be done

In some cases these differing demands were spread across the work and home domains, suggesting that total role overload was the subject. For example, one respondent who described an upcoming busy time at work, involving multiple different demands, added:

And, to compound this, I'm taking the leadership academy course, and it was highly recommended from my director last time, but this time there was an expectation to take the course, plus I have 4 kids at home.

Synchronicity of additional demands to existing workload A second type of synchronous demands was mentioned by respondents when speaking of the situation properties of role overload. In this case, people spoke of the day to day role expectations 
and pressures that ended up competing with an existing workload for the time and attention of the respondents. Also cited in $20 \%$ of the episodes, respondents often talked of the burden of their existing demands when explaining why they felt overloaded:

Because I still had to do my day to day stuff.

Besides, the fact that when you're doing things like that for the organization, you are still expected to continue in your role. You still need to do all your other work.

These comments also suggest that respondents had no spare moments in their daily lives to take on additional demands to their existing workload.

Synchronicity of simultaneous demands A third type of synchronous demands emphasises questions or requests from multiple people, via multiple different communication channels such as face to face interactions, telephones, and pagers, all competing for the respondents immediate attention and action. Typically involving minor events, or demands requiring short responses (such as emails, phone calls, or quick questions from colleagues) this property of role overload featured in $12.5 \%$ of the role overload episodes. The following quote typifies the descriptions of simultaneous demands:

I am in my office in the hospital looking after a patient or working with a patient in a counseling capacity or otherwise and I receive a telephone call on my telephone and a page on my pager and a knock on my door simultaneously from the staff in the clinic or from patients at the door or from a physician whose come by my office. 
Together the three different types of synchronous demands were mentioned in $52.5 \%$ of the total set of 40 episodes (not shown) and notably never occurred together in a role overload episode.

Regularity A small but notable proportion of role overload episodes happened on a repetitive and regular basis (as described in $12.5 \%$ of the episodes discussed).

Respondents claimed previous experience of their role overload situation, which was often described as 'not uncommon':

This isn't an uncommon situation so I kind of do this all the time. .... This happens on a regular basis three or four times a year.

It is an established program so we show up and the schedule is predetermined. For some, these regular role overload situations happened daily, and were also typically triggered by everyday demands:

Well, I guess that situation would arise at work with almost everyday frequency. These comments also suggest that employees facing episodes that reoccur on a regular basis would also have sound knowledge regarding the characteristics of the role overload faced, and thus form a subset of familiar role overload. Comments such as:

...here we go again,

also suggests a degree of frustration brought about by the regularity of these situations, as well as suggesting previous experiences.

Complexity A small number of episodes (10\%) were specifically described as 'complex' by hospital workers. These situations involved intense focus, by the individual, 
on the skills required to achieve a satisfactory outcome. All of the episodes described in this way were set in the work domain (not shown). The example quotes given below demonstrate these features.

....too sick of a patient, too many things could go wrong, that things wouldn't get done and the patient would die, it was pretty complex.

There was a very complicated client who had entered our program.

Not surprisingly for a healthcare organisation, a majority of the situations described as complex relate to patient care.

Novelty This situation property (cited in $10 \%$ of episodes) refers to situations involving new experiences about which respondents claimed to have limited knowledge. The fact that workers in these situations faced a 'big learning curve' suggests novelty may add additional challenges to an already overloaded situation:

It was my first time in that situation, in negotiations, so you almost don't know how to act and what's appropriate and what's not and what to say and what not to say, so that was a big learning curve

I had to learn this new job

Such new or unfamiliar events are consistent with the situation property of the same name as described by Lazarus and Folkmans (1984).

Event uncertainty This situation property (cited in $10 \%$ of episodes) refers to role overload episodes that happened out of the blue, or that could not be predicted:

I am always a little worried about something unexpected coming up 
Such comments suggest that the inability to prepare for such an event was a particular concern for workers in these situations. Lazarus and Folkman (1984) also described the likelihood of an event's occurrence as being influential during the appraisal of potentially stressful events.

\subsubsection{Situation properties and episode assessment}

From the results in Table 8-7 we can make three observations about the relationship between situation properties and episode assessment type. First, time pressures and the volume of demands were the most salient situation properties related to feelings of overload. Moreover, the tendency to cite temporal constraints and burdensome demands as properties of role overload was not related to how the role overload episode was assessed. Similarly, familiar, regular, or novel role overload situations, and those in which demands of a different nature are synchronized or demands arrive simultaneously, also appear to be unrelated to the individual episode assessment.

Second, there are two situation properties that were described more often in role overload episodes assessed as overwhelming: duration (seven of the eight episodes of long duration were assessed as overwhelming), and event uncertainty (all the episodes involving uncertainty were evaluated as overwhelming). Lastly, there are two situation properties that featured more often in not-overwhelming role overload: complexity (all four complex role overload episodes were assessed as not-overwhelming), and having additional demands synchronised to the respondents' existing workload (six of the eight episodes having this property were assessed as not-overwhelming). 


\subsubsection{Situation properties and respondent stress}

The same situation properties are presented in Table 8-8, this time according to the stress level of the employees experiencing overload.

\begin{tabular}{|c|c|c|c|c|c|c|}
\hline \multirow[b]{3}{*}{ Situation Properties } & \multicolumn{6}{|c|}{ Role Overload Episodes by Respondent Stress Level } \\
\hline & \multicolumn{2}{|c|}{ Low $(n=20)$} & \multicolumn{2}{|c|}{ High $(n=20)$} & \multicolumn{2}{|c|}{$\begin{array}{l}\text { Total Episodes } \\
(n=40)\end{array}$} \\
\hline & \multicolumn{6}{|c|}{ \# \& \% of episodes in which this characteristic was mentioned } \\
\hline & \# & $\%$ & \# & $\%$ & \# & $\%$ \\
\hline Time Pressures & 12 & 60 & 8 & 40 & 20 & 50 \\
\hline Volume of demands & 8 & 40 & 8 & 40 & 16 & 40 \\
\hline Ambiguity & 5 & 25 & 7 & 35 & 12 & 30 \\
\hline Familiarity & 3 & 15 & 7 & 35 & 10 & 25 \\
\hline Duration & 3 & 15 & 5 & 25 & 8 & 20 \\
\hline Synchronicity of demands of a different nature & 3 & 15 & 5 & 25 & 8 & 20 \\
\hline $\begin{array}{r}\text { Synchronicity of additional demands to existing } \\
\text { workload }\end{array}$ & 5 & 25 & 3 & 15 & 8 & 20 \\
\hline Synchronicity of simultaneous demands & 3 & 15 & 2 & 10 & 5 & 12.5 \\
\hline Regularity & 1 & 5 & 4 & 20 & 5 & 12.5 \\
\hline Complexity & 3 & 15 & 1 & 5 & 4 & 10 \\
\hline Novelty & 1 & 5 & 3 & 15 & 4 & 10 \\
\hline Event uncertainty & 0 & 0 & 4 & 20 & 4 & 10 \\
\hline Predictable & 1 & 5 & 2 & 10 & 3 & 7.5 \\
\hline
\end{tabular}

Table 8-8 Situation properties of role overload: by stress level of respondent

The findings with respect to the situation properties in Table 8-8 support three observations. First, respondent stress was not associated with the likelihood that a respondent would talk about the following role overload situation properties: volume of demands, ambiguity, duration, all three types of synchronous demands, complexity, and novelty). Second, when describing what made them feel overloaded, low stress hospital workers cited time pressures, such as time constraints and deadlines, more frequently than highly stressed workers (12 episodes to 8 ). Finally, three situation properties were 
talked about more often by highly stressed workers, when they described what made them feel overloaded: familiarity (7 episodes to 3); regularity (4 episodes to 1 ) and event uncertainty (4 episodes to 0). Since regular episodes are also familiar, these results indicate that it is the regular role overload episodes that account for the stress level differences noted for familiar role overload.

\subsubsection{Situation properties by respondent stress and episode assessment}

Table 8-9 allows us to examine the results by episode assessment for each of the stress level respondent groups. Only two situation properties have data patterns worthy of note. First, the tendency for low stress workers to talk more frequently about time pressures was restricted to those episodes subsequently assessed as not-overwhelming. More specifically, time pressures were cited in half of the overwhelming episodes of both low and high stress respondents, whereas low stress respondents mentioned time pressures in more not-overwhelming episodes than the high stress respondents ( 7 episodes to 3).

Second, when describing what made them feel overloaded, low stress workers talked about the difficulties of dealing with additional demands on top of their existing workload in equal numbers of overwhelming and not-overwhelming episodes. High stress workers, on the other hand, seemed to find such role overload episodes consistently less stressful, since all were subsequently assessed as not-overwhelming. 


\begin{tabular}{|c|c|c|c|c|c|c|c|c|c|c|}
\hline \multirow{5}{*}{ Situation Properties } & \multicolumn{8}{|c|}{ Role Overload Episodes by Respondent Stress Level } & & \\
\hline & \multicolumn{4}{|c|}{ Low $(\mathrm{N}=10)$} & \multicolumn{4}{|c|}{ High $(\mathrm{N}=10)$} & & \\
\hline & \multicolumn{2}{|c|}{$\begin{array}{c}\text { Not- } \\
\text { overwhelming } \\
\text { Episodes }(n=10)\end{array}$} & \multicolumn{2}{|c|}{$\begin{array}{l}\text { Overwhelming } \\
\text { Episodes } \\
(\mathbf{n}=\mathbf{1 0})\end{array}$} & \multicolumn{2}{|c|}{$\begin{array}{c}\text { Not- } \\
\text { overwhelming } \\
\text { Episodes } \\
(\mathbf{n}=\mathbf{1 0}) \\
\end{array}$} & \multicolumn{2}{|c|}{$\begin{array}{l}\text { Overwhelming } \\
\text { Episodes } \\
(n=10)\end{array}$} & \multicolumn{2}{|c|}{$\begin{array}{c}\text { Total } \\
\text { Episodes } \\
(n=40)\end{array}$} \\
\hline & \multicolumn{10}{|c|}{ \# \& \% of episodes in which this characteristic was mentioned } \\
\hline & $\#$ & $\%$ & $\#$ & $\%$ & $\#$ & $\%$ & $\#$ & $\%$ & $\#$ & $\%$ \\
\hline Time Pressures & 7 & 70 & 5 & 50 & 3 & 30 & 5 & 50 & 20 & 50 \\
\hline $\begin{array}{r}\text { Synchronicity of add'l } \\
\text { demands to existing } \\
\text { workload }\end{array}$ & 3 & 30 & 2 & 20 & 3 & 30 & 0 & 0 & 8 & 20 \\
\hline
\end{tabular}

Table 8-9 Situation properties of role overload by episode assessment and stress level of respondent

\subsection{Other role factors in the role overload environment}

This section will focus on examining the role domains and other role stresses that characterize the role overload environment of the overloaded female health care employees as displayed in Table 8-10. The first part of this section identifies the role domains in which the chosen role overload events were set (Section 8.2.1). The second part of this section looks at the evidence for the influence of other forms of role conflict in the role overload experiences of hospital employees (Section 8.2.2). The relationship between role factors and episode assessment are presented in Section 8.2.3, whilst the fourth subsection (Section 8.2.4) presents the role factor results by respondent stress level. The fifth and final subsection (Section 8.2.5) presents the results by respondent stress level and episode assessment. 


\begin{tabular}{|c|c|c|c|c|c|c|}
\hline \multirow[b]{4}{*}{ Other Role Factors } & \multicolumn{4}{|c|}{ Role Overload Episode Assessment } & \multirow{2}{*}{\multicolumn{2}{|c|}{$\begin{array}{c}\text { Total Episodes } \\
(n=40)\end{array}$}} \\
\hline & \multicolumn{2}{|c|}{$\begin{array}{c}\text { Not- } \\
\text { overwhelming } \\
\text { Episodes }(n=20)\end{array}$} & \multicolumn{2}{|c|}{$\begin{array}{l}\text { Overwhelming } \\
\text { Episodes }(n=20)\end{array}$} & & \\
\hline & \multicolumn{6}{|c|}{ \# \& \% of episodes in which this characteristic was mentioned } \\
\hline & \# & $\%$ & $\#$ & $\%$ & \# & $\%$ \\
\hline Role Domain & 20 & 100 & 20 & 100 & 40 & 100 \\
\hline Work Situation & 12 & 60 & 13 & 65 & 25 & 62.5 \\
\hline Family - Home Situation & 4 & 20 & 4 & 20 & 8 & 20 \\
\hline Work and Home & 4 & 20 & 3 & 15 & 7 & 17.5 \\
\hline Other Role Stresses & 8 & 40 & 7 & 35 & 15 & 37.5 \\
\hline Intra-Role Conflict & 4 & 25 & 3 & 15 & 7 & 17.5 \\
\hline Work Family Conflict & 4 & 20 & 3 & 15 & 7 & 17.5 \\
\hline
\end{tabular}

Table 8-10 Role factors described in role overload episodes by episode assessment.

\subsubsection{Role domains}

In order to capture information about the most personally meaningful role overload events, respondents were specifically encouraged in the interview to select examples from home or work or any other area of their lives when describing situations in which they felt overloaded. For their contrasting pair of role overload episodes, all the respondents chose from one of three domains: work, home or a combination of the two, as shown in Table 8-10 above. Of the total set of 40 episodes chosen by the twenty hospital workers, $62.5 \%$ occurred at work, $20 \%$ at home and $17.5 \%$ involved the two domains. In other words, $80 \%$ of the role overload episodes presented by the respondents involved a work situation to some degree.

Given that the sample of hospital workers for this study were chosen because they are all suffering from high levels of work, family and total role overload, it is somewhat surprising that overload from the work domain features so prominently for such a large 
proportion of the role overload episodes chosen. Possible explanations for this will be discussed in part six of the thesis. An immediate consequence of this imbalance, however, is that respondent quotes presented in support of the interview analysis in the subsections to come will largely be drawn from work based role overload episodes. Home based examples will be used in proportion to their representation or if they are specifically relevant to the topic under discussion.

\subsubsection{Other Role Stresses}

Two other forms of role stress (intra-role conflict, and work family conflict) were identified from respondent descriptions of role overload. The results in Table 8-10 show that close to forty percent of the role overload episodes also involve some form of role conflict.

The first type of role stress identified in the role overload episodes matched Kahn et al.'s (1964) description of intra-role conflict. Involving incompatible expectations from one (intra-sender conflict) or more (inter-sender conflicts) of the employees' role set members, this role stress was cited in $17.5 \%$ of the episodes discussed. A number of these intra-role conflicts feature a discussion of explicit organizational rules which conflict with work expectations, whilst others involved implicit expectations for respondents to take on extra work outside their stated mandate. Moreover, all of the episodes in which these conflicts were cited were work domain based (not shown in Table 8-10). For example, a number of cases concerned demands that were difficult to 
fulfill within normal work hours conflicting with pressures not to claim overtime. The following quote gives an explanation of this scenario:

There is a pressure to do your job within the parameters of what you are actually getting paid for and yet it is not always a realistic expectation. And then when you do put in that overtime there is a feeling that well if you just manage your time better so there is that pressure to not claim overtime.

Such a conflict increases the pressure on the role incumbent to complete the demands in a timely manner. It is not surprising, therefore, that such conflicts were associated with role overload. Another example of intra-role conflict arose for role incumbents who have more than one manager. For example, one employee complained of her two managers who had conflicting ideas on sanctioning time off in lieu for working overtime:

I negotiated with my manager, and she conceded to let me bank the time off. I have too many managers. When I put in a request for 37.5 of those hours my second manager said "have (the first manager) send me an email saying you have that time", because it was a violation of the CBA (collective bargaining agreement), in theory. If they then refuse it for me I am stuck.

Inability to resolve intra-role conflict seemed to lead to further restrictions on time resources, as suggested by one respondent:

The CBA says we are supposed to take time off during the pay period, which means half a day off a week, which only makes things more stressful.

As well as intra-role conflict, respondents also reported inter-role conflict as defined by Kahn et al. (1964), specifically conflict between the work and family roles. During the analysis process, whenever respondents specifically commented on a conflict between the role expectations from home and those from their work domain, the relevant segment of descriptive text was coded at an NVivo node labeled 'work-family conflict'. 
One respondent commented, for example, on the difficulty of trying to resolve work expectations in a timely manner when faced with this conflict in a role overload situation:

Well it is trying to combine getting back to work with care giving. Getting back to work is the stressful factor.

The results in Table 8-10 show that work-family conflict was mentioned in 17.5 $\%$ of the role overload episodes overall. This finding is consistent with the fact that overload set solely in the workplace dominates the role overload episodes reviewed by these female hospital workers. In fact, in six of the episodes in which work family conflict was mentioned, the role overload was set in both work and home domains (not shown). The relationship between the role overload and work-family conflict also varies in the eight episodes in which this inter-role conflict was cited. The comment above, for example, suggests that the work family conflict for this respondent was part of the role overload experience. In other episodes work family conflict arose as a consequence of role overload, and in others, comments suggest it was just one of the factors leading to role overload. The following comment, mentioned after the respondents' initial description of role overload, illustrates the latter example:

And I guess one part I didn't mention was I had to leave at a certain point in time cause I had to pick up my daughter from day care so I didn't have time to like I felt that the clock was...I couldn't stay longer.

\subsubsection{Other role factors and episode assessment}

Table 8-10 also shows the data needed to examine the relationship between the role factors cited during role overload recollections and how the episodes were subsequently assessed by hospital workers. The results show that neither role overload 
domain setting (i.e., work, home, or across work and home) or other role stresses were related to whether or not the respondent assessed episodes as overwhelming.

\subsubsection{Other role factors and respondent stress}

The role domains and role stresses described in overload episodes are displayed in Table 8-11 according to the perceived stress level of the workers. The role domain results in this table support the following observations. First, the work domain dominates for the episodes chosen by both low and high stressed hospital employees. Second, this observation is particularly strong for the low stress respondents, since work based role overload made up $70 \%$ of their chosen episodes. Third, there appears to be an association between multi-domain based role overload and higher levels of personal stress. Of the twenty role overload episodes chosen for discussion by the highly stressed hospital workers, five involved both the work and home domains. The equivalent figure for the low stress respondents was two.

\begin{tabular}{|c|c|c|c|c|c|c|}
\hline \multirow[b]{4}{*}{ Other Role Factors } & \multicolumn{6}{|c|}{ Role Overload Episodes by Respondent Stress Level } \\
\hline & \multicolumn{2}{|c|}{ Low $(\mathbf{n}=\mathbf{2 0})$} & \multicolumn{2}{|c|}{ High $(n=20)$} & \multicolumn{2}{|c|}{$\begin{array}{c}\text { Total Episodes } \\
(n=40)\end{array}$} \\
\hline & \multicolumn{6}{|c|}{ \# \& \% of episodes in which this characteristic was mentioned } \\
\hline & \# & $\%$ & \# & $\%$ & \# & $\%$ \\
\hline Role Domain & 20 & 100 & 20 & 100 & 40 & 100 \\
\hline Work Situation & 14 & 70 & 11 & 55 & 25 & 62.5 \\
\hline Family - Home Situation & 4 & 20 & 4 & 20 & 8 & 20 \\
\hline Work and Home & 2 & 10 & 5 & 25 & 7 & 17.5 \\
\hline Other Role Stresses & 8 & 40 & 7 & 35 & 15 & 37.5 \\
\hline Intra-Role Conflict & 5 & 30 & 2 & 10 & 7 & 17.5 \\
\hline Work Family Conflict & 3 & 15 & 4 & 20 & 7 & 17.5 \\
\hline
\end{tabular}

Table 8-11 Role factors described in role overload episodes by respondent stress level 
Examining the role stress data in Table 8-11 suggests that high and low stress workers were equally likely to cite other role stresses when describing role overload. When we examine the individual role conflict items, however, one variation of note can be observed. Low stress workers cited intra-role conflict during their recollections of role overload more often than the high stress workers (5 episodes to 2 ).

\subsubsection{Other role factors by respondent stress and episode assessment}

In Table 8-12, role factors described by workers in recollections of role overload are presented by episode assessment type for each stress level group, for those role factors for which the results reveal a notable difference. One observation is made. The suggested association between role overload involving both the work and home domains, and high respondent stress levels, is more notable for overwhelming role overload experiences.

\begin{tabular}{|c|c|c|c|c|c|c|c|c|c|c|}
\hline \multirow[b]{5}{*}{ Other Role Factors } & \multicolumn{8}{|c|}{ Role Overload Episodes by Respondent Stress Level } & & \\
\hline & \multicolumn{4}{|c|}{ Low $(\mathrm{N}=10)$} & \multicolumn{4}{|c|}{ High $(\mathbf{N}=10)$} & & \\
\hline & \multicolumn{2}{|c|}{$\begin{array}{c}\text { Not- } \\
\text { overwhelming } \\
\text { Episodes } \\
(\mathbf{n}=\mathbf{1 0}) \\
\end{array}$} & \multicolumn{2}{|c|}{$\begin{array}{c}\text { Overwhelming } \\
\text { Episodes } \\
(\mathbf{n}=10)\end{array}$} & \multicolumn{2}{|c|}{$\begin{array}{c}\text { Not- } \\
\text { overwhelming } \\
\text { Episodes } \\
(\mathbf{n}=\mathbf{1 0}) \\
\end{array}$} & \multicolumn{2}{|c|}{$\begin{array}{c}\text { Overwhelming } \\
\text { Episodes } \\
(\mathbf{n}=\mathbf{1 0})\end{array}$} & \multicolumn{2}{|c|}{$\begin{array}{c}\text { Total } \\
\text { Episodes } \\
(\mathbf{n}=40) \\
\end{array}$} \\
\hline & \multicolumn{10}{|c|}{ \# \& \% of episodes in which this characteristic was mentioned } \\
\hline & \# & $\%$ & $\#$ & $\%$ & $\#$ & $\%$ & $\#$ & $\%$ & $\#$ & $\%$ \\
\hline \multicolumn{11}{|l|}{ Role Domain } \\
\hline Work and Home & 2 & 20 & 0 & 0 & 2 & 20 & 3 & 30 & 7 & 17.5 \\
\hline
\end{tabular}

Table 8-12 Role factors described in role overload by episode assessment and stress level of respondent 
Specifically, none of the overwhelming episodes presented by low stress employees were set across two domains whereas the high stress respondents chose a double-domain based role overload for three episodes subsequently assessed as overwhelming. In other words, overwhelming role overload in which the home environment is implicated with work seemed to be particularly stressful for the highly stressed female hospital workers.

\subsection{Summary of key findings}

This chapter has examined the factors characterizing the environment of the role overload events described by twenty female hospital employees (10 with low stress, 10 highly stressed) who are overloaded in all areas of their lives. The data presented in this chapter have identified four different facets of the role overload environment: demographics of the female hospital workers; the events triggering the role overload discussed; the underlying situation properties that have the potential to influence role overload appraisal and coping; and role factors specific to the role overload episodes described. The summary of key findings is covered in four brief sections. First the major findings common to all episodes and all workers are summarized. The next two subsections summarise key findings according to the assessment of role overload episodes (overwhelming and not-overwhelming) and according to the stress level of the workers (high and low) respectively. The last subsection summarises key environment factor findings for the four episode groupings: not-overwhelming episodes of low stress workers; not-overwhelming episodes of high stress workers; overwhelming episodes of low stress workers; and overwhelming episodes of high stress workers. 


\subsubsection{Commonality of findings for the role overload environment}

A key finding from the results presented in Chapter 8 was that the role overload events recalled by the sample of hospital employees were predominantly set in the work domain (i.e., $75 \%$ of the role overload episodes discussed pertained to work). This finding is particularly significant in light of the fact that the demographic data examined first in this chapter also showed that many respondents had children at home, which would suggest they experienced many role pressures from home as well as from their work responsibilities.

The sample for this thesis all recorded high levels of work, family, and total role overload. The focus on work role overload by respondents when choosing a role overload event to discuss may thus be because work is actually more problematic and role overload at work consumes so much time and energy that individuals experience role overload at home as well. Another explanation could be that individuals may perceive events at home in a different more favourable light. These tentative speculations suggest that further examination of the survey data collected prior to this thesis may help us to understand why work role overload dominated for these workers. An examination of the role salience data gathered during the prior respondent survey may provide additional information regarding the relationship between role overload domains and stressful outcomes. Role salience scores will be examined in the next chapter (Person Factors), since they specifically reflect the values and commitments of the individual.

The results examining the event types in this chapter suggested that two events in particular were the most common triggers for role overload: everyday demands and 
personal/family crises. Workers cited everyday demands as the trigger for role overload in a third of the role overload episodes recalled. Comments made about everyday demands also suggest that for some workers role overload is a chronic condition in their daily lives. Descriptions of role overload featuring personal or family crises, the second most noted cause of role overload (cited in over one quarter of the episodes recalled), suggest that personal events also have the potential to impact work.

Examination of the underlying qualities of the role overload with situational relevance to the well-being of the hospital worker sample revealed twelve situation properties, each with the potential to influence role overload appraisal and coping. Time pressures and the volume of demands surfaced as the two most common properties of role overload. The presence of ambiguity in 30\% of the episodes recalled was also a notable finding. Collectively, synchronized, competing demands were also a prominent feature for over half of the role overload episodes presented by hospital workers, although three different and distinct types of synchronized demands were noted. The other situation properties were familiarity, duration, regularity, complexity, event uncertainty and novelty.

Finally the results shown in this chapter revealed that intra-role conflict and workfamily conflict each featured in close to a fifth of the forty role overload episodes recounted by hospital workers. Intra-role conflict, described predominantly in role overload set in the work domain, revealed a mismatch in the expectations of the hospitals with regard to workload and overtime allowances leading to time constraints. The nature of the relationship between role overload and work-family conflict, however, was 
somewhat varied across the examples given, in terms of whether work-family conflict preceded or followed or was involved with role overload.

\subsubsection{Key environment findings by role overload episode assessment}

Table 8-15 summarises the key environment factors most closely associated with overwhelming and not-overwhelming role overload episodes for these female hospital workers. The findings are presented without regard to respondent stress level. Event types triggering role overload, and the underlying situation properties of role overload, both differed across the two episode assessment types, but no differences were noted for role domains or other role stresses.

\begin{tabular}{|r|c|c|c|c|}
\hline \multirow{2}{*}{$\begin{array}{c}\text { Role Overload } \\
\text { Episode } \\
\text { Assessment }\end{array}$} & \multicolumn{4}{|c|}{ Role Overload Environment Factors } \\
\cline { 2 - 5 } $\begin{array}{c}\text { Not- } \\
\text { overwhelming }\end{array}$ & N/A & Everyday demands & $\begin{array}{c}\text { Synchronicity - additional } \\
\text { demands to existing workload } \\
\text { Complexity }\end{array}$ & Role Factors \\
\hline Overwhelming & $N / A$ & Interpersonal issues & $\begin{array}{c}\text { Duration } \\
\text { Event uncertainty }\end{array}$ & \\
\hline
\end{tabular}

Table 8-13 Key environment factors of role overload assessed as overwhelming and notoverwhelming disregarding the stress level of the respondents

Findings from this chapter suggest that role overload episodes that were evaluated as not-overwhelming were more likely to be triggered by everyday demands than were overwhelming episodes. Notably, everyday demands were the most common event triggering role overload assessed as not-overwhelming. All episodes in which workers referred to the complexity of the situation, when talking about what made them feel overloaded, were subsequently assessed as not-overwhelming as well. These cases mostly 
involved patient care. Workers also talked more frequently about having to deal with additional demands on top of an existing workload in episodes deemed notoverwhelming. Although they were referenced more often by workers in their recollections of less overwhelming role overload, it is notable that both of these situation properties (complexity and synchronicity of additional demands), are represented in relatively small proportions of not-overwhelming episodes overall. Nevertheless, the results suggest that these environment factors are either personally less bothersome to workers or more easily dealt with in role overload situations. The factors more typical in not-overwhelming episodes suggest an environment in which workers are overloaded with additional everyday tasks on top of an existing heavy workload. For those involved with patient care on a daily basis, complex cases would not be unusual.

The results for overwhelming episodes suggest a somewhat different role overload environment. Episodes of role overload triggered by interpersonal issues, although relatively few in number, were more often than not categorised as overwhelming by workers. Role overload episodes of a lengthy duration also form a relatively small set of the total number of episodes described by respondents. There is a marked bias, however, for people to label such experiences as overwhelming. The data presented in this chapter also supports the idea that role overload episodes featuring event uncertainty almost always turn out to be assessed as overwhelming.

In summary, the key findings in this chapter for overwhelming role overload suggest a role overload environment in which unusual situations overwhelm workers. 


\subsubsection{Key role overload environment findings by respondent stress level}

Table 8-15 summarises the key environment factors most closely associated with respondents with low and high stress. The findings are presented without reference to how individual episodes were assessed.

The demographic data of the hospital employees described a quite different context for the low stress hospital workers. They are generally older than the high stressed respondents and their family structure is somewhat different. Low stress workers tend to have older families with children in their teens or adulthood. In addition, a majority of the workers in the low stress group have administrative or management jobs.

Role factors affecting the environment of role overload were also different for those in the low stress group, in that this group recalled more episodes of work based role overload. Low stress workers also mentioned intra-role conflict (mostly work based) more often as an issue when talking about role overload. The role overload property 'time pressures', indicative of time constraints, also features much more commonly in the role overload of low stress respondents.

In summary, the factors typical in the environment of the older low stress workers, when considered as a whole, suggest a role overload environment set mostly in the work domain peppered with functional and structural issues. In other words, the environment featured role demands that are either incompatible, or involve deadlines or time constraints. Some of these time constraints may be linked to the incompatible demands described in intra-role conflict more common for this group. 


\begin{tabular}{|c|l|l|l|l|}
\hline \multirow{2}{*}{\begin{tabular}{c} 
Respondent $\begin{array}{c}\mid 4 \\
\text { Stress } \\
\text { Level }\end{array}$ \\
\cline { 2 - 5 } Low
\end{tabular}} & $\begin{array}{l}\text { Rele Overload Environment Factors } \\
\text { Demographics } \\
\text { management role } \\
\text { Older families }\end{array}$ & Event Types & Situation Properties & Role Factors \\
\hline High & $\begin{array}{l}\text { Patient focused role } \\
\text { Young family }\end{array}$ & Everyday demands & $\begin{array}{l}\text { Regularity } \\
\text { Event uncertainty }\end{array}$ & $\begin{array}{l}\text { Work domain based } \\
\text { Intra-role conflict }\end{array}$ \\
\hline
\end{tabular}

Table 8-14 Key environment factors of role overload for low and high stress workers, without regard to how episodes were assessed

The environment of high stress respondent role overload differs from that of the low stress workers in a number of ways. First, high stress hospital employers are more likely to be mothers of younger children (typically ten or under), and their work roles are mostly focused on the needs of patients. These findings are consistent with the role factor finding from this chapter that more of the episodes of role overload that involve both home and work domains were recalled by the respondents of this group. The suggested association between higher stress levels and dual domain role overload could also be connected to the relative value of the two roles to the female hospital workers. This gives further support to the decision to examine worker role salience in the next chapter.

The results in chapter 8 also show that 'everyday demands' were a particularly common trigger of role overload for the high stress respondents. Forty-five per cent of the episodes presented by high stress workers mention the everyday role responsibilities that define this type of role overload, suggesting that for many in this group "being overloaded" is a way of life. This finding further suggests that over time being overloaded results in high levels of stress. Finally, the situation properties that were most closely associated with the episodes recalled by this high stress group refer to the 
'regularity', and also to the 'uncertainty' of role overload events. The environment factors associated with high respondent stress levels when considered as a whole, paint a picture of chronic daily role overload that leaves no time or space for unpredictable demands, for these workers with young families.

\subsubsection{Key role overload environment findings in episode groupings}

This chapter also examined environment factors according to worker stress level and episode assessment, for those factors where differences were notable. Three key findings are summarized in Table 8-17. First, time pressures were found to be to be more prevalent in not-overwhelming episodes of low stress hospital workers. Episodes involving everyday demands, a common trigger of role overload for high stress workers, were more often than not subsequently assessed as not-overwhelming by this group. Finally, the synchronicity of additional demands on top of an existing workload was notably never mentioned in the overwhelming episodes of high stress employees, a finding that stands out because it was mentioned with the same frequency by workers in the other three groups.

\begin{tabular}{|c|c|}
\hline Not-overwhelming episodes of low stress workers & Not-overwhelming episodes of high stress workers \\
\hline $\begin{array}{c}\text { Time pressures } \\
\text { Synchronicity - add'l demands }\end{array}$ & $\begin{array}{c}\text { Everyday demands } \\
\text { Synchronicity - add'l demands }\end{array}$ \\
\hline Overwhelming episodes of low stress workers & Overwhelming episodes of high stress workers \\
\hline Synchronicity - add'l demands & \\
\hline
\end{tabular}

Table 8-15 Summary of role overload environment factors by episode grouping 


\section{Person factors in role overload appraisal}

According to Lazarus and Folkman (1984), person factors are those characteristics of the person that will be influential in assessing the personal significance of, and hence the level of perceived stress arising from, a stressor such as role overload. The results so far have given little information that would explain the relationship of person factors with the potentially stressful properties of role overload described in the previous chapter. These characteristics will be the focus of this chapter guided by the following research question, 'What person factors help us to understand the differential stress response under role overload conditions?'

Three sets of person factors are examined in three separate sections. Section 9.1 will explore the person factors cited in the role overload episodes recounted by the respondents. Section 9.2 will examine the personality traits of the respondents. Section 9.3 will examine the role salience results for this thesis sample since the results examined in Chapter 8 suggested that role salience may be influential in assessing the personal significance of the role overload environment. The final section of this chapter (Section 9.4) summarises the key findings with regard to the person factors associated with role overload appraisal for these overloaded female health care workers.

\subsection{Person factors cited in role overload recollections}

Three broad person factor themes resulted from the analysis of the answers to the interview questions designed to draw out person factors relevant to role overload (see 
Table 7-1 in Section 7.1). Overall, person factors were identified in three quarters (30) of the total episodes. Multiple answers were permitted to the interview questions and therefore any role overload episode may feature one or more person factors. The three categories of person factors emerging from the qualitative data (internal role pressures, personal beliefs, and personal attributes) are presented in Table 9-1 and discussed below.

The person factors that recorded the highest count across the role overload episodes presented by hospital workers, fit the description of 'internal role pressures' (as identified by Kahn et al., 1964 and described in Section 2.1.2) since they depict internal drivers to role performance based on personal values and attitudes. Internal role pressures were mentioned in close to two-thirds (62.5\%) of the role overload episodes. The second person factor theme, 'personal beliefs' were mentioned in a $40 \%$ of the role overload episodes reviewed. This theme reflects individual evaluations and notions that the respondents have about themselves or about their environment, and as such fits well with Lazarus and Folkman's (1984) person factor of the same name (see Section 3.2.3). The third person factor theme coded from the role overload recollections, labeled 'personal attributes', collects together specific descriptions that respondents made about their own personal characteristics. Mentioned in a fifth of all episodes and therefore somewhat fewer episodes than the other two person factors, 'personal attributes' appear to be less common to the experience of role overload.

The individual person factors gathered under these three themes are detailed in three separate subsections below. Table 9-1 will also be used in a fourth subsection, Section 9.1.4, to explore the relationship between person factors and episode assessment. 
The fifth subsection explores the results by respondent stress level, whilst Section 9.1.6 examines results by episode assessment for each stress level.

\begin{tabular}{|c|c|c|c|c|c|c|}
\hline \multirow[b]{4}{*}{ Person Factors } & \multicolumn{6}{|c|}{ Role Overload Episode Assessment } \\
\hline & \multicolumn{2}{|c|}{$\begin{array}{c}\text { Not- } \\
\text { overwhelming } \\
(\mathbf{n}=\mathbf{2 0})\end{array}$} & \multicolumn{2}{|c|}{$\begin{array}{l}\text { Overwhelming } \\
(\mathrm{n}=\mathbf{2 0})\end{array}$} & \multirow[t]{2}{*}{ Total } & \multirow[t]{2}{*}{$(n=40)$} \\
\hline & \multicolumn{4}{|c|}{$\#$ and $\%$ of episodes with identified property } & & \\
\hline & $\#$ & $\%$ & $\#$ & $\%$ & $\#$ & $\%$ \\
\hline Internal Role Pressures & 15 & 75 & 10 & 50 & 25 & 62.5 \\
\hline Take pride in being good at my role & 9 & 45 & 5 & 25 & 14 & 35 \\
\hline Opportunity for accomplishment & 3 & 20 & 2 & 10 & 5 & 12.5 \\
\hline Importance of family & 2 & 10 & 2 & 10 & 4 & 10 \\
\hline Enjoy the role & 2 & 10 & 1 & 10 & 3 & 7.5 \\
\hline Other personal goals \& objectives & 2 & 10 & 1 & 5 & 3 & 7.5 \\
\hline Other & 1 & 5 & 2 & 10 & 3 & 7.5 \\
\hline Personal Beliefs & 6 & 30 & 10 & 50 & 16 & 40 \\
\hline Lack of control & 6 & 30 & 7 & 35 & 13 & 32.5 \\
\hline Spiritual beliefs & 0 & 0 & 2 & 10 & 2 & 5 \\
\hline Lack of esteem & 0 & 0 & 1 & 5 & 1 & 2.5 \\
\hline Personal Attributes & 5 & 25 & 3 & 15 & 8 & 20 \\
\hline Calm by Nature & 4 & 20 & 0 & 0 & 4 & 10 \\
\hline Resilient & 0 & 0 & 2 & 10 & 2 & 5 \\
\hline Other & 1 & 5 & 1 & 5 & 2 & 5 \\
\hline
\end{tabular}

Table 9-1 Person factors described in role overload episodes by episode assessment

\subsubsection{Internal role pressures}

Three of the six internal role pressures identified were cited in a substantive number of role overload episodes. The first, 'take pride in being good at my role', was cited in just over one third of role overload episodes and as such is the number one person factor item listed in Table 9-1. Many of the workers giving this response described high levels of commitment to their roles, perhaps not surprising given the sector they work in. Those who share this personal characteristic also revealed strong 
desires to overcome role overload in order to ensure role fulfillment to an acceptable quality:

I pride myself on doing a good job and usually respecting the demands of the higher powers. Just for my own personal satisfaction I like when I can do a good job and complete it.

... and some even tolerated role overload in order to honour role expectations:

I like to learn and (I like) keeping up in my field, but I don't like being forced to do it at home. (Yet) I was glad I had done it. I was proud of myself. I take pride in what I do.

...whilst others hinted at the negative impacts they experienced if they could not meet their obligations:

..sometimes I'm not able to make good on promises and I don't like that.

The second internal role pressure, 'opportunity for accomplishment' was cited in $12.5 \%$ of the role overload episodes. Workers who gave this response in the overload episode under discussion appeared to recognise the potential for personal achievement, and also seemed to be focusing on the strong possibility of a positive outcome:

I do like to see actual work being accomplished. This is something very tangible that I can say yes I did that and that's not always the case in my job sometimes you seem to work all the time and not have an actual product at the end of the day.

The final internal role pressure (cited in $10 \%$ of episodes) was labelled 'importance of family'. In these episodes respondents expressed how important family is and sometimes how difficult situations can be if this commitment to family could not be fulfilled. One respondent explained:

Something more serious could have happened (falling and breaking something). Just not having the time to deal with a family situation like that, being expected to work shift work. Family being so important made it hard. 
The last three individual internal role pressures (enjoy the role, other personal goals and objectives, and other unique responses) were each cited in only three episodes each.

\subsubsection{Personal beliefs}

Under the person factor theme, 'personal beliefs', only one item was described in a substantive number of the role overload episodes recalled by the hospital workers (the other two, lack of self esteem and spiritual beliefs, were each mentioned in one episode each). Often quoted verbatim, 'lack of control' was mentioned in $32.5 \%$ of the role overload episodes:

I don't have a lot of control over when they are scheduled, I don't have a lot of control then of what my current work load is, at the time that I am doing this

Respondents describing a lack of control expressed strong feelings of concern or helplessness when decisions were made about them without consultation or whenever they had little choice about, or control over, a specific course of action:

I kinda felt outta control I felt like I had little support from people around me like in terms of support staff here I felt like I had really no one to give me assistance I felt a little helpless I guess, felt kinda frantic.

I was bumped to nights because of a nurse manager's decision, I was told just before Christmas and there was absolutely no chance to prepare it was just dumped on me and had no recourse whatsoever and the position started in two weeks. And on top of that I was told I had to work every weekend in January.

These comments also suggest that lack of control impacts the respondents' ability to locate or prepare the necessary resources to deal with the event. 


\subsubsection{Personal attributes}

Only one personal attribute was mentioned by respondents in any substantive numbers and then in only four episodes (10\%). In these episodes, hospital workers described themselves as being calm by nature, unemotional, and unlikely to react excessively to the stress from role overload. For example, one respondent explained:

I'm not the type of person to explode and have a meltdown.

The limited number of citations with regard to personal attributes overall may be because direct questions about such characteristics were not asked during the role overload recollection portion of the interviews. Comments about being calm by nature arose during questions about coping with emotions. The personality trait measure at the end of the interviews was intended to provide more information about the personal characteristics of these respondents. Personality traits will be examined in Section 9.2.

\subsubsection{Person factors and episode assessment}

Overall, the frequency with which people mentioned person factors, when describing what made them feel overloaded, varied appreciably with how they ultimately assessed the role overload episode. More specifically, person factors were mentioned in more not-overwhelming than overwhelming episodes (18 to 13 episodes) (not shown). The results in Table 9-1 show specifically that internal role pressures were mentioned in more not-overwhelming episodes (15 episodes to 10), whilst workers describing overwhelming episodes tended to talk more of personal beliefs about themselves or the environment (10 episodes to 6). The third group of person factors, personal attributes, 
was equally likely to be mentioned in both episode types. This result suggests that the personal attributes expressed by these respondents had little relationship with episode assessment.

The individual items within these three person factor themes reveal three results of note. First, despite the marked bias for personal beliefs in general to be talked about more often in overwhelming episodes, the results for the individual item 'lack of control', show little variation with episode assessment. Second, the internal role pressure, 'pride in being good at my role', was mentioned more frequently in role overload episodes evaluated as not-overwhelming (9 episodes to 5). All four episodes in which the personal attribute 'calm by nature' was cited were subsequently assessed as not-overwhelming. There are no person factor items that appear to be associated with overwhelming role overload.

\subsubsection{Person factors and respondent stress level}

Table 9-2 presents person factors cited by hospital workers during recollections of role overload, according to respondent stress level. Overall results (not shown) suggest person factors do not seem to be strongly associated with reported stress. The aggregated results for each of the three person factor themes shown in Table 9-2, reveal that the frequency with which high and low stress workers talked about person factors, when discussing what made them feel overloaded, differs for two of the three themes. Specifically, highly stressed hospital workers talked more often about internal role 
pressures (14 episodes to 11) and personal beliefs (10 episodes to 6) than did those with low levels of stress.

There are two individual person factor items that appear to be associated with reported stress. First, highly stressed workers talked about the pride they take in being good at their role in more role overload episodes than did workers from the low stress group (9 episodes to 5). Similarly, beliefs about lack of control were also cited more often by workers with high stress (8 episodes to 5 ).

\begin{tabular}{|c|c|c|c|c|c|c|}
\hline \multirow[b]{4}{*}{ Person Factors } & \multicolumn{6}{|c|}{ Role Overload Episodes by Respondent Stress Level } \\
\hline & Lov & $=20)$ & Higl & $(n=20)$ & Total & $(n=40)$ \\
\hline & \multicolumn{6}{|c|}{$\#$ and $\%$ of episodes with identified property } \\
\hline & $\#$ & $\%$ & \# & $\%$ & \# & $\%$ \\
\hline Internal Role Pressures & 11 & 55 & 14 & 70 & 25 & 62.5 \\
\hline Take pride in being good at my role & 5 & 25 & 9 & 45 & 14 & 35 \\
\hline Opportunity for accomplishment & 3 & 15 & 2 & 15 & 5 & 12.5 \\
\hline Family First & 3 & 15 & 1 & 5 & 4 & 10 \\
\hline Enjoy the role & 2 & 10 & 1 & 5 & 3 & 7.5 \\
\hline Personal goals \& objectives & 2 & 10 & 1 & 5 & 3 & 7.5 \\
\hline Other & 0 & 0 & 3 & 15 & 3 & 7.5 \\
\hline Personal Beliefs & 6 & 30 & 10 & 50 & 16 & 32.5 \\
\hline Lack of control & 5 & 25 & 8 & 40 & 13 & 27.5 \\
\hline Lack of esteem & 0 & 0 & 1 & 5 & 1 & 2.5 \\
\hline Spiritual beliefs & 0 & 0 & 1 & 5 & 1 & 2.5 \\
\hline Personal Attributes & 5 & 25 & 3 & 15 & 8 & 20 \\
\hline Calm by Nature & 2 & 10 & 2 & 10 & 4 & 10 \\
\hline Resilient & 1 & 5 & 1 & 5 & 2 & 5 \\
\hline Other & 1 & 5 & 1 & 5 & 2 & 5 \\
\hline
\end{tabular}

Table 9-2 Person factors described in role overload episodes by stress level of respondent

\subsubsection{Person factors by respondent stress and episode assessment}

Table 9-3 presents the data by stress level and role overload episode assessment for those row items where differences are notable. The results in this table reveal that the 
tendency for low stress respondents to cite fewer internal role pressures is restricted to episodes assessed as overwhelming. Specifically, low stress respondents talked of internal role pressures in only three episodes assessed as overwhelming whereas high stress workers talked of internal role pressures in eight overwhelming episodes.

The unusual data pattern noted for internal role pressures overall is restricted to one person factor, 'take pride in being good at my role'. Pride felt in doing a good job was rarely mentioned in the overwhelming episodes of low stress employees, a finding that stands out because it was mentioned with the same frequency by workers in the other three groups.

\begin{tabular}{|c|c|c|c|c|c|c|c|c|c|c|}
\hline \multirow[b]{5}{*}{ Person Factors } & \multicolumn{8}{|c|}{ Role Overload Episodes by Respondent Stress Level } & & \\
\hline & \multicolumn{4}{|c|}{$\operatorname{Low}(N=10)$} & \multicolumn{4}{|c|}{ High $(\mathrm{N}=10)$} & & \\
\hline & $\begin{array}{r}\text { over } \\
\text { E } \\
\end{array}$ & ming & \multicolumn{2}{|c|}{$\begin{array}{l}\text { Overwhelming } \\
\text { Episodes } \\
(\mathbf{n}=10)\end{array}$} & \multicolumn{2}{|c|}{$\begin{array}{c}\text { Not- } \\
\text { overwhelming } \\
\text { Episodes } \\
(\mathbf{n}=\mathbf{1 0})\end{array}$} & \multicolumn{2}{|c|}{$\begin{array}{l}\text { Overwhelming } \\
\text { Episodes } \\
(n=10)\end{array}$} & \multicolumn{2}{|c|}{$\begin{array}{c}\text { Total } \\
\text { Episodes } \\
(\mathbf{n}=\mathbf{4 0}) \\
\end{array}$} \\
\hline & \multicolumn{10}{|c|}{$\# \& \%$ of episodes in which this characteristic was mentioned } \\
\hline & $\#$ & $\%$ & $\#$ & $\%$ & $\#$ & $\%$ & $\#$ & $\%$ & $\#$ & $\%$ \\
\hline Internal Role Pressures & 8 & 80 & 3 & 30 & 7 & 70 & 7 & 70 & 25 & 62.5 \\
\hline Take pride in being good at my role & 4 & 40 & 1 & 10 & 5 & 50 & 4 & 40 & 14 & 35 \\
\hline
\end{tabular}

Table 9-3 Person factors described in role overload episodes by episode assessment and stress level of respondent

\subsection{Personality Traits}

Five personality traits (extraversion, agreeableness, conscientiousness, neuroticism, and openness to experience) were measured and scored using ten short questions at the end of each interview. The instrument was developed by Gosling et al. (2003) and a full description of the measure used and the scoring calculation was presented in Section 
7.1.2. For display purposes, the calculated scores for each trait for each respondent were grouped into one of three categories, high, medium and low, as specified in Section 7.2.2. The results for each of the five personality traits for the total set of respondents and for low and high stress workers are presented in Table 9-4. Descriptors of the poles for each trait dimension (as presented in Section 7.1.2) are also repeated in this table. The overall results for the total sample will be reviewed first before discussions turn to the results for the differential stress level groups. The reader should note that only table type two is used to display the results in this section (i.e., for personality traits) and also in Section 9.3 (i.e., for role salience) as both these person factors are not differentiated at the role overload episode level.

Results for the total sample in Table 9-4 show that for all the five personality traits, at least half (and for four of the five, more than half) of the workers scored in the high range of the trait. In addition, relatively few workers scored low for any of the five personality traits. More specifically, ninety per cent of the respondents scored high in the trait, openness to experience whilst four fifths also have high scores on the trait agreeableness measure. On the other hand, only half of the respondents scored high levels of the trait neuroticism. The fact that the other half of the hospital employees were found to have medium or low scores of this trait, suggests that the emotional stability of this set of hospital workers varies more widely than the other traits. 


\begin{tabular}{|c|c|c|c|c|c|c|}
\hline \multirow[b]{4}{*}{ Personality Traits } & \multicolumn{6}{|c|}{ Stress Level of Respondents } \\
\hline & \multicolumn{2}{|c|}{$\begin{array}{c}\text { Low } \\
(n=10)\end{array}$} & \multicolumn{2}{|c|}{$\begin{array}{c}\text { High } \\
(n=10)\end{array}$} & \multicolumn{2}{|c|}{$\begin{array}{c}\text { Total } \\
(n=20)\end{array}$} \\
\hline & \multicolumn{6}{|c|}{ \# and $\%$ of hospital workers with this level of the personality trait } \\
\hline & \# & $\%$ & \# & $\%$ & \# & $\%$ \\
\hline Extraversion & \multicolumn{6}{|c|}{ Reserved, quiet v Extraverted, enthusiastic } \\
\hline Low & 1 & 10 & 2 & 20 & 3 & 15 \\
\hline Medium & 2 & 20 & 2 & 20 & 4 & 20 \\
\hline High & 7 & 70 & 6 & 60 & 13 & 65 \\
\hline Agreeableness & \multicolumn{6}{|c|}{ Critical, quarrelsome v Sympathetic, warm } \\
\hline Low & 0 & 0 & 0 & 0 & $\mathbf{0}$ & $\mathbf{0}$ \\
\hline Medium & 3 & 30 & 1 & 10 & 4 & 20 \\
\hline High & 7 & 70 & 9 & 90 & 16 & 80 \\
\hline Conscientiousness & \multicolumn{6}{|c|}{ Disorganised, careless v Dependable, self-disciplined } \\
\hline Low & 0 & 0 & 1 & 10 & 1 & 5 \\
\hline Medium & 0 & 0 & 7 & 70 & 7 & 35 \\
\hline High & 10 & 100 & 2 & 20 & 12 & 60 \\
\hline Neuroticism & \multicolumn{6}{|c|}{ Calm, emotionally stable v Anxious, easily upset } \\
\hline Low & 4 & 40 & 0 & 0 & 4 & 20 \\
\hline Medium & 4 & 40 & 2 & 20 & 6 & 30 \\
\hline High & 2 & 20 & 8 & 80 & 10 & 50 \\
\hline Openness to Experience & \multicolumn{6}{|c|}{ Conventional, uncreative v Open to new experiences, complex } \\
\hline Low & 0 & 0 & 0 & 0 & $\mathbf{0}$ & 0 \\
\hline Medium & 0 & 0 & 2 & 20 & 2 & 10 \\
\hline High & 10 & 100 & 8 & 80 & 18 & 90 \\
\hline
\end{tabular}

Table 9-4 Personality trait mean scores by stress level of respondent

\subsubsection{Personality traits and respondent stress}

The pattern of the extraversion, agreeableness, and open to experience trait scores for low stress and high stress respondents show no appreciable difference. The scores for conscientiousness and neuroticism displayed in Table 9-4, however, reveal a very different picture. Although overall $60 \%$ of respondents have high levels of conscientiousness, characterised by a dependable and self-disciplined nature, the mix of 
low, medium and high scores on this dimension of personality are quite different for those workers with high stress compared to those with low stress. Specifically, $70 \%$ of highly stressed respondents have medium levels of this trait and only $20 \%$ scored high, suggesting that at best these hospital workers have moderate to high levels of conscientiousness. In contrast, all of the low stress respondents have high levels of this trait. These results suggest an association between high levels of consciousness and low levels of respondent perceived stress.

The results have already suggested that overall the level of the neuroticism personality trait varies more widely than the other four personality traits for these workers. The difference between the two stress level groups is, in fact, notable since four fifths of those hospital workers with low stress levels have medium or low scores of this trait whereas the same proportion of high stress respondents have high levels of neuroticism. This suggests that the association between the high stress respondents and high levels of neuroticism is relatively strong.

\subsection{Role Salience}

Role salience refers to the importance individuals ascribe to roles played out in their various domains (Amatea, Cross, Clarke and Bobby, 1986). In the survey taken by the hospital employees prior to the thesis interview, three life roles were examined for role salience, the occupational, parental, and marital roles. The scales developed by Amatea, Cross, Clark and Bobby, 1986 were used to measure these three life roles. Role salience is measured across two dimensions. The first dimension relates to role value, the 
extent to which the role is an important means of self-definition or personal satisfaction; the second relates to role commitment, the extent to which the respondent is willing to commit personal resources to assure role success (Amatea et al., 1986). Calculated role salience scores are based on a 5-point Likert scale. The calculated scores for each of the respondents were subsequently categorised into one of three groups based on a three way proportionate split of the 5-point scale (i.e., scores less than 2.33 were labelled low, scores from 2.33 to 3.67 , medium, and scores greater than 3.67, high). Categorising the scores in this way also makes the display of role salience results easier to produce and to interpret.

Role salience results for the respondents are presented in Table 9-5 for each dimension of these three life roles, for the total sample and by respondent stress level. Unmarried or divorced respondents or respondents with no children at home or respondents who did not provide role commitment data were omitted from score calculations, as indicated by the numbers specified in the table for the parental and marital roles.

The results for the total sample show that the parental role is by far the most salient for these female hospital workers in terms of value and commitment. The proportion of the sample with high scores for parental role value and role commitment (more than $90 \%$ of respondents for both) are much greater than for the two role salience dimensions for either of the other two roles. 


\begin{tabular}{|c|c|c|c|c|c|c|}
\hline \multirow{4}{*}{ Role Salience } & \multicolumn{6}{|c|}{ Stress Level of Respondents } \\
\hline & \multicolumn{2}{|c|}{$\begin{array}{c}\text { Low } \\
(n=10)\end{array}$} & \multicolumn{2}{|c|}{$\begin{array}{c}\text { High } \\
(n=10)\end{array}$} & \multicolumn{2}{|c|}{$\begin{array}{c}\text { Total } \\
(n=20)\end{array}$} \\
\hline & \multicolumn{6}{|c|}{$\#$ and $\%$ of respondents with role saliance scores } \\
\hline & \# & $\%$ & $\#$ & $\%$ & \# & $\%$ \\
\hline Occupational Role Value & \multicolumn{2}{|c|}{$\mathrm{n}=10$} & \multicolumn{2}{|c|}{$\mathrm{n}=10$} & \multicolumn{2}{|c|}{$\mathbf{n}=\mathbf{2 0}$} \\
\hline Low & 0 & 0 & 0 & 0 & $\mathbf{0}$ & $\mathbf{0}$ \\
\hline Medium & 4 & 40 & 8 & 80 & 12 & 60 \\
\hline High & 6 & 60 & 2 & 20 & 8 & 40 \\
\hline Occupational Role Commitment & \multicolumn{2}{|c|}{$\mathrm{n}=10$} & \multicolumn{2}{|c|}{$\mathrm{n}=10$} & \multicolumn{2}{|c|}{$\mathrm{n}=\mathbf{2 0}$} \\
\hline Low & 0 & 0 & 0 & 0 & $\mathbf{0}$ & $\mathbf{0}$ \\
\hline Medium & 7 & 70 & 8 & 80 & 15 & 75 \\
\hline High & 3 & 30 & 2 & 20 & 5 & 25 \\
\hline Parental Role Value & \multicolumn{2}{|c|}{$\mathrm{n}=9$} & \multicolumn{2}{|c|}{$\mathrm{n}=8$} & \multicolumn{2}{|c|}{$\mathbf{n}=17$} \\
\hline Low & 0 & 0 & 0 & 0 & $\mathbf{0}$ & $\mathbf{0}$ \\
\hline Medium & 0 & 0 & 1 & 12.5 & 1 & 5.9 \\
\hline High & 9 & 100 & 7 & 87.5 & 16 & 94.1 \\
\hline Parental Role Commitment & \multicolumn{2}{|c|}{$\mathrm{n}=8$} & \multicolumn{2}{|c|}{$\mathrm{n}=7$} & \multicolumn{2}{|c|}{$\mathrm{n}=15$} \\
\hline Low & 0 & 0 & 0 & 0 & $\mathbf{0}$ & $\mathbf{0}$ \\
\hline Medium & 0 & 0 & 1 & 14.3 & 1 & 6.7 \\
\hline High & 8 & 100 & 6 & 75.7 & 14 & 93.3 \\
\hline Marital Role Value & \multicolumn{2}{|c|}{$\mathrm{n}=7$} & \multicolumn{2}{|c|}{$\mathrm{n}=8$} & \multicolumn{2}{|c|}{$\mathrm{n}=15$} \\
\hline Low & 0 & 0 & 1 & 12.5 & 1 & 6.7 \\
\hline Medium & 1 & 14.3 & 3 & 37.5 & 4 & 26.7 \\
\hline High & 6 & 85.7 & 4 & 50 & 10 & 66.6 \\
\hline Marital Role Commitment & \multicolumn{2}{|c|}{$\mathrm{n}=7$} & \multicolumn{2}{|c|}{$\mathrm{n}=8$} & \multicolumn{2}{|c|}{$\mathrm{n}=15$} \\
\hline Low & 0 & 0 & 0 & 0 & $\mathbf{0}$ & $\mathbf{0}$ \\
\hline Medium & 2 & 28.6 & 4 & 50 & 6 & 40 \\
\hline High & 5 & 71.4 & 4 & 50 & 9 & 60 \\
\hline
\end{tabular}

Table 9-5 Role salience scores for the occupational, parental and marital roles by stress level of respondent

The results in Table 9-5 also suggest that for those respondents who are married or have partners, the number highly committed to putting resources into the marital role is almost as high as the number of workers who highly value this role ( $9 \mathrm{v} 10$ respondents). Nevertheless, appreciably fewer respondents placed high value on the marital role 
compared to the parental role $(66.6 \% \mathrm{v} 94.1 \%$ of respondents $)$. The occupational role is the least salient of the three roles to these respondents. A minority of respondents valued this role highly (40\%) and even fewer (25\%) showed high levels of occupational role commitment.

\subsubsection{Role salience and respondent stress}

The role salience results for the two stress level groups of workers suggest first that there is little difference between them with regard to the parental role in terms of the extent of role value or commitment. Second, differences between role salience scores for the two stress level groups are most notable for occupational role value. A higher proportion of low stress compared to high stress respondents valued the occupational role highly (60\% to $20 \%)$ suggesting an association between low stress employees and high occupational role value scores. When occupational role values are compared to occupational role commitment, however, low stress employees appeared less willing or less able to commit personal resources to the occupational role to the same extent that they valued the role, whereas for the high stress group there is no discernible difference in the levels of occupational role commitment and value.

Thirdly, although only three quarters of the thesis sample are married, a greater proportion of low stress than high stress workers recorded high scores for marital role value $(85.7 \%$ to $50 \%)$. Marital role commitment, however, does not vary greatly from marital role value for both groups of workers. 


\subsection{Summary of key person factor findings}

The objective of this chapter was to examine the person factors influential in the dynamic relationship between the person and the environment during role overload appraisal. The summary of key findings from Chapter 9 is presented in four short subsections. In the first subsection (Section 9.4.1) the person factors affecting significant numbers of role overload episodes, or affecting all episodes equally is summarised. The next two subsections summarise key person factor findings for each respondent stress level (low and high), and for each episode assessment (not-overwhelming and overwhelming) respectively. The last subsection summaries key person factor findings for the four episode groupings: not-overwhelming episodes of low stress workers; notoverwhelming episodes of high stress workers; overwhelming episodes of low stress workers; and overwhelming episodes of high stress workers.

\subsubsection{Key person factor findings for all episodes}

In the first section of this chapter, the hospital workers described internal role pressures and personal beliefs that they felt influenced their appraisal of role overload and its affect on their wellbeing. Most commonly, respondents talked about the pride they took in doing a good job and their beliefs about lack of control. The data included in this section also highlighted that in a quarter of the episodes recalled, workers made no reference to person factors when describing why they felt overloaded.

The personality trait scale at the end of interviews provided a second source of person factor data to help interpret respondent behaviour during role overload appraisal 
and coping. The results suggest that scores for all five personality traits (extraversion, agreeableness, conscientiousness, neuroticism and openness to experience) are in the high range for a majority of this small sample. In particular, all workers measured moderate to high levels of two of the five personality traits examined in this study: agreeableness and openness to experience. The personality trait data also strongly suggests that certain traits are linked to respondent stress (see below).

To further examine how workers viewed their family and work roles, the last section of this chapter presented role salience data from the surveys completed prior to the respondent interviews, measuring role value and role commitment levels. The data presented suggested that parental role salience (role value and commitment) is much higher than marital or occupational role salience for most of the sample, and varies little with reported stress levels. The occupational role was found to be the least salient role by far. Although the marital role is valued less highly than the parental role, results suggest that workers commit to this role to the same extent they value it.

\subsubsection{Key person factor findings by role overload episode assessment}

The key findings for person factors by role overload episode assessment are presented in Table 9-6 below. Only person factors described during worker interviews are noted since personality traits and role salience, measured for each respondent, are not relevant at the role overload episode level. The data in this table is displayed without regard to respondent stress level. The findings presented in this chapter revealed that hospital employees talked of their pride in doing a good job in more of the episodes 
assessed as not-overwhelming. Also relevant is the fact that none of the person factors described by the female overload workers during the interviews appeared to be associated with the assessment of role overload episodes as overwhelming.

\begin{tabular}{|r|l|l|l|}
\hline \multirow{2}{*}{$\begin{array}{c}\text { Role Overload } \\
\text { Assessment }\end{array}$} & \multicolumn{3}{|c|}{ Role Overload Person Factors } \\
\cline { 2 - 4 } & $\begin{array}{c}\text { Person factors from } \\
\text { recollections of role } \\
\text { overload }\end{array}$ & \multicolumn{1}{|c|}{ Personality Traits } & \multicolumn{1}{|c|}{ Role Salience } \\
\hline $\begin{array}{r}\text { Not- } \\
\text { overwhelming }\end{array}$ & $\begin{array}{l}\text { Pride in being good at my role } \\
\text { Calm by nature }\end{array}$ & N/A & N/A \\
\hline Overwhelming & & N/A & N/A \\
\hline
\end{tabular}

Table 9-6 Key person factors described in role overload episodes assessed as overwhelming and notoverwhelming, disregarding the stress level of the respondents

\subsubsection{Key person factor findings for respondents with low and high stress levels}

The key person factor findings for respondents with low and high stress levels are summarised in Table 9-7. The findings are displayed without regard to how the role overload episodes were evaluated.

Results from this chapter suggest that none of the person factors, described by hospital employees when they talked about how role overload made them feel, were uniquely associated with the low stress level respondent group. The results obtained using a common measure of personality, however, do suggest that low stress hospital employees report higher levels of conscientiousness. Role salience results have also suggested that low stress hospital employees tend to have higher scores for occupational and marital role value than do those respondents with higher levels of perceived stress. 


\begin{tabular}{|c|l|l|l|}
\hline \multirow{2}{*}{$\begin{array}{c}\text { Respondent } \\
\text { Stress } \\
\text { Level }\end{array}$} & $\begin{array}{c}\text { Role Overload Person Factors } \\
\text { Pecollections of role } \\
\text { overload }\end{array}$ & Personality Traits & \multicolumn{1}{|c|}{ Role Salience } \\
\cline { 2 - 4 } Low & \multicolumn{1}{|c|}{ Conscientious } & $\begin{array}{l}\text { Higher marital and } \\
\text { occupational role value }\end{array}$ \\
\hline High & $\begin{array}{l}\text { Pride in being good at my role } \\
\text { Lack of control }\end{array}$ & Neurotic & \\
\hline
\end{tabular}

Table 9-7 Key person factors for role overload of low and high stress respondents, without regard to how the role overload episodes were assessed

Results from this chapter also identified three person factors that were associated with high levels of reported stress. First, the internal role pressure, 'taking pride in being good at my role' appeared to be particularly influential in role overload episodes described by the high stress group. In addition, these highly stress workers were more likely to believe that they had no control over role overload. Lastly, highly stress workers also reported higher levels of neuroticism.

\subsubsection{Key person factor findings by episode groupings}

Table 9-8 summarises the key finding in this chapter for the four episode groupings as labeled in the table. Of note is the data showing that the person factor, "pride felt in doing a good job', was rarely mentioned in the overwhelming episodes of low stress employees, a finding that stands out because it was mentioned with the same frequency by workers in the other three groups. 


\begin{tabular}{|c|c|}
\hline Not-overwhelming episodes of low stress workers & $\underline{\text { Not-overwhelming episodes of high stress workers }}$ \\
Take pride in being good in my role & Take pride in being good in my role \\
\hline$\underline{\text { Overwhelming episodes of low stress workers }}$ & $\underline{\text { Overwhelming episodes of high stress workers }}$ \\
& Take pride in being good in my role \\
\hline
\end{tabular}

Table 9-8 Summary of role overload person factors by episode grouping 


\section{Role overload appraisal}

Chapters 8 and 9 of this thesis have examined the specific characteristics of the environments and the persons involved in the appraisal of role overload suffered by female employees of four major hospitals in eastern Ontario. Lazarus and Folkman (1984, p. 31) explain that cognitive appraisal can be best understood as the process of categorizing an encounter "with respect to its significance for well being". This chapter will examine the results of the dynamic interaction that takes place between the person and the environment during role overload appraisal. As described in Chapter 3, when faced with a potentially stressful event, such as role overload, the appraisal process undertaken by all individuals asks two broad questions, 'What's at stake?' (primary appraisal) and 'What, if anything, can I do about it?' (secondary appraisal) (Lazarus and Folkman, 1984). According to Folkman et al. (1986, p.993), the answers to these questions will determine whether the person-environment transaction will affect wellbeing and if it does, whether it will be "primarily threatening (with the possibility of harm or loss), or challenging (offering the possibility of benefit)". In the short term this process will also produce negative and or positive emotional responses depending on the evaluation result reached. The objective of this chapter is to identify the factors pertinent to role overload appraisal, guided by the two research questions that ask:

- How does primary appraisal impact the differential stress response under role overload conditions?

- How does secondary appraisal impact the differential stress response under role overload conditions? 
The first section of this chapter (Section 10.1) examines how the hospital workers evaluated the role overload with respect to well-being (i.e., harmful, threatening or challenging). The next two sections explore the processes linked to primary (Section 10.2) and secondary appraisal (Section 10.3) of role overload. Section 10.4 examines the emotional outcomes from the role overload appraisal process. A summary of the key findings will conclude the chapter.

It is worthy of repetition that although the names of these two forms of cognitive appraisal (primary and secondary) imply relative ranking and temporal order, Lazarus and Folkman (1984) emphasise that this was not the intended meaning. The two processes are equal in importance and in reality interact with each other to create the degree of stress and the strength and content of the emotional reaction (Lazarus and Folkman, 1984). The data that allows us to examine these processes are presented consecutively for convenience.

\subsection{Harm, threat or challenge?}

According to Lazarus and Folkman (1984), appraisals of stress can take three forms, harm, threat or challenge. An appraisal of harm from role overload would indicate that the hospital worker feels they have already sustained injury or loss, whilst a threat appraisal refers to predicted harm or loss. Challenge appraisals, on the other hand, suggest a possibility of benefit or gain but importantly still require coping efforts. To elicit respondents' views of how their well-being was likely to be affected by role overload, respondents were asked "Did you feel that the situation was harmful to you, or 
potentially threatening in any way, or challenging to you (if none of these how would you describe it)?". Table 10-1 presents the results from answers to this question. Lazarus and Folkman (1984 p. 33) also posit that challenge, threat and harm appraisals are "not necessarily mutually exclusive". In the same way as other interview questions, multiple answers to this question were, therefore, allowed.

Results in Table 10-1 illustrate that all respondents selected one or more of the three descriptions offered. The most common type of appraisal result cited by workers was a challenge appraisal (mentioned in over two-thirds of the episodes). In these episodes respondents frequently described challenges in terms of the efforts required to overcome the overload. The following comments illustrate such responses:

It was a bit of a challenge - trying to get the ticket reduced, and dealing with the police on his behalf, but still kind of letting him fight his own fights.

Challenging - Because I had to move pretty darn fast to get everything sorted out

When describing challenges, workers were unlikely to express concerns about negative outcomes but only occasionally acknowledged the benefits to be gained. For example one respondent commented:

It was a great challenge, an excellent challenge. Within that challenge, I went through several emotions and learning curves.

\begin{tabular}{|r|c|c|c|c|c|c|c|}
\hline \multirow{2}{*}{} & \multicolumn{6}{|c|}{ Role Overload Episode Assessment } \\
\cline { 2 - 7 } & \multicolumn{2}{|c|}{$\begin{array}{c}\text { Not-overwhelming } \\
(\mathbf{n = 2 0 )}\end{array}$} & $\begin{array}{c}\text { Overwhelming } \\
(\mathbf{n = 2 0 )}\end{array}$ & Total & $\mathbf{( n = 4 0 )}$ \\
\cline { 2 - 7 } Form of Appraisal & \multicolumn{6}{|c|}{$\#$ and \% of episodes citing identified appraisal type } \\
\cline { 2 - 7 } & $\#$ & $\mathbf{\%}$ & $\#$ & $\mathbf{\%}$ & $\#$ & $\mathbf{\%}$ \\
\hline Challenge & 16 & 80 & 11 & 55 & $\mathbf{2 7}$ & $\mathbf{6 7 . 5}$ \\
\hline Threat & 9 & 45 & 13 & 65 & $\mathbf{2 2}$ & $\mathbf{5 5}$ \\
\hline Harm & 0 & 0 & 8 & 40 & $\mathbf{8}$ & $\mathbf{2 0}$ \\
\hline
\end{tabular}

Table 10-1 Appraisal of role overload by episode assessment 
The interpretations of challenging role overload focused largely on the steps to be taken, the decisions to be made, and the degrees of difficulty arising from those steps or decisions. Explicit statements about the potential for personal growth or other positive outcomes were not common. Rather, the cessation of the role overload situation was the implied benefit in many cases.

Threat appraisals were cited in over half of the role overload episodes. These appraisals were characterised by descriptions of potential outcomes that workers felt could not be dealt with should they occur:

I just felt totally threatened - The financial aspect and that it was his word against ours (that the furnace needed to be replaced) and there wasn't anything I could do about it.

Threatening, in the knowledge that my backup wasn't there anymore where if there was a patient issue I didn't have anyone in the near vicinity to assist me if something went wrong.

These comments also reveal what was at stake in these encounters. In the two comments above, respondents identified a threat to personal finances and to patient well-being and also hinted at coping option and resource assessment. These comments thus illustrate the close interaction of primary and secondary appraisal. (Both elements of appraisal will be examined more fully in the following two sections).

Harmful appraisals were relatively rare (cited in only a fifth of the episodes recalled). For these episodes, however, respondents clearly recognized that some form of harm had already occurred or was inevitable, regardless of coping efforts:

Very much. I'm going to lose credibility with the physicians or with the director. 
Harmful to me in that I do suffer from high blood pressure and I am quite sure my blood pressure on these occasions was way above normal.

\subsubsection{Appraisal results by episode assessment}

As shown in Table 10-1, role overload episodes assessed as not-overwhelming were more likely than those rated overwhelming to feature challenge appraisals (16 to 11 episodes). In contrast, respondents were more likely to cite threat appraisals in overwhelming role overload than in episodes found not to be overwhelming (13 versus 9 episodes). Most interestingly, however, there was no harm appraisals cited in role overload evaluated as not-overwhelming. All eight such episodes arose in situations assessed as overwhelming.

\subsubsection{Appraisal results by respondent stress}

Appraisal results according to the stress level of the hospital employees are presented in Table 10-2.

\begin{tabular}{|c|c|c|c|c|c|c|}
\hline \multirow{4}{*}{$\begin{array}{c}\text { Form of } \\
\text { Appraisal }\end{array}$} & \multicolumn{6}{|c|}{ Role Overload Episode by Respondent Stress Level } \\
\hline & \multicolumn{2}{|c|}{ Low $(n=20)$} & \multicolumn{2}{|c|}{ High $(n=20)$} & \multirow{2}{*}{\multicolumn{2}{|c|}{$\begin{array}{c}\text { Total }(\mathbf{n}=\mathbf{4 0}) \\
\text { appraisal type }\end{array}$}} \\
\hline & \multicolumn{4}{|c|}{$\#$ and $\%$ of episodes citing identified appraisal type } & & \\
\hline & \# & $\%$ & $\#$ & $\%$ & $\#$ & $\%$ \\
\hline Challenge & 15 & 75 & 12 & 60 & 27 & 67.5 \\
\hline Threat & 11 & 55 & 11 & 55 & 22 & 55 \\
\hline Harm & 3 & 15 & 5 & 25 & 8 & 20 \\
\hline
\end{tabular}

Table 10-2 Appraisal of role overload by respondent stress level

Table 10-2 reveals that low stress respondents were more likely to cite challenge appraisals in their descriptions of role overload than respondents with higher levels of stress (15 episodes to 12$)$. Threat and harm appraisals were both equally likely to be cited 
by both respondent groups. These results suggest that any stress that respondents felt about the potential (or actual) harm in individual role overload episodes was not related to their reported stress levels.

\subsubsection{Appraisal results by respondent stress and episode assessment}

Table 10-3 presents results by respondent stress and episode assessment for those items exhibiting notable data patterns. Examination of Table 10-3 reveals that all but one of the not-overwhelming role overload episodes of the low stress respondents were described as challenging. These results suggest a strong association between challenge appraisals and episodes assessed not to be overwhelming for the low stress hospital employees, even though in four of these nine episodes the respondents also acknowledged the possibility of harm (not shown).

\begin{tabular}{|c|c|c|c|c|c|c|c|c|c|c|}
\hline \multirow{5}{*}{$\begin{array}{c}\text { Type of } \\
\text { Appraisal }\end{array}$} & \multicolumn{8}{|c|}{ Stress Level of Respondents } & & \\
\hline & \multicolumn{4}{|c|}{ Low $(N=10)$} & \multicolumn{4}{|c|}{ High $(\mathrm{N}=10)$} & \multirow{2}{*}{\multicolumn{2}{|c|}{$\begin{array}{c}\text { Total } \\
\text { Episodes } \\
(n=40)\end{array}$}} \\
\hline & \multicolumn{2}{|c|}{$\begin{array}{c}\text { Not- } \\
\text { overwhelming } \\
\text { Episodes }(n=10)\end{array}$} & \multicolumn{2}{|c|}{$\begin{array}{l}\text { Overwhelming } \\
\text { Episodes } \\
(n=10)\end{array}$} & \multicolumn{2}{|c|}{$\begin{array}{c}\text { Not- } \\
\text { overwhelming } \\
\text { Episodes }(n=10)\end{array}$} & \multicolumn{2}{|c|}{$\begin{array}{c}\text { Overwhelming } \\
\text { Episodes }(n=10)\end{array}$} & & \\
\hline & \multicolumn{10}{|c|}{$\# \& \%$ of episodes in which this characteristic was mentioned } \\
\hline & $\#$ & $\%$ & $\#$ & $\%$ & \# & $\%$ & \# & $\%$ & \# & $\%$ \\
\hline Challenge & 9 & 90 & 6 & 60 & 7 & 70 & 5 & 50 & 27 & 67.5 \\
\hline
\end{tabular}

Table 10-3 Appraisal of role overload by respondent stress level and episode assessment

\subsection{Primary appraisal - what's at stake?}

According to Lazarus and Folkman (1984), during the primary appraisal process each hospital worker will evaluate whether she has anything at stake in the role overload encounter being faced. The answers to who or what was at stake were broadly 
categorised according to the focus of who, or what, was being impacted in the role overload episodes: personal well-being, the well-being of others, and/or the well-being of the organization, as shown in Table 10-4. During the analysis of the data it was noted that multiple items, from one or more of these three parent themes, could be at stake or at risk for respondents in any one role overload episode. For example, one hospital worker commented:

I felt only threatened of (losing) my nursing license and am I doing enough, am I doing enough to protect the people who work here?

For this respondent, her own professional credibility was at risk, but also the well-being of others, namely her colleagues, was a concern to her.

Examining the three parent themes in Table 10-4, the data reveals that in a notable majority of the episodes discussed ( $80 \%$ of the total) workers indicated that personal well-being was threatened. The parent descriptor of 'personal well-being' incorporates role failure, the personal health and safety of hospital employees, professional credibility, well-being of non-work environments, personal values, financial well-being, and lastly the status of personal goals.

The well being of others also arose as a concern for workers in substantive number of role overload episodes, reflecting the commitment to patients, colleagues and family that has been evident from earlier results. More specifically, the welfare of patients, staff, or family members (i.e., the well-being of others) was cited as being at risk in $62.5 \%$ of role overload episodes.

Finally, the last theme representing answers to the primary appraisal question of 'What's at stake?' is 'the well-being of the organisation' (mentioned in only $30 \%$ of the 
episodes). Commitments to the organisation as a whole (i.e. the hospital unit) have not been noted to any great extent in the examination of results so far and therefore it is not surprising that threats to the organisation were mentioned in few role overload episodes.

\begin{tabular}{|c|c|c|c|c|c|c|}
\hline \multirow[b]{4}{*}{ What's at Stake? } & \multicolumn{6}{|c|}{ Role Overload Episode Assessment } \\
\hline & \multicolumn{2}{|c|}{$\begin{array}{c}\text { Not- } \\
\text { overwhelming } \\
(\mathbf{n}=\mathbf{2 0}) \\
\end{array}$} & \multicolumn{2}{|c|}{$\begin{array}{l}\text { Overwhelming } \\
(\mathbf{n}=\mathbf{2 0})\end{array}$} & \multirow[t]{2}{*}{ Total } & \multirow[t]{2}{*}{$(n=40)$} \\
\hline & \multicolumn{4}{|c|}{ \# and $\%$ of episodes with identified property } & & \\
\hline & $\#$ & $\%$ & $\#$ & $\%$ & $\#$ & $\%$ \\
\hline Personal Well-being & 14 & 70 & 18 & 90 & 32 & 80 \\
\hline Role failure & 7 & 35 & 7 & 35 & 14 & 35 \\
\hline Personal health \& safety & 6 & 30 & 7 & 35 & 13 & 32.5 \\
\hline My professional credibility & 3 & 15 & 8 & 40 & 11 & 27.5 \\
\hline Home or non-work life & 4 & 20 & 5 & 25 & 9 & 22.5 \\
\hline Personal values & 4 & 20 & 5 & 25 & 9 & 22.5 \\
\hline Financial well-being & 2 & 10 & 4 & 20 & 6 & 15 \\
\hline Other personal goals & 2 & 20 & 0 & 0 & 2 & 5 \\
\hline The Well-being of Others & 13 & 65 & 12 & 60 & 25 & 62.5 \\
\hline Well-being of patients & 6 & 30 & 5 & 25 & 11 & 27.5 \\
\hline Well-being of staff & 5 & 25 & 6 & 30 & 11 & 27.5 \\
\hline Well-being of family member(s) & 3 & 15 & 3 & 15 & 6 & 15 \\
\hline Concern for others & 2 & 10 & 1 & 5 & 3 & 7.5 \\
\hline The Well-being of the Organisation & 5 & 25 & 7 & 35 & 12 & 30 \\
\hline Ability to provide care & 1 & 5 & 3 & 15 & 4 & 10 \\
\hline Legal Liability & 1 & 5 & 2 & 10 & 3 & 7.5 \\
\hline Financial Affairs & 3 & 15 & 0 & 0 & 3 & 7.5 \\
\hline Reputation & 0 & 0 & 2 & 10 & 2 & 5 \\
\hline
\end{tabular}

Table 10-4 What is at stake in role overload by episode assessment?

What was at stake in each of these three parent themes will be examined in the next three subsections (Sections 10.2.1 to 10.2.3). Table 10-4 is also used in Section 10.2.4 to examine what is at stake by role overload episode assessment. The relationship between primary appraisal and respondent stress level will be examined separately in Section 10.2.6, whilst the last subsection (Section 10.2.6) examines primary appraisal by respondent stress and episode assessment. 


\subsubsection{Personal Well-being}

The most important 'personal wellbeing' factor of concern during role overload for these employees was labeled 'role failure' (cited in 35\% of episodes). This item also has the highest count overall (see Table 10-4). Concerns about failure were the recurrent theme in many of these 14 episodes, whether the role overload was at work:

I would have felt like I failed the patient, that I didn't get enough done, that I wasn't doing my job properly.

or at home:

I feel completely inadequate as a parent.

Worries about failure were not restricted to work situations and implied direct challenges to self esteem.

'Physical health and safety' was also a particularly important issue at stake during the role overload described by respondents (cited in $32.5 \%$ of episodes). The comments made about the threats to physical health and safety reflects a variety of different issues. Some hospital employees cited the possible risks inherent in their existing health conditions:

(It is) harmful to me in that I do suffer from high blood pressure and I am quite sure my blood pressure on these occasions is way above normal.

...whilst others mentioned concerns about the negative effects from the demands and burdens of role overload:

I think there is a balance and you have to find that balance. You have to decide, okay, is this really worth it. It is not only about the money it is about your health.

Finally, albeit less often, a small number of respondents talked of fears about personal 
injury:

I always have a concern if I am not at my best there is always (the) potential for myself to get injured

The next personal well-being item (cited in $27.5 \%$ of episodes) involved threats to 'professional credibility'. Such concerns were relevant to the work role only. In most cases, workers also specifically expressed their fear of losing the professional respect of colleagues and management, which they recognized could negatively impact their credibility in their work role and potentially their job status:

I'm going to lose credibility with the physicians or with the director It would have showed a lack of professionalism if I hadn't done it. I would have made mistakes in my work, and I'd hear from Toronto about it.

In rare cases losing one's professional license and hence the ability to practice one's profession was a real worry to some respondents. At worst, the notion of 'professional credibility' being at stake also included the possibility of losing one's job, as demonstrated by the following comment:

...job loss fear, and disappointment to the Director as he's putting a lot of faith in me. Previous to me, there were two projects where nothing happened so I sort of (have) the last kick of the can, so I better get something done or else. It could also be embarrassing, as you like to be thought of as someone who makes things happened.

Risks to the home or non-work life of the respondents were mentioned in $22.5 \%$ of episodes). Many of the respondents citing this threat explained that role overload at work meant they had less time for their home or personal life:

I felt that it was infringing on my home time. 
It leads to an unbalanced lifestyle. The things I'd like to do in the evening, I can't do them. I get home at about a quarter to seven, and most social things start at seven. I have the dogs to walk, I have supper, I have things to get ready for the next day.

How respondents talked about this threat are consistent with earlier conjecture that role overload at work could be one of the causes of role overload at home (see Section 6.2.4). Occasionally, however, respondents mentioned how role overload at home could also negatively impact personal time:

I'd have to put all my stuff aside. I'd have to do all the driving. ...while others were worried about strained relationships:

It would have meant a lot of conflict with my mother especially, and having to deal with a lot of potential unpleasantness in the household

The fifth item in this 'at stake' category was labeled "personal values" to reflect a collection of respondent citations (in $22.5 \%$ of the episodes) in which personal values were at risk of being compromised because of the role demands experienced in the role overload episode. One respondent, for example, was very unhappy about being asked to perform a task outside her mandate:

Being asked to be held accountable for such a sensitive event when I had no reporting control over this person. Didn't like the situation, didn't feel I should be responsible. (I felt) very uncomfortable, both personally and professionally.

The final substantive item in the personal well-being category mentioned in substantive numbers was labeled 'financial well-being'. This item was cited in only 6 (15 $\%$ episodes. When financial security felt threatened, respondents were explicit about this threat: 
My family counts on me heavily both financially and in terms of running the household. So had I not been able to do that ... that would have been catastrophic for them.

\subsubsection{Well-being of others}

Concerns for the 'well-being of others', the second risk category identified, focused primarily on threats to patients, staff, and family members. First amongst these (mentioned in $27.5 \%$ of the episodes) was the concern for the 'well-being of patients'. In some role overload cases, workers felt that patients' physical well-being was directly threatened. In other cases, respondents felt that patients suffered indirectly because of service quality issues arising as a result of their excessive workload. The following comments provide examples of these issues:

Clients have rapidly deteriorating conditions and not seeing them is not life threatening but the clients see it as the end of the world if I can't meet their needs You're under stress to make sure you have the right blood products in stock; the patient on the table is wanting stuff faster than you can get it. You have to thaw it, prepare it, issue it and get it to the room as fast as possible.

Threats to the 'well being of staff' were also recorded in $27.5 \%$ of the total set.

Some respondents expressed concerns about the physical safety of staff:

Personally because it's about violence in the workplace and I was afraid that somebody was going to get killed or very seriously injured. And staff was telling me that they feel like they are a punching bag and that they feel like they're not very safe at work

Other respondents talked about the health risks to subordinates and colleagues from overwork arising directly as a result of the event under discussion. In many cases, staff overload was caused when covering for the respondents: 
I feel guilty then because people are having to cover for me at work because I am not there. All sorts of issues because a lot of my work is crisis driven anyway and if a crisis occurs when I am not there somebody else has to deal with it.

The last item registering substantive numbers that refers to the well-being of others highlights respondents' concerns for family members. Reported in $15 \%$ of role overload episodes, typically this threat involved some form of family crisis or illness. For example:

The work issues were huge to begin with, and then throw the family issues on that, that was the cherry on the sundae. In my job, no one is going to die, so the family part was the major issue.

\subsubsection{Well-being of the organisation}

The final broad category of answers to the primary appraisal question 'What's at stake?', featuring worries about organisational well-being, contains only one sub item recording substantive numbers. Threats to the organisations' 'ability to provide patient care' were mentioned in one out of every four role overload episodes. These concerns reflect the potential impact of tight deadlines, the amount to do, and resource shortages on the primary function of the organisation. A typical comment came in an episode featuring a high profile corporate task:

If these computer systems didn't get going, if we didn't reach our target, then we were going to potentially compromise the ability of the cancer centre to provide care. There were very tight timelines, so that was difficult.

Other concerns under the umbrella of the well-being of the organisation involved financial impacts to the hospitals from missed deadlines and inefficient resource scheduling, as well as negative affects to the reputation of the hospitals because of poor 
service delivery. More serious concerns involved potential threats of litigation.

Fortuitously, such concerns, illustrated in the comment below, recorded low numbers:

.... it could have been harmful to the organization in that the client's family could have potentially been litigious.

\subsubsection{What's at stake and episode assessment}

From the data in Table 10-4, the following observations can be made about the relationship between what is at stake during role overload and episode assessment. Threats to the well-being of the organization and to the well-being of others were as likely to be mentioned in episodes evaluated as not-overwhelming as they were in overwhelming episodes. On the other hand, in overwhelming role overload, concerns involving personal well-being were cited in almost all of the episodes recalled (90\%). Such concerns featured in a lower proportion of role overload assessed not to be overwhelming $(70 \%)$.

The results in Table 10-4 show that there are no individual items of concern mentioned in substantive numbers that appear to be associated with role overload assessed as not-overwhelming. In contrast, in overwhelming role overload, respondents were more likely to cite concerns about their own professional credibility ( 8 episodes to 3). Remaining items (personal health and safety, home or non-work life, financial wellbeing, welfare of patients, staff, or family, role failure, and ability of the organization to provide care) were equally likely to feature in either of the two role overload episode types. 


\subsubsection{What's at stake and respondent stress}

The data presented in Table 10-5 shows what was at stake in role overload, this time for episodes by respondent stress level. Looking at the respondent stress level results for the three parent themes, there are three findings of note. First, concerns about personal well-being were equally cited in episodes recalled by respondents with higher and lower levels of stress. Second, the well-being of others tends to be mentioned more often by low stress respondents (14 episodes to 11), as does the well-being of the organization (8 episodes to 4$)$.

\begin{tabular}{|c|c|c|c|c|c|c|}
\hline \multirow[b]{4}{*}{ What's at Stake? } & \multicolumn{4}{|c|}{$\begin{array}{c}\text { Role Overload Episodes by Respondent } \\
\text { Stress Level }\end{array}$} & \multirow[b]{2}{*}{$\begin{array}{r}\text { Total } \\
(\mathrm{n}=\mathbf{4 0})\end{array}$} & \\
\hline & \multicolumn{2}{|c|}{ Low $(n=20)$} & \multicolumn{2}{|c|}{ High $(n=20)$} & & \\
\hline & \multicolumn{5}{|c|}{$\#$ and $\%$ of episodes with identified property } & \\
\hline & \# & $\%$ & $\#$ & $\%$ & \# & $\%$ \\
\hline Personal Well-being & 15 & 60 & 17 & 80 & 32 & 80 \\
\hline Role failure & 6 & 30 & 8 & 40 & 14 & 35 \\
\hline Personal health \& safety & 4 & 20 & 9 & 45 & 13 & 32.5 \\
\hline My professional credibility & 6 & 30 & 5 & 25 & 11 & 27.5 \\
\hline Home or non-work life & 5 & 25 & 4 & 20 & 9 & 22.5 \\
\hline Personal values & 5 & 25 & 4 & 20 & 9 & 22.5 \\
\hline Financial well-being & 2 & 10 & 4 & 20 & 6 & 15 \\
\hline Other personal goals & 1 & 5 & 1 & 5 & 2 & 5 \\
\hline The Well-being of Others & 14 & 70 & 11 & 55 & 25 & 62.5 \\
\hline Patient well-being & 7 & 35 & 4 & 20 & 11 & 27.5 \\
\hline Staff well-being & 4 & 20 & 7 & 35 & 11 & 27.5 \\
\hline Well-being of family member(s) & 5 & 25 & 1 & 5 & 6 & 15 \\
\hline Concern for others & 1 & 5 & 2 & 10 & 3 & 7.5 \\
\hline The Well-being of the Organisation & 8 & 40 & 4 & 20 & 12 & 30 \\
\hline Ability to provide care & 3 & 15 & 1 & 5 & 4 & 10 \\
\hline Legal Liability & 2 & 10 & 1 & 5 & 3 & 7.5 \\
\hline Financial Affairs & 2 & 10 & 1 & 5 & 3 & 7.5 \\
\hline Reputation & 1 & 5 & 1 & 5 & 2 & 5 \\
\hline
\end{tabular}

Table 10-5 What's at stake in role overload by respondent stress level 
When the individual child items in Table 10-5 are examined, the results show that there is little or no difference between the results for high and low stress level respondents for the following items cited as being at risk in role overload: role failure, professional credibility, home or non-work life, financial well-being, personal goals, and ability to provide care. In contrast, high stress hospital workers were more likely to express concerns about personal health and safety (9 episodes to 4), or about staff welfare (7 episodes to 4), in their appraisals of role overload. The results in Table 10-5 also show that low stress hospital employees were more likely to talk about the 'well-being of patients' (7 episodes to 4) or the 'well-being of family members' (5 episodes to 1 ).

\subsubsection{What's at stake by respondent stress level and episode assessment}

Table 10-6 presents episode assessment data for each respondent stress level, where results are of note. The following observations can be made about the relationship between the parent themes categorising what was at stake in role overload and respondent stress and episode assessment. First, high stress workers cited concerns about some aspect of personal well-being in all of their overwhelming episodes. Second, the tendency for low stress respondents to talk of the well-being of others is more notable for role overload viewed as not-overwhelming. The fact that the welfare of others was an issue in $90 \%$ of the episodes assessed by low stress respondents as not-overwhelming suggests that such concerns on their own would be less likely to be associated with overwhelming role overload or high levels of stress. 


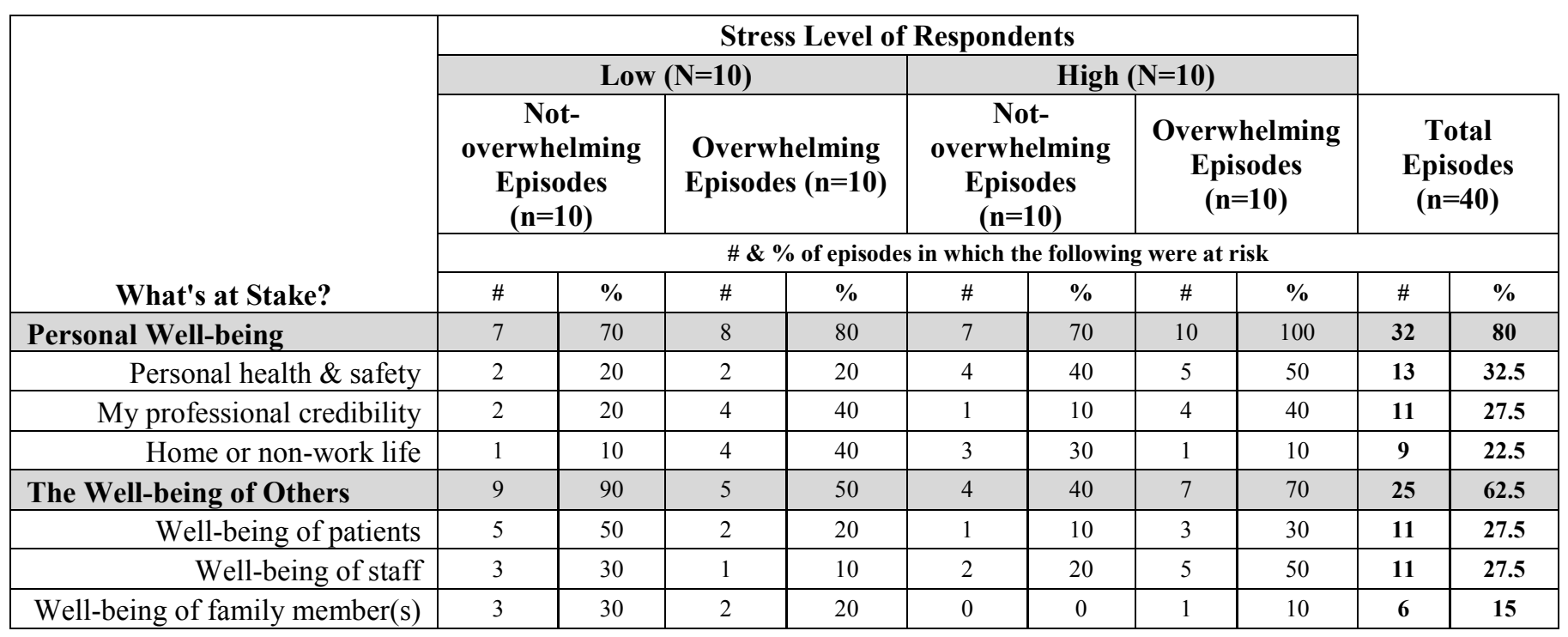

Table 10-6: What's at stake in role overload by respondent stress level and episode assessment

Six further observations are noted from the results in Table 10-6. First, the tendency for personal health and safety to be cited more often as a concern by high stress rather than low stress workers, is more notable for overwhelming episodes ( 5 episodes to 2). Second, although threats to 'professional credibility' and 'home or non-work life' were not strongly associated with respondent stress level, when the episode assessment results are examined as well, other data patterns emerge. In cases where 'professional credibility' was at risk during role overload described by high stress workers, these issues were disproportionally cited in the episodes assessed as overwhelming. Third, the tendency for workers' home or non work lives to be affected in overwhelming role overload (see Section 10.2.5) is more notable for those episodes recalled by low stress employees (4 episodes to 1). Fourth, of the seven episodes in which low stress respondents expressed concern for the 'well-being of patients', five were subsequently assessed as not-overwhelming, suggesting that patient welfare does not normally 
overwhelm hospital employees. Nor was it associated with high levels of perceived stress. Fifth, of the seven episodes in which high stress workers mention risks to staff welfare, five of them were assessed as overwhelming. Concerns for staff welfare expressed by low stress respondents were, however, cited in equivalent numbers of overwhelming and not-overwhelming episodes. This data pattern suggests that staff welfare was associated with overwhelming role overload only for high stress respondents. For the last individual item in Table 10-6, the data suggests that family well-being was almost exclusively cited as a concern by low stress workers and never in the episodes subsequently categorized by high stress respondents as not-overwhelming.

\subsection{Secondary appraisal - what can I do about it?}

In the cognitive appraisal of a potentially stressful event such as role overload, the question posed by secondary appraisal, 'What, if anything, can be done about it?', represents "a judgment of what might or can be done" (Lazarus and Folkman, 1984, p.53). In other words, when faced with excessive role demands, the hospital workers evaluate the possible strategies to overcome the role overload and the availability of coping resources. At the same time, the hospital employees also consider what might or can limit their coping capabilities and what might or can prevent them from trying to resolve the role overload situation, and ultimately how effective or successful the coping strategies will be. The interview questions intended to find the answers to these questions are detailed in Table 7-1 in the methodology chapter. 
This section of the role overload appraisal results chapter will first focus on whether respondents were able to identify coping options and what, if any, coping limitations they noted. Secondly, this section will examine how respondents felt about the potential for coping success. It should be noted that this section does not identify the coping strategies themselves, which are discussed in Chapter 11.

\subsubsection{Coping option appraisal}

The first set of secondary appraisal results, focusing on the evaluation of coping options for role overload, are displayed in Table 10-7. This table displays data for the whole sample, and also displays results according to role overload assessment (i.e., notoverwhelming and overwhelming).

Three coping option appraisal themes were identified from interviewee responses. The first response category, 'restrictions in ability to cope' (cited in over four fifths of episodes), collects together any mention of resource shortages or limitations to coping capabilities emerging from the data. In the second theme, 'coping options' (mentioned in over a half of the episodes), respondents confirmed that coping strategies and resources were identified to help deal with the role overload. The third theme represents the small number of role overload episodes in which the workers claimed not to speculate as to how they would approach the problem (cited in $5 \%$ of episodes). 


\begin{tabular}{|c|c|c|c|c|c|c|}
\hline \multirow{4}{*}{$\begin{array}{c}\text { Secondary Appraisal } \\
\text { Coping Option Appraisal }\end{array}$} & \multicolumn{6}{|c|}{ Role Overload Episode Assessment } \\
\hline & \multicolumn{2}{|c|}{$\begin{array}{c}\text { Not- } \\
\text { overwhelming } \\
(\mathbf{n}=\mathbf{2 0}) \\
\end{array}$} & \multicolumn{2}{|c|}{$\begin{array}{l}\text { Overwhelming } \\
(\mathbf{n}=\mathbf{2 0})\end{array}$} & \multirow[t]{2}{*}{ Total } & \multirow{2}{*}{$\begin{array}{l}(\mathrm{n}=40) \\
\text { ssment }\end{array}$} \\
\hline & \multicolumn{4}{|c|}{ \# and \% of episodes mentioning this coping assessment } & & \\
\hline & $\#$ & $\%$ & $\#$ & $\%$ & \# & $\%$ \\
\hline Restrictions in ability to cope & 18 & 95 & 17 & 85 & 35 & 87.5 \\
\hline Lack of personal resources & 10 & 50 & 9 & 45 & 19 & 47.5 \\
\hline Not sure how to cope & 7 & 35 & 9 & 45 & 16 & 40 \\
\hline Lack of help & 7 & 35 & 8 & 40 & 15 & 37.5 \\
\hline Environmental constraints & 5 & 25 & 8 & 40 & 13 & 32.5 \\
\hline Too much to do & 5 & 25 & 4 & 20 & 9 & 22.5 \\
\hline Coping Options & 16 & 80 & 6 & 30 & 22 & 55 \\
\hline Identified coping strategy & 16 & 80 & 6 & 30 & 22 & 55 \\
\hline Identified Resources & 2 & 10 & 2 & 10 & 4 & 10 \\
\hline Didn't Speculate & 1 & 5 & 1 & 5 & 2 & 5 \\
\hline
\end{tabular}

Table 10-7 Appraisal of coping options for role overload by episode assessment

Multiple coping option appraisal responses could feature in a single role overload episode. In the following comment, for example, the hospital worker identified three possible coping strategies. Nevertheless, she faced restrictions because ultimately overtime payouts must be avoided:

My options are: don't do the group, which isn't very acceptable, do the group and take a half day, or do the group twice and take a full day, which I suppose in the end I might have to do. The idea in the end is to avoid overtime payouts to avoid budget costs.

This comment also illustrates the complexity of the secondary appraisal process as described in the opening paragraph of this section.

The numbers recorded for the three coping option appraisal themes suggest that coping was a preoccupation for most of the respondents faced with excessive role demands. The results confirm that in the vast majority of role overload episodes, respondents spent time speculating about coping options. Having spent time 
contemplating options, in nearly half of the episodes $(45 \%)$ respondents were unable to identify specific options or resources. Moreover, the fact that one or more coping restrictions were identified in so many episodes also means that only in a modest number of situations ( 5 episodes, 5 different people) were respondents unrestricted in their efforts to overcome role overload. In order to further explore what types of coping problems the respondents faced, the detailed child items of the parent themes, "restrictions in ability to cope' and 'coping options', are examined in separate subsections to follow. A third subsection examines the relationship between coping option appraisal and role overload episode assessment. The relationship between coping option appraisal and respondent stress is explored separately in the fourth subsection, whilst the fifth subsection examines coping option appraisal for role overload assessment for each of the respondent stress level groups.

\subsubsection{Restrictions in ability to cope}

Respondents identified five different things they felt restricted their ability to cope with role overload. In the first coping limitation (cited in $47.5 \%$ of the episodes), respondents talked specifically of a lack of personal resources. For many, these resources centred on the lack of time and or energy, and were often associated with commitments to other responsibilities. For example, hospital workers commented:

I'll never be able to do that I am always tired anyway so how am I going to cope with that.

I don't have twenty minutes to track down the supervisor to ask if I could stay and at the same time as trying to call people to come in. 
For others, being physically or mentally below par meant respondents lacked the capacity to tackle role overload. Occasionally this incapacitation (such as anxiety, stress or burnout) was also implicated as a symptom of the event in question, contributing to a vicious circle of role overload:

I could feel my body wanting more and more the holidays I wasn't going to get, and I could feel my brain getting tired but still wanting to work at 100 percent. I was physically tired. I felt a burnout. Physically I'd come home and I was drained, and I'd sleep all the time.

The second coping restriction (cited in $40 \%$ of the episodes) was given the label 'not sure how to cope' based on the often exasperated comments from the hospital employees about their inability to deal with the task causing the role overload:

Well, I was really not sure how I was going to get the work done.

Some respondents explained that they did not know how or where to begin:

It was a complicated thing and I didn't really know how to start. ...whilst others were limited by their personal inability to manage the situation:

There was no resolution; it was out of my domain.

The third coping limitation identified by hospital workers (in $37.5 \%$ of the episodes) was labeled as a 'lack of help'. Such restrictions were referenced mostly in role overload set in the work domain and often involved staff shortages caused by ill-health, vacations, or unfilled vacancies following staff resignations:

My manager was on holidays, there were supposed to be three of us supposed to be covering and we found out that one colleague was now on sick leave for a month

The descriptor 'lack of help' was also coded where respondents complained specifically that there was limited or no help available to them when facing role overload: 
I don't have a lot of resources that I can kind of delegate work to that would help make the whole task more manageable

...or simply because the staff complement was insufficient to deal with the workload:

Not enough resources, too many patients and not enough doctors.

In the few instances in which 'lack of help' arose in role overload in the home, this restriction centred around individuals who had to rely on their own resources to fulfill excessive role demands:

I don't really have family here. I am single and my brothers and sisters live in

\section{Montreal}

Coping restrictions labelled as 'environmental constraints' were cited in a third of role overload episodes. These constraints refer to specific functional characteristics of the role overload environment that limit the use of certain coping strategies or the amount of resources available. For these workers they included hospital rules limiting overtime and unpaid leave, lack of support from colleagues or senior management, and poor management skills:

I was trying to resolve it, but one of the conclusions that I had in the background was that I would never be able to resolve it because I didn't have the support nor the understanding from the upper levels.

Limitations in this case would be they wanted me to fill the cameras, but when something like this happens they don't want me to work overtime, or they want us to request it.

The last role overload coping restrictions was labeled 'too much to do'. Featured in nearly one quarter of the 40 episodes recalled, this restriction suggests an element of 
judgment regarding an inability to fulfill the task(s) because of excessive demands described using phrases such as:-

"too much to do" ; "too many'; "too much to deal with” or "too much on my plate".

Citing 'too much to do' also hints at the potential for coping failure. These concepts are better illustrated by the following more lengthy quote:-

There were far too many expectations to deal with all at the same time. There is no way anyone could deal with them.

This finding also suggests that this idea is recurring in the role overload appraisal process.

\subsubsection{Coping options identified}

The second theme shown in Table 10-7 gathers together two different types of identified coping options described by respondents during the initial appraisal of role overload. The first references discussions of specific planned strategies; the second, any mention of the availability of coping resources.

Respondents confirmed that during initial appraisals they were able to identify coping strategies in just over half ( $55 \%$ ) of the episodes. The hospital employees either specifically stated what they intended to do or they described making mental preparations to overcome the overload or for plans to deal with anticipated problems. The following comments illustrate these responses:

(My initial thoughts were)...to take a step back, first of all, and take a breather, then ... to prioritize the things being bombarded at me. What is the most important, how can I be most efficient. 
I've done this workshop before so I don't know if it is initial thought because you kind of put yourself in a mindset okay this is what I am doing, this is what I need to accomplish so I kind of go through it in my head, kind of psych myself up I guess. I try to anticipate problems and things that might come up that are going to interfere with this being successful and then try to prepare myself for potential solutions should those things arise.

References to coping resources were cited in only $10 \%$ of the total set. Most of the resources noted for use in tackling role overload, took the form of personal skills or knowledge necessary to complete the role demands received by these role incumbents. For example, one worker commented that:

In terms of knowledge base, (I was) very well prepared.

\subsubsection{Coping option appraisal and episode assessment}

Examination of the data in Table 10-7 show that coping restrictions were equally likely to be mentioned in role overload assessed as not-overwhelming as they were in overwhelming episodes (18 versus 17 episodes). Those episodes in which respondents identified coping strategies and or resources were, however, more likely to be subsequently assessed as not-overwhelming (16 episodes to 6).

Examining the individual items cited in substantive numbers, we note that coping strategies were more likely to be identified during appraisals of not-overwhelming role overload (16 episodes to 6). This finding suggests that identifying coping strategies up front is strongly linked to the role overload episode being evaluated as notoverwhelming. 
In contrast to the characteristics of not-overwhelming role overload, environmental constraints were more likely to be a feature of overwhelming episodes of role overload ( 8 episodes to 5). The remaining four coping restrictions (lack of personal resources, lack of help, unsure how to cope, and too much to do) were equally likely to be referenced in both role overload episode assessment types, as was the identification of coping resources.

\subsubsection{Coping option appraisal and respondent stress}

Table 10-8 displays coping restrictions and coping options identified according to the stress level of the hospital employees. The data indicate that high stress employees were just as likely to mention 'restrictions in ability to cope' as were the low stress workers. Similarly, stress level was not associated with the frequency with which respondents identified coping options.

Further examination of the data in Table 10-8 suggest that stress level was not associated with the presence of the following coping restrictions in role overload episodes: lack of personal resources, lack of help, and environmental constraints. The data also show, however, that the high stress group had difficulty deciding how to cope in twice as many episodes as the low stress group (11 versus 5 episodes) whilst the low stress group were much more likely to talk of 'too much to do' (7 episodes to 2). 


\begin{tabular}{|c|c|c|c|c|c|c|}
\hline \multirow{4}{*}{$\begin{array}{l}\text { Secondary Appraisal - } \\
\text { Coping Option Appraisal }\end{array}$} & \multicolumn{4}{|c|}{$\begin{array}{c}\text { Role Overload Episodes by Respondent Stress } \\
\text { Level }\end{array}$} & \multirow[b]{2}{*}{ Total } & \multirow[b]{2}{*}{$(n=40)$} \\
\hline & Low & $(n=20)$ & High & $(n=20)$ & & \\
\hline & \multicolumn{6}{|c|}{$\#$ and $\%$ of episodes mentioning this coping assessment } \\
\hline & \# & $\%$ & \# & $\%$ & \# & $\%$ \\
\hline Restrictions in ability to cope & 17 & 85 & 18 & 90 & 35 & 87.5 \\
\hline Lack of personal resources & 9 & 45 & 10 & 50 & 19 & 47.5 \\
\hline Unsure how to cope & 5 & 25 & 11 & 55 & 16 & 40 \\
\hline Lack of help & 7 & 35 & 8 & 40 & 15 & 37.5 \\
\hline Environmental constraints & 6 & 30 & 7 & 35 & 13 & 32.5 \\
\hline Too much to do & 7 & 35 & 2 & 10 & 9 & 22.5 \\
\hline Coping options identified & 11 & 60 & 11 & 55 & 22 & 55 \\
\hline Identified coping strategy & 11 & 55 & 11 & 50 & 22 & 55 \\
\hline Identified Resources & 2 & 15 & 2 & 15 & 4 & 15 \\
\hline Didn't speculate & 2 & 10 & 0 & 0 & 2 & 5 \\
\hline
\end{tabular}

Table 10-8 Appraisal of coping options for role overload by respondent stress level

The two child items within the coping options identified theme (i.e., coping strategies and coping resources) follow the distribution at the parent level and are identified equally often by both respondent groups.

\subsubsection{Coping option appraisal by respondent stress and episode assessment}

Table 10-9 displays coping option appraisal results by stress level and episode assessment only for those items that show notable differences. Although environmental constraints are equally cited by low and high stress respondents, this particular coping restriction was more prevalent in the overwhelming role overload episodes of the respondents with higher levels of stress. Identified in $60 \%$ of their overwhelming episodes, environmental constraints were cited in only $10 \%$ of the episodes categorised as not-overwhelming by this group and also in only $20 \%$ of the overwhelming role overload 
episodes of low stress hospital workers. Environmental constraints appeared to impact role overload appraisal in the overwhelming episodes of high stress employees to a greater extent.

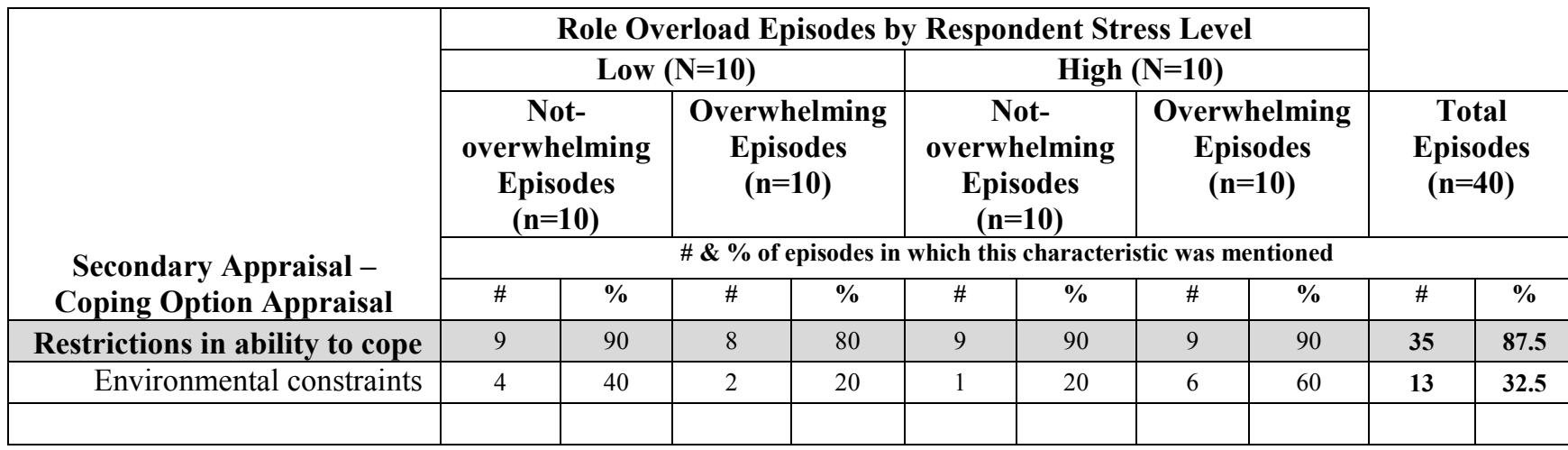

Table 10-9 Appraisal of coping options for role overload by respondent stress level and episode assessment

\subsubsection{Coping prospects appraisal}

The second set of secondary appraisal data is presented in Table 10-10 (for the total set of episodes and by episode assessment), and Table 10-11 (by respondent stress level and episode assessment). Respondents gave three answers to the interview Question 7a , 'To what extent did you believe the situation could be resolved successfully?'.

\begin{tabular}{|c|c|c|c|c|c|c|}
\hline \multirow{4}{*}{$\begin{array}{c}\text { Secondary Appraisal - } \\
\text { Coping prospects appraisal }\end{array}$} & \multicolumn{6}{|c|}{ Role Overload Episode Assessment } \\
\hline & \multicolumn{2}{|c|}{$\begin{array}{c}\text { Not- } \\
\text { overwhelming } \\
(\mathbf{n}=\mathbf{2 0}) \\
\end{array}$} & \multicolumn{2}{|c|}{$\begin{array}{l}\text { Overwhelming } \\
(\mathbf{n}=\mathbf{2 0})\end{array}$} & \multirow[t]{2}{*}{ Total } & \multirow{2}{*}{$(n=40)$} \\
\hline & \multicolumn{4}{|c|}{$\#$ and $\%$ of episodes mentioning this coping assessment } & & \\
\hline & $\#$ & $\%$ & $\#$ & $\%$ & $\#$ & $\%$ \\
\hline Confident of Success & 10 & 50 & 6 & 30 & 16 & 40 \\
\hline Hopeful for success & 8 & 40 & 5 & 25 & 13 & 32.5 \\
\hline Not confident of success & 2 & 10 & 8 & 40 & 10 & 25 \\
\hline
\end{tabular}

Table 10-10 Appraisal of prospects for role overload coping success by episode assessment 
Hospital employees expressed confidence that the role overload could be overcome successfully in $40 \%$ of role overload episodes. In $32.5 \%$ of the episodes, workers were hopeful that the situation could be resolved, but had some reservations about the outcome, while in a quarter of the sample episodes, respondents were not at all confident that they could overcome role overload following their initial appraisal. In other words, for just over half of the episodes discussed the respondents had some degree of reservation that coping restrictions identified would impact the chances of coping success.

\subsubsection{Coping prospects and episode assessment}

The results in Table 10-10 illustrate that in role overload episodes subsequently assessed as not-overwhelming, hospital employees were more likely to believe that they could cope (10 episodes to 6) and more likely to be hopeful for success ( 8 episodes to 5). In overwhelming episodes respondents were more likely to declare that they were not at all confident of resolving the role overload situation ( 8 episodes versus 2 ). These results are not surprising, but nevertheless give further support to the assessment of role overload experiences made by the respondents at the end of their recollections.

\subsubsection{Coping prospects and respondent stress}

From the results in Table 10-11 three observations can be made about the relationship between the appraisal of coping prospects and respondent stress. First, low 
and high stress hospital employees were equally likely to be confident about the prospects for coping success. High stress respondents were more likely to say that they were hopeful for success ( 8 versus 5 episodes). Lastly, low stress and high stress respondents were equally likely to admit that they were not at all confident that the role overload could be resolved.

\subsubsection{Coping prospects by episode assessment and respondent stress}

The data in Table 10-11 suggests two further observations.

\begin{tabular}{|c|c|c|c|c|c|c|c|c|c|c|}
\hline \multirow{5}{*}{$\begin{array}{c}\text { Secondary Appraisal - } \\
\text { Coping Prospects } \\
\text { Appraisal } \\
\end{array}$} & \multicolumn{8}{|c|}{ Role Overload Episodes by Respondent Stress Level } & & \\
\hline & \multicolumn{4}{|c|}{ Low $(N=10)$} & \multicolumn{4}{|c|}{ High $(\mathrm{N}=10)$} & & \\
\hline & \multicolumn{2}{|c|}{$\begin{array}{c}\text { Not- } \\
\text { overwhelming } \\
\text { Episodes } \\
(\mathbf{n}=\mathbf{1 0}) \\
\end{array}$} & \multicolumn{2}{|c|}{$\begin{array}{l}\text { Overwhelming } \\
\text { Episodes } \\
(\mathbf{n}=\mathbf{1 0})\end{array}$} & \multicolumn{2}{|c|}{$\begin{array}{c}\text { Not- } \\
\text { overwhelming } \\
\text { Episodes } \\
(\mathbf{n}=\mathbf{1 0}) \\
\end{array}$} & \multicolumn{2}{|c|}{$\begin{array}{l}\text { Overwhelming } \\
\text { Episodes } \\
(\mathbf{n}=10)\end{array}$} & \multicolumn{2}{|c|}{$\begin{array}{c}\text { Total } \\
\text { Episodes } \\
(\mathbf{n}=40) \\
\end{array}$} \\
\hline & \multicolumn{10}{|c|}{ \# \& \% of episodes in which this characteristic was mentioned } \\
\hline & \# & $\%$ & \# & $\%$ & \# & $\%$ & \# & $\%$ & \# & $\%$ \\
\hline Confident of Success & 5 & 50 & 4 & 40 & 5 & 50 & 2 & 20 & 16 & 40 \\
\hline Hopeful for success & 4 & 20 & 1 & 10 & 4 & 40 & 4 & 40 & 11 & 27.5 \\
\hline Not confident of success & 1 & 10 & 4 & 40 & 1 & 10 & 4 & 40 & 10 & 25 \\
\hline
\end{tabular}

Table 10-11 Appraisal of prospects for role overload coping success by respondent stress level and episode assessment

First, whilst both respondent groups were equally likely be confident about coping success during role overload, high stress respondents were more likely to be confident about coping in the episodes assessed as not-overwhelming (5 episodes to 2). Second, low stress respondents rarely mentioned being hopeful for coping success in episodes they assessed as overwhelming. As a result, these respondents expressed such hope in 
not-overwhelming episodes to the same extent that the high stress group did in role overload assessed as not-overwhelming and in overwhelming role overload.

\subsection{Emotional outcomes from role overload appraisal}

According to Lazarus and Folkman (1984), the primary and secondary appraisal processes examined in the previous two sections are not separate processes, but rather they interact with one other to create the degree of stress and the strength and content of the initial emotional reaction to the potential stressor, in this case a role overload event. Descriptions of how hospital workers appraised role overload (i.e., as harmful, threatening, or challenging) were examined first in this chapter. This section will examine the emotional reactions recalled by hospital employees during their recollections of role overload appraisal. The data draws mainly from responses to the question: 'What was your overriding feelings about the situation?'. These emotions are distinct from the emotional reactions described with regard to the final outcomes of the role overload, which will be examined in the last chapter of the results part of this thesis (i.e., Chapter 12).

The emotions and feelings recalled by the hospital workers when they described how they felt during the initial appraisal of the role overload situation are presented in Table 10-12. 


\begin{tabular}{|c|c|c|c|c|c|c|}
\hline \multirow{4}{*}{$\begin{array}{c}\text { Appraisal Emotions \& } \\
\text { Feelings }\end{array}$} & \multicolumn{6}{|c|}{ Role Overload Episode Assessment } \\
\hline & \multicolumn{2}{|c|}{$\begin{array}{c}\text { Not- } \\
\text { overwhelming } \\
(\mathbf{n}=\mathbf{2 0})\end{array}$} & \multicolumn{2}{|c|}{$\begin{array}{c}\text { Overwhelming } \\
(\mathbf{n}=\mathbf{2 0})\end{array}$} & Tot & \multirow[t]{2}{*}{$(n=40)$} \\
\hline & \multicolumn{5}{|c|}{$\#$ and $\%$ of episodes with emotions or feelings } & \\
\hline & \# & $\%$ & \# & $\%$ & \# & $\%$ \\
\hline Negative Emotions \& Feelings & 19 & 95 & 20 & 100 & 39 & 97.5 \\
\hline Overwhelmed \& Stressed & 11 & 55 & 9 & 45 & 20 & 50 \\
\hline Anxious \& Worried & 9 & 45 & 8 & 40 & 17 & 42.5 \\
\hline Anger \& Irritation & 7 & 35 & 9 & 45 & 16 & 40 \\
\hline Frustration & 7 & 35 & 8 & 40 & 15 & 37.5 \\
\hline Guilt & 4 & 20 & 3 & 15 & 7 & 17.5 \\
\hline Fear & 1 & 5 & 3 & 15 & 4 & 10 \\
\hline Other & 2 & 10 & 4 & 20 & 6 & 15 \\
\hline Positive Emotions \& Feelings & 5 & 25 & 3 & 15 & 8 & 20 \\
\hline Excited & 3 & 15 & 2 & 10 & 5 & 12.5 \\
\hline Other Positives & 3 & 15 & 1 & 5 & 4 & 10 \\
\hline
\end{tabular}

Table 10-12 Emotions and feelings following role overload appraisal by episode assessment

In order to categorise the emotions and feelings specified, the researcher turned to the literature for guidance. Russell's (1980) circumplex model of affect proposed that the underlying structure of affective experience can be represented by affective states arranged on the circumference of a circle in a two dimensional space. The two dimensions of his model describe emotions by valence (positive and negative) and by degree of arousal (high and low). All the emotions and feelings named by workers in substantive numbers at this point in their recollection are consistent with high arousal emotions as presented by Russell (1980). Therefore in Table 10-12 they are categorised by their positive and negative qualities. Negative emotions and feelings featured in all but one episode during role overload appraisal. In sharp contrast, respondents talked about positive emotions and feelings in only one fifth of the total set. The analysis also revealed 
that it is not unusual for multiple emotions and feelings to feature commonly in each episode.

The many negative emotions and feelings expressed by respondents in the role overload episodes were categorised into seven different types and named specifically for the emotions cited. The only exception to this convention was the last type, which was given the label, 'other' and gathered together a number of different emotions or feelings, none of which were cited in more than 3 episodes each. The most commonly cited of the negative emotions and feelings identified (mentioned in half of the episodes), was labelled 'overwhelmed and stressed'. Feelings of 'anxiety and/or worry', 'anger', and 'frustration' were all mentioned slightly less often, but with similar frequency (in approximately $40 \%$ of episodes). For example:

I also felt overwhelmed because it is a huge collective agreement and I knew the impact was huge for the people I was representing there.

Well then we are back in the stressful situation of just trying to... always feeling anxiety, irritation, fluster whatever and worrying about my colleagues.....

I would say "la colere". (In English it is closest to "anger" I guess). I was irate. I was mad. The word in French represents more of an image.

Lots of frustrations after the fact, people assumed I could just re-assign staff, but I couldn't.

The last two negative emotions and feelings were mentioned in considerably fewer episodes: guilt and fear were cited in only $15 \%$ and $10 \%$ of the total set of 40 , respectively. For example:

I don't have a back up as well as the other secretaries you can' $t$ take the time off that you want to take off so then you have family life and you can't take the time off so then you feel guilty. 
Overwhelmed; I felt alone, I felt I was the only person with this mission or with this fear that's bringing it up.

Finally, the most commonly cited positive emotion was excitement, mentioned in only $12.5 \%$ of the role overload episodes:

I was feeling excited because there was things actually that I liked doing that I wanted to do.

Other positive emotions of various descriptions were cited in $10 \%$ of the episodes.

In the remainder of this section, Section 10.4.1 examines the relationship between appraisal emotional outcomes and episode assessment, whilst Section 10.4.2 explores the appraisal emotional outcomes by respondent stress. Finally Section 10.4.3 examines the appraisal emotional outcomes by respondent stress and episode assessment.

\subsubsection{Appraisal emotional outcomes and episode assessment}

Somewhat surprisingly, the assessment of the role overload episodes was not related to how often employees felt 'overwhelming and/or stressed'. This result suggests that when employees are in the middle of role overload it can be overwhelming, but when the situation is over emotions may change in line with episode assessment. All other emotions and feelings presented in Table 10-12 were also equally likely to feature in discussions of both types of episodes. 


\subsubsection{Appraisal emotional outcomes and respondent stress}

Table 10-13 presents the same emotions and feelings expressed during the recollections of role overload by female hospital workers, this time according to the level of respondent perceived stress.

Respondents with higher stress were more likely to mention feeling 'overwhelmed and/or stressed' during role overload than were low stress workers (13 episodes to 7). Similarly, high stress respondents were more likely to mention feelings of anxiety or worry (13 episodes versus 4$)$ and guilt (5 versus 2 episodes). The following comments by high stress respondents illustrate the strength and effects of some of these emotional reactions.

Worry, I worried and to a point it was almost like panic at times. Also, at times, it was kind of like a stupor...I was just paralyzed.

I get worried. I get worried about I mean stress is really the overriding factor. I can end up in tears just because I feel so overwhelmed with it all.

Although only featuring in $20 \%$ of the total episodes for this group, high stress respondents were also the only hospital workers to mention fear when describing role overload. In a situation involving staff and client safety, one respondent expressed the loneliness of fear:

No one is listening no one is doing enough.... I felt alone, I felt I was the only person with this mission or with this fear that's bringing it up.

Finally, with regard to positive emotions, high stress hospital workers were surprisingly also more likely to cite excitement than were the low stress group (4 episodes to 1 ). 
Frustration was the only emotion and feeling that was cited more often by hospital workers with lower levels of stress when respondents were describing how they felt during role overload (9 episodes to 6). All other emotions and feelings (anger \& irritation, guilt, other negative, and other positive emotions and feelings) were equally likely to be cited by both stress level groups

\begin{tabular}{|c|c|c|c|c|c|c|}
\hline \multirow{3}{*}{$\begin{array}{c}\text { Appraisal emotions and } \\
\text { feelings }\end{array}$} & \multicolumn{6}{|c|}{ Role Overload Episodes by Respondent Stress Level } \\
\hline & \multicolumn{2}{|c|}{$\begin{array}{c}\text { Low } \\
(n=20)\end{array}$} & \multicolumn{2}{|c|}{$\begin{array}{c}\text { High } \\
(n=20)\end{array}$} & \multicolumn{2}{|c|}{$\begin{array}{c}\text { Total } \\
(n=40)\end{array}$} \\
\hline & \multicolumn{6}{|c|}{$\# \& \%$ of workers who described this emotion or feeling } \\
\hline Negative Emotions \& Feelings & 19 & 95 & 20 & 100 & 39 & 97.5 \\
\hline Overwhelmed \& Stressed & 7 & 35 & 13 & 65 & 20 & 50 \\
\hline Anxious \& Worried & 4 & 20 & 13 & 60 & 17 & 42.5 \\
\hline Anger \& Irritation & 8 & 40 & 8 & 40 & 16 & 40 \\
\hline Frustration & 9 & 45 & 6 & 30 & 15 & 37.5 \\
\hline Guilty & 2 & 10 & 5 & 25 & 7 & 17.5 \\
\hline Fear & 0 & 0 & 4 & 20 & 4 & 10 \\
\hline Other & 4 & 20 & 2 & 10 & 6 & 15 \\
\hline Positive Emotions \& Feelings & 3 & 15 & 5 & 25 & 8 & 20 \\
\hline Excited & 1 & 5 & 4 & 20 & 5 & 12.5 \\
\hline Other Positives & 2 & 10 & 2 & 10 & 4 & 10 \\
\hline
\end{tabular}

Table 10-13 Emotions and feelings following role overload appraisal by respondent stress

\subsubsection{Appraisal emotional outcomes by respondent stress and episode assessment}

Table 10-14 displays the emotions and feeling expressed by hospital workers during appraisal according to respondent stress and episode assessment, for those row items that show notable differences. These results show that whilst overall positive emotions following appraisal were as likely to be mentioned by both groups of respondents, workers with high levels of stress were more likely to talk positively in 
episodes subsequently assessed as not-overwhelming. Most of those positive emotions referenced excitement.

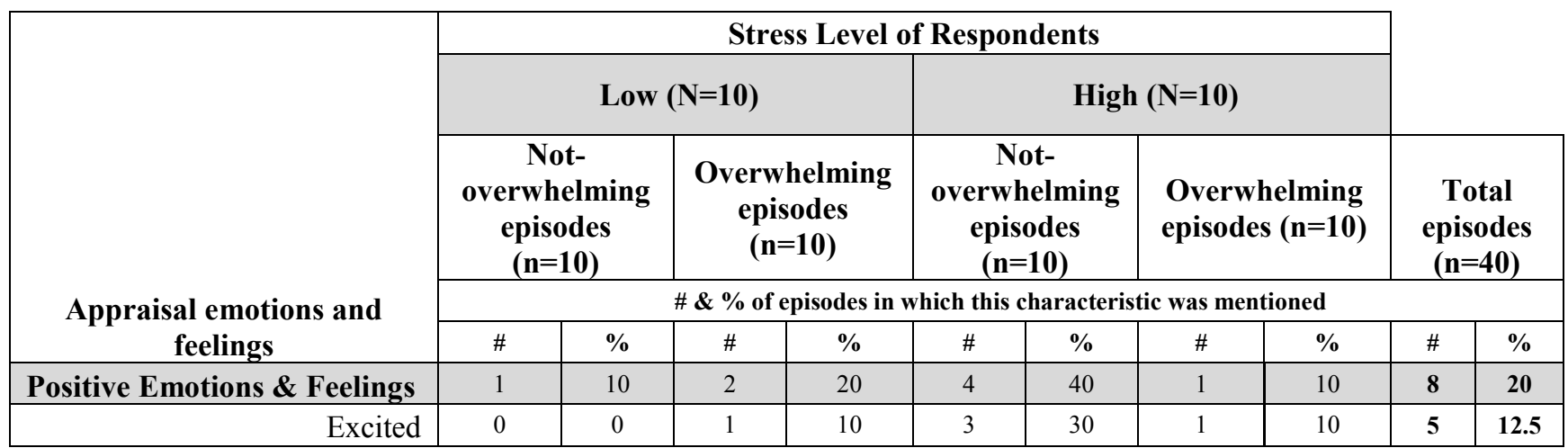

Table 10-14 Emotions and feelings following role overload appraisal by respondent stress level and episode assessment

\subsection{Summary of role overload appraisal key findings}

The focus of this chapter has been on role overload appraisal, the two part process of primary and secondary appraisal by which overloaded hospital workers evaluate the significance of role overload with regard to personal well-being. The summary of key findings from this chapter is presented in four short subsections. In the first subsection (Section 10.5.1) role overload appraisal factors common to many of the episodes is discussed. The next two subsections discuss key role overload appraisal findings for episode assessment (not-overwhelming and overwhelming) and for respondent stress level (low and high) respectively. The last subsection summarises key findings from this chapter for the four episode groupings: not-overwhelming episodes of low stress workers; not-overwhelming episodes of high stress workers; overwhelming episodes of low stress workers; and overwhelming episodes of high stress workers. 


\subsubsection{Key appraisal findings for all episodes}

The first section in this chapter presented results regarding how the hospital workers evaluated role overload with respect to well-being (i.e., as harmful, threatening, or challenging). Results suggest that appraisals of harm, threat or challenge can occur concurrently in any single episode of role overload. Two thirds of role overload events were described as challenges, and over half as threats to well-being. Very few workers described being in a harmful situation from the outset.

The second section of this chapter explored what these workers perceived to be at stake. Workers responses suggested that role failure, personal health and safety, professional credibility, and the wellbeing of staff and of patients were regular concerns during role overload. The third section examined the workers evaluations of coping approaches and resources. Almost all episodes of role overload described by hospital employees referred to some form of coping restriction, with time and energy constraints being the most common. Results suggested that in many episodes workers also had difficulties identifying initial coping plans, and a similar number suggested that the chance of coping success was limited.

The last section of this chapter examined the emotions and feelings workers recalled when they described evaluating the role overload situations. Workers described a wide range of negative emotions in almost all the episodes recalled (in $90 \%$ of episodes), specifically: overwhelmed and stressed, anger and irritation, anxiety and worry, guilt, and fear. Positive emotions were much less common (cited in $20 \%$ of episodes), with excitement the only one described in substantive numbers. 


\subsubsection{Key appraisal findings by role overload episode assessment}

Table 10-15 presents the role overload appraisal factors most closely linked to the assessment as to whether role overload was overwhelming versus not-overwhelming.

\begin{tabular}{|c|c|c|c|c|c|}
\hline \multirow{2}{*}{$\begin{array}{c}\text { Role } \\
\text { Overload } \\
\text { Episode } \\
\text { Assessment }\end{array}$} & \multicolumn{5}{|c|}{ Role Overload Appraisal } \\
\hline & $\begin{array}{l}\text { Appraisal } \\
\text { Results }\end{array}$ & $\begin{array}{c}\text { What's at } \\
\text { Stake? }\end{array}$ & What Can I Do? & $\begin{array}{c}\text { Secondary } \\
\text { Appraisal } \\
\text { Results } \\
\end{array}$ & $\begin{array}{c}\text { Emotions \& } \\
\text { Feelings }\end{array}$ \\
\hline $\begin{array}{c}\text { Not- } \\
\text { overwhelming }\end{array}$ & Challenge & & $\begin{array}{l}\text { Coping option } \\
\text { identified }\end{array}$ & $\begin{array}{c}\text { Confident } \\
\text { Hopeful } \\
\end{array}$ & \\
\hline Overwhelming & $\begin{array}{c}\text { Threatening } \\
\text { Harmful }\end{array}$ & $\begin{array}{c}\text { Professional } \\
\text { credibility } \\
\text { More items at stake } \\
\text { per episode }\end{array}$ & $\begin{array}{l}\text { Environmental } \\
\text { constraints }\end{array}$ & Not confident & \\
\hline
\end{tabular}

Table 10-15 Key appraisal factors and results for role overload assessed as overwhelming and notoverwhelming without regard to respondent stress level

In episodes assessed as not-overwhelming, respondents were more likely to say they felt challenged. More specifically, challenge appraisals featured in almost every episode assessed as not-overwhelming, suggesting strong links with more positive outcomes despite the lack of positive narrative supporting these responses. In role overload categorized as not-overwhelming, respondents also had few if any difficulties identifying coping strategies and were also more confident and hopeful of a successful outcome.

Appraisals of harm were mentioned only in overwhelming role overload episodes. The results from this chapter also suggest that, more often than not, role overload in which the individual reported being threatened were also subsequently assessed as overwhelming. Professional credibility was specifically noted as a concern for workers in a greater number of overwhelming episodes. Results also suggest that environmental constraints, such as inflexible work scheduling or poor management support, impacted 
role overload appraisal in overwhelming episodes. Finally, workers were more likely to express lack of confidence about the prospects for coping success in episodes evaluated as overwhelming.

\subsubsection{Key appraisal findings for respondents with low and high stress levels}

Table 10-16 summarises the role overload appraisal factors most closely associated with low and high stress respondents. These findings are presented without reference to how the respondents subsequently evaluated the role overload episodes.

\begin{tabular}{|c|c|c|c|c|c|}
\hline \multirow{2}{*}{$\begin{array}{c}\text { Respondent } \\
\begin{array}{c}\text { Stress } \\
\text { Level }\end{array}\end{array}$} & $\begin{array}{c}\text { Appraisal } \\
\text { Results }\end{array}$ & What's at Stake? & $\begin{array}{c}\text { What Can I Do? } \\
\text { Coping Option } \\
\text { Appraisal }\end{array}$ & $\begin{array}{c}\text { What Can I } \\
\text { Do? Coping } \\
\text { Prospects }\end{array}$ & $\begin{array}{c}\text { Emotions \& } \\
\text { Feelings }\end{array}$ \\
\cline { 2 - 6 } Low & Challenge & $\begin{array}{c}\text { Well-being of patients } \\
\text { Well-being of family } \\
\text { Well-being of the organisation }\end{array}$ & Too much to do & & Frustration \\
\hline High & $\begin{array}{c}\text { Personal health \& safety } \\
\text { Well-being of staff }\end{array}$ & Not sure how to cope & $\begin{array}{c}\text { Overwhelmed \& } \\
\text { stressed } \\
\text { Anxious \& worried } \\
\text { Excited } \\
\text { Guilty }\end{array}$ \\
\hline
\end{tabular}

Table 10-16 Key appraisal factors and results for role overload of high and low stress respondents without regard to how the episode was assessed

Low stress hospital workers were more likely to express concerns about the wellbeing of patients, the well-being of their families, and the wellbeing of the organization when assessing what was at stake (primary appraisal) in role overload. Findings also suggest that impacts to non-work areas of life were particularly troublesome for the low stress respondents. When describing role overload appraisal, low stress workers were also more likely to declare that there was 'too much to do', thereby expressing a judgment on the demands of the situation and the resources available, which also implies consideration 
of alternative plans. This is consistent with the finding from this chapter that these respondents were more likely to describe their role overload episodes as challenging.

The role overload appraisal factors that dominate for the higher stress group of hospital workers are quite different to those of the low stress respondents. Personal health and safety and staff well-being were at stake for this group more often, particularly with regard to the chronic daily role overload, which typified their environment. Results suggest that there is a strong relationship between being unsure how to cope and high levels of reported stress. This finding may be related to the results showing that moderate levels of uncertainty regarding coping prospects may also be linked to higher stress levels for some hospital workers. More telling are the feelings expressed by this group during role overload appraisal. Highly stressed respondents reported feeling overwhelmed and stressed, expressed anxiety and worry, and talked of being excited in more episodes than the workers with lower levels of reported stress.

\subsubsection{Key appraisal findings by episode groupings}

Table 10-17 summarises the notable findings from this chapter for the four episode groupings. First, results revealed a particularly strong association between notoverwhelming role overload appraised as challenging and low levels of stress as reported by hospital workers. Low stress respondents notably expressed more concerns for patient welfare, particularly in episodes assessed as not-overwhelming. Concerns about home or non work life were talked about more frequently by these low stress respondents in episodes evaluated as overwhelming. Low stress respondents also rarely talked of being 
hopeful of coping success in the role overload they assessed as overwhelming, a finding that stands out when compared to the other three groupings.

Secondly, although the numbers are small, when detailing their initial reactions to role overload, highly stressed workers described feelings of excitement in more of the episodes subsequently assessed as not-overwhelming. These individuals rarely mentioned being confident of coping success in descriptions of overwhelming role overload, a finding that stands out because it was mentioned with the same frequency by workers in the other three episode groupings. High stress individuals also appear to be particularly bothered about personal health and safety, professional credibility, staff well-being and environmental constraints in overwhelming role overload.

\begin{tabular}{|c|c|}
\hline Not-overwhelming episodes of low stress workers & Not-overwhelming episodes of high stress workers \\
\cline { 2 - 2 } Challenging & Confident of coping success \\
Well-being of patients & Hopeful of coping success \\
Confident of coping success & \\
Hopeful of coping success & \\
\hline Overwhelming episodes of low stress workers & Overwhelming episodes of high stress workers \\
\hline Home and non-work life & $\begin{array}{c}\text { Personal health \& safety } \\
\text { Staff well-being } \\
\text { Confident of coping success }\end{array}$ \\
& Professional credibility \\
& Environmental constraints \\
& Hopeful of coping success \\
\hline
\end{tabular}

Table 10-17 Summary of role overload appraisal factors by episode grouping 


\section{Coping with role overload}

In the theory of psychological stress and coping, developed by Lazarus and his colleagues, there are two important processes that mediate stressful person-environment relationships and their short and long term outcomes (Folkman et al., 1986). The first critical process is cognitive appraisal, the second is coping. Cognitive appraisal of any potential stressor includes the evaluation of coping resources and strategies available as well as their potential for success. Cognitive appraisal of the role overload suffered by the fulltime female hospital employees was the focus of discussion in the previous chapter. Results discussed in Chapter 10 suggested that many of these overloaded hospital employees have problems identifying and implementing effective coping strategies. The objective of this chapter is to examine what the hospital employees actually did (or tried to do) to cope with their role overload. The analysis is guided by the research question: What impact do coping strategies have on the level of stress people experience under role overload conditions?

Respondents typically cited multiple coping strategies in their recollections of role overload episodes. The first section of Chapter 11 therefore examines how many coping strategies workers described using per episode as well as presenting the different strategies used to tackle this specific stressor and its effects. The data results presented in this chapter are drawn predominantly from the multipart question 9 of the interview protocol (see Table 7-1 in Section 7.1.1.2). Although the interview protocol included questions aimed at capturing respondents' assessment of coping effectiveness in dealing 
with role overload, the interviewees were unable to capture such data for each individual coping strategy described. Specific coping effectiveness data are therefore not included in this thesis, although we can speculate by looking at the data for overwhelming and notoverwhelming role overload episodes and for high and low stress respondents. The second section of this chapter presents a summary of the key findings for role overload coping.

\subsection{Coping Strategies for Role Overload Episodes}

Analysis of the coping strategies described by the hospital workers suggests these women use two broad approaches to coping with role overload: 'demand-reduction', strategies that are directed at lessening the volume of role demands contributing to role overload, and 'demand-management', strategies that focus on organising, avoiding, or tolerating the demands contributing to role overload. Both coping approaches include strategies involving other people as well as strategies involving only the individuals experiencing the role overload.

Also of note from the analysis is that each coping action used by our sample of hospital workers is consistent with one or other of the two major coping functions defined by Lazarus and Folkman (1984): problem focused (aimed at managing or changing the problem causing the distress) or emotion focused (aimed at regulating emotional reactions to the role overload). According to Folkman and Lazarus (1988 p. 7), this classification may not always fit the data one hundred percent, since "problem focused coping can sometimes be used to regulate emotions" and vice versa. In this thesis the 
researcher was guided by how respondents answered the question "What did you do to deal with the emotional aspects of the situation?" to clarify the few ambiguities that arose. For example, a small number of respondents commented, in answer to this question, that getting on with the job (i.e., focusing on the problem) was the best way to deal with any arising emotions. These coping strategies were labeled 'endure and comply' and are considered to be emotion focused demand-management strategies.

The full list of coping strategies is presented in Table 11-1. Some of these strategies are subdivided further, when the numbers warranted it, into a more detailed description of how the person coped. The resulting categories thus reflect the parent and child nodes of NVivo (where the data references are stored). The terms 'parent strategies' and 'child strategies' will therefore be used to refer to these two level composite groupings in the remainder of the thesis.

An initial review of the full list of coping strategies that emerged from the coding and analysis process also reveals that respondents used a large number of coping strategies of varying forms. Using a similar procedure to that described in Section 7.2.2, the number of strategies cited in each episode for each respondent were counted and summed for each of the total set of 40 episodes. This analysis allowed us to examine and compare the number of coping strategies used per episode. After counting the number of strategies used in each of the episodes, the overall average rate per episode was found to be 4.78. The usage rate data for all 40 episodes also revealed that respondents cited an average of 3.3 demand-management coping strategies and 1.48 demand-reduction strategies per episode. These results suggest that overall, the hospital workers used 
greater numbers of demand-management than demand-reduction strategies per episode to cope with role overload. Counting the problem focused and emotion focused strategies for each episodes showed that that, when describing role overload, workers also tend to cite a greater number of problem focused coping strategies in each episode than emotion focused strategies. The coping strategy usage rate data by episode assessment and by respondent stress level added little additional unique information and as such, are not discussed in detail in this thesis. The interested reader can get the results from the author.

The remainder of this section is structured as follows. The first part explores the demand-reduction coping strategies described by the hospital workers. This is followed by an examination of the strategies used to manage demands. The third part of this section examines the relationship between demand-reduction and demand-management coping strategies, and episode assessment, whilst the fourth examines how these two strategy approaches were used by the two stress level groups of workers. The fifth subsection then focuses on coping strategy results identified by stress level and episode assessment. The sixth and final subsection examines how many different forms of coping were used in the episodes recalled by the hospital workers.

\subsubsection{Demand-reduction coping}

Four different parent coping strategies aimed at reducing the demands contributing to role overload are identified. Three were problem focused, one emotion focused. 
Use others to reduce demands. The first group of demand-reduction strategies (cited in $77.5 \%$ of all episodes) described by hospital workers, involve coping actions provided by others to help the respondent deal with their overload. The tangible help provided by others helped by decreasing the number of role demands triggering the role overload faced by the respondents.

Three child strategies involving others were identified. 'Help from colleagues, family and/or friends' was the first of these and also the most frequently cited coping strategy (in over a half of all episodes). Such help describes practical or informational help freely given by others, often spontaneously and without being actively sought out:

The secretary did some typing for me that I would normally do myself. Team members worked through their breaks too.

Many respondents were able to delegate demands, and some actively sought help by making specific arrangements to get advice or practical assistance. Used to cope with role overload based only in the work domain, this approach ('delegate or seek help') was the second coping strategy involving others aimed at reducing demands, and was cited in one in every five episodes described.

The following example comments illustrate a common outcome from 'delegating or seeking help' - that respondents were subsequently comfortable about concentrating on remaining role demands: 


\begin{tabular}{|c|c|c|c|c|c|c|}
\hline \multirow[b]{4}{*}{ Role Overload Coping Strategies } & \multicolumn{4}{|c|}{$\begin{array}{c}\text { Role Overload Episode } \\
\text { Assessment }\end{array}$} & \multirow{2}{*}{\multicolumn{2}{|c|}{$\begin{array}{c}\text { Total } \\
\text { Episodes } \\
(n=40)\end{array}$}} \\
\hline & \multicolumn{2}{|c|}{$\begin{array}{c}\text { Not- } \\
\text { overwhelming } \\
\text { Episodes } \\
(\mathbf{n}=\mathbf{2 0}) \\
\end{array}$} & \multicolumn{2}{|c|}{$\begin{array}{c}\text { Overwhelming } \\
\text { Episodes } \\
(\mathbf{n}=\mathbf{2 0})\end{array}$} & & \\
\hline & \multicolumn{6}{|c|}{ \# \& \% of episodes in which the coping action was cited } \\
\hline & $\#$ & $\%$ & $\#$ & $\%$ & \# & $\%$ \\
\hline Demand-reduction coping & 19 & 95 & 16 & 80 & 35 & 87.5 \\
\hline \multicolumn{7}{|l|}{ Problem focused: } \\
\hline Use others to reduce demands & 17 & 85 & 14 & 70 & 31 & 77.5 \\
\hline Help from colleagues, family or friends & 13 & 65 & 10 & 50 & 23 & 57.5 \\
\hline Delegate or seek help & 6 & 30 & 2 & 10 & 8 & 20 \\
\hline Professional or paid help & 3 & 15 & 3 & 15 & 6 & 15 \\
\hline Cut demands & 7 & 35 & 3 & 15 & 10 & 25 \\
\hline Compartmentalise & 6 & 30 & 3 & 15 & 9 & 22.5 \\
\hline Other problem focused strategies & 0 & 0 & 2 & 10 & 2 & 5 \\
\hline \multicolumn{7}{|l|}{ Emotion focused: } \\
\hline Positive Thinking & 2 & 10 & 4 & 20 & 6 & 15 \\
\hline Other emotion focused strategies & 1 & 5 & 1 & 5 & 2 & 5 \\
\hline Demand-management coping & 19 & 95 & 20 & 100 & 39 & 97.5 \\
\hline \multicolumn{7}{|l|}{ Problem focused: } \\
\hline Planning \& organisation & 10 & 50 & 8 & 40 & 18 & 45 \\
\hline Organise for efficiency and order & 6 & 30 & 5 & 25 & 11 & 27.5 \\
\hline Take time to research, analyse and plan & 6 & 30 & 5 & 25 & 11 & 27.5 \\
\hline Personal Deprivation & 8 & 40 & 7 & 35 & 15 & 37.5 \\
\hline Work longer \& harder & 7 & 35 & 7 & 35 & 14 & 35 \\
\hline Use personal time & 1 & 5 & 3 & 15 & 4 & 10 \\
\hline Manage Expectations & 5 & 25 & 10 & 50 & 15 & 37.5 \\
\hline Prioritise & 6 & 30 & 4 & 20 & 10 & 25 \\
\hline \multicolumn{7}{|l|}{ Emotion focused: } \\
\hline Involve others in managing demands & 10 & 50 & 12 & 60 & 22 & 55 \\
\hline Talk about it & 7 & 35 & 5 & 25 & 12 & 30 \\
\hline Receive moral support \& friendship & 4 & 20 & 6 & 30 & 10 & 25 \\
\hline Vent to others & 1 & 5 & 4 & 20 & 5 & 12.5 \\
\hline Take my mind off role overload & 6 & 30 & 7 & 35 & 13 & 32.5 \\
\hline Engage in enjoyable activities & 4 & 20 & 6 & 30 & 10 & 25 \\
\hline Engage in potentially unhealthy activities & 2 & 10 & 2 & 10 & 4 & 10 \\
\hline Let the feelings go & 4 & 20 & 6 & 30 & 10 & 25 \\
\hline Positive efforts to prevent stress & 3 & 15 & 3 & 15 & 6 & 15 \\
\hline Endure, comply & 2 & 10 & 3 & 15 & 5 & 12.5 \\
\hline Other strategies to manage demands & 2 & 10 & 2 & 10 & 4 & 10 \\
\hline
\end{tabular}

Table 11-1 Coping strategies used for role overload by episode assessment 
I also, in this case, worked with a trainer I had worked with before who I knew was really experienced. I delegated some stuff to her that I was comfortable that she would certainly be able to do that and I was able to relax about that and let it go.

For example, to get others to go get medication from the pharmacy room while I was at the bedside caring for the patient.

The last demand-reduction coping strategy involving others, 'professional or paid help' (used in 15\% of episodes), described getting help from specialized professionals or institutions, or paying for help:

I'm getting the union and the human rights commission on it to see if we can work out something

Cutting demands. In a quarter $(25 \%)$ of the role overload episodes, hospital workers said they coped by systematically 'cutting demands', by temporarily cutting roles, or by reducing efforts for certain tasks in order to focus on others that were more important or more urgent. In some cases, this problem focused strategy was more of an automatic response and, as a result, demands were implicitly identified as less important or less urgent since they did not get done or they went away. The following comments illustrate this strategy:

Cancelled one meeting, left corporate meeting a little bit early, arrived late at (the) open house. I cut some reasonable corners.

Some of the time you don't get to things because the demands on your time are too many and oddly enough some of the time when you don't get to them, they go away. 
Compartmentalise. The next parent level coping strategy, focused on reducing demands (cited in $22.5 \%$ of role overload episodes), refers to conscious segmentation of some, or all, parts of the respondents' role domains and demands (i.e., 'compartmentalise'). The analysis revealed that this approach allowed workers to bring more focus and less conflict to whatever was the most important task, for however long was needed. The effect was less demands to interfere with the task at hand. This problem focused coping strategy is illustrated by the following quote:

I make a conscious effort not to worry about voicemails and emails while I am doing a teaching session like this. I act as if I am not here in terms of the rest of my responsibilities and I make a conscious effort to let that go.

Other respondent comments suggest that this strategy sometimes proved difficult to implement.

Positive thinking. The only emotion focused demand-reduction coping strategy identified in this study is labeled 'positive thinking' and was cited in $15 \%$ of role overload episodes. This coping strategy involved a conscious decision to cognitively alter the personal meaning of a situation in order to reduce internal role demands. In some cases this involved reducing personal standards:

.......thinking that the world wouldn't end if I didn't get the list done.

In other cases, the focus was on thinking positively to reduce negative connotations:

......moved on and focused on the positive interactions with the CEO from then on, and take it as an evaluation that that one event didn't change his opinion of me 


\subsubsection{Demand-management coping}

Nine coping strategies were grouped in the demand-management category. These strategies were either problem focused and involved efforts to organize, avoid, or tolerate the excessive role demands and time pressures causing the role overload, or were emotion focused attempts to deal with the emotional distress of role overload. The 'demandmanagement' strategies are presented below, beginning first with those that are problem focused.

Planning and organisation In close to a half of the role overload episodes recalled, the hospital employees identified and implemented a range of constructive actions, well thought out and prepared in advance, to manage the role demands causing the overload. These actions (cited in $45 \%$ of episodes) were collectively labeled 'planning and organisation'. Two approaches (i.e., child strategies) to the parent strategy, 'planning and organisation', were used, each in a substantive number of episodes. The first (in $27.5 \%$ of episodes) involved organising workloads or resources to maximize efficiency and structure (labeled 'organise for order and efficiency'), as illustrated by the following comments:

Think methodically, organise myself. Being organised helps you think in a calm measure. I made a little list of things to do, like 'call the insurance company, call Direct Energy'.

I put in place a couple of things to make things quicker and easier at the end. 
The second set of 'planning and organisation' child strategies (labeled 'take time to analyse and plan' and mentioned in $27.5 \%$ of episodes discussed) involved taking the time to step back, gather information, and plan how to deal with the role overload.

I naturally am able to take a step back and analyze.

Make some observations, gather some information and then sit down and discuss.

These quotes suggest that respondents who used these strategies did so because they thought that time spent organizing and planning was worthwhile despite the time pressures they faced.

Personal deprivation. The next parent coping strategy for managing role demands (cited in $37.5 \%$ of episodes described), was given the descriptor 'personal deprivation' and was further divided into two child strategies. Respondents either 'worked longer or harder' (mentioned in $35 \%$ of episodes) or 'used their personal time' (e.g. lunch times or vacations) (mentioned in 10\% of episodes). In both cases, managing the role demands causing the overload impinged on personal resources. The following quotes illustrate the use of these problem focused coping actions involving personal deprivation:

I'm working an hour and a half a day (doing uncompensated work) and sometimes on weekends doing program development

I don't have time to make breakfast at home. I eat through my lunch at my desk because I find even taking an hour out of the day can just put you behind.

Manage expectations. To manage the demands of role overload, hospital workers also used direct communication with either role demand senders and/or others impacted by 
this stressor. This strategy (cited in $35 \%$ of the episodes) was labeled 'manage expectations' to reflect the idea, conveyed by respondents, that this strategy comprised efforts to inform those involved about the circumstances behind the role overload, and its potential impact. Workers who used this problem focused strategy sought to minimize or avoid unrealistic expectations as illustrated by the following quotes:

For the clients we as a group communicated to them that we wouldn't be able to act as quickly as they wanted

I can only do so much ... will do my best to get info to people but knew that there would be pieces missing everywhere.

Prioritising. The next coping strategy used to manage role demands (cited in $25 \%$ of episodes), labeled 'prioritising', was regularly described in the respondent interviews using these exact words. This problem focused strategy was also often mentioned after the workers talked of using planning and organisation strategies. The following quote describes how workers assessed and decided the relative importance of the urgent role demands faced:

What I had to do was prioritize. Take a step back and say, "What are the priorities today, tomorrow, at the end of the week, and so on". That's the only way you can get through that. What can you do right now to be reactive, and then what can you do tomorrow and so on to be proactive.

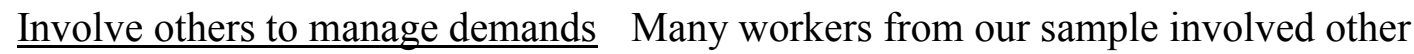
people in their attempts to manage role demands ('involve others to manage demands' was cited in 55\% of all episodes). Three child strategies (all emotion focused) were identified: 'talk about it', 'receive moral support and friendship', and 'vent to others'. 
Workers who coped by 'talking about it', chose to talk about their role overload situation with their family members, friends and/or colleagues. This tactic (cited in $30 \%$ of episodes) enabled respondents to use role set members as a sounding board and to gain validation for their thoughts and feelings, as illustrated by the following quotes :

...talking with team members so we can support each other

... talking with committee members to get a perspective on things - known them for a long time - good sounding board

I also bounce things off my husband and give him my concerns, yes, it helps to

talk.

For the second demand-management coping action involving others, 'receive moral support and friendship', respondents described the importance of moral support given by colleagues, managers, friends and family (in $25 \%$ of episodes). Others were described as providing needed psychological help and support, as the following respondent explained:

I did receive tremendous support at work, from my boss and from my co-workers. I don't want to say this was a surprise, but it was a relief. This is what got me through the time period.

In the last coping activity involving others, workers described venting to other role set members. Cited in $12.5 \%$ of episodes, most respondents specifically used the word 'vent' when talking about this coping strategy. 'Venting to others' was directed at those willing to listen, and sometimes at the individual responsible for triggering the role overload event. The common purpose of this strategy, illustrated by the following quote, appears to be to relieve the stress of the moment:

Just to vent really just an opportunity to get it off my chest. 
I gave him a bit of a tongue lashing and that certainly helped to relieve my stress. Take my mind off role overload. The next parent level demand-management coping strategy (cited in $32.5 \%$ of episodes) is labeled 'take my mind off role overload' to reflect efforts to forget the stress of role overload. Respondents used two approaches: 'engaging in enjoyable activities', and 'engaging in potentially unhealthy activities'. The first (mentioned in a quarter of the episodes), described activities such as going for a walk, reading, gardening or just focusing on a task respondents love, for example :

I put on my mp3 player and listen to some good head banger music.

Went out to garden, something I enjoy.

Engaging in potentially unhealthy activities (cited in 10\% of episodes) involved sleeping more, drinking alcohol, and or taking prescription drugs to deal with role overload. For example, when asked how they dealt with the emotions, one respondent replied:

Slept. I did take a couple of sleeping pills to help me get rest.

These two child strategies, involving ways to stop thinking about role overload, are in direct contrast with actively 'talking about it' to others as described above.

Let the feelings go. Workers in the thesis sample also managed the demands associated with role overload by letting their feelings out privately, rather than to others (cited in a $25 \%$ of episodes):

At the beginning ......there was a temper that blows up and comes out. Sometimes I cry

Sometimes, these emotion focused coping actions appeared to be taken deliberately when 
intense emotions arose:

Take a deep breath; calm down; think your way through the situation.

Similar to venting, 'letting the feelings go' seemed to help relieve the stress of the moment.

Positive efforts to prevent stress. $\quad$ In $15 \%$ of the episodes described, the workers spoke of specific efforts to deal with, or prevent, the emotional stress of role overload. Aimed at living a healthy lifestyle, these emotion focused strategies, labeled 'positive efforts to prevent stress', included physical exercise, yoga and meditation, and other forms of personal care, as illustrated by the following comments:

I run, love yoga, that how I cope with stress. ... and not forgetting to look after myself

Endure and comply The last demand-management coping strategy (cited in $12.5 \%$ of role overload episodes) was given the title 'endure and comply' to reflect what the respondents told the researchers - they just got on with the job (whatever it was) as they had determined this was the best or only way to deal with the associated emotions. Workers often intimated, however, that they used this strategy when no other immediate coping options were available, as illustrated by the following quote:

...then you do the task and sort of buck up and do it. It is not what you want to do but it has to be done this way and you don't feel happy about it or you don't feel satisfied but you do it. 


\subsubsection{Role overload coping strategies and episode assessment}

The table listing the coping strategies described above (Table 11-1) also allows us to examine how individuals coped in role overload assessed as overwhelming, and to compare it to coping in episodes assessed as not-overwhelming. Overall, strategies targeted at reducing demands are cited more often when people talked about coping with role overload assessed as not-overwhelming, rather than overwhelming (19 episodes to 16). Demand-management coping strategies, on the other hand, are cited in all episodes of both types.

The following demand-reduction strategies were mentioned more often in notoverwhelming episodes: 'using help to reduce demands' (17 episodes to 14), specifically 'help from others' (13 episodes to 10) and 'delegate or seek help' (6 episodes to 2); 'cut demands' (7 episodes to 3); and lastly 'compartmentalise' (6 episodes to 3). There are just two individual demand-management strategies that are notably cited more often in overwhelming role overload episodes: 'manage expectations' (10 episodes to 5), and 'vent to others' (4 episodes to 1).

\subsubsection{Role overload coping strategies and respondent stress}

Table 11-2 displays the demand-reduction and demand-management coping strategies used in role overload episodes, this time by respondent stress level. 


\begin{tabular}{|c|c|c|c|c|c|c|}
\hline \multirow[b]{4}{*}{ Role Overload Coping Strategies } & \multicolumn{4}{|c|}{$\begin{array}{c}\text { Role Overload Episodes by Respondent } \\
\text { Stress Level } \\
\end{array}$} & \multirow{2}{*}{\multicolumn{2}{|c|}{$\begin{array}{l}\text { Total Episodes } \\
(n=40)\end{array}$}} \\
\hline & \multicolumn{2}{|c|}{$\operatorname{Low}(n=20)$} & \multicolumn{2}{|c|}{ High $\quad(n=20)$} & & \\
\hline & \multicolumn{6}{|c|}{ \# \& \% of episodes in which the coping action was cited } \\
\hline & $\#$ & $\%$ & $\#$ & $\%$ & $\#$ & $\%$ \\
\hline Demand-reduction coping & 17 & 85 & 18 & 90 & 35 & 87.5 \\
\hline \multicolumn{7}{|l|}{ Problem focused: } \\
\hline Use help to reduce demands & 15 & 75 & 16 & 80 & 31 & 77.5 \\
\hline Help from colleagues, family or friends & 12 & 60 & 11 & 55 & 23 & 57.5 \\
\hline Delegate or seek help & 5 & 25 & 3 & 15 & 8 & 20 \\
\hline Professional or paid help & 2 & 10 & 4 & 20 & 6 & 15 \\
\hline Cut demands & 5 & 25 & 5 & 25 & 10 & 25 \\
\hline Compartmentalise & 5 & 25 & 4 & 20 & 9 & 22.5 \\
\hline Other problem focused strategies & 0 & 0 & 2 & 10 & 2 & 5 \\
\hline \multicolumn{7}{|l|}{ Emotion focused: } \\
\hline Positive thinking & 3 & 15 & 3 & 15 & 6 & 15 \\
\hline Other emotion focused strategies & 0 & 0 & 2 & 10 & 2 & 5 \\
\hline Demand-management coping & 20 & 100 & 19 & 95 & 39 & 97.5 \\
\hline \multicolumn{7}{|l|}{ Problem focused: } \\
\hline Planning \& Organisation & 11 & 55 & 7 & 35 & 18 & 45 \\
\hline Organise for efficiency and order & 8 & 40 & 3 & 15 & 11 & 27.5 \\
\hline Take time to research, analyse and plan & 5 & 25 & 6 & 30 & 11 & 27.5 \\
\hline Personal Deprivation & 8 & 40 & 7 & 35 & 15 & 37.5 \\
\hline Work longer \& harder & 8 & 40 & 6 & 30 & 14 & 35 \\
\hline Use personal time & 2 & 10 & 2 & 10 & 4 & 10 \\
\hline Manage Expectations & 8 & 40 & 7 & 35 & 15 & 37.5 \\
\hline Prioritise & 5 & 25 & 5 & 25 & 10 & 25 \\
\hline \multicolumn{7}{|l|}{ Emotion focused: } \\
\hline Involve others in managing demands & 10 & 50 & 12 & 60 & 22 & 55 \\
\hline Talk about it & 6 & 30 & 6 & 30 & 12 & 30 \\
\hline Receive moral support and friendship & 5 & 25 & 5 & 25 & 10 & 25 \\
\hline Vent to others & 0 & 0 & 5 & 25 & 5 & 12.5 \\
\hline Take my mind off role overload & 6 & 30 & 7 & 35 & 13 & 32.5 \\
\hline Engage in activities I enjoy & 5 & 25 & 5 & 25 & 10 & 25 \\
\hline Engage in potentially unhealthy activities & 2 & 10 & 2 & 10 & 4 & 10 \\
\hline Let the feelings go & 5 & 25 & 5 & 25 & 10 & 25 \\
\hline Positive efforts to prevent stress & 2 & 10 & 4 & 20 & 6 & 15 \\
\hline Tolerate, endure, comply & 3 & 15 & 2 & 10 & 5 & 12.5 \\
\hline Other strategies to manage demands & 3 & 15 & 1 & 5 & 4 & 10 \\
\hline
\end{tabular}

Table 11-2 Coping strategies used for role overload by stress level of respondents 
An examination of the strategies in Table 11-2 shows that overall the likelihood an individual will talk about a particular demand-reduction strategies is not associated with their level of stress, a very different situation than was observed with respect to episode assessment. Overall, both respondent groups also cite demand-management coping strategies in an equivalent number of episodes. There are, however, several different individual demand-management strategies that were used more often by one group than the other.

Low stress respondents talked about using 'planning and organization' strategies to manage the role overload demands in a greater number of episodes than did the high stress workers (11 episodes to 7). In particular, these respondents mentioned the coping strategy 'organise for efficiency and order' more often (8 episodes to 3 ). Workers with high levels of perceived stress mentioned 'venting to others' in more of their role overload episodes. These workers vent to other people as a way to let off steam in five episodes. Low stress respondents never mentioned using this strategy.

\subsubsection{Role overload coping strategies by respondent stress and episode assessment}

Table 11-3 shows the coping strategies used by low and high stress respondents according to episode assessment, for those strategies where notable differences occur across the four episode groupings. From this table the following observations can be made.

First, the lower usage of 'demand-reduction' coping strategies in role overload episodes assessed as overwhelming (see Section 11.1.3 above) is restricted to low stress 
respondents. This data pattern is particularly notable for the individual strategies:

'delegate and seek help'; and 'cut demands'. As a consequence, low stress workers use these two strategies far less in episodes that they find overwhelming compared to the episodes they describe as not-overwhelming. A second observation that can be made from the data above is that, although the strategy 'positive thinking' is equally cited by low and high stress groups, low stress workers never used this strategy to reduce the emotional demands of role overload in episodes assessed as not-overwhelming.

\begin{tabular}{|c|c|c|c|c|c|c|c|c|c|c|}
\hline & & & & Level & Res & ent & & & & \\
\hline & & Low & $\mathrm{N}=10$ ) & & & High & $\mathrm{N}=\mathbf{1 0}$ & & & \\
\hline & $\begin{array}{r}\text { over } \\
\mathrm{E}_{\mathbf{I}} \\
(\end{array}$ & $\begin{array}{l}\text { ming } \\
\text { les } \\
\text { ) }\end{array}$ & $\begin{array}{r}\text { Over } \\
\text { E }\end{array}$ & $\begin{array}{l}\text { lming } \\
\text { les } \\
\text { ) }\end{array}$ & $\begin{array}{r}\text { over } \\
\text { E }\end{array}$ & $\begin{array}{l}\text { Iming } \\
\text { des } \\
\text { 0) }\end{array}$ & $\begin{array}{r}\text { Over } \\
\text { E }\end{array}$ & $\begin{array}{l}\text { Iming } \\
\text { des } \\
\text { 0) }\end{array}$ & & $\begin{array}{l}\text { al } \\
\text { des } \\
\text { (0) }\end{array}$ \\
\hline Role Overload Coping & & & $\& \% 0$ & odes in & iich th & ing stra & gy was & ioned & & \\
\hline Strategies & $\#$ & $\%$ & \# & $\%$ & $\#$ & $\%$ & $\#$ & $\%$ & $\#$ & $\%$ \\
\hline Demand-reduction coping & 10 & 100 & 7 & 70 & 9 & 90 & 9 & 90 & 35 & 87.5 \\
\hline Use help to reduce demands & 9 & 90 & 6 & 60 & 8 & 80 & 8 & 80 & 31 & $\mathbf{7 7 . 5}$ \\
\hline Delegate or seek help & 4 & 40 & 1 & 10 & 2 & 20 & 1 & 10 & 8 & 20 \\
\hline Cut demands & 4 & 40 & 1 & 10 & 3 & 30 & 2 & 20 & 10 & 25 \\
\hline Positive Thinking & 0 & 0 & 3 & 30 & 2 & 20 & 1 & 10 & 6 & 15 \\
\hline $\begin{array}{l}\text { Demand-management } \\
\text { coping }\end{array}$ & 10 & 100 & 10 & 100 & 9 & 90 & 10 & 100 & 39 & 97.5 \\
\hline Planning \& organisation & 5 & 50 & 6 & 60 & 5 & 50 & 2 & 20 & 18 & 45 \\
\hline $\begin{array}{r}\text { Organise for efficiency and } \\
\text { order }\end{array}$ & 3 & 30 & 5 & 50 & 3 & 30 & 0 & 0 & 11 & 27.5 \\
\hline $\begin{array}{l}\text { Involve others in managing } \\
\text { demands }\end{array}$ & 6 & 60 & 4 & 40 & 4 & 40 & 8 & 80 & 22 & 55 \\
\hline Talk about it & 5 & 50 & 1 & 10 & 2 & 20 & 4 & 40 & 12 & 30 \\
\hline Vent to others & 0 & 0 & 0 & 0 & 1 & 10 & 4 & 40 & 5 & 12.5 \\
\hline
\end{tabular}

Table 11-3 Coping strategies used for role overload by respondent stress and episode assessment 
The third data pattern of note in Table 11-3 involves 'planning and organisation' and the associated child strategy, 'organise for efficiency and order'. The reduced usage of these tactics by high stress respondents (noted in the previous section) is concentrated in overwhelming role overload. As a result, high stress respondents use very few 'planning and organisation' strategies in their overwhelming episodes.

Finally, whilst both respondent groups talked equally often about managing the emotional reactions to role overload by involving others, high stress workers cite these strategies in more overwhelming than not-overwhelming episodes ( 8 episodes to 4$)$. This data pattern is not, however, reflected across all the child strategies that involve others to manage demands. 'Vent to others' is a coping action involving others that does contribute to the parent strategy data pattern. Used exclusively by high stress respondents, venting is cited mostly in overwhelming episodes (4 episodes to 1 ). Talking about role overload to others, in contrast, is more equitably cited by high stress workers in the two episode types and moreover, cited by low stress workers in more not-overwhelming than overwhelming episodes (5 episodes to 1$)$.

\subsection{Summary of key findings for coping with role overload}

The objective of this chapter was to examine the coping strategies and specific actions employed by these hospital workers in their efforts to overcome role overload and its effects.

Although these overloaded hospital employees talked about a wide variety of coping strategies, the analysis the data show that they can broadly be divided into those 
directed at reducing role demands and those directed at managing role demands and their effects. The coping strategies cited by these workers can also be described as either problem focused or emotion focused (as defined by Lazarus and Folkman, 1984). Variations in the coping approach were found to have less to do with the reported stress levels of the hospital worker and more to do with episode assessment. A brief summary of the coping strategy findings for all episodes is given in Section 11.2.1. The results by episode assessment and worker stress level are summarised in Sections 11.2.2 and 11.2.3 respectively. The last subsection briefly summarises notable coping strategy findings for the four episode groupings of the two stress levels groups.

\subsubsection{Key role overload coping strategy findings for all epsiodes}

The data in this chapter suggested that, overall, 'involving other people' was a particularly important way for female hospital workers to cope with role overload. The most commonly used role overload coping strategy was to offload demands to other people by accepting practical help. As well as involving others to lessen the demands responsible for triggering role overload, other people were often involved to manage the emotional responses arising from role overload. Hospital workers typically cited more demand-management than demand-reduction strategies per episode, and more problemfocused than emotion focused strategies. Commonly used demand-management problem focused strategies included planning and organisation, personal deprivation and managing expectations. 


\subsubsection{Key coping strategy findings by role overload episode assessment}

Table 11-7 summarises the key findings for coping strategies according to episode assessment, without regard to respondent stress level. The results suggest that hospital workers were more likely to use the problem focused demand-reduction strategies 'help from family, friends and colleagues', 'delegate and seek help', 'cut demands', and 'compartmentalise' to cope with role overload in episodes they subsequently evaluated as not-overwhelming. Thus, all but one of the problem focused demand-reducing strategies (i.e. 'use professional help') appears to be associated with less overwhelming role overload. The data also suggested that the following individual coping strategies were employed more often in overwhelming episodes: 'managing expectations' and 'venting to others', both aimed at managing role demands during role overload.

\begin{tabular}{|c|c|c|c|c|}
\hline \multirow{3}{*}{$\begin{array}{c}\text { Role Overload } \\
\text { Episode } \\
\text { Assessment } \\
\end{array}$} & \multicolumn{4}{|c|}{ Role Overload Coping Strategies } \\
\hline & \multicolumn{2}{|c|}{ Demand Reduction } & \multicolumn{2}{|c|}{ Demand Management } \\
\hline & Problem Focused & Emotion Focused & Problem Focused & Emotion Focused \\
\hline $\begin{array}{c}\text { Not- } \\
\text { overwhelming }\end{array}$ & $\begin{array}{l}\text { Coping actions involving } \\
\text { others to reduce demands } \\
\text { Help from others } \\
\text { Delegate \& seek help } \\
\text { Cut demands } \\
\text { Compartmentalise }\end{array}$ & & & \\
\hline Overwhelming & & & Manage expectations & Vent to others \\
\hline
\end{tabular}

Table 11-4 Key coping strategies for role overload assessed as overwhelming and not-overwhelming without regard to respondent stress level 


\subsubsection{Key coping strategy findings for respondents with low and high stress levels}

The key findings for coping strategies for role overload episodes by respondent stress level are presented in Table 11-8. The table summarises the findings for the types of strategies cited (i.e. demand-reduction, problem and emotion focused, and demandmanagement, problem and emotion focused). These results are summarised with no reference to role overload episode assessment.

Results from this chapter suggest that workers with lower levels of perceived stress tend to use the problem focused 'planning and organization' based strategies (especially 'organise for efficiency and order') more frequently to manage role overload than highly stressed individuals.

The demand-management emotion focused strategy, 'venting to others' was the only specific coping action employed more often by highly stressed workers than by those with lower stress levels.

\begin{tabular}{|c|c|c|c|c|}
\hline \multirow{2}{*}{\begin{tabular}{c} 
Respondent $\begin{array}{c}\text { Role Overload Coping Findings } \\
\text { Stress } \\
\text { Level }\end{array}$ \\
\cline { 2 - 5 }
\end{tabular}} & \multicolumn{2}{|c|}{$\begin{array}{c}\text { Demand Reduction } \\
\text { Strategies }\end{array}$} & \multicolumn{2}{|c|}{$\begin{array}{c}\text { Demand Management Coping } \\
\text { Strategies }\end{array}$} \\
\hline \multirow{2}{*}{ Low } & Problem Focused & Emotion Focused & Problem Focused & Emotion Focused \\
\hline High & & & $\begin{array}{c}\text { Planning \& } \\
\text { organisation } \\
\text { Organise for efficiency } \\
\text { and order }\end{array}$ & \\
\hline & & & Vent to others \\
\hline
\end{tabular}

Table 11-5 Key coping strategies for role overload of high and low stress respondents, without regard to how the episodes were assessed 


\subsubsection{Key coping strategy findings by episode groupings}

Table 10-17 summarises the notable coping strategy findings for the four episode groupings. Two findings were noted in this chapter with regard to coping strategies used in the overwhelming role overload episodes of highly stressed hospital workers. First, although cited relatively infrequently overall, the strategy 'venting to others', was almost exclusively restricted to the episodes of this grouping. In contrast, these highly stressed individuals rarely mentioned the strategy, 'organise for efficiency and order', when talking about coping with overwhelming role overload. This finding is particularly notable because this demand-management problem focused strategy was mentioned equally often by workers in the other three episode groupings.

In the not-overwhelming episodes described by workers with low levels of reported stress, a number of coping strategies stood out. Results suggested that delegating and seeking help, and cutting demands, were more likely to be used to reduce the demands of role overload in the episodes of this grouping. Talking about role overload was also a strategy employed in a greater number of these episodes as a way to relieve emotional stress. The findings in this chapter also suggest that 'positive thinking', a demand-management emotion focused strategy mentioned relatively infrequently overall, was cited more often in the overwhelming role overload described by low stress workers. 


\begin{tabular}{|c|c|}
\hline $\begin{array}{c}\text { Not-overwhelming episodes of low stress workers } \\
\text { Delegate and seek help } \\
\text { Cut demands }\end{array}$ & $\begin{array}{c}\text { Not-overwhelming episodes of high stress workers } \\
\text { Organise for efficiency and order } \\
\text { Talk about it }\end{array}$ \\
\hline $\begin{array}{c}\text { Overwhelming episodes of low stress workers } \\
\text { Positive thinking }\end{array}$ & Organise for efficiency and order \\
\hline $\begin{array}{c}\text { Organise for efficiency and order } \\
\end{array}$ & \\
\end{tabular}

Table 11-6 Summary of role overload coping strategies by episode grouping 


\section{Role overload outcomes}

Lazarus and Folkman's (1984) stress appraisal model suggests that outcomes from the role overload appraisal process can be divided into two groups: short term and long term results. This thesis was not longitudinal and thus we do not have the opportunity to examine long term outcomes from role overload. To complete the analysis of role overload appraisal and coping, the last chapter of the results and discussion part of this thesis focuses on the short term outcomes of the role overload episodes as described by the overloaded female hospital employees in their recollections of personal role overload events.

Emotions and feelings arising during the appraisal of role overload have already been examined in Chapter 10. The data for this chapter is drawn primarily from the last set of interview questions as listed in Table 7-1 (in Section 7.1.1.2), which asked respondents to describe how the role overload situation ended and how they felt about it. Analysis of the responses from these questions suggests that two sets of outcomes resulted from the role overload episodes: outcomes personal to the respondent and outcomes specific to the role overload episode. These role overload outcomes are examined in two sections: Section 12.1, and 12.2 respectively. The third section summarises the key findings from this chapter. 


\subsection{Personal outcomes}

Personal outcomes from role overload involved expressions of emotions and feelings describing how respondents felt as well as specific personal consequences of a more permanent or tangible nature. Guided by the same model of affect referenced in Chapter 10 (Russell's (1980) circumplex model) emotions and feelings were broadly divided into two groups: positive and negative. The personal consequences of role overload can also be categorized as being either positive or negative for individuals. Table 12-1 presents the positive and negative personal outcomes described by female hospital workers. These outcomes are presented in Sections 12.1.1 and 12.1.2 respectively. The relationship between these personal outcomes and role overload assessment is examined next (Section 12.1.3), followed by an examination of personal outcomes according to the stress level of respondents (Section 12.1.4). Section 12.1.5 explores personal outcome results by episode assessment and respondent stress level.

\subsubsection{Positive personal outcomes}

Positive emotions and feelings resulting from role overload were cited in $62.5 \%$ of episodes, whilst specific personal gains from the episodes were mentioned in $17.5 \%$ of episodes. Compared to the emotions and feelings workers described when talking about their initial reactions to the role overload situations, the emotions recalled at this stage of the respondent interviews were found to be more varied on the second dimension of Russell's circumplex model of affect (i.e., degree of arousal). Three positive emotions and feelings were cited in substantive numbers at the end of the interviews: 'relief', 
'good' and 'satisfied'. The associated descriptions, which are discussed below, suggest that all of these can be described as low arousal emotions. Of the remaining singular positive emotions and feelings noted, a small number (cited in $7.5 \%$ of episodes) suggest higher levels of activity or arousal (i.e., pride, surprise, and enjoyment) and were therefore collectively labeled as 'other high arousal positive emotions and feeling' in Table 12-1. An identical number were collected together and labeled as 'other low arousal positive emotions and feelings' (e.g., fine, relaxed, comfortable). Similar variability by arousal was noted for negative emotions and feelings arising at the end of role overload episodes, and will be discussed in the next section. The positive outcomes noted in substantive numbers of episodes are described below.

$\underline{\text { Positive emotions and feelings } \quad \text { Expressions of relief (cited in } 30 \% \text { of episodes) }}$ were the most common of all emotions and feelings talked about by hospital workers when describing how they felt at the end of each role overload episode. Expressions of relief were often specifically associated with the end of stressful feelings:

Relieved... The stress was off my shoulders.

For others, relief implied thankfulness that the situation was over and the job was done. This was particularly notable when multiple positive emotions and feelings were expressed together. For example, whilst good feelings were cited in $20 \%$ of episodes in total, feeling good and relieved at the end of role overload episodes were also sometimes mentioned together:

Good I felt good. I was glad and relieved that it was over and felt it had been totally successful. 
Sometimes I feel very good when you get a task done and then you think I am so relieved.

\begin{tabular}{|c|c|c|c|c|c|c|}
\hline \multirow[b]{4}{*}{ Role Overload Personal Outcomes } & \multicolumn{6}{|c|}{ Role Overload Episode Assessment } \\
\hline & \multicolumn{2}{|c|}{$\begin{array}{l}\text { Not Overwhelming } \\
(n=20)\end{array}$} & \multicolumn{2}{|c|}{$\begin{array}{l}\text { Overwhelming } \\
\qquad(n=20)\end{array}$} & \multicolumn{2}{|c|}{$\begin{array}{c}\text { Total } \\
(n=40)\end{array}$} \\
\hline & \multicolumn{6}{|c|}{$\#$ and $\%$ of episodes with identified property } \\
\hline & $\#$ & $\%$ & $\#$ & $\%$ & $\#$ & $\%$ \\
\hline Positive Personal Outcomes & 18 & 90 & 7 & 70 & 25 & 62.5 \\
\hline Positive emotions \& feelings & 18 & 95 & 7 & 35 & 25 & 62.5 \\
\hline Relieved & 10 & 50 & 2 & 10 & 12 & 30 \\
\hline Good & 7 & 35 & 1 & 5 & 8 & 20 \\
\hline Satisfied & 2 & 10 & 3 & 15 & 5 & 12.5 \\
\hline $\begin{array}{r}\text { Other low arousal positive emotions \& } \\
\text { feelings }\end{array}$ & 2 & 10 & 1 & 5 & 3 & 7.5 \\
\hline $\begin{array}{r}\text { Other high arousal positive emotions and } \\
\text { feelings }\end{array}$ & 2 & 10 & 1 & 5 & 3 & 7.5 \\
\hline Personal gains & 6 & 30 & 1 & 10 & 7 & 17.5 \\
\hline Personal growth & 6 & 30 & 1 & 10 & 7 & 17.5 \\
\hline Negative Personal Outcomes & 6 & 30 & 16 & 80 & 22 & 55 \\
\hline Negative emotions and feelings & 5 & 25 & 16 & 80 & 21 & 52.5 \\
\hline \multicolumn{7}{|l|}{ Low arousal: } \\
\hline Tired and exhausted & 3 & 15 & 5 & 50 & 8 & 20 \\
\hline Other low arousal negative emotions & 1 & 5 & 8 & 40 & 9 & 22.5 \\
\hline \multicolumn{7}{|l|}{ High arousal: } \\
\hline Stressed \& overwhelmed & 1 & 5 & 5 & 25 & 6 & 15 \\
\hline Other high arousal negative emotions & 1 & 5 & 6 & 30 & 7 & 17.5 \\
\hline Negative Consequences & 2 & 10 & 5 & 25 & 7 & 17.5 \\
\hline Role demand spillover & 1 & 5 & 3 & 10 & 4 & 10 \\
\hline Other negative consequences & 0 & 0 & 4 & 20 & 4 & 10 \\
\hline
\end{tabular}

Table 12-1 Role overload personal outcomes by episode assessment

Expressions of satisfaction (cited in $12.5 \%$ of episodes), suggest a tone that is more selfcongratulatory, as illustrated by the following comment:

It was great; there was definitely a sense of satisfaction having done a good job. 
The quietly positive comments described above suggest that many respondents who felt stressed and overwhelmed during role overload, as indicated by the results in Chapter 10, were able to let go of these feelings at the end.

Personal Gains $\quad$ The personal gains arising from role overload (cited in $17.5 \%$ of episodes) specifically related to personal growth. Most comments describing these benefits reflected learning experiences and new skills available to respondents for future use.

\section{I learned a lot of lessons.}

I wrote some tools that we can use for next year that will be helpful.

Other comments about personal growth also hinted at changes to person characteristics, such as beliefs about control and self esteem:

Ifelt more under control.

I was asked challenging questions and was able to handle them. My colleagues were impressed.

\subsubsection{Negative personal outcomes}

When workers described their feelings at the end of the role overload, negative emotions and feelings were cited in $52.5 \%$ of episodes. Specific negative consequences were cited in $17.5 \%$ of the episodes.

Negative emotions and feelings The negative emotions and feelings resulting from role overload varied widely and were further classified into low and high arousal negative 
emotions and feelings, in line with the second dimension of Russell's (1980) circumplex model of affect. Low arousal rather than high arousal negative emotions and feelings were cited more often by respondents. Specifically, tiredness and exhaustion (mentioned in $20 \%$ of episodes) was the most commonly expressed negative emotion and feeling. Other low arousal negative emotions and feelings were cited in $22.5 \%$ of episodes. The mild, less active nature of these low arousal negative emotions and feelings are illustrated in the following comments:

Exhausted, tired... didn't want my job that day.

I still feel disappointed and I'm not as angry - I'm just disappointed

I have to say that after four and a halfyears I am getting increasingly resentful of this kind of situation.

Workers specifically described feeling 'stressed and overwhelmed' in $15 \%$ of the role overload episodes. The other singular high arousal negative emotions and feelings (cited in $17.5 \%$ of episodes) included expressions of anger, frustration, and burnout. Collectively, these intense expressions suggest that for many hospital employees, the negative emotions and feelings arising during role overload appraisal also lingered after the situation ended. The strength of the high arousal negative emotions and feelings are illustrated in the following respondent quotes:

I made it through the shift, but went home feeling completely overwhelmed I still have a lot of anger and bitterness It stresses me just thinking about it to tell you the truth. I count the days until I retire

I feel like a shipwreck. I made it to the shore and I am in pieces 
Negative consequences Most of the negative consequences from role overload suggested by these employees involved the spillover of tasks to another day or another domain (i.e., 'role demand spillover', cited in $10 \%$ of the episodes). For example, one worker commented:

I mean I guess it worked at that point cause I got what I thought was important done but I had many other things to do that I didn' 't' get done to take care of the next day and later on which added more work

'Other negative consequences' represents a collection of negative personal outcomes that include ongoing interpersonal issues and lost vacations.

\subsubsection{Personal outcomes by episode assessment}

For each of the personal outcomes described, the data in Table 12-1 also allows us to compare results in episodes assessed as overwhelming to those that are considered 'not-overwhelming'. This data supports the following observations.

First, at the end of role overload assessed as not-overwhelming, workers talked more often of positive emotions and feelings (18 episodes to 7). This data pattern is repeated for two specific emotions and feelings: expressions of relief (10 episodes to 2) and of good feelings ( 7 episodes to 1). Positive outcomes involving personal growth were also noted more often in role overload deemed not-overwhelming (6 episodes to 1 ).

In contrast, negative emotions and feelings were cited more frequently in role overload episodes deemed overwhelming (16 episodes to 5), as were negative consequences (5 episodes to 2). The following negative emotions and feelings were 
similarly talked about more often at the end of overwhelming episodes: stressed and overwhelmed (5 episodes to 1); other high arousal negative emotions and feelings (6 episodes to 1); and other low arousal negative emotions and feelings (8 episodes to 1 ). Finally, the likelihood a respondent would talk about feeling tired and exhausted was not associated with types of episode assessment.

\subsubsection{Personal outcomes and respondent stress}

Table 12-2 displays personal outcomes from role overload according to respondent stress levels.

\begin{tabular}{|c|c|c|c|c|c|c|}
\hline \multirow[b]{4}{*}{ Role Overload Personal Outcomes } & \multicolumn{6}{|c|}{ Role Overload Episodes by Respondent Stress Level } \\
\hline & \multicolumn{2}{|c|}{ Low $(n=20)$} & \multicolumn{2}{|c|}{ High $(n=20)$} & \multicolumn{2}{|c|}{$\begin{array}{c}\text { Total Episodes } \\
(n=40)\end{array}$} \\
\hline & \multicolumn{6}{|c|}{ \# \& \% of episodes which resulted in this outcome } \\
\hline & \# & $\%$ & \# & $\%$ & \# & $\%$ \\
\hline Positive Personal Outcomes & 12 & 60 & 13 & 65 & 25 & 62.5 \\
\hline Positive Emotions \& Feelings & 12 & 60 & 13 & 65 & 25 & 62.5 \\
\hline Relieved & 7 & 35 & 5 & 25 & 12 & 30 \\
\hline Good & 2 & 10 & 6 & 30 & 8 & 20 \\
\hline Satisfied & 1 & 5 & 4 & 20 & 5 & 12.5 \\
\hline Other low arousal & 1 & 5 & 2 & 10 & 3 & 7.5 \\
\hline Other high arousal & 1 & 5 & 2 & 10 & 3 & 7.5 \\
\hline Personal Gain & 4 & 20 & 3 & 15 & 7 & 17.5 \\
\hline Personal growth & 4 & 20 & 3 & 15 & 7 & $\mathbf{1 7 . 5}$ \\
\hline Negative Personal Outcomes & 11 & 55 & 11 & 55 & 22 & 55 \\
\hline Negative emotions and feelings & 10 & 50 & 11 & 45 & 21 & 52.5 \\
\hline \multicolumn{7}{|l|}{ Low arousal : } \\
\hline Tired and exhausted & 3 & 15 & 5 & 20 & 8 & 20 \\
\hline Other low arousal negative emotions & 5 & 25 & 4 & 20 & 9 & 22.5 \\
\hline \multicolumn{7}{|l|}{ High arousal : } \\
\hline Stressed \& overwhelmed & 2 & 10 & 4 & 20 & 6 & 15 \\
\hline Other high arousal negative emotions & 4 & 20 & 3 & 15 & 7 & $\mathbf{1 7 . 5}$ \\
\hline Negative Consequences & 2 & 10 & 5 & 25 & 7 & $\mathbf{1 7 . 5}$ \\
\hline Role demand spillover & 0 & 0 & 4 & 20 & 4 & 10 \\
\hline Other negative consequences & 1 & 5 & 3 & 15 & 4 & 10 \\
\hline
\end{tabular}

Table 12-2 Role overload personal outcomes by respondent stress level 
Table 12-2 supports the following observations about the relationship between personal outcomes and respondent stress. At the parent level, positive emotions and feelings were cited equally by high and low stress respondents when describing how they felt at the end of role overload. Nevertheless, two of the positive emotions and feelings identified were mentioned more often by workers with higher levels of stress: 'good' (6 episodes to 2) and 'satisfied' (4 episodes to 1).

Negative emotions and feelings were all cited equally by both stress level groups of individuals regardless of whether they were high or low arousal. Negative consequences, in contrast, were cited more often by highly stressed workers. Specifically, the potential for role overload spill-over was mentioned more often by these respondents (4 episodes to 0$)$.

\subsubsection{Personal outcomes by respondent stress level and episode assessment}

Table 12-3 provides further information about the relationships between role overload outcomes and respondent stress, particularly for workers with higher stress levels. Three observations are made.

First, the suggestion that 'good' feelings were more likely to result from role overload assessed as not-overwhelming seemed to be applicable only to those workers with higher stress levels. Second, the suggested association between personal gains and role overload described as not-overwhelming (see Table 12-1) is also restricted to 
workers with higher levels of stress. These data support the idea that high stress workers never achieved personal growth in episodes assessed as overwhelming.

\begin{tabular}{|c|c|c|c|c|c|c|c|c|c|c|}
\hline \multirow{5}{*}{$\begin{array}{c}\text { Role Overload Personal } \\
\text { Outcomes }\end{array}$} & \multicolumn{8}{|c|}{ Stress Level of Respondent } & & \\
\hline & \multicolumn{4}{|c|}{ Low $(N=10)$} & \multicolumn{4}{|c|}{ High $(\mathrm{N}=10)$} & & \\
\hline & \multicolumn{2}{|c|}{$\begin{array}{c}\text { Not- } \\
\text { overwhelming } \\
\text { Episodes } \\
(\mathbf{n}=\mathbf{1 0}) \\
\end{array}$} & \multicolumn{2}{|c|}{$\begin{array}{c}\text { Overwhelming } \\
\text { Episodes } \\
(\mathbf{n}=10)\end{array}$} & \multicolumn{2}{|c|}{$\begin{array}{c}\text { Not- } \\
\text { overwhelming } \\
\text { Episodes } \\
(\mathbf{n}=\mathbf{1 0})\end{array}$} & \multicolumn{2}{|c|}{$\begin{array}{l}\text { Overwhelming } \\
\text { Episodes } \\
(\mathbf{n}=10)\end{array}$} & \multicolumn{2}{|c|}{$\begin{array}{c}\text { Total } \\
\text { Episodes } \\
(\mathbf{n}=\mathbf{4 0}) \\
\end{array}$} \\
\hline & \multicolumn{10}{|c|}{ \# \& \% of episodes which resulted in this outcome } \\
\hline & $\#$ & $\%$ & $\#$ & $\%$ & $\#$ & $\%$ & $\#$ & $\%$ & $\#$ & $\%$ \\
\hline \multicolumn{11}{|l|}{ Positive Personal Outcomes } \\
\hline \multicolumn{11}{|l|}{ Positive Emotions \& Feelings } \\
\hline Good & 2 & 20 & 0 & 0 & 5 & 50 & 1 & 10 & 8 & 20 \\
\hline \multicolumn{11}{|l|}{ Personal Gain } \\
\hline Personal Growth & 3 & 40 & 1 & 20 & 3 & 30 & 0 & 0 & 7 & 17.5 \\
\hline \multicolumn{11}{|l|}{ Negative Personal Outcomes } \\
\hline \multicolumn{11}{|l|}{ Negative Emotions \& Feelings } \\
\hline Tired \& exhausted & 0 & 0 & 3 & 30 & 3 & 20 & 2 & 20 & 8 & 20 \\
\hline Stressed/overwhelmed & 1 & 10 & 1 & 10 & 0 & 0 & 4 & 40 & 6 & 15 \\
\hline
\end{tabular}

Table 12-3 Role overload personal outcomes by respondent stress level and episode assessment

Third, low stress workers never talked about feeling tired or exhausted at the end of not-overwhelming role overload. This result is notable since these feelings were equally expressed in the episodes of the other three groupings. Lastly, feeling stressed and overwhelmed at the end of role overload (see Section 12.1.4) was almost exclusively restricted to the overwhelming episodes of high stress workers.

\subsection{Resolution of role overload episodes}

Table 12-5 presents the episode resolution outcomes described by hospital employees at the end of their role overload recollections, for all episodes and also by episode assessment. The data shows that respondents described one of three types of role 
overload episode resolutions: successful, mixed, or unresolved. The three types of episode resolution are described below in Section 12.2.1, followed by an examination of the results by episode assessment in Section 12.2.2. The relationship between episode resolutions and worker stress levels is examined in Section 12.2.3.

\subsubsection{Episode resolutions}

Forty two and a half per cent of the role overload episodes were successfully and satisfactorily resolved for all concerned, as illustrated by the following respondent comments:

Dad got better, projects were finished, expansion is ongoing and people are happy.

Successful across the board.

The actual orientation session went very well. They were very successful, very well received. Ultimately, the work got done, the staff got trained and they got launched and so far so good.

\begin{tabular}{|c|c|c|c|c|c|c|}
\hline \multirow[b]{4}{*}{ Role Overload Outcomes } & \multicolumn{6}{|c|}{ Role Overload Episode Assessment } \\
\hline & \multicolumn{2}{|c|}{$\begin{array}{c}\text { Not } \\
\begin{array}{c}\text { Overwhelming } \\
(\mathrm{n}=20)\end{array} \\
\end{array}$} & \multicolumn{2}{|c|}{$\begin{array}{c}\text { Overwhelming } \\
(\mathrm{n}=\mathbf{2 0})\end{array}$} & \multicolumn{2}{|c|}{$\begin{array}{c}\text { Total } \\
(\mathrm{n}=\mathbf{4 0})\end{array}$} \\
\hline & \multicolumn{6}{|c|}{ \# and $\%$ of episodes with identified property } \\
\hline & \# & $\%$ & $\#$ & $\%$ & \# & $\%$ \\
\hline Episode Resolution & 20 & 100 & 20 & 100 & 40 & 100 \\
\hline Successful & 11 & 55 & 6 & 30 & 17 & 42.5 \\
\hline Mixed - got through it & 8 & 35 & 9 & 45 & 17 & 42.5 \\
\hline Unresolved - ongoing & 1 & 5 & 5 & 25 & 6 & 15 \\
\hline
\end{tabular}

Table 12-4 Episode resolutions by episode assessment 
The same proportion of episodes ended with a mixed resolution. In many of these 'mixed' episodes, respondents suggested that they managed to get through the situation, although it was a struggle and often involved working long hours. In other cases, whilst some elements of the outcomes were satisfactory, some were described as unsatisfactory, either for the respondent or the role set members:

Temporarily resolved - we got her out of a dangerous situation. We eventually got a bed for her. For me it was ok I think, once the crisis was over and the pressure was off a bit, but not completely.

Things were ok not great not a successful day but got through it-whole lot of things being done but not done completely.

Finally, in $15 \%$ of the episodes the resolution could not be determined as the event trigger for the role overload was unresolved, as illustrated in the comments below:

The people that were off on sick leave came back, but the feeling that we were short staffed is still there.

Well it hasn't ended yet - I'm still working on it, it hasn't ended

The majority of these unresolved role overload episodes were categorized with the situation property 'duration' as identified in Chapter 8.

\subsubsection{Episode resolution and episode assessment}

The data in Table 12-5 allows us to examine the episode resolutions according to how they were subsequently assessed. The data in this table shows that a greater number of episodes categorized as not-overwhelming ended successfully (11 episodes to 6). Unresolved episodes, however, were almost exclusively categorized as overwhelming (5 episodes to 1). 


\subsubsection{Episode resolution and respondent stress}

Table 12-6 presents the episode resolution results by worker stress level and episode assessment.

\begin{tabular}{|c|c|c|c|c|c|c|c|c|c|c|}
\hline \multirow{5}{*}{$\begin{array}{c}\text { Role Overload Episode } \\
\text { Resolution }\end{array}$} & \multicolumn{8}{|c|}{ Role Overload Episodes by Respondent Stress Level } & & \\
\hline & \multicolumn{4}{|c|}{ Low $(N=10)$} & \multicolumn{4}{|c|}{ High $(\mathbf{N}=10)$} & & \\
\hline & \multicolumn{2}{|c|}{$\begin{array}{c}\text { Not- } \\
\text { overwhelming } \\
\text { Episodes } \\
(\mathbf{n}=10) \\
\end{array}$} & \multicolumn{2}{|c|}{$\begin{array}{c}\text { Overwhelming } \\
\text { Episodes } \\
(\mathbf{n}=10)\end{array}$} & \multicolumn{2}{|c|}{$\begin{array}{c}\text { Not- } \\
\text { overwhelming } \\
\text { Episodes } \\
(\mathbf{n}=10)\end{array}$} & \multicolumn{2}{|c|}{$\begin{array}{l}\text { Overwhelming } \\
\text { Episodes } \\
(\mathbf{n}=10)\end{array}$} & \multicolumn{2}{|c|}{$\begin{array}{c}\text { Total } \\
\text { Episodes } \\
(\mathbf{n}=\mathbf{4 0})\end{array}$} \\
\hline & \multicolumn{10}{|c|}{ \# \& \% of episodes which resulted in this outcome } \\
\hline & \# & $\%$ & \# & $\%$ & \# & $\%$ & \# & $\%$ & $\#$ & $\%$ \\
\hline \multicolumn{11}{|l|}{ Episode Resolutions } \\
\hline Successful & 4 & 40 & 3 & 30 & 7 & 70 & 3 & 30 & 17 & 42.5 \\
\hline Mixed - got through it & 5 & 50 & 4 & 40 & 3 & 30 & 5 & 50 & 17 & 42.5 \\
\hline Unresolved & 1 & 10 & 3 & 30 & 0 & 0 & 2 & 20 & 6 & 15 \\
\hline
\end{tabular}

Table 12-5 Episode resolutions by respondent stress level and episode assessment

Whilst mixed resolutions and unresolved episodes were cited equally by the two groups of workers, the results show that highly stressed workers described more episodes as successfully resolved than low stress respondents (10 episodes to 7). Furthermore, highly stressed workers assessed more of the successfully resolved episodes as notoverwhelming ( 7 episodes to 3 ). This finding stands out because the other three episode groupings were equally likely to report successfully resolved role overload.

\subsection{Summary of key findings for role overload outcomes}

The focus of this chapter was the short term outcomes of role overload episodes as described by the overloaded health care workers at the end of semi-structured interviews. This chapter completes the step by step analysis of role overload appraisal and coping. 
The key findings from this chapter are summarised as follows. Findings for all or most role overload episodes and workers are summarised first (Section 12.3.1). The key role findings by role overload episode assessment follows in Section 12.3.2, and by respondent stress level in Section 12.3.3. Finally, a fourth subsection summarises the findings from this chapter by worker stress level and episode assessment.

\subsubsection{Key findings for all episodes}

In the first section of this chapter workers' descriptions of personal outcomes from role overload were examined. The results revealed that positive and negative emotions and feelings were commonly cited in $62.5 \%$ and $52.5 \%$ of episodes respectively, suggesting it is not unusual for both to co-exist during the role overload appraisal and coping process. A notable finding from the first section was that emotions and feelings expressed at this stage of the respondent interviews were more varied than those described when workers initially described appraising role overload. In particular, positive emotions and feelings were more common at this stage of the role overload appraisal and coping process, and mostly mild and low arousal in nature (as defined by Russell, 1980). For many, negative emotions were also consistent with low arousal emotions, whilst for other workers, negative emotions and feelings remained intense and upsetting. Finally, results from the first section also suggested that some of the workers in our sample gained new skills from coping with role overload whilst others suffered negative workload consequences in other areas of their lives as a result of their role overload experience. 
In the second section of this chapter, workers described three types of episode resolution. According to the hospital workers, well over half of the episodes described, involved elements that were not resolved satisfactorily, or that remained unresolved.

\subsubsection{Key role overload outcome findings by episode assessment}

Table 12-8 summarises the key role overload outcome findings according to episode assessment. These results are presented with no regard to respondent stress.

\begin{tabular}{|c|c|c|}
\hline \multirow{2}{*}{$\begin{array}{c}\text { Role } \\
\text { Overload } \\
\text { Episode } \\
\text { Assessment }\end{array}$} & \multicolumn{2}{|c|}{ Role Overload Outcomes } \\
\hline & Personal Outcomes & Episode Resolution \\
\hline $\begin{array}{c}\text { Not- } \\
\text { overwhelming }\end{array}$ & $\begin{array}{l}\text { Positive Emotions \& Feelings } \\
\quad \text { Relieved } \\
\text { Good } \\
\text { Personal growth }\end{array}$ & Successful \\
\hline Overwhelming & $\begin{array}{l}\text { Negative Emotions \& Feelings } \\
\text { Stressed/overwhelmed } \\
\text { Other intense negative emotions } \\
\text { Other mild negative emotions } \\
\\
\text { Negative Consequences } \\
\text { Negative impact to health/wellbeing }\end{array}$ & Unresolved \\
\hline
\end{tabular}

Table 12-6 Key outcomes for role overload assessed as overwhelming and not-overwhelming without regard to respondent stress level

Findings from this chapter suggest that the relationship between role overload outcomes and episode assessment are consistent with what we would expect from the theory. Positive emotions, feelings, and personal growth were more likely to result from role overload in episodes subsequently assessed as not-overwhelming. Such episodes were also more likely to be associated with successful outcomes. Negative emotions and feelings, and negative impacts to health and wellbeing, were more likely to be mentioned 
in role overload episodes evaluated as overwhelming. Respondents were also more likely to report that these episodes were unresolved.

\subsubsection{Key role overload outcome findings by respondent stress level}

Table 12-9 summarises the key personal outcome and episode resolution findings according to hospital worker stress level. These results are presented with no regard to whether the episodes were assessed as overwhelming or not. Notably, there were no role overload outcome factors that were mentioned more often by respondents with low stress.

\begin{tabular}{|c|c|c|}
\hline \multirow{2}{*}{$\begin{array}{c}\text { Respondent } \\
\text { Stress } \\
\text { Level }\end{array}$} & Personal Outcomes & Epole Overload Outcomes \\
\cline { 2 - 3 } Low & & \\
\hline High & $\begin{array}{c}\text { Good } \\
\text { Satisfied } \\
\text { Negative consequences: } \\
\text { Spillover }\end{array}$ & \\
\hline
\end{tabular}

Table 12-7 Key outcomes for role overload of high and low stress respondents without regard to episode assessment

In contrast, the data reported in this chapter revealed that highly stressed workers were more likely to recall feeling good or satisfied at the end of role overload episodes. Negative consequences, especially the spill-over of role overload to other domains, were also noted more often by this group. Finally, the results also support the idea that negative emotions and feelings, and perceptions of episode resolution were unrelated to respondent stress. 


\subsubsection{Key findings by episode grouping}

Table 12-10 summarises the notable coping strategy findings for the four episode groupings. Good feelings arising at the end of role overload were recalled more frequently in the not-overwhelming episodes of highly stressed workers, whilst these workers were stressed and overwhelmed notably more often at the end of episodes they evaluated as overwhelming. In addition, the highly stressed individuals in this sample never mentioned that they had achieved personal growth hen describing episodes of overwhelming role overload. This finding is noteworthy because such gains were mentioned equally often by workers in the other three episode groupings.

\begin{tabular}{|c|c|}
\hline $\begin{array}{c}\text { Not-overwhelming episodes - low stress } \\
\text { Personal growth }\end{array}$ & $\begin{array}{c}\text { Not-overwhelming episodes - high stress } \\
\text { Personal growth } \\
\text { Good } \\
\text { Tired \& exhausted } \\
\text { Successfullv resolved } \\
\end{array}$ \\
\hline Overwhelming episodes - low stress & Overwhelming episodes - high stress \\
\hline $\begin{array}{l}\text { Personal growth } \\
\text { Tired \& exhausted }\end{array}$ & $\begin{array}{l}\text { Stressed \& overwhelmed } \\
\text { Tired \& exhausted }\end{array}$ \\
\hline
\end{tabular}

Table 12-8 Summary of role overload outcome factors by episode grouping

Lastly, low stress workers never complained of feeling tired and exhausted at the end of role overload, a finding that is notable in light of the data showing that these feelings were equally expressed in the episodes of the other three groupings. 


\section{PART SIX: DISCUSSION and REFINEMENT of CONCEPTUAL FRAMEWORK}

The purpose of this research is to explore the factors influencing the appraisal of stress by individuals facing role overload at work and at home. More specifically this study examines the relationship between role overload and perceived stress guided by the framework developed in Part Three. In this part of the thesis the guiding framework is refined to reflect the findings discussed in Part Five.

Part Six is divided into two chapters dedicated to the two research objectives of this thesis. Chapter 13 addresses the first objective with a discussion of a framework for understanding the role overload appraisal and coping process. Chapter 14 discusses the factors that speak to the second research objective of this thesis: why and how some people suffer more stress in role overload situation than others. 


\section{Development of a conceptual framework to explain the relationship between role overload and perceived stress}

The first objective of this thesis was to develop a theoretical framework describing the relationship between role overload and perceived stress. Figure 13-1 uses the findings from this thesis to define a framework that represents the role overload appraisal and coping process as described by female hospital workers. Whilst space does not allow all the details of the process elements to be displayed in the figure below; all relevant details are discussed separately in the subsections to follow.

Central to this framework (Figure 13-1) are the cognitive processes that link the person (Element 1) and the role overload environment (Element 2). More specifically, the framework posits that role overload will be appraised as stressful (i.e. challenging, threatening and or harmful) if the mismatch between role expectations and time and energy resources is appraised as having personal significance (Element 3 - primary appraisal) and or strains or exceeds the individuals resources for coping (Element 4 secondary appraisal). According to our analysis, once the role overload episode is appraised as stressful, coping strategies (Element 5) will be initiated to reduce and manage the demands of role overload resulting in a number of outcomes (Element 6), including emotions and feelings, other short term personal consequences, and episode resolution outcomes. It should be acknowledged that whilst the cognitive appraisal and coping processes included in the framework shown in Figure 13-1 are represented in a sequential manner with a return arrow shown between Elements 6 and 3, this is for the sake of simplicity as primary appraisal, 
Figure 13-1 Conceptual framework of role overload appraisal and coping process

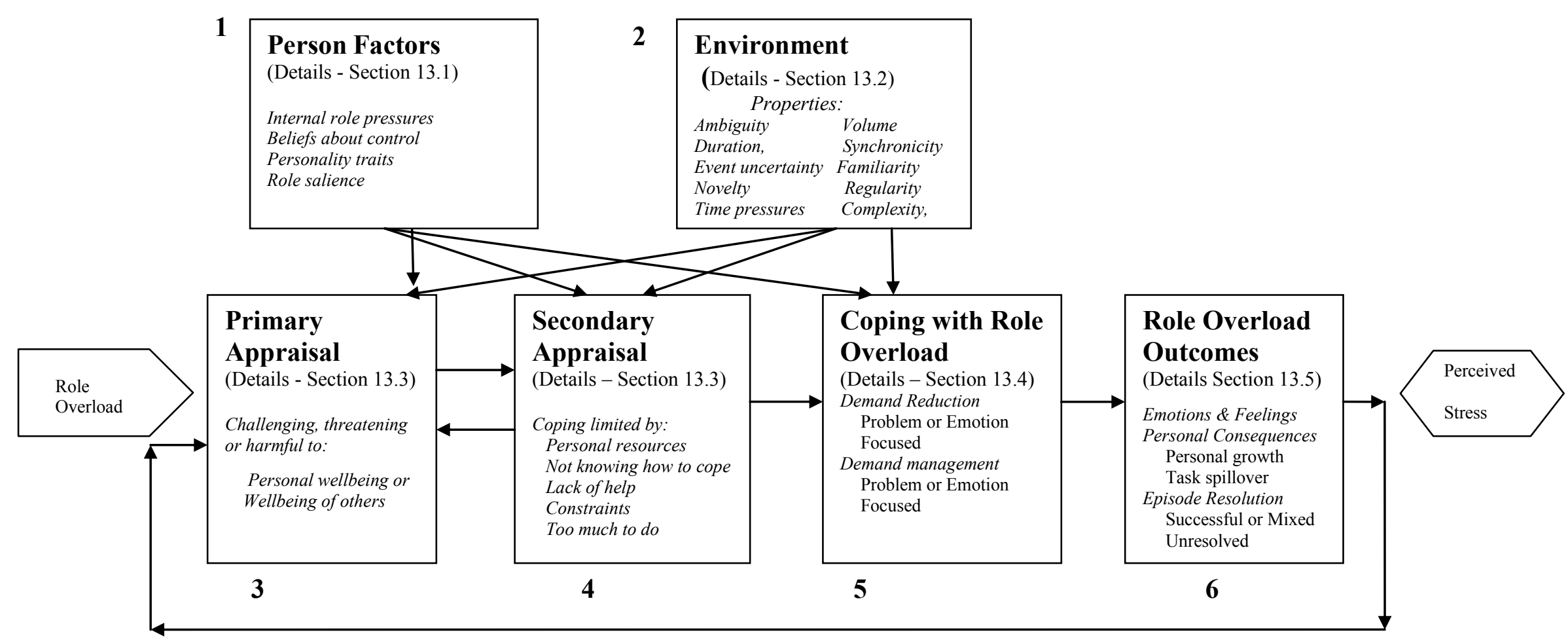


secondary appraisal, coping efforts and the resulting feelings and emotional expressions of stress can overlap and moreover, are repeated in a continuous iterative process.

Overall the refined framework is consistent with the guiding framework for this case study (see Part 3: Section 4.1). This is to be expected given the fact that: (1) the guiding framework was based on Lazarus and Folkman's (1984) view of the stress appraisal coping process and Kahn et al.'s conceptualization of role overload, and (2), role overload is known to be a type of role stress consistently linked to psychological stress (Santos et al. 2003; Pearson, 2008; Glynn, Maclean, Forte and Cohen, 2009). It is important to note, however, that although many of the factors identified in the elements of Figure 13-1 are also consistent with Lazarus and Folkman's (1984) original view of the stress appraisal process, this study has also identified areas that are either unique to role overload appraisal and coping, or that have been modified somewhat by their application to an examination of role overload.

The remainder of this chapter will discuss the findings for each element of the framework focusing on the similarities and differences between the role overload appraisal and coping process presented in Figure 13-1 and the conceptual framework developed in Part 3 of this thesis. Section 13.1 discusses person factors most important in evaluating the significance of role overload to wellbeing whilst the situation properties of the role overload environments that make them potentially stressful are discussed in Section 13.2. Section 13.3 discusses the cognitive processes through which individuals evaluate the implications of role overload for well-being and also how they might deal with the role overload. Role overload coping strategies are discussed in Section 13.4 
followed finally, by a discussion of the short term outcomes arising from these episodes (Section 13.5).

Before beginning the discussion, it is important to re-iterate that the framework of role overload appraisal and coping represents a complex set of interdependent appraisals, emotions and coping responses. As explained by Lazarus and Folkman, discussions of any variable in this process will therefore include references to other variables. For example, whilst Lazarus and Folkman (1984, p. 55) defined 'commitment' as a person factor that expresses what is important to a person, they also note that "for a commitment to influence appraisal, it has to be engaged by a particular encounter". In the sections below, whilst the discussions will focus on the factors found to be most important to the specific element of the process, the relationship between those factors and other elements of the process will sometimes form an essential part of the analysis.

\subsection{Person factors}

The role of person factors in stress appraisal and coping has been the subject of considerable debate in the stress literature for many years (see discussion in Section 3). Most researchers in the area now acknowledge the role of both the person and the environment in the stress appraisal and coping process (Lazarus, 1993; Folkman and Moskovitz, 2004). During role overload appraisal, person factors shape an individual's

view of an event and, as a consequence, the emotional reaction and subsequent coping efforts (Lazarus and Folkman, 1984). Details of the person factors identified in this study are provided in Figure 13-2 below. 
Internal role pressures pushing individuals to meet personal goals and values, and beliefs about control during a role overload episode were the most noted person factors described by hospital workers as being influential during the appraisal of role overload. Together, internal role pressures and beliefs about control were mentioned in three quarters of the role overload episodes described. Lazarus and Folkman's (1984) stress appraisal and coping model identified two person factors (commitments and beliefs), which are very similar in nature to the concepts of internal role pressures and beliefs identified in this study. According to Lazarus and Folkman (1984), commitments are based on what is important to the individual (analogous to internal role pressures), whilst beliefs involve ones relationship with the environment.

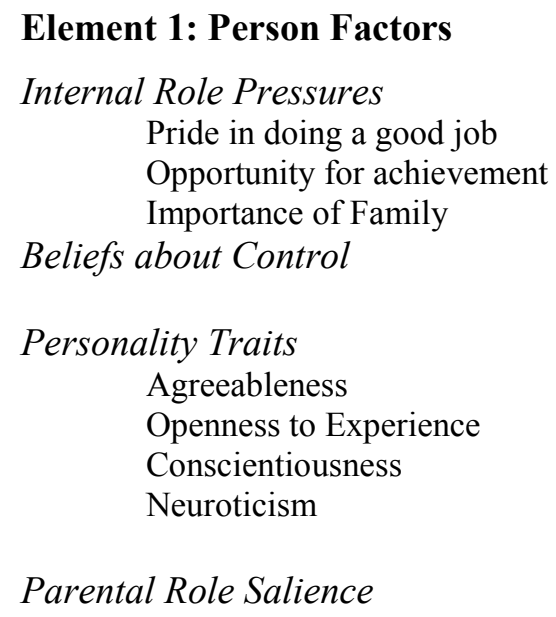

Figure 13-2 Person factors in the role overload appraisal and coping process

It is also important to note that in a quarter of the role overload episodes examined in this thesis, respondents did not mention specific person factors. This finding might imply that for these respondents other factors (e.g. properties of the role overload environment or coping resource availability) were uppermost in their minds when recalling these episodes. Alternatively, it may be that person factors are less relevant to the role overload experience than to the appraisal of other stressors. Personality traits and 
role salience factors, measured for each worker independently of the recollections of role overload episodes, however, reinforce the idea that person factors play an influential part in role overload appraisal and coping as described by Lazarus and Folkman (1984).

Each person factor identified in Figure 13-2 will be discussed, in the four subsections to follow, with reference to the literature, to the data in this case study, and to their influence on role overload appraisal and coping. Throughout the discussion, similarities and differences between the framework presented in Figures 13-1 \& 13-2 and the guiding framework for this study (based on the stress appraisal process defined by Lazarus and Folkman (1984)) will be highlighted.

\subsubsection{Internal role pressures}

Internal role pressures can be described as pressures that individuals place on themselves according to personal expectations about role participation (Kahn et al., 1964). Hospital workers implicated internal role pressures in role overload appraisal in nearly two thirds of the role overload episodes recollected. What stands out from this data is that in over half of these cases, workers described how they 'took pride in being good at their role'. This suggests that concerns about the ability to get work done to personal standards were particularly important, both to these individuals and to the perception of role overload. As described by the women in this study, the personal, internally held pressure to do a good job resulted in appraisals of stress when role overload compromised their ability to meet these personal standards.

Other, albeit less frequently mentioned, internal role pressures, including the opportunity for accomplishment, and the importance of family, were also identified as 
person factors that impact the appraisal of role overload in this study. In line with Lazarus and Folkman's theory of stress appraisal, the data in this case study thus suggests that whilst taking pride in doing a good job is the most notable and influential person factor for many hospital workers, when role overload arose some workers felt stressed because of threats to personal quality standards whilst others felt stressed because of threats to family life.

The data from the study also support the idea that the subjective feelings of role overload and associated psychological stress resulted from a unique confluence of person and environment factors. These findings may help inform the recurring debate in the literature about qualitative versus quantitative role overload (see Section 2.2) as discussed below.

Kahn et al. argued that role overload is a composite of aspects of inter-sender role conflict and person-role conflict. It is the latter aspect of role overload (i.e. person-role conflict) in which internal role pressures, such as taking pride in doing a good job, can play a part. More specifically, for many respondents in the thesis sample, role overload arose because externally sent role expectations could not be completed in line with internal expectations (i.e., personal standards) within a given time limit. This finding is consistent with Katz and Kahn's (1966) comment that role overload often involves a choice between quantity and quality (i.e. the amount that can be done versus the quality of the work that can be done). It is also consistent with the fact that some researchers differentiate between quantitative and qualitative forms of role overload (see Chapter 2).

Based on Lazarus and Folkman's (1984) stress appraisal model, the results of this study suggest that issues about the quality of work to be done arose from internal 
commitments to personal standards rather than from a lack of skills or knowledge as posited in the literature. Moreover, as person factors, internal role pressures depend on what is important to the individual and could vary from person to person (as noted in this thesis). Future research is required to see if the internal role pressure to do a good job is a common factor of role overload for men and for individuals working in other sectors.

\subsubsection{Beliefs about control}

In one third of the episodes recalled, the hospital workers reported that lack of control was influential in the appraisal of role overload. No other references to personal beliefs were noted by respondents in a substantive number of episodes. These findings are consistent with Lazarus and Folkman's (1984) view that beliefs about personal control are a particularly powerful person factor, and are the most relevant beliefs affecting cognitive appraisals of stress and the subsequent emotional reactions and behaviour. Other research has also shown that control over the environment in potentially stressful encounters is important for personal well-being (Barnett and Baruch, 1985; Cooper et al., 2001).

Lazarus and Folkman (1984) specified that beliefs about personal control were either related to generalised dispositional ways of thinking, or were judgments about specific encounters. When a lack of control was mentioned in this study, workers either felt that they were powerless to avoid or manipulate the demands causing role overload or they mentioned a lack of control over the availability of coping resources. In other words, the respondents in this study who mentioned lack of control were referring to specific encounters not personal temperament. 


\subsubsection{Personality traits}

Personality can affect the stress appraisal and coping process by influencing the nature and severity of the stress experienced as well as the selection and effectiveness of coping strategies (Bolger and Zuckerman, 1995; Penley and Tomaka, 2002; Lazarus, 2007). Consistent with the guiding framework based on the stress, appraisal, and coping literature, findings in this case study suggest that the personality traits measured may be influential in the role overload appraisal and coping process. Key findings in this area are discussed below.

First, most respondents reported high levels of agreeableness and openness to experience. The qualities associated with these traits, as defined by Gosling et al. (2003) (i.e., a warm and sympathetic demeanour, and a willingness to take on new challenges), suggests that the overloaded women in our sample may be more likely than those with lower levels of agreeableness and openness to experience to take on too many role demands. It would appear that these two traits seem to be characteristic of individuals with high levels of role overload, such as those selected for this study. This idea is also supported by Lazarus and Folkman (1984) who argue that the greater the strength of personal commitment the more vulnerable the person is to psychological stress in that area. A warm and sympathetic demeanor (i.e. high levels of agreeableness), would be expected in individuals committed to health care, whilst high levels of openness to experience suggest a personal commitment to making extra effort in challenging situations. These commitments may push an individual towards a course of action that would increase role demands and make them more vulnerable to role overload consistent with the selected sample. 
Another important finding in this study relates to the workers' reported levels of conscientiousness and neuroticism. The neuroticism score noted for the whole sample was higher than average, a finding which may be explained by past research that has shown that women tend to score higher than men for neuroticism (Lynn and Martin, 1997). Respondents who reported lower levels of perceived stress, however, tended to report lower levels of neuroticism (i.e., a calmer and more emotionally stable demeanour) than workers with higher levels of stress. The low stress respondent group also tended to report higher levels of conscientiousness (i.e., self disciplined). These findings suggest that secondary appraisals of coping resources, the options for dealing with role overload, their potential outcomes, and how effective they might be, may result in a more positive reaction for such conscientious, less neurotic people. This interpretation of the data is consistent with findings in the literature showing that conscientiousness and neuroticism

are respectively, positively and negatively related to perceived coping capability (Penley and Tomaka, 2002). The relationship between these traits and perceived stress will be discussed further in Chapter 14.

\subsubsection{Role salience}

Role salience, which reflects the importance and the efforts people put into roles determined to be central to their lives (Greer and Egan, 2012), was also identified as a potentially influential person factor in the appraisal of role overload. Although this concept was not specifically mentioned in Lazarus and Folkman's (1984) stress appraisal and coping theory, role salience is nevertheless consistent with their idea of 
commitments, as "an expression of what is important" to an individual that helps determine what is at stake in a stressful encounter (p.80).

A large majority of the females in this study were found to have high levels of parental role salience. Gender role theory prescribes that traditional gender roles impose different levels of importance for men and women such that men place more value on their work and for women the family role is more important (Gutek, Searle and Klepa, 1991; Korabik, McElwain, \& Chappell, 2008). Although there are discrepancies about whether gender factors into parental role salience (Greer and Egan, 2012), gender role theory would suggest that the high levels of parental role salience found in this study are consistent with the fact that all respondents in this study are female.

The data from this study showed that for these women role overload at work was mentioned more often as a source of role overload than either overload at home or role overload across both role domains. Whilst work may just be more troublesome than other roles for these individuals, the findings with respect to role salience suggest that there may be a difference in how these employees view overload at work (negatively impacts performance of parental role) versus parental role overload (time in parental role is accepted and enjoyed). Future research is required to further examine if role overload at work and at home is viewed differently by men and women and to what extent any differences are related to parental role salience. 


\subsection{Role overload environment}

According to Lazarus and Folkman (1984), it is not the substance of the situation that determines whether an event is perceived as stressful; rather, stress levels depend upon the individual's appraisal of the situation's relevance to well-being. This would suggest that in order to determine whether or not role overload creates the potential for appraisals of harm, threat or challenge, we have to understand the form or appearance of the situations that was influential in respondent feelings of overload - what is referred to in this thesis as the 'situation properties' (see Element 2 of Figure 13-1). A taxonomy listing role overload situation properties was developed from the findings presented in Chapter 8. The taxonomy is summarised for discussion purposes in Table 13-1. In keeping with Lazarus and Folkman's focus on what makes the individual appraise an event as stressful, the taxonomy presented here identifies the underlying properties of role overload situations that were described as being influential in determining feelings of role overload and hence some degree of stress. There has been little research into the properties of situations that can lead to stressful appraisals generally speaking. No research whatsoever was found that examined role overload properties specifically. The taxonomy developed in this thesis addresses this gap in the literature and, consistent with the aims of this thesis, increases our understanding of role overload appraisal and its relationship with stress.

The taxonomy developed in this case study includes a wide variety of situation properties that were described by hospital workers as making role overload potentially harmful, threatening or challenging. Descriptions by hospital workers suggest that the presence of any one of the properties in Table 13-1 can create the potential for a stressful 
appraisal of role overload, and as reported by Lazarus and Folkman (1984), multiple properties can be present in a single episode. The variety of properties identified suggests that typically there is a lot more going on during role overload appraisal than a simple evaluation that there is 'too much to do and not enough time to do it'.

In comparing this taxonomy to the guiding framework for this thesis, the role overload properties identified can be divided into three groups: (1) those that are consistent with the situation properties of the same name identified by Lazarus and Folkman (1984); (2) those that share similarities with properties in Lazarus and Folkman's model of stress appraisal and coping and with Kahn et al.'s definition of role overload; and finally, (3) role overload properties that are newly identified from the thesis data. The role overload properties presented in the table are discussed below with reference to the existing literature, the data in this case study, and their potential impact on role overload appraisal. Focusing on the similarities and differences with the guiding framework for this thesis, the properties are discussed in the order they appear in the table beginning first with the role overload properties consistent with properties identified by Lazarus and Folkman (1984). 


\begin{tabular}{|c|c|c|c|c|}
\hline $\begin{array}{l}\text { Role Overload } \\
\text { Property }\end{array}$ & Description & Examples & Link to Kahn et al. (1964) & $\begin{array}{c}\text { Link to Lazarus \& } \\
\text { Folkman (1984) stressor } \\
\text { property }\end{array}$ \\
\hline Group 1 & \multicolumn{4}{|c|}{ Role overload properties consistent with situation properties relevant to the stress appraisal process } \\
\hline Ambiguity & $\begin{array}{c}\text { Lack of clear guidelines made } \\
\text { it difficult to respond to } \\
\text { demands, or set priorities } \\
\text { leading to inadequate role } \\
\text { performance }\end{array}$ & $\begin{array}{l}\text { I had little information from my boss on } \\
\text { what her agenda for this presentation } \\
\text { was. (This led to ) frustration at the lack } \\
\text { of clarity of what was required }\end{array}$ & $\begin{array}{l}\text { Role ambiguity - lack of } \\
\text { information required for } \\
\text { adequate role performance }\end{array}$ & $\begin{array}{c}\text { Matched by Ambiguity - } \\
\text { When information is unclear } \\
\text { or insufficient }\end{array}$ \\
\hline Duration & $\begin{array}{l}\text { An event of lengthy or } \\
\text { continuous duration. Often } \\
\text { there was no foreseeable } \\
\text { resolution to the issue. }\end{array}$ & $\begin{array}{l}\text { I couldn't see an end, even after I } \\
\text { refocused and looked to see what I } \\
\text { could do. }\end{array}$ & N/A & $\begin{array}{l}\text { Matched by Duration - How } \\
\text { long a stressful event persists }\end{array}$ \\
\hline $\begin{array}{c}\text { Event } \\
\text { uncertainty }\end{array}$ & $\begin{array}{l}\text { Unexpected role overload } \\
\text { events happening out of the } \\
\text { blue }\end{array}$ & $\begin{array}{l}\text { I am always a little worried about } \\
\text { something unexpected coming up }\end{array}$ & N/A & $\begin{array}{l}\text { Matched by Event uncertainty } \\
\text { - not knowing whether an } \\
\text { event is going to occur }\end{array}$ \\
\hline Novelty & $\begin{array}{l}\text { Role demands that are either } \\
\text { new to the respondents, or } \\
\text { where the respondent has no } \\
\text { knowledge of the event context }\end{array}$ & I had to learn this new job. & N/A & $\begin{array}{l}\text { Matched by Novelty - } \\
\text { situations with which the } \\
\text { person has not had any } \\
\text { previous experience }\end{array}$ \\
\hline Group 2 & \multicolumn{4}{|c|}{ Role overload properties sharing some characteristics with situation properties influential in the stress appraisal process } \\
\hline Time pressures & $\begin{array}{l}\text { Strict time constraints and } \\
\text { deadlines set by role set } \\
\text { members. Role incumbents } \\
\text { lack spare time to respond to } \\
\text { demands } \\
\end{array}$ & $\begin{array}{c}\text { I guess just sort of chronically feeling } \\
\text { time pressed, and if we had the luxury of } \\
\text { four hours I don't know I would feel so } \\
\text { pressured. }\end{array}$ & $\begin{array}{l}\text { Role overload -Time limits make } \\
\text { the completion of all role } \\
\text { demands virtually impossible }\end{array}$ & $\begin{array}{l}\text { Similar to 'Imminence' - How } \\
\text { much time there is before an } \\
\text { event occurs }\end{array}$ \\
\hline $\begin{array}{l}\text { Volume of } \\
\text { demands }\end{array}$ & $\begin{array}{l}\text { Volume of role demands faced } \\
\text { by role incumbent }\end{array}$ & $\begin{array}{c}\text { It was the sheer volume of looking at ... } \\
\text { the amount of work that needed to be } \\
\text { done. }\end{array}$ & $\begin{array}{l}\text { Role overload -Sizable number } \\
\text { in labor force have too heavy a } \\
\text { workload }\end{array}$ & N/A \\
\hline $\begin{array}{c}\text { Synchronicity } \\
\text { of-demands } \\
\text { of a different } \\
\text { nature }\end{array}$ & $\begin{array}{c}\text { Multiple demands of a different } \\
\text { nature occurring in close } \\
\text { temporal proximity }\end{array}$ & $\begin{array}{l}\text { I had a big presentation, our computer } \\
\text { systems were being merged, there was a } \\
\text { lot of expansion going on, and my dad } \\
\text { had a heart attack all in the same day. }\end{array}$ & $\begin{array}{l}\text { Role overload - uncoordinated } \\
\text { sendings of logical compatible } \\
\text { but different role demands }\end{array}$ & Similar to Timing of event \\
\hline
\end{tabular}




\begin{tabular}{|c|c|c|c|c|}
\hline $\begin{array}{l}\text { Role Overload } \\
\text { Property }\end{array}$ & Description & Examples & Link to Kahn et al. (1964) & $\begin{array}{c}\text { Link to Lazarus \& } \\
\text { Folkman (1984) stressor } \\
\text { property }\end{array}$ \\
\hline $\begin{array}{c}\text { Synchronicity } \\
\text { of-additional } \\
\text { demands } \\
\end{array}$ & $\begin{array}{l}\text { Demand(s) in addition to an } \\
\text { existing work load }\end{array}$ & $\begin{array}{c}\text {.when you're doing things like that, for } \\
\text { the organization, you are still expected } \\
\text { to continue in your role. You still need } \\
\text { to do all your other work. }\end{array}$ & $\begin{array}{l}\text { Role overload - uncoordinated } \\
\text { sendings of logical compatible } \\
\text { and similar role demands }\end{array}$ & Similar to Timing of event \\
\hline $\begin{array}{c}\text { Synchronicity } \\
\text { of - } \\
\text { simultaneous } \\
\text { demands } \\
\end{array}$ & $\begin{array}{l}\text { Multiple demands occurring at } \\
\text { the same instant }\end{array}$ & $\begin{array}{l}\text { I am in my office with a patient ...and I } \\
\text { receive a telephone call .. and a page on } \\
\text { my pager and a knock on my door } \\
\text { simultaneously from staff in the clinic }\end{array}$ & $\begin{array}{l}\text { Role overload - logically } \\
\text { compatible role demands } \\
\text { impossible to complete at the } \\
\text { same time } \\
\end{array}$ & Similar to Timing of event \\
\hline Group 3 & \multicolumn{4}{|c|}{ Unique role overload properties described as influential in the appraisal process } \\
\hline Familiarity & $\begin{array}{l}\text { Format and context of role } \\
\text { overload situation is familiar }\end{array}$ & $\begin{array}{c}\text { It had been a situation I had to deal } \\
\text { with before }\end{array}$ & N/A & $\begin{array}{c}\text { Similar to 'Degree of } \\
\text { Novelty': appraisal inferences } \\
\text { are based on related previous } \\
\text { experience. } \\
\end{array}$ \\
\hline Regularity & $\begin{array}{l}\text { Involving role demands that } \\
\text { happen on a regular basis, often } \\
\text { daily, }\end{array}$ & It was not uncommon & $\mathrm{N} / \mathrm{A}$ & N/A \\
\hline Complexity & $\begin{array}{c}\text { Involving a complex set of role } \\
\text { demands requiring intense } \\
\text { focus }\end{array}$ & $\begin{array}{c}\text {....too sick of a patient, too many things } \\
\text { could go wrong, that things wouldn't } \\
\text { get done and the patient would die, it } \\
\text { was pretty complex. }\end{array}$ & N/A & N/A \\
\hline
\end{tabular}

Table 13-1 Taxonomy of role overload situation properties that create the potential for stressful appraisals 
Table 13-1 is structured as follows. The first and second columns list and describe the twelve role overload situation properties identified from the analysis. An example quote from the hospital workers referencing these properties is included in column three. The fourth column links each of the role overload situation properties identified in this study to the role theories of Kahn et al. (1964). Similarly, the fifth column links each of the role overload properties identified in this research to matching or similar situation properties from Lazarus and Folkman's (1984) taxonomy of properties that create the potential for stressful appraisals.

\subsubsection{Role overload properties consistent with situation properties relevant to the stress appraisal process}

When describing what it was about the episodes recalled that made them feel overloaded respondents identified four properties (ambiguity, duration, event uncertainty and novelty) that are consistent with those identified by Lazarus and Folkman (1984). This finding suggests that these properties, which are especially relevant to the process through which stressful appraisals are determined, are common across multiple stressor types (i.e. appraisal of stress, appraisals of overload).

Ambiguity When talking about what made them feel overloaded, hospital workers referred to ambiguous role demands especially with respect to a lack of guidelines or information about priorities. How these women referred to ambiguity is consistent with Lazarus and Folkman's (1984, p.103) argument that ambiguity occurs "when the 
information necessary for appraisal is unclear or insufficient". The frequency with which this role overload property was cited (in one in three episodes) is also consistent with Lazarus and Folkman's view that ambiguity is a common feature in potentially stressful encounters.

The lack of clear instruction about role responsibilities described by respondents intuitively suggests that hospital workers would experience an increased workload as they seek to discover the exact nature of the expectations demanded of them or have to re-do tasks. The data also indicated that in some episodes, the inability to prioritise due to lack of clear guidelines contributed to difficulties in the identification of coping resources. Lazarus and Folkman's stress appraisal model suggests that in these cases role overload would be appraised more negatively.

Ambiguity, as expressed by these female hospital workers is also consistent with Kahn et al.'s definition of role ambiguity, a form of role stress that co-occurs with role overload according to their studies. Research into the relationship between role overload and role ambiguity is sparse, and this thesis found only one study providing empirical evidence for the co-existence of role ambiguity and role overload for workers in the health sector (Chang and Hancock, 2003). The paucity of research investigating the connection between role ambiguity and role overload (identified in the literature review of this thesis) is unfortunate given that the data from this study support the idea that role ambiguity can be a property of role overload that can create the potential for appraisals of harm, threat, or challenge. 
Duration Lazarus and Folkman (1984) defined 'duration' to be the length of time during which a potentially stressful event is occurring. In this study, the 'duration' of role overload episodes refers to how long the role overload events have been unfolding. In some cases, the role overload was still ongoing when the hospital workers were interviewed. The duration of role episodes was not a property of role overload identified by Kahn et al. (1964).

Lazarus and Folkman (1984) argued that the impact of duration on stress appraisal can be mixed. For example, individuals may become habituated and tolerant of ongoing events, leading to more positive appraisals. Seyle's (1956) research on stress, on the other hand, suggests that long lasting events lead to resistance and possible exhaustion and hence could have a negative impact on appraisal, an explanation more in keeping with the data in this study. In this case study, duration was positively associated with a negative view of the role overload situations. Most problematical was when hospital workers could not see an end to the episode.

Event uncertainty Only a small number of respondents identified the third role overload property linked closely to stress appraisal theory, event uncertainty (mentioned in $10 \%$ of episodes). The feeling of not knowing whether an event will occur is consistent with Lazarus and Folkman's (1984) definition of this situation property. The results from this study also suggest that hospital workers felt unprepared for unexpected events. Whilst event uncertainty does not feature in discussions of role overload in the literature, the idea that inadequate personal resources may contribute to feelings of role overload is consistent with Kahn et al.’s (1964) definition of role overload. 
Novelty When describing what made them feel overloaded, a few hospital workers specifically identified situations which were new to them. In these cases, workers also talked about the learning curves they faced, suggesting that learning to do a new task may contribute to role overload. These unfamiliar situations are consistent with Lazarus and Folkman's concept of 'novelty'. According to Lazarus and Folkman (1984, p. 84) such a situation "encourages appraisal inferences based on related previous experiences or on general knowledge”. While such related experiences may have been positive or negative, in the role overload situations described in this study the data associates novelty with increased overload, suggesting the potential, therefore, for a more threatening appraisal.

This property of role overload was not explored in Kahn et al.'s discussions of role overload. The lack of specific knowledge described by respondents when recalling new situations that contributed to feelings of overload is another factor suggestive of descriptions of qualitative role overload. Defined to be work that exceeds an individual's skills or abilities, qualitative role overload was differentiated from quantitative role overload (work that cannot be completed in the time allowed) in the early studies (Sales, 1969; French and Caplan, 1972) that followed Kahn et al.'s research into organizational stress. In this case study, however, concerns about the lack of knowledge described in novel situations was more about the extra work it created to learn new techniques rather than the lack of skills to do the job. This interpretation is consistent with Beehr and Glazer (2005) who concluded in their recent review of role stress that most role overload research focuses first on the inconsistencies between the amount of work and the time available to do (i.e. quantitative role overload) rather than on the mismatch between available skills and role demands. 


\subsubsection{Role overload properties sharing some characteristics with situation properties influential in the stress appraisal process}

Five role overload properties identified in this thesis overlap, to some degree or another, in content with properties identified in Lazarus and Folkman's (1984) model of stress appraisal and coping. All five (time pressures, volume of demands, and three forms of synchronicity of demands) were also cited in Kahn et al.'s (1964) descriptions of role overload, suggesting they may be role overload specific forms of the situation properties. Details of each of these properties are given below.

Time pressures The most frequent property of role overload described by the female hospital workers in this study involved 'time pressures'. These women talked often about the insufficiency of time available to fulfill role expectations and of being pressed for time. According to Lazarus and Folkman (1984), the cognitive appraisal process is heavily influenced by the amount of time, a property they label 'imminence' and specifically define as the amount of time before a potentially stressful event occurs. While (imminent) deadlines were occasionally mentioned by the respondents, in all cases they were talked about in a way that implied that they contributed to time pressures rather than caused them. In this study, the label of imminence was, therefore, rejected for this role overload property in favour of 'time pressures'.

It is also worthwhile to note that comments offered by hospital workers during the interviews suggesting that the lack of time available impacts the ability to cope with role demands, are consistent with Lazarus and Folkman (1984) who argue that shorter timescales reduce the opportunity for mediating coping processes, thus increasing the likelihood of threat appraisals. 
Volume of demands In this study, the number of episodes in which hospital workers described the perceived amount of demands as being influential in their appraisal of role overload was second only to 'time pressures' in terms of frequency. The results showed that in many episodes respondents found the sheer volume of demands facing them quite daunting, suggesting that the greater the volume of the demand loads the more negative the appraisal. This role overload property has no equivalent in Lazarus and Folkman's (1984) taxonomy of situation properties. The assessment of demands in light of available resources is, however, a key part of the appraisal process according to Lazarus and Folkman (1984), suggesting that the volume of demands will be particularly influential during secondary appraisal of coping capabilities during role overload episodes.

The volume of role demands is an important factor of role overload as originally defined by Kahn et al. (1964). In their study of organisational stress, Kahn et al. (1964 p. 59) specifically noted that nearly half of the male workers they interviewed for their research indicated that they felt disturbed about having "too heavy a workload". Moreover, high or heavy workloads continue to be linked to organisational stress (Dewe et al., 2010). All together, these findings suggest that volume of demands is a role overload specific property that creates the potential for harm, threat or challenge.

\section{Synchronicity Workers described three different ways in which synchronised} role demands made them feel overloaded: (1) when demands are different in nature, (2) when they are added to an existing workload, and (3) when they arrive simultaneously. Taken together, these properties of synchronicity feature in over half of the episodes 
recalled for this thesis, which suggests synchronicity of demands is an important property of role overload. This finding is consistent with Kahn et al.'s (1964) notion of numerous demands competing for the time of a role incumbent.

Lazarus and Folkman (1984) also posit that events may have different personal significance if they occur in juxtaposition to other events. The idea of demands having to be dealt with at the same time as other demands shares some similarities with this concept. The thesis data showed, however, that each of the three properties featuring multiple synchronous demands are conceptually distinct in the data and do not occur together in any single episode. This finding suggests that the three forms of synchronous demands should be included as separate role overload properties in their own right, each with the potential to create challenging, threatening or harmful appraisals. Whilst the concept of event timing, described by Lazarus and Folkman, captures the general idea of synchronous demands competing for time, their focus on the synchronization between potentially stressful events and major life events does not capture the idiosyncrasies of the types and nature of the role demands characterizing these three role overload properties discussed below.

Comments by workers describing the synchronicity of different demands suggest that the different nature of the synchronous demands was the primary factor in their feelings of overload. The demands described in these cases were typically large or important events or projects, or involved crises. They could also arise in multiple domains. The variable characteristics of the multiple demands suggest that the time and effort to cope with them made them more burdensome, increasing the likelihood of an appraisal of harm, threat or challenge. Where the demands arose in multiple domains, the 
data also suggest that work-family conflict was part of the role overload experience. This finding is consistent with Kahn et al.'s contention that role overload can be comprised of both inter-role and person-role conflict.

A common concern described by workers facing additional demands on top of an existing workload (synchronicity of additional demands) was the potential of these new demands to negatively impact their ability to deal with existing demands. This suggests that the additional demands reduce the availability of coping resources for the current task load and therefore, according to Lazarus and Folkman (1984), increases the chance for a negative appraisal of role overload. If the expected outcomes of the existing workload also have some personal significance to the individual, then favouring the additional demands over existing demands will further increase the likelihood of threat appraisals.

The third role overload property focused on synchronous demands involving multiple short tasks received by the role incumbent at the same instant (synchronicity of simultaneous demands). Hospital workers described how difficult it was to respond to simultaneous demands, suggesting that secondary appraisal of coping capabilities was the main reason for the resultant psychological stress arising from role overload featuring this property.

\subsubsection{Unique role overload properties described as influential in the appraisal process}

The final three properties found to be influential during role overload appraisal have not been identified in previous studies. All three (familiarity, regularity and 
complexity) are properties that could be applicable to other potentially stressful situations and, therefore, are potential extensions to Lazarus and Folkman's general taxonomy.

Familiarity Data analysis determined that many role overload situations were familiar to hospital workers, either because the situation had occurred once before or, happened on a regular basis. Comments by workers suggest that appraisal of such role overload situations were based on past experiences. Whilst familiarity is logically the antithesis of Lazarus and Folkman's concept of novelty, we would expect that individuals would also tend to appraise familiar, potentially stressful, role overload experiences by referring to former experiences, as was found to be the case. This further suggests that familiarity is a situation property that creates the potential for harm, threat or challenge in its own right. A familiar role overload situation could, however, be evaluated positively or negatively, since the previous experiences on which appraisal will be based, may have been good or bad.

Focusing on impressions of past experiences rather than on the specifics of the current role overload episodes may also lead to judgment errors similar to the phenomenon referred to as halo error. Halo errors are classically discussed when overall impressions of individuals are based on single characteristics, but similar phenomena have been noted in research on other judgments (Murphy, Jako \& Anhalt, 1993). If respondents had problems with this type of situation in the past, halo errors in judgment may increase the chance that individuals will view current episodes negatively and vice versa. This, in turn, will impact secondary appraisal and potentially lead to more coping efforts than are needed or to ineffective coping respectively. Familiarity with, or previous 
knowledge of, a role episode has not been identified as a typical characteristic of role overload in the literature, suggesting that this situation property is not stressor specific.

Regularity In a small number of the episodes recalled for this thesis, when describing what made them feel overloaded, respondents noted that the role overload event reoccurred on a regular, periodic, basis (sometimes every day). The data also suggested that the qualities of repetitiveness and regularity described could be annoying and burdensome to these women. The regularity of a potentially stressful event was not noted by Lazarus and Folkman (1984) as a property creating the potential for harm threat or challenge. Neither have such qualities been explored in the role overload research literature. Descriptions of regular role overload by hospital workers suggest that these situations are familiar, but cannot always be predicted. Such familiarity suggests that hospital workers will draw on past experiences, good or bad during role overload appraisal as discussed above (i.e. the halo effect). The comments expressed by workers when these situations are repeated again and again suggests, however, that regular role overload has a negative personal significance and thus will increase the likelihood of a more threatening appraisal.

Complexity The final role overload property identified by hospital workers was also described in relatively few episodes. Nevertheless, the data suggest that considerable focus and effort was required on the part of workers when dealing with situations described as 'complex'. This suggests that coping resources may be appraised as inadequate or insufficient, thus creating the potential for increased psychological stress. 


\subsection{Cognitive appraisal of role overload}

The appraisal of role overload and its significance for well-being, as described by hospital workers, is consistent with the interdependent processes of primary and secondary appraisal (Elements 3 and 4 of Figure 13-1) in which individuals ask 'What's at stake?' and 'What can be done about it?' (as defined by Lazarus and Folkman, 1984). Primary and secondary appraisal of role overload will be discussed separately below, highlighting similarities and differences between role overload appraisal identified in this case study and that described by the guiding framework presented in Chapter 4 .

\subsubsection{What's at stake in role overload?}

The full details of the factors involved in role overload primary appraisal are summarized for discussion in Figure 13-3 below. During recollections of personal experiences of role overload, female hospital workers primarily evaluated role overload episodes in terms of their challenge or threat to individual well-being. Appraisals of harm, which were less frequent, referred to physiological symptoms, hurt feelings and damages to credibility. No other descriptors were offered by respondents for these stressful appraisals. Challenges were talked of in a majority of episodes, but the fact that challenges, threats, and harmful appraisals were all mentioned and coexisted in some cases is consistent with claims by Lazarus and Folkman (1984) that such appraisals are not necessarily mutually exclusive.

The narrative used by respondents to describe challenge and threat appraisals suggests different interpretations to Lazarus and Folkman's (1984) original definitions of these constructs. Lazarus and Folkman (1984) argue that the possibilities of personal 
benefits are a key characteristic of challenge appraisals, which would typically be associated with pleasurable emotions such as excitement or exhilaration. Threats, on the other hand, focus on the potential for harm and are characterized by negative emotions such as anxiety, fear and anger.

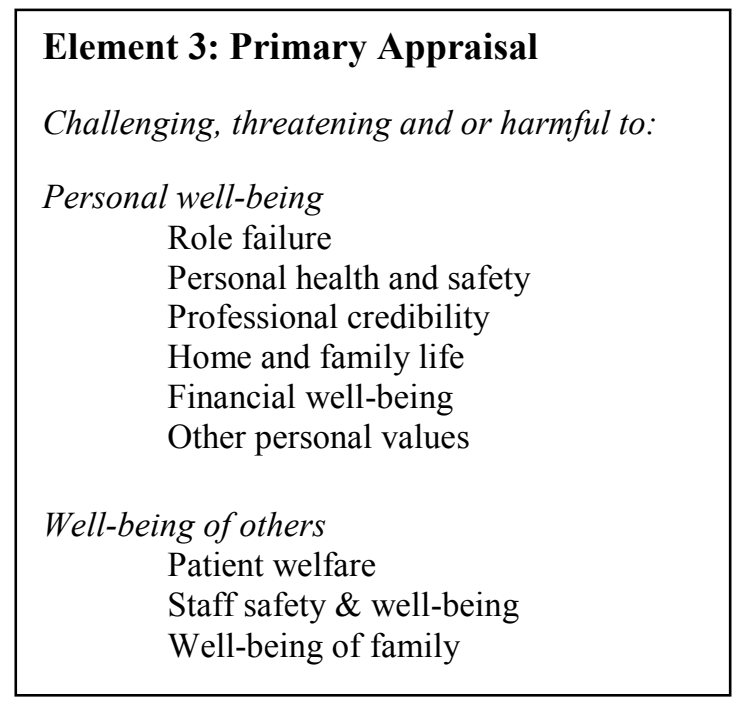

Figure 13-3 Primary appraisal in the role overload appraisal and coping process

Findings from this study deviate from Lazarus and Folkman's definitions and conceptualizations in two ways. First, a paucity of positive emotions and feelings expressed by hospital workers when describing their initial evaluations of role overload episodes reflects a less optimistic narrative associated with challenge appraisals. Whilst potential benefits were mentioned in a few episodes described as challenging, in most episodes, the term 'challenge' seemed to be synonymous with the amount, complexity, and time constraints of the role demands they faced, rather than with the possibilities for personal gain. Second, study respondents also seemed more hesitant than expected, given our review of Lazarus and Folkman's work, to talk about threats, even when describing the potential for harmful outcomes. These findings may say more about contemporary 
usage of this terminology than about the potential for stressful appraisals of role overload, since definitions of these terms were not offered to the respondents during the interview. Furthermore, the reluctance by respondents to describe role overload as threatening were inconsistent with the frequency and strength of negative emotions and feelings used by respondents to describe role overload appraisal. Together, these findings suggest that some episodes described by respondents as challenging might be more appropriately labeled as threatening.

The challenges and threats to well-being described when respondents talked about the potential consequences of role overload were focused primarily on personal wellbeing (i.e., role failure, health and safety, professional credibility, home and family life and financial well-being) and on the well being of others (i.e., patients, staff and family). The well-being of the organization (i.e. the four hospitals) was rarely mentioned by these employees. The fact that personal well-being and the wellbeing of patients, staff and family seemed to be important to hospital workers is consistent with Lazarus and Folkman's (1984) view that stressful appraisals arise when individuals have an investment in the possible outcomes.

Concerns for personal issues varied, but study respondents talked most often about the risks of role failure when talking about the potential impact of role overload to well-being. Such fears of failure were common in work and home situations. The potential for role failure was also mentioned by these women as an underlying worry in descriptions of the coping restrictions faced during role overload episodes (i.e. the outcomes of secondary appraisal, see Section 13.3.2 below). Such concerns suggest conflict between the values of the respondents and the demands from her role set 
members. Person-role conflicts are noted to be a composite element of role overload by Kahn et al. (1964), suggesting that concern about role failure would be particularly important in role overload. The notion that personal values are at stake is also consistent with Lazarus and Folkman's (1984) description of primary appraisal.

The potential for role overload to impact the home and or family life was mentioned in nearly a quarter of the episodes recalled. The nature of the potential impact varied but a plurality focused on work impinging negatively on home life. The idea that work role overload can reduce leisure or family time is consistent with findings in a recent review of work and family research (Eby et al., 2005). According to Lazarus and Folkman (1984), the deeper a person's commitment the greater the potential for threats or harm. The fact that most respondents reported high levels of parental role salience may explain why these women would feel vulnerable to threats to family life whether from work or home based role overload.

Analyses of the remaining issues at stake during the episodes recalled for this study suggest they would not necessarily be exclusive to role overload appraisals. First, concerns expressed about professional credibility (seen by respondents to be potentially at risk in a substantive number of the work role overload episodes recalled) suggest that such worries were more about the importance of professional standards within the health sector than about the nature of role overload.

Second, challenges and threats to personal health and safety, as described by the respondents in a third of the episodes, referred to a variety of issues arising at home and at work, including concerns about personal injuries, as well as the potential for negative impacts on health from the stress arising from the demands and burdens of overload. The 
study sample was selected because they were regularly overloaded. Concerns about ill health arising from role overload might be expected and are consistent with the literature showing a relationship between increased workload and poorer health, in general (Dewe et al. 2010) and in the health sector (Lim et al. 2010).

Lastly, the fact that the well being of patients and staff were also concerns for hospital workers in role overload appraisal is not surprising given the nature of their work and the high levels of the traits, agreeableness and conscientiousness, scored by many respondents. The fact that family well-being was sometimes a worry is consistent with the high levels of parental role salience found for most of these women.

\subsubsection{What can be done about role overload?}

Secondary appraisal of role overload addresses the question "What if anything can be done about the role overload?" The factors found to be influential in answering this question (identified from the results presented in Chapter 10) are summarized in Figure 13-4. Almost all episodes described by the hospital workers in this study included references to some form of restriction to the ability to cope with role overload. Most notable is the fact that, in many episodes, respondents mentioned that they did not have the time or energy needed to cope with role overload. A substantive number also featured respondents explaining that they had 'too much to do', a negative evaluation of the sheer number of demands often faced. These findings are not surprising since time and energy shortages and having too much to do have been associated in the literature with the experience of role overload (Kahn et al., 1964). 
This study did, however, uncover a broad range of coping restrictions that were identified when respondents talked about their evaluations of role overload. Also noteworthy was the fact that in a number of cases, coping restrictions were talked about in respondents' descriptions of challenges and threats, suggesting that they reinforce evaluations of role overload's personal significance to wellbeing, and therefore play an important part in the appraisal process. These findings are consistent with secondary appraisal and its interdependent relationship with primary appraisal as described by Lazarus and Folkman (1984).

\section{Element 4: Secondary Appraisal}

Coping limited by:

Lack of personal resources:

- $\quad$ Time \& energy

Not knowing how to cope Lack of help

Environmental constraints:

- No access to overtime

- Poor management support

Too much to do

Figure 13-4 Secondary appraisal in the role overload appraisal and coping process

Another important finding regarding the secondary appraisal of role overload was that a plurality (40\%) of hospital workers stated that they were 'unsure how to cope'. This uncertainty on how best to cope with overload is echoed in other data examined in Chapter 10. Most notable is the fact that in approximately half of the episodes presented, respondents made no mention of specific coping strategies when describing initial evaluations of role overload. Taken together, these results suggest that the overloaded women in our sample often struggled to identify strategies or resources that they could use to tackle role overload. It may be that these workers consider overload (a given in 
their lives) as something that cannot be controlled. The fact that workers mentioned lack of control in one third of the total episodes recalled gives some support to this explanation and is also consistent with views expressed by Lazarus and Folkman (1984) that controllability is particularly influential in secondary appraisal. An alternative explanation is that coping resources were restricted in some other way. The two remaining coping limitations identified in Figure 13-4 speak to this idea.

The last two coping limitations: 'lack of available help', and 'environmental constraints' (both mentioned in approximately a third of all episodes) were cited only during recollections of role overload involving the work domain. Research has shown that staff shortages in the health sector are linked to stress (Lim et al., 2010; Glazer and Gyurak, 2008). Social support has also been noted as an important type of coping strategy, particularly for women (Dewe et al. 2010; Lazarus and Folkman, 1984; Lease, 1999), suggesting that its absence might be troublesome. These findings from existing stress appraisal and coping research may explain why the female hospital workers would cite lack of help at work as something that makes it more difficult for them to cope with role overload. They also highlight one consequence of understaffing on employees in the health sector (i.e. role overload).

The environmental constraints described by respondents referred first to explicit organisational rules or processes that limit the use of certain coping strategies or the amount of resources available, and second to lack of support from senior management. In a notable example of the former, hospital restrictions imposed on taking overtime sometimes limited the time resources available to these workers. This limitation suggests that when role expectations require work beyond mandated hours, respondents will be 
faced with intra-role conflict (as identified in results chapter 8). In other cases, respondents were not allowed to take time off to deal with family medical issues. These situations lead to work-family conflict because of increased expectations to return to work within certain time limits. Such episodes, in which time pressures create role conflict between otherwise logically compatible expectations, are consistent with Kahn et al.'s definition of role overload. Consistent with Lazarus and Folkman (1984) description of secondary appraisal, these time constraints may make a challenging situation more threatening to well-being.

Finally, the lack of management support described by respondents suggests that no matter how much effort individuals put in, the lack of forethought or upper level support to resolve the underlying issues was a given in certain situations. These underlying constraints may make a challenging situation more stressful.

\subsection{A typology of role overload coping strategies}

Lazarus and Folkman (1984) argued that understanding how coping thoughts or actions function in any particular instance can only be understood by thoroughly examining the context in which they occur, which in this case study, is that of the role overload episode. A typology of role overload coping strategies, developed from the findings presented in Chapter 11, is presented in Figure 13-5 below for discussion purposes. This typology provides details of Element 5 in Figure 13-1. Shown on this typology are all parent and child strategies used to cope with role overload. Characteristics of the typology will be discussed first (Section 13.3.1), following by a discussion of the strategies in each dimension (Section 13.3.2). 
Problem Focused

Emotion Focused

\begin{tabular}{|c|c|}
\hline $\begin{array}{l}\text { Use others to reduce demands: } \\
\text { Help from colleagues, family members or friends } \\
\text { Delegate or seek help } \\
\text { Professional or paid help } \\
\text { Cut demands } \\
\text { Compartmentalise }\end{array}$ & Positive thinking \\
\hline $\begin{array}{l}\text { Planning \& organization: } \\
\text { Organise for efficiency \& order } \\
\text { Research, analyse \& plan } \\
\text { Personal Deprivation: } \\
\text { Work longer \& harder } \\
\text { Use personal time } \\
\text { Manage expectations } \\
\text { Prioritise }\end{array}$ & $\begin{array}{l}\text { Involve others in managing demands: } \\
\text { Talk about it } \\
\text { Moral support \& friendship } \\
\text { Vent to others } \\
\text { Take my mind off role overload: } \\
\text { Engage in activities I enjoy } \\
\text { Engage in potentially unhealthy activities } \\
\text { Let the feelings out } \\
\text { Positive efforts to prevent stress } \\
\text { Endure \& comply }\end{array}$ \\
\hline
\end{tabular}

Figure 13-5 Typology of role overload coping strategies (Element 5 of Figure 13-1)

\subsubsection{Dimensions of the role overload coping strategy typology}

Coping is defined as the "constantly changing cognitive and behavioural efforts to manage specific external and/or internal demands that are appraised as taxing or exceeding the resources of the person" (Lazarus and Folkman, 1984, p.141). The word 'manage' was used by Lazarus and Folkman to avoid confusing coping with mastery. In their words, "managing can include minimizing, avoiding, tolerating, and accepting the stressful conditions as well as attempts to master the environment" (p. 142). Two broad approaches for coping with role overload were identified in this study (shown on the left of Figure 13-5): 'demand-reduction' and 'demand-management'. Demand-reduction 
coping is aimed at reducing the volume of role demands contributing to role overload. Strategies within this grouping have a direct impact on role overload by eliminating role demands. The concept of demand-management coping is defined in this thesis to include strategies that do not reduce demands but rather focuses on managing them, e.g., organising, avoiding, or tolerating (external and/or internal) role overload demands. While these coping approaches are consistent with Lazarus and Folkman's (1984) overall definition of coping, they also suggest two clear coping approaches specific to this stressor. These two approaches are also consistent with the terms used by Lang and Markovitz (1986), who categorised role overload coping as task reduction or task management.

The second pair of role overload coping strategy dimensions (at the top of Figure 13-5) match and are named for the two major coping functions defined by Lazarus and Folkman's (1984): problem focused (directed at the problem causing the role overload) and emotion focused (aimed at dealing with the emotional response to role overload). While the consensus in the literature reviewed for this thesis is that a universal coping classification system remains elusive (Skinner et al. 2003), Lazarus and Folkman's 'problem versus emotion focused' classification is, the most commonly used. As such it was felt to be appropriate to use this framework in this thesis, along with the role overload specific demand-reduction, demand-management dimension, to create the typology shown in Figure 13-5.

Coping strategies mentioned by the respondents in this study as ways they deal with role overload were classified using this typology. Consistent with the coping research of Lazarus, Folkman and colleagues $(1984,1986)$ the data analysis done for this 
thesis reveal that in the vast majority of role overload episodes discussed in this study, workers reported using a mixture of both problem and emotion focused coping strategies. Overall, however, more problem focused strategies were used per episode than emotion focused strategies. Given that the secondary appraisal data revealed that respondents were either hopeful or confident of coping success in nearly three quarters of the episodes recalled, the use of a higher number of problem focused strategies (not shown) is also consistent with the contention by Lazarus and Folkman's (1984) that problem focused forms of coping are more likely when stressful conditions are appraised as amenable to change.

Overall, findings from this case study also show that more demand-management than demand-reduction strategies are used per role overload episode, suggesting that role demands are either more difficult to reduce or demand-reduction strategies are more difficult to implement for some reason. It is particularly notable that only one emotion focused strategy is used by hospital workers to reduce demands. In our study, the fact that a a broader mix of both problem and emotion focused demand-management strategies was used by respondents when coping with role overload suggests that emotion-focused strategies seem to be less appropriate for reducing role overload than they are for reducing stress levels.

Also noteworthy are data showing that the most important way of reducing role demands for these women involved receiving support from other people. This suggests that if more help was available these strategies might be used more often. This idea is consistent with the secondary appraisal findings (in Chapter 10) showing that 'lack of help' was cited as a coping restriction in over a third of episodes described. 
Environmental coping constraints such as lack of senior management support may also make it difficult to get help.

It is also possible that the lower use of demand-reduction strategies by the respondents might be because not all workers have the authority to delegate or seek help, or to cut demands (i.e., use demand-reduction strategies). As such, these women must focus, by necessity, on managing demands. Situations where the overriding needs of others limit people's ability to reduce demands (e.g. when caring for critically ill patients) also necessitates the use of demand-management strategies. Future research is required to see if the ratio of demand-reduction to demand-management strategies observed in this sample varies by role type, gender or sector.

Another important finding obtained by using the typology of role overload coping strategies to classify responses is the tendency the women in this study have to cope by involving other people as a way to reduce or manage role overload demands. Six of the coping strategies identified in this study involved family members, friends, colleagues or other professionals. Three of these strategies involved problem focused techniques (help from others, delegate or seek help, use professional or paid help) used by respondents to reduce demands through the provision of practical and informational assistance. The other three are demand-management strategies (talk about it, receive moral support \& friendship, and vent to others), which provide emotional support during role overload. All these coping activities are consistent with the concept of social support (except 'venting to others', which is used relatively infrequently). Social support typically comprises an interpersonal transaction to provide emotional support, practical assistance, or information (Lazarus and Folkman, 1984). Research by Lazarus and his colleagues 
(1980, 1984 and 1986) also identified social support as an important way of coping that can be either problem or emotion focused. As described by respondents, venting to others as an outlet for stress does not always involve willing recipients, and as such does not fully fit the description of social support offered by Lazarus and Folkman (1984).

A recent review of the coping literature that focuses on stress at work (the most frequent setting for the role overload described for this case study) has also confirmed the importance of social support (Dewe et al., 2010). The high use of strategies that make use of support from other people may also reflect the fact that our sample is restricted to women. Women have been found to make greater use of coping strategies that rely on interpersonal support than men (Ptacek, Smith \& Zana, 1992; Lease, 1999; Higgins, Duxbury, \& Lyons, 2010). Future research is required to see if the high use of social support strategies to cope with role overload holds true for men as well.

\subsubsection{Coping strategies of the role overload coping typology}

The literature review in this thesis revealed that little research has been done on coping with role stress (Dewe et al. 2010). Comparisons between the role overload coping strategies in the typology presented here and the strategies in other coping structures are, therefore, difficult. Many researchers have used Lazarus and Folkman's (1984) 'Ways of Coping' questionnaire (WCQ) to develop coping scales for various stressors using factor analytic procedures (Parker et al., 1993). Lazarus and Folkman (1984) drew from their theoretical framework, from contemporary literature, and from items suggested by their own research to create the 67 item WCQ that describe a broad range of cognitive and behavioural strategies that individuals might use to cope with the internal and external demands in stressful encounters. Since the coping actions in the role 
overload coping typology can be classified using the problem or emotion focused categorization defined by Lazarus and Folkman (1984), the comprehensive WCQ provides a source of comparative literature for the items in the coping typology presented here. Comparing the strategies identified in this case study with a list that represents generic coping actions, which are applicable to a variety of stressors, allows us to highlight similarities with Lazarus and Folkman's stress and coping model and also any distinguishing features of the role overload coping typology.

Lang and Markovitz (1986) report on a study whose objective was to determine which coping strategies would reduce or prevent the stress of short-term role overload. Initially, Lang and Markovitz developed a 35 item closed-end scale based on previous studies of role stress and on pretests for their own study of undergraduates enrolled in a three week intensive evening course. In several classes, students used the scale to indicate how much they used the coping strategies on a five point scale from 'not at all' to 'very much'. Students' not experiencing overload indicated how much they used these techniques to prevent overload or manage their time better. Focusing on the idea that lowering the demands producing the conflict would help reduce strain, Lang and Markoviz's scale was broadly divided into strategies aimed at task reduction and task management. After a number of modifications supplemented by validity and reliability analysis and a classification of the items by judges, the scale was reduced to 28 items. Task reduction was further divided into cooperative or unilateral task reduction, whilst task management differentiated between reactive and planned strategies. As such, Lang and Markovitz's scale provides a comparison for this case study with a rare attempt to develop a role overload specific coping scale. 
Further details of the role overload coping strategies in each of the four dimensions (demand reduction problem focused, demand reduction emotion focused, demand management problem focused, demand management emotion focused), are summarized in Table 13-2 for discussion. This table is structured as follows. The table has four parts, which correspond to the four cells in Figure 13-5. All the coping strategies are listed in the first column of the table. If applicable, column two of Table 13-2 indicates the parent coping strategy that the child strategy is linked to. Coping strategy descriptions and example quotes from the hospital workers are presented in columns 3 and 4. Where relevant, the equivalent coping strategies from Lang and Markovitz's (1986) role overload coping scale, and from the WCQ (Lazarus and Folkman, 1984), are cited in columns 5 and 6 respectively.

Of the 19 items strategies listed in the table, 13 are matched by items in the WCQ, suggesting that whilst the role overload coping strategies identified in this study are broadly consistent with Lazarus and Folkman's model of the coping process, the study identified a number of strategies that may be more important for dealing with role overload. The fact that 53 of the WCQ items have no match to coping used to deal with role overload has more to do with the diversity of a coping instrument designed for use in a variety of stressful situations than with the limitations of items that need to be included in a role overload typology.

Thirteen of the 19 items in Table 13-2 are consistent with items in the role overload coping scale developed by Lang and Markovitz (1986). Some role overload strategies represent a combination of two or more items from the Lang and Markovitz scale, with the result that 21 of the 28 items in this scale have comparators in the role 
overload coping typology. The thesis results therefore, support the idea that the coping typology emerging from this study is focused on role overload. It may be that the items in Lang and Markovitz's scale that are not represented in the role overload coping typology may have been mentioned by respondents in our sample but not in any substantive numbers. The fact that the setting for this thesis is very different to the student focused academic setting used in Lang and Markovitz's (1986) study may be another reason for the discrepancy between the two scales.

Within each of the four parts of the table, coping strategies linked to both Lang and Markovitz's scale and the WCQ are listed first, followed by those represented only in the WCQ, then those represented only in Lang and Markovitz's scale, and finally by those strategies that appear in neither. The implications of these patterns will be discussed in the four subsections to follow 


\begin{tabular}{|c|c|c|c|c|c|}
\hline Coping Strategy & $\begin{array}{c}\text { Parent } \\
\text { Coping } \\
\text { Strategy }\end{array}$ & Description & Example Quotes & $\begin{array}{l}\text { Lang \& Markovitz (1986) Role } \\
\text { Overload Coping Scale }(n=28)\end{array}$ & $\begin{array}{c}\text { Lazarus \& Folkman (1984) } \\
\text { Ways of Coping } \\
\text { Questionaire (WCQ) }(n=67)\end{array}$ \\
\hline \multicolumn{6}{|c|}{$\begin{array}{l}\text { Demand reduction problem } \\
\text { focused strategies }\end{array}$} \\
\hline $\begin{array}{l}\text { Help from } \\
\text { colleagues, family } \\
\& \text { friends }\end{array}$ & $\begin{array}{l}\text { Use others to } \\
\text { reduce } \\
\text { demands }\end{array}$ & $\begin{array}{l}\text { Practical help freely given by } \\
\text { friends, family, colleagues \& } \\
\text { managers }\end{array}$ & $\begin{array}{l}\text { The secretary did some typing for me } \\
\text { that I would normally do myself. }\end{array}$ & $\begin{array}{l}\text { Got help from co-workers, spouse or } \\
\text { others outside work }\end{array}$ & $\begin{array}{l}\text { Talked to someone who could tell } \\
\text { me more about the situation, or, } \\
\text { someone who could do something } \\
\text { concrete about the problem }\end{array}$ \\
\hline $\begin{array}{l}\text { Professional or } \\
\text { paid help }\end{array}$ & $\begin{array}{l}\text { Use help to } \\
\text { reduce } \\
\text { demands }\end{array}$ & $\begin{array}{c}\text { Seek professional or paid help to } \\
\text { overcome overload }\end{array}$ & $\begin{array}{l}\text { I'm getting the union and the human } \\
\text { rights commission on it to see if we can } \\
\text { work out something }\end{array}$ & & I got professional help \\
\hline Cut demands & $\mathrm{n} / \mathrm{a}$ & $\begin{array}{c}\text { Eliminate tasks from workload or let } \\
\text { things slide }\end{array}$ & $\begin{array}{l}\text { Cancelled one meeting, left corporate } \\
\text { meeting a little bit early, arrived late at } \\
\text { (the) open house }\end{array}$ & $\begin{array}{l}\text { I decided not to do some of the things I } \\
\text { had planned to do, or, I reduced the } \\
\text { quality of things I did or negotiated a } \\
\text { reduction in the amount of work required. }\end{array}$ & \\
\hline Compartmentalise & $\mathrm{n} / \mathrm{a}$ & $\begin{array}{l}\text { Consciously segment role domains } \\
\text { or demands to avoid conflict, } \\
\text { increase focus and reduce demands }\end{array}$ & $\begin{array}{l}\text { I act as if I am not here in terms of the } \\
\text { rest of my responsibilities and I make a } \\
\text { conscious effort to let that go. }\end{array}$ & $\begin{array}{l}\text { Concentrated on one thing at a time and } \\
\text { put other things out of my mind }\end{array}$ & \\
\hline $\begin{array}{l}\text { Delegate or seek } \\
\text { help }\end{array}$ & $\begin{array}{l}\text { Use others to } \\
\text { reduce } \\
\text { demands }\end{array}$ & $\begin{array}{l}\text { Delegate work or actively seek help } \\
\text { from others at work }\end{array}$ & $\begin{array}{l}\text { I delegated some stuff to her that I was } \\
\text { comfortable that she would certainly be } \\
\text { able to do }\end{array}$ & Delegate tasks to others & \\
\hline \multicolumn{6}{|c|}{$\begin{array}{c}\text { Demand reduction emotion } \\
\text { focused strategies }\end{array}$} \\
\hline Positive thinking & $\mathrm{n} / \mathrm{a}$ & $\begin{array}{l}\text { Reappraise thoughts to put a positive } \\
\text { view on the overload }\end{array}$ & $\begin{array}{l}\text {...thinking that the world wouldn't end if } \\
\text { I didn't get the list done. }\end{array}$ & $\begin{array}{l}\text { Changed my view of the importance of } \\
\text { the things I had to do or lowered demands } \\
\text { on myself by looking at the positive } \\
\text { things I am doing }\end{array}$ & $\begin{array}{l}\text { I told myself things that helped me } \\
\text { to feel better }\end{array}$ \\
\hline \multicolumn{6}{|c|}{$\begin{array}{c}\text { Demand management problem } \\
\text { focused strategies }\end{array}$} \\
\hline $\begin{array}{l}\text { Work longer \& } \\
\text { Harder }\end{array}$ & $\begin{array}{c}\text { Personal } \\
\text { Deprivation }\end{array}$ & $\begin{array}{c}\text { Work longer hours or increase effort } \\
\text { levels }\end{array}$ & $\begin{array}{l}\text { I'm working an hour and a half a day } \\
\text { (uncompensated) and sometimes on } \\
\text { weekends doing program development }\end{array}$ & $\begin{array}{l}\text { Worked harder than usual or worked } \\
\text { longer than usual }\end{array}$ & Doubled efforts to make things work \\
\hline $\begin{array}{l}\text { Organise for } \\
\text { efficiency \& order }\end{array}$ & $\begin{array}{l}\text { Planning \& } \\
\text { Organisation }\end{array}$ & $\begin{array}{l}\text { Organise task load to give structure } \\
\text { and maximize efficiency }\end{array}$ & $\begin{array}{l}\text { Think methodically, organize myself. } \\
\text { Being organized helps you think in a } \\
\text { calm measure. }\end{array}$ & $\begin{array}{l}\text { Scheduled, organised and planned very } \\
\text { carefully }\end{array}$ & $\begin{array}{l}\text { Changed something so that things } \\
\text { would work out all right }\end{array}$ \\
\hline $\begin{array}{l}\text { Take time to } \\
\text { research, analyse } \\
\& \text { plan }\end{array}$ & $\begin{array}{l}\text { Planning \& } \\
\text { Organisation }\end{array}$ & $\begin{array}{l}\text { Spend time researching, gathering } \\
\text { information and devising a plan of } \\
\text { action }\end{array}$ & $\begin{array}{l}\text { Make some observations, gather some } \\
\text { information and then sit down and } \\
\text { discuss. }\end{array}$ & $\begin{array}{l}\text { Scheduled, organised and planned very } \\
\text { carefully }\end{array}$ & $\begin{array}{l}\text { I made a plan of action and followed } \\
\text { it }\end{array}$ \\
\hline Prioritise & $\mathrm{n} / \mathrm{a}$ & $\begin{array}{l}\text { Examine task load and determine } \\
\text { and decide relative importance }\end{array}$ & $\begin{array}{l}\text { Take a step back and say, "What are the } \\
\text { priorities today, tomorrow, at the end of } \\
\text { the week, and so on". }\end{array}$ & $\begin{array}{l}\text { Set priorities and made sure most } \\
\text { important things got done }\end{array}$ & \\
\hline
\end{tabular}




\begin{tabular}{|c|c|c|c|c|c|}
\hline Coping Strategy & $\begin{array}{l}\text { Parent } \\
\text { Coping } \\
\text { Strategy }\end{array}$ & Description & Example Quotes & $\begin{array}{l}\text { Lang \& Markovitz (1986) Role } \\
\text { Overload Coping Scale }(n=28)\end{array}$ & $\begin{array}{l}\text { Lazarus \& Folkman (1984) } \\
\text { Ways of Coping } \\
\text { Questionaire (WCQ) }(n=67)\end{array}$ \\
\hline $\begin{array}{l}\text { Managing } \\
\text { Expectations }\end{array}$ & $\mathrm{n} / \mathrm{a}$ & $\begin{array}{c}\text { Communicate directly with role set } \\
\text { members to minimize unrealistic } \\
\text { expectations }\end{array}$ & $\begin{array}{l}\text { For the clients we as a group } \\
\text { communicated to them that we wouldn't } \\
\text { be able to act as quickly as they wanted }\end{array}$ & & \\
\hline Use personal time & $\begin{array}{l}\text { Personal } \\
\text { deprivation }\end{array}$ & $\begin{array}{l}\text { Skip lunch, use vacation time to } \\
\text { complete role demands }\end{array}$ & $\begin{array}{l}\text { I eat through my lunch at my desk } \\
\text { because I find even taking an hour out of } \\
\text { the day can just put you behind. }\end{array}$ & & \\
\hline \multicolumn{6}{|c|}{$\begin{array}{c}\text { Demand management emotion } \\
\text { focused strategies }\end{array}$} \\
\hline Talk about it & $\begin{array}{l}\text { Involve } \\
\text { others in } \\
\text { managing } \\
\text { demands }\end{array}$ & $\begin{array}{l}\text { Talk about feelings with friends, } \\
\text { family or colleagues }\end{array}$ & $\begin{array}{l}\text { talking with committee members to get a } \\
\text { perspective on things - known them for a } \\
\text { long time - good sounding board }\end{array}$ & Talked it over with people & $\begin{array}{l}\text { Talked to someone about how I was } \\
\text { feeling }\end{array}$ \\
\hline $\begin{array}{l}\text { Engage in } \\
\text { activities I enjoy }\end{array}$ & $\begin{array}{l}\text { Take my } \\
\text { mind off role } \\
\text { overload }\end{array}$ & $\begin{array}{l}\text { Engage in enjoyable activities to } \\
\text { take my mind of the role overload }\end{array}$ & Went out to garden, something I enjoy. & $\begin{array}{l}\text { Substitute activity to take their mind off } \\
\text { things }\end{array}$ & $\begin{array}{l}\text { Became involved with diversions } \\
\text { (watched TV, etc.) }\end{array}$ \\
\hline $\begin{array}{l}\text { Positive efforts to } \\
\text { prevent or reduce } \\
\text { stress }\end{array}$ & $\mathrm{n} / \mathrm{a}$ & $\begin{array}{l}\text { Healthy living, including exercise } \\
\text { and eating well }\end{array}$ & $\begin{array}{l}\text { I go to the gym after work and do cardio } \\
\text { and weights, which gets rid of my stress. }\end{array}$ & Exercised more than usual & I jogged or exercised \\
\hline $\begin{array}{l}\text { Endure and } \\
\text { comply }\end{array}$ & $\mathrm{n} / \mathrm{a}$ & $\begin{array}{l}\text { Accept the role demands have to be } \\
\text { done and just get on and do the job }\end{array}$ & $\begin{array}{l}\text { then you do the task and sort of buck up } \\
\text { and do it. }\end{array}$ & $\begin{array}{l}\text { Tried to meet all the demands by doing } \\
\text { everything expected of me }\end{array}$ & $\begin{array}{l}\text { Accepted it, since nothing could be } \\
\text { done }\end{array}$ \\
\hline $\begin{array}{l}\text { Engage in } \\
\text { potentially } \\
\text { unhealthy activities }\end{array}$ & $\begin{array}{c}\text { Take my } \\
\text { mind off role } \\
\text { overload }\end{array}$ & $\begin{array}{l}\text { Drank wine or used prescription } \\
\text { drugs or slept too much }\end{array}$ & & $\begin{array}{l}\text { Ate too much or too little or slept too } \\
\text { much or too little or smoked more than } \\
\text { usual or drank more than usual. }\end{array}$ & $\begin{array}{l}\text { Slept more than usual or tried to } \\
\text { make myself feel better by drinking, } \\
\text { smoking, using drugs or medication }\end{array}$ \\
\hline Let the feelings go & $\mathrm{n} / \mathrm{a}$ & Cry, curse and swear in private & $\begin{array}{l}\text { At the beginning ......there was a temper } \\
\text { that blows up and comes out. Sometimes } \\
\text { I cry }\end{array}$ & & Let my feelings out somehow \\
\hline $\begin{array}{l}\text { Receive moral } \\
\text { Support }\end{array}$ & $\begin{array}{l}\text { Involve } \\
\text { others in } \\
\text { managing } \\
\text { demands }\end{array}$ & $\begin{array}{l}\text { Supported by friends family } \\
\text { colleagues }\end{array}$ & $\begin{array}{l}\text { I did receive tremendous support at } \\
\text { work, from my boss and from my co- } \\
\text { workers }\end{array}$ & & $\begin{array}{l}\text { Accepted sympathy and } \\
\text { understanding from someone }\end{array}$ \\
\hline Vent to others & $\begin{array}{l}\text { Involve } \\
\text { others in } \\
\text { managing } \\
\text { demands }\end{array}$ & $\begin{array}{l}\text { Vent about feelings about the role } \\
\text { overload to others }\end{array}$ & $\begin{array}{l}\text { Just to vent really just an opportunity to } \\
\text { get it off my chest. }\end{array}$ & Expressed my feelings & \\
\hline
\end{tabular}

Table 13-2 Typology of role overload coping strategies 


\subsubsection{Problem-focused role overload coping strategies used to reduce role demands}

Five problem focused demand-reduction role overload coping strategies were identified in this case study: help from others, use professional or paid help, cut demands, compartmentalize, and delegate or seek help. The first, help from colleagues, family and friends, is the most frequently used problem-focused strategy for reducing role demands cited in this study and was mentioned in appreciably more episodes by these women than any other role overload coping strategy. Also noteworthy are data showing that such help from other people is used more in role overload episodes described as having a successful outcome than in episodes with mixed outcomes or in unresolved role overload. These findings emphasize how important social support was to these women in dealing with role overload.

Second, this strategy is also the only problem-focused demand-reduction strategy consistent with items in Lang and Markovitz's role overload coping scale and in the WCQ. The links to both coping instruments underscore the findings in the literature discussed above, that social support is an important coping strategy whatever the stressor. In this thesis, help from others is also distinguished by the fact that the help provided was not necessarily sought out by the workers but freely given by others. Given that all the respondents suffered high levels of work, home and total role overload, and most episodes recalled were set at work, these respondents are receiving help from individuals who may well be suffering from role overload themselves.

Occasionally, to get the necessary advice or practical support to reduce role demands hospital workers used professional or paid help instead of using resources from the social environment. This strategy is consistent with a similar item in the WCQ; 
however, it does not have an equivalent in the coping scale developed by Lang and Markovitz (1986). The fact that the case study setting for this thesis is very different to that of the academic setting in Lang and Markovitz's study may help to explain this variation.

The remaining three problem focused demand-reduction strategies (cutting demands, compartmentalizing, and delegate or seek help) are distinguished by the fact that they are consistent with items in the Lang and Markovitz role overload coping scale but are not represented in the broad WCQ. This suggests that these strategies may be unique to coping with role overload.

Cutting demands is a technique used by hospital workers to cancel, drop, postpone, or complete to a lesser standard the role demands faced in an overload situation and as such has the potential to have an immediate reductive effect on role overload. Lang and Markovitz's coping list included three items representing the different ways demands could be cut, consistent with 'cancel or drop', 'postpone', or 'complete to a lesser standard' respectively. In the role overload coping strategy typology developed in this study, these multiple methods have been subsumed under a single label, because individually they were used too infrequently by the women in the thesis sample to be coded separately.

Compartmentalising is a strategy that reduced demands for workers by focusing on the task in hand whilst deliberately ignoring other demands. Compartmentalisation is commonly mentioned in the work family literature, where efforts to compartmentalise the work and family roles is a way to cope with work-family conflict (Wickham and Parker, 2007). Analysis for this thesis suggests that this strategy is not unique to role overload but 
is consistent with coping used for other forms of role stress, such as work-family conflict. In this thesis, compartmentalisation is not just used to cognitively isolate roles, but is also used to separate role demands received from different role set members within a single role.

Delegate or seek help, the last demand-reduction role overload coping strategy that involves others, refers to deliberate efforts to enlist practical and informational assistance to deal with role overload. As such, it is consistent with descriptions in the coping literature of social support as discussed above, but does not feature specifically amongst the social support items of the WCQ. The extent to which this strategy was used by hospital workers suffering role overload (in 1 in every 5 episodes), however, suggested that it warranted a discrete label. Respondents' descriptions also suggested that workers use this strategy to cut demands when they feel confident and comfortable that other people can handle the role demands. The ability to use this strategy may therefore depend on the availability of help and the skills of the help available, rather than solely on the ability of the hospital workers to delegate or seek help. Taken together these findings suggest this strategy can be an important role overload specific coping strategy when the right circumstances exist.

\subsubsection{Emotion focused role overload coping strategies used to reduce role demands}

As articulated by the respondents in our sample, the only emotion focused coping strategy used for demand reduction (positive thinking) involves a conscious decision to cognitively alter or reappraise the personal meaning of a situation in order to lessen internal role pressures. This strategy is consistent with items in the WCQ checklist and in 
Lang and Markovitz's (1986, p. 201) role overload coping scale. The fact that the hospital workers used a variety of problem focused strategies to reduce role demands but only one emotion focused strategy is a notable finding from this study. Also noteworthy are data from this study showing that positive thinking was used in few (only 15\%) of the episodes.

Comparison of findings from this study to the greater body of coping research suggests that these are unexpected results. Descriptions of 'positive thinking' given by workers in our sample are consistent with an important form of emotion focused coping referred to as cognitive reappraisal in the literature. Cognitive reappraisals are strategies in which individuals use "cognitive maneuvers (to) change the meaning of a situation" to lessen emotional distress (Lazarus and Folkman, 1984, p. 151). Thus positive thinking works by changing the meaning of a situation in order to reduce the internal role pressures that may be contributing to role overload. Analysis of the results have suggested that external role demands (e.g. time pressures and volume of demands) and the inability to deal with them (i.e., coping restrictions) contribute more to feelings of role overload than internal role pressures overall. Thinking positively to change the meaning of a situation to reduce internal role pressures is therefore likely to be less effective at reducing the overall number of role demands causing role overload. This makes 'positive' thinking less relevant for coping with role overload than it is for coping with other stressors where the relative meaning of an event to an individual has a greater influence in cognitive appraisal. 


\subsubsection{Problem focused role overload coping strategies focused on managing role demands}

Six problem focused demand-management strategies were identified in this case study. All but one (use personal time) were employed in at least $25 \%$ of episodes, a finding that is consistent with the following: (1) demand-management strategies are cited more often than demand-reduction, and (2) problem focused strategies are used more than emotion focused strategies. The first three strategies in this quadrant (work longer and harder, organise for efficiency and order, and take time to research, analyse and plan) were found to be similar to items in both the WCQ and Lang and Markovitz's scale, suggesting that these coping strategies are not specific to role overload.

'Working longer and harder', the first strategy of this grouping, is the second most frequently cited demand-management coping strategy in the typology. The results from this study suggest, however, that this coping strategy results in respondents working longer hours than mandated whilst depleting energy reserves in order to focus on the problem of role overload. As such it is unfortunate that this strategy is used so frequently. The potential for negative outcomes for individuals using this strategy is consistent with the literature linking role overload with physical and psychological ill-health, and with negative organisational outcome (Gupta and Beehr, 1979; Beehr, King and King, 1986; Osipow and Davis, 1988; Thompson and Blau, 1993; Lease, 1999; Duxbury \& Higgins, 2001, 2003a; Santos et al., 2003; Perrewé et al. 2005; Gilboa et al. 2008; Pearson, 2008; and Glynn, Maclean, Forte and Cohen, 2009).

Two of the other strategies within this quadrant, organise for efficiency and order (i.e., making modifications to improve the effectiveness of efforts to deal with role overload) and taking time to research, analyse and plan (i.e.,devising plans of action) 
are two child coping strategies both collected under the parent strategy "planning and organisation'. The high usage of planning and organisational strategies observed in this study (collectively cited in nearly half of the episodes recalled) is consistent with Folkman et al.'s (1986) research, which identified planful problem solving as one of eight coping scales derived from a factor analysis of items chosen from the WCQ. Lang and Markovitz's (1986) study also identified planned task management (which includes these strategies) as an important factor for coping with role overload. While Lazarus and Folkman also conceptualize these items as two distinct coping strategies in the WCQ, in Lang and Markovitz's scale they are subsumed under a single item as indicated in Table $13-2$.

The next coping strategy, prioritising, is not included in the WCQ but has a close equivalent in Lang and Markovitz's role overload coping scale (i.e., set priorities and made sure the most important things got done), suggesting that coping in this manner is specific to role overload. The contention by Kahn et al (1964), that deciding which role demands should be tackled and which should be left is a strategy for coping with role overload rather than for coping with other types of role stress, is consistent with this conclusion.

The final two strategies listed in this dimension (i.e., manage expectations and use personal time) were not featured in either of the two coping instruments included in Table 13-2. This finding is somewhat surprising, since manage expectations is the second most common strategy cited by hospital workers, suggesting that it is a particularly important way of coping with role overload. As articulated by these workers, this strategy employs communication to mitigate for demands that cannot be fulfilled and to keep role set 
members informed. As such it helps to guard against negative reactions from role set members, implying that this strategy is closely related to the characteristics of role overload.

Using personal time is a less frequently used strategy, cited only in work based episodes, in which workers deliberately sacrifice personal time by working through work breaks or during vacation time to overcome the time restrictions faced during role overload. In this way, this strategy is focused on one of the key characteristics of role overload (time pressures) highlighted in the thesis data. The differences between the thesis hospital setting and the academic setting used by Lang and Markovitz (1986) may account for the absence of these two strategies from their scale. The fact that our sample is female may also account for this discrepancy.

The fact that these two coping actions are the only strategies not mentioned in one or other or both of the coping instruments is an important contribution of this study to the coping with role overload literature.

\subsubsection{Emotion focused role overload coping strategies focused on managing demands}

Eight emotion focused demand-management strategies were identified in this study. Five of the eight are each consistent with items in the WCQ and in Lang and Markovitz's (1986) role overload coping scale (i.e., talking about it, let my feelings out, engage in activities I enjoy, positive efforts to prevent or reduce stress, endure and comply, and engage in potentially unhealthy activities ). This finding is not surprising given that the emotional response to role overload is not expected to be different to that 
arising in any other stressful encounter. It would appear that emotion focused demandmanagement strategies used to cope with role overload are largely consistent with those reported in the broader coping literature.

Talking about it is a coping strategy that proactively involves others in the management of the emotional demands associated with role overload. Talking through the issues of the described role overload with colleagues, friends or family therefore qualifies as a type of social support as described by Lazarus and Folkman (1984). This strategy is the most frequently cited emotion focused demand-management strategy in the typology. It is also more likely to be cited alongside positive outcomes, such as positive emotions, than in episodes that feature negative outcomes. These findings are consistent with the literature discussed earlier that emphasizes the importance of social support to the role overload coping process.

Engaging in enjoyable activities is an approach respondents use to take their mind off role overload, whilst positive efforts to prevent or reduce stress helps to prevent or minimize emotional distress through a healthy lifestyle based around physical exercise. Descriptions of these strategies suggest that respondents were coping in a way that would be beneficial to the individual. In contrast, the way respondents talked about the strategies tolerate, endure and comply and engaging in potentially unhealthy activities, suggest that their use can prove detrimental to individual well-being. The fact that the coping strategy, tolerate, endure and comply (which focuses on putting up with the situation and submitting to the role pressures as the best way to manage emotional demands) is used mostly in unresolved role overload episodes and in episodes with negative outcomes is consistent with this idea. Engaging in potentially unhealthy 
activities involves drinking, smoking or prescription drugs to avoid thinking about role overload. These findings are consistent with a systematic review of coping strategies by Skinner et al. (2003). These authors identified a number of families of coping responses, many of which were derived from the WCQ. They noted that emotion focused coping is characterized by a variety of features, some of which may be positive and some of which may be negative in effect.

The next two emotion focused demand-management strategies in this typology (receive moral support and let the feelings go) were matched only by items in the broad WCQ (i.e., not recognized by Lang and Markovitz) suggesting their use is not specific to coping with role overload. By providing empathy and emotional support for the hospital workers during role overload episodes, receive moral support is another strategy consistent with social support as described by Lazarus and Folkman (1984). It is also the second most frequently used emotion focused demand-management strategy cited by the women in the sample. In this thesis, the number of episodes in which 'receiving moral support' was cited justified differentiating this passive strategy from proactively talking about role overload with others (i.e. the strategy 'talk about it"). Lang and Markovitz may not have taken this approach because receiving moral support was less important within their student sample. Let the feelings go is a less frequently used strategy by which respondents relieve stress privately by crying, cursing, or using breathing exercises. Based on its inclusion in the WCQ, this strategy is consistent with emotion focused coping as defined by Lazarus and Folkman (1984).

Vent to others is the last emotion focused role overload coping strategies in this typology. In this case, respondents deliberately vent to other people as a way to relieve 
the stress of the moment. Notably, this strategy is not closely matched by any items in the two coping instruments listed in Table 13-2. Other items in Lang and Markovotz's (1986) scale (express my feelings) and in the WCQ (take it out on other people) shares some similarities with this strategy but are not the same. More specifically, 'express my feelings' does not convey the explosive nature of venting. Nor does venting involve personal attacks (i.e. taking it out on others). In this thesis, expressing feelings has been separated into private (let the feelings go) and public behaviours (vent to others), because of the distinctive descriptions given by the hospital workers. Moreover, although venting to others is the least used emotion focused role overload coping strategy, this strategy is exclusively used in the overwhelming episodes of role overload of high stress hospital workers, suggesting it is an important strategy for some people in some role overload situations. The relationship between this strategy and stress will be discussed further in Chapter 14.

\subsection{Outcomes from the role overload appraisal and coping process}

According to the Lazarus and Folkman's (1984) theoretical schematization of stress and coping (see Table 3-1), the short term outcomes of the stress and coping process include physiological changes, positive or negative feelings, and qualities of encounter outcomes such as whether permanent resolutions are achieved or not. In this case study, outcomes were reported at two stages of the role overload appraisal and coping process. First, emotions and feelings were reported when respondents described initial reactions to role overload prior to describing the coping strategies used to deal with it. In most cases emotions and feelings cited as occurring during the stage of role overload appraisal were 
negative. Second, when the respondents described how the role overload episode ended and how they felt about it, two types of short term outcomes were noted in every episode recalled: emotions and feelings (positive and negative) and role overload episode outcomes (successful, mixed or unresolved). In addition, in a small number of episodes specific personal consequences (personal gains or negative consequences, consistent with positive and negative emotions and feelings respectively) were also mentioned when respondents described how role overload ended.

The personal gains (noted in $17.5 \%$ of the total episodes) took the form of increases in personal skills and feelings of self-worth. Such personal gains are consistent with Lazarus and Folkman (1984) and Kahn et al. (1964), who both noted the potential for personal growth from the coping process. Such changes in personal attributes have also been confirmed in a recent review of the literature on positive emotion in the stress and coping process (Folkman, 2008). Only one type of negative consequence was cited in any substantive numbers ( $10 \%$ of episodes). In these cases respondents described the potential for role overload to spill over into other role domains.

Physiological changes resulting from role overload were rarely mentioned by the workers in the thesis sample at any point in the recollections of role overload. Overall, the outcomes identified in this research (as listed in Element 5 of Figure 13-1) are largely consistent with those noted by Lazarus and Folkman (1984). Further details of the emotions and feelings expressed by respondents (during appraisal and post episode) and their relationship with the episode resolutions and the other personal outcomes are discussed below. 


\subsubsection{A typology of emotions and feelings associated with role overload appraisal and role overload episode resolutions}

As described in Chapters 10 and 12, Russell's (1980) circumplex model of affective states was used to help classify all the emotions and feelings described by the hospital workers when recalling role overload, whether they were referring to role overload appraisal or how they felt at the end of the episode. Russell's model represents affective states around the circumference of a circle in a two dimensional space.

According to Russell (1980), within this model emotions and feelings can be categorised by valence (positive or negative) and by degree of arousal (high or low). Based on these two dimensions, a typology of emotions and feelings identified in this study was developed from findings in Chapters 10 and 12. This typology is presented in Figure 136. The emotions and feelings referenced either during appraisal or when respondents were talking about how they felt when the episode ended (i.e., post appraisal) were classified according to whether they were negative or positive (shown at the top of Figure 13-6), or high or low arousal (shown on the left of Figure 13-6).

An important finding from this study (see Figure 13-6) is that the personal emotions and feelings recalled by the respondents when describing the role overload appraisal process itself were found to be quite different to those cited when they talked about how they felt at the end of the role overload episode. The negative emotions and feelings expressed when respondents reflected on how they felt during appraisal varied but were also notably all consistent with the high arousal dimension of Russell's model (e.g. overwhelmed angry, frustrated). The only positive emotion cited in substantive, albeit small, numbers (excitement) at this stage of the appraisal process is also categorised as high arousal. The paucity of positive emotions expressed during the 
recollections of appraisal suggests that the negative emotions described are a common feature at this stage of a role overload episode. These findings suggest that role overload appraisal regularly triggered intense mostly negative emotions and feelings for these hospital workers.

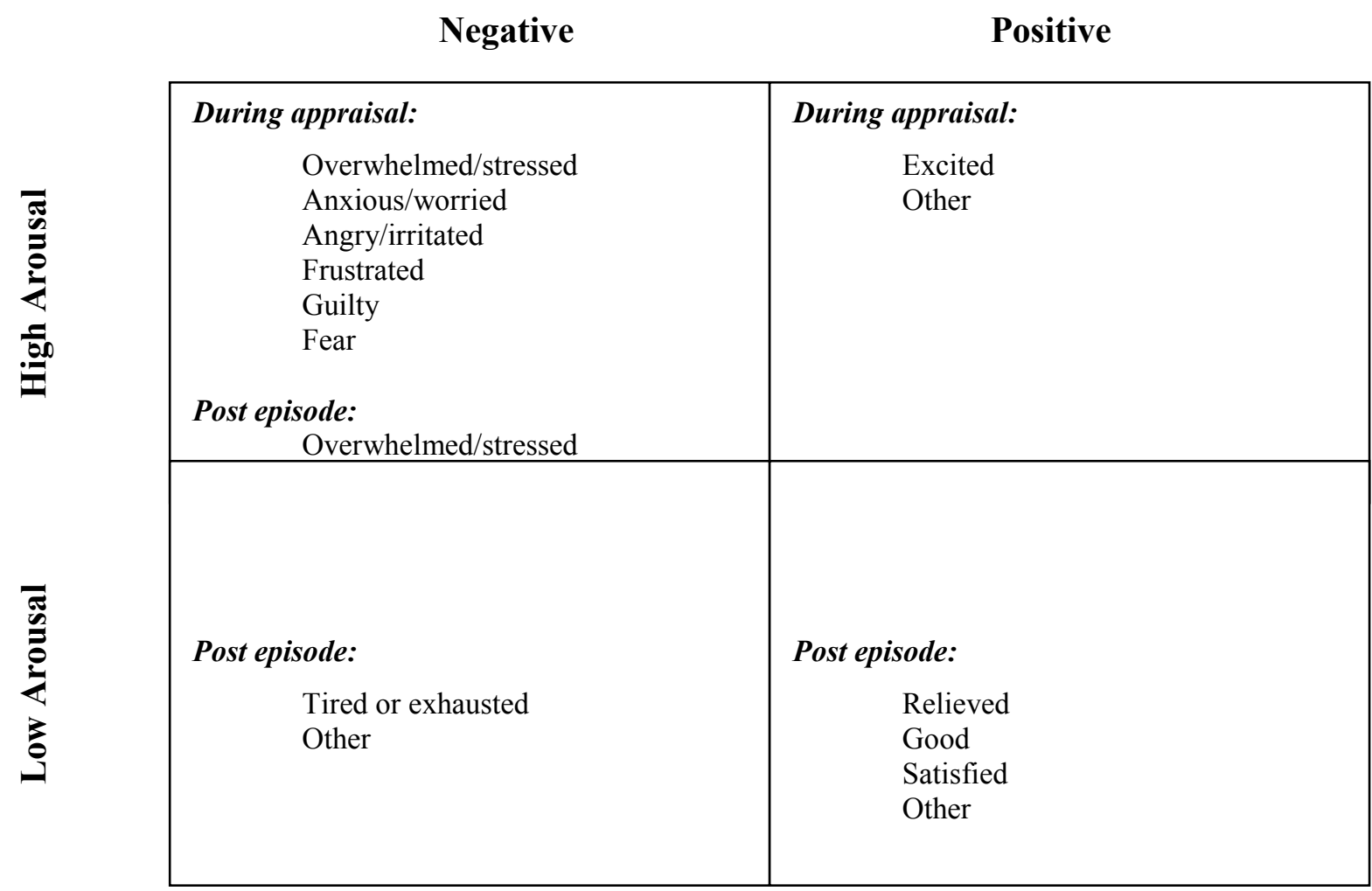

Figure 13-6 Emotions and feelings cited during recollections of appraisal and post episode experiences

From the results presented in Chapter 12 we know respondents felt that most role overload episodes were either resolved successfully or were described as having a mixed resolution (e.g. got through it but results not completely satisfactory). A small number were perceived to be unresolved. Consistent with these variable episode resolutions, when workers talked about their feelings at the end of the role overload, they talked about a broader variety of emotions and feelings: positive and negative, high and low arousal. The results from this study suggest that some respondents remained overwhelmed and 
stressed at the end of role overload episodes (i.e., expressing high arousal negative emotions and feelings), whilst others expressed feeling tired or exhausted (i.e., low arousal negative emotions and feelings). Most positive emotions and feelings expressed during descriptions of how role overload ended were low arousal, such as relief or satisfaction.

Overall, when the respondents recalled the end of role overload, the prevalence of negative emotions cited was lower whilst the number of positive emotions and feelings was much higher than when the workers described how they felt during the appraisal process itself. The relative numbers of episodes featuring negative or positive emotions and feelings expressed when talking about the end of role overload is also consistent with the fact that respondents described two episodes each, one subsequently assessed as overwhelming and one deemed not-overwhelming.

At both points in the recollections of role overload episodes, (i.e., during appraisal and post episode) the results showed that in some cases respondents described both positive and negative emotions and feelings together. The evidence for the coexistence of emotions of opposite valence is consistent with Lazarus and Folkman (1984) who specifically highlight that positive emotion can accompany negative emotion during the stress appraisal and coping process. The tendency for respondents to be more positive at the end of role overload episodes and the change in the intensity of many emotions and feelings also support Folkman and Lazarus's (1985) findings that emotions change over the course of the stress appraisal and coping process. A number of explanations may account for these changes in emotions such as coping effectiveness as well as individual 
and situational differences. Discussions of these possible explanations are reserved for Chapter 14. 


\section{Why do some overloaded workers experience greater levels of stress than others?}

The second research objective of this thesis is to explore why some people suffer stress in role overload situations whilst others do not. The examination of the results by perceived stress level of the hospital workers (low and high), and by episode assessment (not-overwhelming and overwhelming) throughout Part 5 of this thesis, allows us to analyse the role overload appraisal and coping process in three different ways in support of this objective. First, the results allow us to split the role overload episodes examined into four distinct groupings: not-overwhelming episodes of lower stress respondents, notoverwhelming episodes of higher stress respondents, overwhelming episodes of lower stress respondents, and overwhelming episodes of higher stress respondents. Second, we can identify what distinguishes overwhelming from not-overwhelming role overload. Third, we can compare the role overload situation described by respondents with low stress to episodes recalled by those with high stress respondents. This chapter will use these three different approaches as the basis for the discussions about why some overloaded workers experience greater levels of stress than others. This chapter is structured as follows.

The first section of this chapter discusses the underlying factors of the role overload appraisal and coping process that are common across all four episode groupings. Identifying these underlying factors allows us to focus on the distinguishing factors that speak to the second research objective of this thesis in the remaining sections. Section 
14.2 will discuss the four distinct role overload appraisal and coping processes by presenting a hypothetical story that typifies what the employees in each of the four episode groupings told us about role overload. The final two sections will then return to the theoretical framework of role overload (presented in Chapter 13) as a basis for discussing the differences across episode groupings. Specifically, Section 14.3 compares the characteristics of role overload assessed as not-overwhelming to those assessed as overwhelming, whilst Section 14.4 discusses how role overload appraisal and coping differs for low stress workers compared to those with higher stress levels.

\subsection{Underlying factors of the role overload appraisal and coping process}

The common underlying factors of the role overload episodes recalled by the thesis sample are displayed in Figure 14-1. To be included in this figure (i.e., considered as common) the factor had to meet two conditions. First, the frequency with which the factor occurred in each episode grouping was approximately equal. Second, it was cited in at least $40 \%$ of the episodes of each grouping. The reasons for choosing a minimum proportion of $40 \%$ were driven by the need for brevity and to maintain consistency with the data display rules set out at the beginning of the results chapters of this thesis (i.e., Part 5). Additionally, it was felt that representation in four or more of the ten episodes in all four groupings suggests a consistent and substantive feature of role overload for the 40 episodes examined.

All the figures displayed in this chapter use word cloud (or tag cloud) visual representation techniques to focus on the details of the role overload appraisal and coping 
process in a simplified form. A brief description of the characteristics of this figure (using Figure 14.1 as an example) is provided below.

The large downward arrow on the left of the main body of Figure 14-1 is used to indicate that role overload appraisal and coping is a process. While the process is iterative, space does not allow the representation of return arrows. The key elements of the process (as presented in the conceptual framework developed in Chapter 13 - see Figure 13-1) are also listed on the left hand side of the figure, linked by arrows to represent the overall direction of the process and, where appropriate, the interactions between elements. For example, role overload appraisal is represented by three elements: the environment, the person and the two part cognitive appraisal process (i.e., primary and secondary appraisal). They are listed one after the other in Figure 14-1 with bidirectional arrows to indicate the dynamic relationship between them.

Aligned with the elements of the process displayed on the left, the main body of the figure is a formatted word cloud naming the factors of interest, for each process element displayed, in a font size proportional to how frequently the factor is mentioned within the episode grouping. Consistent with recommendations on displaying qualitative data (Patton, 2002), the objective of using word cloud techniques is to represent the relative frequency of concepts identified in the thesis, without focusing on quantitative differences. This is done in this thesis by linking the font size to the number of times a factor was cited. For example, in Figure 14-1, factors cited in 40\% of the episodes in each grouping are displayed with a smaller font size; in $50 \%-60 \%$ of episodes in each grouping with a medium font size; and in $70 \%$ of episodes or more, with a larger font size. 


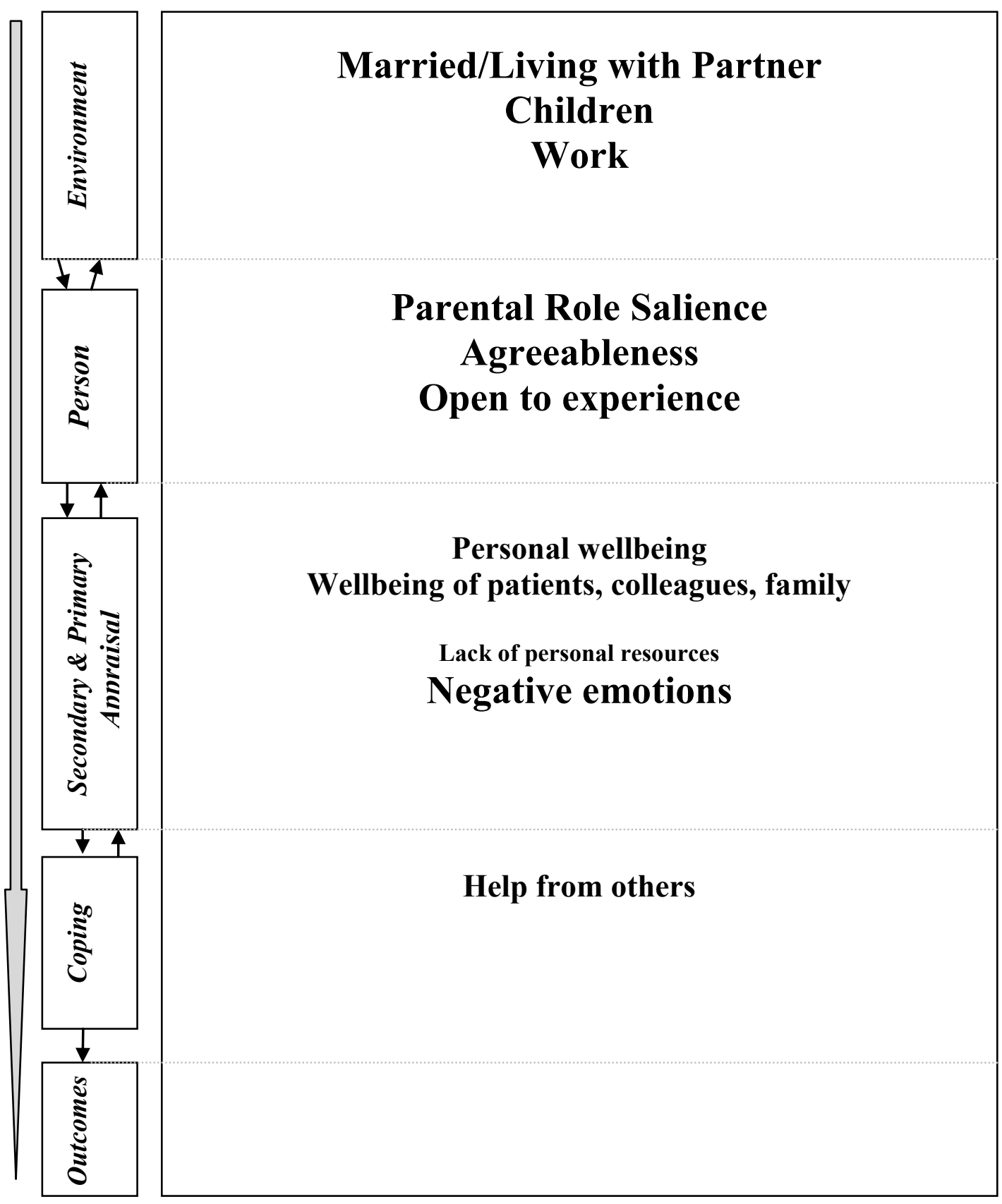

Figure 14-1 Common underlying factors of the role overload appraisal and coping process

In the subsections below, each of the factors identified in Figure 14-1 will be briefly described. The sections will discuss factors in the order in which they appear in the figure, starting with environment factors. Possible relationships between the factors of 
interest, suggested by the data, will also be discussed with reference to their influence in the role overload appraisal and coping process. To ensure parsimony, references to the literature given in Chapter 13 will not be repeated in these sections.

\subsubsection{Underlying environment factors}

A large majority of the women participating in this case study are either married or living with a partner. Most also have children, suggesting that these workers have busy home lives. Work rather than family demands were the primary source of role overload for the workers in this sample. In fact, at least $70 \%$ of the episodes in each of the four groupings involved a work situation. The extent to which these respondents focused on work rather than family role overload is consistent with findings for the Canadian workforce and for the health sector (Higgins et al., 2010). Work-family conflict does not appear to be an underlying property of role overload, as it was mentioned in only one fifth of all the episodes recalled.

\subsubsection{Underlying person factors}

High levels of parental role salience were found to be a common person factor affecting the vast majority of respondents in all four episode groupings. This finding suggests that non-work roles are somewhat more important to these overloaded individuals than their work roles.

The hospital workers interviewed for this thesis also share two personality traits in common. Most scored high levels of the traits agreeableness and 'openness to 
experience'. According to Gosling (2003) these workers therefore have warm and sympathetic demeanours and are willing to take on new challenges.

\subsubsection{Underlying primary and secondary appraisal factors}

Hospital workers, regardless of episode grouping, commonly described role overload as challenging or threatening to personal wellbeing and the well-being of others.

A lack of personal resources (e.g. time and energy) was identified as an influential factor in the secondary appraisal of role overload coping resources by a plurality of respondents in all episode groupings. When asked how they felt during role overload appraisal, hospital workers also mentioned negative emotions and feelings in almost all the episodes they described. No specific negative emotion or feeling was, however, consistently cited across the four episode groupings.

\subsubsection{Underlying coping strategies}

Regardless of worker stress level or how the role overload was assessed, receiving help from colleagues, family or friends was a commonly used strategy for coping with role overload.

\subsubsection{Underlying outcomes}

There are no outcomes from the role overload episodes that are commonly cited in substantive numbers equally across all four episode groupings. Thus, the negative 
emotions cited in most episodes during role overload appraisal are, post episode, no longer an underlying factor of the role overload episodes recalled by the hospital workers.

\subsection{Role overload appraisal and coping by episode assessment and by respondent stress}

This section uses the data from this study to tell the story of role overload appraisal and coping as described by four fictitious hospital workers, Anna, Beth, Cate and Dawn. While Anna and Beth both reported low levels of stress, Beth was overwhelmed by role overload but Anna was not. Cate and Dawn on the other hand both reported high levels of stress. Dawn also found role overload to be overwhelming, whilst Cate did not.

In the sections below, a third person narrative approach is used as follows. Section 14.2.1 narrates a "typical" role overload episode assessed as not-overwhelming by the low stress worker, Anna, whilst Section 14.2.2 tells the story of overwhelming role overload as experienced by the low stress respondent, Beth. The not-overwhelming role overload episodes described by the high stress worker, Cate, is narrated in Section 14.2.3. Finally role overload assessed as overwhelming by the highly stressed hospital worker, Dawn, is described in Section 14.2.4.

For each of the four narratives, the role overload appraisal and coping process is represented by a separate word cloud diagram (Figures 14-2 to 14-5). The format used in these diagrams is similar to that employed in the first section of this chapter. To emphasise the relative frequencies, and any special characteristics, of the factors identified, the following conventions were followed when constructing these figures: 
- Factors cited in $70 \%$ or more of the episode grouping are named in the largest font and positioned in the centre of the main body of the figure.

- Factors cited in $40 \%$ of the episodes are named in the smallest font and positioned furthest away from the centre, at the edges of the main body of the figure.

- Factors cited in 50-60\% of the episodes are named in a medium sized font and are positioned off centre in the main body of the figure.

- Factors named in italics were cited more frequently in the episode grouping represented than in other groupings.

- A pale grey font is used to identify factors that stand out because of their absence. In this case the factor was rarely mentioned in the episode grouping under consideration whilst being mentioned equally often in the other three episode groupings.

The narratives will each follow the approach outlined in the previous section.

\subsubsection{Not-overwhelming role overload episodes described by Anna, a hospital worker who reported low levels of stress}

Figure 14-2 presents the role overload appraisal and coping process for the notoverwhelming episodes described by the low stress respondent Anna.

Anna has an administrative job at the hospital. She lives with her partner and her two children, both in their late teens. Her role as a mother is important to her and she works hard to be a good parent. She also places high value on being a good spouse and on being a good worker for the hospital. Anna appears, however, less willing, or less able, to put the efforts she would like into her role at work. This may be because her family roles are more important to her, or because she faced difficulties committing the necessary resources to her job. 
Not-overwhelming role overload by Anna, a hospital worker with low stress levels

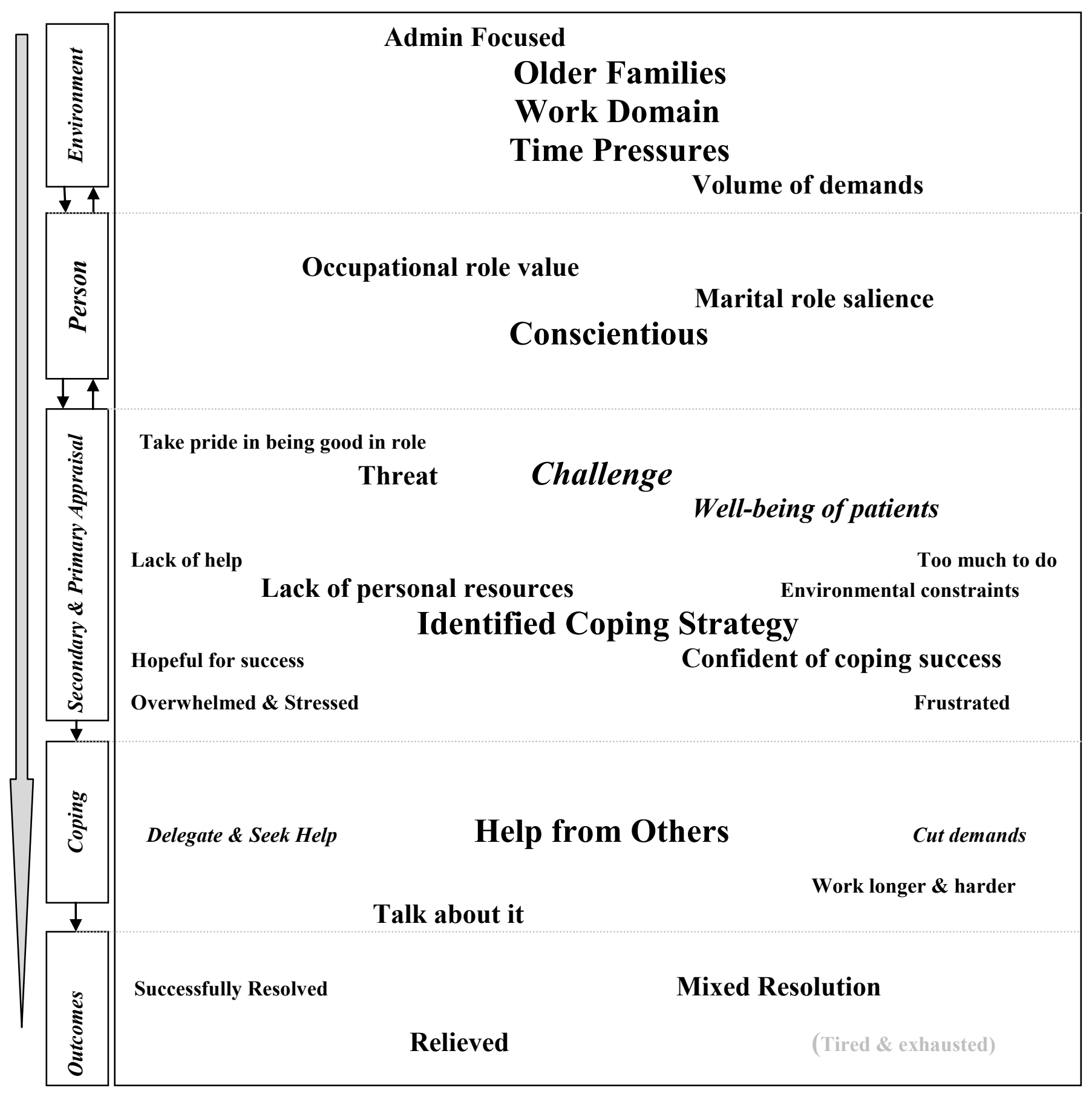

Figure 14-2 Not-overwhelming role overload appraisal and coping of low stress hospital worker Anna 
Results from the personality trait questions that Anna answered at the end of her role overload interview suggest that, compared to her high stress colleagues Cate and Dawn, Anna is more self-disciplined and dependable (typical of someone with higher levels of conscientiousness), and also that she is a calm and emotionally stable person (typical of someone with lower levels of neuroticism) (Gosling et al, 2003).

The role overload episodes subsequently assessed as not-overwhelming by Anna were predominantly set at work. Whilst work may be more troublesome than other roles for Anna, the importance of the family roles to this hospital worker supports the idea that overload at home is accepted and perhaps even enjoyed. When Anna talked about why she felt overloaded during these not-overwhelming episodes at work she spoke of the time pressures she experienced as well as the sheer volume of the role demands she faced:

There were very tight timelines, so that was difficult.

Because of the volume of the things we had to do, you have to take the time to think back and reflect.

Concerns about her ability to get work done to personal standards, were also an important internal role pressure for Anna in these episodes.

I pride myself on doing a good job and usually respecting the demands of the higher powers. Just for my own personal satisfaction I like when I can do a good job and complete it.

The relative importance to Anna of meeting personal quality standards together with the prevalence of time pressure and the high volumes of role demands common in the not-overwhelming role overload she described should not be a surprise given Kahn et al.'s (1964) definition of role overload as demands that cannot be completed within given time limits and quality requirements. These traditional characteristics of role overload 
described by Anna in the episodes she assessed as not-overwhelming suggest that other non-traditional factors may be more prevalent in the overwhelming episodes described by Beth or in the episodes talked about by Anna's high stress colleagues Cate and Dawn. According to Lazarus and Folkman (1984) primary and secondary appraisal are interdependent processes that work together when an individual such as Anna evaluates the extent to which role overload challenges, threatens and or harms their well-being, and ultimately determines the degree of stress experienced. Anna talked about how challenging the role overload episodes were in her descriptions of typical notoverwhelming episodes, a finding that is particularly notable when compared to the overwhelming episodes described by Beth (the other respondent who reported low levels of stress). Anna did, nevertheless, sometimes describe feeling threatened by role overload at the same time. Coexistence of challenge and threat appraisals is consistent with the cognitive appraisal research undertaken by Lazarus and his colleagues $(1984,1986)$. Notoverwhelming role overload was, however, never described as harmful by Anna.

When Anna was asked about the potential consequences of the role overload, she talked only about the risks to patient well-being.

Some of the patients would have shown up and having traveled to do the tests and not being able to do the tests. I can put myself in the shoes of the patient, and it can be frustrating, even if they understand.

We're forgetting the human part because when I call a patient to tell them we have to reschedule we may have to spend ten minutes reassuring them that we are not forgetting about them.

That Anna felt patient welfare was challenged and sometimes threatened in these episodes is consistent with her personality traits (agreeable, open to experience and conscientious) and how important the occupational role is to her. This finding also suggests that patient welfare does not normally contribute to overwhelming role overload 
in hospital employees. Also worthy of note is that only Anna (i.e., not Beth, Cate or Dawn) expressed this degree of worry about her patients, a fact reflected in the use of italics in Figure 14-2.

When Anna talked about how prepared she felt to respond to the situation, she was concerned about having enough time or energy to fulfill demands at work. She also expressed concerns about the lack of available help and mentioned that there was too much to do as well as restrictions at the hospital about what she could do to deal with the extra work (e.g. working overtime was not always allowed):

Just that there was not enough staff to handle the workload and deadlines Limitations in this case would be they wanted me to fill the cameras, but when something like this happens they don't want me to work overtime, or they want us to request it.

Anna indicated that she had no trouble identifying a coping strategy in these episodes and she was confident and/ or hopeful of coping success:

I knew I'd be able to get it done ... the issue was having to put my personal time into it

Finally, Anna expressed few of the negative emotions and feelings when recalling her appraisals during role overload. This finding suggests that negative emotions and feelings may be less common or more varied during role overload appraisal in the notoverwhelming episodes of low stress respondents such as Anna. The challenges and threats to patient well-being noted above reflect what Anna feels is at stake in these episodes (i.e., primary appraisal). Anna also reported a mixture of cautious optimism and concern in her evaluation of what can be done to cope with not-overwhelming role overload (i.e., secondary appraisal), a finding that is consistent with her high levels of conscientiousness. 
Anna used three different approaches to cope with role overload. First, she involved other people in a number of different ways. She received practical help from colleagues and also proactively delegated work or tasks to others:

The team at work were excellent. Once they heard about the mess I was in, they were great. Everyone offered their help

Initially my plan was to get through the day, then I decided to delegate.

Anna also talked about the situation with family, friends and colleagues:

Usually talk about it with the staff I'm working with. Even talking to my manager, if there's an issue about the appropriate level of care.... even at home, I talked to my husband.

Coping strategies based on social support have been shown to be linked to lower levels of stress (Lazarus and Folkman, 1984; Dewe et al., 2010). The approach of talking things through with friends, colleagues or family as a way to deal with any emotions arising in these not-overwhelming episodes is also consistent with Stanton's (2000) research linking the processing and expression of one's emotional experience with beneficial outcomes from stressful situations. The findings in the literature suggest that together these strategies may help to explain the less overwhelming assessment of these episodes and the lower levels of stress that Anna reported.

The second approach Anna took was to use multiple coping strategies to reduce the number of demands making her feel overloaded. As well as receiving practical help from others and delegating some tasks, Anna also decided not to do some of things expected of her (i.e., she 'cut demands'):

I decided to miss out some info that I didn't have time to get Also of note is the fact that Anna coped by using several different strategies to reduce demands while Beth, Cate and Dawn did not. 
Third, Anna worked longer and harder to cope with the role overload:

I was supposed to work till three, but I stayed later.

Work late-come in early

The demand reduction approach (as well as working longer and harder in some episodes) may also help to explain why these episodes were subsequently assessed as not-overwhelming and why Anna has lower levels of stress. It must be said, however, that the low levels of stress reported by Anna and the less overwhelming nature of these episodes may have made it easier for her to implement these approaches to coping with role overload.

When talking about how not-overwhelming role overload episodes ended, Anna described some successes. She also talked about a situation where some aspects of the episodes were satisfactorily resolved while others were not (i.e., mixed resolutions).

Temporarily resolved that we got her out of a dangerous situation. For me it was ok I think, once the crisis was over and the pressure was off a bit, but not completely.

In describing how she felt at that point, Anna did not mention being tired or exhausted (unlike Beth, Cate and Dawn), rather she expressed relief. These findings are somewhat surprising given that these episodes were all assessed as not-overwhelming, however, it may be that Anna who has higher levels of conscientiousness sets high standards for herself and has mixed feelings when outcomes are less than perfect.

The change in emotions over the course of the not-overwhelming episodes, from feeling overwhelmed and stressed, or frustrated, during role overload appraisal, to feeling relieved at the end is consistent with Folkman and Lazarus's (1985) findings that emotions can change over the course of the stress appraisal and coping process. Whilst 
the changes in emotions and feelings and the successes that Anna did acknowledge may be explained by her use of demand-reduction coping strategies, the fact that the role overload episode was over may be the only reason why Anna felt relieved.

\subsubsection{Overwhelming role overload described by Beth, a hospital worker with low levels of stress}

Figure 14-3 presents the role overload appraisal and coping process for the episodes assessed to be overwhelming by the low stress respondent Beth.

Beth also has an administrative job at the hospital and she is married, with two teenage children. As a respondent who recorded low levels of stress Beth shares the same personality traits and role values as Anna. They both also talked about work when recalling a time when they were overloaded. This suggests that other factors in the role overload appraisal and coping process should be examined to find out why Beth felt overwhelmed and Anna did not. The first notable feature of the episodes recalled by Beth is that when she talked about the role overload and coping process, unlike Anna, Beth tended not to focus on single issues (see Figure 14-3), but instead described a mix of factors in her discussions of role overload. This suggests that the overwhelming episodes described by Beth were more varied, and multiple contributing factors were common. 


\section{Overwhelming role overload by Beth, a hospital worker with low stress levels}

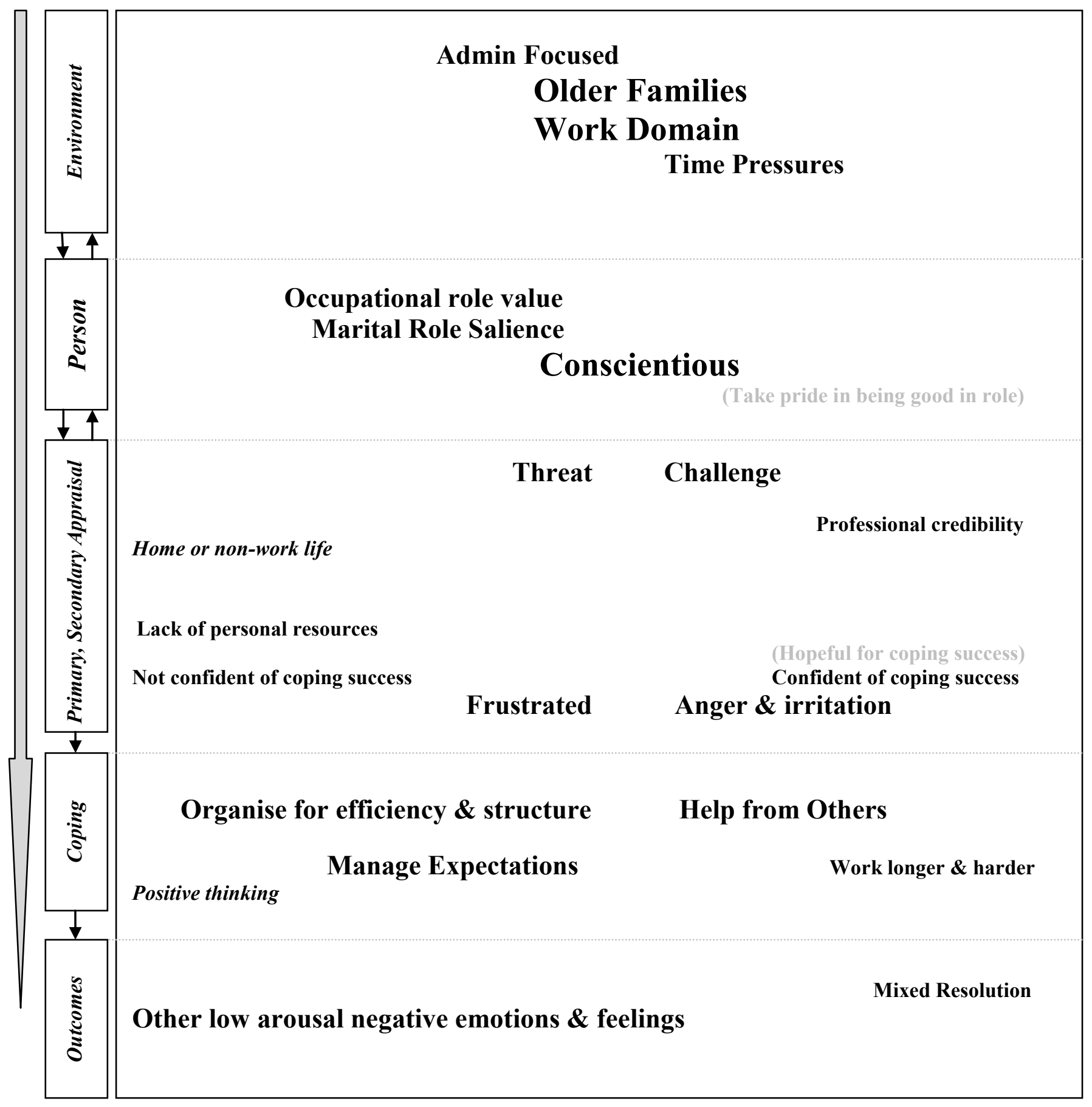

Figure 14-3 Overwhelming role overload appraisal and coping of low stress hospital worker Beth 
The environment and the person factors talked about in the role overload recalled by Beth give little clue as to why these episodes were assessed as overwhelming. The experience she described was not dominated by the traditional characteristics of role overload as were the not-overwhelming episodes described by Anna. Whilst Beth did mention time pressures as a contributing factor to her feelings of overload, the volume of demands or the ability to get work done to personal quality standards did not seem to be important to her description of overwhelming episodes. The fact that Beth rarely talked about how she took pride in being good at her role is a result that stands out since Anna, Cate and Dawn cite this person factor equally often in their recollections of role overload. The reasons for this finding are not immediately obvious. It may be that other issues are more important to well-being for Beth.

Indications of why overwhelming role overload recalled by Beth is different from the not-overwhelming episodes described by fellow low stress hospital worker Anna were more evident when Beth talked about how she appraised the event that made her feel overloaded. First, Beth typically described the role overload experience as both threatening and challenging. Knowing from our discussions in the previous chapter how reluctant these respondents were to talk about threats; this finding suggests that the risk of potential harm was often present for Beth during role overload.

Second, in overwhelming role overload Beth seemed more concerned about the potential consequences for personal well-being than for the well-being of anybody else. Beth specifically noted that she felt her professional credibility and her home or nonwork life to be at risk: 
It would have showed a lack of professionalism if I hadn't done it. I would have made mistakes in my work, and I'd hear from Toronto about it.

Ifelt that it was infringing on my home time.

Most notable, results showed that threats and challenges to home and non-work life were cited more often in the overwhelming episodes described by Beth than in the notoverwhelming episodes described by fellow low stress respondent Anna, or by the overwhelmed high stress respondent Dawn. Intuitively, the possibility of harm to these personal aspects of well-being would be of greater concern to a worker like Beth who values her non-work roles highly, regardless of how important patient care was to her, and hence may help to explain why these episodes were evaluated as overwhelming.

Another aspect of the cognitive appraisal process described by Beth that stands out is that she seemed to be less confident about overcoming role overload in these overwhelming episodes than Anna was in not-overwhelming role overload. Furthermore, whilst Beth did not mention any restrictions on how she was able to cope, neither did she mention any specific coping strategies when describing her initial evaluations of the overwhelming role overload, suggesting that coping plans were difficult to put in place in these episodes. Finally, when Beth described how she felt during the appraisal of role overload she recalled being angry, irritated and frustrated, suggesting that negative emotions and feelings were common during appraisal in most of the episodes that Beth subsequently assessed as overwhelming:

I would say "la colere". (in English it is closest to "anger"). I was irate, I was mad. The word in French represents more of an image.

The frustration of me having to do that on top of everything else. I'm not one to ask "why me?", but quite frankly, why me? 
The factors influencing primary and secondary appraisal suggest that concerns for personal well-being, together with a lack of coping plans may have lead Beth to appraise these episodes as overwhelming/more threatening as suggested by the prevalence of negative emotions. On the other hand, overwhelming role overload may have made it more difficult for Beth to make coping plans, making her more worried about the impact to her home life, which is particularly important to her.

Beth described using four different problem focused coping strategies to cope with overwhelming role overload: 'help from others', 'organize for efficiency and structure', 'manage expectations' and 'work longer and harder':

Think methodically, organize myself.... Support staff member organised equipment for open house.....I sent my regrets for a meeting due to start at 10:30am...... rushed around.

In other words, Beth relied more on demand-management strategies to cope with role overload than did Anna in her not-overwhelming episodes. This difference in coping styles might explain why Beth found her overload to be overwhelming while Anna did not. It may also be possible that Beth just could not use demand reduction coping strategies because she was overwhelmed by her situation.

Interestingly, Beth also used the emotion focused demand-reduction strategy, positive thinking, a finding that stands out particularly when compared to the notoverwhelming episodes described by Anna, who never used this strategy. Why this strategy is associated with overwhelming role overload recalled by low stress respondents such as Beth is not immediately obvious. The overwhelming nature of the role overload may mean that positive thinking, which involves deliberate cognitive manipulations to 
change the meaning of emotional demand, is the only proactive demand-reducing coping strategy open to Beth.

When she was asked about how the overwhelming role overload ended, Beth described mixed outcomes. In other words, whilst she managed to get through it, Beth felt that some aspects of the situation were completed unsatisfactorily or left unfinished:

I was exhausted, long day, late for meeting at 3pm, ... whole lot of things being done but not done completely

In describing how she felt once the role overload was over, Beth also cited various low arousal negative emotions related to feelings of deflation and lack of motivation. These emotions and feelings were very different to the anger and frustration that Beth expressed when describing the appraisal of overwhelming overload. Such changes in emotions and feelings suggest that the coping strategies used had some measure of success despite the episode being assessed as overwhelming. Alternatively, Beth may have just felt deflated because of the unsatisfactory outcomes.

\subsubsection{Not-overwhelming episodes described by Cate, a hospital worker who reported high levels of stress}

Figure 14-4 presents the role overload appraisal and coping process for notoverwhelming episodes described by the high stress respondent Cate. 
Not-overwhelming role overload by Cate a worker with high stress

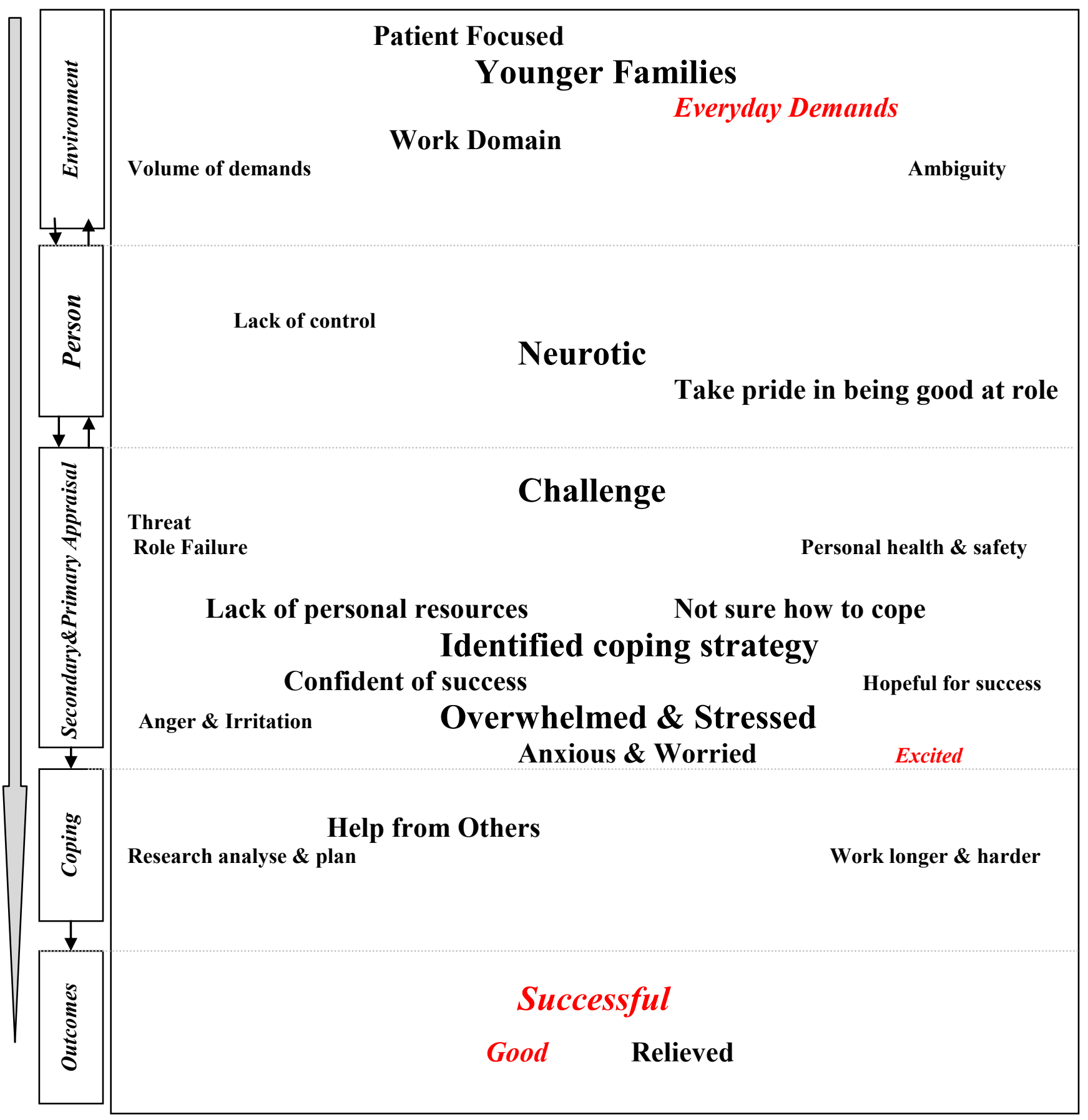

Figure 14-4 Not-overwhelming role overload and coping process of high stress hospital worker Cate

Cate has a job as an occupational therapist at the hospital and is involved with patients on a daily basis. She is married with a young family, having two young children 
under the age of 11. Like Anna and Beth, her role as a parent is very important to Cate and she puts a lot of effort into being a good mother. However, for Cate the marital and occupational roles were somewhat less important than they were for her low stress colleagues, Anna and Beth. Results from the personality trait questions that Cate answered at the end of her role overload interview suggest that some aspects of Cate's personality appear to be quite different to Anna and Beth. Specifically, she reported that she often felt anxious and was easily upset, a trait in keeping with someone who has higher levels of neuroticism. She also described herself as disorganized and less disciplined, traits typical of someone with lower levels of conscientiousness (Gosling et al, 2003).

The descriptions of the role overload appraisal and coping process by Cate suggest that the daily chore of dealing with a busy home and work life is a major cause of overload for her. First, it was not untypical for Cate to talk about role overload set in the home. The fact that Cate recalled more episodes in which role overload at home played a part compared to the low stress respondents Anna and Beth, may reflect the additional demands that come with a younger family. Role overload set in the home may also come to mind more often for Cate because she values her organizational role less than Anna and Beth do. The idea that Cate has a busy home and work life is also supported by the fact that the events most likely to trigger role overload for Cate were described as everyday demands (at home or at work):

Well typically on a normal day coming into work the minute you get in you are on and you are basically running

This finding stands out when compared to the role overload episodes described by Anna, Beth and Dawn. 
When asked about what made her feel overloaded during events that she subsequently assessed as not-overwhelming, relatively few factors of the environment came to mind for Cate. She mentioned only ambiguous role demands as well as the volume of demands she faced. Two person factors are notable in Cate's descriptions of how she appraised role overload. First, Cate talked about the pride she takes in being good in her role(s).

One of my values is to be a competent occupational therapist and to help people This suggests that concerns about the ability to get work done, to personal standards, were particularly important to her perception of role overload in these episodes. Second, Cate talked about the fact that she felt that she had little control of the situation. More specifically, she felt she had no say over the scheduling of the demands creating the role overload or no control over the availability of coping resources to deal with it.

That I don't get to choose. I don't have any control in that circumstance over the intrusion on my time

Cate's description of how she appraised role overload is characterized by contradictory expressions of optimism and pessimism. First, Cate typically described these episodes as challenging. Only a few were described as threatening and, like Anna, Cate never described role overload as harmful. The small number of threatening episodes may help to explain why she assessed these episodes as not-overwhelming, however, the reason why Cate described so few episodes as both challenging and threatening is not immediately obvious. It may be that Cate, being less conscientious and hence less disciplined (Gosling et al., 2003), was just less specific in describing role overload appraisals. An alternative explanation is that as many of these not-overwhelming 
episodes were triggered by everyday demands, which were typical of her life, , they were less likely to be appraised as both challenging and threatening.

Second, when asked to describe the potential consequences of role overload assessed as not-overwhelming, Cate, unlike Anna, seemed to be more concerned about the risks to personal health and safety and role failure than about risks to patients:

I always have a concern if I am not at my best there is always potential for myself to get injured

If I didn't succeed I (would have) failed at doing what I'm paid for

The high level of neuroticism reported by Cate means she tends to get upset more easily. Cate may therefore be more likely to articulate concerns about failing in a role that she wants to do well in. On the other hand, workers like Cate who are probably highly stressed for other reasons may find it more difficult to commit efforts to roles they take pride in, or are genuinely at risk of ill health because of the additional stress of role overload.

When describing secondary appraisal of not-overwhelming episodes Cate mentioned that a lack of personal resources and not being sure what to do also made it hard for her to cope with role overload. Despite this, Cate did identify specific coping plans when she described her initial thoughts about how she would overcome the role overload. Furthermore, Cate was confident or hopeful of coping success, a perception she shared with Anna:

I asked for a vacation day. Asking for it is hard but once I have got through that piece and I feel that everything is organized for the day off than I am okay.

Perhaps with everything else going on in her stressful life and the fact that she lacked control over coping resources, Cate felt initially pessimistic about how to cope with the 
role overload, but once she focused on the role overload she was able to formulate a plan she was confident in.

Lastly, in sharp contrast to Cate's confidence in coping success and to the emotions and feelings expressed by Anna and Beth when describing the appraisal of role overload, Cate stated she felt anxious and worried, and angry and irritated by the role overload she was experiencing. Cate was also the only one of the four who talked about feeling excited about the role overload. The emotional descriptions expressed by Cate when she talked about how she felt during the role overload appraisal in an episode that she subsequently categorized as not-overwhelming may be explained by her neurotic personality. On the other hand, the high levels of stress reported by Cate, which could be caused by other issues in Cate's life, may have made her very sensitive to additional stressors such as role overload over which she has no control.

When asked how she coped with role overload, Cate recalled using the problem focused demand-reduction strategy, 'help from others'. She also described 'researching, analyzing, and planning', as well as 'working longer and harder', both problem focused demand-management strategies. Overall, Cate cited using fewer coping strategies in substantive numbers than Anna and Beth, which suggests that Cate's coping approach was more varied. The emphasis on problem focused demand-reduction strategies, whilst much more limited than Anna's approach, may also help to explain why these episodes were subsequently assessed as not-overwhelming.

The fact that Cate did not talk about using specific emotion focused coping strategies is somewhat surprising given the levels of negative emotions and feelings expressed by Cate during role overload appraisal. This may be because Cate used 
multiple different coping actions to deal with the emotions arising from these episodes of overload. Alternatively, this finding may be attributed to the idea that the negative emotions expressed by Cate when appraising the role overload were transient expressions typical of someone who tends to be easily upset (i.e. neurotic), and therefore fewer emotion focused strategies were needed.

The episode resolution outcomes described by Cate for the role overload she evaluated as not-overwhelming stand out in comparison to the episodes described by Anna, Beth and Dawn. Specifically, Cate explained that the situations ended successfully and she felt good about the outcomes. A reaction that Cate had in common with Anna when describing how they felt at the end of not-overwhelming episodes was that both expressed relief when it was over. The feelings of relief and other generally positive outcomes and feelings expressed by Cate suggest that coping strategies implemented were successful in reducing the levels of stress expressed during cognitive appraisal. Alternatively, as suggested above, the negative emotions talked about during appraisal were transient and as might be expected in not-overwhelming role overload, outcomes were consistently positive.

\subsubsection{Overwhelming role overload episodes described by Dawn, a hospital worker with high levels of stress}

Figure 14-5 presents the role overload appraisal and coping process for the overwhelming episodes as described by the high stress respondent Dawn. 
Overwhelming role overload by Dawn, a worker with high stress

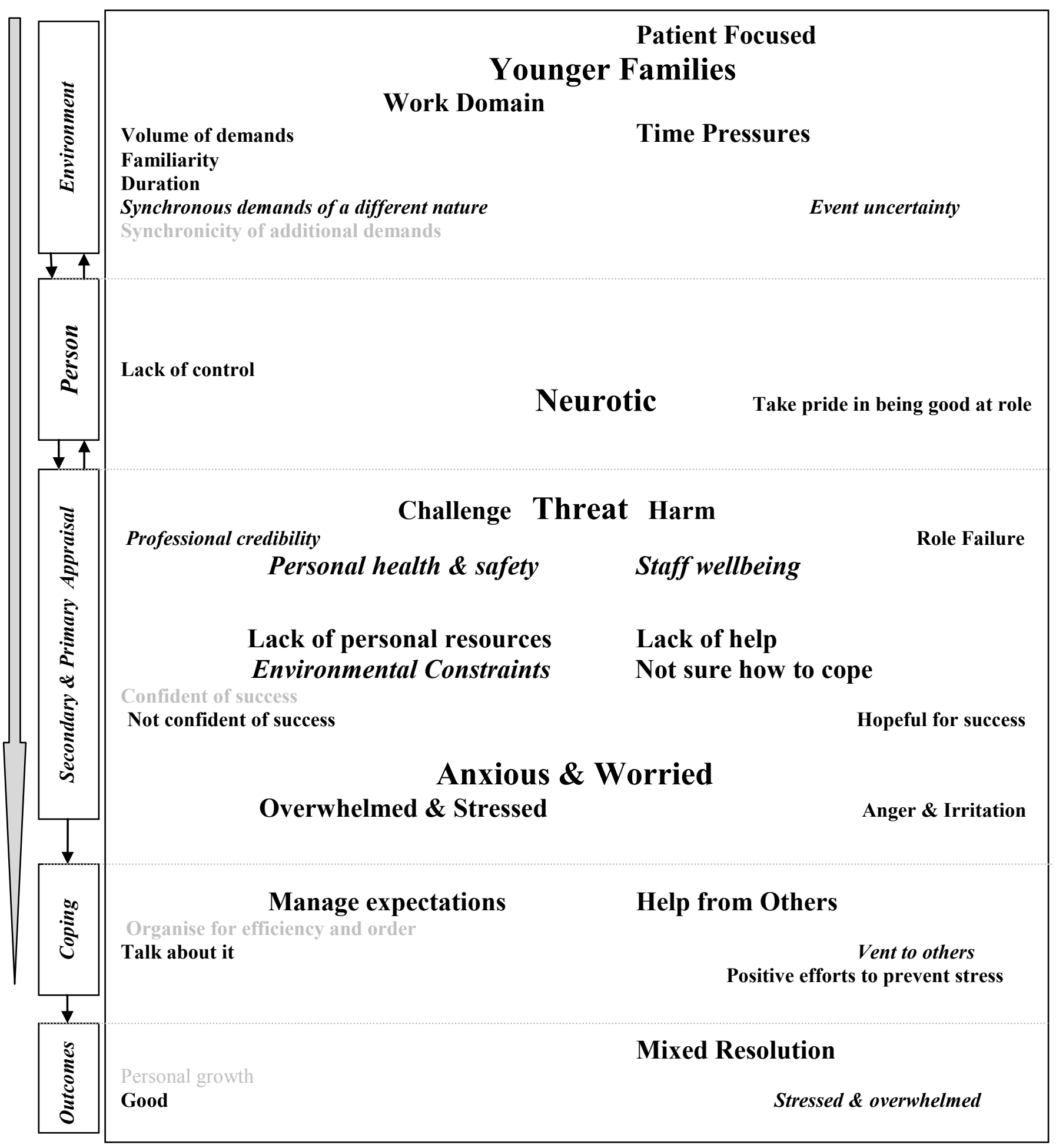

Figure 14-5 Overwhelming role overload appraisal and coping for high stress hospital workers Dawn 
Dawn has a job caring directly for patients at the hospital. She lives with her partner, and has two young children. Dawn had much more to say about her experiences of role overload than her colleagues Anna, Beth and Cate. Like Cate, Dawn was more likely to talk about role overload set in the home than were the low stress respondents, Anna and Beth. The range of role overload factors making Dawn feel stressed when faced with overload, however, suggests that the environment of the overwhelming role overload experienced by high stress workers is complicated and often features multiple characteristics, that have the potential to create appraisals of challenge, threat or harm. When Dawn talked about what made her feel overloaded she did not focus on a single issue. Rather she mentioned several different features of the role overload situation at the same time. For example, Dawn mentioned that she faced time pressures, higher volumes of demands, ongoing role overload (i.e. duration), and circumstances that had overwhelmed her in the past (i.e. familiarity), suggesting that role overload was a chronic condition for her:

It had been a situation I had to deal with before and I think from previous experience just knowing how demanding it can be, I initially was just really kind of just panicking

Well it hasn't ended yet - I'm still working on it, it hasn't ended

Two specific situations were mentioned more often in the role overload recalled by Dawn than in those described by Anna, Beth or Cate (as indicated by the italic font in Figure 14-5). First, Dawn described not knowing whether events were going to occur or not and how uncertain this made her feel:

I am always a little worried about something unexpected coming up because I really don't have any grey area or any play time to deal with a crisis and I never really know what to expect in terms of makeup of the groups either. 
Such event uncertainty was largely exclusive to the overwhelming role overload episodes recalled by high stressed individuals such as Dawn.

Second, Dawn talked about the difficulties of managing multiple projects or crises, often across multiple role domains:

...simultaneous to this which is already a huge workload issue for me I also had people demanding my time and energy to other major corporate initiatives as well.

Whilst uniquely prevalent in the episodes recalled by Dawn, such descriptions (labeled synchronous demands of a different nature) are consistent with Kahn et al.'s $(1964,1966)$ descriptions of role overload. Notably, whilst the synchronicity of different demands is noted more often by Dawn, additional demands synchronized with an existing, similar workload were never mentioned in her descriptions of overwhelming role overload. The reasons for this are not immediately obvious but it may be that role overload featuring synchronous additional demands reflect a less complicated environment than those described in these overwhelming episodes.

Whilst the role overload environment that Dawn described seems to be more varied than that described by Cate, these two high stress individuals shared a number of person factors in common. They reported the same personality traits (high levels of neuroticism, agreeableness and openness to experience and low levels of conscientiousness) and role values (high parental and lower marital and occupational role salience). They both also mentioned a lack of control over the demands causing role overload and over coping resources as an issue as well as the importance doing a good job.

That being said, the appraisal of role overload by Dawn is notably different than the evaluations by Cate, and Anna and Beth, when they discussed their role overload 
experiences. First, it was not untypical for the episodes recalled by Dawn to be described as threatening, harmful, and challenging to well-being:

I think I do find it threatening in a way, but I do find it challenging above all. I don't like to say it's harmful, but stressful, so if stressful, it's harmful so yes you can use that word.

Thus, in contrast to the not-overwhelming episodes described by Cate, multiple appraisals were common in the overwhelming episodes described by Dawn. This supports the idea that there was a lot going on in the role overload episodes she recalled as overwhelming. Furthermore, the frequent appraisals of harm may explain why these episodes were assessed as overwhelming.

Second, Dawn cited a broad range of concerns, for both personal well-being and for the well-being of others, in her discussions of the potential consequences of overwhelming role overload:

I felt only threatened of my nursing license and am I doing enough am I doing enough to protect the people who work here?

Four main consequences of overwhelming role overload were talked about by Dawn. First, concerns about personal health and safety and staff well-being seemed to be particularly important in the role overload she described. Second, Dawn also expressed concerns about professional credibility and role failure. In particular, Dawn mentioned her concerns about professional credibility more often than her high stress colleague Cate. Third, the tendency for high stress respondents to have greater concerns about personal health and safety is more noticeable in the overwhelming episodes described by Dawn than in the not-overwhelming episodes described by Cate. The frequency with which such issues were mentioned by Dawn and the focus on personal well-being (not 
shown in Figure 14-5), when describing these role overload events, was also notably greater than observed for Anna, Beth or Cate. Finally, it is also of note that only Dawn expressed concerns about staff welfare. Why did Dawn appraise overwhelming role overload the way she did? First, it may be that the circumstances she found herself in might increase the chance of stress related ill-health (personally or for other staff) or damages to professional credibility for this overloaded and highly stressed worker. On the other hand, as an easily upset individual (i.e. neurotic), Dawn may be more likely to articulate such worries whenever overwhelming role overload arises over which she has no control.

When Dawn described her initial evaluations on how she might cope with role overload (i.e. secondary appraisal), she identified four potential problems. As well as mentioning a lack of personal resources Dawn felt she lacked help, was frequently faced with environmental constraints at work, and often talked of not knowing how to cope:

I have a problem with energy. I am always tired so to me it is always a big thing to move.

Well, I was really not sure how I was going to get the work done.

(I was concerned) knowing that I was the only one there that there wasn't any backup.

Environmental constraints, such as inflexibilities in work time scheduling, were notably mentioned more often by Dawn than by Beth (low stress) in the episodes evaluated as overwhelming, or by Cate (high stress) in not-overwhelming role overload:

If I didn't have to use all my vacation for things that were not vacation then it would be more comfortable for me. 
When asked how confident Dawn felt about whether she could cope with the role overload, Dawn was equally likely to reply negatively as she was to say she felt hopeful. Dawn rarely talked, however, of being fully confident about her ability to deal with the situations she faced, a finding that stands out because such confidence was commonly expressed by Anna, Beth and Cate in their recollections of role overload

Lastly, when Dawn talked about how she felt during role overload appraisal, negative emotions and feelings were cited. Moreover her emphasis was on feelings of anxiety and worry and she made no mention of any positive emotions and feelings during her discussions of role overload appraisal.

I get worried. I get worried about I mean stress is really the overriding factor. I can end up in tears just because I feel so overwhelmed with it all.

The high levels of anxiety and worry reported by Dawn suggest a link between overwhelming role overload, high stress, perceptions of threats to personal and staff wellbeing and restrictions on the ability to cope. It is possible, however, that as an individual that is highly stressed in other areas of her life and who also tends to get upset more easily, Dawn may be more likely to feel anxious and threatened when faced with overwhelming role overload

The mix of problem focused and emotion focused coping strategies used by Dawn to cope with overwhelming role overload was notably different to the strategies used by Anna, Beth and Cate. First, only two problem focused coping strategy were mentioned by Dawn: 'help from others' and 'manage expectations'. Moreover, Dawn rarely mentioned using the problem focused demand-management strategy, 'organise for efficiency and order' to cope, , a finding that stands out because her three colleagues all talked about using this strategy during their role overload recollections. The lower numbers of 
problem focused strategies, the specific absence of the strategy to organize for efficiency and order, and Dawns uncertainty about coping success are consistent with Lazarus and Folkman (1984) view that problem focused strategies are used less often in stressful conditions appraised as not amenable to change.

A second notable feature of Dawn's coping approach is that she was more likely than Anna, Beth or Cate to use multiple emotion focused strategies. Dawn's greater use of emotion focused strategies suggests that coping difficulties may have contributed to greater levels of stress for her. The overwhelming nature of the role overload experience teamed with her higher levels of stress may also explain why Dawn used more emotion focused strategies than Anna, Beth or Cate.

Dawn had mixed opinions about how her role overload experience ended. On a positive note, she noted that she had "managed to get through it". She did, however, note that in many ways the role overload episode ended unsatisfactorily, a marked contrast to the successful outcomes described by Cate. Dawn was also the only one to cite specific negative emotions at the end of role overload (i.e. she described feeling stressed and overwhelmed). This finding suggests that high arousal negative emotions and feelings remained throughout the entire overwhelming episode. Whilst, Dawn also talked about feeling good at the end of overwhelming role overload she never identified ways she had personally gained from the experience, a finding that contrasts with the episodes of her high stress colleague Cate. 


\subsection{What distinguishes role overload appraisal and coping in not- overwhelming episodes from that in overwhelming episodes}

The interview with the hospital workers for this thesis was constructed so that two descriptions of contrasting role overload episodes were captured, one that was assessed by the respondent as overwhelming and one that was assessed as not-overwhelming. A summary of the key factors of the role overload appraisal and coping processes described by hospital workers in not-overwhelming and overwhelming episodes are presented side by side in Figure 14-6.

Similarly to the word cloud figure used in the previous sections, the factors of the role overload appraisal and coping process highlighted in Figure 14-6 are portrayed in different font sizes in proportion to the number of times they were mentioned by respondents when they talked about not-overwhelming and overwhelming episodes. A small font is used to represent factors cited in $20 \%$ to $40 \%$ (i.e., in $4-8$ ) of the episodes; a medium font represents those cited in 45\%-65\% (i.e., in 9-13) of the episodes; and a large font indicates that a factor was mentioned in $70 \%$ (i.e. in 14) or more episodes.

This section is divided into five separate subsections each of which focus on a different element of the cognitive appraisal and coping process. In each subsection comparisons are between episodes assessed as overwhelming and those assessed as notoverwhelming. The focus of the discussions in these subsections is on factors cited more frequently in one episode type compared to the other. To ensure brevity in these discussions, the episode type in which fewer of the factors are cited will not be mentioned but should be assumed by the reader. 
Not-Overwhelming $(\mathbf{n}=\mathbf{2 0})$ v Overwhelming Episodes $(\mathbf{n}=\mathbf{2 0})$

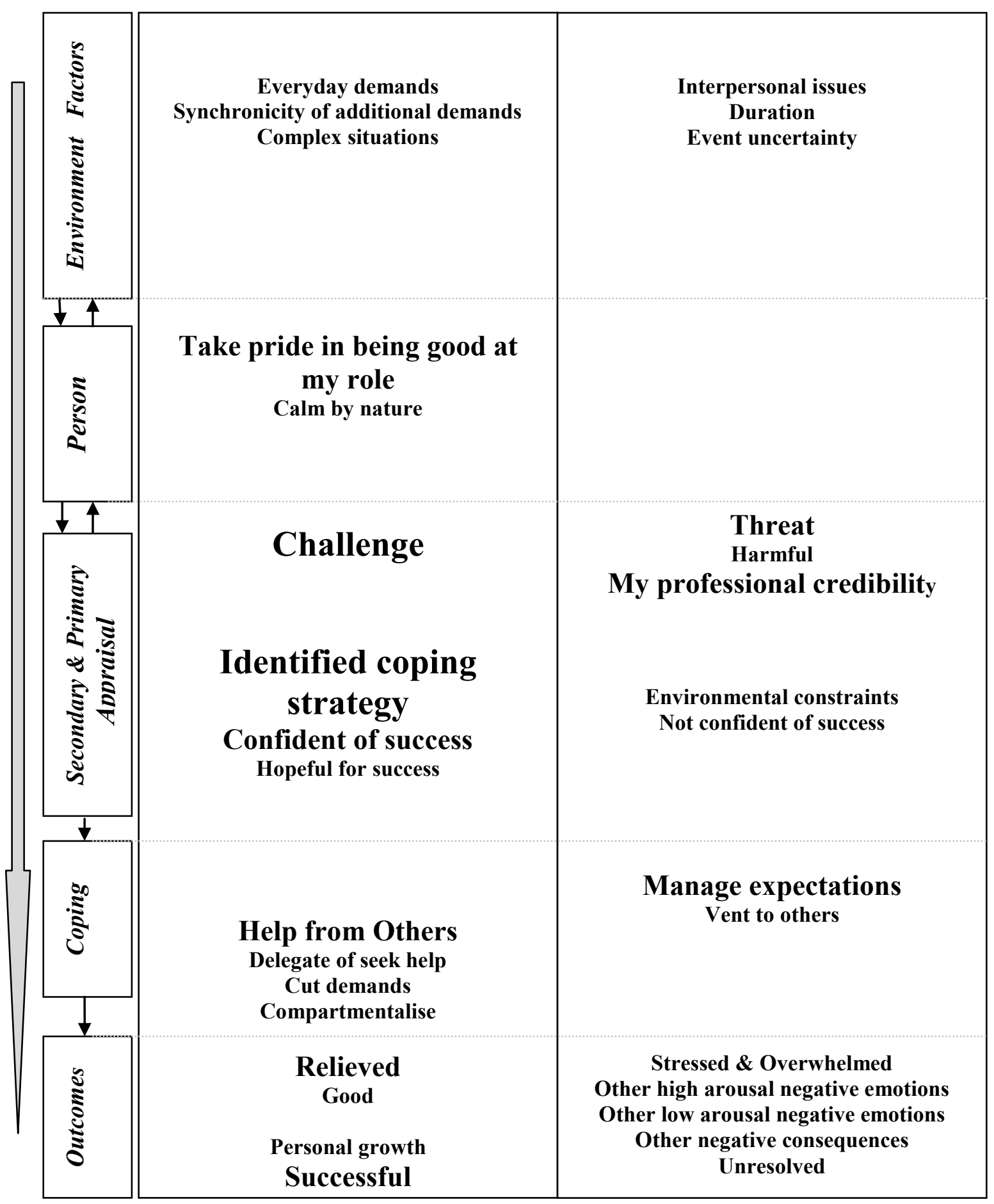

Figure 14-6 Key factors identified in role overload episodes assessed as not-overwhelming and overwhelming 


\subsubsection{Environment factors of not-overwhelming and overwhelming role overload}

Three environment factors were found to be mentioned more frequently by respondents when they talked about role overload assessed as not-overwhelming: the event type labeled 'everyday demands'; and the situation properties, 'synchronicity of additional demands', and 'complexity'. Whilst individually, these three factors refer to different characteristics of the role overload environment, a common feature to all three is that the environment described seems to be one that was normal for many of the female hospital workers in the sample.

First, the respondent descriptions of role overload being triggered by everyday demands (the day to day tasks accepted by these respondents as typical of their role responsibilities) suggest that daily episodes of role overload are a normal part of life for many respondents. Respondent comments about the second environment factor noted more often in not-overwhelming role overload episodes (synchronicity of additional demands) suggest that the synchronised demands involved are the expected and accepted tasks that the individuals associate with their role. Furthermore, when respondents talked about this property of role overload in these episodes, it was concerns about the potential negative impact of the new demands on their ability to deal with the existing workload that seemed to contribute most to the challenges and threats faced by these women.

All the role overload episodes described as complex were assessed as notoverwhelming, even though hospital workers talked about intense experiences during recollections of these events. Whilst it is possible that respondents may assess such episodes as not-overwhelming when these cases turn out well for the patients, the fact that most of these episodes involved specific patient care issues suggests that the health 
care workers describing complex role overload may see such issues as a requirement of the role and therefore a standard part of the job.

The environment findings associated with role overload assessed as notoverwhelmig suggests a pattern of everyday overload, which is consistent with the fact that all these respondents have high levels of work, home and total role overload. The normal or standard environment that seems to characterise role overload assessed as notoverwhelming also suggests that many overwhelming experiences were caused by anomalous factors not typical of the everyday tasks faced by these health care workers. Moreover, the data from this study suggests that non-typical add-ons to the everyday demands could also tip the balance and lead to overwhelming experiences. The event trigger (i.e. interpersonal issues) and the two properties of role overload creating the potential for stressful appraisals that were cited in more episodes assessed as overwhelming (i.e., 'duration' and 'event uncertainty') speak to these ideas.

Interpersonal issues are an example of an atypical event that does not happen on a daily of even regular basis but when added to daily task loads can trigger role overload. The interpersonal issues described by respondents typically involve a breakdown in a working relationship, which threatens task performance and makes coping more difficult suggesting more negative secondary appraisals.

The role overload property, 'duration' is indicative of long lasting or chronic role overload with no obvious solution. As discussed in Chapter 13, the respondent descriptions of these episodes are consistent with Seyle's (1956) model of stress that suggests that stress over time will lead to exhaustion. The fact that all but one of the eight 
episodes in which this role overload property was cited were subsequently assessed as overwhelming supports this argument.

The second role overload property mentioned more frequently by respondents in episodes evaluated as overwhelming, 'event uncertainty', refers to not knowing when an event will occur. As posited by Lazarus and Folkman (1984) (and discussed in Chapter 13), respondents also talked about being unprepared for such unexpected events, suggesting that secondary appraisals in these episodes might be more negative. All four of the episodes in which such concerns were cited were assessed as overwhelming, consistent with this conclusion.

\subsubsection{Person factors of not-overwhelming and overwhelming role overload}

The data in this case study showed that two person factors: (1) the internal role pressure, 'take pride in being good at my role (s)', and (2) the personal attribute, 'calm by nature', were mentioned more often by respondents in role overload episodes that were subsequently assessed as not-overwhelming. The internal role pressure, 'take pride in being good at my role (s)' may affect role overload episodes in two ways. First, the values underlying internal role pressures reflect what is important to respondents. In this case, as described by respondents, the quality of the finished task is important to these workers and may be threatened by role overload, as suggested by Kahn et al. (1964) in their descriptions of role overload, thereby increasing the likelihood of a negative appraisal.

The respondent descriptions of this person factor also suggest that individuals will make extra efforts to respond to this internal role pressure. In other words, these women 
were motivated by their own personal standards to make sure that role expectations were met. This idea is consistent with Lazarus and Folkman (1984, p.80), who described such commitments as having a motivational quality that drives individuals to put more time and energy into roles they are committed to. Whilst this approach may exacerbate role overload, the extra efforts may also help to reduce role overload and explain the subsequent not-overwhelming assessments.

Respondents talked about being 'calm by nature' in only a small number of episodes evaluated as not-overwhelming. These findings suggest that these women were either not troubled by emotions in these episodes or they felt comfortable dealing with them.

Notably none of the person factors described during respondent recollections of role overload was found to be uniquely associated with overwhelming role overload. Since personality traits and role salience are measured for each respondent they are not relevant at the role overload episode level.

\subsubsection{Primary and secondary appraisal in not-overwhelming and overwhelming role overload}

From the respondent descriptions of how they appraised the role overload episodes subsequently assessed as not-overwhelming, two factors stand out (see Figure 14-6). First, in such circumstances the respondents were more likely to describe role overload as challenging. Whilst we know from the study results that when describing role overload as challenging, respondents tended to focus on the efforts required to overcome role overload rather than on the potential for gain (the view of challenge in the literature), 
the link between the perceptions of challenge and the assessment of the role overload episode as not-overwhelming episodes suggest that challenging role overload has strong links with more positive outcomes despite the less positive narrative used to describe them.

The second notable feature of cognitive appraisal in role overload assessed as notoverwhelming is that respondents seemed to have no difficulties identifying strategies to cope with the overload. Furthermore, these women spoke of feeling either confident or hopeful of coping success in these episodes. These findings suggest that coping resources were plentiful and easy to implement in not-overwhelming role overload. It is possible, however, that because these episodes are not overwhelming overload is less threatening and coping resources are just easier to locate.

The appraisal of role overload was talked about quite differently by the female hospital workers when describing episodes subsequently assessed by respondents as overwhelming. More specifically in these cases, role overload was more likely to be described as threatening and the women cited appraisals of harm. Collectively, appraisals of threat and or harm were cited in most of the episodes assessed as overwhelming.

When describing the potential consequences of role overload assessed as overwhelming, respondents were also more likely to express concerns about the risks to professional credibility. Respondent concerns about coping, which underlie a notable property of role overload in overwhelming episodes (i.e. event uncertainty), may account for why respondents feared that role overload could not be resolved successfully and hence might threaten professional credibility. According to Lazarus and Folkman (1984), 
the ongoing symptoms of stress from role overload of a lengthy duration may also lead to the appraisals of threat or harm noted in these episodes.

Whilst respondents identified coping strategies in most not-overwhelming episodes, this was not the case in episodes that were assessed as overwhelming. Respondents were also more likely to say they were not confident about coping success in overwhelming role overload. These findings suggest that coping plans were difficult to put in place in these episodes. Issues with coping resources may account for the absence of coping plans and the higher levels of pessimism about coping outcomes described by respondents in these episodes. Specifically, environmental constraints, such as work scheduling limitations and poor management support were cited more often in overwhelming role overload. Furthermore, atypical role demands leading to lengthy role overload situations or event uncertainty may have made it difficult for respondents to draw on previous experience regarding effective coping strategies. It is possible, however, that overwhelming episodes may make it difficult to identify coping strategies and or make plans for how to deal with the role overload.

\subsubsection{Coping strategies in not-overwhelming and overwhelming role overload}

Four of the five problem focused demand-reduction coping strategies identified in this case study (i.e., help from others, delegate or seek help, cut demands, and compartmentalise) were more frequently mentioned by respondents as strategies they used to deal with role overload in episodes assessed as not-overwhelming. Lazarus and Folkman (1984) argue that a greater use of problem focused strategies is more likely in stressful conditions appraised as amenable to change. The greater use of problem focused 
demand-reduction strategies in not-overwhelming role overload is also consistent with the fact that respondents talked more often about being confident of, and hopeful for, coping success in not-overwhelming episodes. Another explanation, however, is that it may just be easier to use these four demand-reducing coping actions to deal with role overload that proved to be less overwhelming.

In episodes subsequently assessed as overwhelming two specific demandmanagement strategies were used more often by respondents to cope with the role overload in overwhelming episodes: 'manage expectations' and 'vent to others'. Comments by respondents suggest that managing the expectations of role set members is a problem focused strategy used in cases where deadlines are threatened or when difficulties exist in reducing role demands (as might be expected when uncertainty surrounds an event, or in episodes of a lengthy duration). Venting, on the other hand, (mentioned almost exclusively in overwhelming episodes) is often used as a quick and immediate way to relieve the stress of the moment and therefore could reflect the intensity of emotions felt during some of these overwhelming situations.

\subsubsection{Outcomes in not-overwhelming and overwhelming role overload}

Respondents were more likely to mention more successful episode resolutions, more instances of personal growth, and a greater number of references to specific positive emotions and feelings (i.e., relief and good) when talking about role overload assessed as not-overwhelming. Given the high levels of coping confidence expressed during appraisal and the nature of the coping strategies used in these episodes to deal with role overload, these outcome findings give further support to the idea that coping efforts play 
an important part in the more positive outcomes noted in episodes assessed as notoverwhelming. It is possible, however, that less overwhelming role overload may just make it easier for individuals to implement approaches that lead to successful outcomes or workers may have felt more positive simply because the role overload episode was over.

The personal outcomes articulated by respondents when describing overwhelming role overload are all negative and include feelings of stress, other high and low arousal negative emotions and feelings, and other negative consequences. Respondents were also more likely to say that their overwhelming role overload episode was unresolved. These results suggest that the negative emotions and feelings underlying most role overload appraisals described by respondents do not dissipate when the overwhelming role overload episode ends.

\subsection{What distinguishes role overload appraisal and coping described by hospital workers with lower levels of stress from that described by workers with higher stress}

A summary comparing the factors of the role overload appraisal and coping process that distinguish the episodes recalled by respondents with low levels of perceived stress from those described by workers with high levels of perceived stress is presented in Figure 14-7. The factors highlighted in Figure 14-7 are portrayed in the same three font sizes used in Figure 14-6 to proportionally represent the proportion of episodes the factors were cited in, this time as recalled by the two stress level respondent groups (i.e., small font $20-40 \%$; medium $45-65 \%$; large $70 \%$ or more of the 20 episodes described by each group). 
The factors identified in this figure are discussed in the order they are presented (top to bottom) in five separate subsections below. In each subsection comparisons are between episodes recalled by low stress respondents and those recalled by respondents who reported higher levels of stress. The focus of the discussions in these subsections is on factors cited more frequently by one respondent compared to the other. To ensure brevity, the respondent citing fewer of the factors discussed will not be mentioned but should be assumed by the reader.

\subsubsection{Environment factors in role overload recalled by low stress and high stress hospital workers}

The demographic characteristics of respondents in the low stress group were found to be quite different from those observed for respondents in the high stress group. More specifically, low stress workers were found, on the whole, to be older and have older children, and work in administrative or management roles. As the specifics of the role overload and coping process described by this group of workers is examined, the ways in which the demographic characteristics of low stress respondents might influence this process will be discussed as appropriate. 
Low v High Stress

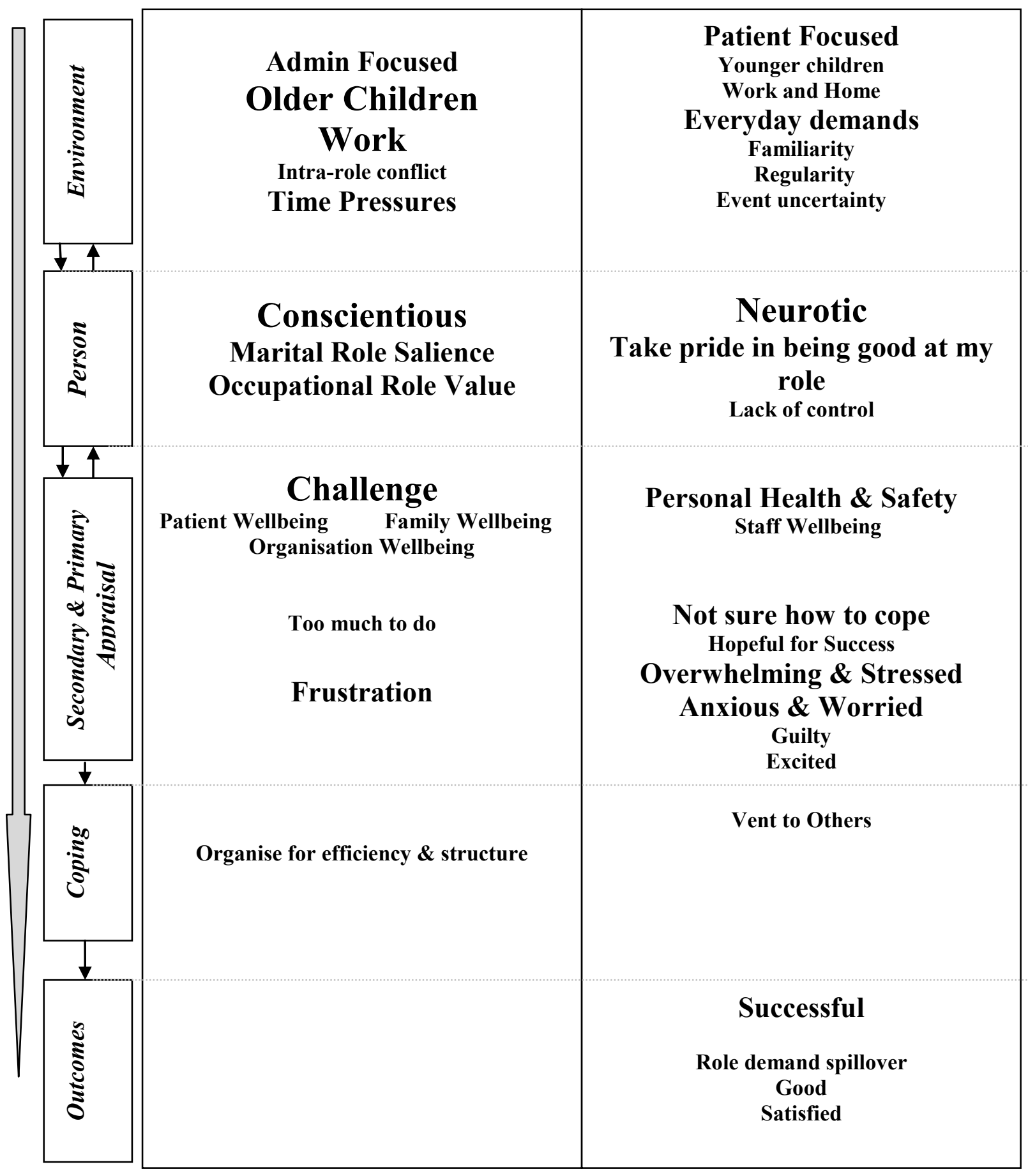

Figure 14-7 Key factors of role overload appraisal and coping as described by respondents with low and high levels of perceived stress 
It is also important to remember at this point that the stress levels of these respondents were measured in the on-line survey undertaken prior to this thesis study. Role overload may not, therefore, be the sole source of stress for these individuals. The low stress group of hospital workers may thus find it easier to cope with role overload because they have lower stress levels in their lives overall. It is also possible, however, that this group of older workers has lower levels of perceived stress because they were more able to cope with the stress they were under (in other words they developed more effective coping skills over time).

Whilst work based role overload is an underlying factor of episodes in all four groupings, when deciding which role overload episodes to discuss, low stress respondents chose to describe more episodes set solely in the work domain. As a result, almost all of the episodes they described involved work. The older children of the low stress group (who would typically be less demanding) may account for this finding. Intra-role conflicts, which occurred almost exclusively in the work domain, were also cited in more of the episodes recalled by the low stress group. Thus, when considering what makes them overloaded, what came to mind for employees in the low stress group was receiving requests to complete extra work when overtime is disallowed or disapproved of (a common characteristic of intra-role conflict), rather than their circumstances at home.

Finally, respondents with low stress also talked more often about time pressures when recalling feelings of overload. Descriptions of time pressures given by respondents indicate that respondents often had little spare time to plan coping actions. Intra-role conflicts can limit respondents' options for dealing with excessive demands and therefore may also contribute to the time pressures felt by low stress respondents. 
The demographic data for the respondents with higher levels of perceived stress revealed that more of these hospital workers had younger children. A majority of this group also worked in jobs involved with direct patient care. These findings offer two possible explanations as to why these individuals reported higher stress levels. First, the younger children of the high stress workers and the patient needs occupying their working roles may create additional daily demands for many of these women. These demands may contribute to greater levels of stress in respondent experiences of role overload, a problem particularly noted for women in an examination of life-stage and gender on balancing work and family life by Higgins, Duxbury and Lee (1994). The observation that role overload regularly reoccurs for some of these respondents is consistent with this explanation. Second, the demanding nature of their home and work lives may help to explain why role overload featuring both domains is more commonly cited by some of the respondents with high stress.

High stress respondents cited everyday demands as a trigger for role overload over twice as often as the respondents with lower stress levels. As well as noting the regularity of some role overload events, these respondents were also more likely to talk about the familiarity of role overload when describing what made them feel overloaded. Together, these findings suggest that for many highly stressed workers this habitual and routine phenomenon is chronic by nature. Duxbury and Higgins $(2001,2003 a)$ report that role overload is a growing problem and increasingly linked to feelings of distress, a finding that supports the notion that such chronic role overload is linked to high stress levels for some of these hospital workers. The finding that everyday demands are an important and regular trigger for role overload for this group is also consistent with the 
fact that employees working in the health sector are more prone to work intensification and overload (Duxbury and Higgins, 2003b, 2009) than are those in other sectors.

There are a number of possible explanations why everyday demands are the trigger for so many of the role overload episodes described by high stress respondents. Descriptions given of familiar role overload that sometimes reoccur on a regular basis suggests that respondents have sound knowledge of such events, as would be expected when dealing with everyday demands. As discussed in Chapter 13, if these events previously resulted in bad experiences, it is reasonable to suggest that such role overload situations might be appraised as more challenging and more threatening than if previous experiences had been positive. The very nature of many of these role overload events (i.e., regular, repetitive and identical) also suggests that any stress from these episodes could cause cumulative problems for these respondents, leading to higher levels of stress. Everyday demands that cannot be accomplished in the time available may similarly contribute to the accumulation of stress on a daily basis.

The final property of role overload cited more frequently by high stress respondents, although in relatively small numbers, is 'event uncertainty'. Event uncertainty can, according to Lazarus and Folkman (1984, p. 109), "deprive a person of the chance to prepare". It may be that for these employees it is the unrelenting pressure of high demand levels (i.e. busy home and work lives, everyday demands, and regular occurrences of role overload) that contribute to perceptions of stress. For a respondent with high stress levels, the presence of event uncertainty as well may increase the intensity of an event threatening something of importance to the respondents. On the 
other hand, for workers who are highly stressed in other areas of their lives, finding the personal resources to deal with uncertain events may just be too difficult.

\subsubsection{Person factors in the role overload recalled by low stress and high stress hospital workers}

An important person factor finding in this study is that low stress workers reported higher levels of conscientiousness and lower levels of neuroticism. This finding is somewhat surprising, since intuitively, it might be expected that high conscientiousness would lead to longer work hours or greater efforts to deal with role overload and hence increased levels of exhaustion and stress. Whilst highly conscientious people may have seen heavy role demands as something that needed to be overcome, the qualities of selfdiscipline characteristic of this personality trait may, however, have made for more informed coping choices and hence better outcomes for role overload. The finding in the literature (noted in Section 13.2) that conscientiousness is associated with perceived coping capability (Penley and Tomaka, 2002) is consistent with this idea. Future research is required to see if the association between conscientiousness and low levels of stress is apparent for respondents who experience low levels of role overload.

Hospital workers with lower levels of stress also tended to have higher scores for marital role salience and occupational role value. The fact that the low stress group are older and generally have older children than the higher stress group may help explain this difference. For example, low stress respondents may no longer feel as stressed about the pressures of maintaining a balance between marital commitment and the time and efforts that young children demand. Moreover, having more time to spend on the roles they 
valued may have made them less stressed. Respondents who are less stressed overall may also have felt less pressured to commit resources to their occupational role or alternatively found it easier to use personal resources more effectively.

We know from examining the underlying factors of role overload in Section 14.1 that most respondents reported high scores for parental role salience. The small but notable difference in marital and occupational role value scores between the two respondent groups means that for respondents with high stress levels the disparity between the value they place on their parental role and their occupational role is greater. This disparity may have made the personal stakes higher when work and family were combined during role overload and therefore may also account for why the dual domain based role overload episodes (i.e. when the home life is added to the mix for role overload) are associated with higher respondent stress levels.

The results which stand out most in Figure 14-7 for highly stressed respondents are that these women reported higher levels of neuroticism and lower levels of conscientiousness. Neuroticism is to do with the ease and frequency with which individuals become distressed or upset (Carver and Connor-Smith, 2010). Since being anxious and easily upset are common characteristics of highly neurotic individuals (Gosling et al. 2003) and feeling nervous or upset are symptomatic of perceived stress (Cohen, Kamarck \& Mermelstein,1983), there is an overlap in the self evaluation instruments for high levels of stress and neuroticism that may explain the difference between the two respondent groups. The level of neuroticism does, however, vary widely for the whole sample and the difference between the two stress level groups is notable. 
Recent research has begun to look at the combined effects of personality traits on the relationship between occupational stress and strain. A study of occupational stress by Grant and Langen-Fox (2006) has demonstrated that low neuroticism combined with high levels of extraversion and conscientiousness is related to lower levels of occupational stress. This gives further support to the notion that the high stress respondents in this case study (with higher neuroticism and lower conscientiousness) may find role overload at work more difficult and hence more stressful. Further research is required to examine the relationship between personality traits and stress arising from role overload.

When talking about role overload, highly stressed hospital workers mentioned taking pride in being good at their roles almost twice as often as the low stress respondents. As discussed in Section 14.3 this association is particularly strong for notoverwhelming role overload and the literature supports various explanations for this difference. Specifically, threats to this internal role pressure may increase the sense of role overload and hence feelings of stress (Kahn et al., 1964; Lazarus and Folkman, 1984). In addition, the energy put into a role to fulfil personal quality standards may increase role overload and lead to increases in stress (Lazarus and Folkman, 1984). Another factor may be particularly influential for high stress respondents. Specifically, workers who are highly stressed for other reasons may find it more difficult to put the effort into roles they take pride in, leading to increases in role overload.

Another important role overload appraisal and coping factor mentioned more often by the high stress respondents involves personal beliefs about 'lack of control'. Lack control is, according to Lazarus and Folkman (1984), more likely to lead to negative secondary appraisals. High stress levels from non-role overload experiences may make 
these respondents more likely to feel that they have no choices when faced with extra demands. The comments made by respondents regarding lack of control, however, suggest that the appraisal of the familiar everyday role overload situations faced by highly stressed workers were also negatively influenced by these beliefs about control because of the perceived impact to the availability of coping options and resources, and or the perceived inability to reduce demands based on similar past experiences.

Subsequent coping may prove to be less effective in these circumstances.

Organizational stress research has shown that individuals experiencing higher levels of perceived control report fewer psychological symptoms than individuals with lower levels of control (Barnett and Baruch, 1985; Amatea and Fong, 1991) whilst other more recent research has shown that the ability to control how and when job demands are completed buffers stress in situations of role overload (Schultz, Wang and Olsen, 2009) Reviews of work stress research have also reported that lack of control (or perceived lack of control) is associated with negative well-being (Cooper et al. 2001; Thompson et al., 2007; Dewe et al., 2010). Consistent with this research the negative secondary appraisals of role overload suggested by the descriptions of low levels of perceived control in this case study may be related to the higher levels of stress reported by these workers.

\subsubsection{Primary and secondary appraisal in role overload recalled by low stress and high stress hospital workers}

When talking about how they appraised role overload, low stress respondents described more of the episodes they recalled as challenging. When describing the challenges of role overload, the fact that family and work based roles seem to be more 
important to these less stressed individuals is consistent with the finding that these respondents were also more likely to express concerns about other people, or the hospital, than about themselves (i.e., patient, family and organisational wellbeing were all cited more often when they were asked about the potential consequences).

Given that the demographic data revealed that these workers predominantly have administrative or management roles in the hospitals, it is reasonable to assume that many of them are particularly knowledgeable about the financial or legal constraints facing the hospital they work in. This could account for the bias in concern for organisational wellbeing from this group. The emphasis on patient well-being by this group may seem surprising since their jobs are largely administrative. The high levels of conscientiousness of these low stress hospital employees and the fact that managers and admin staff are indirectly concerned with patient care are, however, factors that make this result less surprising than at first thought.

During the secondary appraisal process (when evaluating how and if they could cope with role overload) respondents with low levels of perceived stress were more likely to judge that there was just too much to do albeit only in a small number of episodes they described. Whilst such descriptions suggest the potential for coping failure, low stress respondents did not express any other specific concerns about their abilities to cope with role overload. Furthermore, based on the lower levels of neuroticism reported by the low stress respondent group, these workers are expected to be more emotionally stable and generally exhibit calmer behaviour (Gosling et al., 2003) and therefore may be more confident about coping with role overload. We know that negative emotions arising from role overload appraisal were 
common in most episodes of role overload described by the thesis sample. Low stress respondents specifically talked more often about being frustrated during the evaluation of role overload. Intuitively, frustration would not be an unexpected reaction from a conscientious but less neurotic individual who is faced with the challenge of time pressures and too much to.

When high stress respondents were asked to describe their appraisal of what was at stake for them in the role overload episodes recalled, they were more likely to mention personal and staff well-being than were respondents with lower levels of stress. Personal health and safety was a particular concern for these workers. Such risks to personal wellbeing whether from the potential for ill health from the burdens and demands of unrelenting role overload, or from threats of physical harm, would logically increase the likelihood of negative appraisals of role overload, potentially leading to higher levels of stress. On the other hand, individuals who are highly stressed because of other issues in their lives might also be more likely to be at risk of stress related ill health when faced with daily role overload as well.

When appraising what could be done, if anything, about the role overload facing the high stress workers, the tendency for this group of respondents to be unsure how to cope may explain why they are also less confident in their abilities to overcome role overload. These findings are consistent with research linking the neurotic traits reported by these respondents to a lack of perceived coping ability (Penley and Tomaka, 2002). Furthermore, workers who are more disorganised and more easily distracted (i.e., high stress respondents with lower levels of conscientiousness) (Gosling et al. 2003), may find it more difficult to tackle role overload because they either leave things to the last minute 
or they have extra work because they have to do things over again. When describing how they felt during role overload appraisal high stress respondents were also more likely to cite negative emotions and feelings consistent with a negative appraisal of coping capability or threats to personal well-being. In particular, these respondents described being overwhelmed and stressed or anxious and worried during role overload appraisal in close to two thirds of their episodes.

The higher level of neuroticism in the sample of respondents with higher levels of perceived stress is indicative of a more emotional and less calm nature (Gosling et al. 2003). As such, it is not unexpected that these employees are more likely to say they are stressed, overwhelmed, and anxious, as neuroticism has also been associated in prior research with high total negative emotion when coping with stress (Penley and Tomaka, 2002). The emotions and feelings that they typically express during these situations may, however, also be related to the chronic daily nature of role overload suffered by this group of respondents.

Whilst specific negative emotions seem to be common during role overload appraisal for high stress workers, the case study data also showed that this respondent group described feeling excited during role overload appraisal more frequently, albeit in a small number of episodes. High stress respondents are equally likely to cite the internal role pressure, 'opportunity for accomplishment', as are low stress workers. Perhaps the neurotic traits of this group make them more excitable when such opportunities for personal gain arise. 


\subsubsection{Coping strategies used in role overload recalled by low stress and high stress respondents}

The results from this case study suggest that low stress respondents were more likely to use the problem focused demand-management coping strategy 'organise for efficiency and structure' to deal with role overload. With this strategy low stress respondents are taking a systematic and focused approach that reflects their conscientious personality. This notion is consistent with the literature which has shown an association between conscientiousness and the use of problem-focused coping (O'Brien and DeLongis, 1996; Penley and Tomaka, 2002). Whilst these older hospital employees may have learnt to be better at organizational skills with age, the higher proportion of supervisory roles amongst this group may also make it easier for these workers to make organizational changes to increase efficiency. It is possible, however, that the lower levels of stress experienced by this group may simply make it easier to organize for efficiency and order in order to cope with role overload.

The notion that high stress levels recorded for half of the thesis sample are not just about high levels of role overload may be one of the reasons why so few coping strategies appear to be related to worker stress level. The use of 'venting to others' to manage stress by respondents with high stress levels may be related more to the cumulative level of stress from multiple situations rather than to role overload alone. The higher levels of neuroticism associated with this group may also explain the increased usage of coping through such visible expressions of emotion, since such individuals are likely to be easily upset (Gosling et al. 2003). 
Another explanation why venting is used more by high stress respondents as a way to deal with the emotions of role overload is found in the coping literature. First, neuroticism has positively predicted emotional venting (Carver et al., 1989). Second, findings in this study have suggested that high stress respondents cite 'lack of control' as an important factor in their recollections of role overload. Whilst venting to others may just be a particularly effective way of reducing negative emotions, together these findings are consistent with studies that have shown strategies targeted at emotional stress are more likely to be used when demands seem uncontrollable (Lazarus and Folkman, 1984; Folkman et al. 1986; Thoits, 1991)

\subsubsection{Outcomes in role overload recalled by low stress and high stress hospital workers}

There are no personal outcomes (positive or negative) or episode resolution outcomes that were more prevalently reported by the low stress group. Given that both respondent groups described both overwhelming and not-overwhelming role overload in equal numbers, a link between episode outcomes and respondent stress would not be expected. Successful episode outcomes and the positive emotions and feelings, 'good' and 'satisfied' were, however, reported more frequently by the high stress group when they talked about how role overload ended. As suggested in Section 14.2, these findings may be because low stress hospital workers who have higher levels of conscientiousness may be more conservative and expect better outcomes before describing outcomes as completely successful, or before expressing satisfaction and good feelings. In contrast, neurotic high stress respondents may tend to be over enthusiastic when role overload is 
overcome. Furthermore, being less conscientious, high stress workers may be less bothered if role overload episodes are not totally successful and consequently forget to mention some mixed resolutions.

High stress respondents were more likely to mention role overload spill-over as a negative consequence of the role overload they described. A possible explanation for this outcome is that the emphasis on demand-management coping may reduce the time and energy available to these respondents to deal with demands in other role domains, leading to more role overload. Alternatively, high levels of stress arising from non-role overload issues in the lives of these respondents may also limit the resources available for role overload, thereby increasing the chance of spill-over to other domains. 


\section{PART SEVEN: CONCLUSIONS}

\section{Conclusions}

This final chapter is divided into four sections. Section 15.1 discusses the conclusions and contributions of this thesis, whilst the second section acknowledges the limitations of this research. Section 15.3 discusses the potential avenues for future research and the final section discusses implications for practice.

\subsection{Conclusions and contribution of this research}

This case study of role overload in the health sector responds to recommendations for a greater focus on exploratory, qualitative research to support theoretical development in role stress research (Fineman \& Payne, 1981; Hardy \& Hardy, 1988; Ilgen \& Hollenbeck, 1990; King \& King, 1990; Eby, 2005). The focus of this research is role overload, an important but understudied form of role stress (Newton \& Keenan, 1987; Coverman, 1989; Gilboa et al, 2008; Fournier et al. 2010). The review of the literature found that role overload research, which was mostly quantitative in nature, was fragmented and unable to explain the variable psychological stress levels arising from this stressor. The research presented in this thesis was designed to enhance our understanding of the relationship between role overload and perceived stress, thereby addressing this gap in our understanding. The inconsistent results linking role overload 
and perceived stress noted in the literature review and the growing interest in positive and negative outcomes from stressful encounters suggested a different approach was need to examine how and why stress should arise from mismatching role expectations and how and why it might vary (Fineman \& Payne, 1981; MacDermaid \& Harvey, 2006; Thompson et al., 2007; Gilboa et al., 2008). Accordingly this thesis relies on qualitative methods and uses a framework based on cognitive stress theory (Lazarus and Folkman, 1984) to examine this relationship.

The first objective of this case study was to develop a theoretical framework describing the relationship between role overload and perceived stress. This case study concluded that for the most part the role overload appraisal and coping framework developed in fulfillment of the first thesis objective maps closely to Lazarus and Folkman's cognitive model of stress. In other words, researchers can approach the study of role overload knowing that theoretically, the process describing role overload appraisal and coping is consistent with the appraisal and coping processes found for other stressors. Furthermore, future researchers will be able to compare findings from studies that use the framework to examine the relationship between role overload and perceived stress with findings in the extant stress and coping literature.

Key similarities between role overload appraisal and coping and Lazarus and Folkman's stress, appraisal and coping theories, identified in this study, include the following. First, as posited by Lazarus and Folkman (1984), the judgment that role overload is stressful depends on the unique relationship between the role overload environment and the person factors of the individual. Second, the taxonomy developed in this study to describe the situation properties of the role overload environment that have 
the potential to create stressful appraisals overlaps with Lazarus and Folkman's (1984) taxonomy, with matches for four situation properties: the ambiguity and novelty of role demands, the duration of the episode, and event uncertainty. Person factors identified in this study as important to the role overload appraisal and coping process (i.e. what is important to the individual and beliefs about control) are also consistent with those considered most important by Lazarus and Folkman (1984).

Fourth, like Lazarus and Folkman's model, the results from this study showed that the evaluation of the role overload for personal significance (primary appraisal) and the evaluation of options for coping (secondary appraisal), which leads to appraisals of challenge, threat and or harm and the initiation of coping strategies in response to the appraised demands, are at the heart of the relationship between role overload and perceived stress. The data from this study also supports the conclusion that the role overload coping strategies identified are consistent with one or other of the two major coping functions defined by Lazarus and Folkman (1984) (i.e. problem or emotionfocused) and a majority of them are matched by items in Lazarus and Folkman's 'Ways of Coping' checklist. Finally, as predicted by Lazarus and Folkman (1984) the short term outcomes from the role overload and coping process included emotions and feelings, personal consequences and role overload episode resolutions. The links between the role overload appraisal and coping framework and the stress appraisal and coping literatures support the conclusion that this framework provides the opportunity to move the research of role overload forward and in particular may help to increase the understanding of the relationship between role overload and perceived stress. 
Whilst the role overload appraisal and coping framework is identical to the cognitive model of stress (Lazarus and Folkman, 1984) in many ways, this research also determined six key areas where the frameworks differ: situation properties that are specific and unique to role overload; an internal role pressure that appears to play an important part in the perception of role overload and is also specific to this role stressor; modified interpretations of challenge and threat appraisals; a focus on demand reduction versus demand management coping strategies; coping strategies specific to role overload, and four distinct types of role overload episodes. Each of these differences is described more fully below.

First, the findings from this case study identified five situation properties that may make an individual appraise an event as stressful (i.e., time pressures, volume of demands, and three forms of synchronicity of demands) that are role overload specific versions (consistent with Kahn et al., 1984) of properties defined in the cognitive model of stress. The study also concluded that three other properties (familiarity, regularity and complexity) were newly identified situation properties that were influential during role overload appraisal, but were not necessarily specific to role overload. Future research is required to see if the latter three situation properties are applicable to other stressors.

The distinctive taxonomy of role overload situation properties is an important contribution of this study. At the time of writing, it represents the only known classification of the situation properties of the role overload environment that create the potential for a threat, harm, or challenge. The similarities to that defined in the cognitive model of stress together with its distinguishing features suggest that this taxonomy could 
provide a useful tool for researchers and practitioners to examine the environment of role overload.

The second factor that distinguishes the role overload appraisal and coping framework from cognitive stress theory relates to the internal role pressure to 'take pride in being good at my role'. Descriptions of this internal role pressure, which drives individuals to meet personal quality standards, lead to the conclusion that this person factor may be specific to the appraisal of role overload. Discussions in the literature have suggested that during role overload the availability of skills and knowledge is a determining factor when an individual is faced with the choice of how much work can be completed versus the quality of the work that can be done. This case study lends support to the idea, however, that the ability to get work done to personal standards is more important to role overload than lack of skills or knowledge when concerns about quality of the work to be done are threatened.

Third, the thesis research also supports the conclusion that hospital workers interpretations of challenge and threat appraisals differ from the definitions offered by Lazarus and Folkman (1984). Specifically, respondent's reluctance to use the term 'threat' when describing the potential for harm suggests that many situations described as challenging by the respondents correspond to Lazarus and Folkman's (1984) idea of threatening. Future research is required to examine whether this finding is consistent across other samples.

Fourth, unlike the cognitive model of stress, the research supports the idea that coping with role overload focuses primarily on reducing or managing the demands involved, as found by Lang and Markovitz (1986). This is different from Lazarus and 
Folkman (1984 p. 150) who argue that the distinction between problem and emotion focused coping strategies is "of overriding importance". The role overload coping strategies identified in this thesis were also categorized as problem or emotion focused and by combining this pair of coping dimensions with the studies' primary classification of role overload coping (i.e. demand reduction versus demand-management) a two by two typology of role overload coping strategies was developed in this study. The broad applicability of the problem versus emotion focused dimension (Lazarus and Folkman, 1984), suggests that the emerging typology may prove to be a useful tool for examining the coping approaches of individuals facing role overload in a wide range of occupations and industry sectors. Future research is required to see if this typology is applicable to other samples.

Another distinguishing factor of the role overload appraisal and coping framework and an important contribution of this research to the role overload coping literature is the identification of two demand-management, problem focused coping strategies, 'manage expectations' and 'use personal time', that were unmatched in the other two coping instruments (Lazarus and Folkman, 1984; Lang and Markovitz, 1986) examined in this thesis. The study results suggest that these strategies for coping are specific to role overload.

Finally, the role overload appraisal and coping framework helped us distinguish between four types of role overload episodes according to whether the episodes were assessed as overwhelming or not, and whether respondents reported high or low levels of perceived stress. The existence of these four distinct episode types is an important factor 
to consider for researchers when examining the relationship between role overload and stress in the future.

The second objective of this research was to determine why some people who suffer role overload experience symptoms of stress and others do not. Whilst finding differences was the focus of this research objective the study first identified a number of similarities across the four episode groupings that help us to better understand the role overload appraisal and coping process. Specifically, the underlying common factors of this process for the thesis sample included the following:

- dual demands (work and home);

- onerous work demands;

- being a good parent was important to these individuals;

- having a warm and sympathetic personality with a willingness to take on new challenges;

- personal well-being and the well-being of others was threatened or challenged by all they had to do;

- $\quad$ people felt they lacked the time and energy to deal with all the demands;

- appraisal of these circumstances lead to negative emotions and feelings such as anger, worry, or stress and

- people coped with role overload by relying on others to help them reduce the demands.

This research then identified a number of factors that distinguished overwhelming role overload episodes from those assessed as not-overwhelming. When recalling role overload assessed as overwhelming respondents were more likely to describe:

- atypical, out of the blue, and unresolved situations;

- threats and harm to their professional credibility; 
- environmental constraints such as restrictions on work scheduling and poor management support;

- coping by managing expectations and by venting to others to let off steam and;

- feeling stressed and other negative emotions at the end of the role overload episode.

In overwhelming role overload episodes respondents were also less likely to:

- identify coping strategies or feel confident about coping success when appraising these circumstances.

These results support the conclusion that in overwhelming role overload individuals faced unusual situations and difficulties making coping plans, and coped by managing demands and emotions.

Whilst all the hospital workers interviewed for this study reported high levels of work, family, and total role overload this study was also able to identify factors that distinguish the role overload episodes of overloaded individuals who reported high levels of stress from those described by overloaded individuals with low levels of stress. Specifically, when recalling role overload episodes, individuals with high levels of stress were more likely to describe the following:

- a demanding home life;

- familiar and regular everyday role demands at work and home;

- events that happen out of the blue;

- emotionally unstable personality traits;

- a lack of control over role demands and coping choices;

- threats and challenges to personal standards, personal health and safety, and staff well-being;

- feeling unsure about how to cope; 
- intense emotions and feelings such as stress and anxiety when appraising these circumstances;

- coping by venting to others and;

- feeling good and satisfied after successful outcomes.

High stress respondents were also more likely to be:

- hopeful for coping success.

These results support the following conclusions. First, personality is important to role overload appraisal and coping. Second, chronic role overload at work and home and difficulties identifying coping resources are related to higher levels of stress.

Lastly, data from this study supports a number of conclusions with respect to the characteristics of the four types of role overload episode identified. First, the factors that distinguish the episodes that high stress respondents found overwhelming include:

- chronic role overload involving a complicated multi-task environment, which respondents sometimes felt they had little control over;

- emotionally unstable personality and committed to doing a good job;

- personal, professional, and staff well-being were harmed, threatened and/or challenged by everything that had to be done;

- a lack of time, energy, organizational, and people resources and a pessimistic view of the chances for coping success;

- appraisal of these circumstances lead frequently to feelings of anxiety and worry;

- people coped with role overload by managing emotions and expectations as well as receiving help from others;

- feelings of stress often remained from the mixed resolutions.

These results support the conclusion that in overwhelming role overload workers who reported higher levels of stress faced chronic role overload involving multifaceted 
situations and limited resources for coping. They coped by managing emotions and expectations.

The following factors differentiate the episodes that low stress respondents found overwhelming:

- a less demanding home life but not enough time to deal with work demands;

- a self disciplined personality;

- people who valued both their marriage and their job;

- professional credibility and home life were threatened and challenged by what they had to do

- people had difficulties identifying coping strategies;

- appraisal of these circumstances lead to feelings of frustration and anger

- people coped by receiving help from others and using positive thinking to reduce demands, and by organizing, managing expectations and working longer hours to manage demands

- mixed outcomes lead to feelings of deflation and demotivation.

These results support the conclusion that in overwhelming role overload workers who reported lower levels of stress faced time pressures and difficulties making coping plans, and coped by reducing some demands and managing the rest.

The factors that discriminate the episodes that high stress respondents found notoverwhelming are as follows:

- their everyday home and work routine;

- a lack of clarity from ambiguous demands and a lack of control over demands and coping choices

- emotionally unstable personality and committed to doing a good job;

- personal well-being was challenged and occasionally threatened by demands 
- initial concerns about coping were overcome when strategies were identified

- appraisals of these circumstances lead to various feelings such as stress, anxiety, anger and excitement;

- people coped by relying on help from others, planning, and working longer or harder;

- relief and good feelings followed successful outcomes.

These results support the conclusion that in role overload assessed as not-overwhelming, workers who reported higher levels of stress lacked clarity or control over routine tasks in their busy work and home lives and responded with mixed emotions and feelings. They also coped by making plans and working harder as well as receiving help from others.

Finally, the following were the distinguishing factors of the episodes that low stress respondent found not-overwhelming:

- classic work role overload (as defined by Kahn et al., 1964, 1966) with time pressures, too much to do, and concerns about the quality of what could be done ;

- a self disciplined personality;

- people who valued both their marriage and job and were committed to doing a good job;

- patient well-being was challenged and threatened;

- a lack of time, energy, and people to tackle the extra demands;

- coping strategies were easily identified;

- people coped by using help from others, delegating, and cutting demands to reduce demands and by working longer hours and talking about their emotions to manage demands and

- felt relieved at the end of successful and mixed resolutions.

These results support the conclusion that in role overload assessed as not-overwhelming, workers who reported lower levels of stress faced role overload as originally 
conceptualizer by Kahn et al (i.e., classic overload) and coped by using a range of strategies to reduce and manage demands.

In conclusion, the outcomes of this exploratory case study represent a step towards a better understanding of the relationship between role overload and perceived stress. In particular, the development of the conceptual framework of role overload appraisal and coping represents is an initial step in this area of research and provides a foundation for future empirical research in this area.

\subsection{Limitations}

Despite the contributions of the research described above, there are several limitations of this research that must be discussed. The first limitation arises from the decision to use the case study method to examine the role overload appraisal and coping process. This approach will, by definition, limit the ability to predict the characteristics and outcomes of role overload beyond the limits of a large hospital setting. Problems of case study generalizability also come from the unique language and emphasis of the researcher (Peterson et al., 1999) and the use of a purposeful sampling technique. More specifically, our sample includes only female employees with high levels of work, family and total role overload and thus our model may only explain the overload appraisal process for those who are overloaded in all domains. Caution should be used when attempting to generalize the role overload appraisal and coping framework to other populations. The context of role overload may differ greatly between organizations and further research is required to examine role overload appraisal and coping in other contexts. That being said, the strong link between the role overload appraisal and coping 
framework and the stress and coping, and role overload literatures suggests that the model may be largely generalizable. It also means that the framework developed in this thesis has the potential to provide a firm foundation from which future research can increase our understanding of the relationship between role overload and perceived stress. Future research using samples from different industry sectors, which include men and women, should be done to build upon and refine the relationships identified in this thesis.

The second limitation of this research is reflected in the choice of stress model used. As already noted, the selection of the cognitive model of stress is based on its substantial influence on the field of stress and its widespread use in other scholarly fields including management. Other stress models exist, such as LePine et al.'s (2007) challenge stressor-hindrance stressor framework, or French, Rogers and Cobb’s (1974) person environment fit theory of organisational stress, each of which may be equally suitable for the examination of role overload. In this study, it was not practical to use other stress models, however, such an approach could be the subject of future research.

The third limitation of the current thesis research stems from the use of retrospective accounts to capture the data. This is also a common criticism of case studies in which this approach is frequently employed. Recollections may contain inaccuracies and memories may differ according to the types of event or the way they were coped with. Whilst none of the interviewees had any trouble recalling the details of role overload situations, it is impossible to comment on the accuracy of their accounts. Since the interviews were private and confidential, and respondents were asked to choose their own role overload events, there is no reason to believe that the interviewees augmented their recollections to make themselves look better. Nevertheless, the researcher took a 
cautious approach in interpreting findings in order to identify any exaggerated or inconsistent claims on the part of the respondent. Using the within case/cross case analysis approach to build support for the developing theory in the analysis process helped to reduce the impact of such bias or inaccuracy on the results. On balance, given the complexity of the stress approach, the researcher believed that collecting retrospective accounts was the best way to get rich and meaningful data where a longitudinal approach was not an option.

This leads to another, related, limitation of this thesis, which is that this study does not involve an examination of the long term effects of role overload. An important step in future research would be to use a longitudinal study to examine long term effects of role overload. The use of daily process designs in longitudinal research, as recommended by Tennan et al. (2000), would also reduce reliance on retrospective data.

A fifth limitation involves the relationship between the two contrasting retrospective accounts of role overload that the respondents were asked to recall. Specifically, the content of the second role overload event recalled may have been affected by the assessment of the first. For example, those individuals selecting overwhelming episodes as the second role overload event (i.e., following the description of a not-overwhelming event) may have selected an event with less overload and more stress than the first episode. The researcher took several steps during the interview to ensure this did not happen. Interviewees were actively encouraged to think of events in which they were equally overloaded. The overloaded respondents in the thesis sample indicated few problem in recalling two such events. The researcher believes that the cautious approach to interpreting findings and the cross case analysis techniques 
described above also helped to avoid any inconsistencies in the data collected across each pair of recollections.

The sixth limitation of this study is related to the demographics of the respondents involved. This research was restricted to the study of women. This limitation is often reflected in other studies in the work family literature (Pitt-Catsouphes et al., 2006). The restriction to women interviewees was taken for pragmatic and theoretical reasons, but given the evidence of the growth of role overload in Canada it is important to undertake more studies of the experiences of men and women in the future.

Another limitation of this case study revolves around the analysis and data display process that was used to maintain the focus on the objectives of the study. This approach opened up the possibility that other important factors were not identified as relevant. In addition, the large amounts of data produced by this qualitative study and the discussion to focus on differences may mean that key differentiators have been missed. Additional analysis of the data outside the confines of this thesis or future qualitative studies using other populations to refine the framework further may help to mitigate for these possible omissions.

The eighth limitation of this thesis relates to the paper and pencil method used to collect the data for determining respondent levels of role overload and stress in the survey prior to the case study. Specifically, the results from such self report techniques may reflect respondent desirable images rather than actual states. It would have been preferable to verify this data in other ways. The scales used are, however, well used and reliable measures for these constructs (see Duxbury et al., 2010). The purposeful 
sampling technique used in this study also meant that only those with the highest levels of role overload and the highest and lowest levels of perceived stress were interviewed.

Another limitation affecting this research involves the short personality measure used in the interview. The long measures of personality (e.g., Costa and McCrae, 1985) are considered to be more psychometrically sound compared to the brief measure used in this thesis (Gosling, 2003). Since personality was not the primary focus of this study, the relative efficiency of the brief scale (see Section 7.1.2) made it a very attractive research tool for use in the relatively short respondent interviews of this thesis. Caution was also applied when interpreting the results of this measure.

The last limitation of the research is that despite stated intentions, the data may have been biased towards experiences of work role overload. The respondents were originally identified because of their work and most were contacted at their work site. This may have influenced them to talk of work role overload events, even though they were encouraged to pick any situation involving role overload. All the respondents were selected, however, because they reported high levels of work, family and total role overload. The respondents therefore had plenty of home and work role overload examples to choose from, but may have chosen more work episodes for other reasons, as discussed in this thesis.

\subsection{Future research}

The conclusions and contributions of this research offer avenues for further research in a number of areas. The first direction for future research is to test the generalizability of the conceptual framework and the conclusions about differential stress 
experiences presented in this thesis. Researchers can achieve this task by examining the applicability of the framework to other populations in other contexts. This involves replicating the interviews with other samples, including those from other industry sectors. The goal would be to determine if new data fits the conceptual framework emerging from this study, or whether the framework requires further refinement. Such research would also examine if the distinguishing factors of the role overload appraisal and coping framework are consistent across other samples.

A particularly important way to increase the contribution of this research is to extend the sample to include men. The results of this study suggested that a number of factors identified as influential in this research may have emerged because of the women only sample (e.g. parental role salience; personality traits; and the prevalence of social support as a coping strategy). Replicating this study for male professionals in jobs that are similar to those held by the people in the thesis sample (i.e., police, physicians and emergency response) or using a sample in which men and women are equally represented may help to refine the framework in these or other areas.

The second potential direction for future research is to consider hypothesis testing where measures are developed and researchers are confident of the constructs in the framework of role overload appraisal and coping. First, the taxonomy of role overload situation properties and the typology of role overload coping strategies provide opportunities for further testing. Whilst, as expected, time pressures and the volume of demands were the most salient properties of role overload in this study, other salient properties (e.g. ambiguity, familiarity and duration) have received little, if any, attention in the role overload literature. Role overload research could benefit from exploring the 
circumstances in which situations are more likely to be perceived as a challenge or a threat (Gilboa et al., 2008). Future research could also examine the relationship between role overload situation properties and perceived coping capability, coping strategy choices and perceived stress. The moderating effect of person factors, such as beliefs about control or personality, on these relationships could also be examined. The typology of role overload coping strategies developed in this thesis could be used to extend the research of Lang and Markovitz (1986) to determine which coping strategies are effective in reducing stress from role overload across multiple roles. To test the differences associated with overwhelming versus not-overwhelming role overload, and with the episodes of high and low stress respondents, the distinguishing factors suggested during the discussions in Chapter 14 might also form the basis for hypothesis testing in future research.

Role ambiguity was found to be an important indicator of feelings of role overload in this study. As noted above, this construct has received little attention in the role overload literature. The importance Kahn et al $(1964,1966)$ and recent role stress researchers have placed on identifying three distinct forms of role stress (Coverman, 1989; King and King, 1990; Hecht, 2001; Duxbury et al., 2008; Gilboa et al., 2008; Fournier, 2010) suggests that future research should look again at the relationships between role ambiguity, role conflict, and role overload. A survey study including measures of role overload, role conflict, role ambiguity and stress could be used to see how these constructs are related.

According to the thesis literature review, previous role overload research has rarely focused on personality traits and the relationship with stress. The findings in this 
study suggested that certain personality traits (conscientiousness and neuroticism) were associated with variable respondent stress levels and perceived coping ability during role overload. One way to increase the contribution of this research is to include a longer and more psychometrically sound personality measure in the case study. This would have to be administered via a written or internet based questionnaire prior to the semi-structured interview. Future role overload research may also benefit from extending the hypothesis testing suggested above by including such measures in survey research exploring the relationship between role overload, personality, and stress.

Finally, the emotions and feelings identified in the four episode groupings identified in this study suggest that they may vary with perceived levels of stress and by role overload episode assessment. Further research involving a more detailed analysis of the relationship between cognitive appraisal, felt emotions and behavioural outcomes may also provide a better understanding of the relationship between role overload and stress. The existing data could be re-analysed, for example, to more closely examine which emotions and feelings are associated with challenge, threat, and harm appraisals and with the different episode resolution outcomes identified. Alternatively, a short measure of positive and negative affect could be included in the case study interview to more precisely capture the emotions and feelings expressed during recollections of role overload. 


\subsection{Implications for practice}

There are a number of implications for management practice that are suggested by this research. For a good number of hospital workers role overload seems to be a chronic and often daily occurrence. Whilst the volume of tasks and deadlines characteristic of role overload cannot always be controlled in a hospital there are other factors identified in the role overload and coping process as described by hospital workers that could be managed in order to alleviate the occurrence and chronic nature of role overload.

Ambiguous role demands was the third most salient role overload situation property that can create the potential for harm, threat or challenge. Management should be aware that a lack of clear guidelines particularly with regard to priorities can increase levels of role overload. Workers who do not know what is expected of them may also find it difficult to implement effective coping strategies when faced with role overload.

According to Ptacek et al., 1992, examining the relationships between appraisal processes, coping strategies and outcomes can have implications for teaching individuals effective coping skills. Future research involving hypothesis testing once the constructs of the role overload appraisal and coping framework have been adequately refined could be used to examine these relationships. Hopefully this will lead to a better understanding of which coping strategies are more effective in which circumstances and for which people.

Focusing on the factors distinguishing overwhelming from not-overwhelming role overload and those distinguishing the role overload episodes recalled by respondents with high levels of stress, from those of low stress respondents, as identified in this study, may bring the greatest benefit to management practice. For example, the overwhelming 
episodes of high stress respondents are more likely to involve a combination of demands from home and work. It may be worthwhile for management to review and promote work family balance policies to ensure employees are aware of resources that may be useful when role overload is sourced from home and work. The role overload situation properties 'duration' and 'event uncertainty' were both associated with overwhelming role overload, and 'event uncertainty' was also mentioned more often in the episodes of workers with high levels of stress. Management should make it a priority to address ongoing role overload, to reduce the overwhelming feelings that can be associated with such situations of a lengthy duration. Whilst event uncertainty may be impossible to eliminate, especially in the health sector, it may be possible to alleviate the difficulties employees had in identifying coping resources under such circumstances. Specifically, establishing processes to manage events, whose occurrence cannot be predicted with certainty, could help employees obtain appropriate coping resources in a timelier manner when these events do happen.

Problems identifying coping resources have also been noted as an important factor in overwhelming role overload and in episodes recalled by high stress respondents. For example, this research revealed that the coping restriction labeled 'environmental constraints' was more likely to be mentioned in the episodes assessed to be overwhelming. Specifically, restrictive rules on working overtime and on taking family leave prevented some workers from scheduling extra time to deal with role overload. A more flexible approach to scheduling time outside standard work hours might help workers reduce role overload. Workers also felt that management support was sometimes 
lacking when they tried to address the causes of role overload. Management awareness of these issues may help to eliminate this perception.

Finally, findings from this study linking demand-reduction strategies to notoverwhelming episodes described by low stress workers suggest that reducing role demands may be key to coping with role overload. For example, practical help from others is a commonly used strategy for coping with role overload that is aimed at reducing demands. Lack of available help can also contribute to feelings of overload, suggesting understaffing is a cause of role overload. This information can be used by management to maximise people resources at times of most need. 


\section{REFERENCES}

Abramis, D. J. (1994). Work role ambiguity, job satisfaction, and job performance: Meta-analysis and review. Psychological Reports, 75, 1411-1433.

Aldwin, C. M. (2007). Stress, coping and development: An integrative perspective, New York: The Guilford Press.

Amatea, E. S., Cross, E. G., Clark, J. E., \& Bobby, C. L. (1986). The life role salience scale. Journal of Marriage and the Family, 43: 831-383.

Amatea, E. S., \& Fong, M. L. (1991). The impact of role stressors and personal resources on the stress experience of professional women. Psychology of Women Quarterly, 15: 419-430.

Babbie, E. (2001). The Practice of Social Research, Belmont, California: Wadsworth Thompson Learning.

Bacharach, S. B., Bamberger, P. \& Conley, S. (1990). Work processes, role conflict, and role overload: The case of nurses and engineers in the public sector, Work and Occupations, 17 (2), 199-228.

Bacharach, S. B., Bamberger, P. \& Conley, S. (1991). Work-home conflict among nurses and engineers: mediating the impact of role stress on burnout and satisfaction at work. Journal of Organizational Behavior, 12, 39-53.

Barnett, R. C. (1998). Toward a review and reconceptualization of the work/family literature. Genetic, Social and general Psychology Monographs, 124 (2), 125-182.

Barnett, R. C. \& Baruch, G. K. (1985). Women's involvement in multiple roles and psychological distress. Journal of Personality and Social Psychology, 49 (1), 135145.

Barnett, R. C. \& Gareis (2006). Role theory perspectives on work and family. In M. Pitt-Catsouphes, E.E. Kossek and S. Sweet (Eds) The Work and Family Handbook (pp 209-222), New Jersey: Lawrence Erlbaum Associates, Publishers.

Barnett, R. C. \& Hyde (2001). Women, men, work, and family. American Psychologist, 56 (10), 781-796.

Beehr, T. A. (1981). Work-role stress and attitudes towards coworkers, Group and Organizational Studies, 6, 201-210.

Beehr, T. A. (1995). Psychological Stress in the Workplace. New York: Routledge. 
Beehr, T. A. \& Glazer, S. (2005). Organizational role stress. In J. Barling, E. K. Kelloway and M. R. Frone (Eds.) Handbook of Work Stress (pp 7-34), Thousand Oaks, California: Sage Publications.

Beehr, T. A., King, L. A. \& King, D. W., (1986). Theoretical and empirical development of the function of uncertain expectancies in occupational stress. In D J Abramis (Chair), The past, present and future of job stress research: New Thoughts about old concepts, Symposium presented at the annual meeting of the Academy of Management, Chicago.

Beehr, T. A. \& Newman (1978). Job stress, employee health, and organizational effectiveness: A facet analysis, model and literature review.Personnel Psychology, 31, 665-699.

Beehr, T. A. Walsh, J. T. \& Taber, T. D. (1976). Relationship of stress to individually and organizationally valued states: higher order needs as a moderator. Journal of Applied Psychology, 61 (1), 41-47.

Bellavia, G. M. \& Frone, M. R. (2005). Work family conflict. In J. Barling, E. K. Kelloway and M. R. Frone (Eds.) Handbook of Work Stress (pp 113-148), Thousand Oaks, California: Sage Publications.

Biddle, B. J. \& Thomas, J. P. (1966). Role theory: Concepts and research. New York: Wiley.

Biddle, B. J. (1979). Role Theory: Expectations, Identities and Behaviors. New York: Academic Press.

Biddle, B. J. (1986). Recent developments in role theory. Annual Review of Sociology, 12, 67-92.

Bohen, H. H. \& Viveros-Long A. (1981). Balancing Job and Family Life: Do Flexible Work Schedules Help? Philadelphia: Temple University Press.

Bolger, N. \& Zuckerman, A. (1995). A Framework for Studying Personality in the Stress Process. Journal of Personality and Social Psychology, 69 (5), 890-902.

Brown, P. A. (2008). A review of the literature on case study research. Canadian Journal for New Scholars in Education, 1 (1), 1-12.

Byron, K. (2005). A meta-analytic review of work-family conflict and its antecedents. Journal of Vocational Behaviour, 67, 169-198.

Caplan, R. D., Cobb, S. French, J. R. P. Jr., Harrison, R. V. \& Pinneau, S. R. (1975). Job Demand and Worker health: Main effects and occupational differences, Washington, DC: US. Government Printing Office. 
Caplan, R.D., Cobb, S., French, J.R.P., Jr., Harrison, R.V., \& Pinneau, S.R., Jr. (1980). Job demands and worker health: Main effects and occupational differences. Ann Arbor, MI: University of Michigan, Institute for Social Research (Originally published as HEW Publications No. (NIOSH) 75-106, 1975).

Carlson, D. S. \& Grzwacz J. G. (2008). Reflections and future directions on measurement in work-family research In K. Korabik, D.S. Lero and D. L. Whitehead (Eds.) Handbook of Work-Family Integration: Research, Theory and Best Practices, (pp 57-74), Academic Press.

Carpenter, B. N. (1992). Issues and advances in coping research. In Bruce N. Carpenter (Ed.), Personal Coping: Theory, research, and Application, (pp1-14).

Carver, C. S. \& Connor-Smith (2010). Personality and coping. Annual Review of Psychology, 61, 679-704.

Carver, C. S., Scheier, M. F. \& Weintraub, J. K., (1989). Assessing coping strategies: a theoretically based approach. Journal of Personality and Social Psychology, 56 (2), 267-283.

Cast, A. D. \& Burke, P. J. (2002). A theory of self-esteem. Social Forces, 80 (3), 1041-1068.

Chang, E. M., \& Hancock, K. M. (2003). Role stress and role ambiguity in new nursing graduates in Australia. Nursing and Health Sciences, 5, 155-163.

Chang, E. M., Hancock, K. M., Johnson, A., Daly, J. \& Jackson, D. (2005). Role stress in nurses: review of related factors and strategies for moving forward. Nursing and health Sciences, 7, 57-65.

Cohen, S., Kamarck, T. \& Mermelstein, R. (1983). A global measure of perceived stress. Journal of Health and Social Behavior, 24 (4), 385-396.

Cohen, S., Kessler, R. C. \& Underwood Gordon, L. (1995). Strategies for measuring stress in studies of psychiatric and physical disorders. In S. Cohen, R.C. Kessler and L. Underwood Gordon (Eds.) Measuring Stress, (pp 122- 147), New York: Oxford University Press.

Compass, B. E., Connor, J., Osowiecki, D. \& Welch, A. (1997). Effortful and involuntary responses to stress: implications for coping with chronic stress. In Benjamin H. Gottlieb (Ed), Coping with Chronic Stress, (pp105-132). New York: Plenum Press. 
Cooper, C. L. \& Dewe, P. (2007). Stress: A brief history from the 1950s to Richard Lazarus. In A. Monat, R. S. Lazarus, and G. Reevy (eds.) The Praeger Handbook of Stress and Coping, (pp 7-32), Westport: Connecticut: Praegar.

Cooper, C. L. \& Marshall, J. (1976). Occupational sources of stress: A review of the literature relating to coronary heart disease and mental ill health. Journal of Occupational Psychology, 49(1), 11-28.

Cooper, C. L., Dewe, P.J. \& O’Driscoll, M. P. (2001) Organizational Stress, Thousand Oaks, CA: Sage Publications, Inc.

Costa, P. T. \& McCrae, R. R (1985). The NEO personality inventory manual. Odessa, FL: Psychological Assessment Resources.

Costa, Jr. P. T., \& McCrae, R. R, (1990). Personality: Another "hidden factor" in stress research. Psychological Inquiry, 1 (1), 22-24.

Costa, P. T. \& McCrae, R. R (1992). Revised NEO personality inventory (NEO-PI-R) and NEO five factor inventory (NEO-FFI) professional manual. Odessa, FL: Psychological Assessment Resources.

Costa, P. T. \& McCrae, R. R. (2006) Age changes in personality and their origins: Comment on Roberts, Walton, and Viechtbauer (2006). Psychological Bulletin, 132, 28-30.

Coverman (1989). Role Overload, Role Conflict, and Stress: Addressing Consequences of Multiple Role Demands. Social Forces, 67:4, 965-982.

Coyne, J. C. \& Lazarus, R. S. (1980). Cognitive style, stress perception, and coping. In I. L. Kutash and L. B. Sclesinger (Eds.), Handbook on stress and anxiety: Contemporary knowledge, theory and treatment (pp 144-150). San Francisco: JosseyBass.

Coyne, J. C. \& Racioppo, M. W. (2000). Never the twain shall meet? Closing the gap between coping research and clinical intervention research. American Psychologist, $55,655-664$.

Creswell, J. W. (2009). Research Design: Qualitative, Quantitative and Moxed Methods Approaches, Los Angeles: Sage.

De Ridder, D. (1997). What is wrong with coping assessment? A review of conceptual and methodological issues. Psychology and Health, 2, 417-431.

Dewe, P. J. (1993). Measuring primary appraisal: Scale construction and directions for future research. Journal of Social Behavior and Personality, 8 (4), 673-685. 
Dewe, P. J. O'Driscoll, M. P. \& Cooper, C. L. (2010). Coping with work stress: A review and critique, New York: John Wiley \& Sons.

Dooley, L. M. (2002). Case study research and theory building. Advances in Developing Human Resources, 4 (3), 335-354.

Doress-Worters, P.B. Adding elder care to women's multiple roles: A critical review of the caregiver and multiple roles literatures. Sex Roles. 31, 597-616.

Dutton, J. E. Ashford, S. J. O’Neil, R. M. \& Lawrence, K. A. (2001). Moves that matter: Issue selling and organizational change. Academy of Management Journal, 4, 716-736.

Duxbury, L. \& Higgins, C. (2001). Work life in the new millennium: where are we? Where do we need to go? Canadian Policy Research Network (CPRN) Discussion Paper, Vol. W12, Ottawa: CPRN.

Duxbury, L. \& Higgins, C, (2003a). Where to Work in Canada? An Examination of Regional Differences in Work Life Practices, Vancouver, B.C.: B.C. Council of the Families.

Duxbury, L. \& Higgins, C (2003b). Work-life Conflict in Canada in the New Millenium:A Status Report (Report Two). Ottawa: Health Canada.

Duxbury, L. \& Higgins, C (2009). Work-life Conflict in Canada in the New Millenium: Key Findings and Recomendations from the 2001 National Work-Life Conflict Study. (Report Six). Ottawa: Health Canada.

Duxbury, L. Lyons, S. \& Higgins, C (2008). Too much to do and not enough time: an examination of role overload. In K. Korabik, D.S. Lero and D. L. Whitehead (Eds.) Handbook of Work-Family Integration: Research, Theory and Best Practices, (pp 125-140), Academic Press.

Duxbury, L., Higgins, C. \& Lyon, S. (2010). The etiology and reduction of role overload in Canada's health care system. Ottawa, Ontario: Sprott School of Business, Carleton University.

Duxbury, L. \& Higgins, C (2012). Revisiting Work-Life Issues in Canada: The 2102 National Study on Balancing Work and Caregiving in Canada, Ottawa: Carleton University

Eby, L. T., Casper, W. J.. Lockwood, A., Bordeaux, C. \& Brinley, A. (2005). Work and family research in IO/OB: Content analysis and review of the literature (19802002). Journal of Vocational Behaviour, 66, 124-197.

Eisenhardt, K. M. (1989). Building theories from case study research. Academy of Management Review, 16 (3), 620-627. 
Eisenhardt, K. M. (1991). Better stories and better constructs. Academy of Management Review, 14(4), 532-550.

Eisenhardt, K. M. \& Graebner, M. E. (2007). Theory building from cases:

Opportunities and challenges. Academy of Management Review, 50(1) 25-32.

Elloy, D. F. \& Smith, C. R. (2003). Patterns of stress, work-family conflict, role conflict, role ambiguity and overload among dual-career and single-career couples: An Australian study. Cross Cultural Management, 10 (1), 55-66.

Feigin, J. R., Orum, A. M. \& Sjoberg, G. (1991). A case for case study. Chapel Hill, NC:The University of North Carolina Press.

Fineman, S. \& Payne, R. (1981). Role stress - a methodological trap? Journal of Occupational Behaviour, 2, 51-64.

Fink, G. (2000). Encyclopedia of Stress, New York: Academic Press.

Fisher, C. D. \& Gitelson, R. (1983). A meta-analysis of the correlates of role conflict and ambiguity, Journal of Applied Psychology, 68, 320-333.

Folkman, S. (1997). Positive psychological states and coping with severe stress, Social Science Med, 45 (8), 1207-1221.

Folkman, S. (2008). The case for positive emotions in the stress process. Anxiety Stress \& Coping, 21 (1), 3-14.

Folkman, S. \& Lazarus, R. S. (1980). An analysis of coping in a middle aged community sample. Journal of Health and Social Behavior, 21 219-239.

Folkman, S. \& Lazarus, R. S. (1985). If it changes it must be a process: Study of emotion and coping during three stages of a college examination. Journal of Personality and Social Psychology, 48 (1), 150-170.

Folkman, S. \& Moskowitz, J. T. (2000). Positive affect and the other side of coping. American Psychologist, 55, 647-654.

Folkman, S. \& Moskowitz, J. T. (2004). Coping: Pitfalls and promise. Annual Review Psychology 55, 745 -774.

Folkman, S. Lazarus, R. S. Gruen, R. J. \& De Longis, A (1986a) Appraisal, coping, health status, and psychological systems. Journal of Personality and Social Psychology, 50, 571-579. 
Folkman, S., Lazarus, R. S., Dunkel-Schetter, C. DeLongis, A. \& Gruen, R. J. (1986b). Dynamics of a stressful encounter: Cognitive appraisal, coping, and encounter outcomes. Journal of Personality and Social Psychology, 50 (5), 992-1003.

Folkman, S., Chesney, M., McKusick, G. I., Johnson, D. S. \& Coates, T. J. (1991) Translating coping theory into an intervention. In J. Eckenrode (Ed.) The Social Context of Coping, (p. 239-260), New York: Plenum Press.

Fournier, C., Tanner Jr., J. F.Chonko, \& Manolis, C. (2010). The moderating role of ethical climate on salesperson propensity to leave, Journal of Personal Selling and Sales Management, 30 (1) 7-22.

Frantilla, A. (1998). Social Science in the Public Interest: A Fiftieth Year History of the Institute for Social Research, Ann Arbor: Bentley Historical Library, University of Michigan, sourced from http://bentley.umich.edu/research/publications/ISR.pdf on September 18th 2009.

French, J. R. P. \& Caplan, R. D.(1972). Organizational stress and individual strain. In A. J. Marrow (Ed.), The failure of success, New York,: AMACOM.

French, J. R. P., Rogers, W. \& Cobb, S. (1974). A model of person-environment fit. In G. Coelho, D. Hamburg and J. Adams (Eds.) Coping and Adaptation (pp. 316333). New York: Basic Books.

Frone, M. R., Russell, M. \& Cooper, M. L. (1992). Antecedents and outcomes of work-family conflict: Testing a model of the work-family interface. Journal of Applied Psychology, 77, 65-78.

Frone, M. R., Yardley \& Markel, (1997). Developing and testing an integrative model of the work-family interface. Journal of Vocational Behavior, 50, 145-167.

Fu, C. K. \& Shaffer, M. A. (2001). The tug of work and family. Direct and indirect domain-specific determinants of work-family conflict. Personnel Review, 30, 502522.

Galameau, G. (2003, December). Health care professionals. Perspectives on Labour and Income: The online edition, 4 (12). Retrieved from Statistics Canada, http://www.statcan.gc.ca/pub/75-001-x/01203/6699-eng.html

Ganster, D. C., Fox, M. L. \& Dwyer, D. J. (2001). Explaining employees' health care costs: A prospective examination of stressful job demands, personal control, and physiological reactivity. Journal of Applied Psychology, 86 (5), 954-964.

George, (1993). Sociological perspectives on life transitions. Annual Review of Sociology, 19, 353-373. 
Gibbert, M., Ruigrok, W. \& Wicki, B. (2008). What passes as a rigorous case study? Strategic Management Journal, 29,1465-1474.

Gilboa, S., Shirom, A, Fried, Y. \& Cooper, C. (2008). A meta-analysis of work demand stressors and job performance: Examining main and moderating effects. Personnel Psychology, 61, 227-271.

Glaser, B. G. \& Strauss, A. L. (1967). The discovery of grounded theory: Strategies for qualitative research, New Jersey: Transaction Publishers.

Glazer, S. \& Gyurak, A. (2008). Sources of occupational stress in five countries. International Journal of Intercultural Relations, 32, 49-66.

Glynn, K., Maclean, H., Forte, T., \& Cohen, M. (2009). The association between role overload and women's mental health. Journal of Women's Health, 18 (2), 217-223.

Goldberg (1990). An alternative "description of personality". The Big-Five factor structure. Journal of Personality and Social Psychology, 59, 1216-1229.

Gonzalez-Roma, V. \& Lloret, S. (1998). Construct validity of Rizzo et al.'s role conflict and ambiguity scales: A multisample study, Applied Psychology: An International Review, 47 (4), 535-545.

Goode, W. J. (1960). A theory of role strain. American Sociological Review, 25 (4), 483-496.

Gosling, . D. Rentfrow, P. J. \& Swann Jr. W. B. (2003). A very brief measure of the Big-Five personality domains, Journal of research in personality, 37, 504-528.

Grant, S. \& Langen-Fox, J. (2006). Occupational stress, coping and strain: The combined/interactive effect of the Big Five traits. Personality and Individual Differences, 41, 719-732.

Greenhaus, J. H. \& Allen, T. D. (2006). Health consequences of work-family conflict: The dark side of the work-family interface. Research in occupational stress and wellbeing, 5, 61-98.

Greenhaus, J. H. \& Beutell, N. J. (1985). Sources of conflict between work and family roles. Academy of Management Review, 10, 76-88.

Greenhaus, J. H. \& Parasuraman, (1999). Research on work family and gender: Current status and future directions. In G. N. Powell (Ed.) Handbook of gender and work: 391-412. Newbury Park, CA: Sage.

Greenhaus, J. H. \& Powell, G. N. (2006). When work and family are allies: A theory of work-family enrichment, Academy of management review, 31 (1), 72-92. 
Greenhaus, J. H., Parasuramen, S., Granrose, C. S., Rabinowitz, S. \& Beutell, N. J. (1989). Sources of work-family conflict in two career couples, Journal of Vocational Behavior, 34, 133-153.

Greer, T. W. \& Egan, T. M. (2012). Inspecting the hierarchy of life roles: A systematic review of role salience literature. Human Resources Development Review, 11 (4), 463- 499.

Gross, N., Mason, W. S. \& McEachern, A. W. (1958). Explorations and Role Analysis: Studies of the School Superintendency Role, New York, John Wiley \& Sons, Inc.

Gupta, N. \& Beehr, T.A. (1979). Job Stress and employee behaviours, Organizational Behaviour and Human Decision Processes, 23, 373-387.

Gutek, B. A., Searle, S. \& Kleppa, L. (1991).Rational versus Gender Role Explanations for Work-Family Conflict. Journal of Applied Psychology, 76 (4), 560568.

Hardy, M. E. \& Hardy, W. (1988). Role stress and role strain. In M. E. Hardy and M. E. Conway (Eds) Role Theory: Perspectives for Health Professionals (Second Edition) (pp 159-240), Norwalk, Connecticut: Appleton \& Lange.

Hardy, M. E. \& Hardy, W. (1988a). Development of scientific knowledge. In M. E. Hardy and M. E. Conway (Eds) Role Theory: Perspectives for Health Professionals (Second Edition) (pp 159-240), Norwalk, Connecticut: Appleton \& Lange.

Harris, M. M. \& Bladen, A. (1994). Wording effects in the measurement of role conflict and role ambiguity: a multitrait-multimethod analysis. Journal of Management, Winter.

Hecht, L. M. (2001). Role conflict, role overload: Different concepts, different consequences. Sociological Inquiry, 71 (1), 111-121.

Higgins, C., Duxbury, L. E., \& Lee, C. (1994). Impact of life-cycle stage and gender on the ability to balance work and family responsibilities. Family Relations, 43 (2), 144-150.

Higgins, C. Duxbury, L. \& Lyons, S. (2010). Coping with overload and stress: Men and women in dual-earner families. Journal of Marriage and Family, 72, 847-859.

Holahan, C. J., Moos, R. H. \& Schaefer, J. A. (1996) In Moshe Zeidner and Norman S. Endler (Eds.), Handbook of Coping (pp. 24- 43). New York: John Wiley \& Sons. 
House, R.J. \& Rizzo, J. R. (1972). Role conflict and ambiguity as critical variables in a model of organizational behavior. Organizational behaviour and Human Performance, 7, 467-505.

House, R. J. Schuler, R. S. \& Levanoni, E. (1983). Role conflict and ambiguity scales: Reality or artefacts? Journal of Applied Psychology, 68 (2) 334-337.

Ice, G. H. \& James, G. D. (2007). Conducting a field study of stress: general principles. In G. H. Ice, G. D. James (Eds.). Measuring stress in humans, (pp. 3-24). Cambridge, UK: Cambridge University Press

Ilgen, D. R. \& Hollenbeck, J. R. (1991). The structure of work: Job design and roles. In M.D.

Dunnette \& L.M. Hough (eds.), Handbook of industrial and organizational psychology, (Pp. 165-207), Vol. 2. Palo Alto, CA: Consulting Psychologists Press.

Jackson, S. \& Schuler, R. (1985). A meta-analysis and conceptual critique of research on role ambiguity and role conflict in work settings, Organisational behaviour and Human Decision processing, 36, 16-78.

Jerusalem, M. \& Schwarzer, R. (1989). Anxiety and self-concept as antecedents of stress and coping: A longitudinal study with German and Turkish adolescents. Personality and Individual Differences, 10 (7), 785-792.

Johnson, K. L (2007). A case study in boundary spanning role in a multinational enterprise. (PhD). Carleton University.

Jones, E., Chonko, L., Rangarajan, D. \& Roberts, J. (2007). The role of overload on job attitudes, turnover intentions, and salesperson performance. Journal of Business Research, 60, 663-671.

Jones, III, A., Norman, C. S. \& Weir, B. (2010). Healthy lifestyle as a coping mechanism for role stress in public accounting, Behavioral Research in Accounting, $22(1), 21-41$.

Kanter, R. (1977). Work and Family in the United States: A critical review and agenda for research and policy, New York: Russell Sage Foundation.

Kanter, R. (2006). Foreword. In M. Pitt-Catsouphes, E.E. Kossek \& S. Sweet (Eds) The Work and Family Handbook (pp xii). New Jersey: Lawrence Erlbaum Associates, Publishers.

Kahn, R. L., Wolfe, D. M., Quinn, R. P. \& Snoek, J. D. (1964). Organizational Stress: Studies in Role Conflict and Ambiguity, New York: John Wiley \& Sons. 
Kahn, R. L. (1980). Conflict, ambiguity, and overload. Three elements in job stress. In D. Katz, R. Kahn, \& J. Adams (Eds.), The study of organizations (pp. 418-428). San Francisco: Jossey-Bas.

Katz, D, Kahn, R. L. \& Adams, J, S. (1980). Work and health. In D. Katz, R. Kahn, \& J. Adams (Eds.), The study of organizations (pp. 414-417). San Francisco: JosseyBas.

Katz, D., \& Kahn, R. L. 1966. The social psychology of organizations. New York: John Wiley.

Keenan, A. \& McBain, G (1979). Effects of Type A behaviour, intolerance of ambiguity, and locus of control on the relationship between role stress and workrelated outcomes, Journal of Occupational Psychology, 52, 277-285.

Kelloway, E. K \& Barling, J. (1990). Item content versus item wording: Disentangling role conflict and role ambiguity, Journal of Applied Psychology, 75 (6) 738-742.

King, L. A. \& King, D. W. (1990). Role conflict and role ambiguity: A critical assessment of construct validity. Psychological Bulletin, 107 (1), 48-64.

Knowles, E. S. (1982). From individuals to group members: A dialectic for the social sciences. In W. Ickes and E. S. Knowles (Eds.) Personality, roles and Social behaviour, new York, Springer-Verlag.

Kobasa, S. C., Maddi, R. S. \& Kahn, S. (1982). Hardiness and health: a prospective study. Journal of Personal and Social psychology, 42, 168- 180.

Korabik, K. McElwain, A. \& Chappell, D. B. (2008). Integrating gender-related issues into research on work and family. In K. Korabik, D. S. Lero, \& D. L. Whitehead (Eds.) Handbook of Work-Family Integration: Research, Theory and Best Practices, Salt Lake City, Utah: Academic Press.

Lang, D. (1992). Preventing short-term strain through time-management coping. Work and Stress, 6 (2), 169-176.

Lang, D. \& Markowitz, M. (1986). Coping, individual differences, and strain: A longitudinal study of short-term role overload. Journal of Occupational Behaviour, 7, 195-206.

Lazarus, R. S. (1966). Psychological stress and the coping process. New York: McGraw-Hill. 
Lazarus, R. S. (1981). The stress and coping paradigm. In C. Eisdorfer, D. Cohen, A. Kleinman, and P. Maxim (Eds.), Models for Clinical Psychopathology (pp. 174-214). New York: McGraw-Hill.

Lazarus, R. S. (1990). Theory-based stress measurement. Psychological Inquiry, 1(1), 3-13.

Lazarus, R.S. (1991). Foreword. In J. Eckenrode (Ed.) The Social Context of Coping, (p. ix - xi), New York: Plenum Press.

Lazarus, R. S. (1993). Coping theory and research: Past, present and future. Psychosomatic Medicine, 55, 234- 247.

Lazarus, R. S. (1995). Psychological stress in the workplace. In R. Crandell and P. L. Perrewé (Eds.), Occupational stress: A handbook. (pp 3-14). Washington, DC: Taylor \& Francis.

Lazarus, R. S. (1996). The role of coping in the emotions and how coping changes over the life course. In C. Magai and S. H. McFadden (Eds.) Handbook of Emotion, Adult Development, and Aging (pp 289-306). Sandiago: Academic Press.

Lazarus, R. S. (1998a). Fifty Years of the Research and Theory of R. S. Lazarus: An Analysis of Historical and Perennial Issues. New Jersey: Lawrence Erlbaum Associates, Publishers.

Lazarus, R. S. (1998b). The Life and Work of an Eminent Psychologist: Autobiography of Richard S. Lazarus. New York: Springer Publishing Co.

Lazarus, R. S. (1999). Stress and Emotion: A New Synthesis, New York: Springer Publishing Company, Inc.

Lazarus, R. S. (2000). Towards better research on stress and coping. American Psychologist, 55, $665-673$.

Lazarus, R. S. (2007). Stress and emotion: A new synthesis. In A. Monat, R. S. Lazarus, and G. Reevy (eds.) The Praeger Handbook of Stress and Coping, (pp 3352), Westport: Connecticut: Praegar.

Lazarus, R. S. \& Folkman, S. (1984). Stress, appraisal, and coping. New York: Springer Publishing Co.

Lazarus, R. S. \& Launier, R. (1978). Stress-related transactions between person and environment. In L. A. Pervin and M. Lewis (Eds.), Perspectives in Interactional Psychology (pp. 287-327). New York: Plenum. 
Lazarus, R. S., Averill, J. R., \& Opton, E. M., Jr. (1974). The psychology of coping: Issues of research and assessment. In G. V. Coelho, D. A. Hamburg, and J. E. Adams (Eds.), Coping and Adaptation (pp. 249-315). New York: Basic Books.

Lazarus, R. S., Coyne, J. C., \& Folkman, S. (1982). Cognition, emotion, and motivation: The doctoring of Humpty-Dumpty. In R. W. J. Neufeld (Ed.), Psycchological stress and psychopathology (pp. 218-259). New York: McGraw-Hill.

Lazarus, R. S., Kanner, A. D. \& Folkman, S. (1980). Emotions: A cognitivephenomonological analysis. In R. Plutchik and H. kellerman (Eds.), Theories of emotion (pp. 189-217). New York: Springer Publishing.

Lazarus, R. S., DeLongis, A., Folkman, S., \& Gruen, R. (1985). Stress and adaptational outcomes: The problem of confounded measures. American Psychologist, 40, 770-779.

Lease, S. H. (1999). Occupational role stressors, coping, support, and hardiness as predictors of strain in academic faculty: An emphasis on new and female faculty. Research in Higher Education, 40 (3), 285-307.

Leonard-Barton, D. (1990). A Dual methodology for case studies: Synergistic use of a longitudinal single site with replicated multiple sites, Organizational Science, 1 (3), 248-266.

Lee-Baggley, D., Preece, M. \& DeLongis, A. (2005). Coping with interpersonal stress: Role of Big Five traits, Journal of Personality, 75 (5), 1141-1180.

LePine, J. A., LePine, M. A. and Saul (2007). Relationships among work and non work challenge and hindrance stressors and non-work and work criteria: A model of cross domain stressor affects stressors, In P. L Perrewé and D. C. Ganster (Eds.) Research in Occupational Stress and Well Being Volume 6: Exploring the Work and Non-Work Interface, (pp 35-72), Oxford: Elsevier JAI Press.

Levi, L. (1998). Preface - Stress in organizations - Theoretical and empirical approaches. In C. L. Cooper (Ed.) Theories of Organizational Stress (pp v - xii), Oxford: Oxford University Press.

Locke. K. (2001). Grounded theory in management Research, London: Sage.

Lim, J., Bogossian, F. \& Ahern, K. (2010). Stress and coping in Australian nurses: a systematic review. International Nursing Review, 57 (1), 22-31.

Linton, R. (1936). The Study of Man, New York: Appleton-Century-Crofts.

Lynn, R. \& Martin, T. (1997). Gender differences in extraversion, neuroticism, and psychoticism in 37 nations. The Journal of Social Psychology, 137 (3), 369-373. 
MacDermid, S. M. \& Harvey, A. (2006). The work-family construct: Methodological implications. In M. Pitt-Catsouphes, E.E. Kossek and S. Sweet (Eds) The Work and Family Handbook (pp 567-586), New Jersey: Lawrence Erlbaum Associates, Publishers.

Mak, A. S. \& Mueller, J. (2000). Job insecurity, coping resources and personality dispositions in occupational strain. Work and Stress, 14 (4), 312-328.

Marks, S. R. (1977). Multiple roles and role strain: Some notes on human energy, time and commitment. American Sociology Review, 42, 921-936.

Marks, S. R. \& MacDermid, S. M. (1996). Multiple roles and the self: A theory of role balance, Journal of marriage and Family, 58 (2) 417-432.

Mead, G. H. (1934). Mind, self and society, Chicago: University of Chicago Press.

Merton, R. (1957). Social Theory and Social Structure, $2^{\text {nd }}$ Ed., Glencoe, Ill: Free Press.

Michel, J. S., Mitchelson, J. K., Kotriba, L. M., LeBreton, J. M. \& Baltes, B. B. (2009). A comparative test of work-family conflict models and critical examination of work-family linkages. Journal of Vocational Behavior, 74, 199-218.

Miles, M.B. \& Huberman, A.M. (1994). Qualitative Data Analysis: An Expanded Sourcebook, $2^{\text {nd }}$ Ed., Sage Publications: Newbury Park.

Monat A, Lazarus, R. S. \& Reevy, G. (2007). Introduction to "The Stress Concept". In A. Monat, R. S. Lazarus, and G. Reevy (eds.) The Praeger Handbook of Stress and Coping, (pp 2-6), Westport: Connecticut: Praegar.

Monroe, S. M. \& Kelley, J. M. (1995). Measurement of stress appraisal. In S. Cohen, R.C. Kessler and L. Underwood Gordon (Eds.) Measuring Stress, (pp 122- 147), New York: Oxford University Press.

Moos, R. H. \& Holahan, C. J. (2003). Dispositional and contextual perspectives on copng: Toward an integrative framework. Journal of Clinical Psychology, 59 (12) 1387-1403.

Murphy, K. R., Jako, R. A., \& Anhalt, R. L. (1993). Nature and consequences of halo error: A critical analysis. Journal of Applied Psychology, 78 (2) 218-225.

Nakano, K. (1992). Role of personality characteristics in coping behaviours, psychological Bulletin, 71, 687-690. 
Narayanan, L. Menon S. \& Spector, P. E. (1999).Stress in the workplace: A comparison of gender and occupations, Journal of Organizational Behavior, 20 (1), 66-73.

Netemeyer, R. G., Johnston, M. W. \& Burton, S. (1990). Analysis of role conflict and role ambiguity in a structural equations framework, Journal of Applied Psychology, 75 (2), 148-157.

Newton, T.J. \& Keenan, A. (1987). Role stress re-examined: An investigation of role stress predictors, Organizational behaviour and Human Decision Processes, 40, 346368.

Nieman L. J. \& Hughes, J. W. (1951). The problem of the concept of role - A resurvey of the literature. Social Forces, 10 (1), 141-150.

Noor, K. B. M. (2008). Case study: A strategic research methodology. American Journal of Applied Sciences, 5 (11), 1602-1604.

O'Brien, T. B. \& DeLongis, A. (1996). The interactional context of problem-, emotion-, and association-focused coping: the role of the big five personality factors. Journal of Personality, 64, 775-813.

Örtqvist D. \& Wincent, J. (2006).Prominent consequences of role stress: A metaanalytic review, International Journal of Stress Management, 4, 399-422.

Osipow, S. H. \& Davis, A. S. (1988). The relationship of coping resources to occupational stress and strain. Journal of Vocational Behavior, 32 (1) 1-15.

Parasuraman, S. \& Alutto, J. A. (1984). Sources and outcomes of stress in organization settings: Toward the development of a structural model. Academy of Management Journal, 27, 330-350.

Parasuraman, S., Purohit,Y. S., Godshalk,V. M. \& Beutell, N. J. (1996). Work and family variables, entrepreneurial career success and psychological well being. Journal of Vocational Behavior, 48, 275-300.

Paré, G. (2004). Investigating information systems with positivist case study research. Communications of the Association for Information Systems, 13, 233-264.

Parker, J. D. A. \& Endler, N. S. (1996). Coping and defense: A historical overview. In Moshe Zeidner and Norman S. Endler (Eds.), Handbook of Coping (pp. 3-23). New York: John Wiley \& Sons.

Parsons, T. (1951). The Social System, New York: The Free Press. 
Patton, M. Q. (2002). Qualitative Research \& Evaluation Methods, Thousand Oaks, California: Sage Publications.

Patton, E. \& Appelbaum, S. H. (2003). The case for case studies in management research, Management Research News, 26 (5), 60-71.

Pearlin, L. I., Menaghan, E. G., Lieberman, M. A. \& Mullan, J. T. (1981). The Stress Process. Journal of Health and Social Behavior, 22, (4) 337-356.

Pearson, Q. M. (2008). Role overload, job satisfaction, leisure satisfaction and psychological health among employed women. Journal of Counselling and Development, 86, 57-63.

Penley, J. A. \& Tomaka, J. (2002). Associations among the Big Five, emotional responses, and coping wit acute stress Personality and Individual Differences, 32, 1215-1228.

Perrewe, P. L. \& Zellars, K. L. (1999). An examination of attributions and emotions in the transactional approach to the organizational stress process. Journal of Organizational Behavior, 20, 739-752.

Perrewé, P. L., Zellars, K. L., Rossi, A. M., Ferris, G. R., Kacmar, C. J., Liu, Y, Zinko, R. \& Hochwarter, W. A. (2005). Political Skill: An antidote in the role overload-strain relationship. Journal of Occupational Health Psychology, 10 (3), 239250.

Perry-Jenkins, M., Repetti, R. L. \& Crouter, A. C.(2000). Work and family in the 1990s. Journal of Marriage and Family, 62 (4), 981-998.

Peterson, R. S., Owens, P. D. \& Martorana, P. V. (1999). The Group Dynamic Q-Sort in Organizational Research: A new method for studing familiar problems. Organizational Research Methods, 2 (2) 107-130.

Pienaar, J., Sieberhagen, C. F. \& Mostert, K. (2007). Investigating turnover intentions by role overload, job satisfaction and social support moderation. SA Journal of industrial Psychology, 33 (2) 62-67.

Pleck, J.H. (1995). The gender role strain paradigm: An update, In R. F. Levant \& W. S. Pollack (Eds.), A new psychology of men (pp 164-206), New York: Basic Books.

Pitt-Catsouphes, M. Kossek, E. E. \& Sweet, S. (2006). The Work and Family Handbook, New Jersey: Lawrence Erlbaum Associates.

Post, J. E. \& Andrew, P. N. (1982). Case research in corporation and society studies. In L. E. Preston (Ed.) Research in Corporate Social Performance Policy: A Research Annual, (pp 1-34), Greenwich, CT: Jai Press Inc. 
Powell, G. N. \& Greenhaus, J. H. (2006). Managing incidents of work-family conflict: A decision-making perspective. Human Relations, 59, (9), 1179-1212.

Ptacek, J. T. \& Pierce, G. R. (2003). Issues in the study of stress and coping in rehabilitation settings. Rehabilitation Psychology, 48, 2, 113-124.

Ptacek, J. T. Smith, R. E. \& Zanas, J. 1992. Gender, appraisal and coping. A longitudinal analysis. Journal of Personality, 60 (4), 747-770.

Rapoport, R. \& Rapoport, R. (1976). Dual-career Families Re-examined. New York: Harper and Collins.

Repetti, R. L., Matthews, K. A. \& Waldren, I. (1989). Employment and women's health: Effects of paid employment on women mental and physical health. American Psychologist, 44 (11), 1394-1401.

Rizzo (1983) Citation Classic. Current Contents - Social and Behavioral Sciences.

Rizzo, J. R, House, R. J. \& Lirtzman, S. I. (1970). Role conflict and ambiguity in complex organizations, Administrative Science Quarterly, 150-163.

Rotter, J. B. (1966). Generalized expectancies for internal versus external control of reinforcement. Psychological Monographs: general and Applied, 80 (Whole No 609).

Rotter, J. B. (1975). Some problems and misconceptions related to the construct of internal versus external control of reinforcement. Journal of Consulting and Clinical Psychology, 43, 56-57.

Russell, J. A. (1980). A circumplex model of affect. Journal of Personality and Social Psychology, 39 (6), 1161-1178.

Sale, J. E. M. Lohfield, L. H. \& Brazil, K. (2002). Revisiting the quantitativequalitative debate: Implications for mixed method research. Quality and Quantity, 36, 43-53.

Sales, S. M. (1969). Organizational role as a risk factor in coronary disease. Administrative Science Quarterly, 14 (3), 325-336.

Sales, S. M. (1970). Some effects of role overload and role underload. Organizational Behavior and Human Performance, 5 (6), 592-608. 
Santos, S. R., Carroll, C. A. Cox, K. S. Teasley, S. L. Simon, S. D. Bainbridge, L., Cunningham, M. \& Ott, L. (2003). Baby boomer nurses bearing the burden of care: A four site study of stress, strain, and coping for inpatient registered nurses, Journal of Nursing Administration, 33, (4), 243-250.

Saucier, G. (1994). Mini-Markers: A brief version of goldberg's unipolar Big-Five markers, Journal of Personality Assessment, 63 (3) 506-516.

Schaubroek, J. S., Cotton, J. L. \& Jennings, K. R. (1989). Antecedeents and consequences of role stress: a covariance structure analysis, Journal of Organizational Behaviour, 10, 35-58.

Schonfeld, I. S. \& Farrell, E. (2009). Qualitative and quantitative methods in occupation-stress research. In A. M. Rosse, J. C. Quick and P. L. Perrewé (Eds.) Stress and Quality of Working Life, Charlotte, NC:Information Age publishing Inc.

Schuler, R. S., Aldag, R. J. \& Brief, A. P. (1977). Role conflict and ambiguity: A scale analysis, Organizational Behavior and Human Performance, 20 (1), 111-128.

Schultz, S., Wang, M. \& Olsen, D. A. (2009). Role overload and underload in relation to occupational stress and health, Stress and Health.

Schwarzer, R. (1998). Stress and coping from a social cognitive perspective, Annals of the New York Academy of Sciences, 851, 531-537.

Schwarzer, R. \& Taubert, S (2002). Tenacious goal pusuits and striving toward personal growth: Proactive coping. In E. Frydenberg (Ed.) Beyond coping: meeting goals, visions and challenge (pp 19-35), London: Oxford University Press.

Scott W. (1998). Organizations: Rational, Natural, and Open System. New Jersey: Prentice Hall.

Seyle, H. (1956). The Stress of Life, New York: McGraw-Hill.

Seyle, H. (1976). The Stress of Life (Rev). New York: McGraw-Hill.

Shield, M. \& Wilkins, K. (2005). Findings from the 2005 national survey of the work and health of nurses. Statistics Canada: Health Canada and Canadian Institute for Health Information. Located at: http://www.hc-sc.gc.ca/hcs-sss/pubs/nursinfirm/2005-nurse-infirm/index-eng.php Retrieved Jun 4th, 2013.

Sieber, S. D. (1974). Toward a theory of role accumulation, American Sociological Review, 39, 567-578. 
Siegall, M. (2000). Putting the stress back into role stress: Improving the measurement of role conflict and role ambiguity. Journal of Managerial Psychology, $15,(5), 427-435$.

Siggelkow, N. (2007). Persuasion with case studies. Academy of Management Journal, 50, 22-24.

Skinner, E. A., Edge K., Altman, J. \& Sherwood, H. (2003). Searching for the structure of coping: A review and critique of category systems for classifying ways of coping. Psychological Bulletin, 129 (2) 216-269.

Snyder, C. R. \& Dinoff, B. L. (1999). Coping: Where have you been? In C. R. Snyder (Ed.) Coping, (pp 3 - 19). New York: Oxford University Press.

Snyder, C. R. \& Pulvers, K. M. (2001). Dr. Seuss, the coping machine, and "Oh the places you'll go". In C. R. Snyder (Eds.) Coping with Stress: Effective People and Processes, (pp. 3 - 29). New York: Oxford University Press.

Somech, A. \& Drach-Zahavy, A. (2007). Strategies for coping with work family conflict: The distinctive relationships of gender role ideology. Journal of Occupational health Psychology, 12 (1), 1-29.

Stanton, A., Twillman, R.,Cameron, C. , Collins, C. , Kirk, S. \& Sworowski, L. (2000). Emotionally expressive coping predicts psychological and physical adjustment to breast cancer. Journal of Consulting and Clinical Psychology, 68 (5) 875-882.

Suls, J., David, J. P. \& Harvey, J. H. (1996). Personality and coping: Three generations of research. Journal of Personality, 64, 711-735.

Stephens, N. \& Gwinner, K. P. (1998). Why don't some people complain? A cognitive-emotion process model of consumer complaint behavior. Journal of the Academy of Marketing Science, 26 (3), 172-189.

Stryker, S. \& Statham, A. (1985). Handbook of social psychology, New York: Random House.

Taylor, S. E., Helgeson, V. S., Reed, G. M. \& Skokan, L. A. (1991). Self-generated feelings of control and adjustment to physical illness. Journal of Social Issues, 57, 91109.

Tennen, H., Affleck, G. Armeli, S. \& Carney, M. A. (2000). A daily process approach to coping: Linking theory, research, and practice. American Psychologist, 55 (6) 626636.

Terry, D. J. (1991). Coping resources and situational appraisals as predictors of coping behaviour. Personality and Individual Differences, 12 (10), 1031-1047. 
Thoits, (1991) On merging identity theory and stress research, Social Psychology Quarterly, 54, 101-112.

Thoits, P. A. (1995a). Stress, Coping, and Social Support Processes: Where Are We? What Next? Journal of Health and Social Behavior, 35, (3) 53-79.

Thoits, P. A. (1995b) Social psychology: The interplay between sociology and psychology. Social Forces, 73 (4), 1231-1243.

Thompson, C.A., Beauvais, L. L. \& Allen, T. D. (2006). Work and family from an industrial/organizational psychology perspective. In M. Pitt-Catsouphes, E. E. Kossek and S. Sweet (Eds.) The Work and Family Handbook, (pp. 283-308), Mahwah, New Jersey: Lawrence Erlbaum Associates.

Thompson, C. A. \& Blau, G. (1993). Moving beyond traditional predictors of job involvement: Exploring the impact of work-family conflict and overload, Journal of Social Behaviour and Personality, 8 (4), 635-646.

Thompson, C. A., Poelmans, S. A. Y., Allen, T. D. \& Andreassi, J. K. (2007). On the importance of coping: A model and new directions for research on work and family. In P. L Perrewé and D. C. Ganster (Eds.) Research in Occupational Stress and Well Being Volume 6: Exploring the Work and Non-Work Interface, (pp 73-114), Oxford: Elsevier JAI Press.

Tomaka, J. Blascovitch, J., Kelsey, R. M. \& Leiten, C. L. (1993). Subjective, physiological, and behavioural, effects of threat and challenge appraisal, Journal of Personality and Social Psychology, 65, 248-260.

Tomaka, J. \& Blascovitch, J (1994). Effects of justice beliefs on cognitive appraisal of and subjective, psychological, and behavioural responses to potential stress, Journal of Personality and Social Psychology, 67 (4), 732-740.

Tomaka, J., Blascovitch, J., Kibler, J. \& Ernst, J. M. (1997). Cognitive and physiological antecedents of threat and challenge appraisals. Journal of Personality and Social Psychology, 65, 248-260.

Tracy, L \& Johnson, T. W. (1981). What do the role conflict and role ambiguity scales measure? Journal of Applied Psychology, 66, 464-469.

Tubre, T. C. \& Collins, J. M. (2000).Jackson and Schuler (1985) revisited: A metaanalysis of the relationships betwen role ambiguity role conflict and job performance, Journal of Management, 26 (1), 155-169. 
Van Sell, M., Brief, A. P. \& Schiler, R. S. (1981). Role conflict and role ambiguity: integration of the literature and directions for future research, Human relations, 3443.

Van Steenbergen, E. F., Ellermers, N., Haslam, A. \& Urlings, F. (2008). There is nothing either good or bad but thinking makes it so: Informational support and cognitive appraisal of the work-family interface. Journal of Occupational and Organizational Psychology, 81, 349-367.

Virick M., Lilly, J. D. \& Casper, W. J. (2007). Doing more with less: An analysis of work life balance among layoff survivors, Career Development International, 12 (5), 463-480.

Voydanoff, P. (1988). Work role characteristics, family structure demands and work/family conflict, Journal of Marriage and Family, 50 (3), 749-761.

Wallace, J. E. (1999). Work-to-nonwork conflict among married male and female lawyers. Journal of Organizational Behavior, 20, 797-816.

Watson, D. \& Clark, L. A. (1992). On traits and temperament: General and specific factors of emotional experience and their relation to the five-factor model. Journal of Personality, 60 (2), 441-476.

Watson, D. \& Hubbard, B. (1996). Adaptational style and dispositional structure: Coping in the context of the five factor model, Journal of Personality, 64, 737-774.

Webster, J. \& Watson, R. T. (2002). Analyzing the past to prepare for the future: writing a literature review. MIS Quarterly, 26 (2), xiii-xxiii.

Weick, K. E. (2007). The generative properties of richness. Academy of Management Journal, 50 (1), 14-19.

Wells, Y. \& de Vaus, D. (2003). Work stress and caregiver stress. In C. L. Peterson (Ed.) Work Stress: Studies of the Context, Content and Outcomes of Stress, (pp 113138), Amityville, New York: Baywood Publishing Company Inc.

Wickham, M. \& Parker, M. (2007). Reconceptualising organisational role theory for contemporary organisational contexts. Journal of managerial Psychology, 22 (5) 440464.

Yauch, C. A. \& Steudal, H. J. (2003). Complementary use of qualitative and quantitative cultural assessment methods, Organizational Research Methods, 6(4), 465-481.

Yin, R. K. (2009). Applications of case study research, Thousand Oaks, California: Sage Inc. 
Yin, R. K. (2009). Case Study Research: Design and Methods, Thousand Oaks, California: Sage Inc.

Yin, R. K. (2011). Qualitative research from start to finish. New York: Guilford Press.

Yip, B., Rowlinson, S. \& Oi Ling Sui (2008). Coping strategies as moderators in the relationship between role overload and burnout. Construction Management and Economics, 26, 869-880. 


\section{Appendix A Interview Protocol}

\section{Contact Script - Telephone Interview}

May I speak to

My name is from the Sprott School of Business, Carleton University.

Earlier this year you completed a questionnaire as part of a study entitled "Role Overload in Canada: Causes, Consequences and Effective Coping Strategies." You may recall that when you completed the questionnaire you provided your name and telephone number indicating that you would be willing to be interviewed further about work and family issues. That is why I am calling you today.

Do you have a moment to talk? If not, then when would be a better time for me to call back?

Date and time for call back.

On call back, mention the study again and then begin here/OR if they do not want a call back continue with this material:

** On the basis of your demographics, your name was selected as a possible participant in a more detailed study on the way that people manage their work, non-work and family roles.

Your participation in this phase of the study will involve a telephone interview that will take approximately 45 to 60 minutes of your time. The interview asks a series of questions which were designed to help us:

- Understand why people feel overloaded through what is referred to as the appraisal process,

- Understand how being overloaded affects them, their families and their situation at work, and how people behave when they are overloaded (actions as well as emotions)

- Identify effective and ineffective ways to cope or deal with role overload. **

Are you still interested in participating in the interview?

If yes: Thank you very much for agreeing to participate. We would like to schedule your interview at a time that is convenient to you for sometime in the next week. Could you suggest a few times that you would be available and willing to participate in the interview. We will contact you within the next few days with the date and time for the telephone interview (or confirm now if you can). How would you like to be contacted? 
Telephone (at what number)

E-mail (what is your e-mail address)

If you are calling back for an interview appointment,

Thanks again for agreeing to take part in this follow up study. Just as a reminder, this study is a follow up to the previous study in which you participated, and looks specifically at the demands that people face, how and why people become overloaded, and what can be done to reduce role overload. The interview today will be audio taped to support the analysis of the data. Audio files will be stored on a password protected PC and will be deleted once the data has been coded and analysed. Names and other identifying information will not be recorded or shared with the funder WSIB, You will only be identified by a code number assigned to you. Are you happy to go ahead?

If they are unhappy, try to identify and allay their concerns. If you cannot, end the interview and thank them for their time.

If they ask if the interview can be conducted in French, explain that you personally cannot speak French well enough and someone else will call them back to do arrange the interview and thank them.

Turn on the recorder and ask again.

For the benefit of the recording can I ask you to confirm that you are happy to proceed with the interview.

\section{Part I Background Information}

The first couple of questions give us some background information on you and what you do at work. This information will give us the context to help us interpret the data.

1. BRIEFLY describe your role at work.

2. How long have you been in this role?

3. What percentage of your typical work day is spent dealing with patients? $\%$

\section{Part II: Role Overload Scenarios}


In this section of the interview we are going to focus on your experiences of role overload. Role overload is defined as having too much to do and not enough time to do it. In order to understand how people respond and cope with overload I would like you to think about a situation where you felt overloaded - you just had too much to do, and not enough time. This situation can be at work OR at home, whatever comes to mind. I'm going to walk you through the experience by asking you a number of questions to help me understand the situation, how it unfolded, how you felt about the situation, what you did to try and reduce the overload, and how you now evaluate your actions.

\section{Situation A:}

1. So - to start. For this situation from the past six months where you felt overloaded you had too much to do, not enough time to do it in ( or time crunched) - can you give this event a title?

In the instance that you just described:

2. What made you feel overloaded? What was it about the situation itself?

3.a) What were your initial thoughts about the situation?

b) Did you feel that the situation was harmful to you, or potentially threatening in any way, or challenging to you (if none of these - how would you describe it)?

i) In what way?

4. What was your over riding feeling about the situation? (prompt: Excited, worried, overwhelmed, guilty, fearful, annoyed.....)

5. I want to identify the potential consequences of the situation if it were not resolved successfully:

a) Firstly, what did it mean for you? (Prompt: to your goals or values)

b) How was it likely to affect your

organisation/boss/colleagues/patients/family members, and what did THAT

mean to you? (if it is work situation ask them about their organization, their boss, their colleagues, a patient --- if it is a family situation, ask about their family members) 
6. This question is to determine how well prepared you felt to respond to this situation?

a) To what extent did you believe the situation could be resolved successfully?

b) Describe your initial thoughts about how you could overcome the situation?

7. What was the single most important factor that made this situation potentially overwhelming or stressful for you?

8. I now want to ask what you did to cope with the situation?

a) What actions did you take?

b) How did your actions differ from your initial plan?

c) Why these actions?

PROMPT: Did they work or not? In what way?

d) What actions did others take?

PROMPT: Did they work or not? In what way?

d) What did you do to deal with the emotional aspects of the situation? Why this?

Did this work or not?

9. a) How did the situation end ?

b) Was this what you expected to happen or not?

i) Why do you say this?

ii) How did you feel at the end of the situation? (or, if the situation is ongoing, how do you feel about it now) 
Situation B: Thanks for sharing this example with me. It was very useful. You described an overload situation that:

you were able to overcome so that you felt comfortable with at the end of the day

OR

was really(or somewhat) stressful for you and that resulted in your feeling (or somewhat) overwhelmed and you were not happy with how it turned out.

Can you describe a situation within the past six months that went the other way. Where despite feeling overloaded:

you were able to resolve the situation in the end in a very favourable way

OR

the situation resulted in you feeling very overwhelmed - and you were not happy with how it had turned out.

Again, this could be at work or home. I am going to ask you the same set of questions as before, but in this case I want you to focus on this second situation.

1. First, For this second situation where you felt overloaded - can you give this event a title?

In the instance that you just described:

2. What made you feel overloaded? What was it about the situation itself?

3.a) What were your initial thoughts about the situation?

b) Did you feel that the situation was harmful to you, or potentially threatening in any way, or challenging to you (if none of these - how would you describe it)?

i) In what way

4. What was your over riding feeling about the situation?? (prompt: Excited, worried, overwhelmed, guilty, angry.....)

5. I want to identify the potential consequences of the situation if it were not resolved successfully:

a) Firstly, what did it mean for you? (Prompt: to your goals and/or values) 
b) How was it likely to affect your organisation/boss/colleagues/patents/family members and what did THAT mean to you? (if it is work situation ask them about their organization, their boss, their colleagues, a patient --- if it is a family situation, ask about their family members)

6. This question is to determine how well prepared you felt to respond?

a) To what extent did you believe the situation could be resolved successfully?

b) Describe your initial thoughts about how you could overcome the situation?

7. What was the single most important factor that made this situation potentially overwhelming or stressful for you?

8. I now want to ask what you did to cope with the situation?

a)What actions did you take?

b) How did your strategies /actions differ from your initial plan?

c) Why these actions?

PROMPT: Did they work or not? In what way?

d) What actions did others take?

PROMPT : Did they work or not? In what way?

e) What did you do to deal with the emotional aspects of the situation?

PROMPT: Why this? Did this work or not?

9. a) How did the situation end?

b) Was this what you expected to happen or not?

i) Why do you say this?

c) How did you feel at the end of the situation? 


\section{Part III. Personality Characteristics}

The next set of questions is to help us see how personality influences how individuals deal with overload

I am going to read out a number of statements about personality traits that may or may not apply to you. Using a scale of 1 to 7 , where 1 indicates 'Disagree strongly'; and 7 indicates 'Agree strongly', please specify the extent to which you agree or disagree with the statements. Some statements describe two characteristics and you should rate the extent to which both of them apply to you, even if one characteristic applies more strongly than the other.

1. In groups I have belonged to, I am the most enthusiastic and often take the leadership role.

2. I am not interested in any other people's problems and pride myself on my shrewdness in handling people.

3. I like order and plan ahead carefully for major events in my work and home life.

4. I often get upset or worry about things that might go wrong.

5. I am open to new experiences and have a vivid imagination.

6. Many people think of me as quieter and somewhat reserved.

7. I go out of my way to help or sympathise with people if I can.

8. I never seem to be able to get organised and sometimes make a mess of things.

9. It takes a lot to make me mad and I rarely feel fed up.

10. I am not interested in, and sometimes have difficulty understanding abstract ideas. 


\section{Appendix B: Full Data Sets for Results by Respondent Stress}

\section{Level and Episode Assessment}

Table B-1 Event types triggering role overload

\begin{tabular}{|c|c|c|c|c|c|c|c|c|c|c|}
\hline \multirow{5}{*}{ Event Type } & \multicolumn{8}{|c|}{ Hospital Workers - Stress Level } & & \\
\hline & \multicolumn{4}{|c|}{ Low $(N=10)$} & \multicolumn{4}{|c|}{ High $(\mathbf{N}=10)$} & & \\
\hline & \multicolumn{2}{|c|}{$\begin{array}{c}\text { Not- } \\
\text { Overwhelming } \\
\text { Episodes } \\
(\mathbf{n}=\mathbf{1 0})\end{array}$} & \multicolumn{2}{|c|}{$\begin{array}{l}\text { Overwhelming } \\
\text { Episodes } \\
(\mathbf{n}=10)\end{array}$} & \multicolumn{2}{|c|}{$\begin{array}{c}\text { Not- } \\
\text { Overwhelming } \\
\text { Episodes }(n=10)\end{array}$} & \multicolumn{2}{|c|}{$\begin{array}{l}\text { Overwhelming } \\
\text { Episodes } \\
(\mathbf{n}=10)\end{array}$} & \multicolumn{2}{|c|}{$\begin{array}{c}\text { Total } \\
\text { Episodes } \\
(\mathbf{n}=\mathbf{4 0})\end{array}$} \\
\hline & \multicolumn{10}{|c|}{ \# and \% of episodes for which this task was chosen } \\
\hline & $\#$ & $\%$ & \# & $\%$ & $\#$ & $\%$ & $\#$ & $\%$ & $\#$ & $\%$ \\
\hline Everyday demands & 2 & 20 & 2 & 20 & 6 & 60 & 3 & 30 & 13 & 32.5 \\
\hline Personal or Family Crisis & 3 & 30 & 3 & 30 & 3 & 30 & 2 & 20 & 11 & 27.5 \\
\hline $\begin{array}{r}\text { High Profile Corporate } \\
\text { Task }\end{array}$ & 2 & 20 & 1 & 10 & 1 & 10 & 2 & 20 & 6 & 15 \\
\hline Interpersonal Issues & 0 & 0 & 2 & 10 & 1 & 0 & 2 & 20 & 5 & 12.5 \\
\hline Staffing Shortages & 1 & 10 & 2 & 10 & 0 & 10 & 1 & 0 & 4 & 10 \\
\hline Extracurricular Task & 2 & 10 & 1 & 10 & 0 & 0 & 0 & 0 & 3 & 7.5 \\
\hline Client-Patient Issues & 2 & 0 & 1 & 10 & 0 & 0 & 0 & 10 & 3 & 7.5 \\
\hline Other & 0 & 0 & 1 & 0 & 1 & 0 & 2 & 10 & 4 & 10 \\
\hline
\end{tabular}

Table B-2: Other role factors

\begin{tabular}{|c|c|c|c|c|c|c|c|c|c|c|}
\hline & \multicolumn{8}{|c|}{ Hospital Workers - Stress Level } & & \\
\hline & \multicolumn{4}{|c|}{$\operatorname{Low}(N=10)$} & \multicolumn{4}{|c|}{$\operatorname{High}(\mathrm{N}=10)$} & & \\
\hline \multirow[b]{3}{*}{ Role Factors } & $\begin{array}{r}\text { Ove } \\
\text { E }\end{array}$ & $\begin{array}{l}\text { lming } \\
\text { des } \\
\text { 0) }\end{array}$ & \multicolumn{2}{|c|}{$\begin{array}{l}\text { Overwhelming } \\
\text { Episodes } \\
(\mathbf{n}=10)\end{array}$} & \multicolumn{2}{|c|}{$\begin{array}{c}\text { Not } \\
\text { Overwhelming } \\
\text { Episodes } \\
(\mathbf{n}=10)\end{array}$} & \multicolumn{2}{|c|}{$\begin{array}{l}\text { Overwhelming } \\
\text { Episodes } \\
(\mathbf{n}=10)\end{array}$} & \multicolumn{2}{|c|}{$\begin{array}{c}\text { Total } \\
\text { Episodes } \\
(\mathbf{n}=\mathbf{4 0})\end{array}$} \\
\hline & \multicolumn{10}{|c|}{ \# \& \% of episodes in which this characteristic was mentioned } \\
\hline & \# & $\%$ & $\#$ & $\%$ & $\#$ & $\%$ & \# & $\%$ & $\#$ & $\%$ \\
\hline Role Domain & 10 & 100 & 10 & 100 & 10 & 100 & 10 & 100 & 40 & 100 \\
\hline Work Situation & 7 & 70 & 7 & 70 & 5 & 50 & 6 & 60 & 25 & 62.5 \\
\hline Family - Home Situation & 1 & 10 & 3 & 30 & 3 & 30 & 1 & 10 & 8 & 20 \\
\hline Work and Home & 2 & 20 & 0 & 0 & 2 & 20 & 3 & 30 & 7 & $\mathbf{1 7 . 5}$ \\
\hline Other Role Stresses & 5 & 50 & 3 & 30 & 3 & 30 & 4 & 40 & 15 & 37.5 \\
\hline Intra-Role Conflict & 3 & 30 & 2 & 20 & 1 & 10 & 1 & 10 & 7 & 17.5 \\
\hline Work Family Conflict & 2 & 20 & 1 & 10 & 2 & 20 & 2 & 20 & 7 & 17.5 \\
\hline
\end{tabular}


Table B-3: Role overload situation properties

\begin{tabular}{|c|c|c|c|c|c|c|c|c|c|c|}
\hline \multirow{4}{*}{$\begin{array}{c}\text { Role Overload } \\
\text { Situation Properties }\end{array}$} & \multicolumn{8}{|c|}{ Hospital Workers - Stress Level } & & \\
\hline & \multicolumn{4}{|c|}{ Low $(N=10)$} & \multicolumn{4}{|c|}{$\operatorname{High}(\mathrm{N}=10)$} & & \\
\hline & \multicolumn{2}{|c|}{$\begin{array}{c}\text { Not- } \\
\text { Overwhelming } \\
\text { Episodes }(n=10)\end{array}$} & \multicolumn{2}{|c|}{$\begin{array}{l}\text { Overwhelming } \\
\text { Episodes } \\
(\mathbf{n}=\mathbf{1 0})\end{array}$} & \multicolumn{2}{|c|}{$\begin{array}{c}\text { No } \\
\text { Overwhelming } \\
\text { Episodes } \\
(\mathbf{n}=\mathbf{1 0}) \\
\end{array}$} & \multicolumn{2}{|c|}{$\begin{array}{l}\text { Overwhelming } \\
\text { Episodes } \\
(\mathbf{n}=\mathbf{1 0})\end{array}$} & \multicolumn{2}{|c|}{$\begin{array}{c}\text { Total } \\
\text { Episodes } \\
(n=40)\end{array}$} \\
\hline & \multicolumn{10}{|c|}{ \# \& \% of episodes in which this characteristic was mentioned } \\
\hline & \# & $\%$ & \# & $\%$ & $\#$ & $\%$ & \# & $\%$ & \# & $\%$ \\
\hline Time Pressures & 7 & 70 & 5 & 50 & 3 & 30 & 5 & 50 & 20 & 50 \\
\hline Volume of demands & 5 & 50 & 3 & 30 & 4 & 40 & 4 & 40 & 16 & 40 \\
\hline Ambiguity & 3 & 30 & 2 & 20 & 4 & 40 & 3 & 30 & 12 & 30 \\
\hline Familiarity & 2 & 20 & 1 & 10 & 3 & 30 & 4 & 40 & 10 & 25 \\
\hline Duration & 0 & 0 & 3 & 30 & 1 & 10 & 4 & 40 & 8 & 20 \\
\hline $\begin{array}{r}\text { Synchronous demands of } \\
\text { a different nature }\end{array}$ & 2 & 20 & 1 & 10 & 1 & 10 & 4 & 40 & 8 & 20 \\
\hline $\begin{array}{r}\text { Synchronous additions to } \\
\text { existing workload }\end{array}$ & 3 & 30 & 2 & 20 & 3 & 30 & 0 & 0 & 8 & 20 \\
\hline $\begin{array}{r}\text { Synchronicity of } \\
\text { simultaneous demands }\end{array}$ & 1 & 10 & 2 & 20 & 1 & 10 & 1 & 10 & 5 & 12.5 \\
\hline Regularity & 1 & 10 & 0 & 0 & 2 & 20 & 2 & 20 & 5 & 12.5 \\
\hline Complexity & 3 & 30 & 0 & 0 & 1 & 10 & 0 & 0 & 4 & 10 \\
\hline Novelty & 1 & 10 & 0 & 0 & 1 & 10 & 2 & 20 & 4 & 10 \\
\hline Event uncertainty & 0 & 10 & 0 & 0 & 0 & 0 & 4 & 40 & 4 & 10 \\
\hline Predictability & 0 & 10 & 1 & 10 & 0 & 20 & 2 & 20 & 3 & 7.5 \\
\hline
\end{tabular}


Table B-4: Person factors

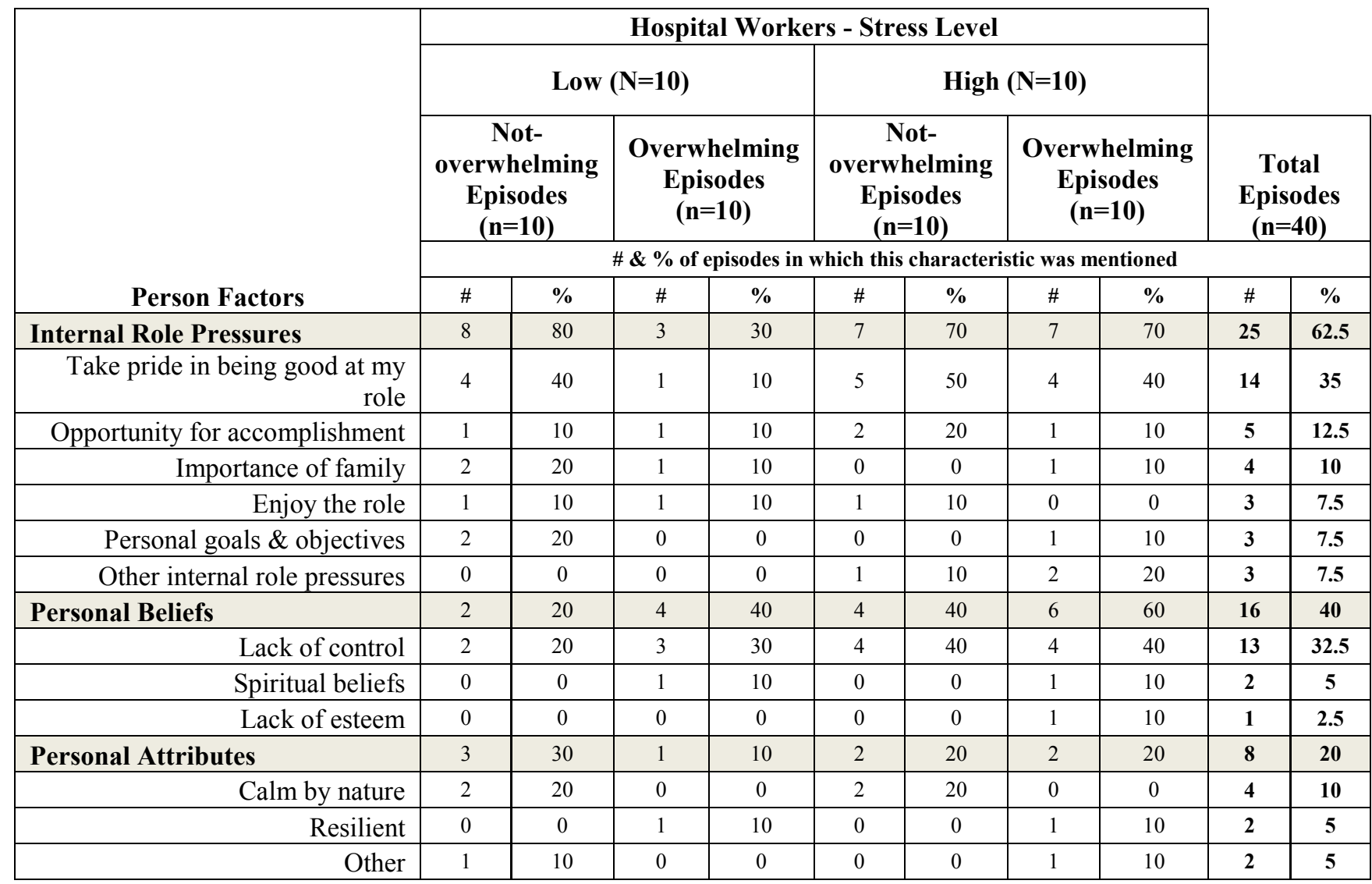


Table B-5: Primary appraisal - Harm, threat, or challenge

\begin{tabular}{|c|c|c|c|c|c|c|c|c|c|c|}
\hline \multirow{5}{*}{$\begin{array}{c}\text { Type of } \\
\text { Appraisal } \\
\end{array}$} & \multicolumn{8}{|c|}{ Hospital Workers - Stress Level } & & \\
\hline & \multicolumn{4}{|c|}{ Low $(N=10)$} & \multicolumn{4}{|c|}{ High $(\mathbf{N}=10)$} & & \\
\hline & \multicolumn{2}{|c|}{$\begin{array}{c}\text { Not } \\
\text { overwhelming } \\
\text { Episodes }(\mathbf{n}=\mathbf{1 0}) \\
\end{array}$} & \multicolumn{2}{|c|}{$\begin{array}{c}\text { Overwhelming } \\
\text { Episodes } \\
(\mathbf{n}=\mathbf{1 0}) \\
\end{array}$} & \multicolumn{2}{|c|}{$\begin{array}{c}\text { Not } \\
\text { overwhelming } \\
\text { Episodes }(\mathbf{n}=\mathbf{1 0}) \\
\end{array}$} & \multicolumn{2}{|c|}{$\begin{array}{l}\text { Overwhelming } \\
\text { Episodes } \\
(\mathbf{n}=\mathbf{1 0}) \\
\end{array}$} & \multicolumn{2}{|c|}{$\begin{array}{c}\text { Total Episodes } \\
(n=40)\end{array}$} \\
\hline & \multicolumn{10}{|c|}{$\# \& \%$ of episodes in which this characteristic was mentioned } \\
\hline & \# & $\%$ & \# & $\%$ & \# & $\%$ & \# & $\%$ & \# & $\%$ \\
\hline Challenge & 9 & 90 & 6 & 60 & 7 & 70 & 5 & 50 & 27 & 67.5 \\
\hline Threat & 5 & 50 & 6 & 60 & 4 & 40 & 7 & 70 & 22 & 55 \\
\hline Harm & 0 & 0 & 3 & 30 & 0 & 0 & 5 & 50 & 8 & 20 \\
\hline
\end{tabular}

Table B-6: What's at stake?

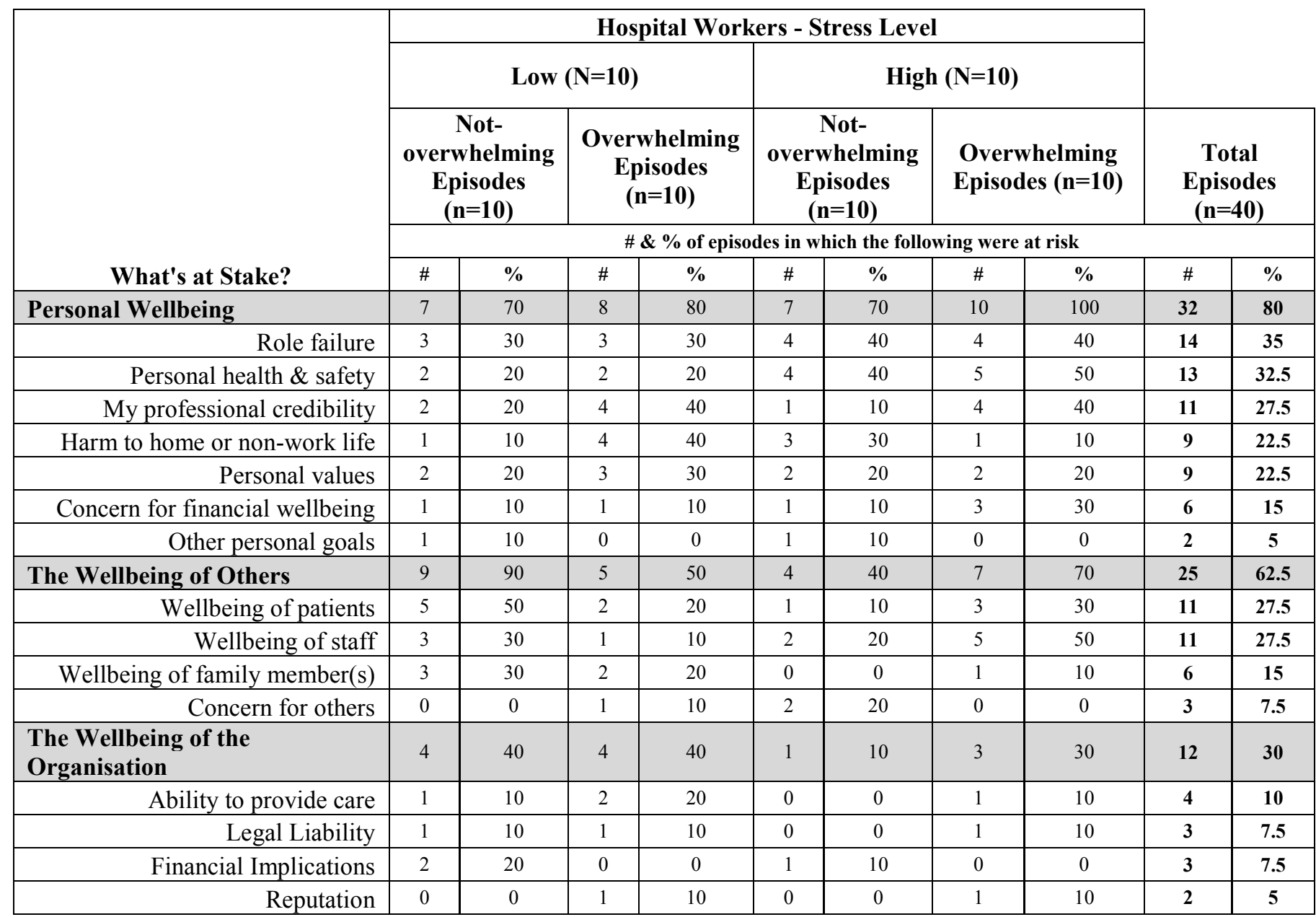


Table B-7: Secondary appraisal - What can I do about it?

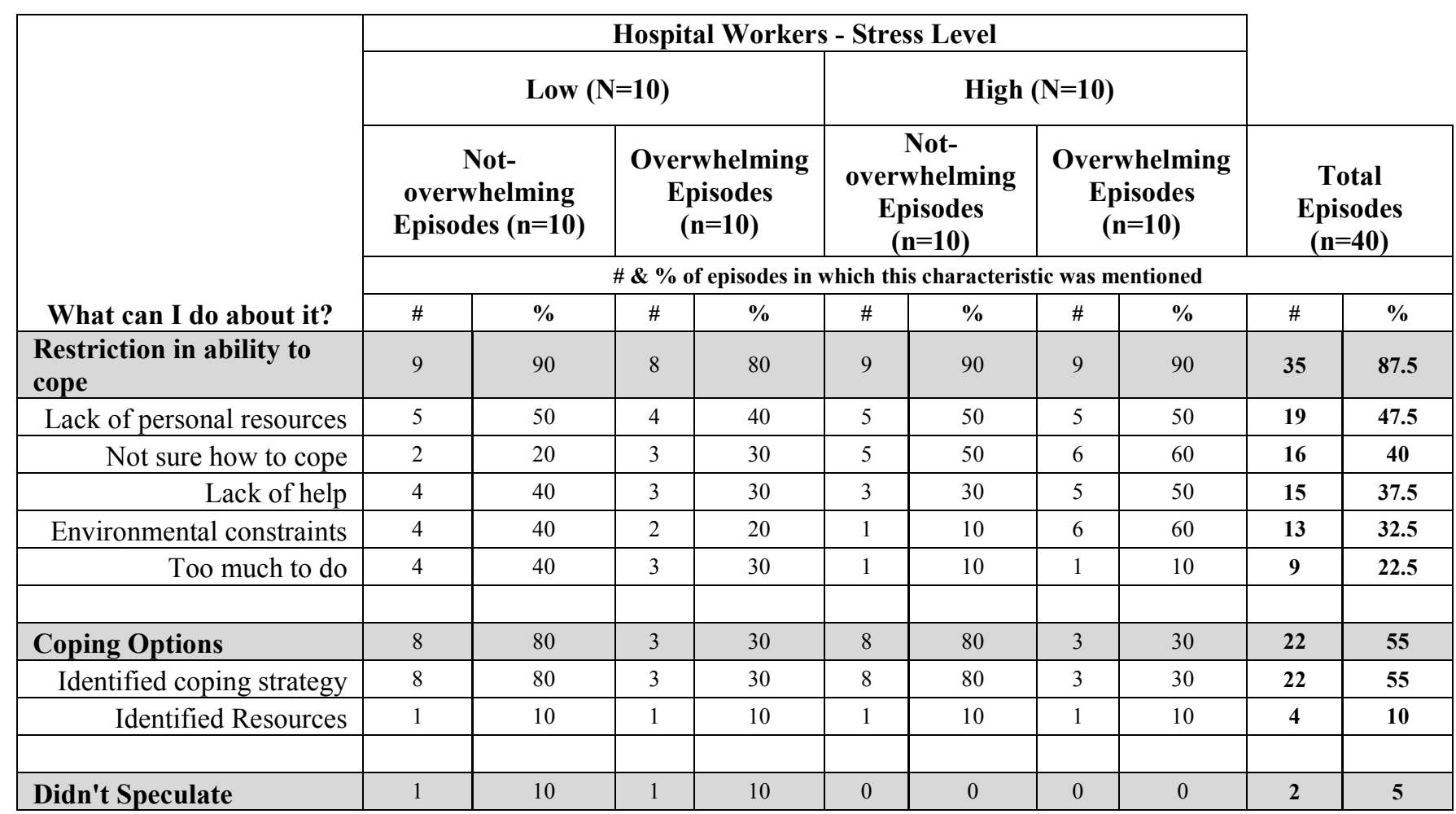

Table B-8: Coping prospects

\begin{tabular}{|c|c|c|c|c|c|c|c|c|c|c|}
\hline \multirow{5}{*}{$\begin{array}{l}\text { To what } \\
\text { extent did you believe } \\
\text { the situation could be } \\
\text { resolved successfully? }\end{array}$} & \multicolumn{8}{|c|}{ Hospital Workers - Stress Level } & & \\
\hline & \multicolumn{4}{|c|}{ Low $(\mathrm{N}=10)$} & \multicolumn{4}{|c|}{ High $(\mathrm{N}=10)$} & & \\
\hline & \multicolumn{2}{|c|}{$\begin{array}{c}\text { Not } \\
\text { overwhelming } \\
\text { Episodes } \\
(\mathbf{n}=\mathbf{1 0}) \\
\end{array}$} & \multicolumn{2}{|c|}{$\begin{array}{l}\text { Overwhelming } \\
\text { Episodes } \\
(\mathbf{n}=\mathbf{1 0})\end{array}$} & \multicolumn{2}{|c|}{$\begin{array}{c}\text { Not } \\
\text { overwhelming } \\
\text { Episodes } \\
(\mathbf{n}=\mathbf{1 0}) \\
\end{array}$} & \multicolumn{2}{|c|}{$\begin{array}{l}\text { Overwhelming } \\
\text { Episodes } \\
(\mathbf{n}=10)\end{array}$} & \multicolumn{2}{|c|}{$\begin{array}{c}\text { Total } \\
\text { Episodes } \\
(n=40)\end{array}$} \\
\hline & \multicolumn{10}{|c|}{ \# \& \% of episodes in which this characteristic was mentioned } \\
\hline & \# & $\%$ & \# & $\%$ & \# & $\%$ & \# & $\%$ & \# & $\%$ \\
\hline Confident of Success & 5 & 50 & 4 & 40 & 5 & 50 & 2 & 20 & 16 & 40 \\
\hline Hopeful for success & 4 & 40 & 1 & 10 & 4 & 40 & 4 & 40 & 13 & 32.5 \\
\hline Not confident of success & 1 & 10 & 4 & 40 & 1 & 10 & 4 & 40 & 10 & 25 \\
\hline
\end{tabular}


Table B-9: Appraisal outcomes - Immediate feelings

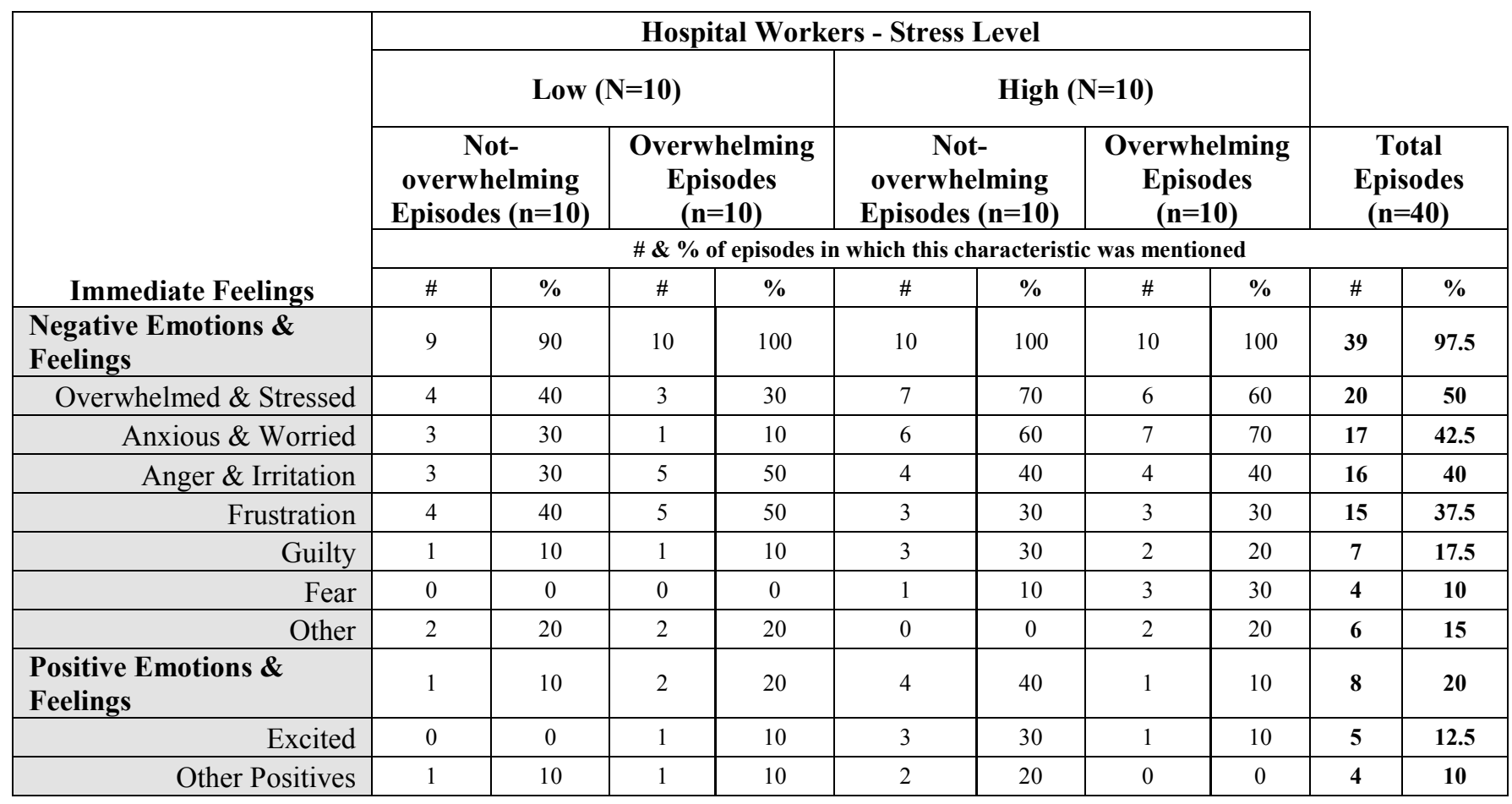




\section{Table B-10: Role Overload Coping Strategies}

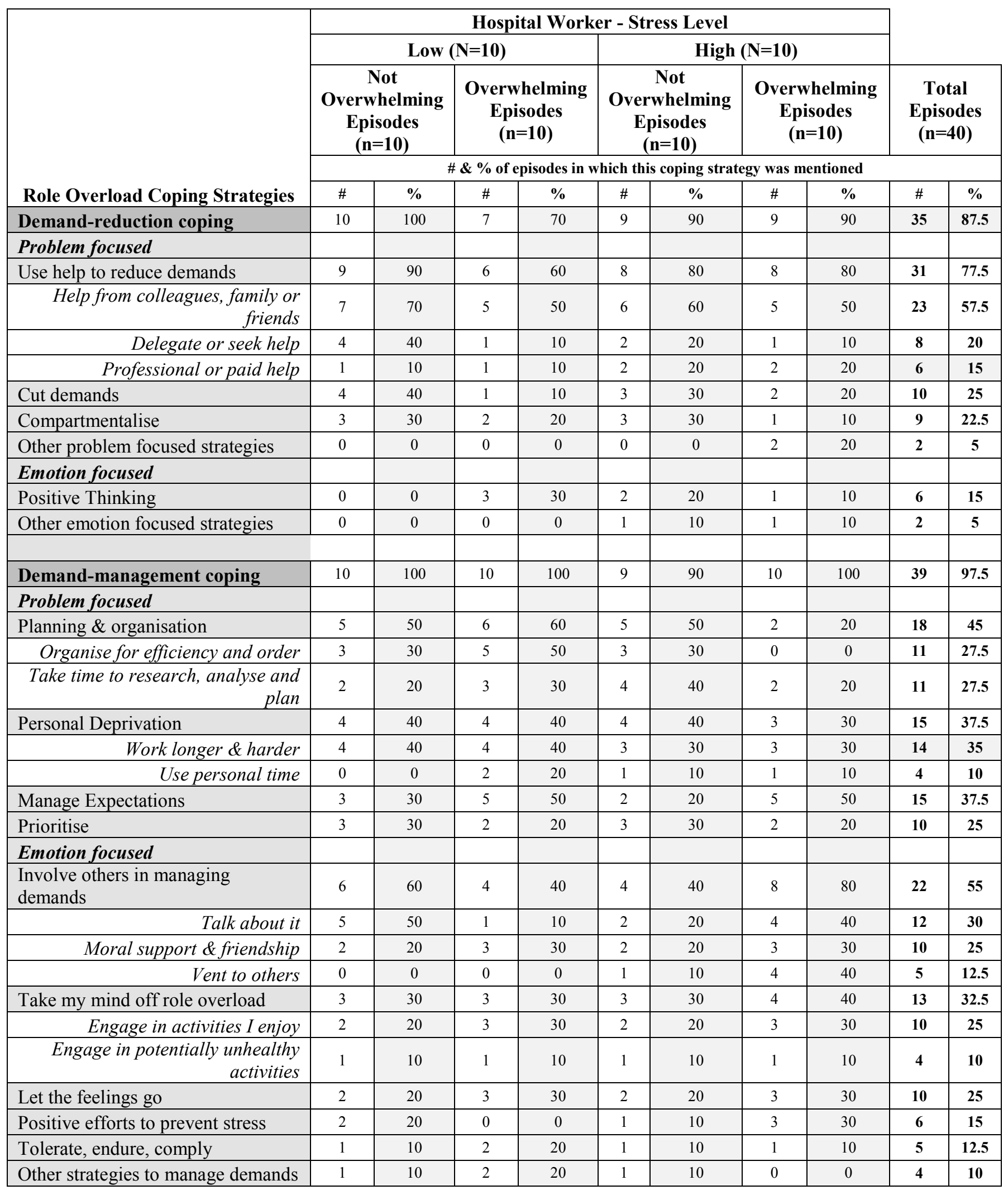


Table B-11: Personal outcomes \& role overload resolution

\begin{tabular}{|c|c|c|c|c|c|c|c|c|c|c|}
\hline \multirow[b]{5}{*}{ Role Overload Outcomes } & \multicolumn{8}{|c|}{ Hospital Workers - Stress Level } & & \\
\hline & \multicolumn{4}{|c|}{ Low $(\mathrm{N}=10)$} & \multicolumn{4}{|c|}{ High $(\mathbf{N}=10)$} & & \\
\hline & \multicolumn{2}{|c|}{$\begin{array}{c}\text { Not } \\
\text { Overwhelming } \\
\text { Episodes } \\
(\mathbf{n}=\mathbf{1 0}) \\
\end{array}$} & \multicolumn{2}{|c|}{$\begin{array}{l}\text { Overwhelming } \\
\text { Episodes } \\
(\mathbf{n}=\mathbf{1 0})\end{array}$} & \multicolumn{2}{|c|}{$\begin{array}{c}\text { Not } \\
\text { Overwhelming } \\
\text { Episodes }(n=10)\end{array}$} & \multicolumn{2}{|c|}{$\begin{array}{l}\text { Overwhelming } \\
\text { Episodes } \\
(\mathbf{n}=10)\end{array}$} & \multicolumn{2}{|c|}{$\begin{array}{c}\text { Total } \\
\text { Episodes } \\
(n=40) \\
\end{array}$} \\
\hline & \multicolumn{10}{|c|}{ \# \& \% of episodes which resulted in this outcome } \\
\hline & \# & $\%$ & $\#$ & $\%$ & \# & $\%$ & \# & $\%$ & \# & $\%$ \\
\hline $\begin{array}{l}\text { Positive personal } \\
\text { outcomes }\end{array}$ & 9 & 90 & 3 & 30 & 9 & 90 & 4 & 40 & 25 & 62.5 \\
\hline $\begin{array}{l}\text { Positive Emotions \& } \\
\text { Feelings }\end{array}$ & 9 & 70 & 3 & 20 & 9 & 90 & 4 & 50 & 25 & 62.5 \\
\hline Relieved & 5 & 50 & 2 & 20 & 5 & 50 & 0 & 10 & 12 & 30 \\
\hline Good, & 2 & 20 & 0 & 0 & 5 & 50 & 1 & 40 & 8 & 20 \\
\hline Satisfied & 1 & 10 & 0 & 0 & 1 & 10 & 3 & 30 & 5 & 12.5 \\
\hline $\begin{array}{l}\text { Other low arousal positive } \\
\text { emotions \& feelings }\end{array}$ & 1 & 10 & 0 & 0 & 1 & 30 & 1 & 20 & 3 & 7.5 \\
\hline $\begin{array}{r}\text { Other high arousal positive } \\
\text { emotions \& feelings }\end{array}$ & 0 & & 1 & & 2 & & 0 & & 3 & 7.5 \\
\hline Personal Gains & 3 & 30 & 1 & 10 & 3 & 30 & 0 & 0 & 7 & 17.5 \\
\hline Personal gains & 3 & 30 & 1 & 10 & 3 & 30 & 0 & 0 & 7 & 17.5 \\
\hline $\begin{array}{l}\text { Negative personal } \\
\text { outcomes }\end{array}$ & 3 & 30 & 8 & 80 & 3 & 30 & 8 & 80 & 22 & 55 \\
\hline $\begin{array}{l}\text { Negative Emotions \& } \\
\text { Feelings }\end{array}$ & 2 & 20 & 8 & 80 & 3 & 30 & 8 & 80 & 21 & 52.5 \\
\hline Low arousal: & & & & & & & & & & \\
\hline Tired \& exhausted & 0 & 0 & 3 & 30 & 3 & 30 & 2 & 20 & 8 & 20 \\
\hline $\begin{array}{r}\text { Other low arousal negative } \\
\text { emotions \& feelings }\end{array}$ & 0 & 0 & 5 & 50 & 1 & 10 & 3 & 30 & 9 & 22.5 \\
\hline High arousal: & & & & & & & & & & \\
\hline Stressed \& overwhelmed & 1 & 10 & 1 & 10 & 0 & 0 & 4 & 40 & 6 & 15 \\
\hline $\begin{array}{r}\text { Other high arousal negative } \\
\text { emotions \& feelings }\end{array}$ & 1 & 10 & 3 & 30 & 0 & 0 & 3 & 30 & 7 & $\mathbf{1 7 . 5}$ \\
\hline Negative Consequences & 1 & 10 & 1 & 10 & 1 & 10 & 4 & 40 & 7 & 17.5 \\
\hline Spillover & 1 & 10 & 0 & 0 & 1 & 10 & 2 & 20 & 4 & 10 \\
\hline Other Negative Effects & 0 & 0 & 1 & 10 & 0 & 0 & 3 & 30 & 4 & 10 \\
\hline $\begin{array}{l}\text { Role Overload } \\
\text { Resolution }\end{array}$ & $\#$ & $\%$ & $\#$ & $\%$ & $\#$ & $\%$ & $\#$ & $\%$ & $\#$ & $\%$ \\
\hline Successful & 4 & 40 & 3 & 30 & 7 & 70 & 3 & 30 & 17 & 42.5 \\
\hline Mixed resolution & 5 & 50 & 4 & 40 & 3 & 30 & 5 & 50 & 17 & 42.5 \\
\hline Unresolved & 1 & 10 & 3 & 30 & 0 & 0 & 2 & 20 & 6 & 15 \\
\hline
\end{tabular}

